Cochrane Database of Systematic Reviews

\title{
A realist review of which advocacy interventions work for which abused women under what circumstances (Review)
}

Rivas C, Vigurs C, Cameron J, Yeo L

Rivas C, Vigurs C, Cameron J, Yeo L.

A realist review of which advocacy interventions work for which abused women under what circumstances.

Cochrane Database of Systematic Reviews 2019, Issue 6. Art. No.: CD013135.

DOI: 10.1002/14651858.CD013135.pub2.

www.cochranelibrary.com

A realist review of which advocacy interventions work for which abused women under what circumstances 
TABLE OF CONTENTS

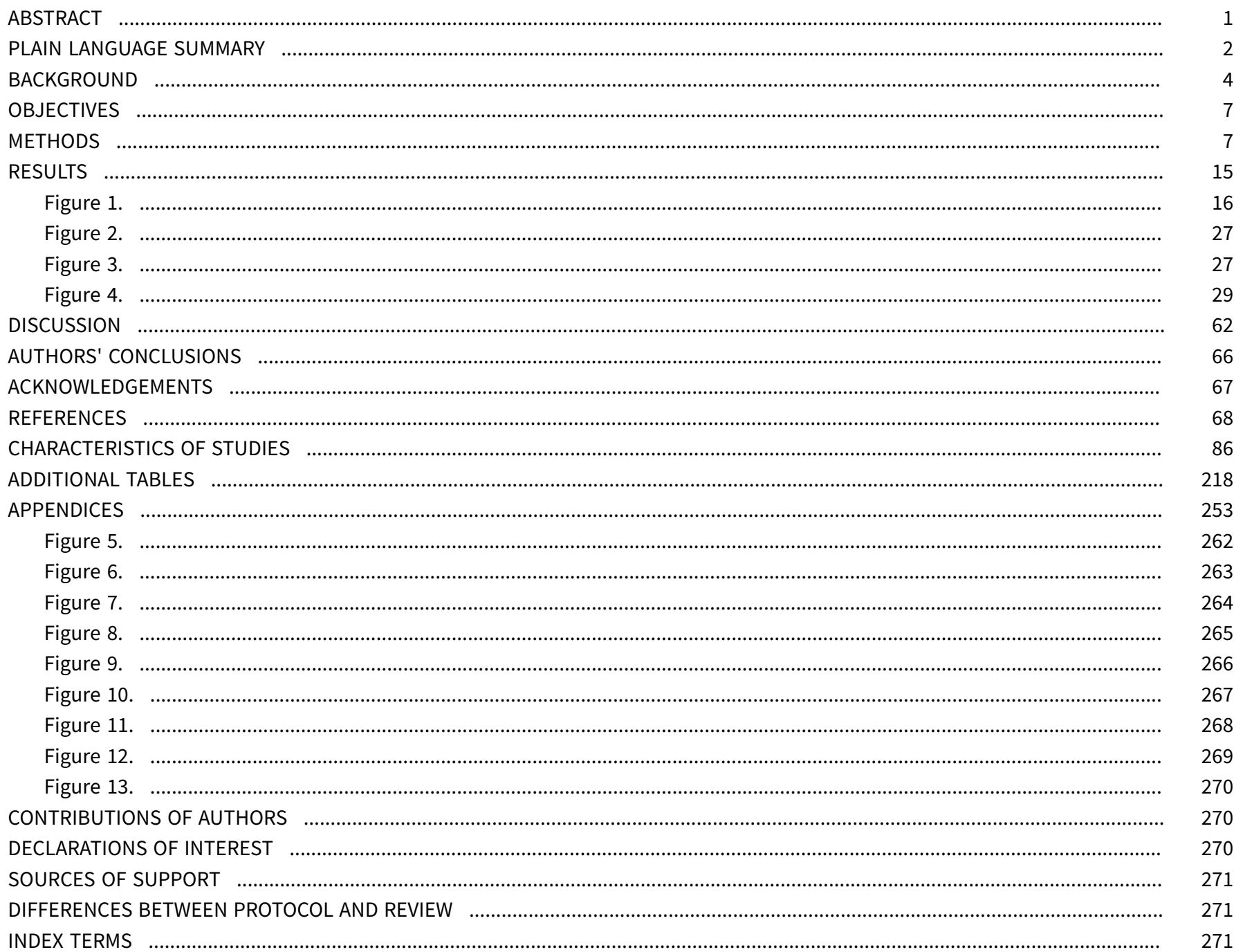


[Prototype Review]

\section{A realist review of which advocacy interventions work for which abused women under what circumstances}

Carol Rivas ${ }^{1}$, Carol Vigurs ${ }^{1}$, Jacqui Cameron 2,3 , Lucia Yeo ${ }^{1,4}$

1Department of Social Science, UCL Institute of Education, University College London, London, UK. 2Department of Social Work, Melbourne School of Health Sciences, The University of Melbourne, Melbourne, Australia. ${ }^{3}$ National Centre for Education and Training on Addiction (NCETA), Finders University, Adelaide, Australia. ${ }^{4}$ Department of Child Development, KK Women's and Children's Hospital, Singapore, Singapore

Contact: Carol Rivas, Department of Social Science, UCL Institute of Education, University College London, 18 Woburn Square, London, WC1H 0NR, UK.C.Rivas@ucl.ac.uk.

Editorial group: Cochrane Developmental, Psychosocial and Learning Problems Group.

Publication status and date: New, published in Issue 6, 2019.

Citation: Rivas C, Vigurs C, Cameron J, Yeo L. A realist review of which advocacy interventions work for which abused women under what circumstances. Cochrane Database of Systematic Reviews 2019, Issue 6. Art. No.: CD013135. DOI: 10.1002/14651858.CD013135.pub2.

Copyright $(2019$ The Cochrane Collaboration. Published by John Wiley \& Sons, Ltd.

\section{A B S T R A C T}

\section{Background}

Intimate partner abuse (including coercive control, physical, sexual, economic, emotional and economic abuse) is common worldwide. Advocacy may help women who are in, or have left, an abusive intimate relationship, to stop or reduce repeat victimisation and overcome consequences of the abuse. Advocacy primarily involves education, safety planning support and increasing access to different services. It may be stand-alone or part of other services and interventions, and may be provided within healthcare, criminal justice, social, government or specialist domestic violence services. We focus on the abuse of women, as interventions for abused men require different considerations.

\section{Objectives}

To assess advocacy interventions for intimate partner abuse in women, in terms of which interventions work for whom, why and in what circumstances.

\section{Search methods}

In January 2019 we searched CENTRAL, MEDLINE, 12 other databases, two trials registers and two relevant websites. The search had three phases: scoping of articles to identify candidate theories; iterative recursive search for studies to explore and fill gaps in these theories; and systematic search for studies to test, confirm or refute our explanatory theory.

\section{Selection criteria}

Empirical studies of any advocacy or multi-component intervention including advocacy, intended for women aged 15 years and over who were experiencing or had experienced any form of intimate partner abuse, or of advocates delivering such interventions, or experiences of women who were receiving or had received such an intervention. Partner abuse encompasses coercive control in the absence of physical abuse. For theory development, we included studies that did not strictly fit our original criteria but provided information useful for theory development.

\section{Data collection and analysis}

Four review authors independently extracted data, with double assessment of $10 \%$ of the data, and assessed risk of bias and quality of the evidence. We adopted RAMESES (Realist and meta-narrative evidence syntheses: evolving standards) standards for reporting results. We applied a realist approach to the analysis. 


\section{Main results}

We included 98 studies (147 articles). There were 88 core studies: 37 focused on advocates (4 survey-based, 3 instrument development, 30 qualitative focus) and seven on abused women ( 6 qualitative studies, 1 survey); 44 were experimental intervention studies (some including qualitative evaluations). Ten further studies ( 3 randomised controlled trials (RCTs), 1 intervention process evaluation, 1 qualitative study, 2 mixed methods studies, 2 surveys of women, and 1 mixed methods study of women and staff) did not fit the original criteria but added useful information, as befitting a realist approach. Two studies are awaiting classification and three are ongoing.

Advocacy interventions varied considerably in contact hours, profession delivering and setting.

We constructed a conceptual model from six essential principles based on context-mechanism-outcome (CMO) patterns.

We have moderate and high confidence in evidence for the importance of considering both women's vulnerabilities and intersectionalities and the trade-offs of abuse-related decisions in the contexts of individual women's lives. Decisions should consider the risks to the woman's safety from the abuse. Whether actions resulting from advocacy increase or decrease abuse depends on contextual factors (e.g. severity and type of abuse), and the outcomes the particular advocacy intervention is designed to address (e.g. increasing successful court orders versus decreasing depression).

We have low confidence in evidence regarding the significance of physical dependencies, being pregnant or having children. There were links between setting (high confidence), and potentially also theoretical underpinnings of interventions, type, duration and intensity of advocacy, advocate discipline and outcomes (moderate and low confidence). A good therapeutic alliance was important (high confidence); this alliance might be improved when advocates are matched with abused women on ethnicity or abuse experience, exercise cultural humility, and remove structural barriers to resource access by marginalised women. We identified significant challenges for advocates in inter-organisational working, vicarious traumatisation, and lack of clarity on how much support to give a woman (moderate and high confidence). To work effectively, advocates need ongoing training, role clarity, access to resources, and peer and institutional support.

Our provisional model highlights the complex way that factors combine and interact for effective advocacy. We confirmed the core ingredients of advocacy according to both women and advocates, supported by studies and theoretical considerations: education and information on abuse; rights and resources; active referral and liaising with other services; risk assessment and safety planning. We were unable to confirm the impact of complexity of the intervention (low confidence). Our low confidence in the evidence was driven mostly by a lack of relevant studies, rather than poor-quality studies, despite the size of the review.

\section{Authors' conclusions}

Results confirm the core ingredients of advocacy and suggest its use rests on sound theoretical underpinnings. We determined the elements of a good therapeutic alliance and how it might be improved, with a need for particular considerations of the factors affecting marginalised women. Women's goals from advocacy should be considered in the contexts of their personal lives. Women's safety was not necessarily at greatest risk from staying with the abuser. Potentially, if undertaken for long enough, advocacy should benefit an abused woman in terms of at least one outcome providing the goals are matched to each woman's needs. Some outcomes may take months to be determined. Where abuse is severe, some interventions may increase abuse. Advocates have a challenging role and must be supported emotionally, through provision of resources and through professional training, by organisations and peers.

Future research should consider the different principles identified in this review, and study outcomes should be considered in relation to the mechanisms and contexts elucidated. More longitudinal evidence is needed. Single-subject research designs may help determine exactly when effect no longer increases, to determine the duration of longitudinal work, which will likely differ for vulnerable and marginalised women. Further work is needed to ascertain how to tailor advocacy interventions to cultural variations and rural and resource-poor settings. The methods used in the included studies may, in some cases, limit the applicability and completeness of the data reported. Economic analyses are required to ascertain if resources devoted to advocacy interventions are cost-effective in healthcare and community settings.

\section{PLAIN LANGUAGE SUMMARY}

\section{A review of which advocacy interventions work to help abused women, and under what circumstances, and which women are likely to benefit}

\section{Background}

Partner abuse (domestic violence) is common worldwide. It includes coercive control, physical, sexual, economic, emotional and/or economic abuse. Trained people, known as advocates, can actively support abused women to make safety plans, cope with and take steps to reduce the abuse, and access community resources. This is known as advocacy. Advocacy can take many forms.

\section{Review question}

We wished to understand which advocacy interventions work to help abused women, under what circumstances, and which women may benefit. This is called a realist approach. It assumes that we often develop a superficial knowledge of how something happens (e.g. how 
advocacy works), based on simple measurements and observations. A realist approach tries to understand what is really happening at a deeper level by exploring different effects in different conditions.

\section{Methods}

We searched scientific literature worldwide up to January 2019 for any relevant studies.

\section{Key findings}

We found 98 studies from 15 countries. Of the 88 core studies, 37 asked advocates about their views and experiences and seven asked abused women about advocacy (two of these also asked staff). The other 44 core studies helped us understand the way advocacy works and how effective it is. We included 10 additional studies that did not fit the original criteria but added useful information, as befitting a realist approach. Of these, three were randomised controlled trials (RCTs; a type of experiment in which participants are randomly allocated to two or more interventions), one was an intervention process evaluation, one was a qualitative (e.g. focus groups, interviews) study, two studies used mixed methods (a combination of qualitative and quantitative research) to explore women's experiences, two were surveys of women, and one was a mixed methods study of women and staff. We were unable to obtain the full texts of two studies that we thought might be core and three further relevant studies are still ongoing.

Advocacy interventions varied considerably in duration, participating staff (e.g. nurses, psychologists, social workers), and setting (e.g. healthcare settings, domestic violence refuges or shelters).

In the studies, women and advocates agreed that the following were all important parts of advocacy: education and information on abuse and on women's rights and sources of help (resources); active referral to, and help in accessing other services; assessment of risk of repeat abuse; and safety planning to avoid it. Trust in the advocate is important and more likely when the advocate and the woman share an ethnic background or the advocate was also abused. Advocates must help women consider their best options, depending on things like ethnicity, immigration status, where they live, the severity and type of the abuse experienced and finances. There are trade-offs when making decisions to reduce the abuse and women's safety was not necessarily at greatest risk from staying with the abuser. Advocacy could potentially have some benefits for abused women, if undertaken for long enough, but its goals need to match each woman's needs. It may take months to have an effect. Two studies (one involving the police and one in an antenatal clinic) found that where abuse is severe to start with, some interventions may possibly prompt the abuser to increase the abuse. Advocates want to help women and can get stressed if they do not feel helpful enough, so they need support from organisations and other advocates, including repeat training, debriefs, and funding to do their job well.

\section{Quality of the evidence}

Our confidence in the key findings varied between moderate and high. However, some themes (the effect on outcomes of women being physically dependent on their abuser, being pregnant or having children) were less well supported by evidence and further, good-quality research is needed to confirm findings. Researchers should be careful when choosing how to measure abuse so that measures have more meaning for advocates and abused women, thus increasing the usefulness of future reviews. Further evidence from studies where participants are followed up for years would be helpful. More economic analyses are needed to establish if current advocacy interventions are the best way of spending money for abused women. 


\section{B A C K G R O U N D}

For a glossary of terms used in this review, please see Appendix 1.

\section{Description of the condition}

\section{Agency definitions}

We used the 2013 England and Wales Government definition of intimate partner abuse as, "any incident or pattern of incidents of controlling, coercive, threatening behaviour, violence or abuse between those aged 16 or over who are, or have been, intimate partners or family members regardless of gender or sexuality. The abuse can encompass, but is not limited to: psychological, physical, sexual, financial, emotional [abuse]" (Home Office 2013). It is important to consider definitions in context when examining specific articles. Partner abuse is recognised as a basic human rights issue (Ingram 2005), but local, national and international institutions have historically disagreed on how to characterise and define it. This is largely due to differences in the ways that agencies work, the outcomes they seek, and the role they play in society (Rivas 2010). The type and efficacy of coherent, multi-agency responses from the judiciary, healthcare and community support services has been affected by different definitions of partner abuse (Felson 2005; Rivas 2010). This is reflected in a lack of consensus on the terms used to describe the phenomenon, which are sometimes employed interchangeably and sometimes used specifically to highlight conceptual nuances or overlap (e.g. domestic violence, battered woman, spouse/wife abuse or (intimate) partner abuse). Overall we have reflected the language used in the different articles and therefore frequently refer to intimate partner abuse as domestic violence, this being the most commonly used term.

As an example of the issues in interpreting studies through the years, in 2005 an England and Wales cross-government agreement established a core definition of domestic violence to facilitate co-ordination between services. However, it was criticised for considering abuse in terms of discrete acts, or single 'incidents', a result of the various government agencies' reactive responses to partner abuse (Rivas 2010). It also ignored the chronicity of partner abuse and the impact on associated social and psychological issues (Feder 2006). Yet these were included in the earlier, international, World Health Organization's (WHO) public health definition of "intimate partner violence" (IPV) as "behaviour within an intimate relationship that causes physical, sexual or psychological harm, including acts of physical aggression, sexual coercion, psychological abuse and controlling behaviours by both current and former spouses and partners" (Heise 2002; WHO 2013a). Therefore, in 2013, the UK Government updated its definition for England and Wales to that which we have used. The Spanish, New Zealand and Hong Kong definitions are similar (Bialy 2018; Department of Justice of Hong Kong 2015; Parliamentary Counsel Office of New Zealand 2018).

There are various variations of this definition worldwide. The official definitions in Scotland and the USA do not include family members and have no age restrictions (Bell 2017; US Office on Violence Against Women 2018). The Northern Ireland, Canadian, Sri Lankan and Mexican definitions do not include patterns of incidents (Bell 2017; Government of Canada 2017; Goonetilleke 2015; Government of Mexico 2016); the Sri Lanka definition also references only physical and emotional abuse (Goonetilleke 2015). In Sweden, the Netherlands and Taiwan the official definition is gender neutral (Nybergh 2013; Netherlands Institute for Human Rights 2018; Lee 2015); the Taiwan definition does not include repeat incidents or coercion. Three countries considered in this review have particularly narrow definitions, Turkey (Uzun 2015), Peru (Women's Rights Division of Human Rights Watch 2000), and Japan (Asia-Japan Women's Resource Center (AJWRC) 2007), as exemplified by the Japanese version: "violence toward the body by one spouse (illegal attacks toward the body threatening the other's life or physical condition. . .) or words and deeds by one spouse that cause comparable psychological or physical harm to the other" (Asia-Japan Women's Resource Center (AJWRC) 2007).

The WHO's definition emphasises the effect of intimate partner abuse while most other definitions emphasise the intent. But there have been moves in some countries to change this. For example, section 76 of the Serious Crime Act ('s 76') of England and Wales makes "coercive or controlling behaviour" that has a "serious effect" on the victim a criminal offence. The provision came into force in England and Wales on 29 December 2015. The Australian definition (of family violence, including partner violence), like the WHO definition, explicitly considers both coercion and effect as "violent, threatening or other behaviour by a person that coerces or controls a member of the person's family, or causes the family member to be fearful" (Phillips 2014; Signorelli 2012).

\section{Review definition}

Since the definition that a study of partner abuse adopts will affect the study in various ways, from its design and participant inclusion criteria to its outcomes and interpretations, this review aims to be broad in its definition and so simply defines intimate partner abuse as the abuse of a woman by a male or female partner who currently is, or formerly was, in an intimate relationship with her. We have not excluded abuse perpetrated by ex-partners from the review. A woman is often at greatest risk when she is preparing to leave or has just left her partner (Brownridge 2006; Wilson 1993), and women often return to an abusive partner several times before leaving for good (Campbell 2002; Campbell 2004; Holt 2015; Mullen 1999; Shalaunsky 1999). This decision, therefore, is reflective of women's actual situations but also practical because it would be impossible for cross-sectional studies at least to determine whether separation from a partner is truly final. Intimate partner abuse perpetrated against male partners or ex-partners also occurs, but is excluded from this review because the outcomes and risks are thought to differ by gender (Henwood 2000; Nybergh 2013; Roe 2010).

We have considered all forms of intimate partner abuse, including physical violence (ranging from slaps, punches and kicks to lifechanging physical injuries or homicide), sexual violence (such as rape, sexual assault and sexual abuse or forced participation in sexual acts), emotionally abusive behaviours (such as stalking; surveillance; intimidation and threats of abuse; involvement of children; prohibition of a woman being away from her partner, leaving the home without a chaperone, or socialising with family and friends; and ongoing belittlement or humiliation), economic control, economic exploitation or employment sabotage (such as preventing a woman from working, determining what work she can do or restricting activities within a work role, confiscating her earnings, restricting access to money or resources in-kind), and other controlling behaviours (Adams 2008; Watts 2002). 


\section{Prevalence of intimate partner abuse}

Worldwide, on average, $30 \%$ of women experience physical or sexual intimate partner abuse at some time in their adult (post 16year-old) lives (WHO 2013a). The 2018 Crime Survey for England and Wales reported a similar figure of 26\% (ONS 2018); this lifetime prevalence rate is echoed in statistics from the USA (Black 2011; NCIPC 2003). An estimated $15 \%$ to $71 \%$ of women experience partner abuse in 10 low- and middle-income countries (Garcia-Moreno 2006). Prevalence rates for psychological abuse are generally considered to be much higher but with variation between studies (Carney 2012). Economic abuse rates are similarly uncertain, though an Australian survey suggested a 15.7\% rate for women across age groups (Kutin 2017). In a review of prevalence rates, $4.5 \%$ of women have been said to experience forced sexual intercourse by a partner (Carney 2012).

\section{Economic burden of intimate partner abuse}

Women experiencing intimate partner abuse are frequent users of healthcare services and require a wide range of medical services that may be linked to the abuse (Campbell 2002; Davidson 2001; Plichta 2007). The economic cost of healthcare for abused women in the UK (including hospital, general practitioner and ambulance services as well as prescriptions) is estimated to be around GBP 1.73 billion (2008 figures; Walby 2009). There are also substantial costs to other public services (GBP 2.13 billion) as well as costs from lost economic output (GBP 1.92 billion) and human suffering (GBP 9.95 billion; Walby 2009). Studies from the USA also suggest considerable economic consequences for society from intimate partner abuse (Bonomi 2009; Jones 2006; NCIPC 2003), as do studies from Australia (Access Economics 2004; NCRVAWC 2009). The economic burden from childhood exposure to interparental partner abuse in the USA for people aged 20 to 64 years has been calculated as over USD 55 billion, or USD 50,000 per person (2016 figures; Holmes 2018). These are costs of healthcare spending, criminal behaviour and loss of labour market productivity (Holmes 2018).

Repeat victimisation accounts for $73 \%$ of all incidents of intimate partner abuse (WHO 2013a), and there is evidence of a positive, linear relationship between severity of abuse and the use of healthcare services (Koss 1991). Therefore, there has been wide interest in the development of interventions to stop repeat victimisation or at least reduce such recidivism or the severity of the abuse, and to help women to overcome the consequences of abuse.

\section{Description of the intervention}

In this review, we focused on one type of intervention aimed to stop or reduce repeat victimisation: advocacy programmes provided directly to women. This review considered all advocacy programmes. The features of advocacy interventions vary both within and between countries, since their precise aims and content, as well as implementation and delivery, depend partly on the setting in which they are delivered and the way they are funded (Rivas 2015), and partly on local historical developments of the advocate role (Feder 2006). Advocacy interventions may, for example, include: advice and support for abused women to access and use a specific service or resource or a range of these, including legal, housing, financial, refuges or shelters, emergency housing; informal counselling; guidance on safety planning; education on relationships; and support to improve the women's physical or psychological health. In some settings, advocates may also have a role in bringing about systemic change in the recognition of abused women by clinicians (WHO 2013b). Advocates may be trained lay mentors; community, healthcare or judicial service employees; or volunteers; and they may deliver advocacy for different time periods and at different intensities as well as in different ways and with different foci.

Advocacy usually aims to empower women and so tends to involve the advocate and woman working in partnership to help the woman set and achieve her own goals and understand and make sense of her situation as an expert in her own life (Campbell 1993). It is therefore an individualised, person-centred approach rather than a prescriptive or directive intervention. Advocacy may be offered as a stand-alone service or as part of a multi-component (and possibly multi-agency) intervention. On the one hand, this individualised and multi-access approach is likely to result in more efficacious advocacy, but on the other hand it makes effectiveness and mechanisms of effect challenging to evaluate, complicating comparisons in evidence syntheses (Rivas 2015). In this review, we compared and contrasted the different approaches to determine what type of contact works for whom, when and where.

Other interventions for women that we considered, but only if they were given within an advocacy intervention programme, included the provision of: psychological therapy; refuge or shelter care; and basic, first-line response by healthcare professionals, as recommended by the WHO, which may have included referral to other services (Bair-Merritt 2014; Colombini 2017; Feder 2013; García-Moreno 2015; Kalra 2017; WHO 2013b). We have also considered some articles that explored a co-ordinated response with advocacy as one component to develop our theory in phase 2 of the review. A co-ordinated response is important to reduce barriers to service access for abused women, and thence the chances of their disempowerment and repeat victimisation from their partner or from disconnected services themselves (Greeson 2013; Shorey 2014).

\section{How the intervention might work}

A realist review usually begins with a draft programme theory, that is described within the protocol, then "tested" and refined against the data included in the review (Wong 2013a). We did not develop a new draft programme theory for our protocol (Rivas 2018), though we described tentative ideas in the narrative text; we only began formal theory development during phase 1 of this review, which is a small departure from the normal process. To date (and Rivas 2015 is no exception), there has been no systematic evaluation of the underlying mechanisms (what makes it work) in stand-alone advocacy interventions, let alone advocacy combined with other interventions. However, there is some indication as to what these mechanisms may be and we began this review with these mechanisms in mind. Empowerment tends to be described as the mechanism that needs to operate for the active ingredients of advocacy interventions to activate. There are several theoretical frameworks that take empowerment as the mechanism for change at the societal, community and individual level. At a societal level, from feminist perspectives, the causes of intimate partner abuse stem from a social and cultural patriarchal ideology that allows men to control women through power and violence. The experience of intimate partner abuse is understood to be fundamentally disempowering (Vigurs 2016; Wood 2015). The proposed solution to this social and cultural problem, then, is one that aims to effect change through the empowerment of women at a social 
and cultural level. Typically in this approach, advocates can be seen to facilitate access to resources that are a woman's right, by connecting and liaising with community supports and services at a community or organisational level for a co-ordinated response to intimate partner abuse. Such resources might include housing, legal aid and welfare advice, for example.

Advocacy approaches that operate at the individual (micro) level to empower women tend to focus on helping her to change behaviours, such as safety planning and help-seeking behaviours. Such approaches will typically include or facilitate access to: cognitive behavioural therapies (CBT); counselling; or motivational interviewing. Not only do these approaches change a woman's ways of thinking, they may also alleviate depressive symptoms and improve mental health and well-being. Also at the individual level, strengths-based approaches aim to work with survivors of abuse to increase their knowledge, agency and self-efficacy. Strengthsoriented advocates empower the people they work with by setting future goals (Wood 2015), enabling women to access their own strengths and skills and apply them to current problems (Black 2003; Howe 2009).

Trauma-informed approaches for advocacy link the safety and resource needs of advocacy from both feminist and strengthsbased approaches. Trauma-informed advocacy involves an understanding of the ways in which trauma is overwhelming, and the ways in which this impacts on beliefs, cognition, memory, emotions and behaviours, constituting a normal response to the trauma rather than a mental health issue.

Trials, and therefore evidence syntheses of complex interventions such as advocacy, increasingly include components from different disciplines, such as psychotherapy (Campbell 2000; Monitor 2013). The coming together of different approaches with different theoretical underpinnings within advocacy programmes (typified by trauma-informed approaches) has not been formally explored to determine additive mechanisms of effect. However, it has been suggested that multi-component interventions that include advocacy are particularly effective because the advocacy addresses an abused woman's immediate needs, which then increases her receptiveness to other interventions, such as psychotherapy or childcare support (Rivas 2015).

\section{Why it is important to do this review}

We believe that this is the first realist review of advocacy interventions in intimate partner abuse, and will offer important insights into how advocacy interventions work. Systematic reviews, such as that by Rivas 2015, typically consider only randomised controlled trials (RCTs) and quasi-experimental studies, and the desired output is a meta-analysis (statistical summary) of the evidence on intervention effectiveness. This is appropriate to determine what works, given that these studies control for external factors to show a clear cause and effect relationship between the intervention itself and specific outcomes. However, meta-analysis of positive effect, negative effect and no effect, and heterogeneity between and within studies, essentially cancel each other out, to little or no effect overall, masking the finding that the intervention may work for some people and not others, or only under some conditions. A realist review draws on more diverse study types, as its aim, the desired output, is a 'programme theory'. This is an interpretative, narrative summary of how and why an intervention works in practice (the mechanisms) in different contexts (Pawson 2005; Pawson 2006; Wong 2012).

Importantly, this review was inspired by a 'what works' or effectiveness review of advocacy interventions for women who have experienced or are experiencing intimate partner abuse (Rivas 2015), and it will specifically inform the next update of that review and partner reviews on psychological interventions for these women. Our realist review will therefore have immediate impact on the developing evidence base for policy and practice.

Past versions of our Cochrane Review to evaluate the effectiveness of advocacy interventions to support abused women focused only on what works. Without contextualisation, and given the individualised approach to advocacy, as well as considerable variation in primary study and intervention design, these versions have been able to draw only limited conclusions and therefore weak recommendations. For example, our 2009 Cochrane Review, Ramsay 2009, excluded studies evaluating advocacy as an adjunct to another intervention if the control arm was not the other intervention alone. In the updated effectiveness review, Rivas 2015, which included 13 studies internationally, we made the decision to include trials where women in the intervention arm may have received advocacy plus some other form of intervention compared with no care or usual care. We felt that important information might otherwise be excluded from the review given the increasing number of trials evaluating advocacy within multi-component interventions. However, we were not able to make use of this information beyond a narrative synthesis of outcomes, as the heterogeneity of combinations made it unclear which components were leading to - or even diluting - effectiveness.

There has been no consideration of the interplay of the different components or mediating factors in such interventions, with components reviewed separately by authors. For example, TiradoMuñoz 2014 considered psychological interventions, and Rivas 2015 considered advocacy interventions. Where there was overlap from studies that combined both types of component, each review considered only those components of direct relevance to their focus. There were some 'grey areas'. In Rivas 2015, where necessary information was lacking in papers and not provided by the primary study authors on request, we had to decide whether terms such as 'counselling', 'supportive listening' or 'peer counselling' described facilitation of access to resources (which fitted our criteria for advocacy) or psychological therapy (which did not). The results of the realist review may better support such decisions, inasmuch as they have made it clearer which mechanisms lead to the effectiveness of advocacy. The realist review has also, we hope, provided more clarity in its approach of drawing more broadly on the literature where this can provide further information (e.g. on theoretical underpinnings).

A further issue that we wished to address is that the outcomes of a complex intervention (and indeed any intervention) are context-dependent, that is, they are affected by various individual, community and societal factors, from the ethnicity and socioeconomic status of the abused women involved to the role and training of the person delivering the advocacy, as well as the setting and the precise content of what is offered to whom (Pawson 2003; Pawson 2009). It is important, therefore, to consider not just what works, but where, in what circumstances, for whom and how, with more focus not only on the interaction of different components of an intervention but also on the contexts in which they are played

A realist review of which advocacy interventions work for which abused women under what circumstances (Review) 
out. These data are available from, and reported by, many of the effectiveness studies of advocacy interventions, but they have not been considered in terms of mechanisms of 'what works'. The realist review approach affords us the structure by which to do so. This realist review approach will also enable politicians, policy makers, practitioners and commissioners of services to understand existing interventions and identify those active ingredients that may be transferable to their local contexts. This is important in the context of funding cuts for intimate partner abuse-specific services (Towers 2012), which our review shows has a material impact on the effectiveness of intimate partner abuse interventions and the well-being of advocates. One further intention was for the inclusion criteria for the effectiveness review to be widened in future updates to include studies that fit with the list of identified 'core ingredients' described in the 'Summary of results' section in the Discussion.

\section{Special features of the realist review}

During the course of a realist review, authors develop, test and refine a programme theory, which ultimately comprises a set of $\mathrm{CMO}$ (context; mechanism; outcome) configurations. A programme theory is an explanation of how a particular programme is expected to work (Papoutsi 2017). To develop the theory, we consider different $\mathrm{CMO}$ configurations during the review. These CMO configurations combine: contexts (C) that are often the 'backdrop' of interventions (Jagosh 2014); mechanisms (M), which are not observed directly but ask what is it about programmes that make them work (Rycroft-Malone 2016; Westhorp 2018); outcomes $(0)$ of the intervention (planned or unplanned, visible or not, proximal or distal, intermediate or final) or strategies of the intervention (e.g. empowerment in the case of many advocacy interventions) (Jagosh 2012). As an example, in a context where an advocate has themselves been previously abused (C) sharing resources or strategies that they have personally found effective (M) helps a woman to believe that the strategy will be effective for her (0).

In developing theory, realist syntheses aim to balance comprehensiveness with theoretical saturation, so they may include fewer and different studies than an effectiveness review on the same topic. Ultimately, a realist review seeks to identify $\mathrm{CMO}$ configurations from the fuzzy reality of complex interventions, based on the expectation that although outcomes will vary in different contexts, there will be some patterning in CMO configurations (Jagosh 2012).

In this realist review we developed theory from qualitative literature as well as from effectiveness and more conceptual studies. The final theory and linked outcomes or impacts, therefore, adds to outcomes already included in the effectiveness review (Rivas 2015), and with more qualitative outcomes that are less commonly measured. This may make it challenging to use theory to evaluate previous effectiveness reviews but we have made recommendations for future studies. There is increasing acknowledgement of the need for more qualitative outcomes within effectiveness studies, and for the use of mixed methods. Additionally, and importantly, we are involved in development of a core outcome set (Williamson 2017), for intimate partner abuse, with a study design that foregrounds the qualitative experiences of abused women and their families. Therefore, any recommendations to include more qualitative outcomes in future effectiveness studies will feed into the development of this core outcome set.

\section{O B JECT IVES}

To assess advocacy interventions for intimate partner abuse in women, in terms of which interventions work for whom, why and in what circumstances.

\section{Research questions}

\section{Higher order question}

How do the key mechanisms associated with the delivery or use of complex interventions that include advocacy as a component interact with contextual influences, and with one another, to explain the successes, failures and partial successes of advocacy as an intervention?

\section{Second order questions}

- What are the active strategies used in advocacy interventions?

- What are the important contexts that determine whether the different mechanisms produce their intended outcomes?

- How are women's experiences, needs and desires met by advocacy services?

- What are women's expectations of advocates?

- How do organisational and system contexts influence implementation by advocates of advocacy interventions?

- Whose perspective matters and does this correspond to the active ingredients of advocacy?

These research questions are modifications of our original list, as specified in our protocol (Rivas 2018). We considered active ingredients, impact and outcomes in relation to qualitatively and quantitatively measured effects. We determined all answers (as much as possible) from existing evidence.

\section{METHODS}

Our realist synthesis follows the steps and procedures outlined in the 'Realist and meta-review evidence synthesis: evolving standards (RAMESES) publication standards for realist synthesis' and associated training materials (Wong 2013a; Wong 2013b; Wong 2017). We used the information management tool, EPPI-Reviewer 4 (Thomas 2010), to extract information systematically from each study. We employed the EMMIE realist evaluation framework to provide fields for data extraction (Johnson 2015). EMMIE codes the effectiveness of the intervention, the mechanism theorised to be at work, moderators that could affect the response to the intervention, implementation issues in practice, and any economic costs and benefits information for each study. The EMMIE framework has been developed from health and criminal justice frameworks to evaluate not only the effectiveness of interventions but also to capture information that explain variation of outcomes. This realist approach to evaluation includes assessing the necessary programme components and implementation issues that are of interest to the policy makers or practitioners who wish to implement such interventions.

The review had three phases and eight operational objectives as follows.

- Phase 1: scoping phase

- Gathered the full texts of all studies included in the existing effectiveness review of advocacy interventions for women experiencing intimate partner abuse (Rivas 2015), as well as

A realist review of which advocacy interventions work for which abused women under what circumstances (Review) 
all studies excluded in the final screening stage of Rivas 2015 on the basis of a single criterion mismatch and cited in its 'Characteristics of excluded studies' tables, or determined from review authors' unpublished records of exclusions.

- Gained familiarity with the data set by close reading.

\section{- Phase 2: theory generation}

- Produced a descriptive summary of the scoping phase data to summarise the kinds of research questions that have been asked, how these questions have been addressed (considering intervention details and all possible contextual factors), and the key findings or outcomes. We included any theories or mechanisms suggested by study authors.

- Identified additional, relevant publications that might contribute to explanatory theory building about what works for whom in what circumstances, on the basis of the first two objectives. This involved the development of a new search (new keywords; not reported here) and selection criteria, informed by our descriptive summary and consideration of the theories, mechanisms and contexts that may be linked to outcomes, rather than effectiveness per se. We added to this from further searches of the literature, as needed, developed from a reading of the new material and rechecks of papers already collated. This iterative and recursive approach is important since studies that may seem less relevant at first could end up providing good evidence on specific areas as the review progresses.

- Developed a realist analysis consisting of candidate theories linking context, mechanism and outcome.

- Phase 3: theory testing and refinement

- Undertook systematic data extraction of diverse qualitative, quantitative and mixed-methods studies identified from the searches to update the existing Cochrane Review (Rivas 2015), and revisited the studies considered in the first phases, to confirm, refute and refine our candidate theories.

- Summarised middle-range theories for which there is strong empirical evidence of what works for whom in what contexts.

- Clarified gaps in the knowledge base and made recommendations for further research.

We kept a full audit trail at all times, with lists of studies included and excluded (along with reasons for exclusion) at all stages.

In all searches in all phases, we excluded many papers at title and abstract screening, but we obtained the full text of all papers that possibly included sufficient detail for our review objectives. A second review author checked a $10 \%$ subsample of included and excluded papers for agreement. We resolved any differences through discussion and there was no need for adjudication by our stakeholder group. This group was comprised of members of an existing stakeholder group with whom we are working on the development of core outcome sets. The group included policymakers, academics, women who have experienced abuse, and advocacy providers, and they had oversight of the study findings. We consulted with the group by email or other remote means (e.g. videoconferencing). Although abused women participated in the group, we did not apply for ethical approval since these women were acting as consultants, rather than study participants. We did, however, maintain ethical principles. We asked this group to check emerging theory and comment on key decisions. Specifically, we asked them to:
- contribute to developing, refining, adjudicating between, and refuting emerging theories; and

- in phase 3 , comment on the credibility and validity of our explanatory theory and its coherence. This included providing advice about and considering any gaps in the theory, as determined from our test of the theory against studies identified in the phase 3 literature search.

This approach ensured our review would have meaning to the relevant stakeholders, including potential end users.

\section{Phase 1: scoping phase}

The aim of this search was to scope for information that could be used to inform the development of our emerging theory. It differed from some realist reviews in that it did not involve a new primary search, but rather:

- consideration of potentially relevant papers from those identified and included or rejected by Rivas 2015, as detailed in the published review and their unpublished records; and

- supplementary searches based on these papers.

As this is a realist review, based on a realist paradigm, we were interested in the nuanced detail as to why a particular intervention has been more or less successful at impacting on its target outcomes or behaviours and its 'critical ingredients' rather than in the actual effectiveness data (i.e. quantitative findings such as outcome scores and effect sizes). This means that we potentially could have excluded studies from the Rivas 2015 review if they did not contain information to contribute to the development or testing (or both) of our explanatory theory about why, how and when advocacy interventions for women experiencing partner abuse might work.

It also meant that some papers identified and subsequently excluded from the Rivas 2015 review were relevant to our realist review. For example, Rivas 2015 excluded some studies because advocacy was not a major component of the intervention, but these could still have contributed key information for the realist review. Others were excluded because they did not fit the study design inclusion criteria; Rivas 2015 only considered randomised controlled trials (RCTs) and before-and-after study designs, whereas we were inclusive in our use of different study types. We considered all such papers in the scoping phase of this realist review since the aim of this phase was to scope for candidate theories, mechanisms and contextual factors that may be linked to outcome, and that we could explore in more detail in phase 2 .

\section{Selecting papers for inclusion}

We began by considering the full text of all articles included in our Rivas 2015 review, or excluded in the final screening stage and cited in its Appendix or, if necessary (e.g. for qualitative studies), noted in their unpublished search records. Thus, we used the Rivas 2015 review as a starting point from which to develop a first iteration of a core list of papers that differed from the final list of papers included in that review. Once we developed this list, we supplemented it with:

- papers identified through citation chaining (through backward citation tracking of reference lists and Google Scholar forward citation tracking) of all papers that we judged as core to our realist question; 
- papers identified through the 'search similar citations' function on PubMed, after entering the title of each key paper in turn; and

- papers linked to the effectiveness studies, identified as part of an integrated mixed-methods study or as a 'sibling study' (e.g. qualitative, economic or process evaluations associated with specific effectiveness studies).

We then repeated the scoping phase process described in this section using the supplementary searches described directly above. We initially used a comprehensive sampling approach, because we did not deem an alternative approach, such as theoretical or extreme case sampling, to be necessary. This was because logistically we could manage the number of papers retrieved so there was no need to delimit them.

In all cases, CV reviewed the full-text papers and made a judgement as to whether the paper included sufficient descriptive detail or theoretical discussion, or both, to contribute to the explanatory theory, excluding those that did not. CR checked a $10 \%$ subsample of included and excluded papers for agreement. At the first check, both review authors expressed uncertainty as to whether to include studies that described advocacy but did not consider intimate partner abuse. After discussion, we agreed that non-consideration of intimate partner abuse should be an exclusion criterion, as the specific goals of other types of advocacy differed (though the goals were similar at the societal level it was not possible to translate these to our research question). The two review authors then reconsidered all papers ( $C V$ reviewed all papers while $C R$ reviewed a subset) and this time found no points of difference.

The review authors identified a number of existing explanatory theories connecting advocacy interventions to both intended and unanticipated outcomes during this phase, including advocacy as part of a multi-component intervention. This enabled us to draw up a preliminary list of possible theories, which we described under the 'How the intervention might work' section in our protocol (Rivas 2018), and then in the Background to this review. These were feminist, psychological, strengths-based and traumainformed approaches to empowerment, which we considered from prior experience to be especially relevant. We further added to this list as the review developed. This list was generated solely in relation to domestic abuse advocacy. While it would have been more in keeping with the realist approach to relate these more broadly to other forms of advocacy, when we tried to do so - and we were open to this throughout the review process - we found that the other forms of advocacy differed considerably in approach and could not, therefore, contribute useful information.

We repeated this process iteratively for all new papers added to our inclusion list until we exhausted this search, at which point we moved to phase 2 .

\section{Phase 2: theory generation}

As we extracted data from studies identified in the scoping phase and generated candidate theories as well as detail on relevant contexts, mechanisms and outcomes, we further augmented our list of included studies. The activities in this phase:

- took contextual or conceptual points from our initial data extraction as stepping stones out to a wider body of relevant literature; and
- led to the iterative formulation of potential theories and search for support or refutation of these in the evidence, adopting, adjudicating between and discarding different versions as we worked.

Our approach therefore differed from the way we obtained supplementary studies in the scoping phase because it had the potential for us to develop new searches that used keywords based on the theories identified in the first stages of the review and any further relevant keywords identified as we iteratively proceeded.

\section{Search methods for identification of studies in phase 2}

This search, led by CR, was a result of identifying candidate theories in the scoping phase literature and also gaps in information. These gaps could potentially be filled from studies that described relevant theories and mediating factors in other settings and for other types of intervention even though they might not specifically include any mention of advocacy or abuse. Such studies could include 'kinship studies' that may share a common theoretical origin with the starting-point paper, links to a common antecedent study or a contemporaneous or spatial context. We used the BeHEMoTh framework to structure this search, where:

- $\mathrm{Be}=$ behaviour of interest: the way the population or patient interacts with the health context; for example, access for a service, compliance or attitude to policy;

- $\mathrm{H}=$ health context: that is the service, policy, programme or intervention (including contexts outside of health settings such as judicial settings, if relevant to our emerging model);

- $E$ = exclusions: to exclude non-theoretical or technical models (dependent on volume; in our case we did not exclude these); and

- MoTh $=$ models or theories: operationalised as a generic "model ${ }^{\star}$ or theor ${ }^{\star}$ or concept ${ }^{\star}$ or framework " strategy together with named models or theories, if required (Booth 2013).

In each case, our iteratively developed explanatory theory guided the inclusion criteria.

As with the scoping phase, we also considered iteratively, and this time recursively too, further papers:

- identified through citation chaining of all papers that we had included in the theory-generation phase;

- identified through the 'search similar citations' function on PubMed for of all papers that we had included in the theorygeneration phase; and

- linked to the studies included in the theory-generation phase, as part of an integrated, mixed-methods study or as a 'sibling study'.

We included a recursive element because, as the theory developed, we needed to revisit previous papers for relevance or further information.

Searching continued until we found sufficient data to enable development of a coherent and plausible theory that was well rounded and could be tested; that is, when 'theoretical saturation' was achieved. This differed from the comprehensive sampling used in the scoping and theory-testing phases. It meant that we generated ideas about our theory and used these ideas to direct further searches and sampling (Emmel 2013); this process was 
iterative as we searched and considered articles, amended our theory and searched some more until we were not able to further develop our theory (because no further changes had been made for which we could find relevant articles).

This approach meant that we undertook multiple different searches in the databases, in an ad hoc way as new research avenues were opened by our reflections on the emerging data, using specific keywords such as 'advocacy + pregnant/pregnancy' or 'conservation of resources + domestic violence [and related terms]' or 'trade(-)off + domestic violence [and related terms]'. We held some meetings to discuss emerging ideas.

\section{Electronic sources}

We searched the following electronic sources. There were no restrictions to these searches. Our search strategies are not reported here being numerous and based on scoping keywords and phrases.

- Cochrane Central Register of Controlled Trials (CENTRAL; 2019, Issue 1), in the Cochrane Library

- MEDLINE Ovid (1948 to 31 January 2019)

- Embase Ovid (1980 to 31 January 2019)

- PsycINFO Ovid (1806 to 31 January 2019)

- PsycArticles American Psychological Association (1894 to 31 January 2019)

- ASSIA Cambridge Scientific Abstracts (Applied Social Sciences Index and Abstracts; 1987 to 31 January 2019)

- CINAHL Plus EBCSCOhost (Cumulative Index to Nursing and Allied Health Literature; 1937 to 31 January 2019)

- Social Science Citation Index Web of Science (1970 to 31 January 2019)

- International Bibliography of Social Sciences ProQuest (1951 to 31 January 2019)

- Database of Abstracts of Reviews of Effects (DARE; 2015, Issue 2. Final Issue), part of the Cochrane Library (searched 8 September 2018)

- OpenGrey (www.opengrey.eu; searched 31 January 2019)

- Dissertations \& Theses ProQuest (1861 to 31 January 2019)

In our protocol (Rivas 2018), we stated that all studies needed to consider advocacy interventions for women who had experienced partner abuse. However, in the final realist review we made the decision to also include some other studies that provided relevant information and have made it clear which these are (see Additional Table 1).

\section{Data extraction and management in phase 2}

We imported records for all studies into EPPI-Reviewer 4 (Thomas 2010), classifying each paper in each of the following four categories where relevant (we did not exclude opinion pieces, letters, editorials, other non-empirical articles or the grey literature): study design, academic discipline (e.g. primary care, legal), country (where the primary study took place) and setting. To these we added references already found in phase 1 .

Using paper annotations and EPPI-Reviewer 4 to aid data management (Thomas 2010), we noted from each study (e.g. from the Discussion sections of the empirical studies), how successful the study was and what explanations for this could be used to develop our candidate theories to be tested further in the next phase of the study. In our preliminary work on this, we identified the following features as potentially important.

- Underlying programme theory

- Length and intensity of the interventions

- Programme fidelity

- Expertise of the person delivering the intervention

- Quality of the relationship between the participant and the advocate

- Stage of change of the participant - whether the participant self-identified as experiencing intimate partner abuse (such as women offered advocacy in a shelter) at the time of recruitment or was identified from a screening process while attending an appointment for an unrelated issue

- Whether the participant was pregnant or had children, and her socioeconomic status

- Availability and quality of the services to which she was referred

- Ethical and safety considerations

Next, we extracted data on each of the following, if relevant, from each paper.

- Study aims and rationale

- Discipline, determined by considering the academic department or workplace role of the lead author (Greenhalgh 2016)

- Country of the primary study if empirical

- Study design

- Actual sample characteristics

- Programme or intervention description

- Programme strategies or underpinning theories

- Comparator type (referral, counselling, advice)

- Outcomes

- Methods - sampling strategy

- Methods - recruitment and consent

- Methods - data collection

- Methods - data analysis

- Length of time to follow-up

- Effectiveness of the interventions or qualitative themes where relevant

- Mechanisms (those aspects that explain how an intervention is to work through the responses of the abused women (or advocates if their professional practice was the focus of a study) that are described by the original study author. These should be described in the Aims and rationale, Methods, and Findings sections of the studies. In addition, we considered the explanations offered by study authors in the Discussion sections of papers, which we tagged as more speculative.

- Context (these include pre-existing characteristics of the participants before entry to the study, such as age or socioeconomic status, whether or not they had children or were pregnant, or level of risk at baseline, which might explain different responses to the same intervention (Kraemer 2002)).

- Mechanisms (factors of potential influence that occur along a causal chain between the intervention and the aimed for outcomes; for example, the level of trust in the advocate, or feelings of self-efficacy (Johnson 2015)). 
- Implementation issues (listing the key components and activities necessary for implementation and the contexts or mechanisms to implementation described in the study, such as information on who delivered the intervention, its intensity (i.e. hours per session), duration (i.e. spread over how much time)).

- Economic costs and benefits

- Risk of bias

- Weight of evidence

Four review authors (CR, CS, JC, LY) analysed articles in this way and extracted relevant passages into EPPI-Reviewer 4 for easier synthesis (Thomas 2010). They gave labels to relevant features not previously identified to aid data management. They mapped or charted the final data to form a tentative $\mathrm{CMO}$ matrix; that is, each study was represented in the rows of a matrix, and the relevant moderator or context, mechanism or mediators, and outcome details for the study were listed in the columns, facilitated by the functions in EPPI-Reviewer 4. We used different extraction procedures for different types of evidence as appropriate. All review authors worked together on this, with double charting of $10 \%$ of the studies, and discussions to ensure agreement on the charted data for all studies.

\section{Phase 3: theory testing and generation}

Phases 1 and 2 resulted in a set of candidate theories linking outcomes with context, mechanisms and implementation. In phase 3 , we undertook a systematic search of the literature for studies that could be used to test, confirm or refute our explanatory theory. In this way we were able to refine it.

We determined which of our small set of candidate theories had most informational value when used with the studies identified in the phase 3 search. We were open to combining theory elements as needed. We checked for gaps in the theory in relation to:

- aspects of intervention delivery, as these affect programme outcomes;

- which moderators or context and mechanisms or mediators are driving different outcomes and implementation events at different times; and

- which active ingredients are relevant in different settings.

We consulted our stakeholder group for suggestions where gaps remained.

We based our phase 3 literature search on the search terms used in Rivas 2015. We included studies that considered abused women and advocacy interventions according to the definitions given earlier (Background, 'Description of the condition', 'Review definition'). To test our theory, effectiveness studies had to consider at least one outcome (planned or unplanned, visible or not, proximal or distal, intermediate or final). We considered textual data in qualitative studies of views and experiences of advocacy interventions for the contextual factors they described and any other relevant information. We excluded studies lacking sufficient descriptive detail. We required qualitative and narrative studies to include a systematic analysis of primary or secondary data.

As in previous phases, we also considered for all papers that we included in this stage:
- papers identified through the 'search similar citations' function on PubMed; and

- papers that are linked to the studies identified, as part of an integrated, mixed-methods study or as a 'sibling study'.

\section{Criteria for considering studies for phase 3}

\section{Types of studies}

We included all empirical studies (e.g. process evaluations, qualitative research, RCTs, before-and-after studies and systematic reviews). Our more inclusive approach, compared with our effectiveness review (Rivas 2015), led to changes in the forms of outcomes evidence that we have listed below under 'Types of outcome measures'; for example, the need to include qualitative research outcomes.

We undertook a separate synthesis of RCT and non-RCT quantitative studies, and of qualitative studies, and then used the realist methodologies described, with CMO charting, to bring the two components together.

\section{Types of settings}

Examination of studies included in our previous review and the grey literature showed that, in general, women are referred to advocates by healthcare clinicians. Sometimes delivery of advocacy may be through other services such as shelters or judicial services (Rivas 2015). Different settings indicate different circumstances for the woman accessing advocacy. Given the wide variety of settings, even within healthcare, we considered the way the intervention was specifically delivered in a specific setting in a specific context, as relevant to our determination of potential mechanisms and moderators or contexts. We therefore considered all settings, as in Rivas 2015 and our scoping phase.

\section{Types of participants}

As with Rivas 2015, we included women aged 15 years and over who had experienced intimate partner abuse (as defined in the Background under 'Description of the condition', 'Review definition'), with no upper age limit. Partner abuse may coexist with other forms of violence within families, such as child abuse or elder abuse, but such abuse is not the focus of this review. Thus despite the lack of upper age limit we excluded studies that defined their advocacy as for elder abuse.

\section{Types of intervention and advocacy activities}

We included all advocacy interventions or multi-component interventions that included advocacy. We developed a list of activities that made up the advocacy components of an advocacy intervention in order to be consistent with our existing review. Thus, for the purposes of this review, we defined the core activities of advocacy as:

- providing legal, housing, or financial advice;

- facilitating access to and use of community resources such as legal, housing, financial advice and help, refuges or shelters, emergency housing, and psychological interventions and counselling;

- giving safety planning advice; and

- providing ongoing support and informal counselling.

- papers identified through citation chaining;

A realist review of which advocacy interventions work for which abused women under what circumstances (Review) 
As the Rivas 2015 review excluded interventions where counselling was a dominant component, our final list of studies is more comprehensive in terms of the interventions included. This list should help to define more focused intervention inclusion criteria for future reviews on advocacy interventions for abused women that are based on consideration of the active ingredients as elucidated in our realist review.

\section{Types of outcome measures}

We organised the outcomes into primary and secondary outcomes in a way that was consistent with those of Rivas 2015. These were determined, in turn, with reference to the WHO definition of intimate partner abuse (WHO 2013a). We added qualitative outcomes to this list.

Abuse may take various forms (see Background, 'Description of the condition', 'Review definition'), and all need to be covered by the programme theory. Thus, we considered outcomes relating to any and all of these forms; there was no restriction on how the outcomes were measured.

\section{Primary outcomes}

- Incidence of any form of abuse

- Physical

- Sexual

- Emotional

- Financial

- Other (such as risk of death, harassment, coercion to have children). Abuse may be assessed using self-report measures or other validated tools, or a single question about continuing abuse or professional observations of abuse such as in healthcare, social service or judicial service records, or from qualitative analyses.

- Psychosocial health

- Quality of life (using validated tools or from qualitative analyses)

- Depression (using validated tools or from reports of prescribed medication)

- Anxiety (using validated tools or from qualitative analyses)

\section{Secondary outcomes}

- Physical health (quantified incidents or descriptions of incidents, including frequency)

- Deaths, all-cause and partner-abuse related (documented in medical or police records, regional and national databases or from study follow-up records)

- Physical injuries, such as fractures and bruises (self-reported or formally documented (e.g. in medical, dental or judiciary records), or from qualitative analyses)

- Any chronic health disorders such as gynaecological problems, chronic pain, or gastrointestinal disorders (selfreported or formally documented (e.g. in healthcare or dental records), or from qualitative analyses)

- Any general measures or observations of physical health (self-reported or formally documented, or from qualitative analyses)

- Birth outcomes (self-reported or formally documented (e.g. in health or social care records), or from qualitative analyses)

- Psychosocial health (both qualitative and quantitative formats)
- Post-traumatic stress (using validated tools or from qualitative analyses)

- Self-efficacy (using validated tools or from qualitative analyses)

- Self-esteem (using validated tools or from qualitative analyses)

- Perceived social support (using validated tools or from qualitative analyses)

- Alcohol or drug abuse (using validated tools, self-reported or formally documented (e.g. in health or social care records), or from qualitative analyses)

- Attempted suicide (self-reported or formally documented (e.g. in health or social care records), or from qualitative analyses)

- Self-harm (self-reported or formally documented (e.g. in health or social care records), or from qualitative analyses)

- Impact on relationships inside and outside the family (using validated tools, self-reported or formally documented (e.g. in health or social care or judiciary records), or from qualitative analyses)

- Any measures of the quantity or quality of network ties not included above

- Socioeconomic outcome measures

- Income

- Housing

- Participation in education

- Participation in work

- Any other socioeconomic outcomes reported in studies

- Benefits applications

- 'Proxy' or intermediate outcome measures (including uptake of referrals to other agencies)

- Use of safety behaviours (e.g. use of coded telephone messages to a friend, keeping clothes at a friend's house, hiding emergency money)

- Use of refuges or shelters

- Use of counselling

- Calls to police

- Filing of police reports

- Solicitation of protection orders

- Maintenance of family ties (i.e. children staying with mother)

- Any other such outcomes

- Other qualitative outcomes

- Improved communication with the intimate partner

- Reduced fear and anxiety and other unwanted feelings, and improvement in desirable feelings

- An improved sense of security

Outcomes were further specified during the realist review. Thus there was scope, for example, for unanticipated outcomes to be recorded, such as advocates experiencing burnout.

Post-intervention, there may be both positive and negative, planned and unplanned outcomes for abused women, and this requires careful interpretation. For instance, increased refuge or shelter usage may reflect proactive behaviour on behalf of abused women, but it could also correlate with - or precipitate - an escalation of violence. Moreover, self-reports and official documentation of outcomes, such as improved relationships, may

A realist review of which advocacy interventions work for which abused women under what circumstances (Review) 
reflect coercive pressures by the perpetrator on the abused woman to report this. We also considered any adverse outcomes from interventions where these were reported by study authors.

\section{Timing of outcome assessment}

Intimate partner abuse can have short-term and long-term negative health consequences for survivors even after the abuse has ended (Campbell 2002), and we incorporated these into our programme theory. Correspondingly, an intervention may also result in some immediate positive outcomes, such as a reduction in physical violence, whereas other benefits, such as positive mental health effects, may take some time to be realised.

Previously, we have been unable to determine the optimal period of follow-up or outcomes trajectory (Rivas 2015). For the purposes of this review, and to conform with our previous work (Rivas 2015), we tentatively defined short term as up to and including 12 months, medium term as more than 12 and up to 24 months, and long term as more than 24 months.

\section{Search methods for identification of studies in phase 3}

We based the phase 3 literature search on the searches reported in Appendix 1 of Rivas 2015 and Appendix 2 in this review. Where necessary, we modified these searches to reflect changes in controlled vocabulary or database syntax. We restricted our searches to English-language studies. We considered any study excluded from the Rivas 2015 review but deemed relevant on the basis of this realist review, hence considering all studies from when databases began. In practice, there are unlikely to be any studies of intimate partner abuse interventions before 1980 (Dobash 1984).

We also followed selected elements of the CLUSTER (citations, lead authors, unpublished materials, scholar searches, theories, early examples, related projects) approach (Booth 2013), which includes backwards and forwards citation checking, as well as kinship- and sibling-paper searches, and the 'search similar citations' function on PubMed for all papers relevant to our realist question that we accrued through the review.

\section{Electronic searches}

We searched the electronic sources listed below, to test the theory in this phase of the realist review, and to update the Rivas 2015 review.

- Cochrane Central Register of Controlled Trials (CENTRAL; 2019, Issue 1), in the Cochrane Library (searched 31 January 2019)

- MEDLINE Ovid (1948 to 31 January 2019)

- Embase Ovid (1980 to 31 January 2019)

- PsycINFO Ovid (1806 to 31 January 2019)

- PsycArticles America Psychological Association (1894 to 31 January 2019)

- ASSIA Cambridge Scientific Abstracts (Applied Social Sciences Index and Abstracts; 1987 to 31 January 2019)

- CINAHL Plus EBCSCOhost (Cumulative Index to Nursing and Allied Health Literature; 1937 to 31 January 2019)

- Social Science Citation Index Web of Science (1970 to 31 January 2019)

- International Bibliography of Social Sciences ProQuest (1951 to 31 January 2019)
- Health Management Information Consortium Ovid (1979 to 31 January 2019)

- Maternity and Infant Care Ovid (1971 to 31 January 2019)

- Cochrane Database of Systematic Reviews (CDSR; 2019, Issue 1), part of the Cochrane Library (searched 31 January 2019)

- Database of Abstracts of Reviews of Effects (DARE; to March 2015), part of the Cochrane Library (searched 8 September 2018).

- UK Clinical Research Network Study Portfolio (www.ukcrc.org/ research-infrastructure/clinical-research-networks/uk-clinicalresearch-network-ukcrn; searched 2 December 2018 and 31 January 2019)

- OpenGrey (www.opengrey.eu; searched 2 December 2018 and 31 January 2019)

- Dissertations \& Theses ProQuest (1861 to 31January 2019)

- UK Clinical Trials Gateway (now called Be Part of Research (bepartofresearch.nihr.ac.uk; searched 2 December 2018 and 31 January 2019).

- WHO International Clinical Trials Registry Platform (ICTRP; www.who.int/ictrp/en; searched 2 December 2018 and 31 January 2019)

\section{Other resources}

We also searched the following websites.

- WHO Violence and Injury Prevention (who.int/topics/violence/ en; searched 31 January 2019)

- Violence Against Women Online Resources (vawnet.org/ publisher/violence-against-women-online-resources; searched 31January 2019)

\section{Final data synthesis}

Data analysis and narrative synthesis is intended to show how the extracted data inform our understanding of the contexts, mechanisms and outcomes for advocacy interventions. We looked for similarities and differences in CMO configurations and refined our initial theory. We discussed tentative findings with each other and invited comments from our stakeholder group, leading to final adjustments to theory.

We explored CMOs in the data in three different ways, which we triangulated. Thus we a) developed a coding tree based on the CMOs and used comparative analysis to compare and contrast the different CMOs, b) drew simple box and arrow diagrams to represent CMOs and c) used an Excel spreadsheet with the coding tree as the columns and studies as the rows.

Data synthesis across stages and across these three approaches drew on:

- juxtaposition of sources in ways that might have provided further insights;

- consolidation of sources when evidence about mechanisms and outcomes was complementary;

- reconciliation of sources where outcomes differed in comparable context;

- situation of sources where outcomes differed in different contexts; and 
- adjudication of sources according to methodological strengths or weaknesses (Gough 2007; Pearson 2015; see also 'Strength and quality of the evidence' section below).

We tested early hypotheses on empirical studies extracted after their development, and fed the analyses back into our developing theory in a process of induction by elimination until we decided that our theory had sufficient explanatory power to answer the research questions. Once coding and hypothesis formation was completed, we reviewed the entire corpus to check consistency of interpretation (Thomas 2008). In developing our theory, we found examples where the outcomes were unanticipated, when considering the contexts and mechanisms. This occurred for example in women from rural or ethnic minority groups, and with some judiciary-based interventions. This led us to a particular focus on the role of settings, support for advocacy, and the tradeoffs involved in women's responses to interventions. This in turn produced new research questions, in which we asked whether women's experiences, needs and desires were met by advocacy services, what were women's expectations of advocates, whose perspective matters and whether this corresponded to the active ingredients of advocacy?

Where there were gaps in the data (i.e. where CMO configurations were incomplete because the mechanisms were not clear), and we were unable to fill these from further searches, we used the 'Final data synthesis approaches' - described directly above- in abductive reasoning to infer mechanisms from the data that were available. This is consistent with the realist approach. In so doing we considered how the mechanism could be explained from what we knew about the context and outcomes and also theoretical commentary or discussions within the literature.

In Results we describe the final realist theory using narrative and summary tables, a logic model and theory graphics, drawing insights from across the sources. In Results we also provide data and extracts from published papers, and views from our stakeholder group, to support our synthesis and developed theory. We have written up the Results according to the RAMESES Publication Standards For Realist Synthesis (Wong 2013a). In Authors' conclusions we use our theory to make recommendations for future research and to highlight the implications for practice.

\section{Strength and quality of the evidence}

\section{Assessment of risk of bias in included studies}

Usual 'Risk of bias' checklists have a focus on the whole study, whereas we were interested in specific data of relevance to our theory. Nonetheless, we decided to use 'Risk of bias' checklists to provide some indication of overall risk of bias for readers of this review. These assessments did not inform our choice of studies to include, but did help us to develop construct tables in which we summarise our key findings. They also helped us to consider the level of confidence we had in our findings.

The different 'Risk of bias' criteria are considered separately in the next three sections. In all cases, we assessed studies as being at overall high risk of bias when there were at least three criteria conferring high risk of bias, or when one or two criteria were associated with high risk of bias and the number of criteria with an unclear risk exceeded the number of criteria with a low risk, an assessment we previously developed for Rivas 2015. We defined low overall risk of bias as occurring when a study had no high-risk indicators and a maximum of two criteria conferring uncertain risk. We classified all other studies as having a moderate or intermediate risk of bias.

\section{Assessment of risk of bias in included experimental studies}

The review authors independently assessed the extent to which each study attempted to control for six potential types of bias and assigned ratings of 'low risk of bias', 'high risk of bias', or 'unclear risk of bias' accordingly. We used the 2011 Cochrane 'Risk of bias' criteria (Higgins 2011), rather than the recently updated version (Higgins 2019), since the earlier version allowed for different trial designs. We considered the following criteria.

- Sequence generation: did the study contain a sufficiently detailed description of the method used to generate the allocation sequence so as to enable an assessment of whether it should have produced comparable groups?

- Allocation concealment: did the study contain a sufficiently detailed description of the method used to conceal the allocation sequence, enabling an assessment of whether participants and staff could have foreseen intervention schedules before or during recruitment?

- Blinding: did the studies describe any measures used to blind outcome assessors in sufficient detail so as to assess possible knowledge of which intervention a given participant might have received?

- Incomplete outcome data: did studies report data on attrition, including the numbers involved (compared with total randomised) and the reasons?

- Selective outcome reporting: did investigators attempt to assess the possibility of selective outcome reporting?

- Other sources of bias: was the study apparently free of other problems that could put it at a high risk of bias?

We specified a priori that we would assess the following three criteria under the heading 'Other sources of bias'.

- Baseline measurement of outcome measures: did studies contain information on the comparability of the groups in terms of the primary outcome measures at baseline?

- Reliability of primary outcome measures: did investigators assess the primary outcomes using reliable measures (e.g. Cronbach's alpha 0.6 or above (Nunnally 1994)?

- Protection against contamination: did investigators describe any measures taken to prevent or minimise the possibility that women in the control arm might receive part or all of the intervention so as to assess possible contamination between groups?

\section{Assessment of risk of bias in cross-sectional (survey) studies}

For cross-sectional survey studies, we considered the following criteria from Agarwal 2017 to inform the 'Risk of bias' tables.

- Representativeness of the sample: this is important to ensure that the survey results provide an unbiased estimate of the attitudes or practices of the target population.

- Adequacy of response rate: this is important to minimise any effect on results of systematic differences between respondents and non-respondents. 
- Missing data within completed questionnaires: even when a survey has a good response rate, a substantial amount of missing data (items that were not answered by survey respondents) is likely to introduce bias.

- Conduct of pilot testing: risk of bias is decreased if investigators have conducted a formal assessment of the comprehensiveness, clarity and face validity of a questionnaire with a field test in a subset of, for example, 5 to 10 individuals drawn from the larger sample.

- Established validity of the survey instrument: the degree to which survey items evaluate the theoretical concept(s) that the survey purports to measure.

\section{Assessment of risk of bias in qualitative studies}

We used the Critical Appraisal Skills Programme (CASP) (caspuk.net/wp-content/uploads/2018/01/CASP-Qualitative-

Checklist-2018.pdf) for assessing qualitative studies, with the following criteria, used to inform the 'Risk of bias' rating insofar as this can be applied to qualitative research (Lincoln 1985),

- Was there a clear statement of the aims of the research?

- Was a qualitative methodology appropriate?

- Was the recruitment strategy appropriate to the aims of the research?

- Had the relationship between researcher and participants been adequately considered?

- Was the research design appropriate to address the aims of the research?

- Had ethical issues been taken into consideration?

- Were the data collected in a way that addressed the research issue?

- How valuable was the research?

- Was the data analysis sufficiently rigorous?

- Was there a clear statement of findings?

\section{Thickness of the data}

We also used the concept of 'thickness' of the sources (Roen 2006), that is, the degree to which they offered explanatory insights on the developing theory and the factors shaping implementation processes. This related to the quality of the content in the study that was relevant to our research questions, rather than the quality of the whole study (as for risk of bias). The criteria we used to assess thickness were:

- detail of the description of relevance to our emerging theory;

- level of consideration of the relevant context and its influence;

- detail on potential mediators or moderators; and

- attempt to explain anomalous results and findings with reference to context and to data.

Data of relevance to our review may have formed only a small part of a paper, rather than being its focus, so this distinction between thickness as a measure of the quality of the data we used and risk of bias as a measure of the overall quality of the study method is an important one. Assesments of thickness were used to choose the papers we considered in this review, in particular when developing our theory and filling in the gaps in our emerging theory, These assessments were also used in our Characteristics of included studies in which we summarise the key findings, alongside risk of bias considerations; we added a line in these tables that asked: How valuable is the research (richness of the data for the review research questions)?

\section{Assessment of confidence in the review findings}

We used the GRADE-CERQual (confidence in the evidence from reviews of qualitative research) approach to summarise our confidence in the evidence (Lewin 2015). CERQual assesses confidence in the evidence, based on the following four key components.

- Methodological limitations of included studies: the extent to which there are concerns about the design or conduct of the primary studies that contributed evidence to an individual review finding and whether the original researchers' inferences had sufficient weight to make a methodologically credible contribution to the testing of the intervention theory.

- Coherence of the review finding: an assessment of how clear and cogent the fit is between the data from the primary studies and a review finding that synthesises those data.

- Adequacy of the data contributing to a review finding: an overall determination of the degree of richness and quantity of data supporting a review finding. We used the concept of 'thickness' of the sources (Roen 2006), that is, the degree to which they offered explanatory insights on the developing theory and the factors shaping implementation processes. The criteria we used to assess thickness were:

- detail of the description of relevance to our emerging theory;

- level of consideration of the relevant context and its influence;

- detail on potential mediators or moderators; and

- attempt to explain anomalous results and findings with reference to context and to data.

- Relevance of the included studies to the review question: the extent to which the body of evidence from the primary studies is applicable to the finding.

After assessing each of the four components, we made a judgement about the overall confidence in the evidence supporting each review finding (our hypotheses), which we report in additional tables. We judged confidence as high, moderate, low, or very low. This assessment provided an indication of the quality of the evidence used to inform our theory rather than the studies or papers themselves; data of relevance to our review may have formed only a small part of a paper, rather than being its focus, so this distinction is important. We also used assessments of relevance, rigour and thickness to choose the papers we considered in this review, in particular when developing our theory and filling in the gaps in our emerging theory.

\section{Changes from protocol}

Changes from the protocol for this review (Rivas 2018) are shown in Table 1.

\section{RES U L T S}

\section{Results of the search}

In phase 1 we included 81 articles after checking the full texts of 177 articles. Twenty-one of the 81 articles came from the 71 articles either excluded or awaiting assessment in Rivas 2015, to which we 
added the 21 reviewed in Rivas 2015. We then undertook a CLUSTER search of these 42 articles, which led us to check the full texts of 106 further articles, of which we included 39.

The phase 2 electronic database search and initial handsearching yielded a total of 6165 unique articles, which we entered into EPPI-4 review management software (Thomas 2010). After screening article titles and abstracts against the pre-specified inclusion and exclusion criteria, we identified 153 potentially relevant articles. Following discussion we decided not to include advocacy for conditions or situations other than intimate partner abuse at this stage. So we screened the titles and abstracts of these 153 articles for a second time, reducing them to 83 potentially eligible articles. We retrieved the full-text copies of these 83 papers and two review authors independently assessed them for relevance, resolving disagreements through discussion; we did not need arbitration with a third review author. This resulted in a total of 31 included articles added to the 81 in phase 1 . We identified 152 additional papers using CLUSTER in phase 2 , of which we included 16 . Iterative searching as we developed our theory resulted in a further 16 included articles from 117 considered. By the end of phase 2 we had found 144 relevant articles (including the phase 1 articles) and identified two studies that are awaiting assessment and three ongoing studies.

In phase 3, we screened a further 1366 articles from database searches as an update to the Rivas 2015 search, and included an additional three articles to add to the 144 full texts obtained.

Thus, we included a total of 147 articles, representing 98 studies, in this review (Figure 1).

\section{Figure 1. Study flow diagram}

\section{Note: 2015 review is Rivas 2015}

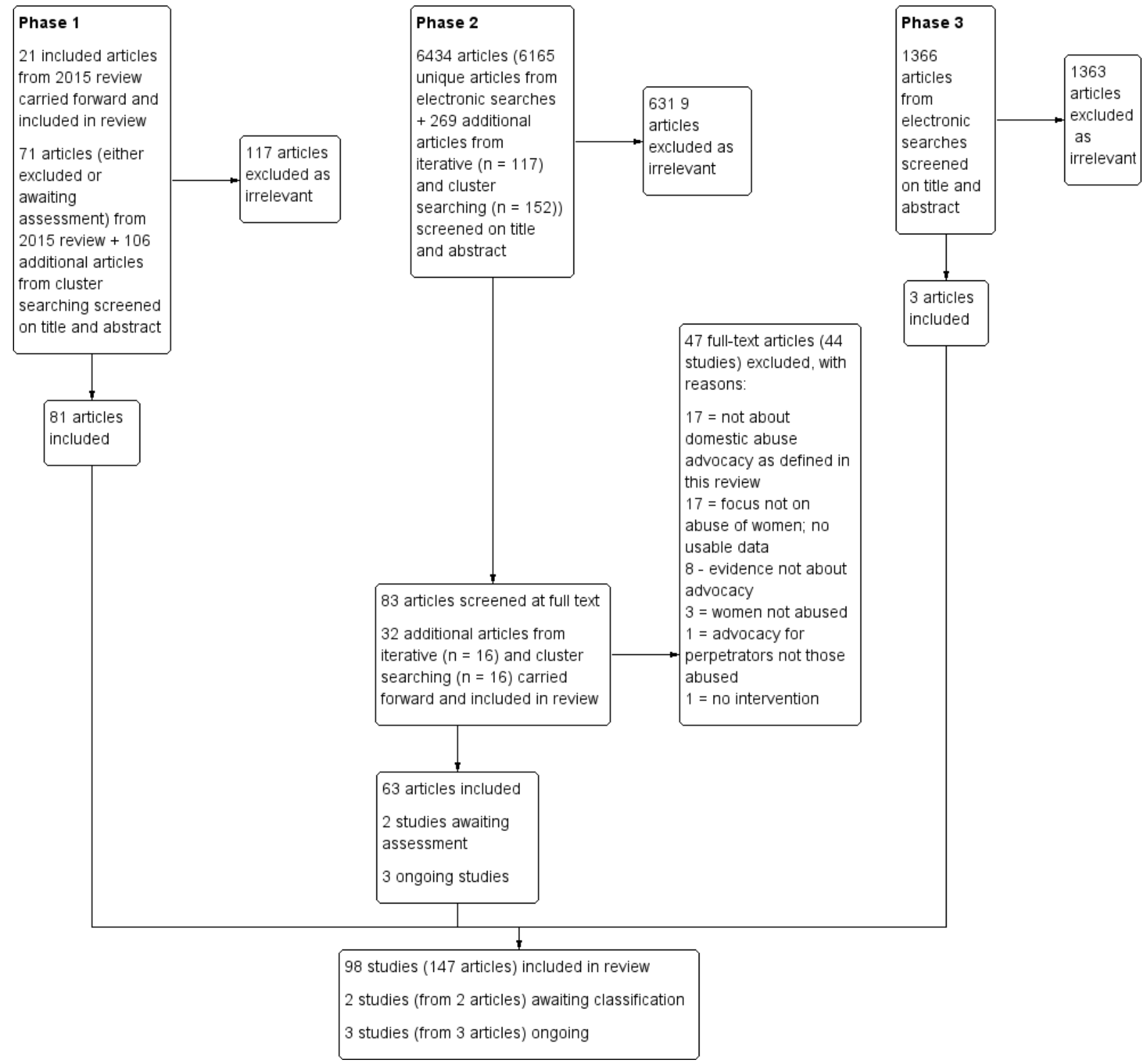

A realist review of which advocacy interventions work for which abused women under what circumstances (Review) 


\section{Description of studies}

In this section we provide summary descriptions of the included studies. These are not ordered in a way that is intended to link with our subsequent realist considerations but by study methods as a reference section for readers. Findings from these studies are discussed in the realist synthesis itself in later sections.

\section{Included studies}

\section{Studies of professionals}

Our scoping exercise showed that the way that advocates undertake their role, and the barriers and facilitators to this, are contextual factors that affect the outcomes of advocacy. We therefore considered 37 studies that focused on staff performance of their professional role in intimate partner abuse services. In keeping with the nature of the realist review, we included studies that sought information from a range of intimate partner abuse service providers and not just advocates, if we judged that they reported findings of relevance to advocacy itself. None of these studies provided effectiveness data - although four survey-based studies undertook correlation and mediation analyses.

Five of the 37 studies used a qualitative focus group or group interview approach. In Goodman 2016b, 38 advocates working in a range of intimate partner abuse programmes across one state in the USA were asked about their use and perceptions of networkoriented strategies. Lynch 2013 conducted focus groups with 24 participants from organisations including women's refuges, family support services, domestic violence outreach services, solicitors, and Domestic Court Advocacy Services (DVCAS); the commonality was that all had assisted women seeking Apprehended Domestic Violence Orders (ADVOs) in New South Wales, Australia. Logan 2018 ran five focus groups with 37 domestic violence and sexual assault advocates from a variety of settings in the USA to explore safety planning strategies. They reported analyses across two companion papers. Similarly, and also in the USA, Murray 2015 explored 62 domestic violence service providers' perspectives toward safety planning. In a very different setting, Infanti 2015 undertook group interviews and ran a participatory workshop with 31 public-health midwife advocates working on the tea estates of Sri Lanka.

Fifteen of the studies in this group were wholly based on semistructured interviews. Three had particularly specific foci. SilvaMartinez 2016 explored the experiences of 19 domestic violence advocates in providing a financial literacy programme across 10 states in the USA. Sudderth 2017 conducted interviews with representatives from 24 refuges in New Zealand, to explore the inclusion of both the abused woman and people from her social networks in her safety planning meetings. Sullivan 2019, in the USA, interviewed 11 advocates from north-western, mid-western and eastern states specifically about their work helping intimate partner abuse survivors obtain housing. The advocates worked in agencies located in large cities, a university town, mid-sized cities, and small, rural communities. Three worked in culturally specific agencies (focused on immigrants and refugees or lesbian, gay, bisexual, transgender and queer (LGBTQ) communities.

Turning now to the other interview-based studies, Donnelly 2005 interviewed the executive directors (rather than advocates) of 44 agencies for battered women in the 'deep south', USA. Dunn 2007 conducted 32 interviews with domestic violence victim advocates working in shelters ( $n=14)$, criminal justice settings ( $n=16)$, or both $(n=2)$, including prosecutors' offices, a police department and a sheriff's office in rural, suburban and urban areas of a large mid-western state in the USA. Garcia-Leeds 2017 interviewed seven health professionals (counsellors and their supervisors who were also equipped for crisis advocacy) working for the Latina Domestic Violence Programme (LDVP) in Philadelphia, USA. Grant 2012 interviewed 10 domestic violence advocates in Connecticut, USA about their day-to-day work. Hidalgo 2016 interviewed a purposive sample of 10 domestic violence advocates (e.g. case manager, director, supervisor, and social worker) who worked in New York City in the social services field, in not-for-profit community organisations. The aim was to consider the barriers to successful advocacy and methods that might overcome these barriers when dealing with community stakeholders such as the police, medical personnel, and public housing agents. Hughes 2017 interviewed six shelter advocates and six shelter residents in a study about the advocates' practices and the impact of shelter stay on women residents. Merchant 2015 used interviews with 19 current and former shelter advocates from nine shelters in the southwestern USA, to develop a grounded theory concerning their role challenges and how these influenced staff satisfaction, retention and turnover. Matthew 2016 interviewed 11 purposefully sampled advocates to explore their knowledge, attitudes, perceptions, perspectives and understanding of US Afro-Caribbean abused women and their historical and structural gender role and cultural and religious beliefs. Owen 2015 focused on the challenges experienced by 49 intimate partner abuse service providers, but these came from criminal justice agencies (police and courts), health and welfare services across 12 local government areas in rural New South Wales in Australia. In Japan, Umeda 2017 aimed to determine what motivated eight intimate partner abuse care experts in their work, while in the USA, White 2019 undertook a series of "focused conversations" with 72 leaders in the fields of domestic violence and sexual assault working in various capacities (service provision, justice responses, advocacy, research), and sometimes also with perpetrators. Wood 2014 developed a grounded theory from interviews with 22 advocates in the USA, working in a domestic violence shelter or non-residential agency, or housed in a legal setting but working specifically with survivors of domestic violence and predominantly survivors of partner abuse.

Nine studies used a mixture of interviews and other forms of data collection. Briones-Vozmediano 2014 undertook 29 interviews and four focus groups with 43 professionals (social workers, psychologists, intercultural mediators, judges, lawyers, and public health professionals) who were involved in providing support for battered immigrant women in Spain. Ganz 2015 used cultural studies, feminist methodology and sociological theory to analyse 21 interviews with advocates plus a survey begun by 382 advocates with a completion rate of 221/382 (58\%). Kapur 2017 recruited 26 domestic violence advocates from 14 South Asian-focused non-profit organisations in the USA (10 providing direct services to women and four acting as legal or advocacy organisations), to explore how they managed the domestic violence-related intersectional needs of Asian-Indian marriage migrants. Sixteen advocates were interviewed face-to-face and five were interviewed by phone; five more attended a group interview. Kolb 2008 undertook non-participant ethnography at an organisation in the USA called SAFE (Stopping Abuse in Family Environments), and interviews with one of the agency's co-directors, four advocates, 
five counsellors and two full-time shelter staff, between July 2005 to August 2006. The shelter director and a family counsellor worked in a shelter, the co-director worked in a small office in the next town and the others all worked at the main agency office. Reina 2015, also in the USA, completed individual, semistructured interviews and a focus group with 10 undocumented Latinas recruited from a non-profit domestic violence and sexual assault agency. In the UK, Lea 2016 undertook case-file analysis ( $n=86)$ of an existing, independent domestic abuse service known as the Domestic Abuse Advocacy Project (DAAP), and interviews with abused women $(n=12)$ and key individuals based in related statutory and community organisations ( $\mathrm{n}=$ 12). DAAP included free specialist legal advice and support with civil action, as well as other advocacy work to facilitate access to a range of social and health-related specialist services. The advocate was a specialist family law solicitor trained in intimate partner abuse advocacy. The evaluation of DAAP was independent and participatory, involving intervention stakeholders, and was aimed at informing strategic decision-making by the local multiagency domestic abuse partnership board. Wies 2008 focused her research on two domestic violence shelters in Kentucky, USA. She used participant observation, working as an advocate to observe other advocates in-role. She also ran four focus groups with the advocates, and collected documentary information from archives, popular media sources. newsletters, regional reports, conference materials and internal paperwork. In addition, she interviewed individuals who self-identified as participants in the Kentucky domestic violence social movement in the past. Burnett 2012 undertook in-depth interviews and focus groups with 37 staff and four executive directors from four shelters in Ontario, Canada, as well as analysing relevant policy documents using critical discourse analysis. Johnson 2014 undertook telephone interviews with 25 intimate partner abuse advocates $(67.5 \%$ of those invited to take part) who worked at 20 of the 28 advocacy locations ( $71.4 \%$ sampled) across 16 rural counties in the USA; six advocates also attended a focus group. Johnson 2014 explored the microsystem and exosystem levels of the ecological model to understand advocates' relationships with abused women and criminal justice personnel.

There were four survey-based studies in this group, all from the USA. Macy 2018 questioned 97 agency directors on recommended delivery practices for six types of services typically offered by domestic violence and sexual assault agencies: crisis; legal advocacy; medical advocacy; support groups; individual counselling; and shelter in rural, suburban or urban locations. Slattery 2009 analysed survey data from a convenience sample of 148 domestic violence advocates working in a variety of settings. Babin 2012 surveyed 69 staff from a domestic violence agency serving women and dependent children under 18 years of age. Bemiller 2010 sent their survey to 30 domestic and sexual assault shelters employing 420 advocates and received 194 complete responses.

There was one study that comprised only non-participant observation, by Stylianou 2018, who analysed 22 recorded family court advocate-survivor sessions.

Three studies differed from the rest in that they focused on instrument-development using an exploratory, sequential mixed-methods design. The PhD dissertation by Magruder 2017 described the development of the Intimate Partner Violence
Responder Collaboration Scale. The qualitative component of this study, semi-structured interviews with 15 intimate partner abuse service delivery staff (victim advocates/victim service providers, law enforcement professionals, prosecutors, and perpetrator intervention providers) in Florida, provided valuable information on what intimate partner abuse responders consider to be important elements of collaboration between organisations. The primary focus of the survey-based study by Kulkarni 2015 was the development and revision of the Survivor-Defined Advocacy Scale (SDAS), to assesses intimate partner abuse service providers' attitudes about their work and clients. In developing this scale, Kulkarni 2015 were able to report on critical mediating variables, but it should be noted that participants represented a range of intimate partner abuse service roles, including advocates or case managers, counsellors and administrators (shelter personnel and outreach or educators). Goodman 2016a surveyed a convenience sample of 370 abused women seeking services at one of 15 urban and suburban intimate partner abuse organisations in five states in the USA about the strength of their alliance with their advocates, and symptoms of depression and post-traumatic stress disorder (PTSD). All the programmes in the Goodman 2016a study provided safety planning, counselling, and referral services for abused women. Furthermore, most provided emergency shelter, with stay lengths of several weeks to six months, and several also offered transitional living programmes, with stay lengths of up to two years. In a separate paper as part of the Goodman 2016a study, the authors built on these findings, developing the SurvivorDefined Practice Scale from the perspective of abused women. They used factor analysis to determine the core elements of safetyrelated empowerment and the link between survivor-defined practice and safety-related empowerment to be incorporated into the scale. The result was a nine-item measure that assessed participants' perception of the degree to which their advocates helped them achieve goals they set for themselves, facilitated a spirit of partnership, and showed sensitivity to their individual needs and styles. The study authors also developed a final, 13-item validated measure, the Measure of Victim Empowerment Related to Safety (MOVERS), from triangulation with other data collected as part of the same broad programme of work. In a third paper, Goodman 2016a and colleagues analysed the quantitative survey data used for the safety subscale of MOVERS and also the associated qualitative free-text answers using content analysis. Most of the analysis in this paper involved the full survey sample but they gave only 215 respondents three questions on retrospective evaluation, and of these, 117 responded; thus, the consideration of whether women would have done things differently in retrospect drew on a smaller subset than other considerations in this paper.

\section{Studies of abused women}

Among the studies we included in this review, only six were qualitative studies of women that were not associated with (and therefore reported under) intervention studies. Two also included agency staff (Gillum 2008; Weisz 1999). Bader 2014 undertook 26 interviews with women who disclosed to service providers that they were experiencing intimate partner abuse, as part of a co-ordinated community response in Canada. Ekstrom 2015 interviewed a convenience sample of six abused women who had all received social support and advice about police investigations and criminal trials concerning the perpetrator from the Relationship Violence Centre (RVC) in Stockholm. The social workers from the RVC were employed by social services, 
but the RVC was located at the regional police office and cooperated closely with social services, the police, and to some extent, also prosecutors. Gillum 2008 held two focus groups with 11 African-American abused women, two interviews with two further African-American abused women, and a separate interview involving two African-American female service providers who worked primarily with African-American abused women. In a separate analysis as part of the same overall programme of work, Gillum 2008 interviewed 13 abused women for a service user perspective of a domestic violence agency in the USA, the co-ordinator of Victim's Services for an administrative perspective, and two of the agency's advocates for a direct service provider perspective. Hathaway 2008 used structured interviews to explore abused women's perceptions of an existing, longterm intimate partner abuse advocacy programme involving five professional advocates and based in the social service department of a large, urban teaching hospital. Advocacy was flexible, survivor-focused and on-demand, and included crisis intervention, risk assessment and safety planning, individual counselling and support groups, assistance connecting to resources, and accompaniment to key appointments. Kenyon 2016 undertook and analysed nine interviews with abused women in shelters in Canada, using the Fraser 2004 narrative method, which situates personal stories within their social context. Weisz 1999 used open-ended interviews and focus groups to explore advocates and abused women's views and experiences of legal advocacy in the USA.

There was one survey-based study exploring the experiences of women abused by their intimate partner; this study, by Song 2012, based in Taiwan, considered their contact with social services and empowerment, perceived changes of self, and life satisfaction. Data were obtained from 191 abused women in shelters as a convenience sample.

\section{Intervention studies}

There were 43 experimental intervention studies, some of which included qualitative evaluations.

Using a single-group pre-post design, Bacchus 2007 evaluated a domestic violence intervention in the maternity and sexual health services department of a UK hospital, which included referral of women disclosing violence to an on-site advocacy service (MOZAIC Women's Well Being Project, provided by a community organisation). They conducted semi-structured interviews with 34 women, one to 22 months after they received support from the service, selected according to clinical setting, living with abuser or not at the time of referral advocacy, length of abuse, pregnant or not, immigration status, access to their own money, ethnic origin and first language (English or Spanish). Three hospital staff who used the advocacy service were amongst those interviewed. They also undertook semi-structured interviews with staff ( 8 midwives and 11 sexual health professionals), as well as six focus group discussions (19 midwives and 6 sexual health professionals); they selected participants according to clinical setting (maternity or sexual health), professional group (doctors, nurses, midwives, health advisors), gender, and time elapsed since training (three months versus more than six months). They reviewed maternity records for 487 (98\%) of the 501 women who gave birth in January 2007. A total of 915 women attended the sexual health clinic in a one-month assessment period. However, of the 879 records available for audit, they analysed only the $644(73 \%)$ records that contained the domestic violence code. Kendall 2009, in a hospital emergency department pre-post study, gave a convenience sample of 350 women an initial session of their advocacy intervention (Domestic Violence Healthcare Project; DVHP) face-to-face, with follow-on telephone contact to see whether women were keeping to their plans at 2, 6, and 12 weeks; they also collected outcomes data during these calls. Part way through the study, they added another phone on day two, to increase retention. In a prepost study, Tutty 1996 evaluated two programmes in which 31 women departing a battered women's shelter received three to six months of "advocacy and counselling" from a bachelor'slevel social worker. Wuest 2015 carried out a feasibility study of a health intervention for abused women. They detailed the intervention development in two separate papers. One described initial qualitative interviews with purposeful samples of women and community stakeholders, and focus groups with nurse home visitors recruited from four sites. They based development of the IPV intervention, which is described in depth in the second development paper, on data from 69 respondents. The study itself as undertaken by Wuest 2015, was conducted in partnership with policy, community and practitioner stakeholders. The study used a mixed-methods design, combining a single-group, prepost intervention study with 52 survivors of IPV, of whom 42 completed data collection, with chart review data and interviews of 18 purposefully sampled participants and all nine interventionists. Krasnoff 2002 reported on an observational case study of an IPV advocacy intervention provided on-site at an urban hospital emergency department with screening for IPV by a nurse, a session with a volunteer advocate from a local human service agency crisis intervention when IPV was identified (given within 30 minutes of screening) and referral to telephone-based followup care from a professional case manager. Of the 528 women identified as experiencing IPV, 475 (84\%) agreed to speak to the advocate, and 258 (54\% of those seen by the advocate) accepted case management lasting 3 to 6 weeks. Pre-post measures were reported.

Muelleman 1999 compared data on a consecutive sample of 117 women before they entered the hospital emergency department BRIDGE advocacy programme with data on a consecutive sample of 105 women (57\% of the 183 asked) who were seen by the BRIDGE advocate for a one-off session of a mean of 1.5 hours). The intention of the programme was to provide a 'bridge' for women from the emergency department to community resources. Song 2010 evaluated the effectiveness of a strengths-based IPV advocacy intervention with 65 abused women in Taiwan using a pre-post study design, with follow-up three and nine months after the intervention or when the intervention was about to end, and with 26 qualitative interviews.

Bell 2001 undertook a quasi-experimental pilot study to evaluate the effectiveness of a legal advocacy programme in which law students worked intensively with abused women to obtain protective orders. The control group received usual care (standard court services). Follow-up was at six weeks. Coker 2012, also using a quasi-experimental study design, investigated the efficacy of clinic-based advocacy for IPV, compared with the usual care of business card referral only, with follow-up to 24 months. Trevillion 2013 undertook a quasi-experimental controlled study within five Community Mental Health Teams (three intervention and two control teams). The intervention comprised domestic violence training for clinicians $(n=29)$ and referral to domestic violence advocacy for service users. They presented quantitative outcomes 
on the 34 service users (27 intervention and 7 control women) who completed interviews at baseline and three months' followup. Semi-structured interviews (number unclear) with service users at three-month follow-up explored the women's experiences of mental health services' response to domestic violence.

Ten randomised controlled trials (RCTs) recruited pregnant women. Thus, Bahadir-Yilmaz 2018 compared an empowerment programme $(n=30)$ with usual care controls $(n=30)$ in two settings: the obstetrics and gynaecology departments of a hospital, and a Family Counselling Centre in Turkey. They followed up women in both groups at week 10, with an additional follow-up for women in the intervention group at week 12. Cripe 2010 reported on an RCT pilot study to evaluate the effectiveness of advocacy for abused pregnant women versus standard care. Women were followed up a week after giving birth, or in the period from 14 to 28 weeks postintervention. Curry 2006 described a small multi-site RCT to evaluate the effectiveness of a screening of a video about IPV plus individualised nursing case management with access for abused pregnant women available 24 hours a day and seven days a week $(n=499)$, versus standard care $(n=$ 501). In this study baseline assessment was prior to 23 weeks of pregnancy and follow-up between 32 weeks and delivery, hence with an interval of at least nine weeks. McFarlane 1997 evaluated an intervention given in public prenatal clinics to increase the safety-promoting behaviour of 132 pregnant women reporting physical or sexual abuse in the year before or during the present pregnancy, using an ethnically stratified design. The intervention comprised three education, advocacy, and community referral sessions that included information on safety behaviours. Half the women were invited to attend three additional counselling and information sessions by workers at the local shelter but attendance at these classes generally required a separate visit to the clinic, and was sporadic at best, and not attempted by $49 \%$ of the women invited to these extra sessions. A comparison group of 67 abused pregnant women were offered a wallet-sized card listing community resources. Follow-up was at 6 and 12 months post-delivery. McFarlane 2000 compared the effectiveness of three interventions specific for Latina women within two urban public health prenatal clinics, in a longitudinal study with follow-up at $2,6,12$, and 18 months post-delivery. The participants were 329 pregnant, physically abused Latina women. The interventions were: a) wallet-sized resource cards ('brief intervention'), b) unlimited access to supportive, nondirective counselling, or c) unlimited counselling plus support from a "mentor mother" (termed the outreach condition). Interventions were provided during the prenatal period only. Over two included papers, Bacchus 2016a described evaluation of the Domestic Violence Enhanced Home Visitation Programme (DOVE), One paper reported on an assessor-blinded multi-site (urban and rural) RCT of 239 women experiencing perinatal IPV. The intervention group $(n=124)$ received a structured abuse assessment and six home visitor-delivered empowerment sessions integrated into perinatal home visits. The control group received standard care (assessment and referral for perinatal IPV during the first perinatal home visit only). Follow-up was at 1, 3, 6, 12, 18 and 24 months postpartum. In the second paper, 26 women from the trial were interviewed in a process evaluation. In a related study by Bacchus 2016b, the researchers were primarily interested in the incorporation of an 'mHealth' (using mobile health technology such as mobile phones or web-based technology) educational and safety planning empowerment tool into DOVE home visiting for pregnant and up to three-month postnatal women. However, they explored the home worker-client relationship as part of the analysis, through 51 interviews (26 women, of whom 19 had used a non-mHealth version of DOVE, 23/45 home-visiting staff at the eight rural and urban sites, and the two DOVE programme designers), and four non-participant observations. Although DOVE is an intervention to prevent IPV during pregnancy in women who have screened positive for IPV in the year before the current pregnancy, 31\% of the sample studied had not experienced IPV over the last year. Tiwari 2005 evaluated the effectiveness of empowerment training in 110 women with a history of IPV in an antenatal clinic in a public hospital in Hong Kong. In this RCT, the intervention was especially designed for Chinese abused pregnant women; the control group was standard care. Tiwari 2005 stated that outcomes were assessed at six weeks post-delivery, but this could represent a follow-up period of anywhere from 16 to 34 weeks MOSAIC (MOtherS' Advocates In the Community) was evaluated by Taft 2011 in a cluster-RCT embedded in general practice and maternal and child health $(\mathrm{MCH})$ nursing services in disadvantaged suburbs of Melbourne, Australia. IPV-trained GPs and MCH nurses from 24 general practices and eight nurse teams referred women who were pregnant or with infants, identified as abused or symptomatic of abuse; in total 63/106 clinics referred 215 eligible culturally and linguistically diverse women. Women in the intervention arm ( $\mathrm{n}=$ 167) received up to 12 months' support from trained and supported non-professional mentor mothers. Women in the control arm ( $\mathrm{n}=$ 91) received usual care. Vietnamese health professionals referred Vietnamese women to bilingual mentors in a substudy. Baseline and 12-month comparisons were made between study arms. Implementation interviews were undertaken with MCH nurses, GPs and mentors, in-depth interviews with participants and mentors, and with follow-up of $\mathrm{MCH}$ nurses and GPs at trial conclusion only. This intervention provided parenting support to all the abused mothers as well as advocacy (Taft 2011). Prosman 2014 adapted the Taft 2011 MOSAIC intervention. They broadened the target group to abused mothers with children under 19 living at home; shortened the weekly mentoring period from 12 months to 16 weeks; provided more intensive training for mentor mothers; only had face-to-face sessions; included more 'therapeutic' content and focused on participation in society. Their programme was called MeMoSA (Mentor Mothers for Support and Advice). Mentor mothers were linked to family practices. Thus overall, MeMoSA was a shorter, but more intensive intervention providing similar content. MeMoSA was evaluated in a pre-post study in 63 identified abused women with children referred to the programme by a family doctor, of whom 43 women completed the programme. In a separate paper, Prosman 2014 described how 14 abused women were interviewed six months later in a process evaluation, with two focus group discussions held with the mentor mothers to evaluate their experiences and needs.

DePrince 2012 described a randomised, longitudinal study that compared community-co-ordinated, victim-focused outreach services (flexibly shaped depending on service availability and on the woman's specific circumstances) with referral, in 236 ethnically diverse women, following police-reported IPV. In the outreach condition, a community-based agency phoned women and gave them detailed information about the resources and services on offer; the agency had been told the woman's circumstances, so she did not have to explain this, and the conversation was confidential. For the referral condition, a system-based advocate from the prosecuting attorney's office or police department contacted 
women and gave them only basic information about communitybased agencies, and the conversation was not confidential. The onus in the referral condition was on the woman to contact the agencies and describe their situation to see whether they could be supported. Random assignment to either arm occurred very soon after an abuse episode. The study measured outcomes within a median of 26 days after police-reported IPV, six months later, and 12 months later. The Gillum 2009 RCT, with follow-up at three months, gave women in the control arm $(n=20)$ information on resources, while advocates actively helped to engage women in the intervention group $(n=21)$ with resources.

In a cluster-RCT, Gupta 2017 randomised 42 public health clinics in Mexico City to intervention or control arms. The intervention group ( $n=470$ women) received a nurse-delivered session of IPV screening, supportive referrals and health/safety risk assessments, and enhanced nurse-delivered advocacy if needed. A booster session was given after three months. The aim was to reduce IPV and reproductive coercion, and improve levels of safety planning behaviours, use of community resources and mental well-being. Control clinics offered women $(n=480)$ screening for IPV and a referral card. Follow-up was post-intervention and at three and 15 months from baseline. Kiely 2010 recruited 1044 women to an RCT comparing the intervention $(n=521)$ to usual care $(n=$ 523). The intervention provided advocacy within the context of two possible further (and separate) interventions, depending on the abused woman's risk profile: cognitive behavioural therapy (CBT) if depression was a risk, and smoking cessation/reduction/passive smoking sessions if smoking or environmental tobacco smoke exposure were identified risks. A subset of 336 women were abused at baseline (169 intervention group, 167 control group). There was no follow-up after completion of the intervention. In a multicentre RCT described over three papers, including the protocol, Lako 2018 compared Critical Time Intervention (CTI), a time-limited (nine-month) outreach intervention, to care-as-usual for abused women making the transition from shelter facilities to supported or independent housing. Participants were recruited from 19 women's shelters in the Netherlands, with questionnaires administered before leaving the shelter and at follow-up at three, six and nine months after leaving the shelter (that is, at end of the intervention). Altogether 136 women were assigned to CTI $(n=70)$ or care-asusual $(n=66)$. In one of the two analysis papers, Lako 2018 used intention-to-treat, three-level, mixed-effects models. The second paper for the same study (Lako 2018), explored fidelity and factors influencing adherence, from chart review $(n=70)$ and two focus groups with CTI workers $(n=11)$. McFarlane 2004 evaluated a telephone intervention intended to increase the safety-promoting behaviours of abused women, with a total of 54 minutes of advocacy being given (six, nine-minute telephone calls) over eight weeks, as well as usual services of the District Attorney's office. This $\mathrm{RCT}$, randomised 75 women to the intervention and 75 to usual care (usual services of the District Attorney's office), with followup calls to assess safety- promoting behaviours at 3,6, 12, and 18 months. McFarlane 2006 completed a two-arm RCT in urban public primary care clinics with 360 abused women who had experienced physical or sexual abuse within the preceding 12 months. The study tested two interventions: a wallet-sized referral card and a 20-minute nurse case-management protocol, with follow-up to 24 months. Rodgers 2017 assessed the feasibility, acceptability, and safety of Community Health Worker (CHW) outreach co-ordinated with enhanced clinic-based IPV advocacy services from a Family Health Advocate (FHA) trained in IPV advocacy and motivational interviewing. The setting for this RCT was four urban community health clinics. Women were randomised to either the $\mathrm{FHA}$ plus $\mathrm{CHW}$ intervention $(n=10)$ or the clinic-based FHA intervention alone $(n=$ 9). As this was a feasibility and acceptability study, Rodgers 2017 do not report clinical outcomes but the experiences and needs of the abused women, drawing on the CHW's field notes and structured interviews with the 10 women in the intervention group.

Hyman 2001 assessed the impact of emergency departmentbased advocacy that included empathic support, safety planning, linkage with community resources, and follow-up. Hyman 2001 randomised 100 women who screened positive for IPV to either the intervention or standard care. Follow-up $(n=53)$ was at three to four months.

Bybee 2005 undertook longitudinal evaluation of women who were leaving a domestic violence shelter after at least one night's stay and were randomly assigned 1:1 to usual care or to four to six hours per week of one-on-one advocacy for 10 weeks. Trained undergraduates undertook advocacy and the focus was on assisting women in accessing needed community resources. Follow-up was immediately upon exit, and at 10 weeks and 6,12 , 18 , and 24 months post-intervention and at 36 months (considered in different papers). The initial sample was 141 women with further data collection resulting in a total sample of 278 women interviewed every six months for two years and a subset of 124 women re-interviewed at three years. A mediation analysis of the data was reported in one of the six papers on the study included in this review. Sullivan 1991 also studied a more intensive advocacy programme in which services were provided by "trained paraprofessionals" for six to eight hours per week over 16 weeks. In a similar study, Sullivan 2002 recruited abused mothers with at least one child from a mid-sized urban city, either after they had exited a domestic violence shelter programme $(79 \%)$ or when they obtained services from a community-based family service organisation (4\%) or a state Social Services department (18\%) (figures taken directly from the report). Mothers randomised to the intervention group ( $\mathrm{n}$ $=80$ ) received the free services of a trained paraprofessional for six to eight hours per week over 16 weeks for IPV advocacy, with an additional 10-week support and education group for the children. They did not specify the control group intervention. Follow-up was to four months after the end of the intervention.

Tiwari 2010 evaluated an empowerment intervention with a complementary focus on accessing resources in an RCT involving 50 women in the intervention arm and 50 in the usual care control group. This intervention included information and education concerning risks of IPV and safety, the cycle of violence, legal protection orders, filing for criminal charges, and community resources, paired with an individualised safety plan. Follow-up was for six months.

Wong 2013 undertook an assessor-blinded RCT of 200 Chinese women with a history of IPV, in a community centre in Hong Kong. The intervention group $(n=100)$ received a 12-week empowerment advocacy intervention with telephone social support. The control group $(n=100)$ received usual community services including child care, health care and promotion, and recreational programmes. Data were collected at three and six months. This study was described across three papers, one of which reported a secondary analysis of the data, considering the impact of immigration status of 60 immigrant women on the efficacy of the advocacy. 


\section{Studies of existing interventions}

Many of the interventions assessed were new and non-established modes of care that the study authors had developed. The exceptions were O'Brien 2016, Casey 2007, Coy 2011. Davis 2006, Davis 2007, Hathaway 2008, Howarth 2016, Infanti 2015, Kelly 1999, Lea 2016, Logan 2018, Macy 2018, Stover 2010 and Thiara 2009, and a trial that evaluated two modes of interventions already in regular use in an emergency room (Hyman 2001). Some of these studies were qualitative and we have considered them above.

Amongst the others, four studies were service evaluations of existing non-police-based interventions. O'Brien 2016 described an internal evaluation of the Doncare Angel for Women Network (DAWN), which trains volunteer mentors to support women in recovery from IPV. Women are routinely asked to complete mental health inventories before and after their DAWN experience and entry and exit interviews are conducted with women and volunteers. Thiara 2009 evaluated the Refuge Southwark Advocacy Project (RSAP), which focused on the provision of court-based support to women going through the criminal justice system. The RSAP was staffed by a full-time court-based advocate, a sixmonth, part-time advocate of black, Asian and minority ethnicity (BAME) and a National Advocacy Manager. The mixed-methods evaluation included interviews and discussions with all strategic and operational project staff and other multi-agency players (n $=15$ ), observations of monthly project team meetings, data from other agencies such as the police and the courts, as well as data specific to the project, and a detailed feedback survey on the service ( $n=38$ women). Coy 2011 evaluated four Independent Domestic Violence Advocacy (IDVA) schemes in London, each based in a different setting: a police station; a hospital emergency department; a community-based domestic violence project; and a women-only violence against women (VAW) organisation. They used a mixed methods approach with prospective case tracking using a bespoke database across all four schemes ( $n=748$ cases); two rounds of interviews with IDVAs and scheme managers ( $\mathrm{n}$ $=27$ ) and with members of local Multi-Agency Risk Assessment Conferences or groups (MARACs; $n=44$ ); observations of IDVAs' work in their own settings and each MARAC; expert interviews with national informants $(n=4)$; questionnaires and interviews with service users $(n=73)$. Howarth 2016 undertook a multi-site evaluation of existing IDVA services for women experiencing severe abuse. The data were collected from women $(n=2427)$ at the point of referral to one of seven IDVA services over 27 months who consented to analysis of their case management data. Data from $48.1 \%$ of women $(n=1167)$ were also collected at the closure of a case or after four months of engagement with the service as an interim marker of case progress (whichever came first). IDVAs also conducted short interviews with 411 women on their exit from the service about the factors that had impacted on their safety during the intervention. Thirty-four women were re-contacted six months after the closure of their case to examine the maintenance of any changes to safety and well-being.

Five studies considered existing advocate-police partnerships (Casey 2007; Davis 2006; Davis 2007; Kelly 1999; Stover 2010). The Domestic Violence Home Visit Intervention (DVHVI) in the USA provides advocate/police officer team home visits following a call to police about IPV as an established service. In four included papers, Stover 2010 assessed 52 women given the DVHVI and 55 controls at 1,6 , and 12 months following a police-reported domestic incident to measure repeat violence, service utilisation, and symptoms. In one of the papers, in a multiple regression analysis, Stover 2010 assessed factors affecting engagement with the DVHVI. They analysed a sample of 204 domestic violence cases referred to the advocate in the DVHVI programme from five police districts during an 18-month period. They particularly considered ethnicity and ethnic matches of women, advocates and police officers. Casey 2007 evaluated an extension of this intervention (the Child Development-Community Policing DVHVI (CD-CP DVHVI) and compared two groups: a) 204 women requiring a police response for IPV who were given the intervention and $b$ ) a group of 102 matched controls sampled from domestic violence cases in police reports after receiving standard police intervention. Intervention group women received law enforcement-advocacy services through a home-visit project conducted by neighbourhood patrol officers and IPV advocates. Results from Casey 2007 led to the Stover 2010 study.

Davis 2006 reported outcomes from a series of analyses of the Domestic Violence Intervention Education Project (DVIEP), a similar home visit intervention involving a social worker and a police officer, but with intervention sites receiving education about family violence, via leaflets through house doors, brochures and posters in communal areas, and at public meetings. In one analysis, the sampling frame was households in designated public housing units in Manhattan where someone had called the police in response to a family violence incident ( $42 \%$ were IPV). Only $7 \%$ of the incidents resulted in arrests, and just $14 \%$ of victims reported any form of injury. Davis 2006 randomly assigned 435 victims to receive a home visit as a follow-up to the initial police response. The control group (number not stated) received only the initial police response. The research team tracked both groups for additional calls for police services over the next six months. Another similar analysis, also in a public housing setting, examined 197 cases (69\% IPV) that involved an arrest and on top of a home visit, the intervention involved community education. They informed victims about services and legal options, especially restraining orders, and made referrals. They provided on-the-spot crisis counselling when indicated. In cases where the complainant was not home in two tries, they left literature or made telephone contact with the household, or both. They interviewed the study participants at the end of the tracking period. Another study, by Davis 2007, evaluated a similar secondresponse intervention involving an officer who was trained in domestic violence advocacy and who tried to ensure that the victim had information about resources and services, practical assistance, a safety plan, and instructions on how to document future abusive or stalking behaviours, as well as a written description of local resources, including housing relocation, counselling, domestic violence shelters, medical help, civil legal assistance, information about the criminal justice process, aid in applying for an order of relief, and emergency financial assistance. Victims were assigned to either a one-day response or a seven-day response and there was also a control arm with no second response.

Kelly 1999 evaluated a pilot crisis intervention for domestic violence undertaken by lay advocates within the police service. This included participant observation; in-depth interviewing; project database creation and maintenance; and questionnaires. Police officers, service users and local agencies were surveyed. An action research model was used, in order that findings could inform subsequent intervention development. 
Interventions with additional components that were not focused on the woman or on her abuse

Four trials included components in addition to advocacy that may have impacted the outcomes reported. The first of these included advocacy and education or support for all the children of mothers who had been abused (Sullivan 2002), while the second and third provided parenting support to all the abused mothers (Prosman 2014; Taft 2011). The fourth study was more complex, providing advocacy within the context of three possible further (and separate) interventions, depending on the abused woman's risk profile: cognitive behavioural therapy (CBT) if depression was a risk, and smoking cessation/reduction sessions if smoking or environmental tobacco smoke exposure were identified risks (Kiely 2010).

\section{Studies not fitting our core inclusion criteria but adding valuable information and hence included in the review}

As we explained in Methods, in line with realist principles, we included some studies that did not fit our core inclusion criteria because we determined their usefulness during our theorybuilding phase, Where there are gaps in theory and CMOs, a wider body of literature may provide important contributory information.

Constantino 2005 is one of the additional 10 studies thus included. It was a core paper in Rivas 2015 but in this realist review, we did not treat studies of group-based advocacy as core, since group work depends on a specific range of behaviour-change techniques (Michie 2013). Constantino 2005 undertook an RCT to evaluate the feasibility and effectiveness of a group intervention that allowed for interaction between women, as well as interaction between the women and the advocate. The study authors labelled this a "social support intervention". Given to 24 women in a shelter in the USA, it focused on accessing community resources, including information on resources and hours of operation for those resources when available. There was no follow-up beyond the intervention. Taha 2015 undertook an RCT to explore the intervention outcomes and mechanisms of a culturally informed intervention, the Grady Nia Project, designed for low-income, African American women with a history of intimate partner abuse and suicide attempt. The intervention was group-based and therefore not fitting our definition of advocacy. However, the study authors used multivariate general linear modelling with mediation analyses of potential mechanisms and outcomes that was relevant to this review, using data from 89 women who completed both preand post-intervention assessments. We included three papers from this project as a result.

Eight studies considered domestic violence services overall (including advocacy) without stratification by type of service so that we could not separate out advocacy data. These studies used a variety of designs. Lyon 2011 collected survey data over nine months from 1408 women and 68 men in 90 community domestic violence programmes in four US states with maximal geographical, population and economic diversity. Only $36 \%$ of the women had received advocacy and without separate analysis, which is why this study did not fit our core criteria. They also held 10 focus groups with 73 of the women. In relation to the programmes, $38 \%$ were independent domestic violence programmes and 23\% were standalone dual domestic violence and sexual assault programmes and $61 \%$ were part of a national social service or community agency. Over half had an annual budget of less than USD 500,000 and 21\% were culturally specific. McDermott 2004 undertook a process evaluation of the services offered by a rural, co-ordinated, interagency programme in the USA, when responding to women who reported an abusive episode to the police. They primarily used qualitative methods (document analysis, unstructured interviews, observations) to evaluate the different components including victim advocacy, a domestic violence clinic at the local law school, community support groups, and police/probation victim safety checks. As such they did not focus on advocacy, but they made several points that are relevant to our research question. Agencies such as a municipal police department, a university public safety department, a county state's attorney's office, county probation, and a local law school delivered the different components. After this intervention ended, a not-for-profit women's centre sent women postal invitations to follow-up advocacy. Perez 2012 collected data over approximately four years from 227 residents of two shelters in a medium-sized city in the USA and used this to explore the impact of resource acquisition and empowerment on the relationship between IPV and PTSD using hierarchical regression. Services offered by the shelters included emergency housing and access to basic resources, case management, support groups, and advocacy programmes. One shelter provided emergency crisis stabilisation, whereas the other provided apartment-style transitional living spaces. Women completed validated outcome questionnaires with the support of a researcher. Zweig 2007 also did not evaluate advocacy itself, but rather community services in general (hence including advocacy) for women who had experienced domestic violence or sexual assault. This was part of the US STOP Violence Against Women Formula Grants Programme, which aimed to improve community responses. This study undertook a telephonebased survey of agency representatives in eight states (five communities per state), chosen because their state-level STOP administrative offices had emphases on creating co-ordinated community responses (CCRs) or collaboratives, which was the study focus. Analysis considered data from 90 representatives of 26 agencies. This was followed by 1509 telephone interviews with women from selected communities served by the 26 agencies; they asked women to rate services on a number of variables. Sullivan 2018 considered the impact of trauma-informed practices (not specifically advocacy) on residents in one of four shelters in Ohio, USA, using a pre-post design. They took baseline data from 57 women on average 11 days into residents' shelter stays (mean = 11.19; standard deviation $(S D)=7.98$ ) and then followed them up approximately 30 days later or at shelter exit if they had stayed in the shelter at least two weeks. They also undertook secondary analysis of survey data completed at two points in time by 565 shelter residents. The original research involved 215 domestic violence programmes across eight states in the USA, and shelter residents completed surveys shortly after they arrived in shelter and again as they were close to exiting. The focus of this study was on women's needs rather than advocacy. Feder 2018 evaluated a modification of Olds' Nurse-Family Partnership (NFP), a nursedelivered home-visiting programme for young, disadvantaged pregnant women in the USA. They added an IPV component comprising the McFarlane 1997 brochure-driven safety planning intervention plus a skills-based curriculum on relationships. Feder 2018 delivered NFP over 2.5 years and also included content relating to the development of the child. Then they randomly assigned 240 women to either the control arm (NFP as usual) or the IPV preventive intervention embedded into NFP (NFP+). They did not consider data separately for the IPV component.

We also included two studies that did not consider IPV but that provided useful information on advocacy and its mechanisms. One 
of these, by Goodman 2009, considered a US programme called ROAD (Reaching Out About Depression). This was designed for women on low income with depression rather than IPV but the exploration of its advocacy element was useful in developing our theory. ROAD paired a mental health counselling student volunteer (master's level) with an interest in social issues with a woman eligible for support. Advocate-woman teams worked together in flexible ways at individual, interpersonal, familial and systemic levels, and advocates provided emotional and instrumental support. The main aims were to alleviate the women's acute crises (e.g. threatened evictions, loss of benefits), and to facilitate their short- and long-term goals, with the women's needs kept central. Advocates often accompanied the woman to community agencies. Pairs met weekly for four to six hours each week for nine months, after which, women had the option of continuing with a new advocate. Goodman 2009 interviewed seven women who had received support from an advocate (two for one year and five for two years) and had subsequently become workshop facilitators within the larger ROAD intervention, six months to three years before interview. In the other study in this category, Scheer 2018 explored mechanisms mediating the relationship between trauma-informed care (TIC) and health in 227 LGBTQ adults, using structural equation modelling (SEM). They recruited participants from national and local online fora and listservs and hence these findings are not relevant to advocacy interventions. Trauma was related to sexuality, and participants were not all women, though all were abused. Nonetheless we have included this study because it provides information on mechanisms of TIC that accords with studies of woman-focused advocacy.

We considered many other studies that explored advocacy but not IPV, or co-ordinated services rather than advocacy per se but these other studies were not able to contribute in any depth to the emerging theory and we did not, therefore, include them in our final selection. We had several meetings to discuss this point, early in the review process. Those studies and articles whose full texts we considered before deciding not to use them are listed in the section on Characteristics of excluded studies. Thus we selected the 10 studies described in the current section as part of a systematic search strategy. Other similar articles may be relevant that we have not considered but we stopped searching when we believed we had sufficient information as per realist approaches, rather than including such additional studies exhaustively. This means that we excluded some studies because they did not entirely match our criteria and we had already gained any relevant additional information they contained from the studies cited here.

\section{Countries in which the studies were set}

This section gives a summary overview of the spread of countries in which studies were set, before we describe the realist synthesis.

SIx studies came from Canada (Bader 2014; Burnett 2012; Hughes 2017; Kenyon 2016; Tutty 1996; Wuest 2015). Four studies came from Australasia; three took place in Australia (O'Brien 2016; Owen 2015; Taft 2011), and one in New Zealand (Sudderth 2017). Three studies by the same team took place in Hong Kong (Tiwari 2005; Tiwari 2010; Wong 2013). There were two studies in Taiwan (Song 2010; Song 2012), and one study apiece from Japan (Umeda 2017), and Sri Lanka (Infanti 2015). Two studies came from South and Central America; one took place in Mexico (Gupta 2017), and one in Peru (Cripe 2010). In Europe, two studies were from the Netherlands (Lako 2018; Prosman 2014), and one apiece from
Turkey (Bahadir-Yilmaz 2018), Spain (Briones-Vozmediano 2014), and Sweden (Ekstrom 2015). There were seven studies from the UK (Bacchus 2007; Coy 2011; Howarth 2016; Kelly 1999; Lea 2016; Thiara 2009; Trevillion 2013). The remaining studies $(n=67)$ were from the USA.

\section{Ethnicity of study participants}

This section gives a summary overview of the ethnicity of study participants, before we describe the realist synthesis. However ethnicity and cultural considerations are also highlighted within the realist synthesis in later sections.

\section{Experimental intervention studies}

A considerable amount of data has been collected about advocacy in women from BAME groups. Only four of the intervention studies we considered, for example, recruited mostly white women $(70.8 \%$ of the total sample in Constantino 2005; $79 \%$ in Curry 2006, 2/3 in stage 1 and 3/4 in stage 2 of the study by Kulkarni 2015; 70.4\% in Sullivan 2018).

In six other studies, the mix of ethnicities was unclear. Thus, in one study set in Australia, the numbers of indigenous and nonindigenous Australians was not specified: $17 \%$ of those recruited to the intervention arm and $7 \%$ of those recruited to the control arm were born in Vietnam, and $17 \%$ and $32 \%$ in the intervention and control arms, respectively, were born in countries outside Australia (Taft 2011). Ethnicity data were not given for Casey 2007, Feder 2018, Thiara 2009 or Wuest 2015. In Tutty 1996, 8/60 of the women were of aboriginal origin, and others had recently immigrated from countries such as the Philippines, India and Germany.

In the majority of intervention studies, minority women predominated or formed the entire study population; generally there was a mix of white, African American and Latina women, reflecting that the studies had been undertaken in the USA. Basic details of these studies are as follows: in Bybee 2005, 46\% of participants were white; in Coker 2012, 69\% of women in the intervention group and $56 \%$ in the control group were AfricanAmerican (no other data given); in DePrince 2012, 58\% of women were from ethnic minority groups; in Garcia-Leeds 2017, four participants were from Puerto Rico and the remaining three were born in the USA (the parents of one of these came from Puerto Rico); in Gillum 2009, 83\% of participants were African American; in Goodman 2016a, only 38\% of participants self-identified as white; in Hyman 2001, 32\% of participants were white; in Kendall 2009, $74 \%$ of participants were from minority ethnic groups; in Krasnoff 2002, 66\% of participants were from minority ethnic groups; in Merchant 2015, 37\% of participants reported their ethnicity as 'Caucasian' (white), seven (37\%) as Hispanic, and 26\% as AfricanAmerican); in McFarlane 2006, $12 \%$ of participants were white and non-Hispanic; in McFarlane 1997, 35\% $(n=70)$ of participants were African American, 33\% were Hispanic (primarily Mexican and Mexican American), and 32\% were white; in McFarlane 2004, 25\% of the intervention group and $28 \%$ of the control group were white; in Muelleman 1999, $75 \%$ of the intervention group and $61 \%$ of the control group were black ( $P=0.05$ ); in Perez 2012, 37\% of participants were white; in Sullivan 1991, 56\% of participants were white; in Sullivan 2002, $49 \%$ of participants were white; in Stover 2010, 13\% of participants were white; in Thiara 2009, 31\% of participants were white British;and in Trevillion 2013, $62 \%$ of participants were of BAME origin. In Bacchus 2016a, 86\% of the urban site participants were African American compared with $23 \%$ 
at the rural site (difference, $P<0.001$ ), whereas in the linked qualitative study, $47 \%$ of women self-defined as not white. While 26 women were interviewed, four non-participant observations were conducted with four African-American women who preferred this to the interviews facilitated by their African American home visitor. Results were combined and there appeared to be no overall difference in themes. Lyon 2011 compared those born outside (9\% white) versus within ( $63 \%$ white) the USA; the remainder came from a range of ethnic groups.

Remarkably, these studies mostly did not consider ethnicity or cultural factors in their results. There were eight exceptions amongst those mentioned above. Kendall 2009 tested for and found no correlation between race and percentage of safety plan completion. DePrince 2012 found that outreach was particularly effective in ethnic minority women. Bybee 2005 reported that there was no clear difference in effects due to race. Goodman 2016a showed different effects of advocacy by race or ethnicity. Lyon 2011 reported differences between women born inside and outside of the USA in use of services, needs and outcomes. Lako 2018 found that the proportion of Dutch-speaking women with unmet care needs declined from $88 \%$ before the intervention to $57 \%$ after, while the proportion of non-Dutch-speaking women with unmet care needs declined from $100 \%$ to $90 \%$. The study authors considered this to reflect the greater barriers to resource use for migrants and their greater number of unmet care needs at baseline. Kulkarni 2015 found that race was negatively associated with a survivor-focused approach but that this did not reach statistical significance. McFarlane 2004 found that use of police and resources varied by ethnicity: a lower percentage of Hispanic women than African American and white women used the police and related resources. Stover 2010, using multivariate analysis of covariance (MANCOVA) found no differences in the number of visits requested or received in outreach based on victim ethnicity in their sample ( $F$ statistic: $F=0.02 ; P=0.98)$. They entered ethnicity and perpetrator criminal histories into repeated measures models and showed no effect on abuse scores over the 12 months of follow-up.

In five studies of interventions, at least $90 \%$ of participants were from single, minority ethnic groups, including a study reporting on Latina women (McFarlane 2000), and four recruiting African American women (Bell 2001; Kiely 2010; Rodgers 2017; Taha 2015). One study included women of both African American (56.5\%) and Hispanic (35\%) descent (Stover 2010). Two other studies had no white participants but a large range of other ethnicities (Prosman 2014; Sullivan 2002), but they did not consider their findings in the context of the women's minority status.

Five studies considered the majority ethnic groups in countries other than North America, the UK and Australasia: one with Turkish women (Bahadir-Yilmaz 2018); one in Taiwan (Song 2010), and three in Hong Kong (Tiwari 2005; Tiwari 2010; Wong 2013), in which all participants were Chinese. A further trial in Peru predominantly (87\%) recruited women identifying their ethnicity as Mestizo (Cripe 2010). Participants in the study by Gupta 2017, which took place in Mexico, were mostly ( $80 \%$ ) born in Mexico. These studies provided general contextual background on ethnicity and abuse but only Cripe 2010 and Song 2010 discussed their findings in light of this. Cripe 2010 noted that their intervention was similar to that used by Tiwari 2005 in Hong Kong but unlike Tiwari 2005 did not result in improved emotional and physical well-being (assessed by the role-physical and role-emotional subscales of the SF-36 (Garratt
1993)). Cripe 2010 suggested that cultural differences in exposure to abuse and coping mechanisms as well as types and extent of social support had led to different outcomes. However, there were differences in study design also; Tiwari 2005 measured depression as an independent psychological outcome whereas Cripe 2010 did not.

\section{Other studies}

Among the qualitative studies of professionals, the Infanti 2015 study took place in Sri Lanka and considered only Tamil women. This study was unusual in considering a specific agricultural setting, tea plantations. Sudderth 2017 reported that New Zealand provided a unique setting for domestic violence interventions because of its formal recognition of the Māoris in all its national policy initiatives. Sudderth 2017 described the National Collective of Independent Women's Refuges (NCIWR), the umbrella organisation for most domestic violence shelters in New Zealand, as a model of good practice in running services that are culturally sensitive to the needs of Māori women, integrated with but often distinct from other services. While Sudderth 2017 explored the provision of NCWIR services for Māori women in depth, they considered that Pacific Island refuges and services were not adequately represented in their sample. Five of the staff in GarciaLeeds 2017 were fluent in Spanish and two could hold a basic conversation in Spanish; four were from Puerto Rico, as were the parents of a fifth - this reflected the demographics of the service users they served. Dunn 2007 gave no information on ethnicity of the women seen by staff but 27/32 staff were white. In Donnelly $2005,57 \%$ of women seen by staff were of non-white ethnicity. In Ganz 2015, 19/21 staff were white. Considering Murray 2015, 37/62 staff were white. In Goodman 2016b, 27/28 advocates were white. In Johnson 2014, 76\% of staff were white. In Merchant 2015, 7/19 staff were white, while Sullivan 2019 reported that $4 / 11$ advocates were white in their study. In Wood 2014, 14/22 advocates were white. Since English was a second language for most staff in Kapur 2017 it can be inferred that they were not native Americans.

Three survey-based studies of professionals explicitly considered mainly white staff (Babin 2012: 71\%; Bemiller 2010: 81\%; Slattery 2009: 77.6\%).

Considering studies exploring the experiences of abused women, in total $46 \%$ of women in Bacchus 2016b were described as white. In Bader 2014, most service users (69\%) identified as white. Reina 2015 only considered migrants from Mexico and Central or South America. Song 2012 only surveyed women from Taiwan. In Ekstrom 2015, two women were Middle Eastern, one was Eastern European and three were Swedish. Women in the Goodman 2009 study were predominantly from minority groups. In total, $51 \%$ of the 49 women recruited by Hathaway 2008 were white (non-Latina) and 35\% were Latina. In Kenyon 2016, only two women self-identified as white. Hughes 2017 in Canada interviewed two women who identified as white and of European descent, one who identified as white, another as German, one as Mennonite, and one as Métis. Gillum 2008 only considered African American women. In Lea 2016, 93\% of women self-identified as white British. In a survey-based study by Perez 2012, 37\% of respondents were white, and in a survey by Zweig 2007 80\% were white. In Scheer 2018, 60\% of participants were white. These studies did not explore issues around ethnicity in their analyses. Other studies did not provide ethnicity data.

We included one systematic review: Shorey 2014. 


\section{Excluded studies}

We excluded 47 reports (44 studies) after examining the full texts. We excluded 17 studies (17 reports) because the interventions did not meet our criteria for being advocacy-based (Camacho 2008; Clarke 2013; Davidson 2012; Giocolea 2015; Goldblatt 2009; Lorenzo 2018; McFeely 2017; McLelland 2008; McNamara 1997; Miller 2011; Miller 2014; Simmons 2011; Valpied 2014; Zlotnick 2011) or were group advocacy interventions (Basu 2009; Kramer 2012; GrahamBermann 2013); 14 studies (17 reports) for lack of available data on the subset of results on partner abuse or the subset of women who had experienced abuse (Andrews 2011; Bair-Merritt 2010; Becker 2008; Bennett 2007; Carlson 2012; Champion 2007; El-Mohandes 2011; Juillard 2016; Kyegombe 2014; Nichols 2013; Rhodes 2014; Saggurti 2014; Samuels-Dennis 2013; Wagman 2015); eight because they did not test or explore advocacy itself (Donovan 2010; Feder 2011; Fox 2018; Hart 2013; Hovmand 2009; Mantler 2017; McKean 2004; Mitchell 2006); three because the women were not abused (Cupples 2011; Howard 2017; Jack 2015); one because it was not associated with an intervention and was not useful in terms of our research questions (Anderson 2012); and one because the advocacy was for perpetrators (Denne 2012). Reasons for exclusion are stated by order of application of criteria so that some studies may have fitted more than one exclusion criterion.

Two further studies await assessment (Harris 2002; Shepard 1991).

Although we excluded El-Mohandes 2011 from this review, the relevant data were published in a subsequent paper that is included in the review (Kiely 2010); see Characteristics of included studies for further details. We did not consider sister papers of other studies if they were not relevant to our main questions but if we were able to exclude them from their abstracts they do not appear in our Characteristics of excluded studies list. Thus this review, unlike Rivas 2015, was not exhaustive in its inclusion if articles, in keeping with our realist approach.

For more information, please see 'Characteristics of excluded studies' tables.

\section{Risk of bias in, and quality of, included studies}

To assess the risk of bias in included experimental studies, we applied specific criteria (see Methods). Overall, we judged 25 of the 41 studies thus assessed to be at high risk of bias, in many cases because they used a pre-post design (Bahadir-Yilmaz 2018; Bell 2001; Casey 2007; Constantino 2005; Davis 2006; Davis 2007; Gillum 2009; Howarth 2016; Kendall 2009; Krasnoff 2002; McFarlane 1997; McFarlane 2000; McFarlane 2004; McFarlane 2006; Muelleman 1999; O'Brien 2016; Prosman 2014; Rodgers 2017; Song 2010; Stover 2010; Sullivan 2018; Taft 2011; Trevillion 2013; Tutty 1996; Wuest 2015). We judged seven studies to be at low risk of bias (Coker 2012; Feder 2018; Gupta 2017; Kiely 2010; Lako 2018; Tiwari 2005; Tiwari 2010). The remainder (nine studies) were at moderate or unclear risk of bias, with selection bias, lack of blinding, risk of contamination, unreliable outcome measures and attrition all common reasons.
We used a separate 'Risk of bias' checklist for other quantitative designs. Overall, we judged four of the eight survey-based studies to be at high risk of bias (Bemiller 2010; Lyon 2011; Slattery 2009; Zweig 2007), because they had not piloted their survey, and in two cases (Bemiller 2010; Zweig 2007), had not validated their instruments. Three were at moderate risk of bias (Babin 2012; Goodman 2016a; Kulkarni 2015), because of lack of representativeness of the sample or missing data and a lack of clarity over other criteria. Two (Perez 2012; Scheer 2018), were at low risk of bias.

We used CASP for qualitative studies. Overall, we judged eight of the CASP-assessed studies to have high risk of bias (Coy 2011; Kelly 1999; Lynch 2013; Lyon 2011; McDermott 2004; Song 2012; Sullivan 2019; Weisz 1999). Coy 2011, Kelly 1999, McDermott 2004 and Lyon 2011 lacked rigorous research designs, Lynch 2013 did not report methods in sufficient detail, Sullivan 2019 and Weisz 1999 had a poor research or recruitment design and did not consider the researcher-participant relationship or ethical issues, and Song 2012 used case managers as researchers and other aspects were unclear. There were 13 qualitative studies at moderate risk of bias. Donnelly 2005 was at moderate risk of bias due to lack of ethical considerations; Ekstrom 2015 because the professionals they interviewed selected the abused women they also interviewed; Gillum 2008 and Hughes 2017 because results were skewed to positive viewpoints without justification; Johnson 2014, Macy 2018 and Murray 2015 because they did not consider the relationship between researcher and participants, and ethical issues, or they were unclear. Owen 2015 had only one analyst; Stylianou 2018 and Sudderth 2017 did not consider the researcher-participant relationship or use appropriate recruitment strategies; a qualitative methodology was not particularly appropriate for Thiara 2009; and in White 2019 and Wies 2008 there was a lack of general clarity over methods. We rated most qualitative studies as of low risk of bias (Bacchus 2007; Bacchus 2016a; Briones-Vozmediano 2014; Burnett 2012; Dunn 2007; Ganz 2015; Garcia-Leeds 2017; Goodman 2009; Goodman 2016b; Hathaway 2008; Infanti 2015; Kenyon 2016; Lea 2016; Logan 2018; Merchant 2015; Silva-Martinez 2016; Umeda 2017; Wood 2014).

It should be noted that, as this is a realist review, our focus was on the relevance of the data in papers, rather than study quality. Therefore, we do not report on quality in more depth here, but include additional tables in which we indicate our confidence in the findings for each essential principle.

\section{Theory development}

We first describe our testing and refining of the theory of 'empowerment' (or similar and related concepts) within advocacy and its influence in generating various improved outcomes in various contexts. We then develop an initial theory (Figure 2) and test this against the CMOs we have identified, before developing our final theory (Figure 3). 


\section{Figure 2. Initial theory}

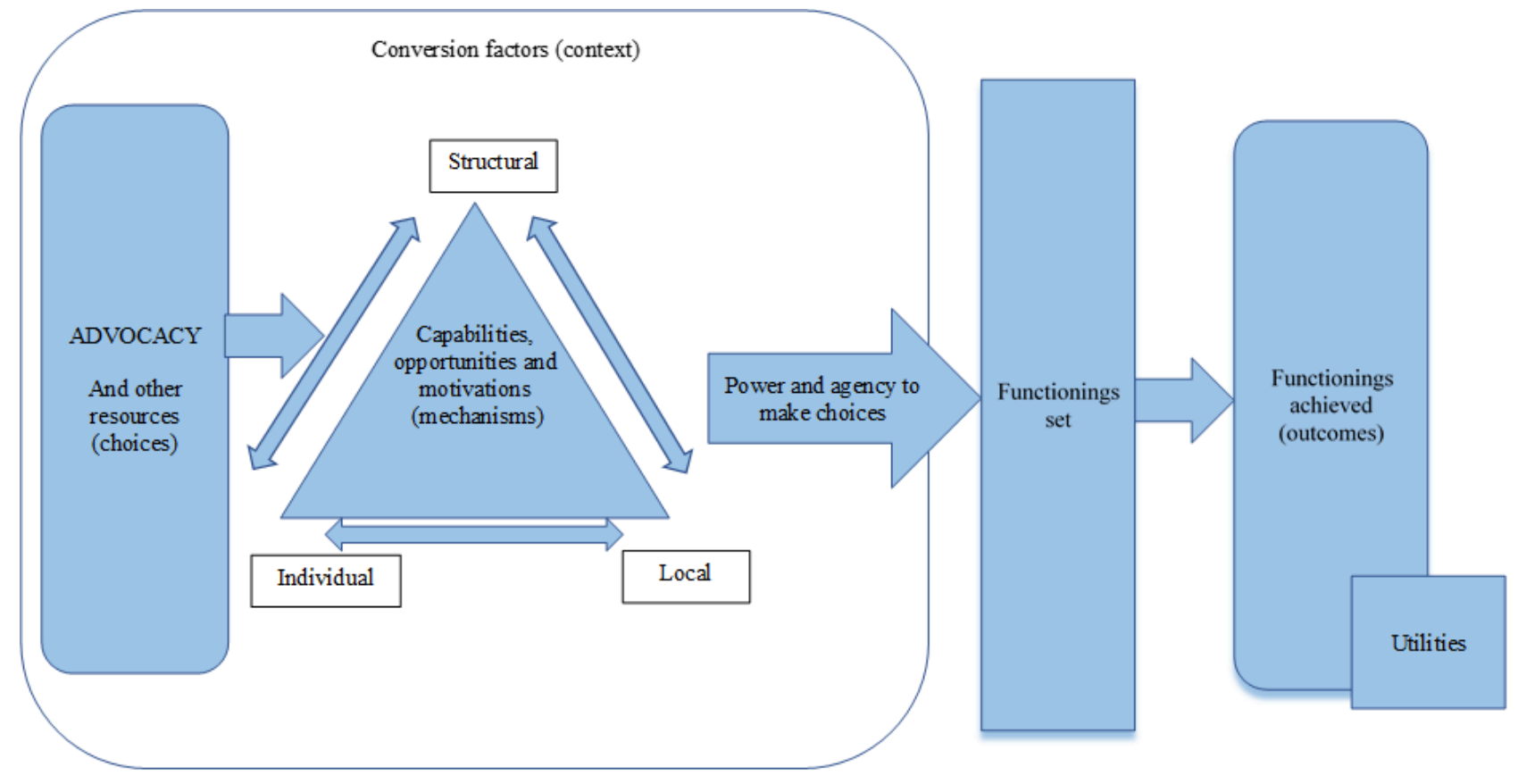

\section{Figure 3. Final theory}

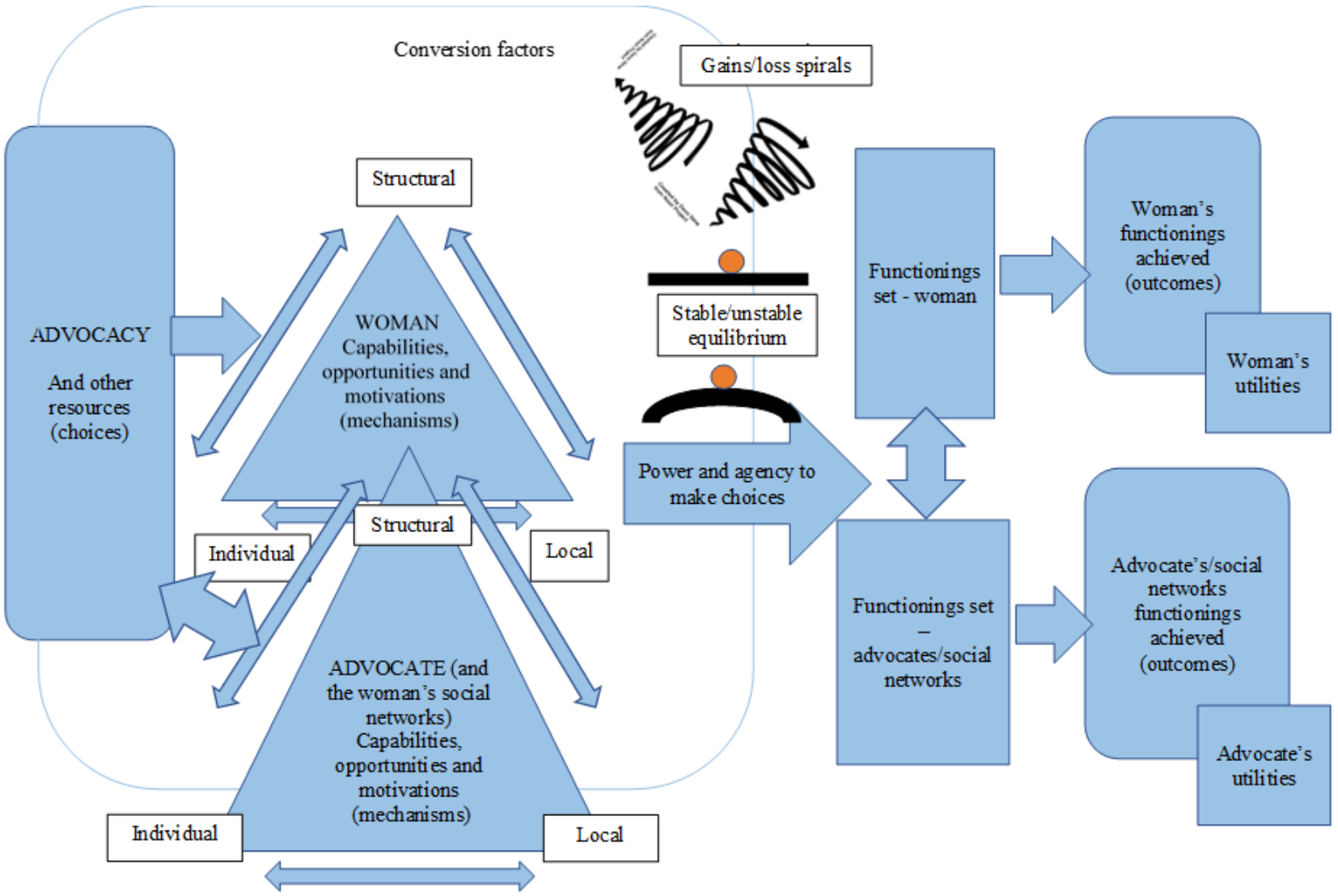




\section{Theoretical considerations of empowerment and testing against outcome}

Empowerment is complex. It can occur at the individual, organisational or community level although advocacy interventions are mainly focused on improving the individual's empowerment through mechanisms such as enhanced selfefficacy and self-esteem or sense of self-worth (Gibbon 2000; Laverack 2006; Wallerstein 2006), broadened social networks and good social support, knowledge and awareness (NICE 2008). These will in turn be developed through advocacy components such as education on relationships and coping skills and other personal skills that can enable abused women to develop greater physical, financial, human, social and natural 'capital' or capability 'assets' (Kabeer 1999), that give them the potential freedom to function outside of the abusive relationship. Individual empowerment interventions are based on the premise that they enhance women's sense of control when abuse aims to take this away. Self-efficacy is the belief in one's own ability to perform the actions needed to achieve desired goals (Bandura 1997). Selfesteem is considered a stable personality characteristic, which reflects an individual's overall sense of self-worth or personal value (Rosenberg 1965). This contrasts with self-efficacy or selfconfidence, which are related to an individual's belief in their ability to do something.

One aim of advocacy is to remove structural barriers for abused women, by supporting them as they access material, human, financial, physical and social resources. Kabeer 1999 describes empowerment as a process that gives the woman greater ability or agency to make strategic life choices relating to such resources. According to Kabeer 1999, resources and agency, when combined, equal capabilities, which will encompass self-esteem, self-efficacy and so forth.

Advocates may create a situation where empowerment may be more likely, through facilitation and support, but the individual is the only one who can realise this empowerment (Wallerstein 2006). It is perhaps for this reason that the mechanisms of effect in empowerment may also be measured as the outcomes of empowerment activities in advocacy.

Advocacy itself may be considered as a resource, one that it is assumed will enable and encourage and empower women to access other resources such as social welfare, housing services, education and employment services. It is assumed that the trade-offs of empowerment and divesting themselves of the abuse are less than the benefits that accrue. However, the women's responses to the advocacy resource will be affected by personal, local and structural factors.

These theoretical assumptions, with their reference to resources and capabilities, align with the capability approach of Sen 1985. The capability approach considers that people aim to achieve the best utility or quality of life they can, as affected by the core concepts of 'functionings' and 'capabilities'. Functionings are states of being and doing, such as being financially independent or going to work. A woman's capabilities represent her effective freedom to choose between different functioning combinations that she values. Resources (such as advocacy) are inputs whose value or impact depends upon an individuals' capabilities to convert them into valuable functionings (the mechanisms of CMOs in realist analysis) and the functionings that they have to choose from. This might be affected by individual factors (such as a person's disability, language ability, economic dependency), local factors (such as subsidies for housing, community norms and attitudes) and structural factors (such as policies and the funding of services); these are the contexts of CMOs in realist analysis. An individual's capabilities set is the set of valuable functionings that an individual has real access to. Achieved functionings (the outcomes of the CMOs in realist analysis) are those they actually select. Hence this framework assumes the individual has choices. Utility is considered as an output, because what people choose to do and to be affects their sense of subjective well-being. Utlity, in the form of subjective well-being (such as feeling happy), is also a valuable functioning in its own right within the capability approach. The relationships between the different elements of this framework are shown in Figure 4. Sen's concept of capabilities has been further developed into the capabilities space by Frediani 2010. This encapsulates an individual's choice, ability and opportunity to transform resources into achieved functionings and the individual, local, and structural factors that affect this. Thus, as our starting point for our realist theory, we developed a framework based on Frediani 2010's development of Sen's work and incorporating a modification (the addition of motivation) based on Michie 2011. The initial theory can be seen in Figure 2. 
Figure 4. Frediani 2010's development of Sen's work

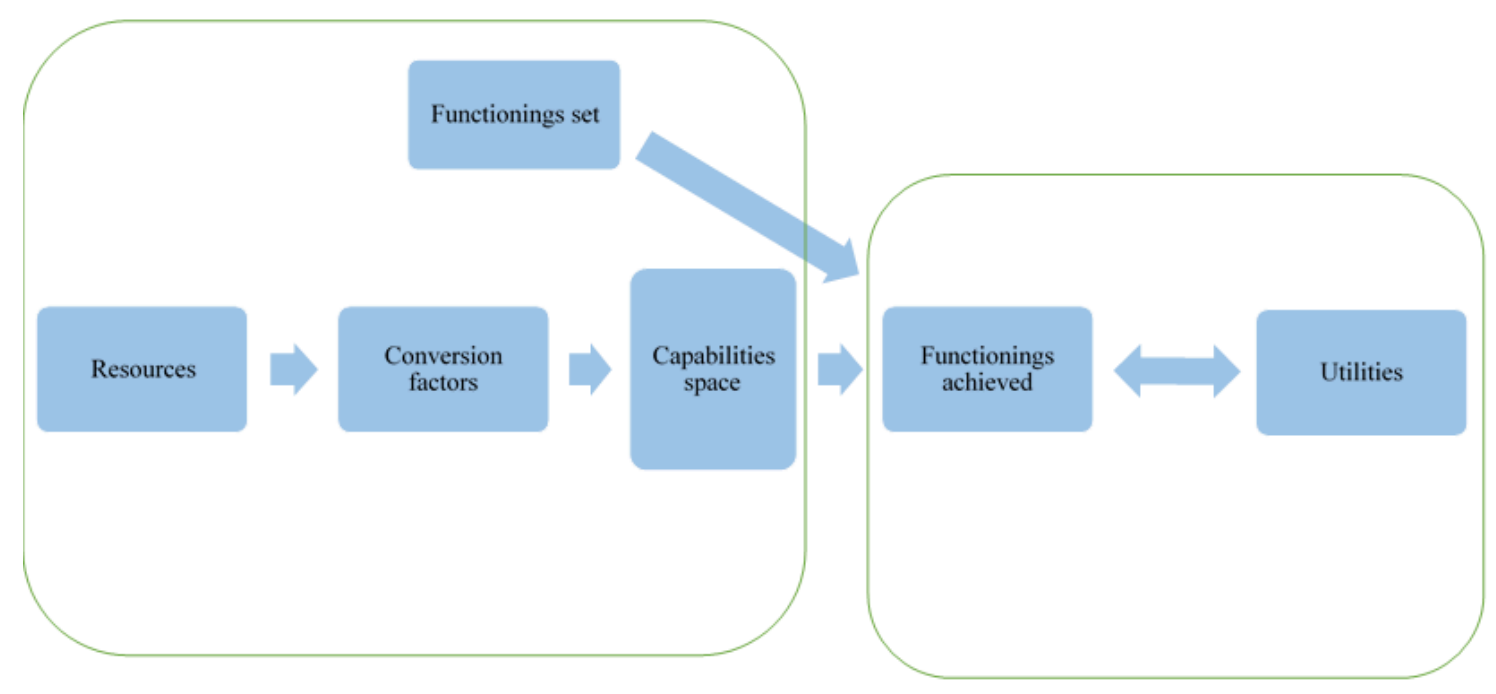

We tested this theory on the 98 studies we included in this review, with a cycle of testing and revision culminating in our final theory and hypotheses.

As suggested in the Background, we found that in all cases the advocacy provided in the studies we included was based on the concept of empowerment, meaning that our theory had general applicability. We also found that across studies, advocacy included education, advice on safety and accessing resources, and empathic support, all of which should improve capabilities. All of the interventions were pragmatic and survivor-focused in that they provided tailored services to meet the wants and needs of the individual women. Six studies cited use of the strengthsbased model (Bahadir-Yilmaz 2018; Lako 2018; Song 2010; Song 2012; Sullivan 2002; Taha 2015), of which all except Bahadir-Yilmaz 2018 were set within shelters, with study authors from a range of disciplines and backgrounds (primary and community care, social work, psychology). Goodman 2016a, Taha 2015 and Sullivan 2019 explicitly used a trauma-informed approach but Goodman 2016a focused on empowerment rather than trauma-informed approaches in their analysis.

Despite the commonality of empowerment across studies, there were implicit differences in researcher definitions of empowerment, or their foci on specific domains of empowerment, and these were reflected in the outcome measures used in the studies. Kenyon 2016 was the only study to explore how abused women themselves defined empowerment. Four interrelated empowerment processes emerged from that study: selfreflection; gaining clarity; acquiring knowledge; and building community. Three key features of shelters that were perceived to impact empowerment and could be measured as outcomes were: increasing safety; forming connections; and promoting self-efficacy (Kenyon 2016). Women's perspectives on what they needed therefore overlapped with what advocacy provided but included the desire for space for self-reflection, which may be seen as a mechanism for renewing self-worth. However it is important to note that women were rarely asked directly about their perspectives, with only seven studies focusing on them. The outcomes women most want are being considered further in a core outcome set development study being undertaken by researchers on intimate partner abuse in which Rivas is involved (with two workstreams, in the Children and Families Policy Research Unit (CPRU) (www.ucl.ac.uk/childrenpolicy-research), and the Violence, Abuse and Mental Health (VAMH) Network (www.vamhn.co.uk), which is funded by UK Research and Innovation (UKRI, formerly Research Councils UK (RCUK)). Perez 2012 operationalised empowerment advocacy for abused women in terms of: positive self-evaluation and selfesteem, a sense of personal control or self-efficacy, self-nurturance and self-care (capabilities or mechanisms); comfort/distress ratio (which may act as a mechanism, outcome or utility); gender role and cultural identity awareness (normative factors and capabilities); effective problem-solving skills, competent use of assertiveness skills, effective access to multiple economic, social, and community resources, gender and cultural flexibility and socially constructive activism (by improving women's achieved functionings or outcomes). This operationalisation thus cuts across the different domains of our model.

Apart from consideration of repeat abuse itself, which was seen as key to well-being, we were interested in testing what seemed to be a clear focus on outcomes associated with theoretical considerations of empowerment across all studies, as demonstrated in particular by the variables considered in several modelling studies. We explored these outcomes at the start of our review to see whether these demonstrated achieved functionings and utilities as a result of empowerment-based advocacy. We detail these here before moving onto our $\mathrm{CMO}$ configurations. 


\section{Finding 1: empowerment and psychosocial and well-being outcomes}

Feelings of helplessness, lack of self-efficacy, reduced mental well-being, and hopelessness were often equated with disempowerment, one or more of which were outcomes in many studies. Increasing women's capabilities through knowledge and skill development was not directly measured in studies, but these were routinely built up within intimate partner abuse advocacy interventions as specific components of practice. Hope involves reflection on a positive future and therefore can provide the motivation for changes in behaviour as well as being important to coping and well-being (Snyder 2002). Measurement of these outcomes is therefore consistent with our initial theory. But did they improve in practice?

Bybee 2005 was unable to find any improvement in self-efficacy after intensive advocacy in women exiting domestic violence shelters compared with controls. Other studies did not measure this. Two showed improved self-esteem after advocacy (BahadirYilmaz 2018, with a clear effect, $P<0.05$ (exact value not specified in the paper); Sullivan 2002 at the four-month follow-up (we are unsure how important this effect on self-esteem was because the results are imprecise).

Song 2010 and Prosman 2014 reported clear reductions in depression after advocacy $(P<0.05$ and $P<0.001$ respectively; exact values not specified in the paper). Rivas 2015 undertook a meta-analysis of dichotomous data from Gillum 2009 and Tiwari 2005 (both in healthcare settings) and showed that fewer women developed depression (number needed to treat for an additional beneficial outcome (NNTB) 4) if they received a brief advocacy intervention.

Wong 2013, in a secondary analysis of their original data (i.e. the research question was post hoc), compared abused legal immigrants from Mainland China to Hong Kong $(n=60)$ and abused, ethnically Chinese women who were born in Hong Kong or who had lived in Hong Kong for at least seven years $(n=137)$. Among the abused Chinese immigrant women, depressive symptoms in the intervention group were higher than that in the control group at baseline. However, at the nine-month follow-up, the converse was true. Immigration status influenced the intervention effect at three months $(P=0.02)$, but not at nine months $(P=0.74)$. In other words, at three months, the advocacy intervention reduced depressive symptoms among non-immigrant women, but not among immigrant women. At nine months the intervention also reduced depression in the immigrant women. Wong 2013 noted that the immigrant participants had severe depression at baseline (an individual contextual factor affecting the capabilities space in our model). Moreover, immigrant women would be likely to have fewer opportunities to make choices and possibly a smaller functionings set. This would all have had an impact on results and explains the more immediate effect for these women but also means longer follow-up might have been advised.

Perez 2012 determined that empowerment attenuates the impact of IPV severity on PTSD at low and moderate levels of violence $(P<0.001$; exact value not specified in the paper). In other words, empowerment advocacy is effective in reducing PTSD when violence is low to moderate in severity. Lako 2018 also showed that advocacy reduced symptoms of PTSD. Wuest 2015 found clear improvements in quality of life, mental health, capacity as indicated by mastery, PTSD and depressive symptom severity between baseline and six months, which were sustained at 12 months. The number of women with symptoms of clinical depression dropped from 32 at baseline to 20 at 12 months, as scored using the Center for Epidemiologic Studies-Depression (CES-D) 20-item scale (Radloff 1977), and the number with symptoms consistent with a diagnosis of PTSD dropped from a score of 22 to 14, as measured using the 17-item Davidson Trauma Scale (Davidson 1997).

There was evidence that advocacy made a clear difference to psychological distress and levels of perceived stress at three to four months' follow-up in the study by Hyman 2001 (see Rivas 2015).

There was evidence from only one paper of benefits in women's coping. Bahadir-Yilmaz 2018 reported some improvements in learned resourcefulness, self-confidence and ways of coping including social support seeking, all $P<0.05$ (exact value not specified in the paper). Song 2010 found their advocacy led to clinically and statistically significant improvements in depression, life satisfaction, helplessness and powerlessness (all $P<0.05$; exact value not specified in the paper) but did not improve coping strategies. They suggested that coping may take more time to change than the other measures, which would accord with our model, particularly if reduced depression is a mechanism rather than an outcome (e.g. leading to the utility-as-outcome of life satisfaction). Thematic analysis of in-depth interviews with the women and the case managers' evaluation summaries suggested that most participants experienced recovery of a sense of self, affirmation of the capacity and potential of their self, and action or realisation of their self in interactions with others, which they called the intrapersonal, interpersonal and transpersonal levels of self, respectively. Song 2010 categorised these levels of self as distal outcomes whereas in our initial theory the first two would be mechanisms (capabilities) and the third would be a functioning. Song 2012 further explored the impact of empowerment in data from a survey of 191 abused women who had received services - including but not exclusively advocacy - from the centres of Prevention and Intervention for Domestic Violence or from the private IPV sector in Taiwan. Empowerment had a direct effect on the two core outcomes considered, life satisfaction (beta $=0.49$ ) and change of self (which included self-affirmation - potentially linked to self-efficacy - and enhanced ability to use resources). In our model life satisfaction would be an outcome, and change of self a mechanism.

Taft 2011 reported clear improvements in women's physical quality of life after advocacy immediately post-intervention, but not their perceived mental quality of life. Pooled data from two studies, Bybee 2005 and Sullivan 2002, indicated a higher quality of life for women in the intervention group at 12 months (Rivas 2015) but Rivas 2015 reported that this did not extend to two- or three-year follow-up. Brief, one-off advocacy sessions to pregnant, abused women did not improve quality of life (Cripe 2010; Tiwari 2005). Lako 2018 found no differences between study arms for quality of life (a primary outcome) over their nine-month outreach work. These results accord with empowerment as a process, with changes taking time, but also that as mechanisms lead to outcomes, the status quo changes and different contexts may operate, different mechanisms may operate and thus different capabilities spaces may open out and different sets of functionings may become available (so that different achieved functionings, i.e. outcomes, may result). 
McDermott 2004 determined that women's age, income, social support, coping strategy, tendency of antisocial personality, and therapeutic relationship with the advocate were all contextual factors that affected well-being, life satisfaction and perceived changes of self of the participants in their study.

\section{Finding 2: empowerment and access to resources}

Tiwari 2010 used an empowerment intervention with a complementary focus on accessing resources in their communitybased study. This was based on Dutton's Empowerment model (Dutton 1992), combined with Cohen's Social Support Theory (Lakey 2000). Tiwari 2010 found that more women in the intervention group than in the control group had begun to engage in divorce processes by six months as one of their outcomes. It is possible that the empowerment intervention, which contained support on making choices from viable options and legal advice on divorce procedures, may have provided the women with the necessary skills, knowledge and resources (i.e. the capabilities) to take this action as the mechanism by which the advocacy worked. However, the intervention was not successful in improving other outcomes. Notably, Tiwari 2010 observed no clear benefit at six months' follow-up in terms of improved physical or mental quality of life. Tiwari 2010 noted that:

- they measured health and quality of life but the women may have been more focused on safety and accommodation;

- cessation of abuse through entry to the shelter may have already reduced depressive symptoms; and

- women in the intervention arm had worse relationships with their partners and experienced more life adversities compared to the controls.

In relation to our model, this can be restated as follows. There was a need for a greater choice of resources for the women before they could achieve improved quality of life, thus while the advocacy may have improved their capabilities (M), their opportunities (M) for achieved functionings $(\mathrm{O})$ were affected by contextual constraints (conversion factors; C). It would appear that divorce processes were more accessible than housing choices. This demonstrates how several mechanisms may need to be simultaneously at play for effect and how different resources may need different capabilities. In agreement with this, results from Bybee 2005 suggest that benefits in accessing most resources were only apparent at two years.

In considering resource use in healthcare settings, McFarlane 2000 employed a summary dichotomous measure of 10 resources, McFarlane 2006 used a summary continuous measure of a revised eight-item version, Gillum 2009 used a 15-item measure, and Cripe 2010 reported individual frequency data for seven items. These studies found no benefit from advocacy on post-intervention resource use. Indeed, in McFarlane 2000, the likelihood of accessing resources dropped below baseline levels in the control group (from $32 \%$ to $17 \%$ ), the advocacy group (from $33 \%$ to $17 \%$ ), and the group with access to a mentor mother (from 23\% to 21\%). These data suggest that advocacy in healthcare may not adequately enhance women's capabilities to access resources, or that access to healthcare support acts as a barrier to other sources of support.

\section{Finding 3: empowerment and safety-related behaviours}

Empowerment is said to be domain-specific; that is, people may be empowered in some parts of their lives but not in others (Cattaneo 2015). The safety domain is often considered a priority in IPV studies (Lyon 2011). Safety-related empowerment can be defined as having the power and control to keep safe from physical and emotional abuse (Cattaneo 2015

Goodman 2016a reported that greater safety-related empowerment was associated with fewer depressive and PTSD symptoms. A SEM analysis supported the hypothesis that safety-related empowerment mediates the association between therapeutic alliance and symptoms of depression and PTSD (Goodman 2016a). Thus, a good therapeutic alliance led to reduced depressive and PTSD symptoms through the mechanism of safetyrelated empowerment, but not on its own. This means that a woman's relationship with the advocate could act as a conversion factor (context) rather than a mechanism.

Stylianou 2018 tried to identify which elements in family court sessions with advocates and abused women were survivor-centred strategies or mechanisms for enhanced safety planning. She described the following elements as being important: open-ended exploration of risks and safety options; paraphrasing survivoridentified risks and safety plans; prioritising immediate risks; understanding safety strategies and resources; exploring support systems; providing survivor-centred information; and discussing the pros and cons of safety options.

Four trials investigated the use of safety behaviours that resulted from these types of mechanism; three recruited within a healthcare setting (Cripe 2010; Gillum 2009; McFarlane 2006), while one recruited within the community (Tiwari 2010). Rivas 2015 pooled data from Gillum 2009, McFarlane 2006 and Tiwari 2010 and found that at follow-up of 12 months or less, when compared with controls (usual care), abused women who received advocacy used a considerably higher number of safety behaviours (outcomes of the advocacy) compared with women receiving usual care. However, this benefit was not apparent at two years in McFarlane 2006. It may be that these behaviours were no longer needed. Cripe 2010 reported that when women were followed up at 14 to 28 weeks postintervention, advocacy was associated with a larger increase in the number of women who adopted safety behaviours (8 of the 13 behaviours) compared with women in the control group (4 of the 13 behaviours ), but there was no clear difference between the two trial arms for any of the 13 safety behaviours investigated.

\section{Finding 4: trauma-informed approach}

Most of the studies we found that used a trauma-informed approach focused on psychotherapy. Three papers reported related findings from an RCT study of an intervention known as Nia (Taha 2015), which provided advocacy for depression rather than PTSD, and which used a trauma-informed approach. In total, 217 women were randomised 2:1 to Nia ( $=130$ ) and treatment as usual $(n=87)$. In the first paper, the study authors tested hierarchical linear regression models with the intent-to-treat sample, which indicated that the Nia intervention reduced depressive symptoms and general distress compared with treatment as usual. The between-group differences were sustained throughout follow-up of up to 12 months. While Nia was not associated with greater reductions than treatment as usual in suicidal ideation or symptoms of PTSD, those women in the Nia

A realist review of which advocacy interventions work for which abused women under what circumstances (Review) 
arm who were exposed to further IPV showed attenuation in suicidal ideation compared to women in the treatment-as-usual arm; thus, the intervention was protective against effects of IPV. The analysis considered total scores of the scale for suicidal ideation. The second paper found that restricting analysis to only one of the two subscales gave clear group differences. In the same paper, the study authors also explored the mechanisms for Nia, by examining the relationships between primary psychological outcomes, religious well-being (as a proxy for religious coping) and existential well-being, and adaptive and maladaptive coping in the same group of women. Bootstrap estimates were used to test two, multiple mediator models. In both models, the intervention condition was the independent variable; and religious well-being, existential well-being, adaptive coping, and maladaptive coping were the parallel mediators. In one model, suicidal ideation was the outcome variable, and in the other, depressive symptoms were the outcome variable. Post-intervention variables were used for the analyses. Results supported the hypothesis that existential well-being mediates the treatment effect on suicidal ideation and depressive symptoms; both symptoms were clearly reduced postintervention compared with treatment as usual (independent t-test $1.91, P=0.06)$, as levels of existential well-being increased. This accords with the capability approach, where utility feeds back into outcomes (achieved functionings).

The third paper by Taha 2015 used multivariate generalized linear modelling (GLM), to determine if there were main effects of group condition (Nia versus treatment as usual) on levels of self-esteem, hopefulness, and also effectiveness in obtaining resources, which should be improved according to the strengths-based approach described in the Background. Positive effects were only found for self-esteem (F-test statistic $=3.80, P=0.05, \eta_{p}{ }^{2}=0.07$ ), but the effect size was small and increases in self-esteem may not have had practical significance. A clear interaction was also found between conditions, women's levels of readiness to change their suicidal behaviour and effectiveness of obtaining resources (F-test statistic $=4.58, \mathrm{P}=0.04, \mathrm{\eta p}^{2}=0.08$ ). Overall then, these data showed that women earlier in the stages of change process (as a mechanism), and so less likely to change their suicidal behaviour, endorsed greater levels of hopefulness and perceived effectiveness of obtaining resources, following Nia.

Sullivan 2019, using multiple regression modelling, found that women's perceptions of the degree to which services were traumainformed was the mechanism associated with improvement in their self-efficacy and safety-related empowerment; traumainformed practices made the greatest contribution to survivors' safety-related empowerment (accounting for $29 \%$ of the variance explained). However, depressive symptoms decreased over time, regardless of receipt of trauma-informed practice or control. Each regression controlled for the length of time survivors had spent in shelter, as those with longer stays had more time to recover from trauma.

Scheer 2018 considered LGBTQ victims of intimate partner abuse and therefore results considered the intersection between sexuality and abuse rather than abuse per se. They found that the direct and indirect effects of trauma-informed care on mental and physical health were not clear. However, trauma-informed care appeared to be the mechanism for greater empowerment and emotion regulation, and lower social withdrawal (which was associated with stigma relating to sexuality). Lower social withdrawal and lower shame predicted better mental health, while lower shame and emotion regulation predicted better physical health.

\section{Finding 5: theories of change}

Four experimental studies (DePrince 2012; Gillum 2008; Rodgers 2017; Taha 2015), drew on the trans-theoretical theory of change (Prochaska 1992). This says that people move through a series of stages (precontemplation, contemplation, preparation, action, maintenance) when modifying their behaviours. The time a person can stay in each stage is variable, and they may move backwards as well as forwards through the stages. Different strategies are suited to each stage to help a person progress through the stages and prevent relapse. Gillum 2008 found no clear relationship between stage of readiness to change behaviours measured by the Stages of Change Scale (Prochaska 1992), and use of safety-promoting behaviours. DePrince 2012 simply reported that women in their advocacy outreach condition reported greater readiness to leave the abuser. Rodgers 2017 only used the model to justify their study design. Using multivariate general linear modelling, Taha 2015 found interactions between being earlier in the stages of change process, being in a current IPV situation and showing suicidal behaviour, with women earlier in the process reporting greater levels of hopefulness and perceived effectiveness of obtaining resources following advocacy. It is not clear whether these positive outcomes decrease if the intervention does not live up to the women's hopes. The study authors concluded that abused, suicidal African American women who are initially reluctant to change their abusive situation and suicidal behaviour may benefit from even a brief, culturally informed intervention. A similar model, used by Curry 2006, is Landenberger's Theory of Entrapment and Recovery (Landenberger 1989). This comprises four phases: binding, enduring, disengaging, and recovering. The process of moving through these phases is cumulative and multidimensional. Similarly, McFarlane 2000 and McFarlane 2004 drew on Walker's three-phase Cycle Theory of Violence (Walker 1979), and Curnow's Open Window Phase of help-seeking and reality behaviours (Curnow 1997). Walker suggested IPV passes through three phases in a repeating cycle: the tension building phase, the acute abusive incident, and a honeymoon (calm) period. Curnow suggested an open window phase, occurring between Walker's phases two and three, in which a woman realises she is unable to stop the violence but should not be experiencing it, and is most likely to reach out for and be receptive to intervention. Each of these theories shaped the design of the interventions rather than the outcomes measured. All of these studies were set in healthcare or criminal justice settings and the main study author in each case came from a medical discipline. Mixed results were seen across these studies as reported above.

\section{Finding 6: other theories}

Wuest 2015 developed and used a grounded theory of health promotion to design their intervention, Strengthening Capacity to Limit Intrusion (SCLI), which was described as, "a theoretical rendering of how survivors of IPV spontaneously promote their health" (quote; Wuest 2015, p 84). Their advocacy was said to work by strengthening the abused women's capacity to limit intrusion from the various issues in their lives, using processes of providing, rebuilding security, regenerating family and renewing self. Wong 2013 included a social support component based on Cohen's Social Support Theory, with improvements across a range of outcomes for the intervention group. Bybee 2005 and Johnson 
2014 used the socioecological framework (Heise 1998), to consider the different aspects that were important in women's lives. Taft 2011 focused on parenting theory; we included this study because of its distinct advocacy component for abused mothers. Hence, these studies used theory to develop their intervention rather than to explore outcomes. None of these studies was set within a criminal justice setting though Johnson 2014 explored the way community advocates worked with criminal justice services.

\section{Programme strategies and outcomes: the complexity of the interventions and mode of delivery shows no clear pattern with outcomes}

Given the complexity of abused women's situations, an integrated model of intervention that combines various elements and with different theoretical underpinnings might be thought to produce better outcomes. We found no evidence that any one type of intervention was more successful than any other, based on the types of theoretical underpinnings and complexity of the different interventions. However, this is partly because of a lack of comparability of outcomes and there are indications that brief advocacy over one session is unlikely to deliver sufficient mechanisms. No formal comparison was undertaken given that this would not adhere to the realist approach; meta-analyses can be consulted in Rivas 2015 and its planned update in 2019.

The basic elements in studies included risk assessment and safety planning with referral cards or active referral, and education on IPV (albeit that this could be brief). Some provided a range of support elements in addition to safety planning and safety-related referrals.

Many interventions included social or emotional support as well as other elements: liaison with family and friends as well as transportation to court (Bell 2001); network-oriented social support (Bybee 2005; Coker 2012 (who included connection to healthcare); Constantino 2005; Cripe 2010; Curry 2006; Goodman 2016a; Ekstrom 2015; Gupta 2017; Hathaway 2008; McFarlane 2006; O'Brien 2016; Prosman 2014; Wood 2014; Wong 2013; Wuest 2015) or support groups (Rodgers 2017; Song 2012; Sullivan 2002; Taft 2011; Trevillion 2013; Tutty 1996).. Lako 2018 were particularly focused on social support. Thus they aimed through advocacy to expand clients' networks, involve their social and professional networks, and maintain continuity of care and support during the transition to independent community living, as well as adding practical support (helping out with, for example, home furnishing) and emotional support. With the exception of Prosman 2014, all studies including a focus on building up social support reported clear effects relative to controls in the use of social support and other outcomes. However, Rivas 2015 pooled the data from Bybee 2005, Sullivan 2002 and Taft 2011, and found no evidence that advocacy had a positive effect on the use of social support in the short-term.

Nine interventions included legal support (Bell 2001; Ekstrom 2015; Kiely 2010; Rodgers 2017; Stover 2010; Thiara 2009; Tiwari 2010; Trevillion 2013; Tutty 1996). Weisz 1999 determined in a qualitative study that women were usually confused, doubtful, and lonely when they tried to cope with the police and the courts; thus, advocates played an important role in legal settings.

Garcia-Leeds 2017 undertook a qualitative study about an intervention that provided families with several services, support in aspects related to other agencies (working with these agencies on the women's behalf), information about laws and services available to undocumented users, a crisis line and community educational workshops related to domestic violence.

There were three very focused studies. Silva-Martinez 2016 considered financial literacy specifically. Infanti 2015's qualitative study and Kendall 2009's cohort study concerned interventions intended to prepare evidence for court and connect women to community services (healthcare in the case of Infanti 2015).

Considering mode of delivery, as with focus and complexity, we could discern no patterns in relation to context or outcomes. Eleven studies included phone-based support as well as at least one faceto-face meeting (Bell 2001; Cripe 2010; Curry 2006; DePrince 2012; Gillum 2008; Krasnoff 2002; McFarlane 2004; Rodgers 2017; Song 2010; Thiara 2009; Wong 2013). These studies represent the full range of settings, outcomes and levels of effectiveness.

For full details of the interventions see the Characteristics of included studies.

\section{CMO development}

For this review, we initially only created CMOs for contexts, mechanisms and outcomes that were explicitly correlated in the papers themselves (in pairs of $\mathrm{C}+\mathrm{O}, \mathrm{M}+\mathrm{O}$ or $\mathrm{C}+\mathrm{M}$ ), for example through regression analyses or study author's discussions. When we began to configure the CMOs in our synthesis and look for patterns, we reviewed these studies' potential explanations alongside alternative possibilities and tested them against the other data in the review (as explained in Methods) and against our emerging theory. As we tested CMOs against our theory, we also populated gaps in the CMOs from the wider literature, so that ultimately, CMOs were developed that differed substantially from our early work and the explanations of some studies. We initially grouped CMO configurations according to intervention and study type; for example, we separated interventions given to pregnant women from those given only to minority women, and from those studies that used interviews or focus groups to explore the experiences of the advocates. From these small sets we developed candidate essential principles (which together make up our overall theory), each supported and shaped by the CMO configurations. We then clustered our tentative essential principles across interventions and studies to ensure that our final principles were underpinned by mechanisms found across the range of interventions and contexts. This led to revisions of the principles. For example, there were four main initial hypotheses resulting from the studies of professionals, all of which were subsequently modified: how the woman's vulnerabilities affected advocacy success; challenges of the advocacy role/resources for advocacy; features of a good advocate; and organisational and programme support. As articles were able to contribute to multiple principles, we also checked that each principle was based on data from several different papers, to ensure the principles were transferable.

Initially we identified 11 essential principles from the literature, which were checked and enriched by the stakeholder group input. This group made the principles more precise, for example in defining terms such as 'vulnerable and marginalised women'. In this review we take the view that marginalisation is both a process and a state. It refers to the relegation to the fringes of society of an individual or group because they cannot access societal 
rights, resources, and opportunities and so cannot fully participate in social, economic and political life. As such, marginalisation is multi-layered and complex, being rooted in interconnected economic, social, environmental, and political systems. It leads to greater vulnerability, vulnerable people being broadly considered as individuals or groups with a status that may adversely impact upon their well-being, which they are unable to adequately protect (Walker 2018; Wrigley 2016). All abused women may be considered as marginalised and vulnerable to at least some degree, but in this review we use these interconnected terms to refer to women who are particularly limited in their access to rights, resources and opportunities through their economic, physical, legal, or geographic states.

Our stakeholder group also suggested amalgamating some CMOs as different variations of the same concept (e.g. combining economic and physical dependence and also advocacy solutions to these rather than having issues and solutions as two separate principles). This led us to reduce the total to six essential principles. Members of the group all considered the essential principles that focused on women's vulnerabilities to be the most valuable, though they also considered the needs of advocates to be important. They found the essential principles 3 and 6 especially interesting.

Below we describe the final essential principles and relevant contributory $\mathrm{CMOs}$ developed from the literature, as hypotheses. Constraining contexts and mechanisms are largely the converse of enabling contexts and mechanisms. For example, if there is a lack of joined-up working between organisations (context) and this leads women and advocates to feel powerless (mechanism), this will be constraining. We have included quotations from participants provided in included articles, or quotations from authors of the articles in some cases, where we feel they demonstrate a particular point clearly and add value to the overall narrative. Figures are provided in Appendix 3.

\section{Essential principle 1: advocacy that takes into account the influence of the abuser on the woman will be more effective in reducing or ending the woman's tolerance of his abuse and coercive control}

Since abuse is characterised by the man's coercive control over the woman (Stark 2007), an intervention might be expected to be more effective if this control and the tolerance of the abuse by the woman is reduced. Poverty, chronic mental or physical illness or disability (Barie 2012), undocumented immigration status or some cultural practices (such as the woman being expected to not work) are examples of relevant context (conversion factors according to Sen 1985), that may increase the risk of experiencing abuse in the first instance and the woman's dependencies on the abuser, and may limit women's help-seeking options and responses. This essential principle is summarised in Table 2 in which we include potential negative outcomes that are well documented in the existing literature, of increased abuse and potentially also death from the abuse. It is estimated that between $65 \%$ and $75 \%$ of women killed by abusive partners are leaving or have already ended the relationship (Wilson 1993). Overall, our confidence in the quality of the evidence for this essential principle varied between moderate and high (Table 3).

\section{Hypothesis 1.1: many women will be economically, physically,} legally or emotionally dependent on the abuser and this needs

\section{to be addressed within advocacy to make it less challenging for} the woman to change the abusive relationship

The Conservation of Resources (COR) theory, used by one of the leading researchers in domestic violence, Cris Sullivan, to underpin her advocacy work, postulates that economic, social and interpersonal resources are important for an individual's well-being (Sullivan 2018). We can apply the lack of these resources to our model, as context (or conversion factors). For example, consider a context in which the woman has chronic mental or physical illness (C1a) or undocumented immigrant status (C1b) and where she has economic or legal or physical dependencies on her abuser (C2). She may decide not to leave her abuser (O1) or prosecute him (O2) because she fears the potential loss of her home and the close ties with family and friends (M) if she does so. In terms of our model, given her limited alternative functionings choice set she may be prevented by the context of her dependency and the mechanism of her fear from taking steps to end the abuse (Breen 1997; Hidalgo 2016). This then means that she does not attain the personal well-being, agency, and freedom that is at the heart of Sen's approach (Sen 1985). Advocacy may reduce the influence of the abuser by shifting the way she feels about him (M1) and increasing her knowledge on how to access resources to reduce her dependency (M2). This may lead to immediate improvement in some outcomes, such as episodes of abuse (O1), but delayed improvement or worsening in others such as mental well-being $((\mathrm{O} 2)$ as an often unanticipated outcome, especially as the woman becomes more aware of her situation), depending on the type of advocacy being offered.

\subsubsection{Affirming studies of physical dependence}

Some confirmation of this has come from the DePrince 2012 RCT, which compared outreach services with referral, though it should be noted that these followed police-reported IPV and hence women had already taken some action in response to the abuse (it is not clear who reported the abuse). The outreach intervention was not specifically designed to reduce physical or economic dependence. Thus, their moderating role on fear could be assessed, using a simple question rated on a five-point scale for dependences, and the Fear scale of the Trauma Appraisal Questionnaire (TAQ). (The TAQ was developed by DePrince 2010 in an earlier paper.) DePrince 2012 found first that women's greater perceptions of physical dependence on the abuser reduced the odds of total to no engagement with prosecution tasks, court attendance, and prosecution outcomes (B (unstandardised regression coefficient) $=-0.62, \mathrm{~W}$ (coefficient of concordance) $=7.66, \mathrm{P}=0.006$ ) similarly across treatment groups. In other words, women with greater perceived physical dependence were less likely to follow through with prosecution. Second, women who reported partial or total physical dependence on the abuser showed no clear decrease in fear across groups at follow-up to 12 months. Dependence appeared to affect fear since those women who reported not being at all physically dependent on the offender showed moderate-tolarge reductions in fear over time regardless of group (outreach: $d$ (difference between the paired data) $0.75,95 \%$ confidence interval (Cl) 0.43 to 1.07 ; referral: $d 0.40,95 \% \mathrm{Cl} 0.01$ to 0.80 ). Over time, outreach led to more reduction in fear but this did not reach statistical significance (outreach to referral, baseline to 12 months: $\mathrm{d} 0.33,95 \% \mathrm{Cl}-0.03$ to 0.68$)$. Third, neither condition had an effect on revictimisation by 12 months, but women in the outreach condition reported greater readiness to leave the abuser irrespective of dependencies, showing that these were not 
the only contextual factors affecting outcomes, and perhaps that over time, outreach had given women more options to choose from. This study suggests that perception of dependence may be as important as actual dependence. This is an important consideration since coercive control aims to disempower women so that they feel a greater dependence on the man than may be necessary. Thus in this regard, advocacy could work not just by removing actual dependence through an increase in potential resources, but through mechanisms that reverse the effects of coercive control itself, such as awareness-forming and increasing self-esteem

\subsubsection{Affirming studies of economic dependence and immigration status (legal dependence)}

Wong 2013 reported how abusive men could make use of their wives' undocumented immigration status to prevent them from choosing legal, financial and emotional support service options to independence, which would be likely to operate through the mechanism of fear of immigration officials and deportation. Similarly, undocumented, abused Latinas interviewed by Reina 2015 reported their financial dependence on their partners as a direct barrier to leaving:

"You know I wasn't working and I was taking care of my girls so I didn't look for jobs for a while. So I was dependent on him and that stopped me. Five years went by before I decided to look for help. I first looked for help with a friend because I was desperate. I wasn't working and I wanted to find a job" (quote; Reina 2015, p 485).

Along the same lines, Bacchus 2016a reported that 12 of the $32(35 \%)$ pregnant women she interviewed or observed had no recourse to welfare benefits because of their immigration status and were completely dependent on the abuser for housing and financial support. In spite of their growing understanding of the abusive situation, most chose to remain in the relationship rather than try to support themselves and their baby illegally in the UK, or return to their country of origin, where they would encounter additional risks and dangers:

"...it's really, really hard to be in this country without your status and you are with somebody who is hitting you or doing bad things to you... He's using that as a weapon to hurt you more because he knows that whatever he [does] to you, you [can't] do anything about it. (Maternity service user 4, $28 \mathrm{yrs}, 9$ months postintervention)" (quote; Bacchus 2016a, p153).

" So we get that woman started, but then we send her out there into a very hostile economy. So we're really also talking about economics here and to be able to provide that, I mean the bigger stroke of the wand makes it a more equitable economy that's based on supporting the needs of everybody in that society and not making a few people rich..." (quote; Wood 2014, p 115).

\subsubsection{Socioeconomic status and income}

Women's perceptions of their economic dependence had no effect on fear in the DePrince 2012 study. It should be noted that they were looking at court outcomes specifically, which may have affected findings. Thus physical dependence may make it challenging for a woman to go to court in the first place, that is to go somewhere independently of the man, particularly when the police have already been involved, as was the case in DePrince 2012, while economic dependence would more likely affect different outcomes.
DePrince 2012 explored the relationship between economic dependence and outcomes further, by developing a measure of socioeconomic status (SES) from their existing data through the application of principal component analysis to education, occupation and income variables. SES did not directly affect engagement with prosecution tasks; however, higher SES was associated with a greater likelihood of this outcome being achieved regardless of group. The higher the SES, the lower the odds of having cases dismissed or not filed relative to having a verdict entered (odds ratio (OR) $0.54 ; \mathrm{B}=-0.61, \mathrm{~W}=2.54$ ). The study authors suggested that SES may affect engagement through, or in conjunction with, mechanisms such as economic dependence or the severity of the abuse or beliefs about the criminal legal system. Given that their measure included occupation and education however, it may also have been that the women of higher SES had more skills to do research into possible actions, more knowledge about their options and more understanding about the systems they had to navigate (hence increased capabilities) and therefore more options at their disposal. In this regard, the advocacy used by Kendall 2009 included support to help women gain education or training to become financially independent and to appreciate where they could access shelters and housing, financial assistance and employment assistance. This approach therefore worked through the mechanism of increasing women's sense or selfperception of capabilities in these areas (self-efficacy). Kendall 2009 reported that over $96 \%$ of women perceived an increase in their safety after the intervention, and approximately 50\% had completed part of their safety plan. They suggested that they may have recruited women with higher SES status who "may have been more likely to implement safety plans and feel safer at follow-up because of intangible factors not readily measured in the demographic data" (quote, Kendall 2009, p293).

The income of participants in the studies we included was mixed, but excepting Kendall 2009, most of the women were on low incomes. Only in two studies, which focused on psychological outcomes, did a reasonably high proportion of the women have an income that was above average for the country (Curry 2006; Tiwari 2005). There is some evidence that women from low SES and poorer families are particularly likely to experience abuse, and it has been suggested that this may be related to the need for men to show their manhood in other ways than through income, or to a tendency for the man (and woman) to alcohol and drug abuse (Heise 2014, p.12), But women who are financially independent and earn more than their partners are also frequently abused; their higher income may be seen as a threat to the man's male status as a provider so that he reasserts his power through violence (Heise 2014). Anderberg 2018 found that higher wages reduce women's overall exposure to partner abuse, but income support and subsidised childcare lead to increased abuse because they encourage childbearing earlier in the relationship (increasing physical dependence) and less accumulated labour market experience. These examples show the complex mechanisms at play. They also show how a change in a conversion factor (context) or in the set of choices available to a woman can lead to unanticipated outcomes. There is a spiral of effect, an example of how changes in resources will create altered contexts for subsequent change mechanisms.

Overall, these findings would predict that women with greater physical and economic dependence need more support than women with greater independence in these, in order to change their situations. This fully supports our final model, shown in Figure 
3. There is some evidence of this from studies of disadvantaged groups, which are considered in essential principle 2 .

\section{Hypothesis 1.2: the trade-offs (balance of the immediate and long-term losses and gains) of the woman leaving the abusive relationship or staying need to be part of the woman's decision- making process and addressed by advocacy}

\subsubsection{Affirming studies on trade-offs}

Many advocacy interventions assume that the woman should leave her abusive partner and many advocates report being frustrated when they do not do so (Briones-Vozmediano 2014; Goodman 2016b; Merchant 2015; Prosman 2014; Wood 2014). As Dunn 2007 argue, minority women are least likely to want to leave but tend to live in countries where individualism would suggest leaving to be the best choice for self-determination.

When women make decisions the advocate considers unsafe, the advocate may try to persuade the woman to their point of view: "We just have to keep saying to them that, Look, that may not be a good idea, and maybe you need to think about this" (quote; Coy 2011, p 36). However, it cannot be assumed that leaving is the best outcome for a woman or indeed that she will be prepared to do so (DePrince 2012; Goodman 2016a; Kelly 1999; Reina 2015; Wood 2014). Women's fears about leaving as noted in hypothesis 1.1. may be valid. Advocates and women, especially those with low reserves of resources (C), need to consider the 'trade-offs' or consequences of such choices as leaving versus staying (balancing benefits and harms (M), where the trade-offs are the immediate and long-term losses and gains ( 0 ) from any action they take). Support in doing so was explicitly asked for by respondents to the survey by Goodman 2016a. Particular issues (problem outcomes) for women who leave their abusive partners are the loss of home and possible severance of close ties with family and friends as a result, financial problems, the health consequences of physical and psychological assault, and a loss of self-identity. Consideration of these issues needs to include the particular contexts of the women themselves as seen in hypothesis 1.1; for example, women who typically have low reserves of resources to start with are those with chronic mental or physical illness or disability, immigrant women, women from rural communities or with financial instabilities.

As illustration, Goodman 2016a reported the women they surveyed wrote comments such as:

"Changing jobs to get away from my abuser meant a pay cut. Also supporting a complete household of bills and children by myself is a financial strain. Court dates mean time lost at work and caused me to worry about losing my new job" (quote; Goodman 2016a, p $6)$; and

"...a new residence, new day care for my son, a new routine (travel to/from work, etc.), new job, and so forth, which are all things that are difficult to change" (quote; Goodman 2016a, p 6).

All $28.2 \%$ of women in the Goodman 2016a study who responded to the question asking if they would have done things differently in retrospect replied 'yes'. Of these, $33(27.2 \%)$ wrote that they would have avoided the relationship, left the relationship earlier, or not returned to the relationship having left it before; $15.2 \%$ wrote that they would have stayed with or worked on their relationship; $12.1 \%$ that they would have avoided formal support systems; and $6.1 \%$ that they would have sought help sooner. Reasons, broadly, that affected their decisions were losses in their: emotional and physical safety (30.3\%); level of social support (20.6\%); financial stability (19.4\%); sense of home and rootedness (19.4\%); ability to parent (15.8\%); and freedom (12.7\%) (e.g. "I can't do the things I want because I always worry about is he going to find me (if I fill out this application) or if I leave my phone number at any job or credit card or anything" (quote; Goodman 2016a p 7).

\subsubsection{Resource gains, losses and spirals}

According to the COR theory, and our theory based on Sen's capability approach (Sen 1985), once a woman has left and extricated herself from her dependencies on the abuser, difficulties associated with a loss of resources - that is problematic outcomes (they have been chosen as the lesser of two evils so we do not call them unwanted) may lead to psychological distress. Resource loss often leads to further resource loss, while gain often results in further gain; this is known as the resource spiral (Sullivan 2018). Resource spirals can be explained by our model drawing on Sen's capability approach. Thus if a person's set of functionings is increased, they may make choices that further add to this set. If their set of functionings is small and their capabilities also, they may be unable to choose options that prevent further resource loss. Given the generative nature of change, spirals not only lead to larger or smaller functioning sets, but different sets with different conversion factors (contexts) and capabilities required and operating. For example a woman who is injured by her abuser after leaving him (a common occurrence as noted by Wilson 1993), may not be able to go to work (a loss of a functioning) and therefore she will have a reduced income. Without an income she may not be able to afford to travel to look for more work unless she borrows money. When she borrows money she pays high rates of interest which leads to further loss. Considering the examples in Hypothesis 1.1, a woman with a good set of resources and a large set of functionings - for example a university professor (hence from a higher SES) - when injured by her former abuser may be able to work from home, which increases the time she can spend on researching acceptable ways to end the abuse. She already has the skills to do this research and quickly develops the knowledge, because of her training in research enquiry. She then decides that with this new knowledge she can achieve a functioning not previously open to her, which is to support other abused women. Because of her connections, her knowledge and skills, and her new public-facing role as an IPV spokesperson she becomes relatively protected from further abuse from her former partner compared with someone with less means and less education. A woman with children and on income support who leaves her abusive partner is likely to find it challenging to re-enter the job market. She is likely to end up in worse accommodation than when she was with her partner as a result, in a different area, which further reduces the resources at her disposal and hence her choices.

Advocacy interventions that improve a woman's capabilities to analyse the trade-offs of her actions can lead to decisions being made that can reverse her psychological distress and increase wellbeing. This is central to the COR theory. For example, turning again to our theory, with COR spirals embedded within, the woman with children and on income support can be supported in accessing employment that includes childcare vouchers or has a workplace nursery or can be supported in joining a housing association and developing community networks. She could then be placed on an upward spiral leading to better mental health and greater wellbeing. Therefore, it could be argued that advocacy that focuses 
on safety plans alone may be insufficient (Goodman 2016a). At the least, a safety plan should not lead to resource loss or should aim to counteract resource loss (Goodman 2016a), while a coordinated community response to IPV may result in a more positive experience and better outcomes for women. It has been argued that programmes may not have sufficient resources to address some of the poverty-related needs of very low-income survivors (Warshaw 2003), especially in communities with few resources, where, for example, stretched funds often mean services limit both what they provide and the number of women they can help. However, according to the COR theory, and our own theory, women may be empowered through being able to use other types of resource, including social support. Once they make changes in these other domains they may develop a well-being and feelings of empowerment in these other domains that may lead to a gain spiral that ultimately improves their economic resources too.

The trick for advocates is to help the women avoid further resource losses, pushing them to a gain spiral rather than a loss spiral. A number of studies, which we now consider, support these arguments, or the obverse, that resource losses that are not reversed by services can lead to reduced effectiveness of advocacy.

\subsubsection{Affirming studies considering resource use capabilities}

Bybee 2005 followed up abused women at 6, 12, 24 and 36 months after half the women had been randomly assigned to receive shelter-based advocacy that focused on increasing their access to community resources and social support once they had left the shelter. Their natural support networks were involved in the advocacy process. Compared with women in the control group (usual care), according to Bybee 2005, women who received this advocacy reported less re-abuse and better social support, effectiveness accessing resources and quality of life. This all agrees with our model. Benefits in effectiveness accessing resources were only found at the two-year follow-up (mean difference (MD) -0.18 , $95 \% \mathrm{Cl}-0.34$ to $-0.02 ; \mathrm{n}=265$ ). This is an important point it takes time to learn new skills and knowledge and develop the mechanisms, such as confidence and self-efficacy, to use them. In a further paper as part of the same study, the authors reported a cluster-analysis of findings. This revealed distinct subgroups of survivors (one focused primarily on activities to acquire housing, one concentrated on education and employment, and one focused on legal issues), while cutting across these were high and low activity groups. GLM showed that women who worked with advocates were more effective overall at accessing needed community resources (F-test value $=42.90, \mathrm{P}<0.001$ (exact $\mathrm{P}$ value not reported)). Across clusters, women in the control group reported a mean level of effectiveness of 2.71 (SD 0.71), compared with 3.26 (SD 0.57) for women in the advocacy group. This difference held true for all clusters when considered separately, except for the high-activity cluster. Women in the high-activity cluster across intervention and control arms perceived themselves as more effective at accessing needed resources than women in the housing cluster (MD 0.52, $\mathrm{P}<0.01$ (exact $\mathrm{P}$ value not reported)). In the terms of our model, women in the high-activity cluster would be likely to be in a gains spiral. The condition by cluster interaction gave uncertain results, suggesting that the effectiveness of the advocacy was not dependent on the types of needs women initially presented with. This may be partly explained by the fact that although women focused their activities in particular areas, they rarely had needs in only one domain. Women in the low-activity cluster engaged in fewer strategies to change their lives but felt effective in meeting their needs and benefited from the advocacy. Bybee 2005 suggested that this may be because some women have fewer needs or are at a different stage of change or come from particular contexts such as an immigrant community or rural area, in which small steps may be more appropriate. This contradicts the perceptions of some advocates that these women are less rewarding or less likely to be helped (see essential principle 5). Considering our model, this suggests that a gains spiral may be built up slowly. This could simply result from small steps being taken that have a smaller incremental effect on functionings choices. However it is possible that these women are also making 'dead-end' or equilibrium choices, that is choices that satisfy their immediate needs without resulting in either a gain or a loss spiral. This is a concept not considered in COR or Sen's approach more generally. As an example, a woman on income support could simply take advantage of resources to ensure that the welfare and legal help she receives enables her to stay in the same house with her children even though she no longer has her partner's income. She may be happy with this, despite there being no other changes in her life, and may not seek other changes, therefore. Equilibrium could be stable or unstable (Figure 4).

The measure used by Bybee 2005 recorded the difficulty that women experienced in accessing resources. This was a composite, 11-item measure especially developed for the study that considered housing, material goods and services, education, employment, healthcare, child care, transportation, social support, legal assistance, financial issues and issues regarding children. Where participants had not directly tried to access a resource, they were asked to answer how difficult they expected it would be. The Bahadir-Yilmaz 2018 study, set within an obstetrics and gynaecology hospital department, measured learned resourcefulness, finding a clear difference in this at twoweek follow-up in the empowerment advocacy group only ( $F$ statistic $=7.43, P<0.05$ (exact $P$ value not reported) versus usual care controls). These measures may be seen as proxies for perceptions of or actual increased capabilities, rather than as outcomes of resource use. Other studies have considered actual resource use as an outcome.

\subsubsection{Affirming studies considering actual resource use}

Howarth 2016 reported a dose-response relationship between access to resources and positive outcomes. They found that women accessing more than one resource had higher odds of both achieving a cessation in abuse and feeling safer, compared with those women accessing no other or only a single form of "other agency" support. Accessing two to five community resources doubled the odds that abuse had ceased (adjusted OR 2.00, 95\% $\mathrm{Cl} 1.32$ to 3.07 ; Wald $=10.57, \mathrm{P}<0.01$ (exact $\mathrm{P}$ value not reported)) and tripled the odds that a woman felt safer (adjusted OR 3.18, 95\% $\mathrm{Cl} 2.11$ to 4.81; Wald $=30.18, \mathrm{P}<0.01$ (exact $\mathrm{P}$ value not reported)) . Thereafter, the odds of achieving either positive outcome increased progressively with the number of resources used. This fully supports the concept of the gains spiral. In McFarlane 2000, use of community resources (a primary outcome) decreased over time in all three intervention groups (brief, 'counselling' (i.e. advocacy), and outreach); differences between these groups were not clear. Although McFarlane 2000 used a broad definition of resources, the difference from Bybee 2005 was explained by the study authors as possibly related to the immigration status of the women; McFarlane 2000 included only women monolingual in Spanish and who were often undocumented. This does not explain however why resource 
use should decrease over time, unless the women have entered a resource loss spiral that the study is merely measuring, with the interventions all having no effect, or if the interventions are having an unanticipated effect. It should be noted that Rivas 2015 pooled data from Cripe 2010, Gillum 2009, McFarlane 2006 and Tiwari 2010, and found that brief advocacy (which simply entailed providing a list of resources without capabilities building) had a minimal effect on subsequent resource use by abused women recruited in healthcare settings and the community. This accords better with Bybee 2005 and again suggests that some women may simply wish to maintain an equilibrium. Once this is reached, they no longer seek further resource use. McFarlane 2000 and Bybee 2005 both suggest this may be more likely in immigrant women and in essential principle 2 we discuss how these women, and women from rural communities, may need or wish to stay in the relationship, or not make big changes because of community pressures, in which case this equilibrium may be sufficient for them.

Coker 2012, in a quasi-experimental controlled study of a brief intervention, reported that women in the intervention arm (inclinic advocacy) were more likely to use community services (police, lawyer, or court systems) than the control group (who had access to an advocate in the community), and this increased use was most likely to occur $(P<0.05$ (exact $P$ value not reported)) soon after seeing the in-clinic advocate. Stover 2010 found that women who received a home visit by the police paired with a domestic violence advocate (the Domestic Violence Home Visit Intervention (DVHVI)) after a police-reported incidence of abuse were more satisfied with the police than the control group and more likely to call the police in the 12 months following the initial incident (OR 5.18). In total, $57.7 \%$ of the DVHVI group calls were for nonphysical incidents (e.g. breach of peace, violation of a protective order) compared with $43.8 \%$ of control calls. Women who received the DVHVI were more likely to use court support services (e.g. legal aid, court-based advocacy) 6 to 12 months following the initial incident and were more likely to have active protective orders at follow-up. Stover 2010 found that the DVHVI group were also more likely than the control group to use social support and mental health services; this further supports the gains spiral. Groups in the McFarlane 1997 study differed at study entry in their use of resources $\left(\mathrm{Chi}^{2}=4.40\right.$, $\mathrm{df}=1, \mathrm{P}=0.04)$, with $45 \%$ of the intervention group and $61.2 \%$ of the comparison group having accessed community resources (i.e. use of a shelter or calls to the police) within the past 12 months. At six months post-advocacy there was no clear difference in resource use (logistic regression controlling for resource use at study entry), but at 12 months, controlling for both entry and six-month resource use, there was a clear difference between the groups $(P=0.01)$; the comparison group was more likely to use resources at 12 months. A reduction in police use could indicate IPV recidivism (a positive outcome), which is also likely to explain the similar patterns of resource use in Coker 2012, and given that this occurred in the intervention group the mechanism is likely to have been through increased capabilities consequent upon the advocacy. DePrince 2012 found no difference between the outreach and referral control groups in access to resources though women in the outreach arm reported greater perceptions of the helpfulness of contacts and women in the control group became increasingly distressed over time. This suggests that women in the intervention group felt empowered by the advocacy, and that their capabilities had increased. Thus they were better able to use resources and gain more satisfaction from them than the control group.

\subsubsection{Affirming studies considering the impacts of resource loss on women who have left an abusive relationship}

Six studies considered the impacts of resource loss once a woman has left the abusive relationship and that need to be considered as trade-offs. Goodman 2016a surveyed women who had been supported by IPV agencies offering multiple services. They found that financial instabilities resulting from a woman's safety-related actions (such as leaving her abusive partner) could damage the therapeutic alliance. The study authors suggest that this could have arisen because the advocate found it challenging to deal with the issues and because the intervention did not change economic circumstance so that the woman perceived advocacy as not helpful. There is the danger when women believe they will be helped and this does not transpire that the woman will develop a learned helplessness (Clarke 2008; Prosman 2014), which can be addressed if advocates clarify with the woman what their powers and the advocacy process entail in relation to trade-offs. The difficulties the advocate faces in this - as considered in essential principle 6 - cannot be divorced from the advocacy processes and may be critical to advocacy effectiveness, and therefore we have modified our initial theory to give factors affecting the woman and the advocate equal and intersecting importance.

The path analysis reported by Song 2012 in their similar survey showed that low or no income had a direct negative effect on empowerment, thus reducing the direct positive effect of empowerment on life satisfaction (beta $=0.49$ ) and on change of self (beta $=0.38$ ). Social support had a positive direct impact on life satisfaction (beta $=0.34$ ), and an indirect effect via empowerment (beta $=0.26$ ). Similarly, Rodgers 2017 found that a higher income post-relationship was associated with greater empowerment regardless of the intensity of community outreach work (though this was only a feasibility study). In other words, women with low or no income are less likely to benefit from empowerment advocacy in terms of changes in self (capabilities) and life satisfaction (wellbeing) unless they received social support as part of the advocacy. The social support operates through a different mechanism to the empowerment component. For example, empowerment could involve education while social support could boost emotional resources. This supports the importance of a gain in one resource leading to an upward spiral of gains in others. It is also in line with theories of social capital (Kabeer 1999), which say that a boost in emotional resources leads to better coping with adversity.

Using hierarchical regression, Perez 2012, analysing data collected from women's shelters, found that resource acquisition had an impact on the relationship between IPV severity and symptoms of PTSD, but that this acted through empowerment. Thus in this study, the act of gaining resources was suggested as the mechanism affecting women's feelings of empowerment and these feelings then led to mechanisms that reduced the abuse. In this study insufficient types of mechanisms and outcomes were considered for us to be confident about the underlying mechanisms but overall this study supports our theory. Women interviewed by Bacchus 2016a and Bacchus 2016b reported practical difficulties such as unsuitable or poor-quality accommodation in undesirable areas, lack of money or support for childcare, and unemployment, along with depression, isolation, residual feelings for the abuser and postseparation abuse, thus supporting Song 2012 and also, given the continuing abuse in particular, Perez 2012, but illustrating how the trade-offs are sometimes inadequately considered: 
"I'd literally sleep with a knife or something next to me because I thought I don't know what's going on. And you know two, three o'clock in the morning my front door would be knocking and it would be him knocking. And because I'm not opening the door, 'Oh, I know you've got a man in there you bitch, open the door.' And he's kicking the door down, banging the windows and he's woken up my son and the baby. (Maternity service user 3, 33 yrs, 5 months postintervention)" (quote; Bacchus 2016a, p 153); and

"In here [the refuge] there's no space... and everything gets to me sometimes... I still think about [him] and I'm scared that once I'm rehoused I'll still wanna see him... I think it has to do with loneliness. I feel really lonely." (Maternity service user 5, 35 yrs, 13 months postintervention)" (quote; Bacchus 2016a, p 153).

\subsubsection{Affirmation and interventions specifically tackling financial independence}

Some interventions are designed to focus specifically on financial independence and its effect on other outcomes. Many of these fall outside our definition of advocacy and we did not consider them; we deemed two to be relevant. One study evaluated advocate provision of financial literacy education as part of their services. The aim was to increase survivor knowledge of, and access to, financial resources and increase survivor confidence in managing their finances (Silva-Martinez 2016). Advocates found this intervention challenging to provide. Encouragingly in this regard, in the second study, the mentor mother intervention (MeMoSA), reported by Prosman 2014 in the Netherlands, was able to effect financial independence through mentor mothers helping women to develop, implement and evaluate a plan for education or find a job, as well as providing other support. At baseline, 11 women (26\%) had a job. At the end of MeMoSA, 20 women (47\%) were employed and 13 women (30\%) had started a new educational programme, two of whom also started a job. IPV decreased from a Composite Abuse Scale (CAS; Hegarty 2005), score of 46.7 points (SD 24.7) to 9.0 points (SD 9.1) $(P \leq 0.001)$, symptoms of depression decreased from 53.3 points (SD 13.7) to 34.8 points (SD 11.5) ( $P \leq$ 0.001 ) and social support increased from 13.2 points (SD 4.0) to 15.2 points (SD 3.5) ( $P \leq 0.001$; exact $P$ value not reported). This was a pre-post study and so a controlled trial is needed to confirm these findings. Goodman 2016a, in a post-hoc mediation analysis, found that financial stability and social support fully mediated the negative relationship between survivor-defined practice and trade-offs (indirect effects beta -0.04 and -0.09 , SE 0.03 and 0.05 respectively, $\mathrm{P}<0.05$; exact $\mathrm{P}$ value not reported). In other words, it is possible that when advocacy helps improve survivors' financial stability and increases their social connections, difficult trade-offs may be diminished. Therefore these studies further support our theory and suggest mechanisms of women gaining the skills to develop, implement and evaluate a plan for education or find a job, resulting in achieved functionings (i.e. outcomes) of enrolment on courses, or employment.

\subsubsection{Affirming studies from interventions specifically tackling social support}

Six studies included social support components (Constantino 2005; Coker 2012; Goodman 2016a; Murray 2015; Lako 2018; Sudderth 2017). Constantino 2005 found that a social support intervention for women in shelters decreased their psychological distress and increased the perceived availability of social support. The study by Coker 2012 showed that when the social support scores of women experiencing IPV increased, anxiety and depression levels, post-traumatic stress disorder symptoms and suicide attempts decreased. Advocates in the Goodman 2016a study encouraged shelter residents to socialise with each other both inside and outside the shelter. They considered that by sharing views and experiences, and by attending social events together, women would become more empowered and confident and better able to reintegrate into society. Goodman 2016a and Murray 2015 also explored how some advocates worked directly with members of the woman's immediate network to ensure they were sources of support and not harm. This necessitated educating network members about IPV and about available services for survivors (including running educational workshops within the local community) but also the provision of emotional support for network members who were themselves emotionally affected by the woman's abuse (Goodman 2016a). As one advocate said:

"It's also letting them know that when it's someone that's close to them that has been victimized, that they are also a victim as well.... And letting them know that our services are there for them as well, to come and talk, and also if they just need support or help that we're there for them too" (quote; Goodman 2016a, p 6).

In parallel, women needed education about relationships with their networks (Goodman 2016a). Women often lost a sense of boundaries and some over-shared while others isolated themselves. Women therefore needed support to develop new 'templates' for thinking about their relationships. According to Goodman 2016a, advocacy work within the woman's networks was facilitated when network members called IPV hotlines themselves, when a woman asked her advocate to talk to a family member, or within support groups. Lako 2018 noted for their shelter-based study that women might engage with networks for support if the connections began while they were in the shelter, but did not feel the need once they had left the shelter and were accessing a continuation of advocacy support within their own community.

We have added other networks to our model in view of these studies, as a central subject along with women and advocates, as well as a contextual factor.

The Sudderth 2017 study from New Zealand provides an example of a politically- and culturally appropriate model of social support intervention and the mechanisms that this entails. In New Zealand, service providers are under a legal obligation to ensure equity for the Māori people through culturally sensitive protocols and practices, so one mechanism here might be advocate's fear of job security or other sanctions if these are not followed. The staff in the Sudderth 2017 study described how the safety plans they developed with women could involve the woman's family if the family was threatened by the abuser, or if she was from a Māori background, since in traditional Māori culture, abuse against one person is treated as a crime against the entire group. Reconnecting with the community, social network and the church was part of the plan for many Pacific women. One educator in a Māori women's refuge said:

"For Māori women, you can't do safety in isolation; culturally, it makes no sense to do things alone. . . . So for Māori women, safety planning includes identifying their support system." (quote; Sudderth 2017, p 235).

Conversely in other communities, advocacy could be less successful because of community constraints; for example, when 
women were undocumented immigrants, or lacked English language skills, or access to resources (McFarlane 2000), or lived in rural communities as shown for the USA (Bacchus 2016a; Johnson 2015), and Australia (Owen 2015). Rural women tend to be marginalised in the employment market, locked out of the inheritance structure and have their education or careers curtailed through family responsibilities. Comments by staff in Owen 2015 were:

"This woman [client] that works on the farm, she puts up with abuse because she feels she has nothing to leave for. The husband owns the farm, she works on the farm, looks after the kids, and she doesn't have any other work experience or qualifications." (quote; Owen 2015, p 235); and

"Just because they have the income doesn't mean [women] can access it, because it's tied up, or the house is in joint names. If you can't live in it, can't sell or can't access in it, fat lot of good it's doing you! All it's doing is stopping them from accessing services." (quote; Owen 2015, p 236).

Advocates across studies reported other major barriers to the provision of programmes that increase resource use, including: a lack of funding and training; lack of adoption into core services; competing priorities, and loss of contact with survivors before completion if they move away; work schedule conflicts; and focus of the service overall (Silva-Martinez 2016).

\section{Hypothesis 1.3: the impact of the woman's risk or severity (or both) of abuse on advocacy outcomes depends on the outcome being considered and hence the goals of the advocacy, but a higher severity of abuse may require extended advocacy}

Risk to the woman's safety from the abuse, such as when the woman still lives with her abuser (C) as well as the type and severity of the abuse (C), could have an effect on the woman's engagement with the advocacy intervention through mechanisms such as fear of abuse (M) and thus outcomes ( 0 ), but the direction of the relationship depends on which outcomes are being considered. This means that potentially advocacy could always have some benefits for an abused woman if it is undertaken for long enough, but that the goals of the advocacy need to match her needs.

\subsubsection{Affirming studies on risks of abuse and the woman's responses to advocacy}

The different ways the relationship between risks of abuse and the woman's responses to an intervention may take effect depending on context are shown by the DePrince 2012 and Howarth 2016 studies, both of which considered the impact on outcomes of living with the abuser for women experiencing severe abuse. DePrince 2012 found that living with the abuser at the time of the incident that led to police involvement, and in the following month, reduced the odds of the cases being dismissed relative to having verdicts entered $(B=-0.52, W=2.91, P=0.09, O R 0.60)$ and the odds that a not guilty versus a guilty verdict was entered $(B=0.57, W=4.40$, $P=0.04)$. There was a clear difference between groups (Chi2 with 2 degrees of freedom $=7.47, P=0.02$ ). Thus, $100 \%$ of women who lived with the abuser and received outreach had at least one guilty verdict entered against the abuser, whereas in the referral control group of women living with their abuser, $33 \%$ of women's cases had a verdict entered, $56 \%$ were dismissed, and $11 \%$ had no charges or were refused. In other words, outreach may be particularly helpful for women who continue to cohabit with their abuser. This seems to contradict the idea as discussed earlier that fear of further abuse can reduce women's use of resources.

Howarth 2016, in a multi-site evaluation of Independent Domestic Violence Advisor (IDVA) services for women, found that women who lived separately from the abuser at baseline in their study were more likely to report positive outcomes at follow-up (i.e. either at the closure of a woman's case or after three months, whichever came first). Figures for living separately versus living together were, respectively, $55.8 \%$ versus $37.4 \%$ for cessation of abuse and $56.0 \%$ versus $39.6 \%$ for felt safer. The study authors conjectured different mechanisms: there may have been less opportunity for continuing physical abuse when the perpetrator and woman did not live under the same roof, or a greater emotional distance may have resulted from the woman having already broken some ties with the perpetrator. These results are more intuitive than those from DePrince 2012 but both sets of results can be explained using our model and are not mutually exclusive. It might be the case that particular characteristics or contexts that were not documented or the severity of abuse meant that criminal justice intervention was effective initially, at least in some studies. Court and police systems may have been easy to navigate in the chosen areas, or the abuse might have been severe enough for women to be motivated to seek convictions (when considering the trade-offs of not doing so, such as being murdered), with support from advocacy to carry their actions through. Indeed it was suggested by Perez 2012 that women who experience severe levels of violence and become empowered and initially appear to end the abuse (so that criminal justice interventions may be perceived as effective) may be at risk for further abuse as their partner tries to reassert his power. This agrees also with Heise 2014. Therefore, Perez 2012 propose that empowerment alone may be insufficient for severely abused women, and that strategies that reduce violence and focus on long-term safety may be more important initially. Criminal justice settings tended to focus on recidivism as an outcome, however.

Song 2012 conducted a survey of 191 abused women who had received services from the centres of Prevention and Intervention for Domestic Violence or from the private IPV sector in Taiwan. Services were not necessarily advocacy, but findings support other studies included in this review. When modelling direct and indirect effects on outcomes, a path analysis showed that one of the three most influential variables on empowerment was negative impact of the abuse (a composite variable that included fear, severity and type of abuse) (beta $=0.27$ ), which had a direct positive effect on change of self (including self-affirmation and enhanced ability to use resources), where the resources were varied and not just related to criminal justice.

Many of the participants in the various intervention studies we considered were living with, or were still intimately involved with, the perpetrator at study entry. However, in a large number of studies $(n=14)$, the majority were specified as living apart (Bacchus 2016a; Bacchus 2016b; Bader 2014; Cripe 2010; Ekstrom 2015; Gillum 2009; Goodman 2016a; Hathaway 2008; Kiely 2010; McFarlane 2000 (44\% lived apart); McFarlane 2006; Sullivan 2019; Sullivan 2002; Taha 2015). There is no clear CMO relationship connecting the different studies, though none of these provided advocacy in a criminal justice setting. Amongst these studies only Cripe 2010, Gillum 2009, McFarlane 2000 and McFarlane 2006 considered safety behaviours as an outcome, while only 5 of 20 experimental studies in which women were mostly living with 
the abuser considered safety behaviours as an outcome measure (Bybee 2005; Kendall 2009; McFarlane 2004; Tiwari 2010; Wong 2013).

Bacchus 2016a reported that some of the women she interviewed considered they had achieved a number of positive changes whilst still living with the abuser, such as making re-assessments of their situation, gaining insights about themselves and the relationship, and gaining confidence in their ability to begin and to sustain changes. These developing capabilities, as mechanisms of change (Bacchus 2016a considered them as outcomes), enabled them to tentatively explore options (within their choice set of functionings) such as temporarily leaving the abusive situation, enquiring about welfare benefits for single parents, contacting the police to diffuse a volatile situation, and seeking advice from a solicitor:

"I was scared and I think that was the thing that came out in the initial meeting, was making sure that they wouldn't just run to the police and get him locked up... It scared me that kind of decision would be taken out of my hands... It was non-judgemental, the counselling was kept very balanced in that way, so I was not being led down an avenue. (aged 52 yrs, 8 months postintervention)" (quote; Bacchus 2016a, p 153).

Decisions were affected by contextual (conversion) factors such as the degree of commitment and emotional attachment and investment women felt towards maintaining the relationship, the quality and availability of alternative options such as a refuge or temporary accommodation, and income. This suggests that when women are educated on abusive relationships and develop insights regarding these, and at the same time develop the capabilities to choose alternative options (which need to be available to them), then their perceptions of commitment to and investment in the abusive relationship may be shifted. Such shifts are at the heart of theories of change (see earlier). In Bacchus 2016a, only three women were still living with their abuser at the post-intervention interview, compared with 22 of the 34 women at baseline.

\subsubsection{Severity of abuse in different settings}

Articles did not always state the severity of the abuse experienced by women, but for the most part, we inferred this from the study setting or the mean baseline abuse scores. This enabled us to consider possible mechanisms that related to the risks or severity of abuse through abductive reasoning. Thus for example, it is likely that women recruited either exclusively or primarily from domestic violence shelters had experienced relatively severe abuse (Bybee 2005; Constantino 2005; Ganz 2015; Goodman 2016a; Goodman 2016b; Hughes 2017; Kenyon 2016; Lako 2018; Merchant 2015; Perez 2012; Song 2010; Song 2012; Stylianou 2018; Sudderth 2017; Sullivan 2002; Sullivan 2019; Tutty 1996; Wies 2008).

In Bybee 2005, for example, $48 \%$ of women were raped by the abuser and 38\% threatened with a gun or a knife. In Tutty 1996, 87\% of women reported having been both physically and emotionally abused before they entered the emergency shelter. Of these, 35\% also reported having been sexually abused by their partner. The police had intervened in 55\% of cases and weapons or objects had been used in the abuse of $41 \%$ of the women.

It is also likely that women recruited after contacting a service within the criminal justice system were likely to have experienced relatively severe abuse (Bell 2001; Casey 2007; Davis 2006; Davis 2007; DePrince 2012; McFarlane 2004; Stover 2010; Thiara 2009), though a proportion in the Stover 2010 analysis reported mild abuse. Using multivariate multiple regression analyses, Stover 2010 showed that severity of charges was a predictor of the amount of time spent with the advocate ( $F$-test value $=5.16, P=0.02$ ). Severity of charges appeared to predict a follow-up visit (beta $=0.17, \mathrm{P}=$ 0.07 ), but this did not reach statistical significance. In the DePrince 2012 study, in the six months prior to the target incident, $86 \%$ of women reported previous instances of psychologically aggressive acts, $61 \%$ reported physical aggression and $53 \%$ reported injuries. These figures support our suggestion that severity may lead women to perceive their lives to be at risk, shifting the trade-offs balance towards taking action, particularly since criminal justice advocacy might be perceived as the most direct way of tackling the risks.

Severe abuse was also probable for women recruited from hospital trauma or emergency departments (Coy 2011; Hyman 2001; Kendall 2009; Krasnoff 2002; Muelleman 1999; Taha 2015). In Hyman 2001, although they could have been there for other reasons than the abuse, $90 \%$ of participants reported having been physically or sexually assaulted, $93 \%$ were experiencing ongoing abuse, and $68 \%$ had received previous medical treatment for IPV-related injuries. Similarly Coy 2011 considered emergency departments of hospitals as one of several settings they explored, to include women in whom the risk of physical injury was substantial. Trevillion 2013 recruited women from a community mental health setting and reported a high severity of abuse, with intervention group participants scoring a median of 32.0 points (range $=23.0$ to 44.0 ) for total violence on the CAS (the cut-off for violence being 3.0 points) and a median of 1.0 points (range $=0.0$ to 4.0 ) for the severe combined abuse subscale, and with respective median values of 30.0 points (range $=$ 11.0 to 47.0 ) and 1.0 points (range $=0.0$ to 9.0 ) for the control group. This may explain why some relatively brief interventions in these settings were still able to have some effect and also suggests that time might be an important resource for some types of advocacy. We return to this in essential principle 6 .

For women recruited from other healthcare settings (antenatal care or mother and child clinics or primary care/public health settings), abuse severity was more variable. In Curry 2006, abused women had Danger Assessment (DA; Campbell 2009), scores ranging from 0 to 12 points, with 22 women exceeding the cut-off for lethality of 7 points. Mean baseline scores indicated that women experienced moderately severe physical and psychological abuse at study entry in three further studies (McFarlane 2000; McFarlane 2006; Taft 2011). In Cripe 2010 and Tiwari 2005, participants reported moderate to severe psychological abuse, but less severe levels of physical and sexual abuse. In Kiely 2010, instances of minor abuse were common, but $55 \%$ of the women had also experienced severe abuse. In Gupta 2017, 35.74\% of women in the intervention arm and $31.67 \%$ of women in the control arm had experienced both physical and sexual abuse from their partner. In Prosman 2014, participants' mean baseline CAS score was 46.7 points (SD 24.7) and the mean score on the CAS severe combined abuse subscale was 6.5 points. In Rodgers $2017,84 \%$ scored 18 points or higher on the DA tool, which is consistent with the highest level of danger.

Three studies in healthcare settings considered the impact of abuse severity and found mixed results. In Gillum 2009, one third of the participants met the criteria for being in lethal danger; those women who reported more non-physical abuse, risk for lethal harm, and PTSD symptoms engaged in more safety-promoting behaviours after the intervention. McFarlane 1997 found that $34.1 \%$ 
of the intervention group and $46.3 \%$ of the comparison group had called the police within the last 12 months. This suggested that the comparison group may have had more severe abuse. Using logistic regression and controlling for prior use of the police at entry and for 6- and 12-month follow-up, the study authors found no clear difference between the intervention and comparison groups in police use ( 6 months: $P=0.76 ; 12$ months: $P=0.70)$. Nonetheless, "resource use" (i.e. use of agencies) was $(P<0.001$; precise value not stated) related to severity of abuse across groups and women using resources at six months (with more severe abuse) also did so at 12 months. McFarlane 2006 stratified violence scores into tertiles; subgroup analyses of these revealed no differences in outcomes between 20-minute nurse case management (the active intervention) versus the referral card condition. All three studies were set in primary care clinics, but the Gillum 2008 intervention was longer, with one onsite counselling session and six telephone counselling sessions over three months. McFarlane 1997 and McFarlane 2000 offered three counselling sessions to abused women in a prenatal setting as the active intervention. Overall, these results suggest that abuse severity has an effect on outcomes and that interventions of longer duration are needed to mitigate some of this effect. This contrasts with the effect in criminal justice, showing that advocacy may depend on different mechanisms and different functionings sets as well as contexts in different settings. This accords with our model and suggests time to be an important conversion factor.

In most community settings - including third sector and specialist IPV organisations - the baseline abuse was of mixed severity; there were only two exceptions where severe abuse was predominant (Howarth 2016; Tiwari 2010). In Tiwari 2010, in the 12 months prior to women's admission to the shelter that was the setting for the study, $75 \%$ of those recruited had been severely assaulted, $70 \%$ had reported severe psychological abuse, $44 \%$ had severe injuries, 29\% revealed that they had endured sexual abuse (minor or severe). At entry to the shelter, $87 \%$ were being threatened, $84 \%$ had expressed fear and $17 \%$ reported they were being stalked. At baseline, $75.9 \%$ of the sample recruited by Howarth 2016 were experiencing serious, potentially life-threatening levels of abuse (as categorised by an IDVA) and $57.4 \%$ of women reported abuse that was escalating in either severity or frequency. In total, $81 \%$ of the sample reported feeling frightened and $83.7 \%$ were afraid of being injured, while $44.0 \%$ feared they would be killed and $48.6 \%$ reported being harassed or stalked. Howarth 2016 also determined that the women who remained engaged with their study at followup (under three months), when compared with those who were not, were more likely to be experiencing physical abuse $(86.7 \%$ versus $81.7 \%$ respectively), harassment/stalking ( $50.3 \%$ versus $47.1 \%$ respectively), or any type of abuse categorised as severe ( $78.3 \%$ versus $73.7 \%$ respectively), including strangulation and use of weapons. This accords with Stover 2010 in a criminal justice setting. The women considered by Howarth 2016, who remained engaged with the study at follow-up, were also more likely to report feeling fearful $(85.5 \%)$ compared to those who were not $(82.0 \%)$, or afraid of being killed ( $47.1 \%$ versus $41.2 \%$, respectively), or that the perpetrator would harm their child(ren) $30.3 \%$ versus $24.3 \%$ respectively). This contrasts with DePrince 2012, where a reduction in fear was associated with greater engagement with services, but, as with the findings of DePrince 2012 and Howarth 2016 regarding living with the abuser, differences may be explained by setting. Thus, in the setting of Howarth 2016's study, use of services is not likely to impact directly on the man, but can provide the woman with useful support to deal with her fear. For example, in the criminal justice system, a woman who is afraid may be worried that her abuser will be released and will punish her for her actions, or as already stated, may be supported to realise that the trade-offs of not seeking help are too great despite her fears.

\subsubsection{Affirming studies regarding types of abuse}

The type of abuse experienced could also affect women's responses, as suggested by the Howarth 2016 and Song 2012 data on severity. In all studies, women were recruited because they were experiencing current (within the last 12 months) physical or sexual (or both) abuse as a minimum. Twenty-four studies extended the inclusion beyond this to also include women experiencing emotional or non-physical abuse (Bader 2014; Bacchus 2016a; Bacchus 2016b; Bahadir-Yilmaz 2018; Bell 2001; Coker 2012; Constantino 2005; Curry 2006; Cripe 2010; DePrince 2012; Gillum 2009; Howarth 2016; Hyman 2001; Lako 2018; Lea 2016; McFarlane 1997; McFarlane 2000; Prosman 2014; Sullivan 2002; Taft 2011; Thiara 2009; Tiwari 2005; Tiwari 2010, Tutty 1996). Bahadir-Yilmaz 2018 was the only study to explicitly include economic abuse.

Howarth 2016 reported that women experiencing physical and sexual abuse showed greater improvements in safety outcomes than women reporting non-physical forms of abuse; they suggest this may have been because they were evaluating a short-term crisis intervention, or because it may have been harder for women to admit to continuing physical abuse, or because women experiencing severe abuse may be more active and more engaged help-seekers, as we have discussed above. Kiely 2010 found that pregnant abused women with minor IPV or experiencing physical IPV and randomised to their healthcare-based intervention were less likely than women in the usual-care group to experience further episodes at postpartum and at first follow-up (extending to all follow-up points at the end of the intervention for women with minor IPV). Women with severe IPV and randomised to the intervention showed a reduced incidence of episodes at postpartum only, compared to the usual-care group. As with other studies reported in this section, this suggests that a longer time is needed within advocacy for women experiencing more severe abuse or that when abuse is severe, empowerment may have only a temporary effect, nullified when the man tries to reassert his power and control (Heise 2014).

\section{Essential principle 2: there is a need for holistic, tailored advocacy intervention plans that recognise the individual risks and needs of vulnerable and marginalised women such as those from minority groups or from rural areas}

In the context in which women have problems in accessing resources for structural reasons (C1) (which is a key factor in leading to marginalisation and vulnerability as we have defined it), or in which advocates lack training in cultural humility (Tervalon 1998), and awareness of intersectionality (C2), it is important for advocates to undertake training or receive guidance so that they have the capabilities to overcome their fear of doing the wrong thing (M1), to reduce some of the difficulties they face doing advocacy work (M2) and to engage with marginalised communities (M2) in order to reduce abuse (O1) or help the woman to address the structural barriers (02). These mechanisms were noted by BrionesVozmediano 2014, Donnelly 2005, Thiara 2009 and White 2019, and made the focus of the grounded theory by Garcia-Leeds 2017. They were also argued by McFarlane 2000, who concluded that a lack of 
increase in resource use in their study may have occurred because they included only women monolingual in Spanish and who were often undocumented. When women are matched with advocates from similar backgrounds some of the problems might be removed but this is not always so. This essential principle is summarised in Table 4. Overall, we had moderate confidence in the quality of the evidence for this essential principle (Table 5).

\section{Hypothesis 2.1: advocates find it challenging to remove structural barriers to resource access despite the value of this}

\subsubsection{Studies affirming the value of removing structural barriers}

Eight studies (Briones-Vozmediano 2014; Garcia-Leeds 2017; Kapur 2017; Lynch 2013; Macy 2018; Owen 2015; Silva-Martinez 2016; Thiara 2009), emphasised that successful advocacy interventions need to take account of the structural barriers to access to resources for vulnerable and marginalised women and help them remove those that can be altered. These barriers are grounded in the contexts of patriarchal norms that affect community support and economic independence as well as bureaucratic processes such as no recourse to government funds. This means that effective mechanisms will either need to work within these norms or challenge the structural barriers. For example, in the USA, Goodman 2016b ran a focus group with white advocates, and while immigration was not a focus in this study, one advocate commented on structural issues as:

"We had a woman come from [name of foreign country] and she couldn't go back into the community. One, she had been isolated from it and two, we didn't know who knew her husband. She was brought over as an immigrant and sponsored by her husband. Which made it impossible for her to access some of the benefits." (quote; Goodman 2016b, p 75).

Some advocates, therefore, specified the need for change-based structural advocacy to improve service provision for vulnerable and marginalised women. Five studies provided in-depth consideration of the structural barriers associated with ethnicity and immigration status (Briones-Vozmediano 2014; Garcia-Leeds 2017; Kapur 2017; Lynch 2013; Reina 2015). It was recognised by all that advocates may act as a critical gateway into services for immigrant women. Prosman 2014 suggested that structural factors that reduced women's access to resources, especially cases of vulnerable and marginalised women, led to a learned helplessness. Their arguments imply that an empowerment-based intervention may have its effect partly by increasing the women's confidence and reducing their learned helplessness irrespective of resource provision, something we have already suggested in essential principle 1 , section 1.2.5.

DePrince 2012 and colleagues concluded from their analyses that outreach may be particularly helpful for ethnic minority women in buffering against beliefs of, or past experiences with, the system. In other words the active mechanisms counteracted the woman's learned helplessness or deeply held beliefs and values that may be culturally shaped. In such cases small step changes may be more effective as mentioned by Bybee 2005 (see section 1.2.3). Moderator analyses in DePrince 2012 showed that outreach was almost three times more effective in reducing fear in ethnic minority women compared with white women. Altogether, $78 \%$ of ethnic minority women assigned to the outreach condition went to court whereas only $53 \%$ assigned to the referral condition did so. Similarly, in a second paper in the same study, ethnicity was shown to moderate the main effects of condition (outreach or referral) on fear. For women identifying as from ethnic minorities, both outreach and referral were associated with moderate to large reductions in PTSD symptoms, with a slightly greater effect with outreach than referral (though this did not reach statistical significance). For white ethnic majority participants, only the referral condition showed a decrease in PTSD symptoms.

\subsubsection{The problems advocates have with structural change}

Structural changes were the focus of advocates in the Kapur 2017 study, who were concerned at the absence of systematic and ongoing culturally sensitive institutional interventions to address the various structural issues for abused ethnic minority women. The study authors suggested that this may be a result of organisational concerns to avoid stigmatising some immigrant groups as being more likely to condone domestic violence (Kapur 2017). Thus here the mechanism preventing change may have been a worry about sanctions from funders, policymakers and the justice system or retribution by or alienation of immigrant groups (including the women advocates wished to help). There were attempts by organisations to train the mainstream non-profit sector - for example to educate them that stigmatisation could be avoided or to give them the tools with which to be more effective but burnout and high attrition rates among trained staff prevented this from being impactful (Kapur 2017). An alternative solution was suggested by Kapur 2017, which was to streamline their organisation, which specialised in helping women from South Asia, and partner with stakeholders across different sectors, with crosstraining, knowledge exchange, shared expertise and formal plans for collaboration, all informed by BAME people, with experiential knowledge of the issues. This could be effective in developing advocates' knowledge, cultural humility and opportunities to engage with different communities.

As a comparable solution, Thiara 2009 considered the introduction of a specialist BAME advocate to Southwark, London, to support women from Bangladeshi and Somali communities to engage with the criminal justice service system, and liaise with agencies to remove structural barriers. She noted that in this case study (with the acronym RSAP), the process of commissioning and funding for these services was fraught with issues, and once the advocates were in place they found it challenging to change the practice of court and other professionals. They spent much time in chasing staff in other services, which required skill and diplomacy. Nonetheless, prosecutors considered they were getting more results with BAME women when they were supported by the RSAP service. This suggests that possibly the efforts of the advocates brought to the fore the issues of the BAME women, so that prosecutors perceived they were getting more results, a perception that may or may not have accorded with reality. Thiara 2009 noted that BAME advocates need to understand the ways of working of the systems and work within them to effect change. This might involve

- formalising partnership work through the development of joint protocols;

- showing how the advocacy service complements and can be differentiated from other services;

- having a local presence (i.e. close to other services); 
- having SDVCs (Specialist Domestic Violence Court systems) to address statutory equality duties and draw up equality impact assessments in relation to the range of communities;

- having SDVCs involve abused women from a range of communities in court visits and consultation to improve performance and public confidence; and

- gaining senior-level support, building links with individual officers, and undertaking training with agencies.

These recommendations suggest that mechanisms for structural change require the formation of solid connections across the different groups and the development of a coherent community of practice. This would also be suggested by implementation theories (Kirk 2016).

\subsubsection{Affirming studies from rural areas and with marginalised women} concerning access to support

Lynch 2013 and Owen 2015 focused on rural areas in Australia, where there were small, closed, male-dominated communities in which the man's treatment of his partner was seen as a private family matter (Owen 2015), as also stated for the rural USA by Bacchus 2016a, Logan 2018 and Johnson 2015. These studies showed that similar barriers existed to those affecting immigrant women. Consideration by advocates of the intersections of the indigenous population and rurality showed several correspondences:

"Fear of children being taken and [family and community service] involvement due to mandatory reporting. Fear of the man in custody, the perpetrator may use the stolen generation against them - they have promised not to bring [family and community services] into their lives. And also there can be a backlash from their community and much isolation especially if they are from a small community. An additional barrier is if the defendant is white - women will think they will immediately side with him, being in court is difficult, fear of police." (quote; Lynch 2013, p 24); and

"I think that the victims in the smaller communities are sometimes, they feel more, they are more pressured into friends and family putting pressure on them to do things. They come in more often and wanting to drop the charges just to please other people." (quote; Johnson 2014, p 12).

Overall, the barriers to effective intervention and access to services in rural settings were caused by intersecting constraints of poor infrastructure, limited services and local social attitudes. Bacchus 2016a reported that rural women were often unaware of resources as a result. Even when they were, they often had to travel long distances to services that were more widely dispersed than in urban areas, leading to transport costs and other transport issues (Bacchus 2016a; Burnett 2012 Lynch 2013; Owen 2015; Rodgers 2017). Issues such as these meant that in the Macy 2018 survey-based study, rural agency directors were more likely than directors serving mixed urban/suburban areas to state the need for services to respond immediately to abused women, and were also more likely to agree that legal advocacy should be available 24 hours a day, 365 days a year. However, directors of fully urban areas also considered these service deliverables important. These recommendations were an ideal rather than being linked to service capacities. Moreover they only addressed (to a limited degree) resource issues and opportunity issues but did not do anything to enable women to access them in the first place.
In some cases, as Briones-Vozmediano 2014, Silva-Martinez 2016 and Lynch 2013 noted, instead of trying to tackle barriers to support women's resource use, service providers directly excluded those women they considered least likely to benefit by refusing them services. This particularly occurred in areas without specialist services or with services that were very under-resourced; for example, with limited possibility of onward referrals:

"It's partly an issue of just the sheer volume of people, I think, too - and inevitably what happens when there's a problem with resourcing and the capacity is it's the people that are the most vulnerable and marginalised that fall through the gaps (solicitor)." (quote; Lynch 2013, p 6).

In an exploration of racial exclusion, Donnelly 2005 quoted one shelter executive director as saying:

"... right now, we're about 50-50, thank goodness. If we could keep it at that, I would be so happy, because what happened in [city] was it [the shelter] became totally Black, and the white women would not go ... we had to close the shelter and move it to another community [so white women would use it]." (quote; Donnelly 2005, p 28).

There was the feeling by advocates in the Lynch 2013 study that some organisations operated an inflexible, protocol-driven, riskmanagement approach to intake. Vulnerable and marginalised women, defined in this study as migrants, the mentally ill and drug and alcohol abusers, were especially likely to be excluded (Lynch 2013); all of these had complex resource needs as well as a low level of current resources. These points were also confirmed by undocumented Latina women themselves in a study by Reina 2015. A concern of this approach is that it could increase the perceived effectiveness of such programmes.

There were two exceptions. Coy 2011 reported that some advocates stretched capacity:

"We don't really have capacity, because there's no other service to refer on to, as we're the only service providing support for high risk clients and we can't say 'can you go on our waiting list?' (IDVA manager, R2)." (quote; Coy 2011, p 35).

In the area of Australia considered by Owen 2015, women-focused services, such as family support services, court support, women's centre, housing and refuges, were flexible when using the income test in rural areas. This was a response to the particular difficulties rural women often had in accessing their money because it was tied up in the family farm (Owen 2015). However, as with immigrant women, service access alone was insufficient; advocacy had to overcome a number of other structural barriers to be effective.

\section{Hypothesis 2.2: once marginalised women access advocacy they are best supported by advocates with cultural humility, knowledge of how to tackle specific issues for these women, and awareness of intersectionalities}

\subsubsection{Studies affirming the need for a holistic approach}

Issues that persist even when structural barriers to access have been removed include women's dependences on their partner, loss of social support after leaving their country of origin, and a lack of familiarity with the processes of their country of immigration. The varying needs of women from different racial and cultural 
groups can be nuanced, so that considerable sensitivity is needed. Lyon 2011 reported on the different needs of immigrant women born outside versus within the USA. Women born outside the USA wanted more help with issues related to their children, reproductive or women's health issues, staying in their relationship safely, and immigration. Hispanic or Latina were most likely to report child-related needs and Asian or Asian Americans least likely. Asian or Asian American women had the highest education and current financial status, while Hispanic or Latina(s) had the lowest. Those born in the USA were more likely to want help related to previous or other abuse, or their pets. A holistic, tailored approach is needed, which recognises these differences and the implications for mechanisms of change. For example, if a woman's immigration status is resolved, some of the abusive man's control of her can be removed, leading to other changes becoming possible (as per the gain spiral that we have incorporated in our model); a Hispanic woman whose child-related needs are supported may be freed up to make other changes in her life, while an Asian woman is likely to prioritise other changes.

Although the advocacy intervention in the Wong 2013 study was delivered in a culturally appropriate manner, taking care not to demand verbal emotional disclosure, which is not part of the Chinese culture, Wong 2013 commented that it was not sufficiently tailored to address the specific and complex needs of immigrant women. Intersectionality is an important consideration. As one advocate interviewed by Sullivan 2019 stated with regard to an abused woman from a minority ethnic group with a visible physical disability:

"But just being seen as a person in the world who is disabled and that may walk differently or just be different in the world in that way, can be a risk factor and increases vulnerability to harm and violence... And in addition to that, the racism and all the other things that go along with being someone who holds multiple identities. So that was another factor that we were definitely just mindful about-how does this person move through the world and what are the experiences that they may have that we want to be really aware of that will increase their risk to just a different experience than maybe other people we work with." (quote; Sullivan 2019, p 6).

In the study by Sudderth 2017, representatives from Māori women's refuges said that, post-colonialism, economic class could be more relevant than ethnicity, again highlighting the issues of intersectionality.

"If you expect to live on the dole and be in a violent relationship, then you may not care so much about what others think of you, because that is not the most important and pressing challenge in your life. But in the middle class, you don't want to draw attention to yourself or to the negative stereotypes. (Refuge manager)" (quote; Sudderth 2017, p 232).

The need for training in cultural humility and intersectionality was propounded by interviewees in the Prosman 2014 study, which included many diverse cultures. The 43 participants of the intervention programme itself (MeMoSA) came from Africa (said to be Algiers, Cape Verde, Goa, (though Goa is actually in India)) ( $\mathrm{n}=$ $3)$, Antilles $(n=3)$, Former Yugoslavia $(n=2)$, Iraq $(n=1)$, Morocco $(n$ $=3)$, Surinam $(n=12)$, the Netherlands $(n=9)$, and Turkey $(n=10)$. The women interviewed about their experience of MeMoSA $(n=14)$ came from the same spread of locations (Africa: $n=1$, Antilles: $n=$
1, Former Yugoslavia: $\mathrm{n}=2$, Iraq: $\mathrm{n}=1$, Morocco: $\mathrm{n}=2$, Surinam: $\mathrm{n}=$ 2 , the Netherlands: $n=2$, Turkey: $n=3$ ).

\subsubsection{Studies reporting problematic attitudes of advocates and other} IPV professionals

The Briones-Vozmediano 2014 project provides a case study of the issues that can arise when service providers lack cultural humility. A range of service providers in Spain (social workers, psychologists, intercultural mediators, judges, lawyers, and public health professionals involved in IPV services) failed to appreciate the factors that led immigrant women to abandon the helpseeking process and mostly assumed that leaving the aggressor was the best solution, regardless of professional role. They expressed disillusionment, frustration and anger, and a reluctance to help the woman again should she return. This was viewed as an inefficient use of stretched resources, including time. Social workers and cultural mediators were the most accommodating but still demonstrated a lack of cultural humility (Briones-Vozmediano 2014; Trevillion 2013), as exemplified by the following extract:

"The worst thing you can say to a woman who withdraws her complaint is you've made a mistake, or don't come asking for help again. You have to ask her, "How're things? I hope it all goes well, you know? I hope we were all wrong, OK?" You can't tell her off or blame her for anything, because that's the worst thing you could do. (Interview 10, mediator, woman)" (quote; Briones-Vozmediano 2014, p 1014).

In this study, there was a tension between the professionals' ambivalence about differences between immigrants and Spanish natives, and their recognition of the particular problems that immigrants encountered.

Advocates in Matthew 2016 criticised as absurd and ludicrous AfroCaribbean abused women's use of informal networks to end the abuse (i.e. family members, friends, church, prayers, and fortunetellers) but acknowledged them as part of the belief system in the Afro-Caribbean community.

Two studies that considered services more broadly provide further context. Donnelly found shelter directors would use the myth of the strong black woman (Collins 2005), or invoke the idea of a close-knit, supportive black community to explain why they did not consider culturally specific needs within their shelter (Donnelly 2005, p 24). Gillum 2008 found that black women had to continuously disprove this myth to get needed shelter services; Gillum 2008 also reported a number of other issues that black women had to face when IPV services were not culturally competent. Such culturally exclusive actions have been termed racial micro-aggressions (Gillum 2008). Some others, such as the rejection of more complex cases because of a lack of resources or fear of stigmatising communities, we have considered in section 2.1. It is likely that there are underlying mechanisms at work, such as a desire to show cultural humility without seeking the relevant knowledge, perhaps as tokenism, or the use of cultural difference as an excuse to avoid actions that would be resource-heavy or burdensome, or as a way of distancing the professional and the organisation from the issues.

\subsubsection{The use of BAME advocates}

Some studies mitigated the issues somewhat by aiming to match advocates to women with similar backgrounds (see essential 
principle 5). The idea was that shared cultural and social understandings would act as a mechanism to help the ethnic minority or immigrant woman believe that a strategy would be effective for her in achieving the outcomes she desired. GarciaLeeds 2017 - in contrast to Briones-Vozmediano 2014 - found that the counsellors they interviewed, five of whom were Latina and some of whom had been abused themselves, had a strong appreciation of the need for services to include spiritual and cultural support that considered the pressures on women to maintain an intact family. They particularly cited the nature of 'Marianism', with Latina women being expected to sacrifice their well-being for that of their children, since their main identity is to be mothers, and the machismo hypermasculinity of Latin culture. These counsellors noted the need for interventions to include child care and money for transportation to activities that did not involve the woman's children.

However, using BAME advocates might not always prevent cultural prejudice. In a focus group in the Prosman 2014 study, mentor mothers (most of whom were immigrants) said many of the predominantly immigrant abused women they saw had a "laissez faire" attitude to repeat attendance that gave the mentor mothers significant extra work in chasing them for appointments. Thiara 2009 found that the BAME advocates in RSAP often encountered hostility from existing mainstream services, where other professionals' lack of insight into domestic violence was often exacerbated by a lack of cultural understanding.

\subsubsection{Apparent disaffirmation and its possible explanation}

Because women from minority groups start off with so few resources at the time they first receive advocacy, they may show benefits from advocacy even when cultural humility is lacking. DePrince 2012 found that outreach was particularly effective in ethnic minority women. Goodman 2016a, using univariate analyses of variance (ANOVAs), revealed that minority groups were more likely to stay in the programme, even though they showed lower levels of alliance with advocates. Latinas reported higher levels of safety-related empowerment than other racial groups but there was no difference in depression and PTSD symptoms by race or ethnicity. Lyon 2011 found that, compared with women born in the USA, abused women born outside the USA were less likely to delay joining their IPV programme, more likely to have heard about the programme from informal sources or from healthcare providers rather than the IPV programme staff, more likely to have accessed more types of services when they first came, and more likely to use legal advocacy services. They also showed greater outcomes ratings for confidence, hope and efficacy. In a consideration of the Coy 2011 evaluation, Hester 2012 explained that it was likely that the specific London project for BME women recorded the lowest levels of severe violence because these women had a wider range of needs than non-BME abused women, but a lack of resources and alternative means of support. Overall, these results suggest that women have confidence in the impact of these services (perhaps contrasting them with what was available in their country of origin or seeing their new country as a land of opportunity) and particular motivations to use them.

\section{Hypothesis 2.3: helping the woman should also prove mutually beneficial for the advocate}

Sometimes service provision that helped women to respond effectively to the various issues in their lives - especially immigrant women - would also support the advocate in their role. For example if structural barriers to access were removed, this improved service efficiencies by freeing the advocate from spending time sorting access out or developing work-around plans. This in turn meant the advocate could focus on helping the woman in other ways and the therapeutic alliance was also likely to benefit.

\subsubsection{The case of immigrant women}

Staff in the Kapur 2017, Logan 2018, Lynch 2013 and Prosman 2014 studies considered language services for immigrant abused women to be critical for effective advocacy, as structural language barriers resulted in isolation, impeded service access and were generally disempowering. However, it was observed that interpreters were not always available (Logan 2018), and might not always maintain confidentiality, and that language lessons would ultimately be more effective than interpreters (Lynch 2013). Garcia-Leeds 2017 noted that informal family interpreters, such as the woman's children, were often used in court, which had particular issues because of the nature of the case against their father. Participants in the Thiara 2009 study considered the difficulties for advocates, for example:

"just constantly trying to work out is it best to meet in person with an interpreter, is it best on the telephone, is it best to get workers who speak those languages involved?" (quote; Thiara 2009, p 29).

Garcia-Leeds 2017, Kapur 2017, Logan 2018, Lynch 2013, Reina 2015 and Wong 2013 described how a married migrant depends on her abusive partner for immigration status and how perpetrators often use the threat of deportation to control her, which we have mentioned in essential principle 1. The US Logan 2018 and Australian Lynch 2013 studies, which considered a wide range of migrants, highlighted such issues as immigrants being scared of officials because of adverse experiences in their country of origin, or accused by officials of fabricating the allegations of abuse so as to get permanent residency. Reina 2015 described similar issues. This was a USA-based study that interviewed immigrant abused women from Mexico and Central or South America who had previously used the services of an anti-violence organisation. Garcia-Leeds 2017 reported on the tensions between fears of deportation and the impossibility of obtaining a court protection order without residency status. These different issues were difficult for advocates to navigate even with training in cultural humility. Participants in the Kapur 2017 study, which considered migrants from South Asia, considered that pro bono immigration services were therefore important.

\subsubsection{The case of rural areas}

Support for abused women in rural areas was also fraught with practical difficulties. Courts and other service buildings were small in rural areas and often inadequate in terms of privacy from the community and separation of the woman from her abuser (Lynch 2013; Owen 2015). Domestic violence court order applications were heard in between other local court business, such as traffic infringements, meaning the women were likely to be seen by people they knew. In addition, without set times, women and their advocates, who usually came from understaffed services, often had to wait most of the day at court. Courts moreover did not provide childcare so that women with small children, and without alternative support, had to take their children into court (Lynch 2013). Participants highlighted the negative effects these issues had for both women and over-stretched and 
under-resourced advocates (Lynch 2013; Owen 2015). Mechanisms reducing the effectiveness of advocacy in these situations were: social controls that meant inadvertent disclosure of the woman's abuse could lead to shaming, embarrassment or humiliation or other community condemnation; children's exposure to the courts leading to concerns that this would harm them or that they might tell the abuser; lack of opportunity to get away for a whole day. The same informal social controls that acted to restrict rural women's help-seeking made IPV advocates themselves marginalised and vulnerable to social stigma and exclusion, overonerous workloads and under-resourced services, not to mention professional isolation (Owen 2015).

\subsubsection{Studies affirming the need for training and other guidance}

Most interventions we included had manuals and protocols because they were being evaluated as research projects. In the field, advocates said they often did not follow strict protocols, although they typically used checklists to undertake safety planning. A lack of protocol can be useful in giving the flexibility required of a survivor-centred approach, as noted by the advocates interviewed by O'Brien 2016 and Song 2010. However, staff in two studies expressed a preference for protocols (Goodman 2016a; Logan 2018), to support advocates in common but risky or complex situations (such as when advocating for rural or immigrant women). Lako 2018 advocates similarly preferred to draw on a protocol but they often deviated from it to improve service delivery. They reported that a checklist of the expected outcomes of each of the three phases of their intervention, which they called anchor points, helped them decide when the client should move to the next phase: "you're also both aware - the client and the practitioner - that you've got that set amount of time to sort out those basic things.... So you just start to work. That's great." (quote; Lako 2018, p 76). Some advocates in Wood 2014 used a matrix of needs as a similar approach. Such protocols and tools would give advocates more confidence in supporting these women appropriately and less fear of doing the wrong thing and the consequences of this, and also provide them with more time to focus on other aspects of their work (see also essential principle 5). Built-in flexibility means they can be adapted not only to suit individual women but also to suit an advocate's individual ways of working.

Similarly, staff in Goodman 2016a, Grant 2012, Hidalgo 2016, Lako 2018, Logan 2018, Wood 2014 and Prosman 2014 commented on the value of training to help them deal with the challenges of their role as well as to introduce new techniques.

"Well, early in my practice, before I worked here I was taught to use an empowerment model that the entire time we're with a client, we're looking for ways to positively empower them so they can begin to take back their power and feel like they have more of a sense of self to take back from that situation than they did, but now is a movement we're moving towards trauma informed care. I think that's the new buzz word that they're using and I've found the neatest chart that one of the coalitions put together that demonstrates where empowerment model and trauma informed cross over into each other so that was kind-of neat because all of my education came early on from the training I got in (another state) on the empowerment model when I first started doing counseling which is all about drop the self out of it and look at this person and recognize that the choices and decisions that they make help them survive the circumstances that were thrown at them." (quote; Wood 2014 p 135).
Advocates interviewed by Silva-Martinez 2016 considered they could not have delivered a financial literacy curriculum without training and that this also benefited their personal lives.

Song 2010 argued that practitioners need continuous supervision and support after training to transform their knowledge and skills into daily practice.

Bacchus 2007 found that regular case supervision for domestic violence advocates was important in developing practice and maintaining consistency as well as providing support for the advocates. Some of the IDVAs in the Coy 2011 interview-based study commented that time pressures occasionally meant the appropriate supervision did not happen.

Effective debriefs were described by participants in the O'Brien 2016 DAWN intervention study:

"I found the debrief last week extremely beneficial... It left me feeling that maybe I had supported her through the worst and therefore given her a bit more strength to move on." (quote; O'Brien 2016, p 55); and

"At times it was exhausting, emotionally draining and left me feeling totally inadequate - but the support from DAWN and my family (plus a scotch) always got me back on my feet." (quote; O'Brien 2016, p 55).

Case supervision and debriefs have different underlying mechanisms to training and protocols. They can provide shared understandings for emotional support, shared strategies for practical support and confidence building, and also could improve performance through advocate fears of being seen by their peers or supervisors to be less competent or lacking in some way. Reflective practice had similar benefits, though this could involve self-reflection that was not shared, as well as sharing sessions (Wood 2014):

" And we began to identify so many things that weren't as client focused as they should be. Things about curfew, things about rules here in the shelter and stuff like that, and so I'm really feeling that the time I've been here we've gone from having really good intentions and also being "controlling" of the clients and our expectations of them, to an empowering place. You know, our clients are adults, they are in charge of their lives, and we need to be respectful of that in every action, to make sure that we're not replicating some kind of power control thing that they came from, even though we have good intentions! So I've seen us on that journey, as an agency, going from doing that to doing much better, in my opinion." (quote; Wood 2014 p 136).

Advocates interviewed by Kapur 2017 and by Garcia-Leeds 2017 further highlighted the need for:

- pathways for advocates to navigate immigration status issues;

- targeted outreach services that recognised the diversity of immigrant cultural, religious and other identity-based groups;

- culturally appropriate housing support; and

- culturally sensitive counselling.

Such enabling contexts or conversion factors would enable advocates to place their reliance on expert services and processes as the active mechanism and thus give the advocates greater 
confidence in supporting women and onward referral (Cauthen 2015; Hidalgo 2016).

\section{Essential principle 3: whether an abused woman was pregnant or had children could affect her engagement with advocacy and possibly also lead to some adverse outcomes}

Children add a layer of complexity to the context of abused women's lives and the decisions they make. For example, when an abused woman has a child/children ( $\mathrm{C} 1$ ) she may be more engaged with advocacy (O1) if she feels that advocacy will provide her with the capabilities to ensure their safety (M1), or drop out of advocacy (02) if an advocacy focus on her children leads her to fear that they will be removed from her care (M2), if it is challenging for her to find childcare (M3) or if she is afraid the children will reveal her help-seeking to the abuser (which may include fear of his harming the children) (M4). Similar mechanisms and outcomes may be expected with pregnant women (C2). However a woman who is pregnant for the first time (C3) may be more likely to attend all advocacy sessions because she does not have to make childcare arrangements (M5) and because the advocacy can be provided within maternity services (M6). In most studies, the majority of women had children or were pregnant or it was not reported whether or not they had children. There were only three exceptions. In Ekstrom 2015 and Trevillion 2013, only one third of women had children. In Thiara 2009 over half $(n=167)$ of the 295 cases whose notes were considered did not have children. This essential principle is summarised in Table 6. Overall, we had low confidence in the quality of the evidence for this essential principle (Table 7).

\section{Hypothesis 3.1: women with children are more likely to engage with advocacy in order to support them}

Using multivariate, multiple regression analyses, Stover 2010 showed that the total number of children a woman had (Pillias's trace $=0.04, P=0.03$ ) predicted the outcome variables of time spent with the advocate and number of intervention services and home visit services provided ( $F$-test value $=5.91, P=0.02$ ). Number of children (beta $=0.32, \mathrm{P}=0.03$ ) predicted a follow-up visit. In other words, the more children a woman had, the more need she had for services within an advocacy programme. Howarth 2016 similarly determined that more women with $(80.4 \%)$ than without children $(76.1 \%)$ remained engaged with services. The overriding mechanism for this appears to be that once the barrier to service use has been broken, women feel that the sharing of their experiences with advocates will lead to greater safety and better outcomes for their children.

\section{Hypothesis 3.2: women may be reluctant to use advocacy services because of perceived risks to themselves and their children from taking action against the abuser}

On the other hand, women have reported weighing the risk of doing nothing against the potential harm, abduction, or removal of their children (Humphreys 2002; Shorey 2014; Sullivan 2006; Thiara 2016), if they take action. Children also make it challenging to make a clean break from the abuser, increasing the perceived risk of harm to them (fuelled perhaps also by occasional stories of men murdering their partners and children when they separate, or abducting the children). Moreover services will want to spend time on the safety and well-being of the children, which may appear to exclude the woman or make her fearful of social welfare services separating her from her children (Hidalgo 2016; Humphreys 2002; Sullivan 2006; Thiara 2016). Some shelters do not allow children; in which case women may have to choose between leaving their abusers or separating from their children (Hidalgo 2016). For these reasons, interventions for pregnant women or those with children might be less effective than interventions in women without children; however, it was impossible to determine the impact of children from the included studies.

\section{Hypothesis 3.3: women are more likely to engage with advocacy when childcare facilities are provided}

Only two studies emphasised the lack of childcare at agencies as presenting consistent challenges, particularly for participants with children who were not yet school age at the time the service was being offered (Macy 2018; Owen 2015). This was also noted by Shorey 2014 in their review. Lack of childcare is likely to reduce engagement when women do not want to talk about the issues in front of their children, or would worry they would be distracted by them, even if they want help for child-related issues as well as for themselves.

\section{Hypothesis 3.4: women who are pregnant are easier for advocates to access}

\subsubsection{Affirming studies in antenatal care}

Nine studies specifically considered pregnant women. These studies had mixed outcomes though only one study showed any intervention effects. McFarlane 2000 compared three types of intervention - brief therapy, "counselling" (i.e. education and advice), and outreach - in pregnant, physically abused Hispanic American women who were receiving assistance at two urban prenatal clinics. Analysing their data using repeated measures ANOVAs, they found that violence scores at two months were lower for the outreach group and the brief therapy group than for the counselling group $(P<0.05$; exact $P$ value not reported). However, no treatment differences remained at 6,12 , and 18 months; severity of abuse had clearly declined for all three groups ( $P<0.001$; exact $P$ value not reported). There was no usual-care control so it cannot be determined whether this outcome would have occurred without any intervention. McFarlane 1997 used the same interventions and control group and found similar effects at 6 and 12 months (they did not follow up beyond this). The study reported by Kiely 2010 was a RCT of pregnant or recently delivered African American women. The intervention group received multiple sessions of individually tailored psycho-behavioural counselling sessions during pregnancy with booster sessions in the postnatal period, and the control group received standard prenatal care. Kiely 2010 found that overall, the women who had been randomly assigned to the counselling group reported fewer episodes of IPV than those assigned to standard prenatal care. Those in the intervention group who had earlier experienced minor IPV had reductions in further IPV episodes both during pregnancy and postpartum, while women who had previously experienced severe IPV reported reductions in further episodes only during the postpartum period. Those given the counselling intervention also had fewer pre-term infants and longer gestation periods. Kiely 2010 concluded that, "A relatively brief intervention during pregnancy had discernible effects on IPV and pregnancy outcomes"(p. 273).

Taft 2011 examined the role of lay mentors in providing social support to pregnant and recent mothers. They focused on women at high risk of abuse. Separate results were not reported for pregnant women. They found that, at follow-up, there was an improvement in mean abuse scores, depression, well-being, and 
social support in the intervention group compared with the control group though none of these differences could be stated with confidence. Importantly this study also provided support for children but the support for the abused women lasted longer, which may have helped to build trust in the advocates, given that this suggested they were concerned for the woman as much as her child(ren).

In the RCT in Peru by Cripe 2010, women in the intervention group either received a single advocacy session given by social workers or usual care plus an IPV assessment and referral card, which was compared with standard care. Cripe 2010 followed up women a week after giving birth, or anywhere from 14 to 28 weeks post-intervention. The setting was a hospital providing maternity services to low-income women residing in Lima, and high-risk obstetric cases nationally. The study authors found that women in the empowerment group were more likely than women in the standard-care group to adopt a range of safety behaviours at post-intervention. Although there were no clear differences in these behaviours, health-related quality of life, or increased use of community resources between groups, Cripe 2010 suggested that, "Simply asking pregnant women about abuse and offering referral could potentially interrupt and prevent further IPV" (quote; p 2072). This study included women with any or all forms of physical, sexual, emotional and non-physical abuse, but did not analyse by abuse subgroups or consider the severity of abuse, which could influence a woman's decision to adopt safety behaviours. The study authors also argue that one abuse scale (injury) and three quality-of-life scales (general health, vitality, and role-emotional) had Cronbach's $a<0.70$. and hence may not have been the most appropriate instruments for Peruvian women.

The Cripe 2010 intervention was adapted from the work of McFarlane 1997, and this was also used in Hong Kong (Tiwari 2005), tailored specifically for Chinese women with an additional 30-minute empowerment session by a trained social worker. This social worker provided emotional support through empathetic, non-judgmental listening; educated women on the cycle of violence; and gave information on what to expect when seeking help from legal resources, shelters, law enforcement or counselling services. Women were given a brochure with a personalised, 13item safety plan to reinforce the session, a list of community resources and strategies for seeking help from these resources, and were then helped to determine if it was safe to keep these materials. The social worker also offered to assist women with telephone calls to social service agencies or women's groups. Tiwari 2005 showed that an increased quality of life decreased levels of depression and frequency of abuse. They found that treatment group participants who had been subjected to minor physical abuse reported less psychological abuse and fewer incidents of minor physical violence after receiving the intervention. However, women experiencing sexual or serious physical abuse reported no clear changes.

In the Curry 2006 study, women in the intervention group received video advice in prenatal clinics, and then individually tailored case management by a nurse with referral as appropriate. They used services more than the control group and had greater reduction in stress but the effect was not clear because the results were imprecise.

In Bacchus 2016a, the usual-care group received standard, prenatal home visitation and the intervention group received the Domestic Violence Enhanced Home Visitation Programme (DOVE), which comprised standard care plus three further prenatal and three postnatal DOVE sessions. DOVE is an empowerment intervention to prevent IPV during pregnancy in women who have screened positive for IPV in the year before the current pregnancy (though $31 \%$ of the sample studied had not experienced IPV over the last year). This was the only intervention for pregnant women that showed clear benefits. There was a decrease in IPV over time ( $F$ test value $=114.23 ; \mathrm{P}<0.001)$ from baseline to $1,3,6,12,18$, and 24 months postpartum (all $\mathrm{P}<0.001$ ). Women in the DOVE treatment group reported a larger mean decrease in IPV scores from baseline (mean Conflict Tactics Scale 2 (CTS2) (Straus 2004), score decline 40.82 ) compared to women in the usual-care group (mean score decline 35.87).

Thus advocacy intervention may be easily combined with other antenatal care in pregnancy. This means that the abuser will not be aware of it and the women does not have to explain any absences that women who use other forms of advocacy will otherwise have to do. This easy assimilation within other care provides one active mechanism - women will trust the advocate who is also their midwife or who in the case of mentor mothers has already been through what she has and managed to deal with it. They will also be likely to be particularly invested in seeking solutions to the abuse because of their maternal instincts or feelings of nurturing.

\subsubsection{A disaffirming study in antenatal care}

There was one antenatal study that may have led to a lack of effect and potential harm (Feder 2018). The reasons are not clear but seem to be related to degree and severity of abuse. Thus, the study authors found some benefits from the intervention in preventing abuse but women already experiencing physical or sexual abuse at start of the study experienced worse abuse by the two-year followup. Those experiencing psychological abuse experienced no effect. In this case it is possible that the man increased his abuse because of factors associated with having a child (poor sleep, jealousy, for example) that led him to reassert his control over the woman.

\section{Essential principle 4: a good advocate-survivor relationship is important for effective advocacy, as predicted by underpinning theories}

When advocates demonstrate empathy and authenticity towards an abused woman and a desire for a collaborative, if not equal, relationship with her $(\mathrm{C} 1)$, the woman will be more likely to trust the advocate (M1) and the advocate will be more likely to avoid power imbalances despite their specialised knowledge and align with the woman's goals and empower her (M2), helping her to gain or regain confidence, self-reliance and autonomy (O) (Bybee 2005; Prosman 2014; Umeda 2017; Wood 2014; Zweig 2007). A similar concept, the therapeutic alliance, has been well studied in psychotherapy. Empowerment, survivor-centred and trauma-informed advocacy approaches and processes depend on this (Bacchus 2016a; Bacchus 2016b; Kulkarni 2015; Prosman 2014; Thiara 2009; Umeda 2017), and may be more critical to advocacy than other aspects such as disciplinary approach or the advocate's level of experience (C2). This helps explain why mentor mothers and other lay people may be successful advocates and why it may be beneficial to the relationship if advocates have themselves experienced abuse. This essential principle is summarised in Table 8. Overall, we had high confidence in the quality of the evidence for this essential principle (Table 9). 


\section{Hypothesis 4.1: qualities of a good advocate-survivor relationship correspond with what women need for empowerment}

4.1.1 Studies affirming the importance of the advocate-survivor relationship

Bacchus 2016a, in their interview-based exploration of the delivery of the DOVE intervention, found that the close bond between home visitors and the mothers they supported was a key factor in success of the intervention and sometimes appeared to replicate bonds of connectedness and affection with people in the women's social networks. Song 2012, in their path analysis of the direct and indirect effects on outcomes of IPV social worker interventions (including advocacy), showed the relationship with the social worker (beta $=0.29$ ) to be second in importance only to empowerment. It had both a direct effect on "change of self" and also mediated empowerment. Goodman 2016a, using SEM, also confirmed that a stronger therapeutic alliance was associated with reduced symptoms of depression and PTSD through the mechanism of empowerment.

Goodman 2016a developed a scale to measure survivor-defined practice. They found that women reporting higher levels of survivor-defined practice also reported higher levels of safetyrelated empowerment; the relationship held after controlling for demographics, financial stability, and social support. Specifically, survivor-defined practice was associated with two of the three dimensions of safety-related empowerment: participants' perception that they had the internal resources necessary to move toward safety (3.1\% of additional variance explained), and participants' perception that they had access to community support to facilitate safety (6.0\% of additional variance explained). When controlling for the effects of demographics, financial stability, and social support, survivor-defined practice was not, however, related to the third dimension, which Goodman 2016a called "Trade-Offs". This supports the idea that the effectiveness of advocacy is often severely limited when women lack basic resources. Possible alternative explanations put forward by Goodman 2016a are that: a) women who feel more empowered are also more likely to consider an approach to be survivor-defined; or b) that a survivor-defined approach is easier when survivors have access to resources. The largest contribution of survivordefined practice to safety-related empowerment was through the dimension "Expectations of Support". This suggested that when survivors experience survivor-defined support from community resources they are more optimistic about this support. To a smaller but definite degree, survivor-defined practice was also positively associated with what Goodman 2016a labelled as "Internal Tools", that is, a survivor's sense that she knows what her goals are and can do what is needed to move ahead. This aspect of safety-related empowerment is the most directly related to what survivor-defined advocates are aiming to achieve in their work with survivors.

\subsubsection{What makes a good therapeutic alliance?}

The suggested qualities of a good therapeutic alliance could be predicted from empowerment theory, and were consistent across studies (Bacchus 2016a; Bacchus 2016b; Curry 2006; Hughes 2017; Johnson 2014; Kelly 1999; Lako 2018; Lyon 2011; O'Brien 2016; Prosman 2014; Song 2010; Umeda 2017; Wood 2014; Zweig 2007), with advocates needing to:
- believe in what abused women say - authentically so (Bell 2001; Goodman 2009; Song 2010; Wood 2014);

- try to understand rather than wrongly interpret the women's stories;

- remain non-judgmental, respecting women's decisions, and believing that they have the capacity to build their life as responsible and autonomous individuals; and

- be positively flexible - varying support according to practical or emotional needs (Bacchus 2016a; Curry 2006; Goodman 2009; Goodman 2016a; Hathaway 2008; Macy 2018; Prosman 2014; Trevillion 2013; Wood 2014).

Zweig 2007 found in their study of 890 women in shelters that when abused women felt they were involved in the advocacy process, or their input was regarded, they were more likely to report service provision as helpful and were more willing to use services (Zweig 2007). Women themselves in two studies specifically commended a strengths-based approach or a woman-focused approach, as nonjudgmentally building up their self-esteem and energising them to move forward (Goodman 2009; Hughes 2017):

"[My advocate] knew my strengths. She would say things like "You're amazing. I could never do what you are doing. Of course you need support." She commented on my strength every time she saw me. It made me feel like a million bucks and that I could do it. Not just "Rah-rah, you can do it," but she was right there with me, pointing out my strengths and building on them in every interaction we had." (quote; Goodman 2009, p 863).

Women interviewed by Bacchus 2016a reported trust - an essential component - as multidimensional and shaped by:

- prior experience or knowledge of the home visitor;

- the home visitor's tone of voice;

- not feeling pressured to discuss details of the abuse;

- reassurances of confidentiality;

- belief in the home visitor's authenticity;

- a lack of apparent discomfort from the home visitor; and

- affective instincts ('gut instincts') or a resemblance to someone the woman had trusted in the past.

Participants interviewed in Goodman 2009, Song 2010 and Wood 2014 also saw advocates as important bringers of hope, an outcome also associated with empowerment theory:

"Having low self, self-esteem, and not seeing any hope ... I mean that just keeps you in depression. . . . Like last year, when I was going through all this, I'm like, "Oh my god, here's this thing that happened, this disaster" ... and the advocates helped me see that there was hope. [Laughs] Oh my god, there is hope. There's somebody out there that's helping me ... and, oh wow, there is hope." (quote; Goodman 2009, p 864).

Advocates had to take care sometimes to make sure they did not cross the boundary from supportive or neutral language to judgmental or attitude, so as not to diminish or blame the woman in any way and lose her trust (Logan 2018):

"You have to be really careful with your words. Words are so important. Any little word can make it feel like it's something they're doing. Even if you say "you're picking these guys" well "that's my fault, what's wrong with me? Why am I picking these 
guys?" I try to say "these guys are drawn to you." I just think about words all the time, my choice of words." (quote; Logan 2018, p 221).

The home workers in Bacchus 2016a and advocates in Curry 2006 were worried that questions about the women's personal lives might disrupt their professional-client relationship. Home workers talked about helping women to feel supported and validated by:

- taking time to let trust develop without forcing disclosures (even if the advocate was worried the woman would be harmed; Bacchus 2016a; Bacchus 2016b; Curry 2006);

- eye contact (Bacchus 2016a); and

- engaging with "the woman's agenda" on the day, which could change the focus or even be out of scope of the main aims of the intervention (Bacchus 2016a), and relates to the need for flexibility mentioned above.

Advocates also had to accept the difficult, time-consuming, nonlinear process of decision-making for those who survived abusive relationships, and develop an understanding that recovery or life choices for IPV survivors could be markedly different from what the advocates themselves might expect (Bacchus 2016a; Umeda 2017; Wood 2014). This could involve difficult self-reflection by advocates:

"But it's just important to understand that there's a cycle that they go through and they don't necessarily want these relationships to end, they just want the violence to stop. The person's not abusive 24 hours a day, seven days a week, so if he shows her the sliver of hope that things are going to change, there's a good chance she's going to try and I think that's important for new advocates to know, that you can't take it personally, you can't take it as a failure because like I said before, when they come back again knowing that they obviously trusted you enough and respected what you told them, that they've come back when they realize that it didn't work because I don't anyone to ever leave here and think, 'oh, I'm too embarrassed to go back and talk to her because I went back to him,' I think it's real important that they understand that we're there to support them no matter what decision that they make." (quote; Wood 2014, p117).

\subsubsection{Studies affirming the benefits of a shared culture or history of abuse to the advocate-survivor relationship}

In keeping with the concept of essential principle 2, Goodman 2016a found lower levels of alliance in black/African American or multiracial/other women compared with white or Latina participants in their intervention study. Tukey post-hoc analyses confirmed that Latinas $(P=0.01)$ and white women $(P=0.001)$ had the highest levels of alliance of any racial or ethnic group. Stover 2010 analysed 204 domestic violence cases referred to a white or Hispanic advocate. Using multivariate multiple regression analyses, Stover 2010 showed that victim-advocate ethnic match (Pillias's trace $=0.04, \mathrm{P}=0.02$ ) predicted the outcome variables of a number of intervention services provided and the amount of time spent with the advocate (F-test statistic $=7.68, \mathrm{P}=0.006$ ). Sixty-three cases were a victim-advocate, Hispanic-Hispanic ethnic match and 11 were a white-white match. Hispanic women who were served by the Hispanic advocate spent the most time with the advocate and were provided with a broader range of services than those who did not have an advocate-victim ethnic match. A successful advocacy relationship was also linked by participants in the Prosman 2014 study (both women and advocates) to a shared culture and language between woman and mentor mother. Pairs were matched on ethnicity, the intensity of the intervention, and the fact that mentor mothers were often survivors of abuse themselves. Bacchus 2016a and Wood 2014 reported similar approaches.

\section{Hypothesis 4.2: advocacy can be offered across diverse disciplines provided that advocate competency is assured through training}

Trained advocates administered all of the interventions, though there was some variability in their professional status. This depended to a large extent on setting. In particular, professional advocates were more likely in a criminal justice setting, whereas advocates from diverse disciplines administered services in other settings. (According to our stakeholder panel, this suggests the need for standardisation of minimum training requirements or competencies.) The context in which an advocate practices - their discipline and setting - will affect the type of advocacy given and approach used $(\mathrm{M})$ and so may have an indirect effect on outcomes $(0)$, but there seemed to be no direct effect of advocate discipline itself (or the disciplines of the authors of included studies) on the effectiveness of advocacy, confirming the idea that the quality of the therapeutic alliance is more important than other contextual factors. Training was explicitly considered by advocates in Hidalgo 2016 to be more important than setting.

A single professional advocate led 12 interventions (DePrince 2012; Garcia-Leeds 2017; Hathaway 2008; Hyman 2001; Kendall 2009; Krasnoff 2002; Lako 2018; Muelleman 1999; Rodgers 2017; Reina 2015; Stover 2010; Trevillion 2013). A second advocate was involved In two studies; the intervention reported by Thiara 2009 involved a court advocate and also a trained BAME advocate, and a nurse supported the IPV advocate in Wuest 2015. Trained nurses administered six others (Bahadir-Yilmaz 2018; Constantino 2005; Curry 2006, Gupta 2017, McFarlane 2006; Tiwari 2005), social workers, sometimes with psychologists provided six others (Cripe 2010; Kiely 2010; Song 2010; Tiwari 2010; Tutty 1996; Wong 2013), and trained paraprofessional students an additional four (Bell 2001; Bybee 2005; Sullivan 1991; Sullivan 2002). One study provided a mixture of face-to-face advocacy with the study investigators plus telephone support from trained community health workers (Gillum 2009). In another study trained midwives provided the intervention (Infanti 2015). Seven studies were different in that they evaluated the effectiveness of advocacy provided by lay mentors (Bacchus 2016a; Bacchus 2016b; McFarlane 1997; McFarlane 2000; O'Brien 2016; Prosman 2014; Taft 2011). In six of these studies (Bacchus 2016a; Bacchus 2016b; McFarlane 1997; McFarlane 2000; Prosman 2014; Taft 2011), these were 'mentor mothers' and the women were pregnant. In McFarlane 1997 and McFarlane 2000, women were offered a referral card at a single session only, advocacy with a trained professional based in the clinic during pregnancy, or advocacy plus the services of a non-professional mentor mother through pregnancy. Trained, non-professional mentor mothers provided the entirety of the intervention in Prosman 2014 and Taft 2011. In O'Brien 2016, the women were not pregnant but lay mentors were used to deliver the intervention. Bacchus 2016a and Bacchus 2016b reported that home visitors delivering the DOVE intervention included nurses or community health workers supervised by nurses.

Considering the range of advocates across these studies, there was no evidence to link advocate discipline with problems with delivery 
of advocacy, or with reduced effectiveness, though advocates who are on site are preferable. Indeed, lay advocates often seem to be successful.

\section{Essential principle 5: advocates have needs and requirements too in striving for effectiveness}

Advocacy was not considered easy to do and advocates had many needs and requirements that were not met for a variety of reasons, compromising their capabilities to overcome the challenges of their work and be effective in their role. This section considers these needs and requirements principally from the viewpoint of advocates though noting that there is intersection with the women's needs and requirements, as shown in our model. For example, there was evidence that advocates feel that they need time to do the work properly (C1) and that inter- and intraorganisation support ( $\mathrm{C} 2$ ) is also important for this, and yet most interventions are designed to be brief and organisational support is often limited, As a result, the advocate is unable to work as effectively as they would like (M), leading to burn-out and high staff turnover (0). This essential principle is summarised in Table 10. Overall, our confidence in the quality of the evidence for this essential principle varied between moderate and high (Table 11).

\section{Hypothesis 5.1: advocacy takes time to be effective}

Advocates often felt ineffective when they only managed brief contact with the woman, because of organisational failures or because the woman stopped her help-seeking or returned to her abuser (Goodman 2016a; Merchant 2015; Prosman 2014; Wood 2014), or because the advocacy was designed to be relatively brief. According to Kolb 2008 advocates sometimes felt that these women had wasted their time by exploiting their good will. In this way they could describe how they refused sympathy or got annoyed with the women and yet still maintained a "moral identity" as good people.

\subsubsection{Studies affirming the need for time for effective advocacy - and a dose-response relationship}

Wong 2013 showed, using linear mixed-effects analyses, that their advocacy intervention was temporarily effective at reducing depressive symptoms in abused, non-immigrant women, with a reduction at three months but not at nine months. Given that their social support intervention lasted for 12 weeks, they suggested that this may not be long enough for a sustained effect. Nor was it long enough for any effect on immigrant women. The stress of both abusive relationships and immigration may not be expected to change dramatically in three or even in nine months; however, in the shorter term, events, such as beginning formal help-seeking, may have led to a temporary abatement of depressive symptoms. An alternative mechanism might be that sharing with and confiding in others gave non-immigrant women a temporary lift in mood or a feeling of hope but when this did not have any further effect the low mood returned. This would go against the notion of a gain spiral that we have included in our model but we cannot preclude it although other evidence in this section leads us to prefer the explanation of time. For example, immigrant women, as we have discussed in previous sections, are more likely to engage with advocacy over longer periods. Further, Kelly 1999 argued that advocates need to take a holistic approach in which all the different issues the woman must consider are reviewed before she can be supported appropriately in decisions; advocates can only gain this level of understanding with longer-term case work.
Song 2012 conducted a survey of 191 abused women who had received various services from the centres of Prevention and Intervention for Domestic Violence or from the private IPV sector in Taiwan. In this sample, mean service duration was 10.91 months (SD 12.61); women with one contact every two to three months showed less perceived change than women with at least one contact per week over the same period, which Song 2012 suggested was the minimum needed for effectiveness. When the direct and indirect effects on outcomes were modelled, a path analysis showed intensity of contact to be the fourth most influential variable (beta $=-0.26$ ), after empowerment (beta $=$ 0.37 ), relationship with social worker (beta $=0.29$ ) and negative impact of the abuse (beta $=0.27$ ). Similarly, Howarth 2016 reported a dose-response relationship between frequency of contact and effectiveness of the advocacy. Specifically, five or more contacts with an IDVA during the course of a case (compared with less frequent contact) tripled the odds that a woman would experience a cessation in abuse (Wald $=45.09, \mathrm{P}<0.01$, exact $\mathrm{P}$ value not specified in the paper; adjusted OR $2.71,95 \% \mathrm{Cl} 2.02$ to 3.62 ) and that they would feel safer (Wald $=48.27, \mathrm{P}<0.01$, exact $\mathrm{P}$ value not specified in the paper; adjusted $\mathrm{OR} 3.05,95 \% \mathrm{Cl} 2.23$ to 4.17$)$. In an earlier analysis Howarth 2016 reported that for $67 \%$ of abused women receiving intensive support there was an overall cessation in abuse, compared to $44 \%$ for those abused women receiving limited support. Howarth 2016 suggest that women receiving more frequent contact with an IDVA may feel more supported, more optimistic about their situations, and thus less likely to be drawn back into abusive relationships. Thus, intensive advocacy may be effective in reducing physical abuse.

There may be a simple explanation: the more sessions a woman has the more skills and knowledge she can gain. Thus time would work by increasing the woman's exposure to advocacy that in turn increases her competencies. Similarly the more time within advocacy the more likely a woman is to be able to satisfy longerterm needs through advocacy. This was indicated by Wong 2013 in relation to depression (see above). It was also shown by Sullivan 2019, who interviewed shelter advocates about women's housing needs specifically, and found that it took from two months to two years to re-house an abused woman, with, at times, 8 to 10 hours per week of advocate time spent on this for a single woman:

"In the very beginning it was very intensive. Daily communication. You know, probably 5-8 $\mathrm{h}$ a week for that particular person... And then it becomes more diffuse to where you've got three or four people supporting her. But probably several hours a week. And then as we brought in other agencies, for example the transitional housing agency that worked with her, we were able to back off a little bit. But still there was always that constant support." (quote; Sullivan 2019, p 4).

However there may be other mechanisms at play as we discuss in the next subsection.

\subsubsection{Studies affirming the need for time to develop the therapeutic alliance}

Goodman 2016a found that the length of time participants spent in their programme was positively related to the strength of their alliance with the advocate and their sense of empowerment, and inversely related to PTSD and depressive symptoms. In other words, participants who had been in their programme for longer reported stronger alliance, empowerment and mental health. In 
emphasising the need to develop a good working relationship with a client for successful advocacy, advocates in the Lako 2018 study also concurred that it could take weeks to months to develop. Evidence from women themselves further supports this. Women interviewed as part of the Bacchus 2016a study considered that trust in their intervention delivery was cultivated through the repeated interactions they had with women through pregnancy even though the total dose of advocacy was low:

"It takes me a long time to trust somebody. When Rachel [home visitor] first started coming here I didn't like her. I didn't like talking or anybody messing with my daughter. I didn't like people talking to me about past things. But she was very graceful with it. She didn't rush me to want to talk to her. She did it at my own speed and that made me know that she cared.... (Joanne, client, 18 years, rural, IPV +)" (quote; Bacchus 2016a, p 302).

A few interventions outside of health-care settings were particularly high-dosage, in terms of both the length of the sessions and the period over which the sessions were offered. These provided advocacy for respective totals of: about 36 hours over six weeks, mostly in person but also by phone (Bell 2001: legal advocacy); 43 hours over 16 weeks plus an additional 80 hours over 10 weeks provided to the children (Sullivan 2002: shelter); 60 to 80 hours over 10 weeks (Bybee 2005; Sullivan 1991: shelter); and 76 to 82 hours over nine months (Lako 2018: shelter). Perez 2012 analysed data from women who had stayed in shelters for a median of 17 days and considered this too brief for support in dealing with severe abuse combined with PTSD. Song 2010 offered women in shelter services advocacy for up to two years but those who remained in the shelter had a mean of 3.31 months of advocacy and those who left the shelter for the community took up a mean of 8.88 months of advocacy. The one study recruiting in healthcare clinics that was of long duration was conducted in Australia (Taft 2011). The total duration of this intervention is not specified in the paper, but abused women were offered a minimum of one hours' advocacy each week for 12 months, for at least 52 total hours of advocacy.

\subsubsection{What this means for follow-up in studies}

According to Lako 2018, given that more intensive advocacy spread over time should lead to greater effectiveness, follow-up in advocacy intervention studies should be repeated over months to years for a more complete picture of effectiveness. Five studies included a follow-up of 12 months postintervention (DePrince 2012; Muelleman 1999; Stover 2010; Taft 2011; Wuest 2015). Only Bybee 2005, Coker 2012, McFarlane 2000, McFarlane 2004 and McFarlane 2006 included longer follow-up periods (18 and in most cases also 24 months). Bybee 2005 reported on the longest follow-up (three years) and stated that there was no direct effect on risk of revictimisation from their intervention though there had been at 24 months. They argued that this could mean the intervention had no long-term effect or that the control group had developed strategies to reduce abuse so that no difference between groups could be seen; however, the end result is similar, suggesting that the optimal follow-up period for recidivism is 24 months. Yet, the intervention group continued to show better quality of life and level of social support than the control group. This would accord with the gain spiral of the COR (Bybee 2005), as also incorporated within our theory. Bybee 2005 suggested that "booster shots" of advocacy, focusing on recidivism and on changes in circumstances, might be a low-cost means of continuing to protect women from re-abuse.

\subsubsection{Improvements without advocacy over time (implications for} studies)

While advocates may need time to work with women, several studies reported that outcomes improved with the passage of time, regardless of whether or not an intervention was given. Similarly resource use could decrease over time. This can make it challenging to determine intervention-related effects, with regression to the mean (Tiwari 2010). For example, some women might abandon safety behaviours over time as they learn to feel safer, have moved, got jobs or otherwise redeveloped their identities (McFarlane 2004). Tiwari 2010 found reduced severity of depression in both their intervention and control groups. McFarlane 2006 reported that, between baseline and 24 months, women in both intervention and control groups showed $(P \leq 0.001$; exact $P$ value not specified in the paper) improvement in threats of abuse (mean number of threats $=14.5,95 \% \mathrm{Cl} 12.6$ to 16.4 ), assaults (mean number of assaults $=15.5,95 \% \mathrm{Cl} 13.5$ to 17.4 ), danger risks for homicide (mean number of risks $=2.6,95 \% \mathrm{Cl} 2.1$ to 3.0 ), work harassment (Mean number of events of work harassment $=2.7,95 \% \mathrm{Cl} 2.3$ to 3.1 ), and practiced safety behaviours (mean safety behaviours $=2.0$, $95 \% \mathrm{Cl} 1.6$ to 2.3 ), and a decline in the use of community resources (mean number of resources used $=0.2,95 \% \mathrm{Cl} 0.4$ to $0.2 ; \mathrm{P} \leq 0.001$; exact $P$ value not specified in the paper). This could result from women becoming more aware of the need to reduce the abuse even without intervention; there is evidence that women reach a turning point in the relationship even without intervention, at which this realisation occurs (Rivas 2010).

\subsubsection{How does the duration of existing interventions match with need?}

Despite the clear need for time, most advocacy interventions that have been studied in healthcare settings were designed to be relatively brief. Eleven were one-off sessions. Feder 2018 delivered a 20-minute intervention in well-child, family planning, or postpartum clinics; Tiwari 2005, a 20- to 30-minute intervention in antenatal clinics; Cripe 2010, a single session of about 30 minutes in hospital obstetrics; and Hyman 2001 and Muelleman 1999, single, 90-minute sessions in hospital emergency departments. Two more interventions were delivered as one session, but the duration of the sessions is not known (Coker 2012 in hospital clinics and DePrince 2012 in community outreach). Gupta 2017 gave an initial, brief session in a public health clinic and a booster at three months. One intervention in a judicial setting also comprised brief, single sessions, lasting 20 minutes (Casey 2007). Also in a judicial setting, outreach visits were offered as needed but $64 \%$ of women only wanted one (Stover 2010). One further community intervention was delivered as a one-off brief phone session but some women were phoned by a legal advocate as well as a health advocate (Howarth 2016).

Mostly brief sessions involved only safety planning and referral. Advocates interviewed by Goodman 2016a confirmed that they were unlikely to do more complex work, such as developing support networks with the woman, with brief interventions. This accords with the recommendations of Kasturirangan 2008 for advocates to understand that empowerment cannot be achieved from a one-off intervention session but needs to be built up and developed over repeated sessions.

Twenty other studies in healthcare settings offered advocacy sessions over a prolonged period but were still of brief duration overall. Some of these were spread over the prenatal period in 
pregnant women (Bacchus 2016a and Bacchus 2016b, six sessions at monthly intervals for six months; Bahadir-Yilmaz 2018: 10 sessions once a week for 90 minutes, thus with a total dose of 15 hours; Curry 2006: a single session followed by flexible case management, with a mean 3.92 hours (range 15 to 14.75 hours) per case-managed woman spread over a mean number of 22.43 (range 3 to 66) contact events). In McFarlane 2000, women were recruited in antenatal clinics and offered advocacy (or advocacy plus the services of a mentor mother) until the time of their delivery. Most sessions lasted about 30 minutes, with women averaging four to five sessions for an accumulated total of approximately 150 minutes of advocacy (Rivas 2015). McFarlane 1997 reported a similar intervention. In McFarlane 2006, conducted in primary care public health clinics and two Women, Infants and Children clinics, women received five separate 20-minute interventions, each six months apart, for a total of approximately 100 minutes of advocacy.

Krasnoff 2002, in a hospital emergency department, offered a single session plus case management for 6 to 10 weeks. Kendall 2009, in the same setting, gave an initial face-to-face session, with followon contact via telephone at two days and 2, 6 and 12 weeks, to see whether the women were keeping to their plans; thus, this was essentially a single session with brief reminders. Taft 2011 offered up to 10 hours of advocacy during pregnancy. In a similar design, Prosman 2014 offered 16 weekly home visits in which advocacy was combined with prenatal health and social care. In Kiely 2010, advocacy for abuse (and where applicable, therapy for depression and smoking or passive smoking) was provided at four to eight prenatal sessions and two postpartum booster sessions. Depending on how many of the four risks were addressed, each session lasted 50 ( \pm 15$)$ minutes, for a total of 3.5 to 8 hours of advocacy.

Another trial, in a primary care health clinic, provided one face-toface session lasting about 30 minutes, followed by six telephone calls over a three-month period, each lasting an average of 20 minutes (range 5 to 60 minutes; Gillum 2009; Rivas 2015); the total dose was thought by Rivas 2015 to be approximately 2.5 hours. Rodgers 2017 gave weekly advocacy sessions in community health clinics for three months. Trevillion 2013 gave flexible support in community mental health clinics; women received a mean of 7 (SD 5.8, range 2 to 26) one-hour meetings and 28, 20-minute telephone conversations with advisors. The average number of sessions arranged by advisors was 9 (SD 6.1, range 1 to 31). The mean total dose was therefore 13.5 hours.

McFarlane 2004, set in a district attorney's office in the USA, provided six telephone calls ranging from 3 to 25 minutes in duration, over eight weeks, with a mean of nine minutes per call; thus, the total dose may have been as little as one hour. Tiwari 2010 delivered one, two-hour session weekly, for three weeks, in the community; six hours in all. In the community, Wong 2013 offered one, 30-minute (range 20 to 45 minutes) face-to-face session of empowerment, and a social support component of 12 scheduled, weekly telephone calls of 10 minutes each, as well as 24-hour access to a hotline, for a total of up to 12 hours of advocacy. A brief shelter intervention described by Constantino 2005 involved eight weekly sessions of 90 minutes, hence a total of 12 hours. Tutty 1996, also in shelters, offered one to two hours of advocacy a week for three to six months.

Brief interventions spread over time should be more effective than single sessions given our hypothesis about the importance of time and our suggested mechanisms. There is limited evidence to support this though even brief advocacy with one-off meetings with an advocate has some limited effect. In their effectiveness analysis, Rivas 2015 concluded that:

"Based on the evidence reviewed, intensive advocacy may improve short-term quality of life and reduce physical abuse one to two years after the intervention for women recruited from domestic violence shelters or refuges. Brief advocacy may provide small short-term mental health benefits and reduce abuse, particularly in pregnant women and for less severe abuse." (quote; Rivas 2015, p 3).

\section{Hypothesis 5.2: economic resources are needed to preserve the consistency and availability of advocacy services}

\subsubsection{Affirmative studies showing the effect on continuity of care of reduced resources}

Lack of resources, overstretched services and difficulties connecting women to resources meant that women were often unable to be supported by the same advocate at different times. This would affect the therapeutic alliance as we have shown in essential principle 4, and survivor-centred care in any case requires the same advocate to work with a woman for an extended period so that her needs and wishes can be determined. Nine of 14 women interviewed by Prosman 2014 and colleagues, and all mentor mothers delivering the MeMoSA intervention in this study, considered that bonds were strengthened when the mentor mothers not only met the women weekly but could be contacted outside of this time also. Similarly, service directors surveyed by Macy 2018 considered that survivors should be able to speak to a crisis, legal service advocacy or medical advocacy service provider, either in person or by telephone, 24 hours a day, seven days a week and 365 days a year.

Goodman 2016b and Kapur 2017 reported that advocates found it challenging to work with the same woman for an extended period, because of resource constraints or organisational processes, but also that this work would save resources in the longer term:

"I think one of the challenges we've been having in the main office is that when someone comes in it's just whatever advocate happens to be there that works with them. So they may be working with a lot of different advocates. So one person may know that maybe their aunt and uncle are very supportive and have in the past, but when they come in and talk to the next person about something, they're not going to know anything about that. So getting everybody to have that whole big picture, you know we may be missing some things on connecting people." (quote; Goodman 2016b, p 82).

\subsubsection{Affirmative studies on the impact of resource constraints on} what advocates can do for the women

Twelve studies reported lack of resources and overstretched services as a barrier to effective advocacy work because women (and advocates) could not access resources that they needed (Briones-Vozmediano 2014; Burnett 2012; Coy 2011; Goodman 2016a; Kapur 2017; Logan 2018; Lynch 2013; Magruder 2017; Merchant 2015; Murray 2015; Silva-Martinez 2016; Wood 2014). Authors of these studies argued that more effective advocacy is facilitated by communities with more access to supportive resources such as domestic violence-specific resources, supports for legal assistance, shelter and transportation. These give the tools for advocates to do their job as already indicated in essential 
principle 1 . These resource needs were relevant in terms of both inter- and intra-agency work; over-stretched advocates could cut corners in their work, as reported by Silva-Martinez 2016:

"I didn't necessarily use all of the activities . . . just because of the time constraint, of you know, trying to get something accomplished." (quote; Silva-Martinez 2016, p 117).

As suggested in the previous section, this could lead to less effectiveness. Burnett 2012's shelter staff argued that resource constraints meant they were forced to respond to women's needs reactively and not proactively. Lako 2018 noted that the fidelity of their intervention was indirectly affected by service cuts due to austerity measures, since these reduced access to services:

"I basically ran into a brick wall trying to refer her. From pillar to post: Go there, try this and that. And at some point that frustrated her so much that she started rejecting everything." (quote; Lako 2018, p 75).

Goodman 2016b argued that drawing on the women's networks could be a cost-effective way of ensuring support for the women at a time of limited funding.

\section{Hypothesis 5.3: the personal nature of advocacy work makes it challenging for advocates to know how to manage personal- professional boundaries safely, which has implications for the well-being of the advocates and the abused women}

The nature of survivor-centred care, with its focus on what is important in the lives of the women themselves, means that advocates wanted guidance on how to separate the professional from the personal, which they often found difficult (Garcia-Leeds 2017; Goodman 2016b; Johnson 2014; Merchant 2015; Wood 2014). In particular, they were concerned to not cross boundaries in ways that were potentially harmful to themselves or to the women. For example, participants in the Garcia-Leeds 2017, Goodman 2016b and Merchant 2015 studies said they struggled with the fine line between empowerment and disenabling; accompanying survivors into the community or to support services might be intended to empower them but could also make them feel unable to act alone. As one said: "We're a stopgap. We're not a solution" (quote; Goodman 2016b, p 82). This could result in withdrawals of support that left women dissatisfied (Clarke 2008; Grant 2012; Goodman 2016b).

\subsubsection{Where should the boundaries be?}

Advocates who found it challenging to avoid crossing boundaries were unclear as to where their duties stopped. Much of the work done by advocates who were interviewed by Burnett 2012 and Goodman 2016b or who took part in a focus group in the Lako 2018 study, especially work within the community, was done through goodwill and often without resources, funding or training.

In the Goodman 2016b intervention, this out-of-protocol work included organising social activities in the local community or giving women lifts to social facilities or settings. Advocates also said survivor-centred care could lead to women preferring to stay with the advocate rather than moving on to other support networks to which the advocate had tried to link them (Lako 2018; O'Brien 2016). In the Goodman 2016b study, advocates also reported problems in determining the divide between encouraging survivors to forge helpful relationships and deterring them from harmful ones. In the Umeda 2017 study in Japan, the comment was made that advocates who were aware of boundaries were more likely to promote and support survivor autonomy.

Wies 2008 reported that advocates could view organisational boundaries as reducing the partnership or therapeutic alliance with the women:

"We come in wearing heels. We dress differently than the women we serve. We're expected to be professionals. We're not expected to be women helping women. So I think that creates a hierarchy and it creates a distance between us and the women we serve." (quote; Wies 2008, p 230).

However, the same study also found that organisational boundaries, when combined with physical boundaries, could be useful, especially in a shelter:

"Basically a boundary is that line between professionalism and becoming maybe too close with a client. It's keeping up that wall of, "Okay, we're not friends. I have to be the professional and you are the client." Even though in some ways it doesn't always feel that way because it's a residential facility. Boundaries are hard; I think harder in a residential facility than maybe just in a counselling session. So I think it's extra important to make sure you're keeping up those boundaries in a place where they can easily be crossed. So that's it, mapping out where you stand and where the client stands in the relationship. I think individually you have to find that line of where their life ends and your personal/professional life begins. I don't know. I struggle with this, getting wrapped up in a particular person's problems and separating that from you personally but still advocating for them and still wanting better for that client." (quote; Wies 2008, p 226).

In this way, boundaries were identified as positively able to promote "the emotional and physical safety of residents and staff," (quote; Wies 2008, p 231), and allow the advocates to maintain a professionalism but also to distance themselves from the emotional work involved in advocacy (Wies 2008).

The advocates in the Kolb 2008 and Wood 2014 studies sometimes found it challenging to stand back and allow women to make decisions they thought of as unwise, dangerous or both but the advocates did not want to disempower the women. They used this argument to reflect on their work and set boundaries to their responsibility. For example:

"Advocate: What came out of me, something I had to check myself for, was that I wanted to rescue her. My rescuer came out, big time. I was like, 'Oh God! That is not my job! I am not being paid to rescue her.' But I really wanted to rescue her. I really wanted to take her and her child and put them in my car and say, 'We're out.'

Interviewer: So why didn't you?

Advocate: That's not my job." (quote; Kolb 2008 p4).

The experience of the mentors of abused women interviewed by O'Brien 2016 was somewhat different in that, "It was interesting forming a relationship with a stranger that sat somewhere between professional and personal." (quote; $p$ 54). The difference may be because these were lay volunteers. 


\subsubsection{Boundaries and advocates who have themselves been abused}

In the Wood 2014 study, the majority of advocates had experienced partner abuse themselves and sometimes worried that in crossing boundaries they might trigger distressing memories of their own experiences. For this reason, advocates with their own experiences of IPV may be encouraged by the organisation to develop clear boundaries, and discouraged from talking about their personal experiences (Wies 2008).

The participants in the Goodman 2009 study, who had been abused women supported by advocates in the intervention and had then become workshop facilitators, commented on the importance of advocates taking the initiative and showing willingness to roll up their sleeves to do what it takes to get things done, to share with the woman on her own terms. This was seen as a mechanism for building up the therapeutic relationship. . Women put it thus:

"You have to be willing to really get your hands dirty. [For example], if you're having a crisis or something with child care or something, "Oh, well I'll watch your kids for an hour" ... or "I'll come with you to the Laundromat" ... "I'll come with you and ... help you get this done because this is overwhelming in your life and making things a nightmare for you." (quote; Goodman 2009, p 861); and

"Okay, they need to read between the lines 'cause we're not very good at saying, "Oh, this is what we need." You know what I mean? We're not like that 'cause we're taking care of other people and that's how we are. We're not very good at expressing our needs.... But if it's obvious ... you can say, "Hey, well maybe there's something I could do to help you with that." (quote; Goodman 2009, p 862).

\section{Hypothesis 5.4: there is a need for advocates to balance competing roles and duties}

Advocates could find it challenging to balance competing roles and duties without good support. For example, advocates had a duty to encourage women to take control of their lives but also to minimise the risks, and lacked confidence in this balance. Participants in the Goodman 2016b study highlighted the tension between wanting to help survivors to develop their social networks and worrying that this carried risks. These risks were articulated as: losing contact with the woman before this was considered appropriate; network members encouraging women to stay with abusive partners; women encountering rejection, disappointment, stigma, and shame; and network members that were themselves abusive. According to advocates in the Lako 2018 study, a new crisis situation (such as a new episode of abuse from the man, or a deterioration in the woman's economic situation), could lead to temporary intensification of advocacy from the advocate but at the same time it could motivate a woman to become more accepting of help from others than the advocate. The issue for advocates was that:

"We don't know those specific people. How do you know that that's a safe place to send them basically? For us to try and have them reach out to that person, what if it really isn't safe for them to do that, but we don't know." (quote; Goodman 2016b, p 79).

Advocates dealt with this issue by focusing on what Coy 2011 and Dunn 2007 refer to as "empowerment through knowledge", providing information and options for the women to use to make evidence-based decisions (Coy 2011):
"It's about ensuring that the women understand what their choices are, so we're not telling them what to do, it's about telling them what their choices are and that they decide. I think that's important because they've been controlled, so it's not for us to then take that [control]". (quote; Coy 2011, p 36); and

"By empowering women do you make the choices for women, do you give them advice, do you give them guiding advice? I've heard it described as "guiding choices". So you're guiding women to make those choices when - when they might not choose to... You're going to give someone the skills to empower them, but actually the choices they make might not be choices that you agree with, and you have to respect them... And I would tell a client "I think that's your choice as a woman, but actually I have to tell you, for this reason and that reason, for your safety, that I actually don't think that's the right decision" (quote; Coy 2011, p 36).

Other balancing issues included how to:

- take a survivor-focused approach in the context of crisis management (in the study by Goodman 2016b); and

- balance the competing roles dictated by clients (Merchant 2015), the advocate's organisation (Lako 2018; Merchant 2015; Silva-Martinez 2016), and the advocate's own beliefs (Merchant 2015; Silva-Martinez 2016), as well as government rules and regulations (Burnett 2012).

For example, some of the advocates interviewed by Silva-Martinez 2016 expressed a sense of frustration at having to implement a financial literacy programme when there were other crises that needed their attention. Further, advocates seemed to view the curriculum as irrelevant to their clients' current hierarchy of needs and considered that only some women would benefit anyway. Advocates cited the following factors as relevant: a woman's age, immigration status, time at the shelter and employment status. These judgments factored into how, when, and to whom they introduced the content. Participants just entering services and deemed "in crisis" were predominately excluded from the curriculum by the advocates. There was a general sense that clients just leaving abusive situations could not gain from this information at that time because they were addressing more immediate issues.

\section{Hypothesis 5.5: advocates take on emotional labour, which needs to be acknowledged}

\subsubsection{Studies affirming the significance of emotional labour and burnout}

Emotional labour refers to the process by which service industry workers are expected to regulate the expression of their emotions during interactions with clients, co-workers and superiors (Hochschild 2003). This includes showing emotions that are not felt as well as suppressing emotions that are felt but are not appropriate to show. Surface acting involves "faking" (Hochschild 2003), emotions through outward expression while leaving internal feelings intact. Employees who do this must wrestle with the problems of feeling inauthentic, which can lead to reduced wellbeing and eventually to burnout. As an example of surface acting, boundaries in the Wies 2008 study, as noted above, were seen as positively able to promote "the emotional and physical safety of residents and staff" (Wies 2008, p227), and allow the advocates to maintain a professionalism but also to distance themselves from the emotional work involved in advocacy. Deep acting occurs when employees change their internal feelings to align with those of the 
organisation. Hochschild showed that burnout could be reduced if employers trained their employees in emotional labour. Even if surface acting is maintained, burnout can be reduced if employees are given scripts or protocols to follow, or supported in using humour to deal with emotional issues (Leidner 1999); Ganz 2015 considered how and why burnout develops when this was not done.

Thirteen studies emphasised the emotional labour of IPV advocacy work (Babin 2012; Burnett 2012; Ganz 2015; Garcia-Leeds 2017; Grant 2012; Kapur 2017; Kolb 2008; Merchant 2015; O'Brien 2016; Prosman 2014; Slattery 2009; Wies 2008; Wood 2014); it was said that burn-out and a hardening of attitude could attenuate a survivor-centred approach (Wood 2014), or reduce the capacity of the advocate to take on work (Prosman 2014). Slattery 2009 found that being a survivor of past violence predicted higher levels of occupational stress as an advocate, such as burnout and secondary traumatic stress (STS). Workplace social support ( $r$ (correlation coefficient) $=-0.23, P<0.01$; exact $P$ value not specified in the paper), the quality of clinical supervision ( $r=-0.23, P<0.01$; exact $P$ value not specified in the paper), and shared power were all inversely related to STS $(r=-0.30, P<0.01$; exact $P$ value not specified in the paper). Bacchus 2016b and Kelly 1999 reported similar findings from qualitative analyses of advocates.

The study by Kulkarni 2015 determined that advocates with a longer time in their current position were less likely to exercise a survivor-centred perspective, whereas a longer time working within IPV was positively associated with a survivor-centred perspective (F-test statistic $=6.04, \mathrm{P}<0.01$; exact $\mathrm{P}$ value not specified in the paper). Adding demographic controls also resulted in a strong model (F-test statistic $=3.49, \mathrm{P}<0.01)$. The analysis also suggested that higher levels of advocate education are positively associated with a survivor-centred perspective and that older age of staff is negatively associated with a survivor-centred perspective. Possible reasons according to Kulkarni 2015 may be that workers who specialise in IPV have short contracts within individual organisations or that they prefer to move around, but burnout could also be a factor.

\subsubsection{Studies affirming the significance of emotional labour and occupational stigma}

Ganz 2015 found that advocates were largely unprepared for occupational stigma by their organisation and that this had an effect on advocates' sense of self, sense of work, and willingness to share their occupational identity. Six of 11 advocates interviewed by Ganz 2015 cited duty and pride in their work as reasons to talk about their work, and to achieve social and interpersonal change. Conversational partners outside of work attributed advocates as having a special disposition ('othering' them), and stereotyping them such that the advocate was said to be doing good work for others and should be provided accolades for the moral superiority of the work that other people are not capable of doing. In so doing, the advocate and their work were marked out as different, that is, stigmatised. For example:

"I hadn't really noticed anyone or anything that has struck me as a pattern. Other than people being like, "Oh, that must be so hard. You must be...you're just doing great work." There's this weird assumption that I must be this great, altruistic saint for doing what I do." (quote; Ganz 2015, p 73).
Advocates also reported hostile responses, including inappropriate humour, applying negative stereotypes about feminism, or using rhetorical questions-sometimes in the form of declarative statements rather than questions-to bait advocates:

"It [negative encounters] happens when they minimize. There was a situation with some man saying, "Deep fried and battered women." He was being funny about it. It's like, 'Okay. I have a sense of humor.' But, I just said, "Okay." Then, he says, "Well, it's their fault, because they are the ones that go back." So, I tried to explain the situation, because you need to walk a mile in their shoes." (quote; Ganz 2015, p 107).

Advocates in the Kolb 2008 study reported how others often downgraded their work as women's work that drew on their innate caring abilities rather than professional skills and training. Advocates responded by backgrounding the emotional support they gave women and emphasising their expertise or qualifications. For example:

"I think he finally realized that I really did work in the criminal justice system doing this stuff and I really did sit in court and watch all these cases; I really did attend legislative hearings and know a little bit about the law. But I still think that [he believes] my knowledge comes from my experience and my heart... that it's just me being emotional. And I think a lot of advocates get that. Here's an example. A woman did a training [presentation] on DV and she was a survivor. And she was presenting it, and in a very professional way. But this one guy, apparently, didn't like it. He got up and said, "I've never heard such BS in my life." And he just wrote her off as an angry victim. And so we get a lot of that. They think that we are just doing this because we are being over emotional." (quote; Kolb 2008, p 5).

Conversational partner's sympathy and focus on the negative impact of working with abused women suggested the advocate to be extraordinarily capable of managing traumatic material, with exceptional resilience. This can prevent the advocate from seeking help for stress and burnout (Ganz 2015). Mostly, however, advocates evaded stereotypes and occupational stigma by hiding their work identities or simply saying they did social work. This had the benefit of giving advocates time away from their work when they have left their jobs for the day, which could help to avoid burnout (Ganz 2015). Similarly, many people avoided talking about the advocate's work when they heard what it was:

"I think that as people, we struggle with, whether we realize it or not, we struggle with trying to maintain a sense of control within a world that is largely outside of our control." (quote; Ganz 2015, p 104); and

"When you look at issues of violence at the hands of another person that pushes the issue of control or lack of control right in someone's face. So, I can understand why people are like, "Oh, wow, this is something I don't want to talk about or don't know how to talk about." That means, who are you if you work with victims? Do you hate men? Or are you afraid of people? Are you a victim yourself and what does that mean? It's kind of opening up a large can of worms, or at least, it can feel that way to strangers." (quote; Ganz 2015, p 105). 
5.5.3 Studies affirming the connection between occupational stigma and burnout

Analysis of the survey data collected by Ganz 2015 showed advocates' experiences of othering, stereotype, and occupational stigma to be important predictive factors for burnout.

Bemiller 2010 used ordinary least squares multiple regression analyses to develop a burnout model. They found that "Hours per week worked" had a positive relationship with burnout and "Having the tools to do the job" a negative relationship. The "Rewards of working in the job" decreased stress and burnout, and the "Positive aspects of the job" outweighed the negatives and also were negatively associated with burnout. This suggests that selfbalancing strategies may be important in managing burnout in IPV advocates, which fits with Hochschild's model. When controlling for a range of other variables in their model, Bemiller found that a one-unit increase in rewards was associated with a four-unit decrease in burnout (beta $=-0.16$ ) and a one-unit increase in positives outcomes? (beta $=-0.38$ ) was associated with a five-unit decrease in burnout. Beta scores for hours per week worked and having the tools to do the job were 0.18 and -0.15 respectively, while another variable "Negotiating collaborations with outside agencies" also had a beta score of 0.15 and led to an average 1.25 increase in burnout. Bemiller 2010 also noted that a perception of coworker stress increased burnout, whereas one's personal stress did not. Bemiller 2010 suggest that advocates acknowledge that their job is stressful, but as individuals perceive themselves as able to adapt, or alternatively, that advocates may balance sympathy for co-workers experiencing stress with positive valuations of the work they do. Two of the three variables negatively related to burnout represent adaptation factors related to what Bemiller 2010 term "good soldiering". Thus Bemiller's work suggests several mechanisms at work.

Babin 2012 reported results from a survey of 69 individuals recruited from a domestic violence agency in the USA. These showed that the combination of communication anxiety, communication competence, perceived emotional support, and perceived informational support accounted for approximately $13 \%$ of the variance in advocates' emotional exhaustion and $21 \%$ of the variance in advocates' experiences of reduced personal accomplishment. Babin 2012 suggested that the more anxiety people feel about interactions in a counselling or advocacy setting, the more likely they are to feel uncertain about their competencies, leading to feelings of reduced personal accomplishment and selfconfidence and thence burnout. Perceived informational support and perceived emotional support were positively related to one another, and both types of support were negatively related to burnout. Informational support was better able than emotional support to mitigate burnout in their model; Babin 2012 argued that this was because it focused on task-related solutions to work-related problems that, if unsolved, might increase emotional exhaustion over time. Babin 2012 also noted that advocates experienced higher rates of burnout than board members or volunteers, supporting hypotheses that time at work and exposure to trauma content increased levels of occupational stress (Babin 2012).

Hypothesis 5.6: in it together. The importance of support, connection and shared understandings within and across organisations (common language, common tools, common goals...)

The challenges of the advocacy role could be reduced through good organisational, programme and peer support that left advocates effective and able to access support in role and more likely to have job satisfaction in adhering to intervention designs (O'Brien 2016; Prosman 2014; Song 2010). This, in turn, meant they were less likely to leave and were more effective in a woman-focused approach. Conversely, advocates who felt isolated, unsupported or ineffective because of organisational constraints were more dissatisfied and more likely to leave (Burnett 2012; Goodman 2016a; Merchant 2015), or else to reduce adherence to an intervention (i.e. not maintain fidelity to any protocol) (Kulkarni 2015; Lako 2018; Merchant 2015). Kulkarni 2015 found job satisfaction contributed nearly all of the $20 \%$ variance in their overall model of variables affecting staff survivor-centred attitudes. It may be that job satisfaction leads staff to have positive perceptions of their clients or that survivor-centred approaches enhance job satisfaction. According to Merchant 2015's shelter-based study, job satisfaction did not correlate with shelter size, location, budget or access to resources, but was influenced by the impact of various factors on effectiveness in the role.

Institutional support within the organisation where the advocate was based was rarely discussed in intervention studies; this might involve investment, approval, and support for the integration or institutionalisation of the programme at higher levels within health care settings or institutions, and occasionally making linkages with community resources. However, we found seven examples. Bell 2001 's advocates worked with law faculty attorneys. DePrince 2012 reported a successful collaboration between research, systembased partners, and community-based partners. Howarth 2016 commented that IDVAs are formally recognised by service-level agreements and standards as a vital player in multi-agency initiatives. Infanti 2015 described how Sri Lanka's Ministry of Health strengthened the capacity of the public health sector to respond to domestic violence by developing a four-day training module on IPV for primary health care professionals. The RCTs undertaken by Lako 2018 were initiated by the Academic Collaborative Centre for Shelter and Recovery and designed in consultation with this platform's steering committees and working groups, which consisted of directors, managers, and practitioners from the member organisations. Thiara 2009's work was part of the wider development of national advocacy services planned by the charity, Refuge. In Tiwari 2010, the site investigator was an experienced social worker in charge of the family services unit in the centre.

Two studies reported a specific lack of support. Goodman 2016a found a lack of support for their community networks and Kapur 2017 a lack of systemic change.

Participants in five studies highlighted the importance of peer support from co-workers, which they had benefited from, to job satisfaction (Lako 2018; Merchant 2015; O'Brien 2016; SilvaMartinez 2016; Wood 2014). As one participant in Lako 2018's focus groups explained, both "Reflection [during team meetings]. But also right in the middle of your work when someone suddenly reminds you" (quote; Lako 2018, p 76) could improve intervention delivery. 
In terms of external support, multi-agency networks could treat advocates as equal partners and mitigate their less powerful position as voluntary sector agencies (Coy 2011):

"People still have this attitude that if you work for a voluntary organisation it's voluntary and amateur, and I think people still believe that, and I think the only reason certain people attend [multi-agency networks meetings] from Social Services and housing is because they actually have to do it. I think that's why the IDVAs get the co-operation that they perhaps get (Stakeholder, Newham, R2)." (quote; Coy 2011, p 88).

Mostly, however, participants across 11 studies focused on the need for better, effectively co-ordinated interagency collaborations (Burnett 2012; Coy 2011; Kapur 2017; Kelly 1999; Lako 2018; Lynch 2013; Magruder 2017; Murray 2015; Silva-Martinez 2016; White 2019; Wood 2014). This would:

- enable support and knowledge exchange across agencies (Kapur 2017; Silva-Martinez 2016);

- lead to more efficient resource use (Kapur 2017); and

- avoid the frustrations and time-wasting caused by unresponsive external services (Lynch 2013).

It would also help advocates to feel more useful in role, which could mitigate against burnout and other issues. As a participant in the Lynch 2013 study said:

"I feel like I spend a lot of my time helping people negotiate through systems and the work of other workers who haven't picked stuff up or done what they actually should be doing ... We actually spend a lot of our time helping women negotiate systems which should be doing a much better job, you know. And that is a little frustrating. It's frustrating enough when you're dealing with Courts and maybe police, but it's more frustrating when we're dealing with services that are actually established [for victims] - domestic violence services and refuges." (quote; Lynch 2013, p 21).

\section{Essential principle 6: the type of setting affects the theoretical underpinnings and the type and focus of the advocacy being offered and the women's responses}

Setting may be indicative of the severity of the abuse (see essential principle 1) or the woman's multiple needs (not all of which may relate to the abuse) (C1), and different settings operate different constraints on what advocacy is likely or feasible (C2), and therefore the mechanisms and functionings choices that are enabled $(M)$, and the intended and unintended outcomes $(0)$ that may result. No study formally considered the impact of setting on outcomes but a number of study authors made relevant observations, including Casey 2007, and the authors of seven studies that obtained qualitative data focused on setting (Johnson 2014; Lea 2016; Lynch 2013; Macy 2018; McDermott 2004; Owen 2015; Thiara 2009). This essential principle is summarised in Table 12. Overall, we had high confidence in the quality of the evidence for this essential principle (Table 13).

\section{Hypothesis 6.1: advocacy delivered across different settings offers its own specific characteristics, advantages and issues}

\subsubsection{Healthcare}

Sixteen studies recruited women in healthcare settings. Five studies recruited women from hospital trauma or emergency departments
(Hyman 2001; Kendall 2009; Krasnoff 2002; Muelleman 1999; Taha 2015), which Coy 2011 suggest enables early intervention in cases where the risk of physical injury is substantial. Trevillion 2013 recruited women from a community mental health setting. Thirteen studies were based in antenatal care or mother and child clinics (Bacchus 2016a; Bacchus 2016b; Bahadir-Yilmaz 2018; Cripe 2010; Curry 2006; Feder 2018; Infanti 2015; Kiely 2010; McFarlane 1997; McFarlane 2000; McFarlane 2006; Taft 2011; Tiwari 2005), two of which were qualitative (Bacchus 2016b; Infanti 2015) and one of which included a qualitative evaluation as well as an RCT (Bacchus 2016a). There were seven studies in primary care or public health settings (Coker 2012; Gillum 2008; Gupta 2017; McFarlane 2006; Prosman 2014; Rodgers 2017; Wuest 2015).

It is possible that women recruited in non-critical healthcare settings (antenatal care, mother and child clinics, primary care or public health) may not yet be ready to accept help (Zink 2004). Alternatively, they may feel validated by the recognition and acknowledgment of the abuse by someone they respect and may begin to reflect on the seriousness of their situation and the need to change it (Coy 2011; Gillum 2008), which was something we discussed in essential principle 3. Thus, the potential for empowerment in these settings may be affected by the woman's assessment of her current situation and risk. In either case, an important option may be to leave the door open for women to access intervention services in healthcare settings when they are ready to do so. This was articulated by a woman interviewed by Bacchus 2016a after being given the DOVE intervention:

"DOVE really helped a lot...Some women could tell you right off the bat "look he beat me." But some women could be just like me and it takes time. I think if they do it and the home visitor comes in and they're graceful and supportive, I think it will help [women] a lot. I feel like it helped me a lot and to trust people again. (Joanne, client, 21 years, rural, IPV+)" (quote; Bacchus 2016a, p e309).

Most interventions in healthcare were of less than 12 hours' total duration (one session or spread over several sessions), combined with healthcare and sometimes opportunistic (for example, when women presented in an emergency department of a hospital); the exceptions being Taft 2011 and Wuest 2015. The issues of brevity were discussed in essential principle 5.

\subsubsection{Shelters}

Nine studies recruited women either exclusively or primarily from domestic violence shelters, two of which were qualitative studies (Merchant 2015; Sudderth 2017), and the others experimental studies (Bybee 2005; Constantino 2005; Goodman 2016a; Lako 2018; Song 2010; Sullivan 2002; Tutty 1996).

Women in shelters can be assumed to have left the home, if not necessarily the relationship, and to have experienced severe abuse, which may impact on the effectiveness of the intervention (see essential principle 1). Shelter-based interventions were the interventions with the longest duration, reflecting the opportunities the advocates have to spend time with the women and perhaps also their needs (for example, housing).

\subsubsection{Criminal justice services}

Ten studies recruited women who had contacted criminal justice services such as the police (Bell 2001; Casey 2007; Davis 2006; Davis 2007; DePrince 2012; Lea 2016; McFarlane 2004; Stover 2010; 
Thiara 2009; Weisz 1999). Two of these studies were qualitative and supported the importance of legal advocacy, particularly within a multi-agency model (Lea 2016; Weisz 1999). Studies in a criminal justice setting mostly considered recidivism but also considered psychological outcomes and the use of judicial resources. Casey 2007 evaluated home-visit outreach, which involved unannounced home-visits by police officers accompanied by an advocate. The local advocacy community expressed their concerns that this would antagonise the perpetrators of the abuse and compromise the women's safety. However, abused women gave positive feedback about the service. Casey 2007 moreover reported an effect of the intervention on calls to the police about domestic violence 12 months after the target incident ( $\mathrm{Chi}^{2}$ with two degrees of freedom $=12.1, \mathrm{P}<0.001$; exact $\mathrm{P}$ value not specified in the paper). Of the 102 women in the intervention group, 20 $(20 \%)$ received police intervention during this 12 -month period, compared with 43 (42\%) in the comparison group. Casey 2007 reported that police-advocacy relations were strengthened by this success. However, the study authors themselves noted that calls to the police are a proxy for recidivism and a reduced number of calls could reflect reduced desire to involve the police rather than recidivism. Moreover, participants were not randomised to the two study arms, and the total sample size was small. In Stover 2010, women who received advocacy were more satisfied with the police and likely to call them to report a non-physical domestic dispute in the 12 months following the initial incident than women in the comparison group, but Stover 2010 also reported that there was no effect on recidivism.

The results from Davis 2006 and Davis 2007 provide more depth and also support the initial fears of the advocates in Casey 2007 regarding antagonism as a mechanism driving abuse. Davis 2006 found that households receiving the home-visit or public education intervention were more likely to call the police within six months than those not receiving the interventions. Multivariate analyses showed that those assigned to receive home visits were more likely to experience more frequent abuse and to report it sooner than those not assigned to receive home visits. In a doseresponse analysis, Davis 2006 also compared, for the Domestic Violence Intervention Education Project (DVIEP) study, recidivism rates between households assigned to receive home visits in which these visits were actually made versus those in which educational literature only on IPV was posted through their doors. In five of the six comparisons, those receiving home visits reported more abuse in terms of frequency of complaints made to the police (1.25 reports versus 0.81 for those receiving educational literature only) frequency or severity of abuse reports made to research interviewers ( 7.05 reports versus 3.55 for the educational literature group), and prevalence of abuse reports made to research interviewers (48\% versus 35\% for those receiving educational literature). Davis 2007 found that victims who received the DVIEP intervention were more likely to call the police, and to call more rapidly to report abuse, in the six months following the intervention than those assigned to the comparison group (45\% versus $39 \%$, respectively). However, considering victim reports from $72 \%$ of the sample at the six-month follow-up period, there were no group differences in severity of abuse reported on the Conflict Tactics Scale, with high rates of recidivism reported in both groups.

Specific mechanisms for the effectiveness of advocacy delivered as part of the criminal justice system could be a trust in the 'strong arm of the law' and the use of force available to police to protect the woman from her abuser; however, according to Davis 2007, this often appeared to have a negative effect. Partners were aware that the home visit occurred in $46 \%$ of cases, and in $28 \%$ of these the partner had a negative reaction to the visit. In $73 \%$ of cases where the victim said that the perpetrator was aware of the home visit, victims reported subsequent physical abuse. In contrast, among cases where victims were sure that the abuser was not aware of the visit, just $5 \%$ reported subsequent physical abuse. Therefore, it appears that more frequent reporting means more abuse, rather than greater sensitisation to abuse. Thus, in this case, the intervention had a serious unwanted effect; however, it could also mean that victims who were having trouble with their partner were more likely to use the visit as a means of discouraging him from committing new abuse. Among six cases where victims said that the perpetrator was home during the second response, none reported new abuse of any kind. Goodman 2016a reported findings that support the possibility of increased danger to some women from taking a criminal justice approach:

"I have a restraining order against my ex-husband, which works to keep me safe. However, it doesn't keep my son safe. I still have to share custody of my son with my ex-husband, which makes staying safe harder. I worry about my son telling his father where we live, and I worry about my ex-husband following me home. I can honestly say the R.O. doesn't make me feel safer because it doesn't cover my son. It actually has made me feel less safe because now my ex-husband is even more angry with me." (quote; Goodman 2016a, p 6).

It is possible that a further mechanism is the extent to which the woman or her perpetrator believe they can maintain control over the degree of involvement of criminal justice services. In Kelly 1999 's evaluation of an IDVA service in a police station, advocates said it was important for them to make clear to women that they were independent of the police. It was notable that most interventions in a criminal justice setting, like healthcare-based interventions, were of brief duration (less than 12 hours; the one exception was Bell 2001), and often comprising a single session.

\subsubsection{Community services}

Twenty-four studies were set in other community services, including third sector and specialist IPV organisations; most of these studies were qualitative and often considered the advocates rather than the women (Briones-Vozmediano 2014; Burnett 2012; Dunn 2007 (included both criminal justice and shelter advocates); Ekstrom 2015; Ganz 2015; Garcia-Leeds 2017; Johnson 2014; Kapur 2017; Kelly 1999; Kulkarni 2015; Logan 2018; Lyon 2011; Macy 2018; Murray 2015; O'Brien 2016; Owen 2015; Reina 2015; Silva-Martinez 2016; Umeda 2017; Wood 2014). Only Howarth 2016, Tiwari 2010 and Wong 2013 were experimental studies, while Babin 2012 was survey-based. In Australia and the UK at least, survivors do not usually approach such services directly for support, being not ready or not able to, at the time they first seek help ( $O$ 'Doherty 2016). Amongst formal services, health professionals tend to be the gateway to other forms of support; indeed, the WHO guidelines for IPV and sexual violence consider that primary care plays a key role in first-line responses, particularly in countries where there are no specialised services (WHO 2013b). Community-based services, like shelter services, are more likely to apply a subsequent, longer-term approach. Howarth 2016 commented however that the two need to be linked as in their evaluation of IDVAs there were lower rates of referral to health services than might be expected based on other 
studies of the prevalence of physical and mental ill health in abused women.

McDermott 2004 described the issues that arose when an advocacy service made unsolicited contact with women. The main intervention offered was a rural, co-ordinated, interagency programme in the USA (that included advocacy but also other components), which was given to women who contacted the police to report abuse. This advocacy was acceptable to the women, but when this intervention ended, a not-for-profit women's centre sent women postal invitations to follow-up advocacy. It was this advocate's services that were mostly rejected; for example, in one six-month period, the advocate received 89 referrals from the prosecutor's office or the mental health agency but saw only four or five of these women. McDermott 2004 suggested that this was because the advocacy offer was not only unsolicited but it represented an often unwanted intrusion into the lives of the women that intersected negatively with broader societal structures:

- official organisations, including child welfare putting the woman or her parenting 'under the spotlight';

- safety checks and follow-up advocacy that were not requested or seen as necessary by the women;

- 'advice giving' that suggested the professional knew best what was in the woman's interest;

- coercion of the woman 'for her own good', such as: to testify in court; to shape her story to make a better case for the prosecution; to accept the abusive man's arrest against her wishes; to provide evidence against the man such as photographs of her injuries, medical records; to attend a support group; and

- contacting network members, such as neighbours, when the woman would prefer privacy.

The conclusions of McDermott 2004 have been strongly critiqued, for example by Flanakin 2005, for confusing the role of advocates with the interventions of the criminal justice system in which they may be embedded. These criticisms highlight the importance of considering context and setting when evaluating whether or not an intervention works. Coy 2011 found that when an advocate was located in a formal setting, such as 'REACH', based at St Thomas' hospital Accident and Emergency department, and 'DVSS' at Barnet police station, credibility by association was conferred on the advocate. This credibility could be with the women the advocates served but also with other agencies with whom the advocates worked (Coy 2011).

"I feel, especially in multi agency settings, at meetings, that I've got a bit more credibility, because as soon as you say that you are at the hospital, I think people are a lot more open to sharing information with you... So I think that's one really good thing that's come out of being based in a hospital (IDVA, Reach, R1)." (quote; Coy 2011, p 22).

Thiara 2009 similarly reported how RSAP advocates, situated within the courts, had a specialist knowledge and insight into the way the courts and the criminal justice service operated that other sources of support for the women would not have. This was reported by advocates to have improved the support that women received and the numbers of women getting the support, with rapid feedback about the progress of the women's cases considered to be the most valuable aspect (Thiara 2009).

\section{Hypothesis 6.2: urban and rural areas are very differently resourced and have very different community cultures, which will shape the form of advocacy services}

Survivors' needs and responses will also vary depending on whether they live in rural or urban communities. Umeda 2017 pointed out that their study, for example, having been undertaken in one metropolitan city in Japan, had limited generalisability to rural settings or even smaller urban areas. This was because information and resources concerning the care of IPV victims was more abundant in the city of their study than in any other area of Japan. Coy 2011 and Thiara 2009 considered a range of services in London and thus similar observations would apply to their findings. Most studies were undertaken in urban areas.

Seven studies considered rurality as a factor affecting the effectiveness of services. Survivors in rural areas face a number of obstacles in obtaining services not faced by their urban counterparts, such as geographic isolation, unavailable or limited supportive networks, lack of services within the immediate area, local norms about family privacy, and lack of anonymity (Johnson 2014). The multiple constraints of poor infrastructure, limited services and local social attitudes not only created barriers to effective intervention and access to services in rural settings for the women, but also placed various constraints on advocates, including those caused by exclusion, over-onerous workloads and under-resourced services (Owen 2015). Wuest 2015 noted that their intervention cost more to deliver in rural than in urban areas because of additional transport costs for the advocates. The issues this causes and the various mechanisms at play have already been largely discussed in essential principle 1, Burnett 2012 reported that being the "only game in town" (quote, Burnett 2012, p 526) meant that rural shelters had to deliver services well beyond their mandate and stretch their resources, which made strategic partnerships with other rural organisations a matter of survival:

"... in a rural community it's critical that we figure out how to collaborate, which means we don't necessarily totally agree with the philosophy of other organizations ... but in a rural community if we can't figure out how to work together, nobody succeeds. So the need for cooperation and collaboration is hugely increased in a rural community (manager, rural shelter)." (quote; Burnett 2012, p 528).

In relation to women's housing needs, the shelter advocates interviewed by Sullivan 2019 described that they would mostly network within formal community councils in more urban areas, while in rural areas they often relied on more informal connections. This may be because of looser formal connections over widely dispersed areas or because that is how things get done in rural areas.

Lynch 2013 and Owen 2015 both focused on rural areas in Australia, where there were small, closed, male-dominated communities in which personal troubles were seen as private family matters (Owen 2015). This made women reluctant to use services (Lynch 2013; Owen 2015), and they isolated themselves while injuries healed (Owen 2015), because of such issues as: fearing the involvement of statutory child protection services (Lynch 2013; Owen 2015); fearing their partner being taken into custody and the consequent backlash from their community (Lynch 2013); and gossip and stigma associated with having problems (Owen 2015). Such issues were particularly evident in white communities where generations 
of families had typically built up farms or businesses or were highly respected for supporting the cultural development of the area (Owen 2015). Abused women often 'othered' different abused women as "them" to distance themselves from any issues (Owen 2015). According to the advocate participants in the Owen 2015 study, these same informal social controls made IPV advocates themselves also marginalised and vulnerable to social stigma and exclusion, and also to professional isolation. (Owen 2015).

Women in rural areas often had to travel long distances to services, which were more widely dispersed than in urban areas, with lack of transport and transport costs creating additional barriers (Lynch 2013; Owen 2015). Courts and other services were small in rural areas and often inadequate in terms of privacy from the community and separation of the woman from her abuser (Lynch 2013; Owen 2015). Court order applications were heard in between other local court business, such as traffic infringements, meaning the women were likely to be seen by the people they knew and, without set times, women and their advocates, who usually came from understaffed services, often had to wait most of the day at court. Moreover, childcare was not available so women with small children and without alternative support had to have their child with them in court (Lynch 2013). Participants in the Lynch 2013 study highlighted the negative effects these issues had for both women and over-stretched and under-resourced advocates. We have previously considered these issues in essential principle 2 in particular. A multivariate ANOVA by Macy 2018 showed that directors from agencies in mixed areas were less likely than rural directors to believe that crisis and medical advocacy services should be provided by staff rather than volunteers, likely because of lack of staff and resources.

Survivor-centred services, such as family support services, court support, women's centre, housing and refuges, took some account of the issues. Some services were flexible when using the income test of eligibility in rural areas, given the scarcity of support and since rural women might have difficulties accessing any money they might have due to rural economic structures, making the women economically dependent on the man (see essential principle 1) (Owen 2015). It is of interest that Bacchus 2016a found that higher proportions of women from rural sites remained in the study than those from urban locations at three time points (three months: $\mathrm{Chi}^{2}$ $=3.95, P<0.05$, exact $P$ value not specified in the paper; 18 months: $\mathrm{Chi}^{2}=5.33, \mathrm{P}<0.05$, exact $\mathrm{P}$ value not specified in the paper; 24 months: $\mathrm{Chi}^{2}=10.66, \mathrm{P}<0.01$, exact $\mathrm{P}$ value not specified in the paper).

Lyon 2011 found that women in rural programmes were more likely to be white/Caucasian, have less education and have adequate income, compared with women in urban or suburban programmes. They were also more likely to hear about the programme from family members, the police, criminal prosecution services or state support services, while women from urban or suburban programmes were more likely to hear about it from IPV staff, a social service agency or healthcare provider. Women from rural areas were less likely to use counselling and legal advocacy and more likely to use support groups. There were no clear differences in satisfaction with services or outcomes. This suggests some differences from the Australian studies, which may mean findings are country-specific. However, as with the Australian studies, the smallest programmes (with five or fewer staff) reported by Lyon 2011 were more likely to be in rural areas.

\section{DISCUSSION}

\section{Summary of results}

In our final theory we offer a conceptual explanation of advocacy interventions for abused women and the contextual elements that influence abused women's and advocates' capabilities space as the mechanisms for change. That space is constrained or expanded by context which is represented by the individual, local and structural conversion factors in our figure. We have chosen to use the term conversion factor in the model to highlight the effect of context on mechanisms. Our provisional model highlights the complex way that factors combine and interact for effective advocacy.

At the individual level, access to and control over resources is a significant feature of the theory. CMOs in the literature highlight three key resources, which are explored within our essential principles. These are: financial resources (essential principle 1, essential principle 6), social resources (essential principles 1 to 6), and knowledge, awareness and information (which also cuts across essential principles). To these we add emotional resources and legal resources (essential principles 1 and 2). We have shown how it is insufficient to simply provide access to these resources; women also need the capabilities to use them. The social position of women influences their capabilities space, for example women of higher SES may have greater capabilities to use resources even without advocacy, while undocumented migrants may have particularly limited capabilities and may also fear or distrust officials and providers. The severity and type of abuse experienced also affects women's capabilities space.

We have also shown how the generative nature of change applies in the context of advocacy for abused women. In other words, actions lead to changes in actual functionings but also the capabilities and conversion factors required for further action. A change in one conversion factor can lead to a change in capabilities and through this, further action that again leads to a change in another conversion factor and in capabilities. The concept of spirals of gains and losses relates to this and it is critical to understand this when considering the longer-term effectiveness of advocacy. It means that the woman's situation is dynamic - the new change in conversion factors may be a setback for the woman (and may be something that is not easy to change directly through advocacy) or it may be a positive effect. Negative effects may beget other negative effects through the generative nature of change, and positive effects may beget further positive effects. This dynamism means that unanticipated as well as anticipated outcomes may result from advocacy.

For example, economic and social dependence on the abuser may affect whether or not a woman has children early (essential principle 3 ), and this may then feed back into levels of dependency in a circle of effect. This is demonstrated by the feedback arrows as well as the gain and loss spirals in our model. The complexity of the situation is highlighted by the evidence concerning women's vulnerabilities and intersectionalities and the trade-offs that must be considered in advocacy-related decisions in the contexts of the individual women's lives (essential principle 2). It is also shown in the evidence that women may be hopeful about the impact of advocacy in the short term and not realise the longer-term issues of any change (essential principle 6). 
At the local (community) level, we have shown how communities may affect abused women's use of advocacy (essential principle 1 , essential principle 2, essential principle 6). We consider communities as geographically and culturally defined, each differently characterised by the availability of facilities or resources and prevailing social norms and values that can support or constrain abused women, advocacy and advocates. We have also considered how members of the woman's social networks may assume an advocacy role by placing them explicitly within our theory figure (Figure 3). In addition, we have explored how advocates belong to different professionally and physically defined communities of practice, such as legal advocates within a courthouse, or healthcare clinic advocacy separated from other services (essential principles 2, 5 and 6). Within each setting the human and economic resources, ways of working, structural and organisational contexts, existence and type of training or guidance, and linking to other organisations all influence the advocates' and abused women's capabilities space (essential principles 5 and 6). Significant challenges for advocates were identified in inter-organisational working, vicarious traumatisation, and lack of clarity on how much support to give a woman, given the individualised approach (essential principles 5 and 6). Social interactions between the woman and the advocate - the therapeutic alliance - also influence this (essential principle 4).

At the structural level, the key conversion factor is funding, with policy also being important. We have shown how women who are marginalised and made particularly vulnerable to abuse by their dependencies or geographies, which act as barriers to an extension of their capabilities spaces and hence resource use, are affected at all three levels of our model and how the structural level may be especially challenging for advocates to manage.

The spirals in Figure 3 show the interconnectedness of influences on women's/advocate's capabilities space. The two phases of equilibrium (stable and unstable) may alternate over time demonstrating the key significance of time to the development of the capabilities space.

Our panel considered our most important principle to be that advocacy work must take into account the influence of the abuser on the woman, as it may lead the woman to tolerate his abuse and coercive control if the trade-offs of the alternatives are perceived by the woman as worse. Our second most important principle according to our panel highlighted the importance of developing holistic, tailored advocacy intervention plans that recognise the individual risks and needs of vulnerable and marginalised women such as those from minority groups or from rural areas. It is possible that this should include consideration of whether an abused woman was pregnant or had children. More broadly, according to our findings, more effective advocacy was likely when:

- having a 'one-stop' person (a dedicated advocate for each woman) to turn to for advice and information (Coy 2011), for a consistent, coherent and individualised service;

- considering the possible consequences and trade-offs of different decisions;

- speaking on behalf of women where they felt unable to do so;

- following up and ensuring rights and entitlements were realised;

- including action to remove structural barriers to resource access for vulnerable and marginalised women; and

- including cultural humility.
Our findings show that the risk to the woman's safety from the abuse, such as when the woman still lives with her abuser as well as the type and severity of the abuse, could have an effect on the woman's engagement with the advocacy intervention and thus outcomes, but they also provide nuance as to what this entails. Thus, our review shows that the direction of the relationship depends on which outcomes are being considered. For example, a woman living with her abuser could engage more with court action but could be more depressed. This means that advocacy could potentially always have some benefits for an abused woman if it is undertaken for long enough, but that the goals of the advocacy need to match the woman's needs rather than those the advocate might see as preferable.

Our review has also shown that the focus should not be solely on the interventions themselves, but should encompass the interaction between advocates and the women they are supporting, and the support needs and requirements of advocates. The therapeutic alliance was repeatedly demonstrated in studies to be important for woman/survivor-centred care. However, with the focus of this care on valuing what was important in the lives of the women themselves, and on trust and empathy, advocates often found it difficult to separate the professional from the personal. In particular, they were concerned to not cross boundaries in ways that were potentially harmful to themselves or to the women, and they found it challenging to balance competing roles and duties such as encouraging women to take control of their lives whilst minimising the risks. It was clear from our review that being an advocate is not easy, and this can have an impact on effectiveness too. Advocate burnout and a hardening of attitude could attenuate a survivor-centred approach or reduce the capacity of the advocate to take on work, especially if the advocate was a survivor of abuse herself; though being a survivor (or of matched ethnicity) conversely improved the therapeutic alliance. Solving some challenges, such as support for marginalised women, and with additional resources for this, could therefore help both advocates and abused women. Advocates also valued training, protocols for difficult situations, debriefs, and peer and organisational support. This was true of advocates from IPV agencies as much as from other organisations; there was no clear effect of discipline of the advocate on advocacy success.

Advocates often felt ineffective when they only managed brief contact with the woman because of organisational failures or because the woman stopped seeking help or returned to her abuser. One or two contacts with a woman were viewed as never sufficient for empowerment, though they may change safety behaviours. Although brief intensive advocacy was often effective, the evidence suggests that a longer time is needed within advocacy for women experiencing more severe abuse. Nonetheless, women experiencing more severe abuse and women from vulnerable and marginalised groups with few resources at the start of advocacy may show greater benefits in some outcomes in the short term. These benefits indicate a low starting point rather than a 'good outcome'. Smaller changes than expected in other outcomes may mean the women have fewer needs, are at an early 'stage of change' or come from particular contexts (such as an immigrant community or rural area) in which small steps may be more appropriate. Moreover, we have provided some evidence that the outcomes evaluated in studies of interventions are driven by theoretical considerations of empowerment and may not represent women's views of a good outcome. It is noteworthy that there were few

A realist review of which advocacy interventions work for which abused women under what circumstances (Review) 
studies exploring this with the women themselves in relation to advocacy. However, it is encouraging that marginalised women are more likely to remain engaged with services.

We also found that the setting for the advocacy affected the theoretical underpinnings and the type and focus of the advocacy being offered and the women's possible responses (as constrained by the types of services offered), and that this, in turn, drove the decision in studies as to which outcomes to evaluate. Different understandings of the nature of empowerment lead to differences in research design (Cattaneo 2015; Morgan 2013). Empowerment may be experienced as either a perceived sense of control or an actual increase in control over relevant situations and resources (Rappaport 1987). What this means may differ with context. For example, it may lead some women to leave their abuser whereas ethnic minority women with issues such as immigration status and cultural and economic dependencies may need to become empowered to reject options presented by services with limited cultural humility. It was often unclear whether researchers were considering empowerment as a process, an outcome, or both. This made the logic model challenging to construct.

We determined that all IPV advocacy included the following core ingredients to at least some degree:

- education on the dynamics of IPV and on relationships (though in many one-off, brief interventions, including police first-response interventions such as described by Davis 2006 amongst others, there seems limited time for this to have been achieved and it was often only implied as being done);

- explaining rights and possible resources with reference to the legal and social welfare systems (which appeared very successful in the short term);

- referral and liaising with other services (which advocates considered vital but problematic); and

- risk assessment and safety planning (which is popular across interventions, which often incorporate the approach developed by McFarlane 1997).

A study in which abused women were trained to be informal advocates within their community claimed that education alone empowered women and increased their self-efficacy, confidence, assertiveness, and hope for the future (Ross 2012), but this may have been related to their projected roles. Consistent with Rivas 2015, we found that when studies use the terms counselling or peer counselling they may not be describing psychotherapy; though unlike Rivas 2015, we believe these terms to be alternatives to 'advice giving' (i.e. giving counsel). Dunn 2007 note that advice and education "explicitly presumes that there is a version of reality that advocates have access to but to which victims are unaware" ( $p$ 989), whereas our findings make it clear that advocates may often lack understanding of the women's day-to-day realities. Nonetheless, advocates, if not women, considered education critical to success and this is also suggested by our CMOs.

Five studies used the brief, brochure-driven intervention developed by McFarlane 1997. This contains the following core features of advocacy despite its brevity.

- The advocate provides information about IPV and discusses the issues of power and control.
- The brochure given to abused women contains a shortened version of the Danger Assessment Scale, which assesses variables shown to be predictive of severe injury and lethal abuse (Campbell 2009). It also provides information on safety planning and effective safety behaviours.

- The advocate uses the information of component 2 (the brochure and scale that women complete) to discuss the woman's situation and work with the woman to develop a woman-focused, individualised safety plan. In high-risk cases, the advocate assists the woman in phoning relevant services.

- The brochure provides a list of available community resources.

Generally, studies incorporated additional elements. According to the Conservation of Resources Theory (Alvaro 2010), which has similarities with the more well-known theory by Maslow 1943 called the Hierarchies of Need, once a woman has left and extricated herself from her dependencies on the abuser, difficulties associated with a loss of resources may lead to psychological distress. Hence, advocacy interventions that help the woman to access resources and balance resource losses or at least prevent further resource loss, should lead to better mental health and well-being, while a co-ordinated community response should be more effective. This is broadly indicated by the evidence, though we did not explore co-ordinated community responses, and there are particular issues for very low-income women. Nonetheless, improving abused women's social support could lead to a gain spiral that would ultimately improve their economic resources too. Shorey 2014 suggested that empowerment advocacy has its effects largely through social support though they do not evidence this. Coy 2011 argued that "empowerment through knowledge" about resources can be more powerful than the provision of material resources or practical support. Prosman 2014 suggested structural factors that reduced women's access to resources, especially in the case of vulnerable and marginalised women, led to a "learned helplessness". Their arguments imply that an empowerment-based intervention may have its effect partly by increasing the women's confidence and reducing their learned helplessness irrespective of resource provision. Overall, the evidence supports the idea that there are several active ingredients of advocacy, and that advocacy based on empowerment has the potential to affect diverse outcomes, benefiting abused women in a number of ways. We used the CMOs we developed to produce a tentative logic model of advocacy interventions for abused women (see Appendix 5), which differs from that presented in Rivas 2015 in being grounded in a more diverse evidence base. We also developed a theory unifying the different CMOs and essential principles in a way that can be easily understood by practitioners and researchers (see Appendix 5).

Nonetheless, despite finding clear CMO patterns in the data, there are gaps in the evidence. There is a lack of clarity as to how different components of an intervention may interact. We started with the premise that a multi-component intervention that added elements to the core features of advocacy, perhaps including psychotherapy, might be more effective than an advocacy-only intervention, but could find no support for this from the evidence. This may be because of the woman-focused (individually tailored) nature of the interventions, such that elements were added to the core advocacy features depending on need and setting. This flexibility, and also the increased use of co-ordinated responses in which different agencies work together, make it challenging to be certain of the impact of advocacy itself isolated from other interventions 
though our study has shown how advocacy itself may work in different contexts and for whom. McFarlane 2000 compared three different types of intervention that differed from each other in one or two components, but it is not possible from their study design to understand either additive or summative effects. Shorey 2014 lament the dearth of research on the interaction between different elements of multi-component interventions that include advocacy, though they suggest that better understanding of the different components themselves may be the priority. Further complicating understandings, 10 interview, survey and intervention studies have considered women in a shelter environment and none tried to determine from them their functioning beforehand. Just being in a shelter may have led to improvements even before advocacy, and leaving the safety of the shelter may lead to trade-offs that are not considered in studies. There is a lack of clarity concerning what improvements in outcomes might occur even without advocacy and how this might affect advocacy, given the generative nature of change. Several studies noted that some outcomes improved with time regardless of whether or not the woman received an intervention. This suggests that single-subject research designs could be usefully employed to test the time component of our model by determining between-condition differences in effect size in subgroups of women (Smith 2012). There also needs to be a more nuanced consideration of how to measure outcomes from advocacy interventions and what these outcomes should be.

\section{Overall completeness and applicability of evidence}

The sampling approach we used in this review was geared towards developing theory from CMO combinations. In contrast to an effectiveness systematic review, a realist review aims to build an understanding of why and how things work and for whom, rather than providing a comprehensive synthesis of the literature. Therefore, we did not include papers that provided minimal information on the factors of interest to our theory building, even if they were associated with papers that were included, and searches in phase 2 of our strategy were intended to be explanatory. Whilst a realist review does not require a comprehensive search, the availability of data made a quasi-comprehensive approach feasible for us. The realist methodology allowed much broader engagement with the theory underlying advocacy interventions for women experiencing IPV. Our review has drawn from a diverse literature, including both grey literature and peer-reviewed papers, and has been supplemented by insights from the reference panel. There were gaps within the literature; for example, there were insufficient data for an economic consideration or evidence capturing more distal outcomes, or the effects of demographic variables such as the age of abused women or the expertise of advocates. Most studies in this review are from urban settings. It is unclear what impact this has had on the overall completeness of the evidence; the rich studies on rural settings that were included suggest more intervention studies should compare settings as a factor. We also excluded any article for which both child abuse and adult abuse had been assigned as keywords from the MEDLINE search to ensure a single focus on adult abuse only, on the basis of results from a scoping search but did not so implement this for other database searches. Despite the gaps and limitations, this review has provided some clear indications of the mechanisms and contexts/conversion factors for effectiveness in advocacy for abused women. As the Rivas 2015 review excluded interventions that did not adhere strictly to their definition of advocacy and interventions in which advocacy was not key, our final list of studies is more comprehensive in terms of the interventions included. This list should help to define more focused intervention inclusion criteria for future reviews on advocacy interventions for abused women that are based on consideration of the active ingredients as elucidated in our realist review.

\section{Confidence in the findings}

We have moderate-to-high confidence in essential principle 1: advocacy that takes into account the influence of the abuser on the woman will be more effective in reducing or ending the woman's tolerance of his abuse and coercive control. Our stakeholder panel also considered this to be the most important essential principle. It has been the explicit focus of 53 high-quality studies.

We have moderate confidence in essential principle 2: there is a need for holistic, tailored advocacy intervention plans that recognise the individual risks and needs of vulnerable and marginalised women such as those from minority groups or from rural areas. Our panel also considered this to be important, and although fewer studies explored this than essential principle 1 (i.e. only 19), there were rich data from qualitative studies and coherence across countries.

We had low confidence in essential principle 3: whether an abused woman was pregnant or had children could affect her engagement with advocacy and possibly also lead to some adverse outcomes. While there is a small amount of evidence to support this, only five studies considered this, none of which were set in antenatal clinics. Our panel found the idea interesting but did not rate it as high in importance.

Considering essential principle 4: a good advocate-survivor relationship is important for effective advocacy, as predicted by underpinning theories, we had high confidence in this finding with strong concordance and detailed consideration across 41 studies.

We had moderate-to-high confidence in essential principle 5: advocates have needs and requirements too in striving for effectiveness. Our panel found this an interesting and relatively important theme (third most important), which they considered to be neglected in intervention studies.

We had high confidence in essential principle 6: the type of setting affects the theoretical underpinnings and the type and focus of the advocacy being offered and the women's responses.

\section{Agreements and disagreements with other studies or reviews}

This review broadly concurs with the findings of previous effectiveness reviews of, or including, advocacy interventions (Abel 2000; Hender 2001; Jahanfar 2011; Jonker 2015; Ramsay 2005a; Ramsay 2005b; Rivas 2015), which are that advocacy interventions may have some effect but that more work is needed. We have added to these findings in teasing out contextual effects and mechanisms. We have not, however, sought to demonstrate effectiveness and where we have included information on effect sizes, this is to aid the interpretation of results and not intended to be definitive. We will be undertaking an update of Rivas 2015 for a more contemporary effectiveness analysis. 


\section{AUTHORS' CONCLUSIONS}

Our strategic objectives were to explain successes, failures, partial successes and small effect sizes in published, empirical studies of advocacy interventions delivered in different settings, and, in particular, to explain mechanisms of effect in heterogeneous, complex, advocacy-containing interventions. This reflects our higher-order (realist) question (Greenhalgh 2016). We were only partially able to meet this as we had low confidence in the evidence regarding complexity. Nonetheless we have been able to clarify the active ingredients of advocacy and to develop hypotheses linking contexts, mechanisms and outcomes. Our findings should enable researchers and developers to design more effective advocacy interventions in the future, determine which outcomes to include, and improve their reporting and evaluation. In addition, this review will lead policy makers and practitioners to a better understanding of advocacy interventions, and their likely benefit in the local contexts in which they operate.

\section{Implications for practice}

Our results suggest that the use of advocacy rests on sound theoretical underpinnings, and confirms that its core ingredients are: education and information on intimate partner abuse; rights and resources; active referral and liaising with other services; risk assessment and safety planning. Our analysis has also enabled us to make some recommendations. Our panel prioritised our first recommendations (the essential principles), which were that:

- the influence of the abuser on the woman must always be taken into account, and that

- advocates should always exercise cultural humility and bear in mind intersectionalities in considering women's vulnerabilities and the trade-offs of abuse-related decisions in the contexts of the individual women's lives.

It was important to consider:

- the severity and type of abuse,

- whether the woman still lived with the abuser, and

- the women's economic and legal dependencies.

These were relevant across recommendations and may necessitate advocates working to remove structural barriers to resource access by marginalised women.

The evidence is less clear for physical dependencies and seemed indicated, but not certain, for pregnant women and women with children.

Women's safety was not necessarily at greatest risk from staying with the abuser.

Potentially, advocacy could always have some benefits for abused women, if it is undertaken for long enough, but the goals of the advocacy need to match the woman's needs; there may be an impact trajectory and some outcomes may take months to be determined.

A good therapeutic alliance was important and might be improved when advocates are matched with the women they see on ethnicity or abuse experience. We identified significant challenges for advocates that related to intra- and inter-organisational working, vicarious traumatisation, and lack of clarity on how much support to give women, given the need for an individualised approach. To work effectively, advocates need ongoing training, role clarity, access to resources and peer and institutional support.

Our final model (Figure 3) highlights the complex way that factors combine and interact for effective advocacy.

\section{Implications for research}

We have presented throughout this review hypotheses that need to be tested in further research that might extend our theory to different situations or types of advocacy or empowerment interventions.

Future interventions and studies should consider the different mechanisms and contexts identified in this review and the way they link to outcomes and unanticipated effects. In relation to abused women, contextual factors to consider include: ethnicity and race; immigration difficulties; resource loss and gain and resource balances; social and geographic locations; economic dependence (and the difference from low socioeconomic status or low income). There needs to be more consideration of these different contexts when interpreting outcomes, and more understanding of the effects on outcomes when women have children. The ways that advocates can be supported need to be better understood.

There should be more consistency in studies with regard to the outcomes used. These should be explicitly theoretically driven, and should be measured using more standardised scales where this is relevant. Some studies develop an outcome measure specifically for their study, which leads to problems in interpreting the evidence. Study outcomes should be considered in relation to the mechanisms and contexts elucidated in this review and that consider the trajectories of impact in different groups of women.

More longitudinal research is needed since some effects are likely to attenuate over time and others may not emerge until years after an intervention has ended. It may be that two years is optimal for follow-up and that beyond that it is challenging to disentangle the effects that external factors may have on outcomes. However, this may depend on what is being evaluated, and singlesubject research designs may be helpful (Smith 2012), which can determine exactly when equilibrium in effect is reached for different outcomes, that is, when effect plateaus rather than continuing to increase, and follow-up may become unhelpful. It is likely that this will be different for vulnerable and marginalised women.

Longitudinal research is also needed to determine longer-term mechanisms of effect; for example, considering recidivism, mental health, resource acquisition and empowerment, all of which appear central to advocacy interventions.

Further work is needed to ascertain how advocacy interventions can be tailored to cultural variations between ethnic groups (Rodriguez 2006), and for abused women living in rural communities, resource-poor settings, or both. More studies should be undertaken in lower- and middle-income countries. In the development of the WHO guidelines on the healthcare response to intimate partner abuse and sexual abuse (WHO 2013c), applying the evidence on advocacy outside of resource-rich countries was problematic, though since then Sri Lanka (Infanti 2015), and Mexico (Gupta 2017), have each been represented by one qualitative study among the studies we included and other countries are the focus of studies in our Characteristics of excluded studies.

A realist review of which advocacy interventions work for which abused women under what circumstances (Review) 
The methods used in the included studies may, in some cases, limit the applicability and completeness of the data reported. Most of the included qualitative studies made use of individual or group interviews and focus group discussions as their main method of data collection, and none used long-term ethnographic methods; although one study did record interactions, they used thematic rather than ethnographic analysis (Stylianou 2018). Such methods would enable researchers to explore the therapeutic alliance and also organisational contexts in more detail.

Finally, more economic analyses are required to ascertain if the resources devoted to advocacy interventions are cost-effective in different settings. However, to a large extent, the value of such analyses depends on improvement in the outcomes measured, to ensure that the benefits side of the equation is more accurate than is currently possible.

\section{ACKN OWLEDGEMENTS}

This review was funded, in part, by the National Institute for Health Research (NIHR) Cochrane Incentive Scheme 2017 (project reference $17 / 62 / 37$ ).
The original protocol (Rivas 2018), was peer reviewed by Dr Andrew Booth, School of Health and Related Research (ScHARR), University of Sheffield, UK; Dr Chrysanthi Papoutsi, Nuffield Department of Primary Care Health Sciences, University of Oxford; and Professor Jane Barlow, Editor with Cochrane Developmental, Psychosocial and learning Problems (CDPLP). The review was peer reviewed by DrAndrew Booth, School of Health and Related Research (ScHARR), University of Sheffield, UK; Claire Duddy, Nuffield Department of Primary Care Health Sciences, University of Oxford; Mhairi Mackenzie, Professor of Public Policy, Member of CRSU, University of Glasgow; and Faiza Coleman-Salako. We thank these individuals for their contributions to this review.

We are also grateful to Professor Jane Noyes (Convenor of the Cochrane Qualitative and Implementation Methods Group) who liaised with Dr Andrew Booth, Dr Jackie Chandler (former Methods Co-ordinator with the Cochrane Central Executive) and Joanne Duffield at CDPLP; and to Professor Geraldine Macdonald (Coordinating Editor) and Dr Joanne Duffield (Managing Editor), both of CDPLP, for their support.

Disclaimer: The views expressed are those of the authors and not necessarily those of the NIHR or the Department of Health and Social Care. 


\section{R E F E R E N C E S}

\section{References to studies included in this review}

Babin 2012 \{published data only\}

Babin EA, Palazzolo KE, Rivera KD. Communication skills, social support, and burnout among advocates in a domestic violence agency. Journal of Applied Communication Research 2012;40(2):147-66.

\section{Bacchus 2007 \{published data only\}}

* Bacchus LJ, Aston G, Vitolas CT, Jordan P, Fairley Murray SA. Theory-based Evaluation of a Multi-Agency Domestic Violence Service Based in Maternity and Genitourinary Services at Guy's \& St Thomas' NHS Foundation Trust. London: Kings College London, 2007.

Bacchus LJ, Bewley S, Torres Vitolas C, Aston G, Jordan P, Murray SF. Evaluation of a domestic violence intervention in the maternity and sexual health services of a UK hospital. Reproductive Health Matters 2010;18(36):147-57.

\section{Bacchus 2016a \{published data only\}}

* Bacchus L.J, Bullock L, Sharps P, Burnett C, Schminkey D, Buller AM, et al. 'Opening the door': a qualitative interpretive study of women's experiences of being asked about intimate partner violence and receiving an intervention during perinatal home visits in rural and urban settings in the USA. Journal of Research in Nursing 2016;21(5-6):345-64.

Burnett C, Crowder J, Bacchus LJ, Schminkey D, Bullock L, Sharps P, et al. "It doesn't freak us out the way It used to": an evaluation of the Domestic Violence Enhanced Home Visitation Program to inform practice and policy screening for IPV. Journal of Interpersonal Violence 2019;0886260519827161:e1. .

Sharps PW, Bullock LF, Campbell JC, Alhusen JL, Ghazarian SR, Bhandari SS, et al. Domestic violence enhanced perinatal home visits: the DOVE randomized clinical trial. Journal of Women's Health 2016;25(11):1129-38.

\section{Bacchus 2016b \{published data only\}}

Bacchus LJ, Bullock L, Sharps P, Burnett C, Schminkey DL, Buller AM, et al. Infusing technology into perinatal home visitation in the United States for women experiencing intimate partner violence: exploring the interpretive flexibility of an mHealth intervention. Journal of Medical Internet Research 2016;18(11):37-53.

\section{Bader 2014 \{published data only\}}

Bader D. Back to Basics: Service Users Report on the Importance of Material Resources and Empowering Responses: A Community Engaged Evaluation Research Study of an Ontario Sexual Assault and Domestic Violence Protocol [Dissertation]. Guelph (Ontario, Canada): University of Guelph, 2014.

\section{Bahadir-Yilmaz 2018 \{published data only\}}

Bahadir-Yilmaz E, Oz F. The effectiveness of empowerment program on increasing self-esteem, learned resourcefulness, and coping ways in women exposed to domestic violence. Issues in Mental Health Nursing 2018;39(2):135-41.
Bell 2001 \{published data only\}

Bell ME, Goodman LA. Supporting battered women involved with the court system: an evaluation of a law school-based advocacy intervention. Violence against Women 2001;7(12):377404.

\section{Bemiller 2010 \{published data only\}}

Bemiller M, Williams LS. Good soldiering: the role of adaptation in advocate burnout. Violence against Women 2010;17(1):89-110.

\section{Briones-Vozmediano 2014 \{published data only\}}

Briones-Vozmediano E, Goicolea I, Ortiz-Barreda GM, GilGonzalez D, Vives-Cases C. Professionals' perceptions of support resources for battered immigrant women: chronicle of an anticipated failure. Journal of Interpersonal Violence 2014;29(6):1006-27.

\section{Burnett 2012 \{published data only\}}

Burnett C, Ford-Gilboe M, Berman H, Wathen N, Ward-Griffin C. The day-to-day reality of delivering shelter services to women exposed to intimate partner violence in the context of system and policy demands. Journal of Social Service Research 2016;42(4):516-32.

* Burnett CJ. Examining the Effects of Policies on the Delivery of Shelter Services to Women Who Have Experienced Intimate Partner Violence [Thesis]. Ontario (Canada): Electronic Thesis and Dissertation Repository. 635, 2012. [ir.lib.uwo.ca/etd/635]

Bybee 2005 \{published data only\}

Allen NE, Larsen S, Trotter JL, Sullivan CM. Exploring the core components of an evidence-based community advocacy program for women with abusive partners. Journal of Community Psychology 2013;41(1):1-18.

* Bybee DI, Sullivan CM. Predicting re-victimization of battered women 3 years after exiting a shelter program. American Journal of Community Psychology 2005;36(1-2):85-96.

Bybee DI, Sullivan CM. The process through which an advocacy intervention resulted in positive change for battered women over time. American Journal of Community Psychology 2002;30:103-32.

Sullivan CM. Understanding how domestic violence support services promote survivor well-being: a conceptual model. Journal of Family Violence 2018;33:123.

Sullivan CM, Bybee DI. Reducing violence using communitybased advocacy for women with abusive partners. Journal of Consulting and Clinical Psychology 1999;67(1):43-53.

Sullivan CM, Campbell R, Angelique H, Eby KK, Davidson WS. An advocacy intervention program for women with abusive partners: six month follow-up. American Journal of Community Psychology 1994;22(1):101-22.

Sullivan CM, Tan C, Basta J, Rumptz M, Davidson WS. An advocacy intervention program for women with abusive 
partners: initial evaluation. American Journal of Community Psychology 1992;20(3):309-32.

Tan C, Basta J, Sullivan CM, Davidson WS. The role of social support in the lives of women exiting domestic violence shelters: an experimental study. Journal of Interpersonal Violence 1995;10(4):437-51.

\section{Casey 2007 \{published data only\}}

Casey RL, Berkman M, Stover CS, Gill K, Durso S, Marans S. Preliminary results of a police-advocate home visit intervention project for victims of domestic violence. Journal of Psychological Trauma 2007;6(1):39-49.

\section{Coker 2012 \{published data only\}}

Coker AL, Smith PH, Whitaker DJ, Le B, Crawford TN, Flerx VC. Effect of an in-clinic IPV advocate intervention to increase help seeking, reduce violence, and improve well-being. Violence against Women 2012;18(1):118-31.

\section{Constantino 2005 \{published data only\}}

Constantino R, Kim Y, Crane PA. Effects of a social support intervention on health outcomes in residents of a domestic violence shelter: a pilot study. Issues in Mental Health Nursing 2005;26(6):575-90.

\section{Coy 2011 \{published data only\}}

Coy M, Kelly L. Islands in the Stream: An Evaluation of Four London Independent Domestic Violence Advocacy Schemes. London (UK): Child and Woman Abuse Study Unit London Metropolitan University, 2011. [cwasu.org/resource/islandsin-the-stream-an-evaluation-of-four-london-independentdomestic-violence-advocacy-schemes/]

\section{Cripe 2010 \{published data only\}}

Cripe SM, Sanchez SE, Sanchez E, Ayala Quintanilla, B, Hernández Alarcon, C, Gelaye B, et al. Intimate partner violence during pregnancy: a pilot intervention program in Lima, Peru. Journal of Interpersonal Violence 2010;25(11):2054-76.

\section{Curry 2006 \{published data only\}}

Curry MA, Durham L, Bullock L, Bloom T, Davis J. Nurse case management for pregnant women experiencing or at risk for abuse. Journal of Obstetric, Gynecologic, and Neonatal Nursing 2006;35(2):181-92.

\section{Davis 2006 \{published data only\}}

* Davis RC, Maxwell C, Taylor B. Preventing repeat incidents of family violence: analysis of data from three field experiments. Journal of Experimental Criminology 2006;2:183-210.

Davis RC, Taylor B. Evaluating a proactive police response to domestic violence: the results of a randomized experiment. Criminology 1997;35:307-33.

\section{Davis 2007 \{published data only\}}

Davis RC, Weisburd D, Hamilton EE. Preventing repeat incidents of family violence: a randomized field test of a second responder program in Redlands, CA. Report to the US Dept of Justice 2007.
DePrince 2012 \{published data only\}

* DePrince AP, Belknap J, Labus JS, Buckingham SE, Gover AR. The impact of victim-focused outreach on criminal legal system outcomes following police-reported intimate partner abuse. Violence against Women 2012;18(8):861-81.

DePrince AP, Labus J, Belknap J, Buckingham S, Gover A. The impact of community-based outreach on psychological distress and victim safety in women exposed to intimate partner abuse. Journal of Consulting and Clinical Psychology 2012;80(2):211-21.

\section{Donnelly 2005 \{published data only\}}

Donnelly DA, Cook KJ, Wilson LA. Provision and exclusion: the dual face of services to battered women in three Deep South states. Violence Against Women 1999;5(7):710-741.

* Donnelly DA, Cook KJ, van Ausdale D, Foley L. White privilege, color blindness, and services to battered women. Violence Against Women 2005;11(1):6-37.

\section{Dunn 2007 \{published data only\}}

Dunn JL, Powell-Williams M. "Everybody makes choices": victim advocates and the social construction of battered women's victimization and agency. Violence against Women 2007;13(10):977-1001.

\section{Ekstrom 2015 \{published data only\}}

Ekstrom V. Violence against women-social services support during legal proceedings. European Journal of Social Work 2015;18(5):661-74.

\section{Feder 2018 \{published data only\}}

Feder L, Niolon PH, Campbell J, Wallinder J, Nelson R, Larrouy $\mathrm{H}$. The need for experimental methodology in intimate partner violence: finding programs that effectively prevent IPV. Violence against Women 2011;17(3):340-58.

* Feder L, Niolon PH, Campbell J, Whitaker DJ, Brown J, Rostad W, et al. An intimate partner violence prevention intervention in a nurse home visitation program: a randomized clinical trial. Journal of Women's Health 2018;27(12):1482-90. [DOI: 10.1089/jwh.2017.6599]

Niolon P, Whitaker D, Feder L, Campbell J, Wallinder J, SelfBrown S, et al. A multi-component intervention to prevent partner violence within an existing service intervention. Professional Psychology 2009;40:264-71.

\section{Ganz 2015 \{published data only\}}

Ganz JJ. Contested Titles: Gendered Violence Victim Advocacy and Negotiating Occupational Stigma in Social Interactions [PhD Thesis]. Bowling Green (OH, USA): Bowling Green State University, American Culture Studies, 2015.

\section{Garcia-Leeds 2017 \{published data only\}}

Garcia-Leeds CB, Schneider L. Systemic approach in Latino families surviving domestic violence in the United States of America. Propositos y Representaciones 2017;5(2):277-319. 
Gillum 2008 \{published data only\}

* Gillum TL. Community response and needs of African American female survivors of domestic violence. Journal of Interpersonal Violence 2008;23(1):39-57.

Gillum TL. The benefits of a culturally specific intimate partner violence intervention for African American survivors. Violence against Women 2008;14(8):917-43.

Gillum 2009 \{published data only\}

Gillum TL, Sun CJ, Woods AB. Can a health clinic-based intervention increase safety in abused women? Results from a pilot study. Journal of Women's Health 2009;18(8):1259-64.

Goodman 2009 \{published data only\}

Goodman LA, Glenn C, Bohlig A, Banyard V, Borges A. Feminist relational advocacy: processes and outcomes from the perspective of low-income women with depression. Counseling Psychologist 2009;37(6):848-76.

\section{Goodman 2016a \{published data only\}}

Goodman LA, Fauci JE, Sullivan CM, DiGiovanni CD, Wilson JM. Domestic violence survivors' empowerment and mental health: exploring the role of the alliance with advocates. American Journal of Orthopsychiatry 2016;86(3):286-96.

* Goodman LA, Thomas K, Cattaneo LB, Heimel D, Woulfe J, Chong SK. Survivor-defined practice in domestic violence work: measure development and preliminary evidence of link to empowerment. Journal of Interpersonal Violence 2016;31(1):163.

Thomas KA, Goodman L, Putnins S. I have lost everything: trade-offs of seeking safety from intimate partner violence. American Journal of Orthopsychiatry 2015;85(2):170-80.

\section{Goodman 2016b \{published data only\}}

Goodman LA, Banyard V, Woulfe J, Ash S, Mattern. Bringing a network-oriented approach to domestic violence services: a focus group exploration of promising practices. Violence against Women 2016;22(1):64.

\section{Grant 2012 \{published data only\}}

Grant TM. Advocates' Experiences with Victims of Domestic Violence: An Examination of Personal Biases and Attitudes and Their Impact on Service Delivery [Doctoral dissertation]. Minneapolis (MN, USA): School of Public Service Leadership, Capella University, 2012.

\section{Gupta 2017 \{published data only\}}

Falb KL, Diaz-Olavarrieta C, Campos PA, Valades J, Cardenas R, Carino $\mathrm{G}$, et al. Evaluating a health care provider delivered intervention to reduce intimate partner violence and mitigate associated health risks: study protocol for a randomized controlled trial in Mexico City. BMC Public Health 2014;14:772.

* Gupta J, Falb KL, Ponta O, Xuan Z, Campos PA, Gomez AA, et al. A nurse-delivered, clinic-based intervention to address intimate partner violence among low-income women in Mexico City: findings from a cluster randomized controlled trial. BMC Medicine 2017;15(1):128.

\section{Hathaway 2008 \{published data only\}}

Hathaway JE, Zimmer J, Willis G, Silverman JG. Perceived changes in health and safety following participation in a health care-based domestic violence program. Journal of Midwifery \& Women's Health 2008;53(6):547-55.

Hidalgo 2016 \{published data only\}

Hidalgo A. Domestic Violence Advocates: Understanding the Barriers in Collaborating with Stakeholders in New York City [Doctoral dissertation]. Denver (CO): University of the Rockies, 2016.

\section{Howarth 2016 \{published data only\}}

* Howarth E, Robinson A. Responding effectively to women experiencing severe abuse: identifying key components of a British advocacy intervention. Violence against Women 2016;22(1):41-63.

Howarth E, Stimpson L, Barran D, Robinson A. Safety In Numbers: A Multi-site Evaluation of Independent Domestic Violence Advisor Services. London: The Hestia Fund and The Henry Smith Charity, 2009.

\section{Hughes 2017 \{published data only\}}

Hughes J. Women's advocates and shelter residents: describing experiences of working and living in domestic violence shelters. Journal of Interpersonal Violence 2017:1-20. [doi.org/10.1177/0886260517707307]

Hyman 2001 \{published data only\}

Hyman K. Impact of Emergency Department Intimate Partner Violence (IPV) Advocacy: a Longitudinal, Randomized Trial [PhD thesis]. Pittsburgh (PA): University of Pittsburgh, 2001.

\section{Infanti 2015 \{published data only\}}

Infanti JJ, Lund R, Muzrif MM, Schei B, Wijewardena K. Addressing domestic violence through antenatal care in Sri Lanka's plantation estates: contributions of public health midwives. Social Science \& Medicine 2015;145:35-43.

\section{Johnson 2014 \{published data only\}}

Johnson M, McGrath SA, Miller MH. Effective advocacy in rural domains: applying an ecological model to understanding advocates' relationships. Journal of Interpersonal Violence 2014;29(12):2192-217.

\section{Kapur 2017 \{published data only\}}

Kapur S. Individual, Organizational, and Policy Related Aspects of Immigration and Domestic Violence: the Perspectives of Advocates Working with Asian Indian Marriage Migrants [Doctoral dissertation]. Fayetteville (AR): Public Policy Department, University of Arkansas, 2014.

Kapur S, Zajicek AM, Gaber J. Nonprofit organizations serving domestic violence survivors: addressing intersectional needs of Asian Indians. Affilia 2017;32(1):50-66.

\section{Kelly 1999 \{published data only\}}

Kelly L, Bindel J, Burton S, Butterworth D, Cook K, Regan L. Domestic Violence Matters: An Evaluation of a Development Project. Home Office Research Study 193. London: HMSO, 1999. 
Kendall 2009 \{published data only\}

Kendall J, Pelucio MT, Casaletto J, Thompson KP, Barnes S, Pettit E. Impact of emergency department intimate partner violence intervention. Journal of Interpersonal Violence 2009;24(2):280-306.

\section{Kenyon 2016 \{published data only\}}

Kenyon KM. How Women in Domestic Violence Shelters Experience Empowermen [MA thesis]. Winnipeg: Faculty of Graduate Studies of The University of Manitoba, 2016.

\section{Kiely 2010 \{published data only\}}

Kiely M, El-Mohandes AA, El-Khorazaty MN, Blake SM, Gantz MG. An integrated intervention to reduce intimate partner violence in pregnancy: a randomized controlled trial. Obstetrics and Gynecology 2010;115(2 Pt 1):273-83. [DOI: 10.1097/ AOG.0b013e3181cbd482; NCT00381823; PMC2917915; PUBMED: 20093899]

\section{Kolb 2008 \{published data only\}}

Kolb KH. Identity and Emotion Management Among Advocates and Counselors for Victims of Domestic Violence and Sexual Assault [Doctoral dissertation]. Chapel Hill (NC): Department of Sociology, University of North Carolina, 2008.

\section{Krasnoff 2002 \{published data only\}}

Krasnoff M, Moscati R. Domestic violence screening and referral can be effective. Annals of Emergency Medicine 2002;40(5):48592.

\section{Kulkarni 2015 \{published data only\}}

* Kulkarni S, Herman-Smith R, Ross TC. Measuring intimate partner violence (IPV) service providers' attitudes: the development of the Survivor-Defined Advocacy Scale (SDAS) Journal of Family Violence 2015;30(7):911-21.

Kulkarni SJ, Bell H, Hartman J, Herman-Smith R. Exploring individual and organizational factors that contribute to compassion satisfaction, secondary traumatic stress, and burnout in domestic violence service providers. Journal of Society of Social Work and Research 2013;4(2):114-130.

Kulkarni SJ, Bell H, Rhodes D. Back to basics: essential qualities of services for survivors of intimate partner violence. Violence against Women 2012;18(1):85-101.

Kulkarni SJ, Bell H, Wylie L. Why don't they follow through?: intimate partner survivors' challenges in accessing health and social services. Family \& Community Health 2010;33(2):94-105.

\section{Lako 2018 \{published data only\}}

De Vet R, Lako DA, Beijersbergen MD, Van den Dries L, Conover S, Van Hemert AM, et al. Critical time intervention for people leaving shelters in the Netherlands: assessing fidelity and exploring facilitators and barriers. Administration and Policy in Mental Health 2015;44(1):67-80.

* Lako DA, Beijersbergen MD, Jonker IE, De Vet R, Herman DB, Van Hemert AM, et al. The effectiveness of critical time intervention for abused women leaving women's shelters: a randomized controlled trial. International Journal of Public Health 2018;63(4):513-23.
Lako DA, De Vet R, Beijersbergen MD, Herman DB, Van Hemert AM, Wolf JR. The effectiveness of critical time intervention for abused women and homeless people leaving Dutch shelters: study protocol of two randomised controlled trials. BMC Public Health 2013;13:555.

\section{Lea 2016 \{published data only\}}

Lea SJ, Callaghan L. "It gave me my life back": an evaluation of a specialist legal domestic abuse service. Violence against Women 2016;22(6):704.

\section{Logan 2018 \{published data only\}}

* Logan TK, Walker R. Advocate safety planning training, feedback, and personal challenges. Journal of Family Violence 2018;33(3):213-225.

Logan TK, Walker R. Looking into the day-to-day process of victim safety planning. Journal of Family Violence 2018;33(3):197-211.

\section{Lynch 2013 \{published data only\}}

Lynch D, Laing L. 'Women get lost in the gaps' - service providers' perspectives on women's access to legal protection from domestic violence. Report, Faculty of Education and Social Work, University of Sydney 2013.

\section{Lyon 2011 \{published data only\}}

Lyon E, Bradshaw J, Menard A. Meeting survivors' needs through non- residential domestic violence services and supports: results of a multi-state study. Harrisburg, PA: National Resource Center on Domestic Violence; 2011 November. Report No.: 2373282011.

\section{Macy 2018 \{published data only\}}

* Macy RJ, Martin SL, Ogbonnaya IN, Rizo CF. What do domestic violence and sexual assault service providers need to know about survivors to deliver services?. Violence against Women 2018;24(1):28-44.

Macy RJ, Ogbonnaya IN, Martin SL. Providers' perspectives about helpful information for evaluating domestic violence and sexual assault services: a practice note. Violence against Women 2015;21(3):416-29.

Macy RJ, Rizo CF, Johns NB, Ermentrout DM. Directors' opinions about domestic violence and sexual assault service strategies that help survivors. Journal of Interpersonal Violence 2013;28(5):1040-66.

\section{Magruder 2017 \{published data only\}}

Magruder LN. Working the Front Lines of Intimate Partner Violence: Responders' Perceptions of Interrole Collaboration [Dissertation]. Denver (CO): University of Denver, 2017.

\section{Matthew 2016 \{published data only\}}

Matthew M. Exploring Advocates' Work Experiences with Female Victims of Domestic Violence from the Afro-Caribbean Community [Doctoral dissertation]. Minneapolis (MN): School of Public Service Leadership, Capella University, 2016.

A realist review of which advocacy interventions work for which abused women under what circumstances (Review) 
McDermott 2004 \{published data only\}

* McDermott MJ, Garofalo J. Final report: Responding to domestic violence in Southern Illinois Center for the Study of Crime, Delinquency, \& Corrections.. Final report: Responding to domestic violence in Southern Illinois. Carbondale, IL, USA: Center for the Study of Crime, Delinquency, \& Corrections, 2002.

McDermott MJ, Garofalo J. When advocacy for domestic violence victims backfires: types and sources of victim disempowerment. Violence against Women 2004;10(11):124566.

\section{McFarlane 1997 \{published data only\}}

* McFarlane J, Soeken K, Reel S, Parker B, Silva C. Resource use by abused women following an intervention program: associated severity of abuse and reports of abuse ending. Public Health Nursing 1997;14(4):244-50.

Parker B, McFarlane J, Soeken K, Silva C, Reel S. Testing an intervention to prevent further abuse to pregnant women. Research in Nursing and Health 1999;22(1):59-66.

\section{McFarlane 2000 \{published data only\}}

McFarlane J, Soeken K, Wiist W. An evaluation of interventions to decrease intimate partner violence to pregnant women. Public Health Nursing 2000;17(6):443-51.

\section{McFarlane 2004 \{published data only\}}

McFarlane J, Malecha A, Gist J, Watson K, Batten E, Hall I, et al. An intervention to increase safety behaviors of abused women: results of a randomized clinical trial. Nursing Research 2002;51(6):347-54

* McFarlane J, Malecha A, Gist J, Watson K, Batten E, Hall I, et al. Increasing the safety-promoting behaviors of abused women. American Journal of Nursing 2004;104(3):40-50.

McFarlane 2006 \{published data only\}

McFarlane JM, Groff JY, O'Brien JA, Watson K. Secondary prevention of intimate partner violence: a randomized controlled trial. Nursing Research 2006;55(1):52-61.

\section{Merchant 2015 \{published data only\}}

Merchant LV, Whiting JB. Challenges and retention of domestic violence shelter advocates: a grounded theory. Journal of Family Violence 2015;30(4):467-78. [DOI: 10.1007/ s10896-015-9685-y]

\section{Muelleman 1999 \{published data only\}}

Muelleman RL, Feighny KM. Effects of an emergency department-based advocacy program for battered women on community resource utilization. Annals of Emergency Medicine 1999;33(1):62-6

\section{Murray 2015 \{published data only\}}

Murray CE, Horton GE, Johnson CH, Notestine L, Garr B, Pow AM, et al. Domestic violence service providers' perceptions of safety planning: a focus group study. Journal of Family Violence 2015;30(3):381-92
O'Brien 2016 \{published data only\}

O'Brien C. An angel at your table: mentoring and domestic violence recovery. Australian Community Psychologist 2016;28(1):46-58.

\section{Owen 2015 \{published data only\}}

Owen S, Carrington K. Domestic violence (DV) service provision and the architecture of rural life: an Australian case study. Journal of Rural Studies 2015;39:229-38.

Perez 2012 \{published data only\}

Perez S, Johnson DM, Wright CV. The attenuating effect of empowerment on IPV-related PTSD symptoms in battered women living in domestic violence shelters. Violence against Women 2012;18:102.

Prosman 2014 \{published data only\}

* Prosman GJ, Lo Fo Wong SH, Lagro-Janssen AL. Support by trained mentor mothers for abused women: a promising intervention in primary care. Family Practice 2014;31(1):71-80.

Prosman GJ, Lo Fo Wong SH, Romkens R, Lagro-Janssen AL. 'I am stronger, I'm no longer afraid', an evaluation of a homevisiting mentor mother support programme for abused women in primary care. Scandinavian Journal of Caring Sciences 2014;28(4):724-31.

Reina 2015 \{published data only\}

Reina AS, Lohman BJ. Barriers preventing Latina immigrants from seeking advocacy services for domestic violence victims: a qualitative analysis. Journal of Family Violence 2015;30(4):479-88.

\section{Rodgers 2017 \{published data only\}}

Rodgers MA, Grisso JA, Crits-Christoph P, Rhodes KV. No quick fixes: a mixed methods feasibility study of an urban community health worker outreach program for intimate partner violence. Violence against Women 2017;23(3):287-308.

Scheer 2018 \{published data only\}

Scheer JR. Trauma-Informed Care for Sexual and Gender Minority Survivors of Intimate Partner Violence [PhD thesis]. Boston MA: Boston College, 2018.

Shorey 2014 \{published data only\}

Shorey RC, Tirone V, Stuart GL. Coordinated community response components for victims of intimate partner violence: a review of the literature. Aggression and Violent Behavior 2014;19(4):363-71.

Silva-Martinez 2016 \{published data only\}

Silva-Martinez E, Stylianou AM, Hoge GL, Plummer S, McMahon S, Postmus JL. Implementing a financial management curriculum with survivors of IPV: exploring advocates' experiences. Affilia 2016;31(1):112-28.

\section{Slattery 2009 \{published data only\}}

Slattery SM, Goodman LA. Secondary traumatic stress among domestic violence advocates: workplace risk and protective factors. Violence against Women 2009;15(11):1358-79. 


\section{Song 2010 \{published data only\}}

Song $\mathrm{LY}$, Shih CY. Recovery from partner abuse: the application of the strengths perspective. International Journal of Social Welfare 2010;19(1):23-32.

\section{Song 2012 \{published data only\}}

Song L. Service utilization, perceived changes of self, and life satisfaction among women who experienced intimate partner abuse: the mediation effect of empowerment. Journal of Interpersonal Violence 2012;27(6):1112-36.

\section{Stover 2010 \{published data only\}}

* Stover CS, Berkman M, Desai R, Marans S. The efficacy of a police-advocacy intervention for victims of domestic violence: 12 month follow-up data. Violence against Women 2010;16(4):410-25

Stover CS, Poole G, Marans S. The domestic violence homevisit intervention: impact on police-reported incidents of repeat violence over 12 months. Violence and Victims 2009;24:591-606.

Stover CS, Rainey A, Berkman M, Marans S. Factors associated with engagement in a police-advocacy home-visit intervention to prevent domestic violence. Violence against Women 2008;14:1430-50.

\section{Stylianou 2018 \{published data only\}}

Stylianou AM. Family court survivor-centered practice: a qualitative study of advocate-IPV survivor safety planning interactions. Journal of Family Violence 2018;34(3):245-59.

\section{Sudderth 2017 \{published data only\}}

Sudderth L. An uneasy alliance: law enforcement and domestic violence victim advocates in a rural area. Feminist Criminology 2006;1:329-53.

* Sudderth LK. Bringing in "the ones who know them": informal community and safety planning for victims of intimate partner violence in New Zealand. Violence against Women 2017;23(2):222-42.

\section{Sullivan 1991 \{published data only\}}

Sullivan CM, Davidson WS 2nd. The provision of advocacy services to women leaving abusive partners: an examination of short-term effects. American Journal of Community Psychology 1991;19(6):953-60.

\section{Sullivan 2002 \{published data only\}}

Sullivan CM, Bybee SI. Findings from a community-based program for battered women and their children. Journal of Interpersonal Violence 2002;17(9):915-36.

\section{Sullivan 2018 \{published data only\}}

* Sullivan CM, Goodman LA, Virden T, Strom J, Ramirez R. Evaluation of the effects of receiving trauma-informed practices on domestic violence shelter residents. American Journal of Orthopsychiatry 2018;88(5):563-70.

Sullivan CM, Virden T. An eight state study on the relationships among domestic violence shelter services and residents' self-efficacy and hopefulness. Journal of Family Violence 2017;32:741.

\section{Sullivan 2019 \{published data only\}}

Sullivan CM, López-Zerón G, Bomsta H, Menard A. 'There's just all these moving parts:' helping domestic violence survivors obtain housing. Clinical Social Work Journal 2019;47(2):198-206. [DOI: 10.1007/s10615-018-0654-9]

Taft 2011 \{published data only\}

Kerr CP. Project Manual: Setting Up a Mentor Project for Mothers Experiencing Intimate Partner/ Family Violence. Melbourne: La Trobe University, 2009.

Taft AJ, Small R, Hegarty KL, Lumley J, Watson LF, Gold L. MOSAIC (MOthers' Advocates In the Community): protocol and sample description of a cluster randomised trial of mentor mother support to reduce intimate partner violence among pregnant or recent mothers. BMC Public Health 2009;9:159.

* Taft AJ, Small R, Hegarty KL, Watson LF, Gold L, Lumley JA. Mothers' advocates In the community (MOSAIC)-nonprofessional mentor support to reduce intimate partner violence and depression in mothers: a cluster randomised trial in primary care. BMC Public Health 2011;11:178.

\section{Taha 2015 \{published data only\}}

Kaslow NJ, Leiner AS, Reviere SL, Jackson E, Bethea K, Bhaju J, et al. Suicidal, abused African American women's response to a culturally-informed intervention. Journal of Consulting and Clinical Psychology 2010;78:449-58.

* Taha F, Zhang H, Snead K, Jones AD, Blackmon B, Bryant RJ, et al. Effects of a culturally informed intervention on abused, suicidal African American women. Cultural Diversity \& Ethnic Minority Psychology 2015;21(4):560-70.

Zhang H, Neelarambam K, Schwenke TJ, Rhodes MN, Pittman DM, Kaslow NJ. Mediators of a culturally-sensitive intervention for suicidal African American women. Journal of Clinical Psychology in Medical Settings 2013;20(4):401-14.

Thiara 2009 \{published data only\}

Thiara RK. Providing Court Based Support: An evaluation of the Refuge Southwark Advocacy Project. London: Refuge, 2009.

Tiwari 2005 \{published data only\}

Tiwari A, Leung WC, Leung TW, Humphreys J, Parker B, Ho PC. $A$ randomised controlled trial of empowerment training for Chinese abused pregnant women in Hong Kong. BJOG 2005;112(9):1249-56.

Tiwari 2010 \{published data only\}

* Tiwari AF, Salili F, Chan RY, Chan EK, Tang D. An Evaluation of the Effectiveness of an Empowerment Intervention in a Group of Abused Chinese Women. Final Report. Hong Kong, CN: Food and Health Bureau, The Government of the Hong Kong Special Administrative Region, 2010.

Tiwari AF, Salili F, Chan RY, Chan EK, Tang D. Effectiveness of an empowerment intervention in abused Chinese women. Hong Kong Medical Journal 2010;16 Suppl 3:S25-8.

A realist review of which advocacy interventions work for which abused women under what circumstances (Review) 
Trevillion 2013 \{published data only\}

Trevillion K, Byford S, Cary M, Rose D, Oram S, Feder G, et al. Linking abuse and recovery through advocacy: an observational study. Epidemiology and Psychiatric Sciences 2013;23(1):99-113.

\section{Tutty 1996 \{published data only\}}

Tutty LM. Post-shelter services: the efficacy of follow up programs for abused women. Research on Social Work Practice 1996;6(4):1425-4.

\section{Umeda 2017 \{published data only\}}

Umeda M, Kataoka Y, Miller E. Principles of care for women experiencing intimate partner violence: views of expert Japanese health professionals and advocates. Health Care For Women International 2017;38(11):1219-33.

\section{Weisz 1999 \{published data only\}}

Weisz AN. Legal advocacy for domestic violence survivors: the power of an informative relationship. Families in Society 1999;80(2):138-47.

\section{White 2019 \{published data only\}}

White JW, Sienkiewicz HC, Smith PH. Envisioning future directions: conversations with leaders in domestic and sexual assault advocacy, policy, service, and research. Violence against Women 2019;25(1):105-27.

\section{Wies 2008 \{published data only\}}

Wies JR. Professionalizing human services: a case of domestic violence shelter advocates. Human Organization 2008;67(2):221-33

\section{Wong 2013 \{published data only\}}

Tiwari A, Fong DY, Wong JY, Yuen K-H, Yuk H, Pang P, et al. Safety-promoting behaviors of community-dwelling abused Chinese women after an advocacy intervention: a randomized controlled trial. International Journal of Nursing Studies 2012;49(6):645-5.

Tiwari A, Fong DY, Yuen KH, Yuk H, Pang P, Humphreys J, et al. Effect of an advocacy intervention on mental health in Chinese women survivors of intimate partner violence: a randomized controlled trial. JAMA 2010;304(5):536-43.

Tiwari A, Yuk H, Pang P, Fong DY, Yuen F, Humphreys J, et al. Telephone intervention to improve the mental health of community-dwelling women abused by their intimate partners: a randomised controlled trial. Hong Kong Medical Journal (xianggang yi xue za zhi) 2012;18(6):14-17.

* Wong JY, Tiwari A, Fong DY, Yuen KH, Humphreys J, Bullock L. Intimate partner violence, depressive symptoms, and immigration status: does existing advocacy intervention work on abused immigrant women in the Chinese community?. Journal of Interpersonal Violence 2013;28(11):2181-202.

\section{Wood 2014 \{published data only\}}

* Wood L. Domestic Violence Advocacy. Indiana USA: School of Social Work, Indiana University, 2014
Wood L. "I look across from me and I see me": survivors as advocates in intimate partner violence agencies. Violence against Women 2017;23(3):309-29.

\section{Wuest 2015 \{published data only\}}

Ford-Gilboe M, Merritt-Gray M, Varcoe C, Wuest JA. A theorybased primary health care intervention for women who have left abusive partners. Advances in Nursing Science 2011;34(3):198-214.

Jack SM, Ford-Gilboe M, Wathen CN, Davidov DM, McNaughton DB, Coben JH. Development of a nurse home visitation intervention for intimate partner violence. $B M C$ Health Services Research 2012;12:50.

* Wuest J, Merritt-Gray M, Dube N, Hodgins MJ, Malcolm J, Majerovich JA, et al. The process, outcomes, and challenges of feasibility studies conducted in partnership with stakeholders: a health intervention for women survivors of intimate partner violence. Research in Nursing \& Health 2015;38(1):82-96.

\section{Zweig 2007 \{published data only\}}

Zweig JM, Burt MR. Predicting women's perceptions of domestic violence and sexual assault agency helpfulness: what matters to program clients?. Violence against Women 2007;13(11):1149-78.

\section{References to studies excluded from this review}

Anderson 2012 \{published data only\}

Anderson KM, Renner LM, Danis FS. Recovery: resilience and growth in the aftermath of domestic violence. Violence against Women 2012;18(11):1279-99.

\section{Andrews 2011 \{published data only\}}

Andrews CM, Cao D, Marsh JC, Shin HC. The impact of comprehensive services in substance abuse treatment for women with a history of intimate partner violence. Violence against Women 2011;17(5):550-67.

\section{Bair-Merritt 2010 \{published data only\}}

Bair-Merritt MH, Jennings JM, Chen R, Burrell L, McFarlane E, Fuddy $L$, et al. Reducing maternal intimate partner violence after the birth of a child: a randomized controlled trial of the Hawaii Healthy Start home visitation program. Archives of Pediatric and Adolescent Medicine 2010;164(1):16-23.

\section{Basu 2009 \{published data only\}}

Basu A, Malone JC, Levendosky AA, Dubay S. Longitudinal treatment effectiveness outcomes of a group intervention for women and children exposed to domestic violence. Journal of Child \& Adolescent Trauma 2009;2:90.

\section{Becker 2008 \{published data only\}}

Becker KD, Mathis G, Mueller CW, Issari K, Atta SS. Communitybased treatment outcomes for parents and children exposed to domestic violence. Journal of Emotional Abuse 2008;8(1-2):187204. 
Bennett 2007 \{published data only\}

Bennett L, O'Brien P. Effects of coordinated services for drugabusing women who are victims of intimate partner violence. Violence against Women 2007;13(4):395-411.

\section{Camacho 2008 \{published data only\}}

Camacho CM, Alarid LF. The significance of the victim advocate fordomestic violence victims in municipal court. Violence and Victims 2008;23(3):288-300.

\section{Carlson 2012 \{published data only\}}

* Carlson CE, Chen JH, Chang MW, Batsukh A, Toivgoo A, Riedel $\mathrm{M}$, et al. Reducing intimate and paying partner violence against women who exchange sex in Mongolia: results from a randomized clinical trial. Journal of Interpersonal Violence 2012;27(10):1911-31.

Witte SS, Altantsetseg B, Aira T, Riedel M, Chen J, Potocnik K, et al. Reducing sexual HIV/STI risk and harmful alcohol advocacy use among female sex workers in Mongolia: a randomized clinical trial. AIDS and Behavior 2011;15(8):1785-94.

\section{Champion 2007 \{published data only\}}

Champion JD, Shain RN, Korte JE, Holden AE, Piper JM, Perdue ST, et al. Behavioural interventions and abuse: secondary analysis of reinfection in minority women. International Journal of STD \& AIDS 2007;18(11):748-53.

\section{Clarke 2013 \{published data only\}}

Clarke C, Wydall S. 'Making Safe': a coordinated community response to empowering victims and tackling perpetrators of domestic violence. Social Policy and Society 2013;12(3):393-406.

\section{Cupples 2011 \{published data only\}}

Cupples ME, Stewart MC, Percy A, Hepper P, Murphy C, Halliday HL. A RCT of peer-mentoring for first-time mothers in socially disadvantaged areas (the MOMENTS Study). Archives of Diseases in Childhood 2011;96(3):252-8.

\section{Davidson 2012 \{published data only\}}

Davidson MM, Nitzel C, Duke A, Baker CM, Bovaird JA, DePrince. Advancing career counseling and employment support for survivors: an intervention evaluation. Journal of Counseling Psychology 2012;59(2):321-8.

\section{Denne 2012 \{published data only\}}

Denne S, Coombes L, Morgan M. Problematising effectiveness: the inclusion of victim advocacy services in living without violence programme provision and evaluation. Proceedings of Doing Psychology: Manawatu Doctoral Research Symposium 2012;2:27-34.

\section{Donovan 2010 \{published data only\}}

Donovan C, Griffiths S, Groves N, Johnson H, Douglass J. Making Connections Count: An Evaluation of Early Intervention Models for Change in Domestic Violence 2004-2009.. Newcastle: Northern Rock Foundation, 2010.

\section{El-Mohandes 2011 \{published data only\}}

El-Mohandes AA, Kiely M, Joseph JG, Subramanian S, Johnson AA, Blake SM, et al. An intervention to improve postpartum outcomes in African-American mothers: a randomized controlled trial. Obstetrics and Gynecology 2008;112(3):611-20.

* El-Mohandes AE, Kiely M, Gantz MG, El-Khorazaty MN. Very preterm birth is reduced in women receiving an integrated behavioral intervention: a randomized controlled trial. Maternal and Child Health Journal 2011;15(1):19-28.

Feder 2011 \{published data only\}

Feder G, Davies RA, Baird K, Dunne D, Eldridge S, Griffiths C, et al. Identification and referral to improve safety (IRIS) of women experiencing domestic violence with a primary care training and support programme: a cluster randomised controlled trial. Lancet 2011;378(9805):19-25.

Fox 2018 \{published data only\}

Fox KA, Fisher BS, Decker SH. Identifying the needs of American Indian women who sought shelter: a practitioner-researcher partnership. Journal of Family Violence 2018;33:251-6.

Giocolea 2015 \{published data only\}

Goicolea I, Hurtig A, San Sebastian M, Marchal B, Vives-Cases C. Using realist evaluation to assess primary healthcare teams' responses to intimate partner violence in Spain. Gaceta Sanitaria / S.E.S.P.A.S 2015;29:431-6.

\section{Goldblatt 2009 \{published data only\}}

Goldblatt H, Buchbinder E, Eisikovits Z, Arizon-Mesinger I. Between the professional and the private: the meaning of working with intimate partner violence in social workers' private lives. Violence Against Women 2009;15(3):362-84. [DOI: 10.1177/1077801208330436; PUBMED: 19131561]

\section{Graham-Bermann 2013 \{published data only\}}

Graham-Bermann SA, Miller LE. Intervention to reduce traumatic stress following intimate partner violence: an efficacy trial of the Moms' Empowerment Program (MEP). Psychodynamic Psychiatry 2013;41(2):329-49.

\section{Hart 2013 \{published data only\}}

Hart BJ, Klein AF. Practical Implications of Current Intimate Partner Violence Research for Victim Advocates and Service Providers. Washington (DC): National Institute of Justice, Office of Justice Programs, National Criminal Justice Reference Service, 2013.

\section{Hovmand 2009 \{published data only\}}

Hovmand PS, Ford DN. Sequence and timing of three community interventions to domestic violence. American Journal of Community Psychology 2009;44(3-4):261-72.

\section{Howard 2017 \{published data only\}}

Howard BN, Van Dorn R, Myers BJ, Zule WA, Browne FA, Carney $T$, et al. Barriers and facilitators to implementing an evidence-based woman-focused intervention in South African health services. BMC Health Services Research 2017;17(1):746.

\section{Jack 2015 \{published data only\}}

Jack SM, Sheehan D, Gonzalez A, et al. British Columbia Healthy Connections Project process evaluation: a mixed methods 
protocol to describe the implementation and delivery of the Nurse-Family Partnership in Canada. BMC Nursing 2015;14:47.

Juillard 2016 \{published data only\}

Juillard C, Cooperman L, Allen I, Pirracchio R, Henderson T, Marquez R, et al. A decade of hospital-based violence intervention: benefits and shortcomings. Journal of Trauma and Acute Care Surgery 2016;81(6):1156-61.

Kramer 2012 \{published data only\}

Kramer A, Nosbusch JM, Rice J. Safe mom, safe baby: a collaborative model of care for pregnant women experiencing intimate partner violence. Journal of Perinatal \& Neonatal Nursing 2012;26(4):307-16.

\section{Kyegombe 2014 \{published data only\}}

Kyegombe N, Abramsky T, Devries KM, Starmann E, Michau L, Nakuti J, et al. The impact of SASA!, a community mobilization intervention, on reported HIV related risk behaviours and relationship dynamics in Kampala, Uganda. Journal of the International AIDS Society 2014;17:19-32.

\section{Lorenzo 2018 \{published data only\}}

Lorenzo RA. The act of listening to "battered" women: an ethnographic comparison of police and emergency responses in Sao Paulo, Brazil. Latin American Research Review 2018;53(1):96-110.

\section{Mantler 2017 \{published data only\}}

Mantler T, Wolfe B.J. A rural shelter in Ontario adapting to address the changing needs of women who have experienced intimate partner violence: a qualitative case study. Rural and Remote Health 2017;17(1):3987.

\section{McFeely 2017 \{published data only\}}

McFeely CW. The Health Visitor Response to Domestic Abuse [PhD thesis]. Glasgow: University of Glasgow, July 2016.

\section{McKean 2004 \{published data only\}}

McKean L. Self-sufficiency and safety: the case for onsite domestic violence services at employment services agencies. www.impactresearch.org/documents/DVpolicyreport.pdf (accessed 10 January 2019) 2014.

\section{McLelland 2008 \{published data only\}}

McLelland S. Evaluation of a Liaison Domestic Violence Nurse in the Emergency Unit of Cardiff and Vale NHS Trust. Pontypridd: Health Economics and Policy Research Unit, University of Glamorgan, 2008.

\section{McNamara 1997 \{published data only\}}

McNamara JR, Ertl MA, Marsh S, Walker S. Short-term response to counseling and case management intervention in a domestic violence shelter. Psychological Reports 1997;81(3):1243-51.

\section{Miller 2011 \{published data only\}}

Miller E, Decker MR, McCauley HL, Tancredi DJ, Levenson RR, Waldman J, et al. A family planning clinic partner violence intervention to reduce risk associated with reproductive coercion. Contraception 2011;83(3):274-80.
Miller 2014 \{published data only\}

Miller LE, Howell KH, Graham-Bermann SA. The effect of an evidence-based intervention on women's exposure to intimate partner violence. American Journal of Orthopsychiatry 2014;84(4):321-8.

\section{Mitchell 2006 \{published data only\}}

Mitchell MD, Hargrove GL, Collins MH, Thompson MP, Reddick TL, Kaslow NJ. Coping variables that mediate the relation between intimate partner violence and mental health outcomes among low-income, African American women. Journal of Clinical Psychology 2006;62(12):1503-20.

Nichols 2013 \{published data only\}

Nichols AJ. Meaning-making and domestic violence victim advocacy: an examination of feminist identities, ideologies, and practices. Feminist Criminology 2013;8:177-201.

\section{Rhodes 2014 \{published data only\}}

Rhodes KV, Rodgers M, Sommers M, Hanlon A, Crits-Christoph P. The Social Health Intervention Project (SHIP): protocol for a randomized controlled clinical trial assessing the effectiveness of a brief motivational intervention for problem drinking and intimate partner violence in an urban emergency department. BMC Emergency Medicine 2014;14:10.

\section{Saggurti 2014 \{published data only\}}

Raj A, Saggurti N, Battala M, Nair S, Dasgupta A, Naik DD, et al. Randomized controlled trial to test the RHANI Wives HIV intervention for women in India at risk for HIV from husbands. AIDS and Behavior 2013;17(9):3066-80.

* Saggurti N, Nair S, Silverman JG, Naik DD, Battala M, Dasgupta A, et al. Impact of the RHANI Wives intervention on marital conflict and sexual coercion. International Journal of Gynaecology and Obstetrics 2014;126(1):18-22.

Samuels-Dennis 2013 \{published data only\}

Samuels-Dennis J, Bailey A, Killian K, Ray SL. The mediating effects of empowerment, interpersonal conflict, and social support on the violence-PTSD process among single mothers. Canadian Journal of Community Mental Health 2013;32(1):09-124.

Simmons 2011 \{published data only\}

Simmons CA, Farrar M, Frazer K, Thompson MJ. From the voices of women: facilitating survivor access to IPV services. Violence against Women 2011;17(10):1226-43.

Valpied 2014 \{published data only\}

Valpied J, Cini A, O'Doherty L, Taket A, Hegarty K. "Sometimes cathartic. Sometimes quite raw": benefit and harm in an intimate partner violence trial. Aggression and Violent Behavior 2014;19(6):673-85.

\section{Wagman 2015 \{published data only\}}

Wagman JA, Gray RH, Campbell JC, Thoma M, Ndyanabo A, Ssekasanvu J, et al. Effectiveness of an integrated intimate partner violence and HIV prevention intervention in Rakai, Uganda: analysis of an intervention in an existing cluster randomised cohort. Lancet 2015;3(1):e23-e33. 
Zlotnick 2011 \{published data only\}

Zlotnick C, Capezza NM, Parker D. An interpersonally based intervention for low-income pregnant women with intimate partner violence: a pilot study. Archives of Women's Mental Health 2011;14(1):55-65.

\section{References to studies awaiting assessment}

Harris 2002 \{published data only\}

Harris MH, Weber M. Providing crisis counselors on-site to victims of domestic violence in the emergency department:a report of a local pilot project. South Dakota Journal of Medicine 2002;55(4):147-9.

\section{Shepard 1991 \{published data only\}}

Shepard MF. Advocacy for battered women: implications for a coordinated community response. In: Shepard MF, Pence EL editor(s). Coordinating Community Responses to Domestic Violence: Lessons from Duluth and Beyond. Thousand Oaks, CA: Sage, 1999:115-25.

\section{References to ongoing studies}

NCT03016481 \{published data only\}

NCT03016481. Realizing opportunities for self-supported improvement (ROSSI) (ROSE-SAFE) [Healing through education, advocacy and law (HEAL) in response to violence]. clinicaltrials.gov/ct2/show/NCT03016481 (accessed 31 January 2019).

\section{NCT03259646 \{published data only\}}

NCT03259646. The IPV Provider Network: engaging the health care provider response to interpersonal violence against women. clinicaltrials.gov/ct2/show/NCT03259646 (accessed 31 January 2019).

\section{Tol 2017 \{published data only\}}

Tol WA, Greene MC, Likindikoki S, Misinzo L, Ventevogel P, Bonz AG, et al. An integrated intervention to reduce intimate partner violence and psychological distress with refugees in low-resource settings: study protocol for the Nguvu cluster randomized trial. BMC Psychiatry 2017;17(1):186. [DOI: 10.1186/ s12888-017-1338-7; PMC5437564; PUBMED: 28521751]

\section{Additional references}

\section{Abel 2000}

Abel EM. Psychosocial treatments for battered women: a review of empirical research. Research on Social Work Practice 2000;10(1):55-77.

\section{Access Economics 2004}

Access Economics. The Cost of Domestic Violence to the Australian Economy: Part 1. Canberra: Office for the Status of Women, 2004. [ISBN: 1-877-042-74-9; www.dss.gov.au/sites/ default/files/documents/05_2012/cost_of_dv_to_australian_ economy_i_1.pdf]

\section{Adams 2008}

Adams AE, Sullivan CM, Bybee D, Greeson MR. Development of the scale of economic abuse. Violence against Women 2008;14(5):563-88. [DOI: 10.1177/1077801208315529; PUBMED: 18408173]

\section{Agarwal 2017}

Agarwal A, Guyatt GH, Busse JW, Busse JW, Consistent KT. Methods commentary: risk of bias in cross-sectional surveys of attitudes and practices [unpublished position paper]. www.evidencepartners.com/wp-content/uploads/2017/04/ Methods-Commentary-Risk-of-Bias-in-cross-sectional-surveysof-attitude....pdf (accessed 10 January 2019).

\section{Alvaro 2010}

Alvaro C, Lyons RF, Warner G, Hobfoll SE, Martens PJ, Labonté R, et al. Conservation of resources theory and research use in health systems. Implementation Science 2010;5(79):1-20.

\section{Anderberg 2018}

Anderberg D, Mantovan N, Sauer RM. The dynamics of domestic violence: learning about the match. London (UK): Institute for Fiscal Studies; Report No.: IFS Working Paper W18/12 2018.

\section{Andrews 1976}

Andrews F, Withey S. Social Indicators of Well-being: Americans' Perceptions of Life Quality. New York, NY: Plenum Press, 1976.

\section{Asia-Japan Women's Resource Center (AJWRC) 2007}

Asia-Japan Women's Resource Center (AJWRC) of the World Organisation Against Torture. Violations of women's rights In Japan: alternative report to the United Nations Committee Against Torture, 38th session. www.refworld.org/ pdfid/46af4d1f0.pdf (accessed 29 April 2019).

\section{Bair-Merritt 2014}

Bair-Merritt MH, Lewis-O'Connor A, Goel S, Amato P, Ismailji T, Jelley $\mathrm{M}$, et al. Primary care-based interventions for intimate partner violence: a systematic review. American Journal of Preventive Medicine 2014;46(2):188-94. [DOI: 10.1016/ j.amepre.2013.10.001; PUBMED: 24439354]

\section{Bandura 1997}

Bandura A. Self-efficacy: toward a unifying theory of behavioral change. Psychological Review 1977;84(2):191-215.

\section{Barie 2012}

Barie DJ. Empowering the Disempowered: Exploring How Advocates Perceive Resources and Services Available for Deaf Victims of Domestic Violence and Sexual Assault [Master's dissertation]. Indiana (PA): Sociology Department, Indiana University of Pennsylvania, 2012.

\section{Bell 1977}

Bell JM. Stressful life events and coping methods in mental-illness and -wellness behaviours. Nursing Research 1977;26(2):136-41. [PUBMED: 584378]

\section{Bell 2017}

Scottish Government. Domestic Abuse Recorded by the Police in Scotland, 2016-17. www.gov.scot/Publications/2017/10/3700

A realist review of which advocacy interventions work for which abused women under what circumstances (Review) 
(accessed prior to 13 August 2018). [ISBN: 978-1-78851-235-0; www.gov.scot/Resource/0052/00526358.pdf]

\section{Bialy 2018}

Bialy B. A Comparison of Issues About Domestic Violence in the United States and Spain (Una Comparación de Asuntos Sobre la Violencia Doméstica en los Estados Unidos y España) [Master's thesis]. Brockport (NY): The College at Brockport, 2018.

\section{Black 2003}

Black CJ. Translating principles into practice: implementing the feminist and strengths perspective in work with battered women. Affilia 2003;18(3):332-49. [DOI: $10.1177 / 0886109903254578]$

\section{Black 2011}

Black MC, Basile KC, Breiding MJ, Smith SG, Walters ML, Merrick MT, et al. National Intimate Partner and Sexual Violence Survey (NISVS): 2010 Summary Report. Atlanta (GA): National Center for Injury Prevention and Control, Centers for Disease Control and Prevention, 2011. [www.cdc.gov/ violenceprevention/pdf/nisvs_report2010-a.pdf]

\section{Bonomi 2009}

Bonomi AE, Anderson ML, Rivara FP, Thompson RS. Health care utilization and costs associated with physical and nonphysicalonly intimate partner violence. Health Services Research 2009;44(3):1052-67. [DOI: 10.1111/j.1475-6773.2009.00955.x; PMC2699921; PUBMED: 19674432

\section{Booth 2013}

Booth A, Harris J, Croot E, Springett J, Campbell F, Wilkins E. Towards a methodology for cluster searching to provide conceptual and contextual "richness" for systematic reviews of complex interventions: case study (CLUSTER). BMC Medical Research Methodology 2013;13:118. [DOI: 10.1186/1471-2288-13-118; PMC3819734; PUBMED: 24073615]

\section{Breen 1997}

Breen EM. The Long-Term Emotional Effects after Domestic Violence Crisis Intervention: A Process Mode [Doctoral dissertation]. Greeley (CO): University of Northern Colorado, 1997.

\section{Brownridge 2006}

Brownridge DA. Violence against women post-separation. Aggression and Violent Behavior 2006;11(5):514-30. [DOI: 10.1016/j.avb.2006.01.009]

\section{Campbell 1986}

Campbell JC. Nursing assessment for risk of homicide with battered women. Advances in Nursing Science 1986;8(4):36-51. [PUBMED: 3089133]

\section{Campbell 1993}

Campbell JC, Humphreys JC. Nursing Care of Survivors of Family Violence. 2nd Edition. St Louis (MO): Mosby, 1993. [ISBN: 0-801-663-784]

\section{Campbell 2000}

Campbell M, Fitzpatrick R, Haines A, Kinmonth AL, Sandercock P, Spiegelhalter D, et al. Framework for design and evaluation of complex interventions to improve health. $B M J$ 2000;321(7262):694-6. [PMC1118564; PUBMED: 10987780]

\section{Campbell 2002}

Campbell JC. Health consequences of intimate partner violence. Lancet 2002;359(9314):1331-6. [DOI: 10.1016/ S0140-6736(02)08336-8; PUBMED: 11965295]

\section{Campbell 2004}

Campbell JC. Helping women understand their risk in situations of intimate partner violence. Journal of Interpersonal Violence 2004;19(12):1464-77. [DOI: 10.1177/0886260504269698; PUBMED: 15492060]

\section{Campbell 2009}

Campbell JC, Webster DW, Glass N. The danger assessment: validation of a lethality risk assessment instrument for intimate partner femicide. Journal of Interpersonal Violence 2009;24(4):653-74.

\section{Carney 2012}

Carney MM, Barner JR. Prevalence of partner abuse: rates of emotional abuse and control. Partner Abuse 2012;3(3):286-335 [DOI: 10.1891/1946-6560.3.3.286]

\section{Cattaneo 2015}

Cattaneo LB, Goodman LA. What is empowerment anyway? A model for domestic violence practice, research, and evaluation. Psychology of Violence 2015;5(1):84-94.

\section{Cauthen 2015}

Cauthen MC. Identifying and Overcoming Barriers Domestic Violence Victims Face: A Victim Advocate Perspective [Doctoral dissertation]. Minneapolis (MN): Capella University, Department School of Public Service Leadership, 2015.

\section{Clarke 2008}

Clarke DE. Silencing the Voices of Women. A Case Study on the Effects of the "Supporting People" Programme on Survivors of Domestic Abuse in a Support and Housing Association [Doctoral thesis]. Bradford: University of Bradford Department of Social Sciences and Humanities, 2008.

\section{Collins 2005}

Collins PH. Black Sexual Politics: African Americans, Gender, and the New Racism. New York: Routledge, 2005.

\section{Colombini 2017}

Colombini M, Dockerty C, Mayhew SH. Barriers and facilitators to integrating health service responses to intimate partner violence in low- and middle-income countries: a comparative health systems and service analysis. Studies in Family Planning 2017;48(2):179-200. [DOI: 10.1111/sifp.12021; PMC5518204; PUBMED: 28422291] 


\section{Curnow 1997}

Curnow SA. The open window phase: helpseeking and reality behaviors by battered women. Applied Nursing Research 1997;10(3):128-35.

\section{Davidson 1997}

Davidson JR, Book SW, Colket JT, Tupler LA, Roth S, David D, et al. Assessment of a new self-rating scale for post-traumatic stress disorder. Psychological Medicine 1997;27(1):153-60.

\section{Davidson 2001}

Davidson LL, King V, Garcia J, Marchant S. What role can the health services play?. In: Taylor-Browne J editor(s). What Works in Reducing Domestic Violence: A Comprehensive Guide for Professionals. London: Whiting \& Birch Ltd, 2001:95-122. [ISBN-10: 1-861-770-367]

\section{Department of Justice of Hong Kong 2015}

Department of Justice, The Government of the Hong Kong Special Administrative Region. The policy for prosecuting cases involving domestic violence. www.doj.gov.hk/eng/public/ pubppcdv.html (accessed 29 April 2019).

\section{DePrince 2010}

DePrince AP, Zurbriggen EL, Chu AT, Smart L. Development of the Trauma Appraisal Questionnaire. Journal of Aggression, Maltreatment \& Trauma 2010;19:275-99.

\section{Diemer 2013}

Diemer MA, Mistry RS, Wadsworth ME, López I, Reimers F. Best practices in conceptualizing and measuring social class in psychological research. Analyses of Social Issues and Public Policy 2013;13(1):77-113. [DOI: 10.1111/asap.12001]

\section{Dobash 1984}

Dobash RE, Dobash RP. The nature and antecedents of violent events. British Journal of Criminology 1984;24(3):269-88. [APA PsycNet record: 1985-14778-001]

\section{Dutton 1992}

Dutton MA. Empowering and Healing the Battered Woman: A Model for Assessment and Intervention. New York: Springer Publishing Co, 1992.

\section{Emmel 2013}

Emmel N. Sampling and Choosing Cases in Qualitative Research: A Realist Approach. London (UK): Sage, 2013.

\section{Feder 2006}

Feder G, Ramsay J, Zachary MJ. Clinical response to women experiencing intimate partner abuse: what is the evidence for good practice and policy?. In: Roberts G, Hegarty K, Feder G editor(s). Intimate Partner Abuse and Health Professionals: New Approaches to Domestic Violence. Edinburgh: Churchill Livingstone, 2006:93-110. [ISBN: 0-443-074-933]

\section{Feder 2013}

Feder G, Wathen CN, MacMillan HL. An evidence-based response to intimate partner violence: WHO guidelines. JAMA 2013;310(5):479-80. [PUBMED: 10.1001/jama.2013.167453; PUBMED: 23925614]

\section{Felson 2005}

Felson RB, Ackerman JM, Gallagher C. Police intervention and the repeat of domestic assault. Criminology 2005;43(4):563-88. [DOI: 10.1111/j.0011-1348.2005.00017.x]

\section{Flanakin 2005}

Flanakin N, Walsh C. Letter to the editor. Violence against Women 2005;11:822-7.

\section{Fraser 2004}

Fraser H. Doing narrative research: analysing personal stories line by line. Qualitative Social Work 2004;3(2):179-201.

\section{Frediani 2010}

Frediani AA. Sen's Capability Approach as a framework to the practice of development. Development in Practice 2010;20(2):173-87. [DOI: 10.1080/09614520903564181]

\section{Garcia-Moreno 2006}

Garcia-Moreno C, Jansen HA, Ellsberg M, Heise L, Watts CH, WHO Multi-country Study on Women's Health and Domestic Violence against Women Study Team. Prevalence of intimate partner violence: findings from the WHO multi-country study on women's health and domestic violence. Lancet 2006;368(9543):1260-9. [DOI: 10.1016/S0140-6736(06)69523-8; PUBMED: 17027732$]$

\section{García-Moreno 2015}

García-Moreno C, Hegarty K, Lucas d'Oliveira AF, KoziolMcLain J, Colombini M, Feder G. The health-systems response to violence against women. Lancet 2015;385(9977):1567-79. [DOI: 10.1016/S0140-6736(14)61837-7; PUBMED: 25467583]

\section{Garratt 1993}

Garratt AM, Ruta DA, Abdalla MI, Buckingham JK, Russell IT. The SF-36 health survey questionnaire: an outcome measure suitable for routine use within the NHS. BMJ 1993;306:1440-44.

\section{Gibbon 2000}

Gibbon M. The health analysis and action cycle: an empowering approach to women's health. Sociological Research Online 2000;4(4):391.

\section{Goodman 2015}

Goodman LA, Thomas KA, Heimel D. A guide for using the Measure of Victim Empowerment Related to Safety (MOVERS). www.dvevidenceproject.org/wp-content/uploads/MOVERS_v6Goodman-20153.pdf (accessed 20 June 2019).

\section{Goonetilleke 2015}

Goonetilleke SME. Domestic violence: is the Sri Lankan woman still trapped in the private sphere?. OUSL Journal 2015;8:101-23. [DOI: 10.4038/ouslj.v8i0.7321]

\section{Gough 2007}

Gough D. Weight of evidence: a framework for the appraisal of the quality and relevance of evidence. Research Papers in Education 2007;22(2):213-28. [DOI: 10.1080/02671520701296189]

A realist review of which advocacy interventions work for which abused women under what circumstances (Review) 


\section{Government of Canada 2017}

Department of Justice, Government of Canada. Enhancing safety: when domestic violence cases are in multiple legal systems (criminal, family, child protection). A family law, domestic violence perspective. www.justice.gc.ca/eng/rp-pr/flIf/famil/enhan-renfo/p3.html\#p543 (accessed prior to 27 May 2019).

\section{Government of Mexico 2016}

Government of Mexico. Review of reports submitted by the States party to article 18 of the Convention on the Elimination of All Forms of Discrimination against Women [Examen de los informes presentados por los Estados partes en virtud del artículo 18 de la Convención sobre la eliminación de todas las formas de discriminación contra la mujer]. tbinternet.ohchr.org/Treaties/CEDAW/Shared\%20Documents/ CAN/CEDAW_C_OP-8_CAN_1_7643_E.pdf (accessed 29 April 2019).

\section{Greenhalgh 2016}

Greenhalgh T, Macfarlane F, Steed L, Walton R. What works for whom in pharmacist-led smoking cessation support: realist review. BMC Medicine 2016;14:209. [DOI: 10.1186/ s12916-016-0749-5]

\section{Greeson 2013}

Greeson MR, Campbell R. Sexual assault response teams (SARTs): an empirical review of their effectiveness and challenges to successful implementation. Trauma, Violence \& Abuse 2013;14(2):83-95.

\section{Hegarty 2005}

Hegarty K, Bush R, Sheehan M. The Composite Abuse Scale: further development and assessment of reliability and validity of a multidimensional partner abuse measure in clinical settings. Violence and Victims 2005;20(5):529-47.

\section{Heise 1998}

Heise LL. Violence against women: an integrated, ecological framework. Violence against Women 1998;4(3):262-90.

\section{Heise 2002}

Heise L, García-Moreno C. Violence by intimate partners. In: Krug EG, Dahlberg LL, Mercy JA, Zwi AB, Lozano R editor(s). World Report on Violence and Health. Geneva: World Health Organization, 2002:87-122. [ISBN: 92-4-154561-5; apps.who.int/iris/bitstream/handle/10665/42495/9241545615 eng.pdf;jsessionid=E26A1CD5EE2A406EEFC9D9C5C6489EF7? sequence $=1$ ]

\section{Heise 2014}

Heise L, Fulu E. What Works to Prevent Violence Against Women and Girls? State of the field of violence against women and girls: what do we know and what are the knowledge gaps?. Geneva (CH): World Health Organization, 2014.

\section{Hender 2001}

Hender K. Is Therapy/Counselling/Group Work More Effective than No Treatment for Women who are Victims of Domestic Violence? Evidence Report. Clayton: Centre for Clinical
Effectiveness. Monash, Australia: Monash University Medical Centre, 2001.

\section{Henwood 2000}

Henwood M. Domestic Violence: A Resource Manual for Health Care Professionals. London: Department of Health, 2000.

\section{Hester 2012}

Hester M. Domestic violence advocacy and support in a changing climate - findings from three recent evaluations. Newcastle: Northern Rock Foundation, 2012.

\section{Higgins 2011}

Higgins J, Altman, DG, Gøtzsche PC, Jüni P, Moher D, Oxman AD, et al. The Cochrane Collaboration's tool for assessing risk of bias in randomised trials. BMJ 2011;343:d5928.

\section{Higgins 2019}

Higgins JP, Sterne JA, Savović J, Page MJ, Hróbjartsson A, Boutron I, et al. A revised tool for assessing risk of bias in randomized trials. In: Chandler J, McKenzie J, Boutron I, Welch V (editors). Cochrane Methods. Cochrane Database of Systematic Reviews 2016, Issue 10 (Suppl 1). www.cochranelibrary.com/ documents/20182/64256496/Cochrane+Methods +2016/9cd61dd1-04c1-338c-2279-7aa30953451f. [DOI: 10.1002/14651858.CD201601]

\section{Hochschild 2003}

Hochschild A. The commercialization of intimate life: notes from home and work. San Francisco and Los Angeles: University of California Press, 2003.

\section{Holmes 2018}

Holmes MR, Richter FG, Votruba ME, Berg KA, Bender AE. Economic burden of child exposure to intimate partner violence in the US. Journal of Family Violence 2018;33(4):239-49. [DOI: 10.1007/s10896-018-9954-7]

\section{Holt 2015}

Holt S. Post-separation fathering and domestic abuse: challenges and contradictions. Child Abuse Review 2015; Vol. 24, issue 3:210-22. [DOI: 10.1002/car.2264]

\section{Home Office 2013}

Home Office. Domestic violence and abuse. www.gov.uk/ guidance/domestic-violence-and-abuse (accessed prior to 13 August 2018).

\section{Howe 2009}

Howe D. A Brief Introduction to Social Work Theory. London: Palgrave Macmillan, 2009. [ISBN: 0-230-233-120]

\section{Humphreys 2002}

Humphreys C, Thiara R. Routes to Safety: Protection Issues Facing Abused Women and Children and the Role of Outreach Services. Bristol: Women's Aid, 2002.

\section{Ingram 2005}

Ingram JK. Statement to the UN on violence against women. web.worldbank.org/WBSITE/EXTERNAL/TOPICS/ 
EXTGENDER/0,,contentMDK:20779823 menuPK:336914 pagePK:64060R620166K:149114 theSitePK:336868,00.html

(accessed prior to 13 August 2018).

\section{Jagosh 2012}

Jagosh J, Macaulay AC, Pluye P, Salsberg J, Bush PL, Henderson J, et al. Uncovering the benefits of participatory research: implications of a realist review for health research and practice. Milbank Quarterly 2012;90(2):311-46. [DOI: 10.1111/ j.1468-0009.2012.00665.x; PMC3460206; PUBMED: 22709390]

\section{Jagosh 2014}

Jagosh J, Pluye P, Wong G, Cargo M, Salsberg J, Bush PL, et al. Critical reflections on realist review: insights from customizing the methodology to the needs of participatory research assessment. Research Synthesis Methods 2014;5(2):131-41. [DOI: 10.1002/jrsm.1099; PUBMED: 26052652]

\section{Jahanfar 2011}

Jahanfar S, Janssen PA, Howard LM, Dowswell T. Interventions for preventing or reducing domestic violence against pregnant women. Cochrane Database of Systematic Reviews 2011, Issue 11. [DOI: 10.1002/14651858.CD009414]

\section{Johnson 2015}

Johnson SD, Tilley N, Bowers KJ. Introducing EMMIE: an evidence rating scale to encourage mixed-method crime prevention synthesis reviews. Journal of Experimental Criminology 2015;11(3):459-73. [DOI: 10.1007/ s11292-015-9238-7]

\section{Jones 2006}

Jones AS, Dienemann J, Schollenberger J, Kub J, O'Campo P, Gielen AC, et al. Long-term costs of intimate partner violence in a sample of female HMO enrollees. Women's Health Issues 2006;16(5):252-61. [DOI: 10.1016/j.whi.2006.06.007; PUBMED: $17055378]$

\section{Jonker 2015}

Jonker IE, Sijbrandij M, Van Luijtelaar MJ, Cuijpers P, Wolf JR. The effectiveness of interventions during and after residence in women's shelters: a meta-analysis. European Journal of Public Health 2015;25(1):15-19.

\section{Kabeer 1999}

Kabeer N. Resources, agency, achievements: reflections on the measure- men of women's empowerment. Development and Change 1999;30:435-64.

\section{Kalra 2017}

Kalra N, Di Tanna GL, García-Moreno C. Training healthcare providers to respond to intimate partner violence against women. Cochrane Database of Systematic Reviews 2017, Issue 2. [DOI: 10.1002/14651858.CD012423]

\section{Kasturirangan 2008}

Kasturirangan A. Empowerment and programs designed to address domestic violence. Violence against Women 2008;14:1465-75.
Kirk MA, Kelley C, Yankey N, Birken SA, Abadie B, Damschroder L. A systematic review of the use of the Consolidated Framework for Implementation Research. Implementation Science 2016;11:72-85. [DOI: 10.1186/ s13012-016-0437-z]

\section{Koss 1991}

Koss MP, Koss PG, Woodruff WJ. Deleterious effects of criminal victimization on women's health and medical utilization. Archives of Internal Medicine 1991;151(2):342-7. [PUBMED: 1992961]

\section{Kraemer 2002}

Kraemer HC, Wilson GT, Fairburn CG, Agras WS. Mediators and moderators of treatment effects in randomized clinical trials. Archives of General Psychiatry 2002;59(10):877-83. [PUBMED: 12365874]

\section{Kutin 2017}

Kutin J, Russell R, Reid M. Economic abuse between intimate partners in Australia: prevalence, health status, disability and financial stress. Australian and New Zealand Journal of Public Health 2017;41(3):269-74. [DOI: 10.1111/1753-6405.12651; PUBMED: 28245514]

\section{Lakey 2000}

Lakey B, Cohen S. Social Support and Theory. In: S. Cohen, L. G. Underwood, \& B. H. Gottlieb editor(s). Social Support Measurement and Intervention: A Guide for Health and Social Scientist. New York: Oxford University Press, 2000:29-52.

\section{Landenberger 1989}

Landenberger K. A process of entrapment in and recovery from an abusive relationship. Issues in Mental Health Nursing 1989;10:209-27.

\section{Laverack 2006}

Laverack G. Improving health outcomes through community empowerment: a review of the literature. Journal of Health, Population and Nutrition 2006;24(1):113-20.

\section{Lee 2015}

Lee FH, Yang YM, Wang HH, Huang JJ, Chang SC. Conditions and patterns of intimate partner violence among Taiwanese women. Asian Nursing Research 2015;9(2):91-5. [DOI: 10.1016/ j.anr.2015.05.004; PUBMED: 26160235]

\section{Leidner 1999}

Leidner R. Emotional labor in service work. Annals of the American Academy of Political and Social Science 1999;561(1):81-95.

\section{Lewin 2015}

Lewin S, Glenton C, Munthe-Kaas H, Carlsen B, Colvin CJ, Gülmezoglu M, et al. Using qualitative evidence in decision making for health and social interventions: an approach to assess confidence in findings from qualitative evidence syntheses (GRADE-CERQual). PLoS Medicine 2015;12(10):e1001895. [DOI: 10.1371/journal.pmed.1001895; PMC4624425; PUBMED: 26506244] 


\section{Liang 2002}

Liang B, Tracy A, Taylor CA, Williams LM, Jordan JV, Miller JB. The Relational Health Indices: a study of women's relationships. Psychology of Women Quarterly 2002;26(1):25-35. [DOI: 10.1111/1471-6402.00040]

\section{Lincoln 1985}

Lincoln YS, Guba EG. Naturalistic Inquiry. Newbury Park, CA, USA: Sage Publications, 1985.

\section{Maslow 1943}

Maslow AH. Conflict, frustration, and the theory of threat. Journal of Abnormal and Social Psychology 1943;38(1):81-6.

\section{Michie 2011}

Michie S, Van Stralen MM, West R. The behaviour change wheel: a new method for characterising and designing behaviour change interventions. Implementation Science 2011;6:42-53.

\section{Michie 2013}

Michie S, Richardson M, Johnston M, Abraham C, Francis J, Hardeman W, et al. The behavior change technique taxonomy (v1) of 93 hierarchically clustered techniques: building an international consensus for the reporting of behavior change interventions. Annals of Behavioral Medicine 2013;46(1):81-95.

\section{Monitor 2013}

Monitor. Closing the NHS funding gap: how to get better value healthcare for patients. www.gov.uk/government/ publications/closing-the-nhs-funding-gap-how-to-get-bettervalue-healthcare-for-patients (accessed 16 December 2017).

\section{Moos 1994}

Moos RH. Work Environment Scale manual. Work Environment Scale: Manual. 3rd Edition. Palo Alto, CA: Consulting Psychologists Press, 1994.

\section{Morgan 2013}

Morgan M, Coombes L. Empowerment and advocacy for domestic violence victims. Social and Personality Psychology Compass 2013;7/8:526-36.

\section{Mullen 1999}

Mullen PE, Pathé M, Purcell R, Stuart GW. Study of stalkers. American Journal of Psychiatry 1999;156(8):1244-9. [DOI: 10.1176/ajp.156.8.1244; PUBMED: 10450267]

\section{NCIPC 2003}

National Center for Injury Prevention and Control. Costs of Intimate Partner Violence Against Women in the United States. Atlanta (GA): Centers for Disease Control and Prevention, 2003. [www.cdc.gov/violenceprevention/pdf/IPVBook-a.pdf]

\section{NCRVAWC 2009}

National Council to Reduce Violence against Women and their Children. The Cost of Violence Against Women and their Children. Greenway, Canberra (AU): Department of Families, Housing, Community Services and Indigenous Affairs (FaHCSIA), 2009. [ISBN: 978-1-921380-31-0; www.dss.gov.au/sites/default/ files/documents/05_2012/vawc_economic_report.pdf]

\section{Netherlands Institute for Human Rights 2018}

Netherlands Institute for Human Rights. Written contribution to the Group of Experts on Action against Violence against Women and Domestic Violence. publicaties.mensenrechten.nl/ file/7c4685f9-6600-4445-bdc5-54d4876eb631.pdf (accessed prior to 27 May 2019).

\section{NICE 2008}

National Institute for Health and Clinical Excellence (NICE). Community engagement to improve health. London: National Institute for Health and Clinical Excellence (NICE), 2008.

\section{Nunnally 1994}

Nunnally JC, Bernstein IH. The assessment of reliability. Psychometric Theory. 3. New York (NY): McGraw-Hill, 1994:248-92.

\section{Nybergh 2013}

Nybergh L, Taft C, Enander V, Krantz G. Self-reported exposure to intimate partner violence among women and men in Sweden: results from a population-based survey. BMC Public Health 2013;13:845. [DOI: 10.1186/1471-2458-13-845; PMC3848440; PUBMED: 24034631]

\section{O'Doherty 2016}

O'Doherty LJ, Taft A, McNair R, Hegarty K. Fractured identity in the context of intimate partner violence: barriers to and opportunities for seeking help in health settings. Violence against Women 2016;22(2):225-48.

\section{ONS 2018}

Office for National Statistics. Statistical bulletin: crime in England and Wales: year ending December 2017. www.ons.gov.uk/peoplepopulationandcommunity/ crimeandjustice/bulletins/crimeinenglandandwales/ yearendingdecember2017 (accessed prior to 13 August 2018).

\section{Papoutsi 2017}

Papoutsi C, Mattick K, Pearson M, Brennan N, Briscoe S, Wong G. Social and professional influences on antimicrobial prescribing for doctors-in-training: a realist review. Journal of Antimicrobial Chemotherapy 2017;72(9):2418-30. [DOI: 10.1093/jac/dkx194; PMC5890780; PUBMED: 28859445]

\section{Parliamentary Counsel Office of New Zealand 2018}

Parliamentary Counsel Office of New Zealand. Family Violence (Amendments) Act 2018. legislation.govt.nz/act/ public/2018/0047/8.0/LMS113435.html (accessed prior to 27 May 2019).

\section{Pawson 2003}

Pawson R, Boaz A, Grayson L, Long A, Barnes C. Types and Quality of Knowledge in Social Care. London: Social Care Institute for Excellence, 2003. [www.scie.org.uk/publications/ knowledgereviews/kr03.asp]

\section{Pawson 2005}

Pawson R, Greenhalgh T, Harvey G, Walshe K. Realist review - a new method of systematic review designed for complex policy interventions. Journal of Health Services Research \& Policy 
2005;10(Suppl 1):21-34. [DOI: 10.1258/1355819054308530; PUBMED: 16053581]

\section{Pawson 2006}

Pawson R. Evidence-Based Policy: A Realist Perspective. London: Sage Publications Ltd, 2006. [ISBN: 1-4129-1059-5]

\section{Pawson 2009}

Pawson R, Tilley N. Realist evaluation. In: Otto H-U, Polutta A, Ziegler H editor(s). Evidence-based Practice: Modernising the Knowledge Base of Social Work?. Opladen \& Farmington Hills (MI): Barbara Budrich Publishers, 2009. [ISBN: 978-3-86649-121-2]

\section{Pearson 2015}

Pearson M, Chilton R, Wyatt K, Abraham C, Ford T, Woods H, et al. Implementing health promotion programmes in schools: a realist systematic review of research and experience in the United Kingdom. Implementation Science 2015;10:149. [DOI: 10.1186/s13012-015-0338-6; PMC4625879; PUBMED: 26510493]

\section{Phillips 2014}

Phillips J, Vandenbroek P. Domestic, Family and Sexual Violence in Australia: An Overview of the Issues. Canberra: Department of Parliamentary Services, 2014. [ISSN: 2203-5249; parlinfo.aph.gov.au/ parllnfo/download/library/prspub/3447585/upload_ binary/3447585.pdf;fileType=application/pdf]

\section{Plichta 2007}

Plichta SB. Interactions between victims of intimate partner violence against women and the health care system: policy and practice implications. Trauma, Violence \& Abuse 2007;8(2):226-39. [DOI: 10.1177/1524838007301220; PUBMED: $17545576]$

\section{Prochaska 1992}

Prochaska JO, DiClemente CC, Norcross JC. In search of how people change: applications to addictive behaviors. American Psychologist 1992;47(9):1102-14.

\section{Radloff 1977}

Radloff L. The CES-D scale: a self-report depression scale for research in the general population. Applied Psychological Measurement 1977;1:385-401.

\section{Ramsay 2005a}

Ramsay J, Rivas C, Feder G. Interventions to reduce violence and promote the physical and psychosocial well- being of women who experience partner violence: a systematic review of controlled evaluations. bit.ly/ 1geoXU6. London: University of London. Queen Mary's School of Medicine and Dentistry, (accessed 31 Jan 2019).

\section{Ramsay 2005b}

Ramsay J, Feder G, Rivas C, Carter Y, Davidson LL, Hegarty K, et al. Advocacy interventions to reduce or eliminate violence and promote the physical and psychosocial wellbeing of women who experience intimate partner abuse. Cochrane Database of Systematic Reviews 2005, Issue 1. [DOI: 10.1002/14651858.CD005043]

\section{Ramsay 2009}

Ramsay J, Carter Y, Davidson L, Dunne D, Eldridge S, Hegarty K, et al. Advocacy interventions to reduce or eliminate violence and promote the physical and psychosocial wellbeing of women who experience intimate partner abuse. Cochrane Database of Systematic Reviews 2009, Issue 3. [DOI: 10.1002/14651858.CD005043.pub2]

\section{Rappaport 1987}

Rappaport J. Terms of empowerment/exemplars of prevention: toward a theory for community psychology. American Journal of Community Psychology 1987;15:121-48.

\section{Rivas 2010}

Rivas C. "I don't feel like I'm in an abusive relationship": women's naming of their partner's abusive behaviours and how this may be affected by institutional definitions. Psychology of Women Section Review 2010;12(2):2-10.

\section{Rivas 2015}

Rivas C, Ramsay J, Sadowski L, Davidson LL, Dunne D, Eldridge $S$, et al. Advocacy interventions to reduce or eliminate violence and promote the physical and psychosocial wellbeing of women who experience intimate partner abuse. Cochrane Database of Systematic Reviews 2015, Issue 12. [DOI: 10.1002/14651858.CD005043.pub3]

\section{Rodriguez 2006}

Rodriguez MA, Saba G. Cultural competence and intimate partner abuse: health care interventions. In: Roberts G, Hegarty $\mathrm{K}$, Feder $\mathrm{G}$ editor(s). Intimate Partner Abuse and Health Professionals. New Approaches to Domestic Violence. London: Elsevier, 2006.

\section{Roe 2010}

Roe S. Intimate Violence: 2008/09 British Crime Survey. London (UK): Home Office, 2010.

\section{Roen 2006}

Roen K, Arai L, Roberts H, Popay J. Extending systematic reviews to include evidence on implementation: methodological work on a review of community-based initiatives to prevent injuries. Social Science \& Medicine 2006;63(4):1060-71. [DOI: 10.1016/ j.socscimed.2006.02.013; PUBMED: 16574289]

\section{Rogers 1997}

Rogers ES, Chamberlin J, Ellison ML, Crean T. A consumerconstructed scale to measure empowerment among users of mental health services. Psychiatric Services 1997;48(8):1042-7. [DOI: 10.1176/ps.48.8.1042; PUBMED: 9255837]

\section{Rosenberg 1965}

Rosenberg M. Society and the Adolescent Self-Image. Princeton, NJ: Princeton University Press, 1965.

\section{Ross 2012}

Ross AR. Impact of Psychoeducational Advocacy Training as Compared to Psychoeducational Support Group as an Empowering Tool for Female Survivors of Domestic Violence [Doctoral thesis]. New York (NY): Fordham University, Department Social Work, 2012.

A realist review of which advocacy interventions work for which abused women under what circumstances (Review) 


\section{Rycroft-Malone 2016}

Rycroft-Malone J, Burton CR, Williams L, Edwards S, Fisher D, Hall B, et al. Improving skills and care standards in the support workforce for older people: a realist synthesis of workforce development intervention. Health Services and Delivery Research 2016;4(12):6:e011964. [DOI: 10.3310/hsdr04120]

\section{Sen 1985}

Sen A. Commodities and Capabilities. New York: Elsevier Science, 1985.

\section{Shalaunsky 1999}

Shalaunsky C, Ericksen J, Henderson A. Abused women and child custody: the ongoing exposure to abusive ex-partners. Journal of Advanced Nursing 1999;29(2):416-26. [PUBMED: 10197942]

\section{Signorelli 2012}

Signorelli MC, Taft A, Gomes Pereira PP. Intimate partner violence against women and healthcare in Australia: charting the scene. Ciência \& Saúde Coletiva 2012;17(4):1037-48. [DOI: 10.1590/S1413-81232012000400025; www.scielo.br/scielo.php? script=sci_arttext\&pid=S1413-81232012000400025]

\section{Smith 2012}

Smith JD. Single-case experimental designs: a systematic review of published research and current standards. Psychological Methods 2012;17(4):510-50.

\section{Snyder 2002}

Snyder CR. Hope theory: rainbows in the mind. Psychological Inquiry 2002;13(4):249-75.

\section{Stamm 2005}

Stamm BH. ProQOL R-IV: Professional Quality of Life. Compassion Satisfaction and Fatigue Subscales, Revision IV. tinyurl.com/y3dkghy3 (accessed 20 June 2019).

\section{Stark 2007}

Stark E. Interpersonal Violence. Coercive Control: How Men Entrap Women in Personal Life. New York (NY): NUS: Oxford University Press, 2007.

\section{Straus 2004}

Straus MA, Douglas EM. A short form of the Revised Conflict Tactics Scales, and typologies for severity and mutuality. Violence and Victims 2004;19:507-20.

\section{Sullivan 2006}

Sullivan CM. Evaluating parenting programs for men who batter: current considerations and controversies. In: Edleson JL, Williams $\mathrm{O}$ editor(s). Parenting by Men Who Batter Women: New Directions for Assessment and Intervention. New York (NY): Guilford Press, 2006.

\section{Tervalon 1998}

Tervalon M, Murray-García J. Cultural humility versus cultural competence: a critical distinction in defining physician training outcomes in multicultural education. Journal of Healthcare for the Poor and Underserved 1998;9(2):117-25.

\section{Thiara 2016}

Thiara RK, Harrison C. Safe Not Worry: Supporting the Campaign for Safer Child Contact: University of Warwick. Bristol: Women's Aid, 2016.

\section{Thomas 2008}

Thomas J, Harden A. Methods for the thematic synthesis of qualitative research in systematic reviews. BMC Medical Research Methodology 2008;8:45. [DOI: 10.1186/1471-2288-8-45; PMC2478656; PUBMED: 18616818]

\section{Thomas 2010 [Computer program]}

Thomas J, Brunton J, Graziosi S. EPPI-Reviewer 4: software for research synthesis. Version accessed 30 April 2019. London: Social Science Research Unit, UCL Institute of Education, 2010.

\section{Tirado-Muñoz 2014}

Tirado-Muñoz J, Gilchrist G, Farré M, Hegarty M, Torrens M. The efficacy of cognitive behavioural therapy and advocacy interventions for women who have experienced intimate partner violence: a systematic review and metaanalysis. Annals of Medicine 2014;46(8):567-86. [DOI: 10.3109/07853890.2014.941918; PUBMED: 25211469]

\section{Towers 2012}

Towers J, Walby S. Measuring the Impact of Cuts in Public Expenditure on the Provision of Services to Prevent Violence Against Women and Girls. London: Northern Rock Foundation and Trust for London, 2012.

\section{US Office on Violence Against Women 2018}

Office on Violence Against Women. Domestic violence. www.justice.gov/ovw/domestic-violence (accessed prior to 13 August 2018).

\section{Uzun 2015}

Uzun GO, Uzunboylu $\mathrm{H}$. A survey regarding of domestic violence against women. Procedia - Social and Behavioral Sciences 2015;190:24-31. [DOI: 10.1016/j.sbspro.2015.04.911]

\section{Vigurs 2016}

Vigurs CA, Schucan Bird K, Quy K, Gough D. The Impact of Domestic Violence Perpetrator Programmes on Victim and Criminal Justice Outcomes: A Systematic Review of Reviews of Research Evidence. Coventry: College of Policing, 2016.

\section{Walby 2009}

Walby S. The cost of domestic violence: up-date 2009. www.google.co.uk/url? $\mathrm{sa}=\mathrm{t} \& \mathrm{rct}=\mathrm{j} \& \mathrm{q}=\& \mathrm{~s} r \mathrm{c}=\mathrm{s} \&$ source=web\&cd=2\&ved=0CDMQFjAB\&url=http:// www.lancaster.ac.uk/fass/doc_library/ sociology/Cost_of_domestic_violence_ update.doc\&ei=qKFVU7GwHoug7AbWl4DoDg\&usg=AFQjCNG0joG5UNkUYh (accessed 16 December 2017).

\section{Walker 1979}

Walker LE. The Battered Woman. New York: Harper \& Row, 1979. 


\section{Walker 2018}

Walker AK, Fox EL. Why marginalization, not vulnerability, can best Identify people in need of special medical and nutrition care. AMA Journal of Ethics 2018;20(10):E941-947.

\section{Wallerstein 2006}

Wallerstein N. What is the Evidence on Effectiveness of Empowerment to Improve Health? Report for the Health Evidence Network (HEN). Copenhagen: World Health Organization, 2006.

\section{Ware 1997}

Ware JEJ, Snow KK, Kosinski M, Gandek B. SF-36 Health Survey Manual and Interpretation Guide. Boston, MA: The Health Institute, New England Medical Center, 1997.

\section{Warshaw 2003}

Warshaw C, Gugenheim AM, Moroney G, Barnes H. Fragmented services, unmet needs: building collaboration between the mental health and domestic violence communities. Health Affairs 2003;22(5):230-4.

\section{Watts 2002}

Watts C, Zimmerman C. Violence against women: global scope and magnitude. Lancet 2002;359(9313):1232-7. [DOI: 10.1016/ S0140-6736(02)08221-1; PUBMED: 11955557]

\section{Weiss 2007}

Weiss DS. The Impact of Event Scale: Revised. In: Wilson JP, Tang CS-K editor(s). International and Cultural Psychology. Cross-Cultural Assessment of Psychological Trauma and PTSD. New York, NY: Springer Science + Business Media, 2007:219-38. [DOI: 10.1007/978-0-387-70990-1_10]

\section{Westhorp 2018}

Westhorp G. Understanding mechanisms in realist evaluation and research. In: Emmel N, Greenhalgh J, Manzano A, Monaghan M, Dalkin S editor(s). Doing Realist Research. London (UK): SAGE Publications Ltd, 2018:41-57.

\section{WHO 2013a}

World Health Organization, London School of Hygiene, Tropical Medicine. South African Medical Research Council. Global and Regional Estimates of Violence Against Women: Prevalence and Health Effects of Intimate Partner Violence and Non-Partner Sexual Violence. Geneva: WHO, 2013. [ISBN: 978-92-4-156462-5; apps.who.int/iris/bitstream/ handle/10665/85239/9789241564625_eng.pdf?sequence=1]

\section{WHO 2013b}

World Health Organization. Responding to Intimate Partner Violence and Sexual Violence Against Women: WHO Clinical and Policy Guidelines. Geneva: World Health Organization, 2013. [ISBN: 978-92-4-154859-5; apps.who.int/iris/bitstream/ handle/10665/85240/9789241548595_eng.pdf?sequence=1]

\section{WHO 2013c}

World Health Organization. Responding to intimate partner violence and sexual violence against women: WHO clinical and policy guidelines. bit.ly/1wkpds6. Geneva: WHO, (accessed 31 January 2019).

\section{Williamson 2017}

Williamson PR, Altman DG, Bagley H, Barnes KL, Blazeby JM, Brookes ST, et al. The COMET Handbook: version 1.0. Trials 2017;18(Suppl 3):280. [DOI: 10.1186/s13063-017-1978-4; PMC5499094; PUBMED: 28681707]

\section{Wilson 1993}

Wilson M, Daly M. Spousal homicide risk and estrangement. Violence and Victims 1993;8(1):3-16. [8292563]

\section{Women's Rights Division of Human Rights Watch 2000}

Women's Rights Division of Human Rights Watch. Peru: law of protection from family violence. www.hrw.org/ news/2000/03/31/peru-law-protection-family-violence (accessed 29 April 2019).

\section{Wong 2012}

Wong G, Greenhalgh T, Westhorp G, Pawson R. Realist methods in medical education research: what are they and what can they contribute?. Medical Education 2012;46(1):89-96. [DOI: 10.1111/ j.1365-2923.2011.04045.x; PUBMED: 22150200]

\section{Wong 2013a}

Wong G, Greenhalgh T, Westhorp G, Buckingham J, Pawson R. RAMESES publication standards: realist syntheses. $B M C$ Medicine 2013;11:21. [DOI: 10.1186/1741-7015-11-21; PMC3558331; PUBMED: 23360677]

\section{Wong 2013b}

Wong G, Westhorp G, Pawson R, Greenhalgh T. Realist Synthesis: RAMESES Training Materials. London (UK): The RAMESES Projects, 2013. [www.ramesesproject.org/media/Realist_ reviews_training_materials.pdf]

\section{Wong 2017}

Wong G, Westhorp G, Greenhalgh J, Manzano A, Jagosh J, Greenhalgh T. Quality and Reporting Standards, Resources, Training Materials and Information for Realist Evaluation: The RAMESES II Project. Health Service and Delivery Research, No. 5.28. Southampton (UK): NIHR Journal Library, 2017. [ISSN: 2050-4349; www.ncbi.nlm.nih.gov/books/NBK459059/]

\section{Wood 2015}

Wood L. Hoping, empowering, strengthening: theories used in intimate partner violence advocacy. Journal of Women and Social Work 2015;30(3):286-301. [DOI: 10.1177/0886109914563157; journals.sagepub.com/doi/ pdf/10.1177/0886109914563157]

\section{Wrigley 2016}

Wrigley A, Dawson A. Vulnerability and marginalized populations. In: Barrett DH, Ortmann LH, Dawson A, Saenz C, Reis A, Bolan G editor(s). Chapter 7: Public Health Ethics: Cases Spanning the Globe. Cham (CH): Springer, 2016.

\section{Zink 2004}

Zink T, Jacobson C, Regan S, Fisher B, Pabst S. Hidden victims: the healthcare needs and experiences of older women in abusive relationships. Journal of Women's Health 2004;13(8):898-908.

A realist review of which advocacy interventions work for which abused women under what circumstances (Review) 


\section{References to other published versions of this review}

\section{Rivas 2018}

Rivas C, Vigurs C. A realist review of which advocacy interventions work for which abused women under what

\section{CHARACTERISTICS OF STUDIES}

Characteristics of included studies [ordered by study ID]

Babin 2012

\begin{tabular}{ll}
\hline Methods & -Online survey \\
-Self-reported measures \\
-6-point item scale
\end{tabular}

Participants

Country: USA

Setting: part of larger study examining communication practices at a DV agency that serves women and dependent children $<18$ years of age

Inclusion criteria: staff, volunteers and board members at the DV agency

Exclusion criteria: NS

Participant numbers: 69

Experience: NS

Education and training: bachelor's degree $(n=28,41 \%)$

Age: 35.76 years $(S D=11.85$, range $=21-60)$

Ethnicity: $71 \%$ = white, non-Hispanic, $18 \%$ = white, Hispanic, $2 \%=$ African American, $2 \%=$ Asian, $2 \%$ Native American or Alaskan Native, and $4 \%=$ "Other"

\begin{tabular}{|c|c|c|}
\hline Interventions & \multicolumn{2}{|c|}{$\begin{array}{l}\text { Intervention: no specific intervention } \\
\text { Controls: not relevant }\end{array}$} \\
\hline Notes & \multicolumn{2}{|c|}{ Examined burnout among DV advocates from communication perspective } \\
\hline \multicolumn{3}{|l|}{ Risk of bias } \\
\hline Bias & Authors' judgement & Support for judgement \\
\hline $\begin{array}{l}\text { How valuable is the re- } \\
\text { search (richness of the da- } \\
\text { ta for the review research } \\
\text { questions)? }\end{array}$ & Unclear risk & Limited perspective \\
\hline $\begin{array}{l}\text { Representativeness of the } \\
\text { sample }\end{array}$ & High risk & $\begin{array}{l}\text { One agency, no details specified. Participants were recruited either in person } \\
\text { at a staff meeting or via email. The sample contained only DV advocates asso- } \\
\text { ciated with a DV agency and did not contain advocates who work with victims } \\
\text { in other settings (e.g. justice system) who might be more or less vulnerable to } \\
\text { burnout responses due to system structures or constraints }\end{array}$ \\
\hline Adequacy of response rate & Unclear risk & NS but small sample acknowledged \\
\hline $\begin{array}{l}\text { Missing data within com- } \\
\text { pleted questionnaires }\end{array}$ & Unclear risk & NS \\
\hline Conduct of pilot testing & Low risk & NS, validated instruments used \\
\hline $\begin{array}{l}\text { Established validity of the } \\
\text { survey instrument }\end{array}$ & Low risk & Validated instruments used \\
\hline
\end{tabular}

A realist review of which advocacy interventions work for which abused women under what circumstances (Review) 
Bacchus 2007

$\begin{array}{ll}\text { Methods } & \text { 2-year evaluation } \\ & \text { - Interview, focus groups the main focus, with case review also as a single-group, pre-post design }\end{array}$

Country: UK
Participants
Inclusion criteria: women were selected according to clinical setting, living with abuser or not at the
time of referral advocacy, length of abuse, pregnant or not, immigration status, access to their own
money, ethnic origin and first language (English or Spanish). Staff participants were selected according
to clinical setting (maternity or sexual health), professional group (doctors, nurses, midwives, health
advisors), gender, and time elapsed since training ( 3 months vs $6+$ months)
Exclusion criteria: NS
Participant numbers: 34 women (1-22 months after they received support from the service). 3 hos-
pital staff who used the advocacy service were amongst those interviewed. Staff interviewed $=8$ mid-
wives and 11 sexual health professionals. 6 focus group discussions (19= midwives and $6=$ sexual
health professionals); maternity records were reviewed for 487 (98\%) of the 501 women who gave birth
in January 2007 . A total of 915 patients attended the sexual health clinic in a 1 -month assessment pe-
riod. However, of the 879 records available for audit, only 644 (73\%) contained the DV code and were
analysed.

Interventions NS. Main intervention referred women to an on-site advocacy service. Post-advocacy data reported but advocacy not described

Notes

Other aspects of study not relevant to this review but included staff interviews, analysis of patient records, and staff pre/post-training questionnaires

Potential and actual harm occurred, including breaches of confidentiality and failure to document evidence, limiting women's ability to access civil and legal remedies

\section{Risk of bias}

Bias Authors' judgement Support for judgement

How valuable is the re- Low risk

search (richness of the da-

ta for the review research

questions)?

Was there a clear state- Low risk

ment of the aims of the re-

search?

\section{Was the research design Low risk}

appropriate to address the

aims of the research?

Was the recruitment strat- Low risk

egy appropriate to the

aims of the research?

Is a qualitative methodol- Low risk

ogy appropriate?

Have ethical issues been Low risk

taken into consideration? 
Bacchus 2007 (Continued)

Were the data collected in

Low risk a way that addressed the research issue?

Was the data analysis suf- Low risk

ficiently rigorous?

Is there a clear statement Low risk

of findings?

\section{Bacchus 2016a}

Methods - Assessor-blinded, multisite RCT of 'DOVE' intervention with sample size calculation, and

- Nested qualitative study: interviews and nonparticipant observation

Country: USA
Setting: prenatal home visits in urban/rural setting
Inclusion criteria: English-speaking pregnant women aged $\geq 14$ years, low income (i.e. Medicaid eli-
gible), $<32$ weeks' gestation, experiencing perinatal IPV by a current or past partner, and enrolled in a
perinatal home visiting programme of a participating agency were eligible for study participation.

Participant numbers: participating agencies referred 689 women, and 352 women were ineligible due to gestational age $>31$ weeks or no IPV

Of the 337 eligible women, 64 women refused the study mostly due to lack of time or a preference to maintain privacy regarding their IPV status.

Thus, 239 women were randomised to DOVE $(n=124)$ or usual care $(n=115)$ and completed the baseline assessment.

Exclusion criteria: NS

Ethnicity: African American DOVE (54); usual care (59); white DOVE (55); usual care (46); other DOVE (14); usual care (10)

Age: (mean) 24 (DOVE); 23 (usual care)

Abuse: physical assault: IPV abuse status from screening IPV in year before current pregnancy

Participants nested qualitative study (Bacchus 2016a):

Numbers; 26 low-income, high-risk mothers

Ethnicity: white $=12$ (46\%); African/African American/black = 8 (31\%); mixed ethnic origin = $4(15 \%)$; not reported $=2(8 \%)$

Age: between 25 and 66 years

Abuse: physical assault: IPV abuse from screening IPV in year before current pregnancy =69\%; no IPV in year before current pregnancy $=31 \%$

Children: $17 \%$ already had children, but all were pregnant

Relationship status: married $=4 \%$; single $=65 \%$; partnered, not married $=31 \%$

- DOVE is a structured brochure-based IPV empowerment intervention based on the March of Dimes Protocol for prevention of battering during pregnancy

- DOVE intervention was delivered 6 times within regularly scheduled home visits, 3 sessions occurring during pregnancy and 3 during the postpartum period. 
Bacchus 2016a (Continued)

- 15-25 min average session time

Controls: all women received the usual care of the home-visiting programme, which, on average, included $4-6$ visits prenatally and 6-12 visits up to 2 years postpartum.

Timing of follow-up: $1,3,6,12,18$, and 24 months postpartum

Notes

All home-visiting programmes targeted low-income, high-risk mothers such as single, young mothers or families with low birth weight or preterm infants who are presumed to be at highest risk for poor physical and behavioural child outcomes

\section{Risk of bias}

\begin{tabular}{lll}
\hline Bias & Authors' judgement & Support for judgement \\
\hline $\begin{array}{l}\text { Random sequence genera- } \\
\text { tion (selection bias) }\end{array}$ & Low risk & $\begin{array}{l}\text { The urban site randomised participants using computer-generated number } \\
\text { assignments in blocks. The rural sites used cluster randomisation because } \\
\text { each health agency was small enough that intervention contamination was } \\
\text { likely if women were the unit of randomisation }\end{array}$
\end{tabular}

\begin{tabular}{|c|c|c|}
\hline $\begin{array}{l}\text { Allocation concealment } \\
\text { (selection bias) }\end{array}$ & Low risk & $\begin{array}{l}\text { The data managers, database development team, and statistical analysis team } \\
\text { members were blinded to group assignment }\end{array}$ \\
\hline
\end{tabular}

Blinding (performance
bias and detection bias):

Incomplete outcome da- Low risk ta (attrition bias): all outcomes

\begin{abstract}
For the overall study, retention rates ranged from $70 \%-90 \%$ between each time point, with missing data ranging from $9 \%-30 \%$ across all time points, mostly due to attrition. More rural than urban women remained in the study at 3 months w2 $=3.95, \mathrm{P}<0.05 ; 18$ months $\mathrm{w} 2=5.33, \mathrm{P}<0.05$; and 24 months $\mathrm{w} 2$ $=10.66, \mathrm{P}<0.01$. There were significantly higher mean physical abuse scores for women who did not remain in the study at 6 months $(T=2.02, P<0.05)$ and a similar although non-significant difference at 12 months $(T=1.74, \mathrm{P}<$ $0.10)$. Women who remained in the study were less likely to have the father of the baby in the house at 3 months ( $w 2=4.12, P<0.05$ ) with non significant trends at 1 and 6 months. In all $22.6 \%$ of women did not complete their 18 or 24-month assessments due to recruitment being extended beyond the initial time frame and staff running out of capacity. These women ( $n=33$ urban; $\mathrm{n}=21$ rural) were compared to the larger group as well as other women who did not complete all study measures. No significant differences across groups were found on baseline study variables of interest (all $P>0.05$ ). Missing data were addressed using multiple imputation procedures, producing 100 fully imputed datasets used in all hypothesis- testing models
\end{abstract}

Selective reporting (re- Low risk
porting bias)

\begin{tabular}{lll}
\hline $\begin{array}{l}\text { Baseline measurement of } \\
\text { outcome measures }\end{array}$ & Low risk & Minimal differences in baseline demographics \\
\hline $\begin{array}{l}\text { Reliability of outcome } \\
\text { measures }\end{array}$ & Low risk & \\
\hline $\begin{array}{l}\text { Protection against conta- } \\
\text { mination }\end{array}$ & Low risk & $\begin{array}{l}\text { Rural sites used cluster randomisation because each health agency was small } \\
\text { enough that intervention contamination was likely if women were the unit of } \\
\text { randomisation }\end{array}$ \\
\hline
\end{tabular}

$\begin{array}{lll}\text { How valuable is the re- } & \text { High risk } & \text { High value } \\ \text { search (richness of the da- } & \end{array}$
search (richness of the da-

A realist review of which advocacy interventions work for which abused women under what circumstances (Review) 
Bacchus 2016a (Continued)

ta for the review research

questions)?

Bacchus 2016b

Methods $\quad$ - Nested qualitative interpretive study to explore perinatal home visitors' and women's perceptions and experiences of the DOVE programme using mHealth technology

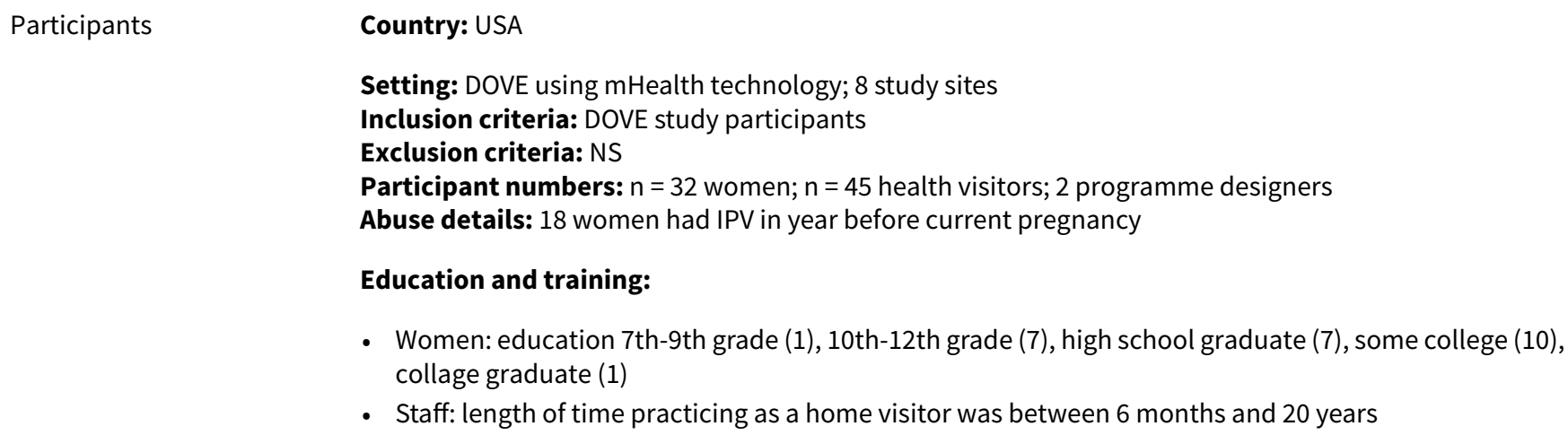

Participants Country: USA

Setting: DOVE using mHealth technology; 8 study sites

Inclusion criteria: DOVE study participants

Exclusion criteria: NS

Participant numbers: $\mathrm{n}=32$ women; $\mathrm{n}=45$ health visitors; 2 programme designers

Abuse details: 18 women had IPV in year before current pregnancy

\section{Education and training:}

- Women: education 7th-9th grade (1), 10th-12th grade (7), high school graduate (7), some college (10), collage graduate (1)

- Staff: length of time practicing as a home visitor was between 6 months and 20 years

Age of women: 16-19 (4), 20-23 (11), 24-27 (7), 28-35 (4)

Ethnicity of women: 23 English; 3 Spanish

Children: all were pregnant

\begin{tabular}{ll}
\hline Interventions & DOVE with mHealth (tablets with support information) \\
\hline Notes & $\begin{array}{l}\text { Part of an RCT but this paper is focused on mhealth rather than advocacy. Although DOVE is an inter- } \\
\text { vention to prevent IPV during pregnancy in women who have screened positive for DV in the year be- } \\
\text { fore the current pregnancy, 31\% of the sample studied had not experienced DV over the last year. }\end{array}$
\end{tabular}

\title{
Risk of bias
}

Bias Authors' judgement Support for judgement

How valuable is the re-

Low risk

search (richness of the da-

ta for the review research

questions)?

\begin{abstract}
Was there a clear state-
ment of the aims of the re-

search?
\end{abstract}

Low risk

Was the research design Low risk

appropriate to address the

aims of the research?

\footnotetext{
Was the recruitment strat- Low risk

egy appropriate to the

aims of the research?
Is a qualitative methodol- Low risk ogy appropriate?


Bacchus 2016b (Continued)

Were the data collected in

Low risk a way that addressed the research issue?

Was the data analysis suf- Low risk

ficiently rigorous?

Is there a clear statement Low risk

of findings?

Bader 2014

Methods

Semi-structured interviews on the barriers and challenges experienced by women in their attempt to access and receive services following community protocol implementation

\section{Participants}

\section{Country: Canada}

Setting: sexual assault and DV services; $96 \%$ of participants lived in the city of Guelph $(n=25)$ and $4 \%$ lived in the county $(n=1)$

Inclusion criteria: women service users, aged $>18$, with their first contact with counselling agencies, the police, shelters, the courts and/or other social services since 2010 for sexual assault or DV

Exclusion criteria: family violence

Participant numbers: 33 interviews, 26 analysed (saturation of themes)

Education: $4 \%(n=1)$ had < high school, $15 \%(n=4)$ completed some high school, $12 \%(n=3)$ completed high school, $19 \%(n=5)$ completed some college and/or university, $23 \%(n=6)$ received a college diploma, $23 \%$ had a university undergraduate degree $(n=6)$, and $4 \%(n=1)$ had a university graduate degree

Age: $61 \%$ of service users were aged $35-54$ years $(n=16), 11 \%(n=3)$ were $18-24,8 \%(n=2)$ were $25-34$, $15 \%(n=4)$ were $55-64$ and $4 \%(n=1)$ was $65-74$

Ethnicity: 24 were born in Canada, and 2 were born outside of Canada. 25 service users spoke English as their first language and 1 participant spoke English and Lithuanian. The majority (69\%) of service users identified as "White" ( $n=18), 8 \%$ identified as Aboriginal $(n=2), 8 \%$ were mixed race $(n=2), 4 \%$ identified as South Asian ( $n=1), 8 \%$ identified as other $(n=2)$

Relationship status: nearly half (46\%) of service users were separated/divorced ( $n=12), 27 \%(n=7)$ were single, $27 \%(n=7)$ were married/common-law

Children: $72 \%$ of service users had children $(n=18)$, while $28 \%$ did not have children $(n=7)$. Children's ages ranged from 7 months- 42 years

Socioeconomic status indicators: $79 \%$ of service users were low-income and reported a total annual income of $\angle$ CAD 20,000, 8\% reported an annual income of CAD 20,000-39,000, $8 \%$ had an annual income of CAD 40,000-59,000 and 4\% reported an annual income between CAD 60,000-79,000

Abuse: the majority of the women interviewed experienced DV, encompassing physical, emotional, psychological, sexual, financial and spiritual violence

Notes Not advocacy per se but useful information

\section{Risk of bias}

Bias Authors' judgement Support for judgement




\section{Bader 2014 (Continued)}

How valuable is the re-

Low risk search (richness of the da-

ta for the review research questions)?

\section{Was there a clear state- Low risk} ment of the aims of the research?

\section{Was the research design appropriate to address the aims of the research?}

Low risk

Was the recruitment strat- Low risk

egy appropriate to the

aims of the research?
The research team developed an exclusion criteria for specific contacts between a woman and service provider. There were some situations in which it was unclear if the relationship between the woman and service provider was a service user/service provider relationship and these women were excluded. Reflexivity also used.

\section{Is a qualitative methodol- Low risk ogy appropriate?}

\section{Were the data collected in Low risk} a way that addressed the research issue?

\section{Is there a clear statement Low risk} of findings?

\section{Bahadir-Yilmaz 2018}

$\begin{array}{ll}\text { Methods } & \begin{array}{l}\text { Experimental RCT with a pre-test, post-test control group, and follow-up, with random allocation to } \\ \text { groups }\end{array}\end{array}$

\section{Participants Country: Turkey}

Setting: family counselling centre at Giresun Maternity Hospital

Participants: $60(30=$ intervention, $30=$ control $)$

Inclusion criteria: included:

- being exposed to physical, psychological, and economic violence

- being aged 18-65 years

- being literate

Exclusion criteria: included:

- being illiterate

- not participating in 2 intervention sessions

- having hearing or perceptual problems

- having mental diseases 
Bahadir-Yilmaz 2018 (Continued)

Employment: unemployed control 73.3\% intervention 83.3\%

\section{Abuse details}

- Economic violence: intervention $86.7 \%$; control $70 \%$

- Physical violence: intervention $100 \%$; control $86.7 \%$

- Psychological violence: intervention 100\%; control $100 \%$

- Duration of violence: extended intervention 30\%; control 33.3\%; < 5 years: intervention $10 \%$; control $30 \%$

Age: mean age of the women in the intervention group was $40.7 \pm 11.0$, mean age of the women in the control group was $34.6 \pm 11.4$

Ethnicity: NS

Children: $(\mathrm{M} \pm \mathrm{SD})$ intervention $2.2 \pm 1.3$; control $1.7 \pm 1.2$

Socioecomomic status indicators: NS

Relationship status: living with abuser

Education: primary, intervention 50\%, control 76\%; high school, intervention $46.7 \%$, control 20\%; university, intervention $3.3 \%$, control $3.3 \%$

- empowerment programme was based on individual counselling and consisted of 10 sessions

- each session lasted for 90 min once a week, and it was essential that each woman participated in all sessions

- the sessions were carried out by first study author, with advanced education in psychiatric nursing

\section{Content of the intervention:}

- developing self-awareness

- increasing self-esteem

- decreasing learned helplessness

- education

- resources

- learned resourcefulness or self-efficacy

- managing and coping with violence

Controls: NS

Notes Only intervention group was analysed

\section{Risk of bias}

\begin{tabular}{lll}
\hline Bias & Authors' judgement & Support for judgement \\
\hline $\begin{array}{l}\text { Random sequence genera- } \\
\text { tion (selection bias) }\end{array}$ & Unclear risk & $\begin{array}{l}\text { Study authors say randomly allocated by participant characteristics such as } \\
\text { marriage age, educational status etc. Generation methods not stated }\end{array}$ \\
\hline $\begin{array}{l}\text { Allocation concealment } \\
\text { (selection bias) }\end{array}$ & Low risk & Women randomly assigned \\
\hline $\begin{array}{l}\text { Selective reporting (re- } \\
\text { porting bias) }\end{array}$ & High risk & Controls not reported \\
\hline $\begin{array}{l}\text { Baseline measurement of } \\
\text { outcome measures }\end{array}$ & Unclear risk & No significant differences between the groups \\
\hline
\end{tabular}


Bahadir-Yilmaz 2018 (Continued)

Reliability of outcome Low risk
measures

Protection against conta- Unclear risk mination

Not clear if the control and intervention were sampled from the 2 centres or altogether (it appears together in the flow diagram). If altogether, women from the 2 groups may have encountered each other

How valuable is the re- High risk Meant to be high value
search (richness of the da-
ta for the review research
questions)?

Bell 2001

\begin{tabular}{ll}
\hline Methods & Experiment with non-random allocation to groups \\
\hline Participants & Country: USA \\
& Setting: court-based advocacy services \\
& Inclusion criteria: 18 -year-old women seeking temporary restraining orders at the DV intake centre \\
& with low income \\
& Exclusion criteria: women with private legal support, women with substance use, women with sup- \\
& port from other DV services, women who had initiated the violence and women without a telephone \\
& Participant numbers: $81 / 157$ invited; $n=81$ baseline (22 advocacy; 59 comparison); $n=57$ follow-up \\
& (21 advocacy; 36 comparison)
\end{tabular}

Age: average 30 (range 19-50)

Ethnicity: 93\% African American

Employment status: 42\% employed full time, 26\% employed part-time 31\% unemployed and looking for work, $25 \%$ receiving some kind of public assistance

Socioecomomic status indicators: personal incomes below poverty guidelines of approximately USD 18,670/year for 1 adult, USD 21,700/year for 1 adult with 1 child, USD 27,300 for 1 adult and 2 children; $91 \%$ earned < USD 20,000 (low income was an eligibility criteria)

Children: $52 \%$ had children

Abuse: $75 \%$ never previously tried to obtain a protective order; length of time of abusive relationship, average 4.5 years

Education: 63\% formal education; 32\% schooling at college level

Relationship status: ex boyfriend $40.4 \%$, boyfriend $36.8 \%$, married $15.8 \%$, separated $7 \%$; $68.4 \%$ of participants had contact with abuser during the study

Interventions

Legal advocacy programmes in which law students worked with women to obtain Civil Protective Orders (CPO)

\section{Content of the intervention:}

- conversation by phone

- in-person meetings

- emotional support

- prepare evidence for court

- referrals/connection to community agencies

- safety planning 
Bell 2001 (Continued)

- supervision of people delivering advocacy

- talked to family and friends on their behalf

- provide information about DV

- provided transportation

Women had unlimited access to the advocate in person/by phone for the approximately 6 weeks it took for the CPO

Notes

Women in the intervention group had already been selected for usual care advocacy intervention at the intake centres; those in the control groups had not yet been screened for this

\section{Risk of bias}

\begin{tabular}{|c|c|c|}
\hline Bias & Authors' judgement & Support for judgement \\
\hline $\begin{array}{l}\text { Random sequence genera- } \\
\text { tion (selection bias) }\end{array}$ & High risk & $\begin{array}{l}\text { Non-randomisation; recruiting people in court lobbies, and waiting areas may } \\
\text { have been a little insensitive to their immediate context }\end{array}$ \\
\hline $\begin{array}{l}\text { Allocation concealment } \\
\text { (selection bias) }\end{array}$ & Unclear risk & $\begin{array}{l}\text { Participants were selected for either the control or advocacy based on eligibil- } \\
\text { ity }\end{array}$ \\
\hline $\begin{array}{l}\text { Blinding (performance } \\
\text { bias and detection bias): } \\
\text { all outcomes }\end{array}$ & High risk & \\
\hline $\begin{array}{l}\text { Incomplete outcome da- } \\
\text { ta (attrition bias): all out- } \\
\text { comes }\end{array}$ & High risk & $\begin{array}{l}81 \text { recruited and measured at baseline. } 57 \text { with follow-up data. There were sig- } \\
\text { nificant differences between the dropouts and completers in the advocacy } \\
\text { condition likely to affect the outcomes }\end{array}$ \\
\hline $\begin{array}{l}\text { Selective reporting (re- } \\
\text { porting bias) }\end{array}$ & Low risk & \\
\hline $\begin{array}{l}\text { Baseline measurement of } \\
\text { outcome measures }\end{array}$ & Low risk & Groups comparable at baseline \\
\hline $\begin{array}{l}\text { Reliability of outcome } \\
\text { measures }\end{array}$ & Low risk & \\
\hline $\begin{array}{l}\text { Protection against conta- } \\
\text { mination }\end{array}$ & Low risk & $\begin{array}{l}2 \text { different groups; participants unlikely to have met each other during the } \\
\text { course of the programme }\end{array}$ \\
\hline $\begin{array}{l}\text { How valuable is the re- } \\
\text { search (richness of the da- } \\
\text { ta for the review research } \\
\text { questions)? }\end{array}$ & Low risk & Low value \\
\hline
\end{tabular}

Bemiller 2010

\begin{tabular}{ll}
\hline Methods & $\begin{array}{l}\text { Survey to better understand agency challenges and concerns among advocates in a state in the Mid- } \\
\text { west USA }\end{array}$
\end{tabular}

\section{Participants}

\section{Country: USA}

\section{Setting:}

- survey was mailed to the 30 domestic and sexual assault shelters in the state 
Bemiller 2010 (Continued)

- these agencies employed 420 advocates who served both sexual assault and DV victims

Inclusion criteria: NS

Exclusion criteria: NS

Participant numbers: $n=194$; out of 420 surveys, 194 surveys were completed, resulting in a $46 \%$ re-

sponse rate

Education and training: $45 \%$ bachelor's degree

Age: average age 38 years

Ethnicity: $81 \%$ white

\begin{tabular}{ll}
\hline Interventions & DV and sexual assault shelter advocacy NS \\
\hline Notes & $\begin{array}{l}\text { Personal experience with victimisation (self or others): yes 51.5\%; no 48.5\% } \\
\text { Although this study was not designed to elicit in-depth qualitative data, respondents were provided } \\
\text { the opportunity to offer open-ended comments. }\end{array}$ \\
\end{tabular}

\section{Risk of bias}

\begin{tabular}{lll}
\hline Bias & Authors' judgement & Support for judgement \\
\hline $\begin{array}{l}\text { How valuable is the re- } \\
\text { search (richness of the da- } \\
\text { ta for the review research } \\
\text { questions)? }\end{array}$ & High risk & \\
\hline Adequacy of response rate & Low risk & \\
\hline Conduct of pilot testing & High risk & Questions not validated \\
\hline
\end{tabular}

Established validity of the High risk

survey instrument

Was there a clear state-

High risk

ment of the aims of the re-

search?

\section{Briones-Vozmediano 2014}

\begin{tabular}{ll}
\hline Methods & Qualitative 1-1 interviews and focus groups \\
\hline Participants & Country: Spain \\
& Setting: public institutions and NGOs \\
& Participants numbers: 29 in-depth interviews and 4 focus group discussions with 43 professionals in- \\
volved in providing support to battered immigrant women; participants came from different cities in \\
Spain (Barcelona, Madrid, Valencia, and Alicante) \\
Experience: social educators, psychologists, social workers, lawyers, judges, policy officers, NGO vol- \\
unteers, intercultural mediators, sociologists and health workers. All professionals employed in NGOs, \\
public institutions, and specialised services aimed at providing legal, social, health, work, family, and \\
psychological support to immigrant women
\end{tabular}

Interventions

Type of advocacy: unclear; professionals talked about accessing resources, protective orders, lodging complaints

Notes Theoretical sampling

A realist review of which advocacy interventions work for which abused women under what circumstances (Review) 
Briones-Vozmediano 2014 (Continued)

Risk of bias

\begin{tabular}{|c|c|}
\hline Bias & Authors' judgement Support for judgement \\
\hline $\begin{array}{l}\text { How valuable is the re- } \\
\text { search (richness of the da- } \\
\text { ta for the review research } \\
\text { questions)? }\end{array}$ & High risk \\
\hline
\end{tabular}

Was there a clear state- Low risk

ment of the aims of the re-

search?

\section{Was the research design Low risk}

appropriate to address the

aims of the research?

$\begin{array}{ll}\text { Was the recruitment strat- } & \text { Low risk Theoretical sampling; a range of different professionals were consulted } \\ \text { egy appropriate to the } & \end{array}$

aims of the research?

Is a qualitative methodol- Low risk

ogy appropriate?

Were the data collected in Low risk
a way that addressed the
research issue?

research issue?

Was the data analysis suf- Low risk

ficiently rigorous?

Is there a clear statement Low risk

of findings?

\section{Burnett 2012}

In-depth interviews and focus groups to better understand how structural factors including policies
shape shelter service delivery and reveal systemic and structural complexities that influence those ser-
vices and women's ability to rebuild their lives, along with a critical discourse analysis of salient policy
texts in order to explore how salient policies and structures shape shelter service delivery and may indi-
rectly contribute to the health and quality of life of women who access services

Participants

\section{Country: Canada}

Setting: 4 shelters for women exposed to violence, located in Ontario

Inclusion criteria: staff of shelters

Exclusion criteria: NS

Participant numbers: 41 , all women, which reflects the mostly female composition of staff working in shelters.

Education and training: participants from urban centres included 30 frontline staff members/managers and 2 directors; from rural locations, participants included 7 staff members/managers and 2 directors

Age: NS

Ethnicity: NS 
Burnett 2012 (Continued)

Interventions
Shelters typically offer advocacy in the form of counselling, legal advice, crisis intervention, and system navigation to help abused women access needed resources and restore their lives.

Notes

\section{Risk of bias}

Bias Authors' judgement Support for judgement

Was there a clear state- Low risk ment of the aims of the research?

\section{Was the research design Low risk} appropriate to address the aims of the research?

\section{Was the recruitment strat- Low risk}

egy appropriate to the

aims of the research?

Has the relationship be- Low risk
tween researcher and par-
ticipants been adequately
considered?

Is a qualitative methodol- Low risk ogy appropriate?

Were the data collected in Low risk
a way that addressed the
research issue?

\section{Was the data analysis suf- Low risk} ficiently rigorous?
Is there a clear statement Low risk
of findings?

\section{Bybee 2005}

\begin{tabular}{ll} 
Methods & Randomisation method: only states 'sealed envelopes' \\
& Analysis by ITT: no \\
Power calculation: no & Qualitative 1-1 interviews \\
\hline Participants & Country: USA \\
& Setting: women in community who had exited shelters \\
& Inclusion criteria: abused women who stayed at least 1 night in shelter and intended to stay in area for \\
at least 3 months post-shelter \\
Exclusion criteria: none stated
\end{tabular}


Participant numbers: at 3-year follow-up 124 had sought refuge from a battered women's shelter 3 years earlier, and half the sample had been randomly assigned to receive free, short-term advocacy services immediately upon exit from the shelter

All data below based on original sample of women (see notes)

Numbers recruited: 146 (76 intervention group, 70 control group)

Number of dropouts: 22 (14 intervention group, 8 control group)

Numbers analysed (and percentage of recruited): 124 (62 (82\%) intervention group, 62 (89\%) control group)

Ethnicity: at 3-year follow-up 46\% = white; $42 \%=$ African American; $7 \%=$ Latina; $2 \%=$ Asian American, and the remainder were Native American, Arab American, or of mixed heritage. All spoke English as their first language. Original data (baseline) 42\% African American, 46\% white, 7\% Latina, 2\% Asian American, $3 \%$ other

Age: at baseline, mean 28 years (range 17-61 years)

Education: 64\% educated to high school level, 34\% some college education

Socioecomomic status indicators: at baseline, $61 \%$ unemployed, $81 \%$ received government aid. Education background: $64 \%$ educated to high school level, $34 \%$ some college education

$\begin{array}{ll}\text { Interventions } & \text { Intervention group } \\ \text { Intensive 1-1 services with an advocate who assisted women to devise a safety plan and access com- } \\ \text { munity resources; } 10 \text { weeks of advocacy, usually meeting twice a week: mean } 7 \mathrm{~h} \text { contact/week for } 10 \\ \text { weeks after leaving shelter } \\ \text { Control group } \\ \text { Women received standard shelter services provided to all residents, and then usual after-shelter care } \\ \text { (if any) }\end{array}$

\section{Risk of bias}

\begin{tabular}{lll}
\hline Bias & Authors' judgement & Support for judgement \\
\hline $\begin{array}{l}\text { Random sequence genera- } \\
\text { tion (selection bias) }\end{array}$ & Unclear risk & $\begin{array}{l}\text { Quote: "Group selection was random, stratifying for order and for whether or } \\
\text { not a woman was romantically involved with her assailant." } \\
\text { No further information was provided }\end{array}$ \\
\hline $\begin{array}{l}\text { Allocation concealment } \\
\text { (selection bias) }\end{array}$ & Low risk & $\begin{array}{l}\text { Quote: "The interviewer opened a sealed envelope which indicated whether } \\
\text { the woman would receive the services of an advocate. The interviewer did not } \\
\text { know to which group a woman would be assigned until after the interview was } \\
\text { completed ..." }\end{array}$ \\
\hline
\end{tabular}

\begin{tabular}{|c|c|c|}
\hline $\begin{array}{l}\text { Blinding (performance } \\
\text { bias and detection bias): } \\
\text { all outcomes }\end{array}$ & Unclear risk & $\begin{array}{l}\text { Blinding of participants and key personnel (people providing the intervention) } \\
\text { was not possible. The outcome assessors were not the same people providing } \\
\text { the intervention, and they were separately trained, but no other information } \\
\text { was provided }\end{array}$ \\
\hline
\end{tabular}

\section{Incomplete outcome da- High risk} ta (attrition bias): all outcomes
14/76 (18\%) missing from intervention group, $8 / 70$ (11\%) missing from control group in the original sample. Reasons given for attrition: 5 participants in the intervention arm were no longer 'eligible' as they had to receive at least 3 weeks of services ( 4 dropped out and returned to their abusers and 1 woman was murdered. This may affect outcome effect size; the remainder (in either group) could not be located. We note that intervention attrition is a function of 
Bybee 2005 (Continued)

the study design, where women had to attend at least 3 advocacy sessions to be eligible, which could not apply to the controls

\begin{tabular}{lll}
\hline $\begin{array}{l}\text { Selective reporting (re- } \\
\text { porting bias) }\end{array}$ & Low risk & $\begin{array}{l}\text { All measures discussed in the 'Methods' section of the paper were also dis- } \\
\text { cussed in the 'Results' section. }\end{array}$ \\
\hline $\begin{array}{l}\text { Baseline measurement of } \\
\text { outcome measures }\end{array}$ & Low risk & $\begin{array}{l}\text { Baseline measures taken. No further information provided for the original } \\
\text { sample, but for the larger sample the investigators stated there were no statis- } \\
\text { tical differences for the } 5 \text { main outcome variables }\end{array}$ \\
\hline $\begin{array}{l}\text { Reliability of outcome } \\
\text { measures }\end{array}$ & Low risk & Outcome measures were reliable \\
\hline $\begin{array}{l}\text { Protection against conta- } \\
\text { mination }\end{array}$ & Unclear risk & $\begin{array}{l}\text { It is possible that there might have been communication between women in } \\
\text { different arms of the trial, as all were recruited from the same shelter }\end{array}$ \\
$\begin{array}{l}\text { How valuable is the re- } \\
\text { search (richness of the da- } \\
\begin{array}{l}\text { ta for the review research } \\
\text { questions)? }\end{array}\end{array}$ & High risk & High value \\
\hline
\end{tabular}

Casey 2007

\begin{tabular}{ll}
\hline Methods & Case-control study \\
\hline Participants & Country: USA \\
& Setting: community policing \\
& Inclusion criteria: battered women who received immediate police intervention at the time of a DV in- \\
cident resulting in an arrest and who were residing with their children at the time of the violence. \\
Exclusion criteria: NS \\
Participant numbers: 204 (102 women = DVHVI, $102=$ comparison group) \\
Age: NS \\
Ethnicity: NS
\end{tabular}

Interventions Intervention

Outreach: the DVHVI is a neighbourhood-based, home-visit, follow-up project with a police officer and a DV advocate visit within 7 days of initial incident. The police-advocate teams conducted unannounced home visits, checking on the safety of victims and their children, explaining court orders, and identifying areas requiring further advocacy or other assistance (e.g. housing, employment, state welfare assistance). Any further home visits or follow-up contact between the women and the DVHVI teams was based on the individual needs of each woman.

Controls

102 DV cases were identified from a review of police reports and selected as a comparison group.

$\begin{array}{ll}\text { Notes } & \text { No significant differences were found between the intervention group and the comparison group for } \\ \text { seriousness of charges or history of DV with police intervention. Similarly, no differences were noted for } \\ \text { relationship type, ethnicity of victim, and ethnicity of perpetrator. }\end{array}$

\section{Risk of bias}

Bias Authors' judgement Support for judgement




\section{Casey 2007 (Continued)}

Random sequence genera- High risk The control group was selected from police records and matched "across a setion (selection bias)

Allocation concealment High risk (selection bias) ries of variables"

Participants were selected from women "who received immediate police intervention at the time of a domestic violence incident resulting in an arrest and who were residing with their children at the time of the violence"

Blinding (performance

bias and detection bias):

High risk

all outcomes

$\begin{array}{ll}\text { Incomplete outcome da- } & \text { Low risk } \\ \text { ta (attrition bias): all out- } & \text { than women in the comparison group } \mathrm{Chi}^{2}(2)=12.1, \mathrm{P}<.001\end{array}$
comes

Selective reporting (re- High risk $\quad$ Measure was number of police calls, not reduction of violence
porting bias)

\section{Baseline measurement of High risk}

outcome measures

Quote: "No significant differences were found between the intervention group and the comparison group for seriousness of charges or history of domestic violence with police intervention. Similarly, no differences were noted for relationship type, ethnicity of victim, and ethnicity of perpetrator."

This is because selection bias may have been present from the start.

How valuable is the re- Low risk value
search (richness of the da-
ta for the review research
questions)?

Coker 2012

Methods $\quad$ Rani-experimental study
Ranisation at the clinic level

Participants

Country: USA

Setting: coalition of clinics against IPV (intimate partner violence); clinic-based advocacy

Inclusion criteria: clinics were recruited to participate if they

- were located in the referral range of the coalition

- provided primary care to lower-income women

- had a patient volume of at least 1000 women/year

- were willing to participate

Women: eligibility was defined based on

- age $\geq 18$

- in an intimate relationship in the past 5 years

- mentally competent to provide consent

Exclusion criteria: NS

Participant numbers: clinics were allocated to the in-clinic advocate intervention versus the usual care such that there would be similar numbers of participants in the intervention and usual care groups 
Coker 2012 (Continued)

The assessment results for each woman were coded as either IPV positive (IPV+) or IPV negative (IPV-). Women who were assessed as positive for any form of IPV within the past 5 years were offered the advocate intervention in the intervention clinics or external referral in the usual care clinics. Approximately $25.6 \%$ of eligible and assessed women were IPV+. Among the screened women who had experienced IPV in the past 5 years, 429 (46\%) agreed to follow-up contact

Numbers with data: intervention $n=447$; control $n=304$

Ethnicity: $68.8 \%$ of women in the intervention and $55.9 \%$ in the control group were African-American (no other data given)

Age: intervention mean age 42.62; control mean age 38.08

Socioecomomic status indicators: NS

Relationship status: NS

All women who were assessed as IPV+ in clinics allocated to the intervention were encouraged by the nurse to meet with the advocate immediately after their appointment

Content of the intervention:

- emotional support

- referrals/connection to community agencies

- safety planning

- in-person meetings

- provide information

- connect to healthcare

- education

\section{Control clinics}

Usual care; women who were assessed as IPV+ were given the business card of their health care provider with the coalition hotline number

Timing of follow-up: followed up for up to 24 months

Notes Women who were assessed as IPV+ were invited into a longitudinal cohort study to prospectively as-
sess outcome

\section{Risk of bias}

\section{Bias} tion (selection bias)
Random sequence genera-

\section{Authors' judgement Support for judgement}

Unclear risk

Not stated. Clinics from a coalition of clinics were randomised

$\begin{array}{ll}\begin{array}{l}\text { Allocation concealment } \quad \text { Low risk } \\ \text { (selection bias) }\end{array} & \begin{array}{l}\text { Randomisation of the intervention was done at the clinic level rather than the } \\ \text { individual level. } \\ \text { Quote: "(the) response rate for participation in the cohort study was less than } \\ 50 \% \text {. It is difficult to recruit participants into a cohort study of } 24 \text { months dura- } \\ \text { tion, and this may be even more difficult among abused women." }\end{array}\end{array}$

\begin{tabular}{|c|c|c|}
\hline $\begin{array}{l}\text { Blinding (performance } \\
\text { bias and detection bias): } \\
\text { all outcomes }\end{array}$ & Low risk & $\begin{array}{l}\text { Women were likely unaware which staff were advocates and which were clinic } \\
\text { staff }\end{array}$ \\
\hline
\end{tabular}


Coker 2012 (Continued)

Incomplete outcome da-

Low risk ta (attrition bias): all outcomes

Selective reporting (re- Low risk
porting bias)

\begin{tabular}{lll}
\hline $\begin{array}{l}\text { Baseline measurement of } \\
\text { outcome measures }\end{array}$ & Unclear risk & Main difference between the groups was age \\
\hline $\begin{array}{l}\text { Reliability of outcome } \\
\text { measures }\end{array}$ & Low risk & \\
\hline $\begin{array}{l}\text { Protection against conta- } \\
\text { mination }\end{array}$ & Low risk & $\begin{array}{l}\text { Randomised by clinic, rather than individual. However, women could talk to an } \\
\text { advocate in the usual care clinics }\end{array}$ \\
\hline $\begin{array}{l}\text { How valuable is the re- } \\
\text { search (richness of the da- } \\
\begin{array}{l}\text { ta for the review research } \\
\text { questions)? }\end{array}\end{array}$ & Low risk \\
\hline
\end{tabular}

\section{Constantino 2005}

Design: pilot study for feasibility of the intervention, $2 \times 2$ group - repeated measures randomised con-
trol design
Randomisation method: a permuted block randomisation with a 1:1 ratio
Analysis by ITT: no
Power calculation: no

Setting: recruited as first-time residents in DV shelter

Inclusion criteria: none stated

Exclusion criteria: none stated

\section{Participant numbers}

Numbers recruited: 30 (15 intervention group, 15 control group)

Number of dropouts: 6 ( 2 intervention group, 4 control group)

Numbers analysed (and percentage of recruited): 24 (13 (87\%) intervention group, 11 (73\%) control group)

Ethnicity: $71 \%$ white not Hispanic, $29 \%$ African Americans

Age: $28-43$ years

Employment: employed full time, $41.7 \%$ employed part time, $7.7 \%$ unemployed, $50 \%$ retired $4.2 \%$

Socioecomomic status indicators: annual household income: 58\% < USD 10,000, 17\% USD $10,000-19,999,21 \%$ USD 20,000-29,999, 4\% > USD 30,000

Education: $12 \%$ up to junior high, $67 \%$ up to high school, $4 \%$ up to trade school, $17 \%$ up to degree

Relationship status: similar distribution between the types of relationships they had with their assailants: never been married, cohabitating, married, divorced, separated, and widowed 
Constantino 2005 (Continued)

Interventions

\section{Intervention}

- A structured group social support intervention to provide and include information on resources, hours of operation to access these, and an environment in which to chat with counsellor (trained nurse) and friends (usual shelter care also provided); safety information

- 8 weekly sessions of 90 -min duration

\section{Control}

No treatment control: usual shelter care and unstructured chats with the principal investigator

Notes

Funded by the University of Pittsburgh Central Research Development Fund

Data were collected for the study July 2003-June 2004

\section{Risk of bias}

\begin{tabular}{|c|c|c|}
\hline Bias & Authors' judgement & Support for judgement \\
\hline $\begin{array}{l}\text { Random sequence genera- } \\
\text { tion (selection bias) }\end{array}$ & Unclear risk & $\begin{array}{l}\text { Randomisation after baseline data collection using a permuted block ran- } \\
\text { domisation scheme with a ratio of 1:1. No other information provided }\end{array}$ \\
\hline $\begin{array}{l}\text { Allocation concealment } \\
\text { (selection bias) }\end{array}$ & Unclear risk & $\begin{array}{l}\text { The medical advocate directed recruitment and retention, but there was no in- } \\
\text { formation about who performed the allocation or the procedure used. }\end{array}$ \\
\hline $\begin{array}{l}\text { Blinding (performance } \\
\text { bias and detection bias): } \\
\text { all outcomes }\end{array}$ & Unclear risk & $\begin{array}{l}\text { Blinding of participants and key personnel (person providing the intervention) } \\
\text { was not possible. No information on blinding for outcome assessment was } \\
\text { provided. }\end{array}$ \\
\hline $\begin{array}{l}\text { Incomplete outcome da- } \\
\text { ta (attrition bias): all out- } \\
\text { comes }\end{array}$ & High risk & $\begin{array}{l}\text { Large difference between groups: } 2 / 15 \text { (13\%) missing from intervention group; } \\
4 / 15(27 \%) \text { missing from control group. Reasons given for attrition (difficulties } \\
\text { with transportation, living conditions, child care issues) were not reported by } \\
\text { trial arm; some may be secondary to being in a particular arm (e.g. not having } \\
\text { advocacy) }\end{array}$ \\
\hline $\begin{array}{l}\text { Selective reporting (re- } \\
\text { porting bias) }\end{array}$ & Low risk & $\begin{array}{l}\text { In the paper, full statistical data were only reported for the significant findings } \\
\text { while other outcomes were only mentioned as being near-significant or non- } \\
\text { significant. These missing data were subsequently provided by the investiga- } \\
\text { tors. }\end{array}$ \\
\hline $\begin{array}{l}\text { Baseline measurement of } \\
\text { outcome measures }\end{array}$ & Low risk & $\begin{array}{l}\text { No specific information on between-group differences was provided, although } \\
\text { the means and standard deviations seemed comparable. (Study investigators } \\
\text { calculated mean change from baseline scores) }\end{array}$ \\
\hline $\begin{array}{l}\text { Reliability of outcome } \\
\text { measures }\end{array}$ & Low risk & Outcome measures were reliable \\
\hline $\begin{array}{l}\text { Protection against conta- } \\
\text { mination }\end{array}$ & Unclear risk & $\begin{array}{l}\text { There might have been communication between women in different arms of } \\
\text { the trial. Also, some participants in the control group had the opportunity to } \\
\text { talk with the principal investigator, although not with the person delivering } \\
\text { the advocacy intervention. This may have had an effect, but would, if anything, } \\
\text { have diminished any positive effects. }\end{array}$ \\
\hline
\end{tabular}

Coy 2011

\begin{tabular}{ll}
\hline Methods & An evaluation of 4 IDVA schemes: narrative, observation-based evaluation from grey literature \\
\hline Participants & Country: UK \\
\hline
\end{tabular}

A realist review of which advocacy interventions work for which abused women under what circumstances (Review) 
Coy 2011 (Continued)

Setting: each based in a different setting in London: a police station; hospital ED; a community-based DV project; and a women-only violence against women organisation

Participants numbers: number of cases across the 4 schemes $=748$

\begin{tabular}{ll}
\hline Interventions & $\begin{array}{l}\text { Independent DV advocacy; content varied across the schemes and the main focus of the report is their } \\
\text { description }\end{array}$ \\
\hline Notes & $\begin{array}{l}\text { The schemes were initially funded from 2007-2010, and the data collection took place over a 2-year pe- } \\
\text { riod from 2007-2009 }\end{array}$
\end{tabular}

\section{Risk of bias}

\begin{tabular}{|c|c|c|}
\hline Bias & Authors' judgement & Support for judgement \\
\hline $\begin{array}{l}\text { How valuable is the re- } \\
\text { search (richness of the da- } \\
\text { ta for the review research } \\
\text { questions)? }\end{array}$ & Low risk & $\begin{array}{l}\text { Low value: specifically focusing on Multi-Agency Risk Assessment Conferences } \\
\text { (MARACs) and the local Co-ordinated Community Response (CCR) }\end{array}$ \\
\hline $\begin{array}{l}\text { Was there a clear state- } \\
\text { ment of the aims of the re- } \\
\text { search? }\end{array}$ & Low risk & \\
\hline $\begin{array}{l}\text { Was the research design } \\
\text { appropriate to address the } \\
\text { aims of the research? }\end{array}$ & High risk & $\begin{array}{l}\text { A multi-method narrative evaluation. This used surveys and interviews for } \\
\text { views of service users and managers but not with the rigour of research design }\end{array}$ \\
\hline $\begin{array}{l}\text { Was the recruitment strat- } \\
\text { egy appropriate to the } \\
\text { aims of the research? }\end{array}$ & Unclear risk & NS \\
\hline
\end{tabular}

Has the relationship be-

High risk

tween researcher and par-

ticipants been adequately considered?

Is a qualitative methodol- High risk No clear consideration of methodology - ad hoc
ogy appropriate?

Were the data collected in Low risk
a way that addressed the
research issue?

research issue?

Was the data analysis suf- High risk Not a research study
ficiently rigorous?

\section{Is there a clear statement Low risk} of findings?

\section{Cripe 2010}

$\begin{array}{ll}\text { Methods } & \text { Design: } 2 \text {-armed RCT using } 2 \text { levels of abuse intervention } \\ \text { Randomisation method: a randomised block design } \\ \text { Analysis by ITT: no }\end{array}$


Cripe 2010 (Continued)

Power calculation: no

Country: Peru
Setting: public hospital prenatal clinic
Inclusion criteria
- Prenatal care women (12-26 weeks gestation)
- Aged 18-45 years
- Spoke and understood Spanish
- Screening positive for IPV in past 12 months

Exclusion criteria: none stated

Participant numbers

Numbers recruited: of 1035 pregnant women, 220 recruited (110 intervention group, 110 control group) Number of dropouts: 16 (6 intervention group, 10 control group) (8 refused to participate, and 2 requested referral for psychological support and were excluded) Numbers analysed (and percentage of recruited): 204 (104 (95\%) intervention group, 100 (91\%) control group)

Age: mean 26 years intervention group, 27 years control group

Ethnicity: $87 \%$ Mestizo (representative of the distribution of racial groups in the Lima region)

Socioecomomic status indicators: $63 \%$ unemployed, $51 \%$ limited access to basic foods, $64 \%$ limited access to medical care

Education: $54 \% \leq 6$ years; $11 \%$ 7-12 years; $34 \%>12$ years

Children: all were pregnant

\section{Interventions Intervention}

1-1 empowerment counselling provided by trained hospital-based social workers: empathic listening and acknowledgement of woman's perceptions and feelings, supportive counselling and education (including helping women to understand the cycle of violence), advice in areas of safety (including a review of safety plan components and an appraisal of the safety of adopting each behaviour), provision of a 13-item safety plan brochure (as well as other prenatal brochures on other topics), a list of community resources (such as emergency shelter, legal aid, law enforcement, and counselling) and what to expect when seeking help from these services, and advice on strategies for seeking help (including assistance with telephone calls to social service agencies or women's groups); 1 session lasting about 30 $\min$

Control

Women received standard care: a wallet-sized referral card listing agencies providing IPV services (e.g. legal, social services, law enforcement). No counselling, advocacy, education, or other services offered

Notes

The study recruitment dates were from 9 January-26 July 2007

This project was funded by Puget Sound Partners for Global Health

\section{Risk of bias}

\begin{tabular}{lll}
\hline Bias & Authors' judgement & Support for judgement \\
\hline $\begin{array}{l}\text { Random sequence genera- } \\
\text { tion (selection bias) }\end{array}$ & Unclear risk & $\begin{array}{l}\text { Randomisation was conducted before baseline data collection "using a ran- } \\
\text { domised block design at recruitment". No other information was available }\end{array}$ \\
\hline $\begin{array}{l}\text { Allocation concealment } \\
\text { (selection bias) }\end{array}$ & Unclear risk & $\begin{array}{l}\text { The project manager assigned participants. No other information was avail- } \\
\text { able about allocation concealment }\end{array}$ \\
\hline
\end{tabular}


Cripe 2010 (Continued)

Blinding (performance Low risk Blinding of participants and key personnel (people providing the intervention) bias and detection bias): was not possible. Outcome assessors at follow-up were different from those all outcomes collecting baseline data and providing the intervention

\begin{tabular}{|c|c|c|}
\hline $\begin{array}{l}\text { Incomplete outcome da- } \\
\text { ta (attrition bias): all out- } \\
\text { comes }\end{array}$ & Low risk & $\begin{array}{l}6 / 110(5 \%) \text { missing from intervention group; } 10 / 110(9 \%) \text { missing from con- } \\
\text { trol group. No missing women from either group could be located at follow-up; } \\
\text { this was unlikely to bias the results }\end{array}$ \\
\hline
\end{tabular}
comes

All measures discussed in the 'Methods' section of the paper were also dis-

Selective reporting (re- Low risk porting bias) cussed in the 'Results' section

\begin{tabular}{lll}
$\begin{array}{l}\text { Baseline measurement of } \\
\text { outcome measures }\end{array}$ & Low risk & $\begin{array}{l}\text { For resource use, no specific information was provided, but the group frequen- } \\
\text { cies appear comparable. For other outcomes, appear comparable }\end{array}$ \\
\hline $\begin{array}{l}\text { Reliability of outcome } \\
\text { measures }\end{array}$ & Unclear risk & $\begin{array}{l}\text { A reliable quality-of-life measure was utilised. No data were provided on the } \\
\text { reliability of the modified scales used to measure either the use of safety be- } \\
\text { haviours or the use of community resources }\end{array}$
\end{tabular}

Protection against conta- Unclear risk There might have been communication between women in different arms of
mination the trial as all recruited from 1 clinic

\begin{tabular}{ll}
\hline Methods & Cluster-randomised experiment with random allocation to groups \\
\hline Participants & Country: USA \\
& Setting: 2 prenatal clinics in the Pacific Northwest and rural Midwest (clinics A and B) \\
& Inclusion criteria: women who spoke English and were $13-23$ weeks pregnant at time of recruitment \\
& Exclusion criteria: adolescents who could not obtain signed consent from parent or guardian \\
& Participant numbers: $\mathrm{n}=1000$
\end{tabular}

501 in clinic A control and 499 in the intervention, clinic B

Ethnicity: white control $=338(67.6 \%)$, intervention $=410(82 \%)$ African American control $=81(16.2 \%)$, intervention $=61(12.2 \%)$, Hispanic control $=22(4.4 \%)$ intervention $=7(1.4 \%)$, Asian/Pacific Islander control $=19(3.8 \%)$ intervention $=13(2.6 \%)$ Native American control $=5(1.2 \%)$ intervention $=0$, other control $=34(6.8 \%)$ intervention $=8(1.6 \%)$

Overall: white 79\%, African American 11\%, Hispanic 3\%; Asian/Pacific Islander 3\%; other 4\%

Age: age 14-46 years, control M (SD) 29.72 (5.91) intervention M (SD) 27.73 (5.87), overall M 29 Socioecomomic status indicators: monthly income control USD 3871 (USD 2889) intervention USD 2893 (USD 2919)

Level of education: (years) control 15.00 (2.86) range 8-27; intervention 14.56 (3.31) range 6-26

Abuse: the majority (74\%) were high risk because of abuse; the rest were high risk because of high stress scores without abuse disclosure. All women reporting abuse by an intimate partner or ex-partner $(n=97)$ were further assessed for risk factors for lethality with the Danger Assessment Scale (DAS) (Campbell 1986). The DAS ranged from 0-12

Pregnant: $13-23$ weeks pregnant at time of recruitment

Relationship status: $n$, \%: married, living with partner control 338 (67.6); intervention 318 (63.6). Married, living alone control 4 (0.8); intervention 8 (1.6). Single, living with partner control 85 (17); intervention $104(20.8)$ 
Curry 2006 (Continued)

Interventions

\section{Intervention}

The Connections study strengths-based nurse case management intervention

All intervention group women $(n=499)$ were offered an abuse video and had access to a nurse $24 / 7$ Additionally, participants at risk for or in abusive relationships received individualised nursing care management throughout the pregnancy

\section{Content of the intervention:}

- conversation by phone

- emotional support

- safety planning

- in-person meetings

- education

- referral card

\section{Control}

Usual care

Notes Included adolescents

\section{Risk of bias}

\begin{tabular}{|c|c|c|}
\hline Bias & Authors' judgement & Support for judgement \\
\hline $\begin{array}{l}\text { Random sequence genera- } \\
\text { tion (selection bias) }\end{array}$ & Unclear risk & Random sequence generation method not stated \\
\hline $\begin{array}{l}\text { Allocation concealment } \\
\text { (selection bias) }\end{array}$ & Low risk & $\begin{array}{l}\text { Women were randomly allocated after a recruitment and a baseline assess- } \\
\text { ment taken }\end{array}$ \\
\hline $\begin{array}{l}\text { Blinding (performance } \\
\text { bias and detection bias): } \\
\text { all outcomes }\end{array}$ & Low risk & $\begin{array}{l}\text { Study authors say they aimed to "provide an intervention that was intact and } \\
\text { distinct from the clinics in order to measure the effects of our NCM [nurse case } \\
\text { management] on outcomes without potential confounders" }\end{array}$ \\
\hline $\begin{array}{l}\text { Incomplete outcome da- } \\
\text { ta (attrition bias): all out- } \\
\text { comes }\end{array}$ & Unclear risk & Numbers suggest there were dropouts, but there is no discussion of these \\
\hline $\begin{array}{l}\text { Selective reporting (re- } \\
\text { porting bias) }\end{array}$ & Low risk & Measures changes in stress and associated clinical outcomes \\
\hline $\begin{array}{l}\text { Baseline measurement of } \\
\text { outcome measures }\end{array}$ & Low risk & $\begin{array}{l}\text { Sample was limited to those who had sought early prenatal care. Study au- } \\
\text { thors point out that women who seek later prenatal care may typically have } \\
\text { more risk factors than those seeking early prenatal care }\end{array}$ \\
\hline $\begin{array}{l}\text { Reliability of outcome } \\
\text { measures }\end{array}$ & High risk & $\begin{array}{l}\text { Study authors say a limitation of the study was the lack of sensitive measures } \\
\text { of outcomes such as service use and trust }\end{array}$ \\
\hline $\begin{array}{l}\text { Protection against conta- } \\
\text { mination }\end{array}$ & High risk & $\begin{array}{l}\text { Randomised by individual at } 2 \text { different clinics. It is possible the women could } \\
\text { encounter each other }\end{array}$ \\
\hline $\begin{array}{l}\text { How valuable is the re- } \\
\text { search (richness of the da- } \\
\text { ta for the review research } \\
\text { questions)? }\end{array}$ & Unclear risk & Low value \\
\hline
\end{tabular}

A realist review of which advocacy interventions work for which abused women under what circumstances (Review) 


\begin{tabular}{ll}
\hline Methods & Pre-post with control \\
\hline Participants & Country: USA \\
Setting: community policing \\
Inclusion criteria: any family reporting family violence in specific areas during the study based on \\
Housing Authority property in the study area \\
Exclusion criteria: NS \\
Participant numbers: 436 incidents reported on Housing Authority property \\
No separate data on partner abuse subgroup (40\% of total)
\end{tabular}

Interventions

\section{Intervention}

Domestic Violence Intervention Education Project [DVIEP]

2 levels: public education and home visits

- The leaflets contained information on the legal rights of victims, locations of emergency and longterm services, and the importance of the police in ending family violence. This intervention consisted of a leaflet and poster campaign and presentations at community and tenant association meetings.

- The leaflets were slipped under the door of every apartment in housing projects assigned to the public education treatment.

- Posters and additional leaflets were placed in common areas, such as around mailboxes and in housing managers' offices.

- The leaflets contained information on the legal rights of victims, locations of emergency and long-term services, and the importance of the police in ending family violence.

- Presentations at community meetings.

- Assignment to levels of public education by housing project: 64 individual housing projects (total population 93,000) were matched for size and sociodemographic characteristics (projects ranged in size from 100 households to more than 2000 households).

- 1 member of each pair was randomly assigned to receive the public education intervention and the other served as a control

- The second treatment was home follow-up: participants either received or did not receive a follow-up visit from a police officer and social worker within several days of the initial police response to a complaint of family violence (see below for details).

- The units assigned to levels of home visits were households that generated police complaints:

- Households were randomly assigned on an individual basis to levels of home visits or designated incidents

- A team consisting of a social worker and a police officer visited households within a few days of a domestic com- plaint.

- The team attempted to educate the victim and perpetrator (if present) about the criminal and escalating nature of family violence and encourage the household to seek change through the use of courts and other services.

Observations by researchers indicated that home visits generally lasted 10-30 min, depending on whether the batterer was present and on the victim's receptiveness to assistance. During that time, victims were informed about services and about pursuing legal remedies, especially obtaining restraining orders. Referrals were made, most commonly to counselling services and support groups, drug and alcohol treatment programmes for batterers, battered women shelters, home security improvement services, health care assessment and assistance, emergency financial assistance, assistance with relocating to other public housing units, and respite assistance for caregivers who batter seniors. On-the-spot crisis counselling was provided when indicated.

\section{Controls}


Davis 2006 (Continued)

Households were assigned to 1 of the 2 levels of the home visit treatment according to a log sheet that was prenumbered with sequential ID numbers and a corresponding treatment group designation for each number.

Notes

Incidents were fairly equally divided among those involving romantic intimates (40\%), children abusing parents (35\%), and others ( $25 \%)$. Although no separate data on partner abuse subgroup ( $40 \%$ of total), included to cover gaps in theory

\section{Risk of bias}

\begin{tabular}{lll}
\hline Bias & Authors' judgement & Support for judgement \\
\hline $\begin{array}{l}\text { Random sequence genera- } \\
\text { tion (selection bias) }\end{array}$ & Unclear risk & $\begin{array}{l}\text { Households were assigned to } 1 \text { of the } 2 \text { levels of the home visit treatment ac- } \\
\text { cording to a log sheet that was prenumbered with sequential ID numbers and } \\
\text { a corresponding treatment group designation for each. Does not say how } \\
\text { prenumbering was done. }\end{array}$
\end{tabular}

Allocation concealment High risk
(selection bias)

Blinding (performance High risk

bias and detection bias):

all outcomes

Selective reporting (re- Low risk
porting bias)

Baseline measurement of Low risk
outcome measures

\begin{tabular}{ll}
\hline $\begin{array}{l}\text { Reliability of outcome } \\
\text { measures }\end{array}$ & High risk \\
\hline $\begin{array}{l}\text { Protection against conta- } \\
\text { mination }\end{array}$ & High risk \\
\hline $\begin{array}{l}\text { How valuable is the re- } \\
\text { search (richness of the da- } \\
\text { ta for the review research } \\
\text { questions)? }\end{array}$ & Low risk \\
\hline
\end{tabular}

\section{Davis 2007}

\begin{tabular}{ll}
\hline Methods & Pre-post with controls; power calculation \\
\hline Participants & Country: USA \\
Setting: community policing \\
Inclusion criteria: households in designated public housing units in Manhattan where someone had \\
called the police in response to a family violence incident. Victims who called the Redlands police with \\
a domestic abuse complaint were randomly assigned to receive: \\
- a second response within $24 \mathrm{~h}$ \\
- a second response within 7 days, or \\
- receive no second response
\end{tabular}


Davis 2007 (Continued)

Participant numbers: $\mathrm{n}=308$ cases; 75 cases assigned to the 24 -h response condition, 77 cases assigned to the 7-day response condition, 148 cases assigned to the control condition and 8 cases that had been assigned to the control condition that were reassigned to receive an immediate response

\begin{abstract}
Interventions

\section{Content of the intervention}

The intervention was delivered as part of the Domestic Violence Intervention Education Project [DVIEP]. The goals of home visits were to ensure that the victim had information about and access to resources and services, to answer any questions they had about the complaint or the justice process, and to encourage a sense of trust in the police and the criminal justice system as a whole.

The programme model involves a social worker or specially trained DV police officer going to the homes of victims who have reported domestic abuse sometime after the initial patrol response to the call for service. The second responder talks with victims about the nature of DV, helps them develop a safety plan, and informs them about help available for counselling needs, relocation, civil legal assistance, restraining orders, and other social services.
\end{abstract}

\title{
Comparators
}

Study designed to determine whether the timing of the intervention is a key determinant of its effects. This study, compared conditions in which the second response occurred within $24 \mathrm{~h}$, within 7 days, or not at all.

\section{Notes}

This could be violence between romantic intimates, sibling violence, elder abuse, or other forms of violence between persons related or living under the same roof. Although no separate data on partner abuse subgroup, included to cover gaps in theory; data collected 1 January 2005-3 December 2005

\section{Risk of bias}

\begin{tabular}{lll}
\hline Bias & Authors' judgement & Support for judgement \\
\hline $\begin{array}{l}\text { Random sequence genera- } \\
\text { tion (selection bias) }\end{array}$ & Unclear risk & NS \\
\hline
\end{tabular}

Allocation concealment High risk
(selection bias)

(selection bias)

\section{Blinding (performance \\ bias and detection bias): \\ all outcomes}

High risk

\begin{tabular}{|c|c|c|}
\hline $\begin{array}{l}\text { Incomplete outcome da- } \\
\text { ta (attrition bias): all out- } \\
\text { comes }\end{array}$ & High risk & \\
\hline $\begin{array}{l}\text { Selective reporting (re- } \\
\text { porting bias) }\end{array}$ & High risk & $\begin{array}{l}\text { Victims who were interviewed were more likely to be involved in non-violent } \\
\text { incidents relative to those not interviewed }\end{array}$ \\
\hline $\begin{array}{l}\text { Baseline measurement of } \\
\text { outcome measures }\end{array}$ & Low risk & \\
\hline $\begin{array}{l}\text { Reliability of outcome } \\
\text { measures }\end{array}$ & High risk & \\
\hline $\begin{array}{l}\text { How valuable is the re- } \\
\text { search (richness of the da- } \\
\text { ta for the review research } \\
\text { questions)? }\end{array}$ & Low risk & Low value \\
\hline
\end{tabular}


DePrince 2012

\begin{tabular}{|c|c|c|}
\hline Methods & \multicolumn{2}{|c|}{ Randomised, longitudinal design } \\
\hline \multirow[t]{10}{*}{ Participants } & \multicolumn{2}{|l|}{ Country: USA } \\
\hline & \multicolumn{2}{|l|}{ Setting: community } \\
\hline & \multicolumn{2}{|c|}{$\begin{array}{l}\text { Inclusion criteria: recruited from the population of publicly accessible, nonsexual assault IPA incident } \\
\text { reports referred to the triage team in Denver (CO) } 5 \text { December 2007-14 July 2008, that involved a het- } \\
\text { erosexual couple, adult victim and offender, and no cross-arrest }\end{array}$} \\
\hline & \multicolumn{2}{|c|}{$\begin{array}{l}\text { Participant numbers: of } 827 \text { women randomised, only } 236(28.5 \%) \text { could be contacted and enrolled in } \\
\text { the study. }\end{array}$} \\
\hline & \multirow{6}{*}{\multicolumn{2}{|c|}{$\begin{array}{l}84 \% \text { of women from the original sample }(n=236) \text { were retained at either } 6 \text { - or } 12 \text {-month follow-up. } \\
\text { Specifically, } 6 \text {-month retention rate of } 81 \%(n=192) \text {, and } 12 \text {-month retention rate of } 80 \%(n=189) \text { of } \\
\text { the original sample } \\
\text { Ethnicity: } 47 \% \text { white, } 30 \% \text { black or African American, } 2 \% \text { Asian/Asian American, } 1 \% \text { Pacific Islander, } \\
11 \% \text { American Indian or Alaskan Native, } 6 \% \text { other, and } 39 \% \text { Hispanic or Latina } \\
\text { Age: } 18-63 \text {, with an average age of } 33.4(S D=11.0) \\
\text { Education: } 3 \% 1 \text { st-8th grade; } 27 \% \text { some high school; } 26 \% \text { high school; } 25 \% \text { some college; } 8 \% \text { asso- } \\
\text { ciate's degrees; } 7 \% 4 \text {-year college degree; } 2 \% \text { postgraduate education; and } 1 \% \text { other (e.g. trade school) } \\
\text { Relationship status: } 49 \% \text { ever been married, } 9 \% \text { married, } 8 \% \text { living with someone, } 18 \% \text { divorced, } 12 \% \\
\text { separated, } 2 \% \text { widowed, } 40 \% \text { single and never married, and } 7 \% \text { other } \\
\text { Socioecomomic status indicators: women's median income was USD } 7644 \text { (range USD } 0-108,000) \text { and } \\
\text { average occupational prestige was } 31.91 \text { (SD } 21.59)\end{array}$}} \\
\hline & & \\
\hline & & \\
\hline & & \\
\hline & & \\
\hline & & \\
\hline Interventions & \multirow{2}{*}{\multicolumn{2}{|c|}{$\begin{array}{l}\text { Community-based advocates initiating outreach by phone, using survivor-defined practice } \\
\text { Co-ordinated outreach intervention compared to the treatment-as-usual (referral) condition }\end{array}$}} \\
\hline & & \\
\hline \multicolumn{3}{|l|}{ Notes } \\
\hline \multicolumn{3}{|l|}{ Risk of bias } \\
\hline Bias & Authors' judgement & Support for judgement \\
\hline $\begin{array}{l}\text { Random sequence genera- } \\
\text { tion (selection bias) }\end{array}$ & Low risk & $\begin{array}{l}\text { A team leader on the triage team used an algorithm to randomly assign } \\
\text { women to the outreach (intervention) or referral (usual care) condition during } \\
\text { the study period }\end{array}$ \\
\hline $\begin{array}{l}\text { Allocation concealment } \\
\text { (selection bias) }\end{array}$ & Low risk & $\begin{array}{l}\text { The assignment was not revealed to anyone else on the review team until after } \\
\text { the team's risk assessment (to assure that risk was not evaluated differently } \\
\text { depending on whether the woman was assigned to intervention or usual care } \\
\text { conditions). At the review team meeting, the team evaluated women's risk in } \\
\text { each case. If the review team believed that the victim was at grave risk (guided } \\
\text { by research on risk factors), she was determined to be ineligible for the study } \\
\text { and automatically referred for outreach. }\end{array}$ \\
\hline
\end{tabular}

Incomplete outcome da-

Low risk

ta (attrition bias): all out-

comes

Selective reporting (re- Low risk porting bias)

Baseline measurement of Low risk outcome measures 
DePrince 2012 (Continued)

Reliability of outcome Unclear risk Mixed
measures

How valuable is the re-
search (richness of the da-

High risk

High value over series of papers

ta for the review research

questions)?

Donnelly 2005

$\begin{array}{ll}\text { Methods } & \begin{array}{l}\text { Action research: telephone interviews on average } 45 \text { min long to explore service provision with execu- } \\ \text { tive directors of shelters }\end{array}\end{array}$

Country: USA
Petting: 44 battered women's shelters with state funding
Inclusion criteria: all battered women's shelters with state funding in Alabama $(\mathrm{n}=17)$, Georgia $(\mathrm{n}=$
32) and Mississippi $(\mathrm{n}=11)$
Exclusion criteria: shelters without state funding
Participant numbers: $70 \%$ response rate with $44 / / 60$ phone interviews completed
Experience: shelters were on average running for 11 years; 17 beds/shelter; mean numbers of staff, 5
full-time and 3 part-time; average 266 women in shelter/year
Ethnicity: NS for staff; shelters: $57 \%$ women of different ethnicity
Age: NS

Interventions Legal advocacy to empower women

Notes

\section{Risk of bias}

Bias Authors' judgement Support for judgement

How valuable is the re- High risk

search (richness of the da-

ta for the review research questions)?

Was there a clear state- Low risk
ment of the aims of the re-
search?

Was the research design Low risk
appropriate to address the
aims of the research?

Was the recruitment strat- Low risk egy appropriate to the aims of the research?

Has the relationship be- Low risk
tween researcher and par-
ticipants been adequately
considered?

Yes. Authors state their recognising of their own white privilege whilst seeking to study it 
Donnelly 2005 (Continued)
Is a qualitative methodol-
Low risk
ogy appropriate?

\title{
Have ethical issues been High risk \\ taken into consideration?
}

\author{
Were the data collected in Low risk \\ a way that addressed the \\ research issue?
}

Was the data analysis suf- Low risk

ficiently rigorous?

Is there a clear statement Low risk

of findings?

Dunn 2007

\begin{tabular}{ll}
\hline Methods & Semi-structured interviews \\
\hline Participants & Country: USA \\
& $\begin{array}{l}\text { Setting: shelters and in criminal justice settings, including prosecutors' offices, a police department, } \\
\text { and a Sheriff's office in a large Midwestern state; geographically dispersed counties in rural, suburban, } \\
\text { and urban areas }\end{array}$
\end{tabular}

and urban areas

Participant numbers: 32 DV victim advocates

Gender: all of the advocates interviewed were female

Ethnicity: 4 were African American, 1 Mexican American, and the remaining 27 were white

Age: $23-69$ years

Experience: 6 months-25 years. 14 of the advocates worked in shelters, 16 in the criminal justice system, and 2 in both

\begin{tabular}{ll}
\hline Interventions NS & NS \\
\hline Notes
\end{tabular}

\section{Risk of bias}

\begin{tabular}{lll}
\hline Bias & Authors' judgement & Support for judgement \\
\hline $\begin{array}{l}\text { Was there a clear state- } \\
\text { ment of the aims of the re- } \\
\text { search? }\end{array}$ & Low risk & \\
\hline $\begin{array}{l}\text { Was the research design } \\
\text { appropriate to address the } \\
\begin{array}{l}\text { aims of the research? } \\
\text { Was the recruitment strat- }\end{array}\end{array} \quad$ Low risk \\
$\begin{array}{l}\text { egy appropriate to the } \\
\text { aims of the research? }\end{array}$ & $\begin{array}{l}\text { May have been some bias in shelters. Initially contacted shelter co-ordinators } \\
\text { and victim advocates in criminal justice settings by phone. Shelter co-ordina- } \\
\text { tors provided contact numbers for advocates, and arrangements with advo- } \\
\text { cates in criminal justice settings were made directly with them. }\end{array}$ \\
\hline
\end{tabular}


Dunn 2007 (Continued)

Has the relationship be- Low risk_ Previous experience as researchers in the field of DV victim advocacy and as tween researcher and participants been adequately considered? a victim advocate (1 study author has been a victim advocate for more than 4 years)

\section{Is a qualitative methodol- Low risk} ogy appropriate?

$\begin{array}{lll}\text { Have ethical issues been NS } & \text { Unclear risk } \\ \text { taken into consideration? }\end{array}$

taken into consideration?

Low risk

Were the data collected in

a way that addressed the

research issue?

Was the data analysis suf- Low risk

ficiently rigorous?

Is there a clear statement Low risk

of findings?

\section{Ekstrom 2015}

\begin{tabular}{|c|c|}
\hline Methods & 6 women interviewed \\
\hline \multirow[t]{7}{*}{ Participants } & \multirow{2}{*}{$\begin{array}{l}\text { Country: Sweden } \\
\text { Setting: judicial services support centre }\end{array}$} \\
\hline & \\
\hline & $\begin{array}{l}\text { Participants: } 4 \text { women who had contacted the support centre were interviewed between November } \\
2008 \text { and January 2009. } 2 \text { additional interviews were conducted in January and February } 2010 \text {. }\end{array}$ \\
\hline & Ethnicity: Middle Eastern x 2, Swedish x 3, Eastern European x 1 \\
\hline & Ages: $40,60,30,20,30,40$ \\
\hline & Children: 2 had children \\
\hline & $\begin{array}{l}\text { Relationship status: } 5 \text { of the women stated during the interview that they had no present relation to } \\
\text { the man and } 1 \text { woman stated that she had started seeing her boyfriend again. }\end{array}$ \\
\hline Interventions & $\begin{array}{l}\text { A support centre staffed by social workers providing social support and advice for female victims in } \\
\text { connection with the police investigations and criminal trials in cases of DV }\end{array}$ \\
\hline \multicolumn{2}{|l|}{ Notes } \\
\hline \multicolumn{2}{|l|}{ Risk of bias } \\
\hline Bias & Authors' judgement Support for judgement \\
\hline $\begin{array}{l}\text { How valuable is the re- } \\
\text { search (richness of the da- } \\
\text { ta for the review research } \\
\text { questions)? }\end{array}$ & High risk \\
\hline
\end{tabular}


Ekstrom 2015 (Continued)

\begin{abstract}
Was there a clear statement of the aims of the research?
\end{abstract}

Low risk

$\begin{aligned} & \text { Was the research design Low risk } \\ & \text { appropriate to address the } \\ & \text { aims of the research? }\end{aligned}$
$\begin{aligned} & \text { Was the recruitment strat- High risk } \\ & \text { egy appropriate to the } \\ & \text { aims of the research? }\end{aligned}$
$\begin{aligned} & \text { Has the relationship be- } \\ & \text { tween researcher and par- } \\ & \text { ticipants been adequately } \\ & \text { considered? }\end{aligned}$
Is a qualitative methodol- Low risk
ogy appropriate?

Have ethical issues been Low risk
taken into consideration?

Were the data collected in Low risk a way that addressed the research issue?

Was the data analysis suf- Low risk

ficiently rigorous?

Is there a clear statement Low risk

of findings?

Feder 2018

\begin{tabular}{ll}
\hline Methods & RCT \\
\hline Participants & Country: USA \\
Setting: NFP, which provides regular home visits conducted by community health nurses for first-time \\
mothers-to-be from the time they are in their 2nd trimester through the child's 2nd birthday. \\
Inclusion criteria: 1 st pregnancy, eligible for the nutrition programme Women, Infants, and Children \\
(WIC), English or Spanish speaking, and at least 15 years of age \\
Participant numbers: 240 invited. The final sample consisted of 238 women completing baseline as- \\
sessments; retention was $81 \%$ at 2-year follow-up
\end{tabular}

Interventions Intervention

3 components intended to be embedded within the existing NFP home visiting programme curriculum:

- a structured assessment for IPV early in the programme as well as continued assessments throughout the women's enrolment

- a brochure-driven intervention for women who indicate that they are experiencing IPV

- a skills-based curriculum delivered to all participants that focuses on making smart relationship decisions and improving relationship outcomes. 
Feder 2018 (Continued)

In each of these intervention components, relationship issues and partner violence are discussed within the context of the mother's and baby's health, with the intent of making the clients more invested in the intervention. The brochure-driven intervention utilised in this intervention was originally developed by McFarlane 1997.

\section{Control}

NFP without abuse (IPV) intervention

Notes Participants enrolled over a 20-month period

\section{Risk of bias}

\begin{tabular}{lll}
\hline Bias & Authors' judgement & Support for judgement \\
\hline $\begin{array}{l}\text { Random sequence genera- } \\
\text { tion (selection bias) }\end{array}$ & Unclear risk & $\begin{array}{l}\text { As women are referred into NFP, they are randomly assigned to receive either } \\
\text { the experimental intervention embedded within NFP (the NFP+ experimen- } \\
\text { tal intervention) or NFP as usual (the control condition). Participants' assign- } \\
\text { ment determines the nurse who takes their case and first contacts the client. } \\
\end{array} \quad \begin{array}{ll}5 \text { trained experimental nurses administer NFP+, and 11 trained NFP nurses ad- } \\
\text { minister NFP to the control clients. }\end{array}$ \\
& \\
\hline
\end{tabular}

Allocation concealment Low risk Nurses not part of allocation process

(selection bias)

Low risk

Blinding (performance

bias and detection bias):

all outcomes

\begin{tabular}{ll}
\hline $\begin{array}{l}\text { Incomplete outcome da- } \\
\text { ta (attrition bias): all out- } \\
\text { comes }\end{array}$ & Low risk \\
\hline $\begin{array}{l}\text { Selective reporting (re- } \\
\text { porting bias) }\end{array}$ & Low risk \\
\hline $\begin{array}{l}\text { Baseline measurement of } \\
\text { outcome measures }\end{array}$ & Low risk \\
\hline $\begin{array}{l}\text { Reliability of outcome } \\
\text { measures }\end{array}$ & Low risk \\
\hline $\begin{array}{l}\text { How valuable is the re- } \\
\text { search (richness of the da- } \\
\text { ta for the review research } \\
\text { questions)? }\end{array}$ & Low risk \\
\hline
\end{tabular}

Ganz 2015

$\begin{array}{ll}\text { Methods } & \begin{array}{l}\text { Qualitative interviews using semi-structured and unstructured, conversational strategies and online } \\ \text { survey that measured similar themes to the interview data }\end{array}\end{array}$
survey that measured similar themes to the interview data

Participants

Setting: DV agencies and coalitions; 12 participants self-identified that they worked in rural communities, 7 worked within suburban areas, and 3 were employed in urban settings 
Inclusion criteria: purposive sampling technique, providing direct service work within the last 2 years and in paid employment, to concentrate on respondents who were fully engaged in the work of advocacy as their primary occupation

Participant numbers: purposive sampling of 251 local, state, national, and identity-based agencies and coalitions

21 interviewees and 387 survey respondents

Gender: 20 identified as female and 1 identified as male

Age: $23-69$ years of age $(M=42)$

Ethnicity: racially, 1 participant identified as Mestizo, 1 identified as Native American, and 19 identified as white.

Experience: participants' length of time spent as a direct-service advocate spanned from 1 to $>30$ years $(M=9)$, while working between 4 and $50 \mathrm{~h}$ a week providing services to victims of gendered violence $(M=26)$.

\begin{tabular}{ll}
\hline Interventions & No details - various advocacy \\
\hline Notes & Data collection began on 4 January 2014 and concluded on 31 May 2014 \\
\hline
\end{tabular}

\section{Risk of bias}

\begin{tabular}{lll}
\hline Bias & Authors' judgement & Support for judgement \\
\hline $\begin{array}{l}\text { How valuable is the re- } \\
\text { search (richness of the da- } \\
\text { ta for the review research } \\
\text { questions)? }\end{array}$ & High risk & Rich data though only relevant to some parts of review \\
\hline
\end{tabular}

Was there a clear state- $\quad$ Low risk
ment of the aims of the re-
ment of the aims of the research?

Was the research design Low risk
appropriate to address the
ss the aims of the research?

\begin{tabular}{|c|c|c|}
\hline $\begin{array}{l}\text { Was the recruitment strat- } \\
\text { egy appropriate to the } \\
\text { aims of the research? }\end{array}$ & Low risk & $\begin{array}{l}\text { Maximal diversity sampling of } 251 \text { agencies, also sought out several racial and } \\
\text { religious identity-based coalitions in order to reach a wider and more repre- } \\
\text { sentative sample. None of these organisations responded }\end{array}$ \\
\hline
\end{tabular}

Has the relationship be- $\quad$ Low risk
tween researcher and par-
ticipants been adequately
considered?

\begin{tabular}{l} 
Is a qualitative methodol- Low risk \\
ogy appropriate? \\
\hline $\begin{array}{l}\text { Have ethical issues been } \\
\text { taken into consideration? }\end{array}$ \\
$\begin{array}{l}\text { Were the data collected in } \quad \text { Low risk } \\
\text { a way that addressed the } \\
\text { research issue? }\end{array}$ \\
\hline
\end{tabular}


Ganz 2015 (Continued)

Was the data analysis suf- Low risk ficiently rigorous?

Is there a clear statement Low risk

of findings?

Garcia-Leeds 2017

\begin{tabular}{ll}
\hline Methods & Qualitative interviews \\
\hline Countricipants USA & Setting: community \\
Inclusion criteria: employee in the LDVP and to provide direct services to the users \\
Exclusion criteria: none \\
Participant numbers: 5 counsellors and 2 supervisors of LDVP \\
Ethnicity: 4 participants were from Puerto Rico and the remaining 3 participants were born in the \\
the parents of 1 of them were from Puerto Rico. \\
English speaking; 5 fluent in Spanish 2 could have basic conversation \\
Experience: average time in the programme was 4.8 months and the standard deviation was 3.5 \\
months
\end{tabular}

Education: high school diplomas to master's degrees

Interventions an organization providing the Latino community of Philadelphia with social services targeted at victims of domestic abuse

\section{Notes}

\section{Risk of bias}

\begin{tabular}{|c|c|}
\hline Bias & Authors' judgement Support for judgement \\
\hline $\begin{array}{l}\text { How valuable is the re- } \\
\text { search (richness of the da- } \\
\text { ta for the review research } \\
\text { questions)? }\end{array}$ & High risk \\
\hline
\end{tabular}

\section{Was there a clear state- Low risk} ment of the aims of the research?

Was the research design Low risk
appropriate to address the
aims of the research?

Was the recruitment strat- Low risk
egy appropriate to the
aims of the research?

Has the relationship be- Low risk tween researcher and par- 
Garcia-Leeds 2017 (Continued) ticipants been adequately considered?

Is a qualitative methodol- Low risk
ogy appropriate?

Have ethical issues been
taken into consideration?

taken into consideration?

Were the data collected in Low risk

a way that addressed the

research issue?

\title{
Was the data analysis suf- Low risk \\ ficiently rigorous?
}

Is there a clear statement Low risk
of findings?
Is there a clear statement Low risk
of findings?

\section{Gillum 2008}

\begin{tabular}{ll}
\hline Methods & Focus groups and individual interviews \\
\hline Participants & Country: USA
\end{tabular}

\section{Participants}

Setting: recruited from a DV programme in a large urban Midwestern city and from a pre-existing National Institute of Mental Health-funded DV research project at a large Midwestern university

Inclusion criteria: African American users of the services

\section{Exclusion criteria: NS}

Participant numbers: 13 African American female survivors of DV and 2 African American female service providers who work primarily with African American female survivors: there were 2 focus groups with survivors, 2 individual interviews with survivors, and a separate paired interview with providers (i.e. the 2 providers in 1 interview)

Interventions

DV programme in a large urban Midwestern city and from a pre-existing National Institute of Mental Health-funded DV research project at a large Midwestern university

\section{Notes}

\section{Risk of bias}

Bias Authors' judgement Support for judgement

How valuable is the re-

Low risk

search (richness of the da-

ta for the review research

questions)?

\begin{abstract}
Was there a clear state-
ment of the aims of the re-

search?
\end{abstract}

Low risk 
Gillum 2008 (Continued)

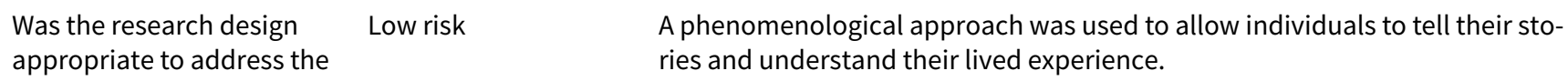
aims of the research?

$\begin{array}{ll}\begin{array}{l}\text { Was the recruitment strat- } \quad \text { High risk } \\ \text { egy appropriate to the }\end{array} & \begin{array}{l}\text { The study gives important insights to the usefulness of culturally sensitive in- } \\ \text { terventions. However, few of the respondents were African American, who } \\ \text { aims of the research? }\end{array} \\ \text { were dissatisfied with the service and left. Views may have been skewed to the } \\ \text { positive. }\end{array}$

Has the relationship be- Low risk tween researcher and par-

ticipants been adequately considered?

Is a qualitative methodol- Low risk
ogy appropriate?

\begin{tabular}{ll}
\hline $\begin{array}{l}\text { Have ethical issues been } \\
\text { taken into consideration? }\end{array}$ & Low risk \\
& $\begin{array}{l}\text { Interviews took place at the site of the participant's choosing. Results from the } \\
\text { thematic analysis were presented to the participants to check accuracy of the } \\
\text { findings. }\end{array}$
\end{tabular}

Were the data collected in Low risk

a way that addressed the

research issue?

\section{Was the data analysis suf- Low risk \\ ficiently rigorous?}

Is there a clear statement Low risk
of findings?

of findings?

Gillum 2009

Design: random experiment with random allocation to groups
Randomisation method: papers with assignments were placed in sealed opaque envelopes and shuf-
fled, women chose a sealed envelope to determine their group assignment.
Analysis by ITT: no
Power calculation: yes

Participants
Setting: a primary care clinic for the uninsured
Country: USA

\section{Inclusion criteria}

- Women aged $>18$ years

- English speaking

- Identified as abused by an intimate partner in last 12 months

\section{Exclusion criteria}

- Women aged $<18$ years

- Could not speak English

- Not experiencing current (last 12 months) abuse 
Gillum 2009 (Continued)

Participant numbers: numbers recruited: 41 (21 intervention group, 20 control group); number of dropouts: 2 ( 2 intervention group, 0 control group); numbers analysed (and percentage of recruited): 39 (19 (90\%) intervention group, 20 (100\%) control group)

Age: mean 43 years (range 23-65 years)

Ethnicity: $83 \%$ African American, $15 \%$ white, 2\% Latina

Socioeconomic status indicators: $39.5 \%$ unemployed, $58.5 \%$ employed, $2 \%$ disabled

Education: $15 \%<$ high school, $41.5 \%$ high school/GED test/trade school, $41.5 \%$ college, $2 \%$ other

Abuse: $56 \%(n=23)$ experienced physical abuse and 95\% ( $n=39)$ nonphysical abuse; $34 \%(n=14)$ of the sample met the criteria for being in lethal danger.

Relationship status: half the participants were single, 7 reported being currently married

Interventions

\section{Intervention}

- 1-1 personalised counselling session provided by the investigators (a discussion around safety-promoting behaviours and women's self-identified individual needs); typical session lasted $30 \mathrm{~min}$

- Series of 6 phone calls over 3 months (at weeks 1, 2, 4, 6, 8, and 10) conducted by a trained CHW (consisted of goal setting, discussion of safety- promoting behaviours, and identifying needs); duration of calls ranged from $5 \mathrm{~min}-1 \mathrm{~h}$, with an average duration of $20 \mathrm{~min}$

\section{Control}

Women received health information brochures and a list of community resources. They were also telephoned by the $\mathrm{CHW}$ once a month, but this was only to confirm contact information for ease of follow-up CES-D. $61 \%(n=25)$ met the criteria for PTSD as assessed by the DTS.

Other: stage of change: 5 women (12.2\%) in pre-contemplation, $12(29.3 \%)$ in contemplation, 4 (9.7\%) in preparation, $10(24.4 \%)$ in action, and $10(24.4 \%)$ in maintenance

Data collected 2005

No external funding

\section{Risk of bias}

\begin{tabular}{lll}
\hline Bias & Authors' judgement & Support for judgement \\
\hline $\begin{array}{ll}\text { Random sequence genera- } \\
\text { tion (selection bias) }\end{array}$ & Low risk & $\begin{array}{l}\text { Quote: "Papers with either intervention or control group assignments were } \\
\text { placed in privacy 'no show through' envelopes, sealed, and the envelopes } \\
\text { shuffled by a nurse colleague not associated with the study. After informed } \\
\text { consent, women were randomly allocated to either the intervention group or } \\
\text { usual care group via their choice of a sealed envelope with group assignment } \\
\text { inside" }\end{array}$
\end{tabular}

\begin{tabular}{|c|c|c|}
\hline $\begin{array}{l}\text { Allocation concealment } \\
\text { (selection bias) }\end{array}$ & Low risk & $\begin{array}{l}\text { Neither the investigators nor the participants knew which envelopes con- } \\
\text { tained which assignments. }\end{array}$ \\
\hline
\end{tabular}

\begin{tabular}{lll}
\hline $\begin{array}{l}\text { Blinding (performance } \\
\text { bias and detection bias): } \\
\text { all outcomes }\end{array}$ & High risk & $\begin{array}{l}\text { Blinding of participants and key personnel (people providing the intervention) } \\
\text { was not possible. The outcome assessors also collected baseline data and pro- } \\
\text { vided the initial face-to-face counselling session, hence assessment was not } \\
\text { blinded. }\end{array}$ \\
\hline $\begin{array}{l}\text { Incomplete outcome da- } \\
\text { ta (attrition bias): all out- } \\
\text { comes }\end{array}$ & Low risk & $\begin{array}{l}2 / 21(10 \%) \text { missing from intervention group, } 0 / 20(0 \%) \text { missing from control } \\
\text { group. Reason given for attrition was that the participants moved out of the } \\
\text { catchment area for eligibility at the clinic. The dropouts were not significant- } \\
\text { ly different in demographic variables from the participants remaining in the } \\
\text { study }\end{array}$ \\
\hline \hline
\end{tabular}

A realist review of which advocacy interventions work for which abused women under what circumstances (Review) 
Gillum 2009 (Continued)

Selective reporting (re- High risk 1 of the 2 stated outcomes of interest was not reported in the 'Results'. porting bias)

Baseline measurement of Low risk outcome measures

There were no significant differences on any of the outcome measures (including the mediating variables)

\begin{tabular}{|c|c|c|}
\hline $\begin{array}{l}\text { Reliability of outcome } \\
\text { measures }\end{array}$ & Low risk & Outcome measures were reliable \\
\hline $\begin{array}{l}\text { Protection against conta- } \\
\text { mination }\end{array}$ & High risk & $\begin{array}{l}\text { There was only } 1 \text { clinic serving a relatively small population, so contact be- } \\
\text { tween the women was possible. In addition, intervention and control 'care' } \\
\text { within the clinic was provided by the same people, and the com- munity health } \\
\text { worker made all intervention phone calls, as well as the monthly call to con- } \\
\text { firm contact details for controls }\end{array}$ \\
\hline
\end{tabular}

Goodman 2009

Methods Qualitative interviews

Participants

Country: USA

Setting: urban community

Inclusion criteria: women who helped to facilitate workshops for other women in the community were eligible to work with an advocate and be recruited

Participant numbers: of the 8 women who had become workshop facilitators by the start of this research, all had worked with an advocate, and 7 agreed to participate in this study.

Experience: participants had served as ROAD facilitators for 6 months-3 years. 2 had worked with an advocate for 1 year and 5 for 2 years.

Age: $25-58$ years old, with a mean age of 47

Ethnicity: 3 identified as African American, 3 as white, and 1 as biracial.

Relationship status: 2 were married at the time of the interview; and 3 were divorced.

Children: all the women were mothers with either 1 or 2 children.

Socioeconomic status indicators: 4 received supplemental security income or social security disability income. All but 1 qualified for food stamps, and all but 1 lived in public housing.

Depression: 3 participants had therapists. based on peer support, empowerment, and community organising for low-income women living in an urban setting on the East Coast and who were struggling with self-identified symptoms of depression. ROAD comprised 2 major programmes:

- Supportive Action Workshop Series

- 12-session workshop run by women also struggling with self-identified poverty and depressive symptoms (facilitators)

- Open to women who reported these difficulties

- Focused on a range of topics related to depression and poverty

- At the end of each series, participants took part in a social action event, such as advocating for specific pieces of state legislation or conducting a workshop for mental health providers on poverty and mental health. 
- Women who had participated in 2 sets of workshops were eventually able to move into facilitator roles for subsequent workshop series.

- Advocacy Resource Team

- Advocacy component of ROAD

- A mental health counselling student volunteer (master's level) was paired with a "partner." Advocate-partner teams worked together in flexible ways at individual, interpersonal, familial, systemic levels and different forms of support (emotional and instrumental), to alleviate partners' acute crises (e.g. threatened evictions, loss of benefits, debt, layoffs, health problems, parenting difficulties), and facilitate their short- and long-term goals.

- Advocates and their partners worked together to determine the focus of their work, with the women's needs kept central.

- Pairs meet weekly at participants' homes or other easily accessible settings and they may have visited various community agencies.

- In general, advocates worked with their partners for 4-6 h each week for 9 months; after which, partners had the option of continuing with a new advocate.

Notes

Low-income women who were living in an urban setting on the East Coast and who were struggling with self-identified symptoms of depression. Not abuse but relevant data for our theory

\title{
Risk of bias
}

Bias Authors' judgement Support for judgement

How valuable is the re-

High risk

search (richness of the da-

ta for the review research

questions)?

Was the research design Low risk appropriate to address the aims of the research?

Was the recruitment strat- Low risk egy appropriate to the aims of the research?

\begin{tabular}{|c|c|c|}
\hline $\begin{array}{l}\text { Has the relationship be- } \\
\text { tween researcher and par- } \\
\text { ticipants been adequately } \\
\text { considered? }\end{array}$ & Low risk & $\begin{array}{l}\text { Part of the social context that serves as a backdrop for participants' observa- } \\
\text { tions and are thus able to understand women's descriptions at a deeper level } \\
\text { than would be possible otherwise }\end{array}$ \\
\hline
\end{tabular}

Is a qualitative methodol- Low risk
ogy appropriate?

Have ethical issues been Low risk
taken into consideration?

Were the data collected in Low risk
a way that addressed the
research issue?

Was the data analysis suf- Low risk Used constant comparison, reflexivity and other quality checks
ficiently rigorous?

\author{
Is there a clear statement Low risk \\ of findings?
}




\begin{tabular}{|c|c|}
\hline Methods & Survey \\
\hline \multirow[t]{5}{*}{ Participants } & Country: USA \\
\hline & Setting: community \\
\hline & Participant numbers \\
\hline & $\begin{array}{l}\text { - Paper 1: from the original sample of } 388 \text { eliminated data from } 18 \text { participants who responded to }<50 \% \\
\text { of the entire survey, leaving a sample of } 370 \text {. }\end{array}$ \\
\hline & $\begin{array}{l}\text { - Paper 2: from the original sample of } 309 \text {, first eliminated data from the } 4 \text { men who participated in } \\
\text { the study, and from } 4 \text { participants who responded to }<50 \% \text { of the entire survey. Then, excluded } 46 \\
\text { participants who had }>50 \% \text { of unusable responses within at least } 1 \text { of the measures in this study either } \\
\text { due to their endorsement of "not applicable" or a lack of response. The final sample }=255 \text { women }\end{array}$ \\
\hline
\end{tabular}

\section{Socioecomomic status indicators}

- Paper 1: participants' socioeconomic status was mixed, with $38.8 \%$ reporting they attended at least some college or graduated college and $51.6 \%$ reporting that they were unemployed. Half (53.1\%) reported either that they couldn't pay their bills or that they had trouble doing so.

- Paper 2: participants' socioeconomic status was mixed, with about half (55.7\%) reporting they attended at least some college or graduated college, $53.7 \%$ reporting that they were unemployed, and $3 / 4$ (71.4\%) receiving some type of government assistance.

\section{Ethnicity}

- Paper 1: most of the sample (74.9\%) was born in the USA and most (86.2\%) completed the survey in English.

- Paper 2: a racial and ethnic composition of 37.6\% white, $26.7 \%$ black/African American, $24.7 \%$ Hispanic/Latino, and $11 \%$ other. Most of the sample (69.8\%) was born in the USA.

Age

- Paper 1: mean age $36.4(\mathrm{SD} \pm 12.0)$

- Paper 2: mean age 36.1

\section{Children}

- Paper 1: most women (67.6\%) had children; of those, $51.9 \%$ reported that their abuser was their children's father.

- Paper 2: most women (89.8\%) had children; of those, $43.7 \%$ reported that their abuser was their children's father.

\section{Relationship status}

- Paper 1: the majority (87.7\%) identified as heterosexual and $16.5 \%$ reported being in a relationship.

- Paper 2: the majority (88.6\%) identified as heterosexual; $22.4 \%$ reported being in a relationship, $31.6 \%$ of whom reported the relationship was with their abusive partner. states across Midwestern and Northeastern USA. All the programmes provided safety planning, counselling, and referral services to survivors. Most provided emergency shelter, with stay lengths of several weeks to 6 months. Several programmes also offered transitional living programmes, with stay lengths of up to 2 years. 
Goodman 2016a (Continued)

\begin{tabular}{|c|c|c|}
\hline Bias & Authors' judgement & Support for judgement \\
\hline $\begin{array}{l}\text { How valuable is the re- } \\
\text { search (richness of the da- } \\
\text { ta for the review research } \\
\text { questions)? }\end{array}$ & High risk & Includes consideration of mechanisms \\
\hline $\begin{array}{l}\text { Representativeness of the } \\
\text { sample }\end{array}$ & Unclear risk & Convenience sample \\
\hline Adequacy of response rate & Low risk & $255 / 388$ \\
\hline $\begin{array}{l}\text { Missing data within com- } \\
\text { pleted questionnaires }\end{array}$ & High risk & $\begin{array}{l}\text { Paper 1: from the original sample of } 388 \text { eliminated data from } 18 \text { participants } \\
\text { who responded to }<50 \% \text { of the entire survey, leaving a sample of } 370 \text {. }\end{array}$ \\
\hline & & $\begin{array}{l}\text { Paper } 2 \text { From the original sample of } 309 \text {, first eliminated data from the } 4 \text { men } \\
\text { who participated in the study, and from } 4 \text { participants who responded to }< \\
50 \% \text { of the entire survey. Then, excluded } 46 \text { participants who had }>50 \% \text { of un- } \\
\text { usable responses within at least } 1 \text { of the measures in this study either due to } \\
\text { their endorsement of "not applicable" or a lack of response }\end{array}$ \\
\hline
\end{tabular}

Established validity of the Low risk survey instrument

Goodman 2016b

Methods Qualitative focus groups $\times 3$

Participants

Country: USA

Setting: community

Participant numbers: 28 DV service advocates working in 1 of the 14 agencies belonging to 1 state's Domestic and Sexual Violence Coalition

Gender: all participants were women

Ethnicity: all but 1 (a Native American woman) were white.

Experience: average number of years at their jobs was 12.9, with a range of $<1$-35 years

Interventions

Provided community education and outreach, emergency shelter and/or transitional housing, support groups, and direct service to survivors living in the community. All built on an empowerment philosophy that involved informing survivors of the community- based programmes and legal strategies available to them and then helping them access the resources they needed. Given the small size of each programme, all programme administrators, with no exception, also provided direct services to survivors.

\section{Notes}

\section{Risk of bias}

Bias Authors' judgement Support for judgement

How valuable is the re-

High risk

search (richness of the da- 
Goodman 2016b (Continued) ta for the review research questions)?

\section{Was the research design appropriate to address the aims of the research?}

Convenience sample. Out of 33 executive directors, shelter managers, and direct service co-ordinators in the state, a total of 28 participated in 1 of the 3 focus groups: 10 executive directors, 10 shelter directors, and 8 direct service coordinators egy appropriate to the aims of the research?

Low risk

Low risk

\section{Is a qualitative methodol- Low risk ogy appropriate?}

Have ethical issues been Low risk

taken into consideration?

Groups homogeneous in terms of job title to avoid a situation where participants would be in a group with someone who had greater or lesser job-related power

\section{Were the data collected in Low risk}

a way that addressed the

research issue?

Was the data analysis suf- Low risk Included peer review
ficiently rigorous?

Is there a clear statement Low risk

of findings?

\section{Grant 2012}

\begin{tabular}{ll}
\hline Methods & Qualitative interviews \\
\hline Participants & 10 DV advocates in Connecticut, USA \\
\hline Interventions & Advocacy \\
\hline Notes &
\end{tabular}

\section{Risk of bias}

Bias Authors' judgement Support for judgement

How valuable is the re- Low risk search (richness of the da-

ta for the review research questions)?

Was there a clear state- Low risk ment of the aims of the research?

Was the research design Low risk appropriate to address the aims of the research? 
Grant 2012 (Continued)

Was the recruitment strat- Low risk egy appropriate to the aims of the research?

Is a qualitative methodol- Low risk ogy appropriate?

Have ethical issues been Low risk

taken into consideration?

Were the data collected in Low risk a way that addressed the research issue?

Was the data analysis suf- Low risk

ficiently rigorous?

Is there a clear statement Low risk

of findings?

Gupta 2017

Methods Cluster-RCT

Participants

Country: Mexico

Setting: healthcare

Inclusion criteria: aged 18-44 years, in a heterosexual relationship, reporting experiences of physical and/or sexual IPV within the past year in research assistant- administered screening, and were not pregnant or were pregnant in their first trimester.

Exclusion criteria: included having plans to relocate in the next 2 years or having an easily recognisable cognitive impairment

Participant numbers: clinics were eligible to participate if they were Type III clinics, which are larger government-led community health clinics that provide more comprehensive care and services (in comparison to smaller Types I and II clinics). These Type III clinics serve a lower-income population within Mexico City. A total of 60 Type III clinics were assessed for eligibility. 3 clinics were excluded from the study; 2 were eliminated to reduce the threat of contamination (i.e. were located in close proximity to a Ministry of Health hospital that offered IPV programming) and 1 was eliminated given that it was located within a small catchment area with few community services. Of these 57 eligible clinics, 42 were randomly selected using Excel, based on sample size calculations. Health centres selected were stratified by city zone and borough using an Excel file. Health centres were assigned a number using the RAND command to randomly select the health centres. Specifically, to randomly select the 42 health centres, all centres were assigned random numbers in Excel and sorted from smallest to largest; health centres were selected based on city zone and in order of their random number.

Randomised 42 public health clinics in Mexico City to treatment or control arms; 29,947 women presenting at the participating 42 clinics were screened for eligibility; 750 (470 = intervention (in treatment clinics), $480=$ control)

Age: mean 30

Abuse: physical violence 97\%, sexual violence intervention, $39.5 \%$, control 33.9\%; physical and sexual violence, intervention $35.75 \%$ control $31.67 \%$; reproductive coercion, intervention $34.64 \%$ control $34.7 \%$

Children: mean 2 children/woman 
Education: $42 \%$ up to secondary school

Relationship status: 66 intervention and 68 control common law marriage

Socioeconomic status indicators: income: $62 \%$ intervention and 54\% control income < USD 2000/ month

Interventions

\section{Intervention}

In treatment clinics, women received the nurse-delivered session (IPV screening, supportive referrals, health/safety risk assessments) at baseline (T1), and a booster counselling session after 3 months (T2).

\section{Controls}

In control clinics, women received screening and a referral card from nurses.

Timing of follow-up: $\mathrm{T} 1, \mathrm{~T} 2$, and 15 months from baseline.

Notes Data collected 2012-2015

\section{Risk of bias}

\begin{tabular}{lll}
\hline Bias & Authors' judgement & Support for judgement \\
\hline $\begin{array}{l}\text { Random sequence genera- } \\
\text { tion (selection bias) }\end{array}$ & Low risk & $\begin{array}{l}\text { Of } 57 \text { eligible clinics, } 42 \text { were randomly selected using Excel, based on sam- } \\
\text { ple size calculations. Health centres selected were stratified by city zone and } \\
\text { borough using an Excel file. Health centres were assigned a number using the } \\
\text { RAND command to randomly select the health centres. Specifically, to ran- } \\
\text { domly select the } 42 \text { health centres, all centres were assigned random num- } \\
\text { bers in Excel and sorted from smallest to largest; health centres were selected } \\
\text { based on city zone and in order of their random number. }\end{array}$ \\
\end{tabular}

\begin{tabular}{ll}
\hline $\begin{array}{l}\text { Allocation concealment } \\
\text { (selection bias) }\end{array}$ & Low risk \\
\hline $\begin{array}{l}\text { Blinding (performance } \\
\text { bias and detection bias): }\end{array}$ & Low risk \\
all outcomes & \\
\hline
\end{tabular}

\begin{tabular}{|c|c|c|}
\hline $\begin{array}{l}\text { Incomplete outcome da- } \\
\text { ta (attrition bias): all out- } \\
\text { comes }\end{array}$ & Low risk & $\begin{array}{l}\text { ITT analyses were conducted via 3-level random intercepts models to evaluate } \\
\text { the interaction term for treatment status by time }\end{array}$ \\
\hline
\end{tabular}

Selective reporting (re- Low risk
porting bias)

Baseline measurement of Low risk

outcome measures

\begin{tabular}{|c|c|c|}
\hline $\begin{array}{l}\text { Reliability of outcome } \\
\text { measures }\end{array}$ & Unclear risk & Not fully validated, but were piloted \\
\hline $\begin{array}{l}\text { Protection against conta- } \\
\text { mination }\end{array}$ & Low risk & $\begin{array}{l}\text { Clinics were eligible to participate if they were Type III clinics, which are larg- } \\
\text { er government-led community health clinics that provide more comprehen- } \\
\text { sive care and services (in comparison to smaller Types I and II clinics). These } \\
\text { Type III clinics serve a lower-income population within Mexico City. A total of } \\
60 \text { Type III clinics were assessed for eligibility. } 3 \text { clinics were excluded from the } \\
\text { study; } 2 \text { were eliminated to reduce the threat of contamination (i.e. were locat- } \\
\text { ed in close proximity to a Ministry of Health hospital that offered IPV program- }\end{array}$ \\
\hline
\end{tabular}




How valuable is the re- Unclear risk Moderate value

search (richness of the da-

ta for the review research

questions)?

Hathaway 2008

\begin{tabular}{ll}
\hline Methods & Structured interviews in English and Spanish \\
\hline Participants & Country: USA \\
Setting: healthcare-based DV advocacy services \\
Inclusion criteria \\
- Able to respond to interviews in English \\
- Had participated in health-care based advocacy for $\geq 6$ months \\
- $\geq 18$ years \\
- $\geq 3$ in-person contacts with programme advocates \\
- Being safe to contact by phone
\end{tabular}

Participant numbers: 49 women

Age: $20-29 n=9(18 \%), 30-39 n=22(45 \%), 40-49 n=1(20 \%), 50-59 n=6(12 \%) \geq 60 n=2(4 \%)$

Ethnicity: white, non-Latino $n=25(51 \%)$, Latino $n=17(35 \%)$, Asian $n=3(6 \%)$, other $n=4(8 \%)$

Relationship status: separated or divorced $n=31(63 \%)$; not married, living apart $n=10(20 \%)$; mar-

ried, living together $n=2(4 \%)$; married, living apart $n=1(2 \%)$; widowed $n=1(2 \%)$

Children: $n=41(84 \%) ;$ multiple abusive partners $n=11(22 \%)$

Abuse: number of years abused by partner $1-3 n=9(18 \%) ; 4-6 n=10(20 \%) ; 7-9 n=5(10 \%) ; \geq 10 n=25$ $(51 \%)$

Emotionally harmed by partner $n=49(100 \%)$; physically hurt by partner $n=39(80 \%)$; coerced/forced sexual activity by partner $n=24(59 \%)$; still experiencing partner abuse $n=23(59 \%)$

\begin{tabular}{ll}
\hline Interventions & Health-care based advocacy \\
\hline Notes & Interviews were conducted August 1999-January 2001 \\
\hline
\end{tabular}

\section{Risk of bias}

Bias Authors' judgement Support for judgement

Was there a clear state- Low risk

ment of the aims of the re-

search?

Was the research design Low risk
appropriate to address the
aims of the research?

Was the recruitmentstrat- Low risk

Was the recruitment strat- Low risk

egy appropriate to the

aims of the research? 
Hathaway 2008 (Continued)

Has the relationship between researcher and participants been adequately considered?
Low risk Yes. Study authors considered the need for participants to feel that they could trust the researchers.

\section{Is a qualitative methodol- Low risk ogy appropriate?}

\section{Have ethical issues been Low risk}

taken into consideration?

\section{Were the data collected in Low risk} a way that addressed the research issue?

\section{Was the data analysis suf- Low risk} ficiently rigorous?
Is there a clear statement
Low risk
of findings?

\section{Hidalgo 2016}

\begin{tabular}{ll}
\hline Methods & Qualitative interviews \\
\hline Participants & $\begin{array}{l}10 \text { DV advocates (e.g. case manager, director, supervisor, and social worker) who worked in New York } \\
\text { City in the social services field, in not-for-profit community organisations }\end{array}$ \\
\hline Interventions & Advocacy \\
\hline Notes & \\
\hline
\end{tabular}

\section{Risk of bias}

Bias Authors' judgement Support for judgement

How valuable is the re- Low risk

search (richness of the da-

ta for the review research

questions)?

\footnotetext{
Was there a clear state-

ment of the aims of the re-

search?
}

Low risk

Was the research design Low risk
appropriate to address the
aims of the research?

Was the recruitment strat- Low risk
egy appropriate to the
aims of the research?
Is a qualitative methodol- Low risk ogy appropriate? 
Hidalgo 2016 (Continued)

Have ethical issues been taken into consideration?

Were the data collected in a way that addressed the research issue?
Low risk

Low risk

Was the data analysis suf- Low risk ficiently rigorous?

Is there a clear statement Low risk

of findings?

Howarth 2016

Methods Case management system (pre-post own controls)

Participants

Country: UK

Setting: community IDVA for crisis advocacy

Inclusion criteria: individuals accessing services were eligible for inclusion in the sample if they

- wished to receive support from the IDVA service and they had experienced recent abuse

- consented to the use of their anonymised information for research and monitoring purposes

- were assessed as being at high risk for further serious abuse.

In practice, survivors were deemed to be at high risk if $10 / 20$ risk factors on a standard risk checklist were endorsed and/or the IDVA perceived the level of risk to be high, irrespective of the risk score. The contribution of data was variable by service, reflecting the relative sizes of the sites.

Participant numbers: only data pertaining to female survivors were retained in the sample $(n=2427$ female survivors at T1). T2 data were available for $48.1 \%$ of the T1 sample $(n=1167)$.

Socioeconomic status indicators: $50.4 \%$ employed

Etthnicity: $73.0 \%$ were white British or Irish with $27.0 \%$ being of BAME status (Asian $=9.2 \%$, black = $7.7 \%$, mixed heritage $=1.8 \%$, other $=8.3 \%$ ).

Age: from $15-83$ years $(M=33.4$ years, $S D=10.4)$

Abuse: experience of physical assault: $83.9 \%$ abuse at the point of referral. $75.9 \%$ of the sample experienced serious, potentially life-threatening levels of abuse (as categorised by an IDVA) $57.4 \%$ of women reported abuse that was escalating in either severity or frequency. $80 \%$ frightened or afraid of being injured $(83.7 \%)$ and just < half of women (44.0\%) were fearful of being killed.

Experience of psychological abuse: nonphysical forms of abuse were also prevalent with the majority $(86.6 \%)$ subject to the perpetrators' jealous and/or controlling behavior; just < half $(48.6 \%)$ reported being harassed or stalked,

Experience of sexual assault: $23.1 \%$ at the point of referral.

Children: $78.1 \%$ had at least 1 child. $27.2 \%$ reported being fearful that their children would be harmed. In $11.1 \%$ of cases, perpetrators had made threats to kill children. $41.3 \%$ of mothers reported that they were contending with ongoing disputes about child contact.

Relationship status: $65.9 \%$ of women reported being abused by an ex-partner and $54.8 \%$ were living separately from the abuser. 
Howarth 2016 (Continued)

Interventions
The IDVA model of intervention is specifically targeted at high-risk individuals. It is designed to be delivered from the point of crisis over a relatively short period of time.

$\mathrm{T} 1$ = baseline, $\mathrm{T} 2$ = either at the closure of a woman's case or after 3 months, as an interim marker of case progress for longer cases (this was substituted for subsequent information gathered at case closure where possible).

Data were not gathered on a second occasion if a woman had disengaged from the service and/or the IDVA had no recent contact with her. Anonymised data were extracted from the case management system and submitted to the evaluation team on a 3-month basis. Unique reference numbers assigned to individuals allowed for the matching of data across time points.

Notes proportion reported having a physical or learning disability (3.7\%).

Data collection: January 2007-March 2009

\section{Risk of bias}

\begin{tabular}{|c|c|c|}
\hline Bias & Authors' judgement & Support for judgement \\
\hline $\begin{array}{l}\text { Random sequence genera- } \\
\text { tion (selection bias) }\end{array}$ & High risk & Not randomised \\
\hline $\begin{array}{l}\text { Allocation concealment } \\
\text { (selection bias) }\end{array}$ & High risk & Not relevant \\
\hline $\begin{array}{l}\text { Blinding (performance } \\
\text { bias and detection bias): } \\
\text { all outcomes }\end{array}$ & High risk & \\
\hline $\begin{array}{l}\text { Incomplete outcome da- } \\
\text { ta (attrition bias): all out- } \\
\text { comes }\end{array}$ & High risk & $\begin{array}{l}\text { Owing to substantial levels of missing data on some of the demographic and } \\
\text { abuse characteristics, descriptive statistics were derived based on those cases } \\
\text { where a response was given (i.e. missing data were excluded). }\end{array}$ \\
\hline $\begin{array}{l}\text { Selective reporting (re- } \\
\text { porting bias) }\end{array}$ & Low risk & \\
\hline $\begin{array}{l}\text { Baseline measurement of } \\
\text { outcome measures }\end{array}$ & Low risk & \\
\hline $\begin{array}{l}\text { Reliability of outcome } \\
\text { measures }\end{array}$ & High risk & Some produced for the study, none were validated measures \\
\hline $\begin{array}{l}\text { Protection against conta- } \\
\text { mination }\end{array}$ & Low risk & Pre-post \\
\hline $\begin{array}{l}\text { How valuable is the re- } \\
\text { search (richness of the da- } \\
\text { ta for the review research } \\
\text { questions)? }\end{array}$ & Unclear risk & Moderate value \\
\hline
\end{tabular}

Hughes 2017

\begin{tabular}{ll}
\hline Methods & Qualitative interviews conducted by telephone \\
\hline Participants & Country: Canada \\
\hline
\end{tabular}

A realist review of which advocacy interventions work for which abused women under what circumstances (Review) 
Setting: various shelters across rural Manitoba

Participant numbers: 6 women's advocates and 6 shelter residents

Ethnicity: 2 of the 6 women's advocates identified as white and of European descent, another as German and the fourth as white. The other 2 women described themselves as Métis and Mennonite.

Experience: advocates had varying levels of experience working within a women's shelter, from 9 months to 16 years in a variety of different positions including counsellors, follow-up counsellors (after women leave the shelter), and children's counsellors. Most had counselling degrees from community colleges (applied counselling and social service worker degrees) with some university-level courses. They had also attended many workshops and professional development training courses, for example, nonviolent crisis intervention and mediation training.

Age: 2 of the 6 residents were about 60 years old. The 4 remaining women were $30-40$ years old.

Ethnicity: 3 women identified as white and of European descent. 1 identified as Mennonite, another as German, 1 as Metis.

Children: 2 women had adult children, 3 had their children with them at the shelter, while the 4th woman did not have children.

\begin{tabular}{ll}
\hline Interventions & Shelter advocacy, details not specified \\
\hline Notes & $\begin{array}{l}\text { Information about the study and its purpose was distributed to each of the executive directors who } \\
\text { then in turn provided this information to shelter advocates and former residents. In determining which } \\
\text { residents to recruit, the executive directors were asked to contact women who had recently left the } \\
\text { shelter to live on their own (not return to partners) and those who they believed would be able to com- } \\
\text { plete an interview about their time at the shelter without re-experiencing trauma. }\end{array}$ \\
\hline
\end{tabular}

\section{Risk of bias}

\begin{tabular}{lll}
\hline Bias & Authors' judgement & Support for judgement \\
\hline $\begin{array}{l}\text { How valuable is the re- } \\
\text { search (richness of the da- } \\
\text { ta for the review research } \\
\text { questions)? }\end{array}$ & High risk & $\begin{array}{l}\text { The study is highly relevant to this review in examining the practices in shel- } \\
\text { ters and including the views of women in the making of the research }\end{array}$ \\
\hline
\end{tabular}

Was there a clear state- Low risk ment of the aims of the research?

Was the research design Low risk
appropriate to address the
aims of the research?

\begin{tabular}{|c|c|c|}
\hline $\begin{array}{l}\text { Was the recruitment strat- } \\
\text { egy appropriate to the } \\
\text { aims of the research? }\end{array}$ & High risk & $\begin{array}{l}\text { Via shelter directors as gatekeepers, there may be selection bias, skewed to- } \\
\text { wards more positive/successful experiences }\end{array}$ \\
\hline
\end{tabular}

Has the relationship be- $\quad$ High risk
tween researcher and par-
ticipants been adequately
considered?
Is a qualitative methodol- Low risk ogy appropriate?


Hughes 2017 (Continued)

Were the data collected in Unclear risk Interviews conducted by telephone. Accuracy of data dependent on accurate a way that addressed the recall of events by participants research issue?

Was the data analysis suf- Low risk Content analysis. Drafts were also read by participants.

ficiently rigorous?

Is there a clear statement Low risk

of findings?

Hyman 2001

Methods

Randomisation method: a 1:1 ratio using random numbers table

Analysis by ITT: no

Power calculation: yes, but dropout higher than expected

\section{Participants}

Country: USA

Setting: ED of level-1 trauma centre

\section{Inclusion criteria}

- Women

- Aged $>18$ years

- Self-identified as currently involved in an abusive relationship

\section{Exclusion criteria}

- Could not speak English

- $<7$ th grade education

- Unable to answer questions

- Intoxicated

- Already in shelter

- Experience of trauma unrelated to their abuse within the last year

\section{Participant numbers}

- Numbers recruited: 102 (51 intervention group, 51 control group)

- Number of dropouts: 49 (20 intervention group, 29 control group)

- Numbers analysed (and percentage of recruited): 53 (31 (61\%) intervention group, $22(43 \%)$ control group)

Age: mean 31 years

Ethnicity: $68 \%$ non-white; $32 \%$ white

Socioeconomic status indicators (annual household income): 19\% < USD 5000; 24\% USD

$6000-10,000 ; 25 \%$ USD 11,000-20,000, 32\% > USD 21,000 (values not discrete)

Education: $11 \%$ < high school; $39 \%$ high school diploma/GED test; $25 \%$ some college, $25 \%$ college degree

Interventions

\section{Intervention}

EDVA programme based on empowerment counselling (empathic support, education, safety planning, linkage with community resources, 48 -h follow-up) to enable the woman to assess her situation, help her to identify signs of danger, and to establish 'back-ups' if the violence escalated; typically, the intervention lasted $1.5 \mathrm{~h}$

Control

Women received standard Social Service care 
Hyman 2001 (Continued)

Notes

Both EDVA and standard social service care already established in ED at time of evaluation $19 \%$ of intervention group received control group care and $23 \%$ of control group received the intervention

\section{Risk of bias}

\begin{tabular}{|c|c|c|}
\hline Bias & Authors' judgement & Support for judgement \\
\hline $\begin{array}{l}\text { Random sequence genera- } \\
\text { tion (selection bias) }\end{array}$ & Low risk & Participants were randomised on a 1:1 ratio using a random numbers table \\
\hline $\begin{array}{l}\text { Allocation concealment } \\
\text { (selection bias) }\end{array}$ & Unclear risk & $\begin{array}{l}\text { No information was provided about who performed the allocation or the pro- } \\
\text { cedure used }\end{array}$ \\
\hline $\begin{array}{l}\text { Blinding (performance } \\
\text { bias and detection bias): } \\
\text { all outcomes }\end{array}$ & Low risk & $\begin{array}{l}\text { Blinding of participants and key personnel (person providing the intervention) } \\
\text { was not possible. Assessors were not provided with any information about ran- } \\
\text { domisation status at either follow-up }\end{array}$ \\
\hline $\begin{array}{l}\text { Incomplete outcome da- } \\
\text { ta (attrition bias): all out- } \\
\text { comes }\end{array}$ & High risk & $\begin{array}{l}\text { High attrition warrants caution in interpreting the study results: } 20 / 51(39 \%) \\
\text { missing from intervention group; } 29 / 51(57 \%) \text { missing from control group. Pri- } \\
\text { mary reasons given for attrition were that participants either could not be con- } \\
\text { tacted or withdrew (not reported by trial arm; withdrawal could have been re- } \\
\text { lated to intervention type). Initial randomisation status did not differentiate } \\
\text { between completing and non-completing participants }\end{array}$ \\
\hline $\begin{array}{l}\text { Selective reporting (re- } \\
\text { porting bias) }\end{array}$ & Low risk & $\begin{array}{l}\text { All measures discussed in the 'Methods' of the thesis were also discussed in } \\
\text { the 'Results' }\end{array}$ \\
\hline $\begin{array}{l}\text { Baseline measurement of } \\
\text { outcome measures }\end{array}$ & Low risk & $\begin{array}{l}\text { No specific information on between-group differences was provided, although } \\
\text { the means and standard deviations seemed comparable }\end{array}$ \\
\hline $\begin{array}{l}\text { Reliability of outcome } \\
\text { measures }\end{array}$ & Low risk & Outcome measures with usable data were reliable \\
\hline $\begin{array}{l}\text { Protection against conta- } \\
\text { mination }\end{array}$ & High risk & $\begin{array}{l}19 \% \text { of women in the intervention arm of the trial only received usual care, } \\
\text { and } 23 \% \text { of women in the control group received the intervention }\end{array}$ \\
\hline
\end{tabular}

Infanti 2015

\begin{tabular}{|c|c|}
\hline Methods & $\begin{array}{l}\text { Individual interviews with women and group interviews, and a participatory workshop with staff to dis- } \\
\text { cuss findings, at health clinic; incorporated visual prompts into the staff conversations, such as the im- } \\
\text { age of abuse }\end{array}$ \\
\hline
\end{tabular}

Participants $\quad$ Country: Sri Lanka
Setting: tea estates

Participant numbers: 31 public health midwives in 6 separate groups, Sinhala-speaking

7 pregnant women at the health clinics; women were randomly chosen by the midwives attending group discussions; some were living with DV, others not.

Experience: some of the midwives were recently appointed to the role, but many had nearly 30 years of experience in the profession.

Interventions No official protocol but midwives respond to need in their partner abuse advocacy work


Infanti 2015 (Continued)
Notes
Data collected over 2 months in late 2014

\section{Risk of bias}

Bias Authors' judgement Support for judgement

How valuable is the re- High risk

search (richness of the da-

ta for the review research

questions)?

Was there a clear state- Low risk
ment of the aims of the re-
search?

\begin{tabular}{|c|c|c|}
\hline $\begin{array}{l}\text { Was the research design } \\
\text { appropriate to address the } \\
\text { aims of the research? }\end{array}$ & Low risk & Used participatory methods \\
\hline $\begin{array}{l}\text { Was the recruitment strat- } \\
\text { egy appropriate to the } \\
\text { aims of the research? }\end{array}$ & Unclear risk & Staff recruited appropriately but some women were not abused \\
\hline
\end{tabular}

\section{Has the relationship be- Low risk}

tween researcher and par-

ticipants been adequately considered?

Is a qualitative methodol- Low risk
ogy appropriate?

Have ethical issues been Low risk
taken into consideration?
Were the data collected in Low risk
a way that addressed the
research issue?

\section{Was the data analysis suf- Low risk}

ficiently rigorous?

Is there a clear statement Low risk
of findings?

\section{Johnson 2014}

Methods

Telephone interviews, open- and closed-ended questions, and focus group on solutions to issues identified in interviews

\section{Country: USA}

Setting: rural Mississippi Delta

Participant numbers: of the 37 eligible advocates i.e. in the area, able to interview 25 victim advocates $(67.5 \%)$ who worked at 20 of the 28 advocacy locations (71.4\%) - in shelters and other various justice agencies serving 16 disadvantaged and rural counties 
Gender: all female

Age: $28-69($ median $=43)$

Ethnicity: $76 \%$ white

Education: $92 \%$ had at least some college

Socioeconomic status indicators: $62 \%$ considered themselves middle or upper-middle class

Experience: working in the field anywhere between 10 months and 32 years (median $=9$ years). Most were from shelter services $(n=17)$ while there was 1 advocate who worked within a police department and 7 legal advocates serving DV victims. Of the legal advocates, 6 worked in the state's attorney's office while 1 worked for a legal clinic serving economically disadvantaged DV victims. The shelter advocates were located in 7 different locations. The shelters were for victims of DV, and sexual assault and rape.

\begin{tabular}{ll}
\hline Interventions & Varied types of advocacy \\
\hline Notes &
\end{tabular}

\section{Risk of bias}

Bias Authors' judgement Support for judgement

How valuable is the re- Low risk search (richness of the data for the review research questions)?

$\begin{aligned} & \text { Was there a clear state- } \\ & \text { ment of the aims of the re- } \\ & \text { search? }\end{aligned}$
$\begin{aligned} & \text { Was the research design } \\ & \text { appropriate to address the } \\ & \text { aims of the research? }\end{aligned}$
aims of the research?

Was the recruitment str
egy appropriate to the aims of the research?

Has the relationship beHigh risk tween researcher and participants been adequately considered?

Is a qualitative methodol- Low risk
ogy appropriate?

\section{Have ethical issues been High risk}

taken into consideration?

Were the data collected in Low risk a way that addressed the research issue? 
Johnson 2014 (Continued)

Was the data analysis suf- Low risk ficiently rigorous?

Is there a clear statement Low risk

of findings?

Kapur 2017

\begin{tabular}{ll} 
Methods & Interviews \\
\hline Participants $\quad$ Country: USA
\end{tabular}

Participants

Setting: 14 non-profit organisations providing services to Asian Indian women experiencing DV; 10 provided direct services to clients and 4 were legal/advocacy organisations

Participant numbers: 26 advocates; 16 interviewed face-to-face, 5 in face-to-face group setting, and 5 participants interviewed on the phone

Ethnicity: English was a second language for most participants. Hence, it can be inferred that most of the participants were not native Americans

\begin{tabular}{ll}
\hline Interventions & Varied \\
\hline Notes & First selected organisations based on purposeful sampling, using 2 criteria: \\
- & organisations working with Asian Indian clients \\
- & organisations located in the states with the highest population of Asian Indians. \\
& These criteria were met by 12 organisations located in California, New Jersey, New York, and Texas, \\
the 4 states that account for almost 50\% of the total Asian Indian population residing in the USA, then \\
snowball sampling
\end{tabular}

\section{Risk of bias}

Bias Authors' judgement Support for judgement

Was there a clear state- Low risk

ment of the aims of the re-

search?

Was the research design Low risk

appropriate to address the

aims of the research?

\begin{tabular}{|c|c|c|}
\hline $\begin{array}{l}\text { Was the recruitment strat- } \\
\text { egy appropriate to the } \\
\text { aims of the research? }\end{array}$ & Unclear risk & $\begin{array}{l}\text { Not clear. Women were recruited from service providers, but the methods of } \\
\text { recruitment are not known }\end{array}$ \\
\hline
\end{tabular}

aims of the research?

High risk

Has the relationship be-

tween researcher and par-

ticipants been adequately

considered?

Is a qualitative methodol- Low risk

ogy appropriate? 
Kapur 2017 (Continued)

Have ethical issues been Low risk taken into consideration?

Were the data collected in Unclear risk a way that addressed the

Unclear risk Partly. Some interviews were conducted by phone so confidentiality/safety research issue? could not be guaranteed. Study would have been limited to women with a fair command of English, although lack of English was considered risk factor

Was the data analysis suf- Low risk

ficiently rigorous?

Is there a clear statement Low risk

of findings?

Kelly 1999

Methods A 3-phase, multi-methodological strategy, including: participant observation; in-depth interviewing; project database creation and maintenance; and questionnaires. Police officers, service users and local agencies were surveyed. An action research model was also used, in order that findings could inform subsequent development.

Participants Participant numbers: DVM provided crisis intervention responses to 1236 individuals, in relation to 1542 incidents.

Interventions

Domestic Violence Matters (DVM) was an adaption of a Canadian project - the Family Consultancy Service (London, Ontario) and involved locating a team of skilled civilian crisis interveners within the police service to follow up police responses to domestic violence. Crisis intervention was available $16 \mathrm{~h} /$ day (10 am-2 am), 7 days/week, 52 weeks/year. DVM was to be called in the event of arrest, in the hope that support would increase the woman's ability and willingness to pursue prosecution, and was to be offered to all other victims.

Notes DVM went 'live' in February 1993 and the pilot involved a total of 32 months direct service.

\section{Risk of bias}

Bias Authors' judgement Support for judgement

How valuable is the re- High risk

search (richness of the da-

ta for the review research

questions)?

Was there a clear state- Low risk

ment of the aims of the re-

search?

\section{Was the research design Low risk}

appropriate to address the

aims of the research?

Was the recruitment strat- Unclear risk NS
egy appropriate to the
aims of the research?

Has the relationship be-
tween researcher and par-


Kelly 1999 (Continued) ticipants been adequately considered?

\section{Is a qualitative methodol- Low risk ogy appropriate?}

\begin{tabular}{ll}
$\begin{array}{l}\text { Have ethical issues been } \\
\text { taken into consideration? }\end{array}$ & Unclear risk \\
\hline $\begin{array}{l}\text { Were the data collected in } \\
\text { a way that addressed the } \\
\text { research issue? }\end{array}$ &
\end{tabular}

\begin{tabular}{lll}
\hline $\begin{array}{l}\text { Was the data analysis suf- } \\
\text { ficiently rigorous? }\end{array}$ & Unclear risk \\
\hline $\begin{array}{l}\text { Is there a clear statement } \\
\text { of findings? }\end{array}$ & Unclear risk & Most of the women's views were from survey answers. Few direct quotes \\
\hline
\end{tabular}

\section{Kendall 2009}

\begin{tabular}{|c|c|}
\hline Methods & Observational study using a convenience sample \\
\hline \multirow[t]{5}{*}{ Participants } & $\begin{array}{l}\text { Country: USA } \\
\text { Setting: hospital ED in a mixed-class, multicultural city; the study hospital was an urban level-1 trau- } \\
\text { ma centre }\end{array}$ \\
\hline & Participant numbers: 360 patients, $350(97 \%)$ women and $10(3 \%)$ men \\
\hline & $\begin{array}{l}\text { Ethnicity: with Latin descent being used as a "race," the racial composition of ED patients during the } \\
\text { study was } 55 \% \text { African American, } 31 \% \text { white (Caucasian), } 10 \% \text { Latin origin, } 1 \% \text { Asian, } 3 \% \text { other. The } \\
\text { racial breakdown of the participating victims was as follows: } 219 \text { (64\%) African American, } 88(26 \%) \\
\text { white, } 28(8 \%) \text { Latin, } 4(1 \%) \text { Asian, and } 3(1 \%) \text { mixed races, with race not documented in } 18 \text { women }\end{array}$ \\
\hline & Age: mean victim age was $32 \pm$ SD years \\
\hline & $\begin{array}{l}\text { Relationship status: } 205 \text { (62\%) heterosexual partners, } 69 \text { (21\%) spouses, } 52(15 \%) \text { ex-partners, and } 5 \\
(2 \%) \text { homosexual partners/other, with relationship data not documented in } 29 \text { women }\end{array}$ \\
\hline Interventions & $\begin{array}{l}\text { ED patients with risk factors were offered consultation with trained IPV advocacy counsellors who com- } \\
\text { pleted safety assessments, provided resource referrals, and helped participants develop safety plans. } \\
\text { Participants were contacted after ED intervention to assess progress and further assist in IPV coun- } \\
\text { selling. }\end{array}$ \\
\hline Notes & $\begin{array}{l}\text { Conducted from } 1 \text { October 2002-1 August 2004; the study commenced shortly after the inception of a } \\
\text { new ED Domestic Violence Healthcare Project (DVHP), which was linked to a large local community ser- } \\
\text { vice organisation. }\end{array}$ \\
\hline
\end{tabular}

\section{Risk of bias}

\begin{tabular}{lll}
\hline Bias & Authors' judgement & Support for judgement \\
\hline $\begin{array}{l}\text { Random sequence genera- } \\
\text { tion (selection bias) }\end{array}$ & High risk & Convenience sample \\
\hline
\end{tabular}

Allocation concealment High risk (selection bias) 
Kendall 2009 (Continued)

Blinding (performance bias and detection bias): all outcomes
High risk

\begin{tabular}{|c|c|c|}
\hline $\begin{array}{l}\text { Incomplete outcome da- } \\
\text { ta (attrition bias): all out- } \\
\text { comes }\end{array}$ & High risk & Took steps to reduce this part way through study \\
\hline $\begin{array}{l}\text { Selective reporting (re- } \\
\text { porting bias) }\end{array}$ & Low risk & \\
\hline $\begin{array}{l}\text { Baseline measurement of } \\
\text { outcome measures }\end{array}$ & Low risk & \\
\hline $\begin{array}{l}\text { Reliability of outcome } \\
\text { measures }\end{array}$ & High risk & \\
\hline $\begin{array}{l}\text { Protection against conta- } \\
\text { mination }\end{array}$ & Low risk & Not relevant \\
\hline $\begin{array}{l}\text { How valuable is the re- } \\
\text { search (richness of the da- } \\
\text { ta for the review research } \\
\text { questions)? }\end{array}$ & Low risk & Low value \\
\hline
\end{tabular}

Kenyon 2016

\begin{tabular}{ll}
\hline Methods & Interviews by shelter (ex-)volunteer \\
\hline Participants & Country: Canada \\
Setting: shelter & Participant numbers: 9 \\
Inclusion criteria: shelter residents aged $>18$ years, residing in shelter for $\geq 10$ days \\
Age: mean participant age 28.8 (range 23-39) \\
Ethnicity: 2 of the women identified as white, 2 identified as Métis, 4 identified as Status First Nations, \\
and 1 woman was an immigrant from South Asia \\
Education: ranged from grade 10 to some college \\
Children: 7 of the nine women had children, and 1 was pregnant for the first time. Of the mothers, 2 \\
had experienced the death of a child. \\
Abuse: the number of times the participants had previously been in shelter ranged from zero to 4 \\
Shelter stay and advocacy \\
\hline Interventions
\end{tabular}

\section{Risk of bias}

Bias Authors' judgement Support for judgement


Kenyon 2016 (Continued)

How valuable is the research (richness of the data for the review research questions)?
Unclear risk The study contributes to the understanding of women's own definition of empowerment and experiences of entering the shelter

\section{Was there a clear state- Low risk} ment of the aims of the research?

\section{Was the research design appropriate to address the aims of the research?}

Was the recruitment strat- Low risk egy appropriate to the aims of the research?
Low risk

(a)
This is a PhD thesis and small in scope. The author had been a volunteer at the shelter although author states that selection bias unlikely as this had been on and off. A purposive sampling method was used to reflect a range of women and experiences
Yes: the author had been a volunteer at the shelter although author states that selection bias unlikely as this had been on and off tween researcher and participants been adequately considered?

\section{Is a qualitative methodol- Low risk} ogy appropriate?

Have ethical issues been Low risk
taken into consideration?

Were the data collected in Low risk a way that addressed the research issue?

Was the data analysis suf- Low risk ficiently rigorous?

Is there a clear statement Low risk
of findings?

of findings?

\section{Kiely 2010}

\section{Methods}

Randomisation method: a computer-generated, permuted block randomisation scheme that considered all possible risk combinations within each recruitment site

Analysis by ITT: no (study authors state it was ITT as analyses based on original allocations and not on care received, but only data for the women completing the study were analysed)

Power calculation: yes

\section{Participants}

Setting: 6 community-based prenatal care sites serving mainly minority women

\section{Country: USA}

\section{Inclusion criteria}

- English speaking

- Female residents of District of Columbia

- Self-identified as minority (African American) 
Kiely 2010 (Continued)

- Aged $\geq 18$ years

- $\leq 28$ weeks pregnant

- Screening positive for at least 1 of 4 risk factors (IPV, depression, cigarette smoking, environmental tobacco smoke exposure), so women did not have to be abused, but findings in this paper are specific to abused subset. No details on any inclusion criteria for the clinics

Exclusion criteria: women excluded if suicidal, also "participation exclusion"

Participant numbers: 1044 women (521 intervention group, 523 control group), with 336 abused at baseline and analysed as a subset (169 intervention group, 167 control group); number of dropouts: 30 abused subset (19 intervention group, 11 control group); numbers analysed (and percentage of recruited): 306 abused subset (150 (89\%) intervention group, 156 (93\%) control group)

Age: mean 25 years (intervention and control group)

Ethnicity: all African American

Socioeconomic status indicators: working now or before pregnancy: $74 \%$ intervention group, $76 \%$ control group; enrolled in Medicaid: $80 \%$ intervention group, $78 \%$ control group; WIC (Women, Infants, and Children) supplemental nutrition programme recipient: $44 \%$ intervention group, $46 \%$ control group; other supplemental food programme recipient: $99 \%$ intervention group, $97 \%$ control group; public assistance/TANF (Temporary Assistance for Needy Families) recipient: $43 \%$ intervention group, $41 \%$ control group

Education: < high school: 32\% intervention group, 32\% control group; high school graduate/GED: 46\% intervention group, $40 \%$ control group; some college: $23 \%$ intervention group, $28 \%$ control group

Interventions

\section{Intervention}

Multi-component intervention: 4 interventions depending on risk:

- advocacy for abuse

- CBT for depression

- cessation/reduction therapy for smoking or passive smoking

- IPV intervention was based on empowerment theory and emphasised safety behaviours: - information about types of abuse and cycle of violence

- a danger assessment to assess risks and consider preventive options

- development of safety plan

- and a list of community resources

- delivered over 4-8 prenatal sessions plus 2 postpartum booster sessions, each session averaged $50 \pm$ $15 \mathrm{~min}$ (depending on how many of the 4 risk conditions were addressed)

\section{Control}

Women received usual prenatal care, as determined by the standard procedures at the each prenatal care clinic

\section{Risk of bias}

Bias Authors' judgement Support for judgement

Random sequence genera- Low risk tion (selection bias)
Quote: "Site- and risk-specific permuted block randomisation to the intervention or usual care was conducted. A computer generated randomisation scheme was utilised to consider all the possible risk combinations within each of the recruitment sites"

\begin{tabular}{ll}
\hline $\begin{array}{l}\text { Allocation concealment } \\
\text { (selection bias) }\end{array}$ & Wow risk \\
& $\begin{array}{l}\text { When a woman was recruited “... staff would call the data coordinating cen- } \\
\text { and field workers were blinded to block size }\end{array}$
\end{tabular}


Kiely 2010 (Continued)

\begin{tabular}{|c|c|c|}
\hline $\begin{array}{l}\text { Blinding (performance } \\
\text { bias and detection bias): } \\
\text { all outcomes }\end{array}$ & Low risk & $\begin{array}{l}\text { Blinding of participants and key personnel (person providing the intervention) } \\
\text { was not possible. Baseline data were collected pre-randomisation. Follow-up } \\
\text { data were collected by telephone interviewers blinded to participants' ran- } \\
\text { domisation group (as were their supervisors) }\end{array}$ \\
\hline
\end{tabular}

\begin{tabular}{|c|c|c|}
\hline $\begin{array}{l}\text { Incomplete outcome da- } \\
\text { ta (attrition bias): all out- } \\
\text { comes }\end{array}$ & Unclear risk & $\begin{array}{l}19 / 169 \text { (11\%) missing from intervention group; } 11 / 167(7 \%) \text { missing from con- } \\
\text { trol group. Reasons given for attrition not stated, although completers ran- } \\
\text { domised to the intervention did not differ significantly from completers in the } \\
\text { control group }\end{array}$ \\
\hline $\begin{array}{l}\text { Selective reporting (re- } \\
\text { porting bias) }\end{array}$ & Low risk & $\begin{array}{l}\text { All measures discussed in the 'Methods' section of the paper were also dis- } \\
\text { cussed in the 'Results' section }\end{array}$ \\
\hline $\begin{array}{l}\text { Baseline measurement of } \\
\text { outcome measures }\end{array}$ & Low risk & $\begin{array}{l}\text { The investigators stated that the groups did not differ on any of the abuse sub- } \\
\text { scales (pregnancy outcomes not relevant at baseline) }\end{array}$ \\
\hline $\begin{array}{l}\text { Reliability of outcome } \\
\text { measures }\end{array}$ & Low risk & Outcome measures were reliable \\
\hline $\begin{array}{l}\text { Protection against conta- } \\
\text { mination }\end{array}$ & Unclear risk & $\begin{array}{l}\text { There might have been communication between women in different arms of } \\
\text { the trial, as individual clinics recruited women allocated to intervention and } \\
\text { control groups }\end{array}$ \\
\hline
\end{tabular}

Kolb 2008

\begin{tabular}{ll}
\hline Methods & Non-participant ethnography and interviews \\
\hline Participants & $\begin{array}{l}\text { The shelter director and a family counsellor worked in a shelter, co-director worked in a small office in } \\
\text { the next town and the others all worked at the main agency office }\end{array}$ \\
\hline Interventions & Advocacy at organisation in the US called SAFE (Stopping Abuse in Family Environments) \\
\hline Notes &
\end{tabular}

\section{Risk of bias}

\begin{tabular}{|c|c|}
\hline Bias & Authors' judgement Support for judgement \\
\hline $\begin{array}{l}\text { How valuable is the re- } \\
\text { search (richness of the da- } \\
\text { ta for the review research } \\
\text { questions)? }\end{array}$ & High risk \\
\hline
\end{tabular}

\section{Was there a clear state- Low risk} ment of the aims of the research?

Was the research design Low risk
appropriate to address the
aims of the research?

Was the recruitment strat- Low risk egy appropriate to the aims of the research? 
Kolb 2008 (Continued)

Has the relationship be-
tween researcher and par-
ticipants been adequately

Low risk considered?

\section{Is a qualitative methodol- Low risk ogy appropriate?}

Have ethical issues been Low risk
taken into consideration?

Were the data collected in Low risk
a way that addressed the
research issue?

\begin{tabular}{l}
$\begin{array}{l}\text { Was the data analysis suf- Low risk } \\
\text { ficiently rigorous? }\end{array}$ \\
\hline $\begin{array}{l}\text { Is there a clear statement } \quad \text { Low risk } \\
\text { of findings? }\end{array}$
\end{tabular}

\section{Krasnoff 2002}

$\begin{array}{ll}\text { Methods } & \begin{array}{l}1 \text { group post-test only. Retrospective chart review and use of a standardised middle form. The project } \\ \text { co-ordinator reviewed medical records to obtain ED data, which were collected without the patient's } \\ \text { name. }\end{array}\end{array}$

Country: USA
Setting: ED of hospital
Inclusion criteria: all English-speaking women aged $18-65$ years presenting $24 \mathrm{~h}$ a day, 7 days a week.
Participant numbers: 6939 (22\%) women were screened for IPV. A total of 528 women representing
562 ED visits disclosed that they had experienced physical IPV within the past year ( $2 \%$ of the popula-
tion of women).
Of the 528 women identified as IPV victims, 475 ( $84 \%)$ agreed to speak to the advocate, and 258 (54\% of
those seen by the advocate) accepted case management follow-up.
Age: mean ( \pm SD) 31.8 (8.6)
Ethnicity: number (\%) black 320 (61\%); white 184 (35\%); other 24 (4\%)
Socioeconomic status indicator: number (\%) Medicaid 224 (42\%); Medicare 2 (1\%); none 197 (37\%);
private 104 (20\%)
On-site IPV advocacy intervention. Once IPV was identified by means of screening or self-disclosure, the
nurse notified a volunteer advocate from a local human service agency, who came to the ED within 30
min, conducted a crisis intervention, and encouraged the woman to follow-up with the case manager;
telephone-based counselling by an IPV case manager to help the client reduce her exposure to addi-
tional violence.
Follow-up after the case management process, lasting 3-6 weeks

Notes

From 1 July 1997-31 December 1999

\section{Risk of bias}

A realist review of which advocacy interventions work for which abused women under what circumstances (Review) 
Krasnoff 2002 (Continued)

\begin{tabular}{lll} 
Bias & Authors' judgement & Support for judgement \\
\hline $\begin{array}{l}\text { Random sequence genera- } \\
\text { tion (selection bias) }\end{array}$ & High risk & 1 group pre-post \\
\hline
\end{tabular}

\section{Allocation concealment High risk}

(selection bias)

Blinding (performance High risk
bias and detection bias):
all outcomes

all outcomes

Incomplete outcome da- High risk Low numbers agreed to be followed up
ta (attrition bias): all out-
comes

\begin{tabular}{|c|c|c|}
\hline $\begin{array}{l}\text { Selective reporting (re- } \\
\text { porting bias) }\end{array}$ & Low risk & \\
\hline $\begin{array}{l}\text { Baseline measurement of } \\
\text { outcome measures }\end{array}$ & Low risk & \\
\hline $\begin{array}{l}\text { Reliability of outcome } \\
\text { measures }\end{array}$ & High risk & \\
\hline $\begin{array}{l}\text { Protection against conta- } \\
\text { mination }\end{array}$ & Low risk & No control \\
\hline $\begin{array}{l}\text { How valuable is the re- } \\
\text { search (richness of the da- } \\
\text { ta for the review research } \\
\text { questions)? }\end{array}$ & Low risk & Low value \\
\hline
\end{tabular}

Kulkarni 2015

Methods Cross-sectional survey. Pilot at stage 1, statewide, followed by national survey, stage 2

Participants Country: USA

Setting: statewide IPV service provider organisations in the South and Southwestern USA. IPV service providers were recruited through targeted and snowball sampling Inclusion criteria: all respondents were current employees of an IPV service organisation and had been for a minimum of 6 months

\section{Participant numbers}

- Stage 1: 260 responded to the survey link. Of those, 13 were not eligible, 11 were not included in analysis because they had missing data on $>5 \%$ of the survey items. Thus the final sample analysed comprised 236 respondents

- Stage 2: 660 respondents; but 99 respondents not in analysis because $>5 \%$ of the survey items were missing. Thus the final sample was 561.

\section{Ethnicity}

- Stage 1 : about $2 / 3$ of the sample $(n=158)$ indicated they were white; $20.9 \%(n=49)$ Hispanic; $13.4 \%$ $(n=32)$ African American; and just over $2 \%$ other 
- Stage $2: 75.2 \%$ identified as white $(n=422) ; 7.5 \%$ as Latina $(n=42) ; 5.9 \%$ as African American $(n=33)$; 2. $\%$ as Asian ( $n=13) ; 2.5 \%$ as American Indian $(n=14)$; and $4.6 \%$ as other $(n=26)$

Age: approximately half were aged 31-50, with the remainder almost evenly split between those aged $\leq$ 30 and those $>50$

\section{Education:}

- Stage 1: $28 \%$ had < a bachelor's degree, 38\% held a bachelor's degree, and about 1/3 had a Master's degree or higher

- Stage 2: almost $40 \%$ had a graduate degree, $37 \%$ had a bachelor's degree, and the remainder had < a bachelor's degree.

\section{Experience}

- Stage 1: $49.0 \%$ reported working in their current agencies for $\geq 5$ years, 38.9\% from 1-5 years, and $12.3 \%$ for $<1$ year; $30.2 \%$ were advocates or case managers, $24 \%$ were counsellors, $21.5 \%$ administrators, $16.5 \%$ shelter personnel, $6.6 \%$ outreach/educators, with $33.9 \%$ working with clients $<15 \mathrm{~h} /$ week, $34.3 \%$ working with them $15-30 \mathrm{~h} /$ week, and $31.8 \%$ working directly with clients $>30 \mathrm{~h} /$ week. Almost half (46.8 \%) worked in a private nonprofit IPV agency, $38.7 \%$ worked in private nonprofit social service agencies serving clients with various needs, and $14.5 \%$ worked in a publicly operated and funded IPV-specific social service

- Stage 2: $25 \%$ were administrators, $19.1 \%$ shelter personnel, $14.4 \%$ counsellors, $14.1 \%$ advocates and $26.9 \%$ outreach/educators; $23.7 \%$ reported working in their current agencies for $\leq 1$ year, and only $11.7 \%$ had worked in IPV services for $<1$ year; $37.6 \%$ had worked in their current positions from 1-5 years, and about $1 / 3$ had worked in the IPV arena for $1-5$ years; $40 \%$ had worked in their agencies for $>5$ years, and $60 \%$ had worked in the IPV field for $>5$ years. Almost $1 / 3$ of the sample had worked in IPV services for $>10$ years. Around $41 \%$ reported working with clients $<15 \mathrm{~h} /$ week, $27 \%$ were with clients $15-30 \mathrm{~h} /$ week, and about $1 / 3>30 \mathrm{~h} /$ week. $49.6 \%$ worked in a private nonprofit IPV agency, and $33.0 \%$ worked in private nonprofit social service agencies serving clients with various needs. Only $3.6 \%$ worked in public IPV agencies.

In all 3.4\% worked in professional legal service agencies, $2.5 \%$ worked in a law enforcement agency, $7.7 \%$ chose "Other".

\begin{tabular}{ll}
\hline Interventions & Survivor-defined advocacy not otherwise specified \\
\hline Notes & Funding not reported
\end{tabular}

\section{Risk of bias}

\begin{tabular}{lll}
\hline Bias & Authors' judgement & Support for judgement \\
\hline $\begin{array}{l}\text { Representativeness of the } \\
\text { sample }\end{array}$ & Unclear risk & $\begin{array}{l}\text { Good range of respondents; nationwide staff survey, but representativeness } \\
\text { was unclear given that the population sample (all staff of these services) was } \\
\text { not known }\end{array}$ \\
\hline
\end{tabular}

Adequacy of response rate Unclear risk While a response rate was not available, the study authors say the survey invitation was likely sent to several hundred DV service providers in the 2-state pilot study (final $n=236$ ), and several thousand in the national follow-up study (final $n=561$ ).

\begin{tabular}{lll}
\hline $\begin{array}{l}\text { Missing data within com- } \\
\text { pleted questionnaires }\end{array}$ & Unclear risk & $\begin{array}{l}\text { Missing data in national sample was }<2 \% \text { and imputed using mean substitu- } \\
\text { tion - but all responses (approx. } 1 / 7 \text { th) in the } 2 \text { surveys with }>5 \% \text { missing data } \\
\text { were dropped from analysis to achieve this state }\end{array}$ \\
\hline Conduct of pilot testing & Low risk & $\begin{array}{l}\text { Piloted between } 2 \text { phases. Results from the initial phase revealed a fairly weak } \\
\text { factor structure, thus the second study was undertaken to revise scale items to } \\
\text { refine and strengthen the scale. }\end{array}$
\end{tabular}


Kulkarni 2015 (Continued)

Established validity of the Low risk Appropriate tests survey instrument

Lako 2018

Methods

Multicentre, parallel-group RCT. Analysed data using ITT 3-level mixed-effects models. De Vet reported a fidelity check (see Lako 2018 secondary reference)

Participants

Country: Netherlands

Setting: shelters

Inclusion criteria: women were eligible if they:

- were aged $>18$

- stayed at the shelter due to IPV or honour-related violence (violence committed to restore or prevent violation of the family honour)

- stayed at the shelter for $>6$ weeks

- had a set date of departure from the shelter or received priority status for social housing

- were moving to housing without daily supervision or support where they would have to pay rent or housing costs

Exclusion criteria: moving to a region where none of the participating organisations provided services

Participant numbers: 9 women's shelters across the Netherlands. Of 486 women assessed for eligibility, 239 (49\%) women were eligible. 136 women assigned to intervention (CTI) ( $n=70)$ or control (careas-usual) $(n=66)$. Fidelity assessment for a representative subsample of 35 women assigned to intervention

Ethnicity: Dutch-speaking and non-Dutch-speaking women (first generation migrants, totaling 56\% of the intervention group and $65 \%$ of the control group, were from Morocco (22\%), Turkey (15\%), Iran (6\%), Poland (5\%), Surinam (5\%) and other countries (11\% Western and 37\% non-Western); second generation migrants contributed $14 \%$ of the intervention and $12 \%$ of the control group). Dutch native: intervention $21(30 \%)$ control 39 (56\%), First-generation migrant, intervention $10(14 \%)$ control 15 (23\%), Second-generation migrant: intervention $43(65 \%)$ control $8(12 \%)$

Age: mean 34

Abuse: prior to shelter:

- emotional: intervention $n=68(100 \%)$ control $n=62(98 \%)$

- physical: intervention $n=52(77 \%)$ control $n=52(83 \%)$

- sexual: intervention $n=30(44 \%)$ control $n=17(27 \%)$

Duration of violence, years $\leq 1$ : intervention $n=6(9 \%)$ control $n=10(16 \%) ; 1-5$ years intervention $n=$ $34(51 \%)$ control $n=30(48 \%) ; 5-10$ years intervention $n=14(21 \%)$ control $n=13(21 \%), \geq 10$ intervention $n=13(19 \%)$ control $n=9(15 \%)$

More women in the intervention group experienced sexual violence

Children: $90 \%$ of the intervention group and $89 \%$ of controls had children

Education: low: intervention $47(68 \%)$ control $16(23 \%)$; intermediate: intervention 6 (9\%) control 41 (63\%); high: intervention 14 (22\%) control $10(15 \%)$

Relationship status: the control group contained more married women 
Lako 2018 (Continued)

CTI is a time-limited, strengths-based intervention designed to support vulnerable people during transitions in their lives by expanding their social and professional networks, and reassuring continuity of care and support during the transition. Case managers trained in CTI provide practical and emotional support during the transition. At least 3 meetings with client and her children (if any) before discharge, with no more than 1 month between each meeting ( $10 \mathrm{~h}$ in total) between discharge and 3 months after discharge.

- Phase 1

- Transition to the community - aims to build a relationship by working in the community; assess client's needs and resources; choose priority areas of intervention; mobilise support resources and link client to them

- Risk and needs assessment, strengths assessment, personal recovery plan, activity log

- Average of $3 \mathrm{~h} /$ week ( $36 \mathrm{~h}$ in total)

- Phase 2

- 3-6 months after discharge

- Less frequent contact, with personal recovery plan, activity log and optional strengths assessment.

- Average of $2 \mathrm{~h} /$ week ( $24 \mathrm{~h}$ in total)

- Phase 3

- Transfer of care: 6-9 months after discharge

- Transfer client to other services with personal recovery plan and activity log and optional risk and needs assessment, strengths assessment

- Average of $0.5-1 \mathrm{~h} /$ week $(6-12 \mathrm{~h}$ in total)

\section{Control}

Care-as-usual; all organisations provided services after discharge, except for 1 that referred women returning to their (ex-)- partner to other services. Most organisations provided support during regular meetings (range of average intensity: 1-3 h/week), average duration range: $13-52$ weeks. All organisations used a strengths-based approach.

Notes

Fidelity was measured using the CTI fidelity scale. The scale items are rated on a 5-point scale ranging from not implemented (1) to ideally implemented (5). The intervention in this study obtained a fidelity score of 3, indicating that CTI was fairly implemented

\section{Risk of bias}

\begin{tabular}{lll} 
Bias & Authors' judgement & Support for judgement \\
\hline $\begin{array}{l}\text { Random sequence genera- } \\
\text { tion (selection bias) }\end{array}$ & Low risk & $\begin{array}{l}\text { Computer random number generator. Randomisation was stratified by shelter } \\
\text { with a } 1: 1 \text { allocation in blocks of } 4 \text {. The numbers were saved in a secured digi- } \\
\text { tal file and concealed until assignment. }\end{array}$ \\
\hline $\begin{array}{l}\text { Allocation concealment } \\
\text { (selection bias) }\end{array}$ & Unclear risk & $\begin{array}{l}1 \text { of the researchers ascertained condition assignment and informed shelter } \\
\text { staff. Shelter staff and the research assistant who scheduled the baseline in- } \\
\text { terviews were unaware of condition assignments in advance. Women were } \\
\text { unaware of condition assignment until they met their CTI worker/case man- } \\
\text { ager. Condition assignment also witheld from the research assistants who } \\
\text { conducted follow-up interviews, but some became aware of the assigned con- } \\
\text { dition of a few women because the women told them about the services re- } \\
\text { ceived. }\end{array}$
\end{tabular}

$\begin{aligned} & \text { Blinding (performance } \\ & \text { bias and detection bias): } \\ & \text { all outcomes }\end{aligned}$
$\begin{aligned} & \text { Incomplete outcome da- } \\ & \begin{array}{l}\text { ta (attrition bias): all out- } \\ \text { comes }\end{array}\end{aligned}$


Lako 2018 (Continued)

Selective reporting (re- Low risk porting bias)

Baseline measurement of Low risk

outcome measures

\begin{tabular}{ll}
\hline $\begin{array}{l}\text { Reliability of outcome } \\
\text { measures }\end{array}$ & Low risk \\
& $\begin{array}{l}\text { Women who did not speak Dutch were interviewed by multilingual research } \\
\text { preters by translating the interview via telephone (baseline: } 24 \% ; 9 \text { months: }\end{array}$ \\
& $23 \%$ ). Because the translation would considerably lengthen these interviews, a \\
shortened version of the questionnaire was used, but for quality-of-life scale at & baseline and 9 months, Cronbach's alpha was 0.61 and 0.80, respectively. \\
& But re-abuse question not validated
\end{tabular}

Protection against conta- Low risk Randomised by shelter
mination

How valuable is the re- High risk High value

search (richness of the da-

ta for the review research

questions)?

Lea 2016

Methods Case file analysis, surveys and interviews: service user and service providers' perspectives

\section{Participants}

Country: UK

Setting: 3rd sector domestic abuse service, community legal advice service

Participant numbers: case-file analysis ( $n=86 ; 1$ was male (1.2\%): surveys $(n=22)(25.6 \%$ response rate) and interviews $(n=12)$ with victims, and interviews with key individuals $(n=12)$ based in related statutory and community organisations.

The following data are from case files only.

Ethnicity: the majority in case file analysis ( $n=80,93 \%$ ) self-classified as white British; 6 victims had diverse ethnic backgrounds.

Age: $20-60$ years, mean 38 years $(S D=9.66)$

Education: to school level (76.7\%), 19.8\% had achieved college or further education, and $2.3 \%$ had attended university; data were missing for 1 participant.

Socioencomic status indicators: using the Office for National Statistics (ONS) Standard Occupation Classification System (2000), participants were employed in a diverse range of jobs from manual to professional. A small number were unemployed (11.6\%), 'stay-at-home mums' (9.3\%), or students (7\%); in $12.8 \%$ of cases, no data

Relationship status: married (51.2\%) or unmarried (38.4\%) partner

Abuse: $92 \%$ suffered multiple forms of abuse, often in combination, $8 \%$ reported psychological abuse exclusively.

Children: $58 \%$ had children (either their own, $12 \%$; the perpetrator's, 3.7\%; or their joint offspring, $83.3 \%$; 1 child was a grandchild of the perpetrator). Victims had 108 children among them, ranging in age from a few months to 18 years. Just over half the children (53.3\%) were reported to have suffered physical, psychological, or a combination of abuse. Thirty participants $(34.9 \%)$ indicated that children were present at the time of their own abuse. 
Lea 2016 (Continued)

Interventions

\section{Intervention}

The Domestic Abuse Advocacy Project (DAAP), a specialist domestic abuse service that sought to offer entirely free specialist legal advice and fast, effective civil action (where appropriate) in conjunction with more holistic victim-focused care and advocacy.

Victims were referred to the DAAP by a range of statutory and 3rd sector organisations including the police (although civil or criminal action was not a prerequisite for service entry) and women's organisations. Victims met with the caseworker to establish their needs and consider their options. The number of meetings varied according to the needs of the victim from a single meeting to a set of meetings, and victims could self-refer back to the service as needed thereafter. The caseworker provided information, facilitated decision making, and supported the decision, which was wholly the victim's, irrespective of the route that decision took. Due to her specialist training, the caseworker could provide informed, empathic support and practical and appropriate legal advice, and facilitate interagency work to address the victim's diverse needs. Thus, referrals were made to other services, such as housing and the police, as appropriate.

Notes just partner abuse; other familial perpetrators were the participant's child (4.7\%), sibling (2.3\%),
parent (1.2\%), parent-in-law (1.2\%), and ex-spouse (1.2\%). Funding: study authors received no financial
support

\section{Risk of bias}

Bias Authors' judgement Support for judgement

How valuable is the re-

High risk

search (richness of the da-

ta for the review research questions)?

\section{Was there a clear state- Low risk} ment of the aims of the research?

\section{Was the research design Low risk} appropriate to address the aims of the research?

Was the recruitment strat- Low risk egy appropriate to the aims of the research?

Has the relationship be- Low risk
tween researcher and par-
ticipants been adequately
considered?

Is a qualitative methodol- Low risk ogy appropriate?

\begin{tabular}{|c|c|c|}
\hline $\begin{array}{l}\text { Have ethical issues been } \\
\text { taken into consideration? }\end{array}$ & Unclear risk & $\begin{array}{l}\text { Anonymity and confidentiality were critical, with implications for how partic- } \\
\text { ipants were contacted and where interviews took place. It is not clear how it } \\
\text { was established that it was safe to send information on the project by post. }\end{array}$ \\
\hline
\end{tabular}

\footnotetext{
Were the data collected in Low risk a way that addressed the

research issue? 
Lea 2016 (Continued)

Was the data analysis suf- Low risk ficiently rigorous?

Is there a clear statement Low risk

of findings?

Logan 2018

Methods Qualitative: focus groups with advocates

Participants

Country: USA

Setting: large combined shelter/rape crisis agency in the largest city in the state, a statewide agency that provides case management for individuals referred, but independent, from the state Child Protective Services agency, and through a community co-ordinating council in the second largest city in the state.

Participant numbers: 5 focus groups with 37 participants

Gender: 33 women and 4 men

Age: $23-63$ years, with an average age of 40 years $(S D=11.3)$

Ethnicity: mostly white (91.9\%) with 5.4\% who identified as black, and 2.7\% identified as Middle Eastern.

Experience: overall, $56.8 \%$ had a Master's degree and 37.8\% had a bachelor's degree. Experience ranged from 6 months-32 years (average of 9.3 years' experience, $S D=8.66$ )

\begin{tabular}{|c|c|}
\hline Interventions & Various not specified \\
\hline \multirow[t]{2}{*}{ Notes } & $\begin{array}{l}2 \text { focus groups were conducted at } 1 \text { shelter location on } 2 \text { different occasions in } 2016 \text { and the other } 3 \text { fo- } \\
\text { cus groups were conducted in a public space (an urban county government conference room, } 2 \text { differ- } \\
\text { ent extension office conference rooms, } 1 \text { in the rural eastern part of the state and } 1 \text { in the rural western } \\
\text { part of the state). }\end{array}$ \\
\hline & Funding: Department of Behavioral Science at the University of Kentucky \\
\hline
\end{tabular}

\section{Risk of bias}

Bias Authors' judgement Support for judgement

How valuable is the re-

High risk

search (richness of the da-

ta for the review research

questions)?

Was the research design
appropriate to address the
aims of the research?

Low risk

aims of the research?

Was the recruitment strat- Low risk
egy appropriate to the
aims of the research?
Has the relationship be- $\quad$ Low risk
tween researcher and par-


Logan 2018 (Continued) ticipants been adequately considered?

\section{Is a qualitative methodol- Low risk ogy appropriate?}

\section{Were the data collected in Low risk a way that addressed the} research issue?

Was the data analysis suf- Low risk ficiently rigorous?

Is there a clear statement Low risk of findings?

Lynch 2013

Methods Focus groups with workers who assisted women seeking Apprehended Domestic Violence Orders (ADVOs) in New South Wales

\begin{tabular}{ll}
\hline Participants & Country: Australia \\
& Setting: community \\
& Participant numbers: 24 participants working in organisations including women's refuges, family sup- \\
port services, DV outreach services, solicitors, and DV Court Advocacy Services \\
No other details
\end{tabular}

\section{Risk of bias}

Bias Authors' judgement Support for judgement

How valuable is the re-
search (richness of the da-
ta for the review research
questions)?

Was there a clear state-
ment of the aims of the re-
search?

Was the research design Low risk
appropriate to address the
aims of the research?

\begin{tabular}{l}
$\begin{array}{l}\text { Was the recruitment strat- Unclear risk } \\
\text { egy appropriate to the } \\
\text { aims of the research? }\end{array}$ \\
$\begin{array}{l}\text { Has the relationship be- Unclear risk } \\
\text { tween researcher and par- }\end{array}$ \\
\hline
\end{tabular}


Lynch 2013 (Continued) ticipants been adequately considered?

\begin{tabular}{ll}
\hline $\begin{array}{l}\text { Is a qualitative methodol- } \\
\text { ogy appropriate? }\end{array}$ & Low risk \\
\hline $\begin{array}{l}\text { Have ethical issues been } \\
\text { taken into consideration? }\end{array}$ & Unclear risk NS \\
\hline $\begin{array}{l}\text { Were the data collected in } \\
\begin{array}{l}\text { a way that addressed the } \\
\text { research issue? }\end{array}\end{array}$ & NS lear risk \\
\hline
\end{tabular}

Was the data analysis suf- Unclear risk No methods of analysis stated
ficiently rigorous?

Is there a clear statement Low risk

of findings?

Lyon 2011

Methods Survey over 9 months and 10 focus groups

\section{Participants}

\author{
Country: USA \\ Setting: 90 community domestic violence services in four US states, which were chosen to maximize \\ geographical, population, rural/urban and economic diversity. \\ Inclusion criteria: overall (including advocacy) without stratification by type of service so that we \\ could not separate out advocacy data (though $36 \%$ had received advocacy previously
}

\section{Exclusion criteria: NS}

\section{Participant numbers:}

- Survey: 1467 participants (1408 women, 68 men). Sampling by services type, immediate outcomes, and experiences by race/ethnicity, immigration status, gender and other characteristics of survivors. Response rate: NS

- Focus groups: 10 focus groups with 73 women (not stated whether or not in addition to the 1467 but says sampled survivors from marginalized groups

Age: median 31 to 40 years

Education: $33.4 \%$ had not completed high school or received their GED. The median level of education completed was high school graduate or GED; $18.3 \%$ of survivors were college graduates or held advanced degrees

Ethnicity: Nearly half of survey respondents reported being born outside of the USA. $31 \%$ of surveys were completed in 8 languages other than English. The largest racial/ethnic groups were white/Caucasian (39.2\%), Hispanic/Latino/a (32.1\%), African American/Black (15.1\%), and Asian (6.8\%); these 4 racial/ethnic groups accounted for $93.2 \%$ of respondents. $46 \%$ came to the USA from another country, $5.5 \%$ reported that at least one parent came to the USA from another country.

Gender: $96 \%$ female, $4 \%$ male (of which two were transgender). $93.8 \%$ self-described their sexual orientation as heterosexual/straight.

SES and finance: only $8.1 \%$ reported that they did not need to worry about paying for things they wanted and needed

Other: $21 \%$ in survey reported having a disability or disabling conditions; over one fifth of survivors came from a predominantly rural program 
Lyon 2011 (Continued)

Interventions
Women-specific services; $38 \%$ were independent domestic violence programmes, $23 \%$ stand-alone dual domestic violence and sexual assault programmes and $61 \%$ were part of a national social service or community agency

Notes

\section{Risk of bias}

\begin{tabular}{lll}
\hline Bias & Authors' judgement & Support for judgement \\
\hline $\begin{array}{l}\text { Representativeness of the } \\
\text { sample }\end{array}$ & Low risk & $\begin{array}{l}\text { Research goals included obtaining a large and diverse sample of survivors } \\
\text { and programs, to permit meaningful comparisons of services, immediate out- } \\
\text { comes, and experiences by race/ethnicity, immigration status, gender and } \\
\text { other characteristics of survivors, while also taking program capacity into ac- } \\
\text { count. }\end{array}$ \\
\hline Adequacy of response rate & High risk & NS, but it is possible that the program population size is much greater \\
\hline $\begin{array}{l}\text { Missing data within com- } \\
\text { pleted questionnaires }\end{array}$ & Unclear risk & NS \\
\hline $\begin{array}{l}\text { Conduct of pilot testing } \\
\text { Established validity of the }\end{array}$ & High risk risk & Not done \\
\hline \begin{tabular}{l} 
survey instrument \\
\hline
\end{tabular} & No
\end{tabular}

Was there a clear state- Low risk

ment of the aims of the research?

\begin{tabular}{|c|c|c|}
\hline $\begin{array}{l}\text { Was the research design } \\
\text { appropriate to address the } \\
\text { aims of the research? }\end{array}$ & High risk & $\begin{array}{l}\text { Views were collected via survey with some open-ended questions, and focus } \\
\text { group }\end{array}$ \\
\hline $\begin{array}{l}\text { Was the recruitment strat- } \\
\text { egy appropriate to the } \\
\text { aims of the research? }\end{array}$ & Unclear risk & $\begin{array}{l}\text { Every effort was made to include the views of participants, including provid- } \\
\text { ing translation of surveys, delivered in paper or orally. Focus group questions } \\
\text { were developed to be culturally sensitive. Nonetheless lacked rigorous re- } \\
\text { search design. }\end{array}$ \\
\hline
\end{tabular}

\begin{tabular}{|c|c|c|}
\hline $\begin{array}{l}\text { Has the relationship be- } \\
\text { tween researcher and par- } \\
\text { ticipants been adequately } \\
\text { considered? }\end{array}$ & Unclear risk & $\begin{array}{l}\text { NS; however, every effort was made to include the views of participants, in- } \\
\text { cluding providing translation of surveys, delivered in paper or orally. Focus } \\
\text { group questions were developed to be culturally sensitive. }\end{array}$ \\
\hline
\end{tabular}

Is a qualitative methodol- Low risk ogy appropriate?

Have ethical issues been High risk

taken into consideration?

Were the data collected in Low risk

a way that addressed the

research issue?

Was the data analysis suf- High risk

ficiently rigorous?

Answers to open-ended questions were reviewed for themes, but not examined by formal methods 
Lyon 2011 (Continued)
Is there a clear statement
Low risk
of findings?

Macy 2018

Methods Survey

Participants

Country: USA

Setting: DV agencies

Participant numbers: the study's sampling frame consisted of the list of all directors in the state $(\mathrm{n}=$ 103).

The response rate was $94 \%(n=97)$.

Experience: most participants were agency directors $(n=80,83 \%) .7(7 \%)$ described working in a leadership role at their agencies. $7 \%$ reported they were "advocates" or "educators."

$n=52,54.7 \%$ employed in their current position for $\leq 5$ years, $45 \%$ reported serving in their current position for $>5$ years, $23.2 \%$ reported 6 - 10 years, 5 participants $(5.3 \%)$ reported $11-15$ years, and 16 participants $(16.8 \%)$ reported $\geq 16$ years in their current position.

8 full-time employees, 5 part-time employees, and 38 volunteers. Agency staff numbers ranged from 1-100 full-time employees, 0-20 part-time employees, and 0-300 volunteers.

Most participants worked for agencies that provided both DV and sexual assault services $(n=67$, $69.8 \%)$. Participants also came from single-focused agencies that provided DV services only $(n=18$, $18.8 \%)$ or sexual assault services only $(n=11,11.5 \%)$.

Education: graduate degrees $n=40,41.2 \%$; 4-year college degrees $n=32,32.9 \% ; 14.4 \%$ had completed community college or associate degrees, and 11 (11.3\%) had completed high school and/or some college.

- legal advocacy

- medical advocacy

\section{Content of the intervention:}

- crisis

- support group

- individual counselling

Notes Country: USA

Setting: DV shelters; most sampled agencies provided services to survivors in rural communities ( $\mathrm{n}=$ $66,68.0 \%$ ), whereas 13 agencies (13.4\%) provided services in urban/suburban communities and 16 agencies (16.5\%) provided services to both rural and urban/suburban communities

\section{Risk of bias}

Bias Authors' judgement Support for judgement

How valuable is the re- High risk

search (richness of the da- 
Macy 2018 (Continued)

ta for the review research

questions)?

\section{Was there a clear state- Low risk}

ment of the aims of the re-

search?

Was the research design Low risk

appropriate to address the

aims of the research?

Was the recruitment strat- Low risk

egy appropriate to the

aims of the research?

Has the relationship be-

High risk

tween researcher and par-

ticipants been adequately

considered?

Is a qualitative methodol- Low risk
ogy appropriate?

Were the data collected in Low risk a way that addressed the

research issue?

Was the data analysis suf- Low risk

ficiently rigorous?

Is there a clear statement Low risk

of findings?

Magruder 2017

Methods Mixed methods: an instrument-development variant of a fixed, exploratory, sequential mixed-methods design. Interviews with service providers

Country: USA
Setting: community service provision
Inclusion criteria: victim advocates/victim service providers, law enforcement professionals, prosecu
tors, and batterer intervention programme providers
Participant numbers: 15

\begin{tabular}{ll}
\hline Interventions & Advocacy details not specified \\
\hline Notes & Funding: NS \\
\hline
\end{tabular}

\section{Risk of bias}

Bias Authors' judgement Support for judgement

How valuable is the re-

search (richness of the da-

High risk 
Magruder 2017 (Continued)

ta for the review research

questions)?

\section{Was there a clear state- Low risk}

ment of the aims of the re-

search?

Was the research design Low risk

appropriate to address the

aims of the research?

Was the recruitment strat- Low risk

egy appropriate to the

aims of the research?

Has the relationship be-
tween researcher and par- $\quad$ Low risk Study author discussed credibility and trustworthiness of the researcher

ticipants been adequately

considered?

Is a qualitative methodol- Low risk
ogy appropriate?

Have ethical issues been Low risk

taken into consideration?

\section{Were the data collected in Low risk}

a way that addressed the

research issue?

Was the data analysis suf- Low risk

ficiently rigorous?

Is there a clear statement Low risk

of findings?

Matthew 2016

\begin{tabular}{ll}
\hline Methods & Qualitative interviews \\
\hline Participants & 11 advocates interviewed about Afro-Caribbean abused women in the USA \\
\hline Interventions & Cultural advocacy \\
\hline Notes &
\end{tabular}

\section{Risk of bias}

\section{Bias}

Authors' judgement Support for judgement

Was there a clear state- Low risk ment of the aims of the re-

search? 
Matthew 2016 (Continued)

Was the research design

Low risk appropriate to address the aims of the research?

Was the recruitment strat- Low risk egy appropriate to the aims of the research?
Has the relationship be-
Low risk
tween researcher and par-
ticipants been adequately
considered?
Is a qualitative methodol- Low risk
ogy appropriate?

Have ethical issues been Low risk
taken into consideration?

Were the data collected in Low risk

a way that addressed the

research issue?

\section{McDermott 2004}

\begin{tabular}{ll}
\hline Methods & $\begin{array}{l}\text { Process evaluation, mainly qualitative document analysis, unstructured and structured interviews, and } \\
\text { observations. Limited quantitative data are also presented in the analysis of prosecution of domestic } \\
\text { battery in Jackson County and the evaluation of the Domestic Violence Clinic }\end{array}$ \\
\hline Participants & In urban Carbondale and Jackson County, USA; whole service \\
\hline Interventions & $\begin{array}{l}\text { Coordinated community response to domestic violence including advocacy services for victims as one } \\
\text { of many components }\end{array}$
\end{tabular}

\section{Notes}

\section{Risk of bias}

\begin{tabular}{lll}
\hline Bias & Authors' judgement & Support for judgement \\
\hline $\begin{array}{l}\text { Was there a clear state- } \\
\text { ment of the aims of the re- } \\
\text { search? }\end{array}$ & Low risk & \\
\hline $\begin{array}{l}\text { Was the research design } \\
\text { appropriate to address the } \\
\text { aims of the research? }\end{array}$ & Low risk & $\begin{array}{l}\text { Though the authors are concerned that a mainly qualitative analysis of a small } \\
\text { service limits usefulness }\end{array}$ \\
\hline $\begin{array}{l}\text { Was the recruitment strat- } \\
\text { egy appropriate to the } \\
\text { aims of the research? }\end{array}$ & Low risk & Whole service process evaluation \\
\hline
\end{tabular}

\footnotetext{
Has the relationship be- High risk tween researcher and par-
} 
McDermott 2004 (Continued) ticipants been adequately considered?

\begin{tabular}{lll}
\hline $\begin{array}{l}\text { Is a qualitative methodol- } \\
\text { ogy appropriate? }\end{array}$ & Low risk \\
\hline $\begin{array}{l}\text { Have ethical issues been } \\
\text { taken into consideration? }\end{array}$ & Unclear risk & $\begin{array}{l}\text { There was reporting of individual cases and it was not clear whether these } \\
\text { were identifiable or not }\end{array}$ \\
\hline $\begin{array}{l}\text { Were the data collected in } \\
\begin{array}{l}\text { a way that addressed the } \\
\text { research issue? }\end{array}\end{array}$ & Low risk & A process evaluation \\
\hline $\begin{array}{l}\text { Was the data analysis suf- } \\
\text { ficiently rigorous? }\end{array}$ & High risk & $\begin{array}{l}\text { Findings are from the perspective of service providers with no systematic con- } \\
\text { sideration of alternative explanations }\end{array}$ \\
\hline $\begin{array}{l}\text { Is there a clear statement } \\
\text { of findings? }\end{array}$ & High risk & $\begin{array}{l}\text { The statements of findings are vague, the necessary understanding can only } \\
\text { be obtained by careful reading of the full report }\end{array}$ \\
\hline
\end{tabular}

\section{McFarlane 1997}

\begin{tabular}{ll}
\hline Methods & Quasi-experimental; power calculation \\
\hline Participants & Country: USA
\end{tabular}

\section{Participants}

\section{Country: USA}

Setting: public clinics in Texas and Virginia that offer a variety of health services for women and children

Inclusion criteria: physically or sexually assaulted by their male partners in the year prior to or during their pregnancy; women who considered themselves still in a relationship (primarily because the abuser was the father of the baby)

Participant numbers: 228 women met the eligibility criteria, 12 refused to enter the study and 17 were lost to follow-up, leaving 199 women for analysis. The primary reason given for refusal to enter the study was fear of retaliation by the abuser.

132 in intervention group, 67 in 'control' (brief intervention) group

Ethnicity: 35\% ( $n=70)$ African American, 33\% $(n=66)$ Hispanic (primarily Mexican and Mexican American $)$, and $32 \%(n=63)$ white

Age: $14-42$ years (mean 23.2, SD $=5.64$ )

Education: $2-16$ years $(M=10.4, S D=2.37)$

Socioeconomic status: all had incomes below the poverty level as defined by each state's eligibility criteria for the Women, Infants and Children (WIC) Program.

Children: first pregnancy for $35.2 \%$ of the sample

Relationship status: $45.7 \%$ were married or living with their partner, $29.6 \%$ were separated, $19.1 \%$ were in a relationship but not living together, and 5.5\% reported 'other' relationship status.

\section{3 intervention groups}

- The main intervention comprised 3 education, advocacy, and community referral sessions that included information on safety behaviours.

- Half the intervention group women were invited to attend 3 additional counselling and information sessions by workers at the local shelter 
- 'Brief' - control women offered wallet-sized card with information on community resources and a brochure

Sessions took approximately $30 \mathrm{~min}$ to complete (10 min for the empowerment protocol and $20 \mathrm{~min}$ for data collection).

Notes $29.6 \%$ were teens (aged $\leq 19$ years)

\section{Risk of bias}

\begin{tabular}{|c|c|c|}
\hline Bias & Authors' judgement & Support for judgement \\
\hline $\begin{array}{l}\text { Random sequence genera- } \\
\text { tion (selection bias) }\end{array}$ & High risk & Quasi-experimental \\
\hline $\begin{array}{l}\text { Allocation concealment } \\
\text { (selection bias) }\end{array}$ & High risk & Quasi-experimental \\
\hline $\begin{array}{l}\text { Blinding (performance } \\
\text { bias and detection bias): } \\
\text { all outcomes }\end{array}$ & High risk & Quasi-experimental \\
\hline $\begin{array}{l}\text { Incomplete outcome da- } \\
\text { ta (attrition bias): all out- } \\
\text { comes }\end{array}$ & Low risk & \\
\hline $\begin{array}{l}\text { Selective reporting (re- } \\
\text { porting bias) }\end{array}$ & Low risk & \\
\hline $\begin{array}{l}\text { Baseline measurement of } \\
\text { outcome measures }\end{array}$ & Low risk & $\begin{array}{l}\text { No significant differences between the women in the intervention and com- } \\
\text { parison groups on age, ethnicity, marital status, parity, income, education, } \\
\text { employment status, or number of people in the household }\end{array}$ \\
\hline $\begin{array}{l}\text { Reliability of outcome } \\
\text { measures }\end{array}$ & Low risk & \\
\hline $\begin{array}{l}\text { Protection against conta- } \\
\text { mination }\end{array}$ & Low risk & $\begin{array}{l}\text { The comparison group was recruited first because of concern that the women } \\
\text { in the intervention group would share information with women in the compar- } \\
\text { ison group. }\end{array}$ \\
\hline $\begin{array}{l}\text { How valuable is the re- } \\
\text { search (richness of the da- } \\
\text { ta for the review research } \\
\text { questions)? }\end{array}$ & Low risk & Low value \\
\hline
\end{tabular}

McFarlane 2000

Design: cluster-RCT
Randomisation method: random determination of the specific intervention into which each clinic
would enter women in the first month of the trial, with each clinic rotating through a specified se-
quence of 3 interventions, entering all participants for a given month into the same intervention
Analysis by ITT: no
Power calculation: yes

Setting: 2 antenatal clinics 
McFarlane 2000 (Continued)

\section{Country: USA}

Inclusion criteria: women physically or sexually abused by current or former male partner in year pri-

or to or during pregnancy

Exclusion criteria: none stated

Participant numbers:

Numbers recruited: 329 (118 'outreach', 98 'counselling', 113 'brief')

Number of dropouts: 70 (26 'outreach', 25 'counselling', 19 'brief ')

Numbers analysed (and percentage of recruited): 259 (92 (78\%) 'outreach', 73 (74\%) 'counselling', 94

(83\%) 'brief')

Age: mean 24 years (range 15 to 42 years); $24.4 \%$ were teenagers

Ethnicity: all Latina

Socioeconomic status indicators: $23 \%$ employed; $53 \%$ financially supported themselves; annual

household income: $66 \%<$ USD 10,000; 6\% > USD 20,000

Education: mean 8.4 (SD 3.2) years of education

Children: (66\%) had $\geq 1$ children living with them. The age of youngest child living with the women varied from infancy to age 16 with a mean of 3 years. Some $64 \%$ of the women had at least 1 child < 6 years of age.

Relationship status: $56 \%$ living with the abusive partner

\section{3 intervention groups}

- 'Counselling': unlimited access during clinic opening times to onsite bilingual DV advocate offering support, education, referral, assistance in accessing resources; available by appointment or drop-in for the duration of pregnancy

- 'Outreach': as 'counselling', plus the services of a bilingual trained, non-professional mentor mother offering support, education, referral, assistance in accessing resources; achieved through personal visits and telephone contact

- 'Brief': women offered wallet-sized card with information on community resources and a brochure

(The 'outreach' and 'counselling' interventions are equivalent to other forms of advocacy intervention reported in this review)

(The 'brief ' intervention is equivalent to the control group care provided in other trials reported in this review)

Notes Supported in part by the U.S. CDC, National Center for Injury Prevention and Control, and by the Houston Department of Health and Human Services (HDHHS)

Some were teenagers

\section{Risk of bias}

\section{Bias \\ Authors' judgement Support for judgement}

Random sequence genera- Unclear risk tion (selection bias)
Quote: “... [I]nitiated by randomly determining the specific intervention into which each clinic would enter during the first month of the study ... each clinic then rotated through a specified sequence of the 3 interventions, entering all participants for a given month into the same intervention."

Additionally, the investigators state "the procedure ... did not involve individually randomising women using a technique such as a random numbers table or computer generated random numbers."

No information on the technique actually used was provided.

\begin{tabular}{l}
$\begin{array}{l}\text { Allocation concealment } \\
\text { (selection bias) }\end{array}$ High risk Key personnel may have foreseen the assignments \\
\hline
\end{tabular}


McFarlane 2000 (Continued)

Blinding (performance Unclear risk Blinding of participants and key personnel (person providing the intervention) bias and detection bias): was not possible. No information was provided in relation to the blinding of all outcomes the outcome assessor(s)

\begin{tabular}{|c|c|c|}
\hline $\begin{array}{l}\text { Incomplete outcome da- } \\
\text { ta (attrition bias): all out- } \\
\text { comes }\end{array}$ & Unclear risk & $\begin{array}{l}26 / 118(22 \%) \text { missing from 'outreach' intervention group, } 25 / 98 \text { ( } 26 \%) \text { missing } \\
\text { from 'counselling' intervention group, } 19 / 113(17 \%) \text { missing from 'brief' con- } \\
\text { trol group. Reasons given for attrition were not discussed but } 2 \text { analyses con- } \\
\text { ducted to estimate any effects due to loss of follow-up showed no significant } \\
\text { differences among the groups }\end{array}$ \\
\hline
\end{tabular}

Selective reporting (re- Low risk Both measures discussed in the 'Methods' section of the paper were also disporting bias) cussed in the 'Results' section

\begin{tabular}{|c|c|c|}
\hline $\begin{array}{l}\text { Baseline measurement of } \\
\text { outcome measures }\end{array}$ & Low risk & $\begin{array}{l}\text { The investigators stated that the groups did not differ on any of the outcome } \\
\text { variables }\end{array}$ \\
\hline
\end{tabular}

Reliability of outcome Low risk
measures

The primary outcome (physical and emotional abuse) was reliable. No data were provided on the reliability of the scale used to measure the use of community resources. The investigators stated that recall bias may have resulted from an interruption in funding that delayed the completion of some of the follow-up interviews, but this was not related to trial arm

Protection against conta- Unclear risk
mination

Not addressed explicitly, although the study design may have minimised potential contamination as women in the different arms of the trial were unlikely to meet, and the treatments were not provided by the same people

\section{McFarlane 2004}

\begin{tabular}{ll}
\hline Methods & Quasi-experimental: a 2-group, repeated measures design. Power calculation: yes \\
\hline Participants & Country: USA \\
& Setting: a special family-violence unit of a large urban District Attorney's office that serves an ethnical- \\
& ly diverse population of 3 million \\
Inclusion criteria: all English- or Spanish-speaking women who applied and qualified for a protection \\
order against an intimate partner were invited \\
Participant numbers: 75 women in each of the intervention and control groups
\end{tabular}

Age: intervention 30.25 (SD \pm 7.87$)$; control $34.61(S D \pm 9.91)$

Ethnicity: intervention: African American $n=23$ (30.7\%), white $n=19$ (25.3\%), Latino $n=33$ (44\%); control: African American $n=26$ (34.7\%), white $n=21(28 \%)$, Latino $n=28(37.3)$

Language spoken; English: intervention $n=62(82.7 \%)$, control $n=64(85.3 \%)$; Spanish: intervention $n=$ $13(17.3 \%)$, control $n=11(14.7 \%)$

Relationship status: spouse or common-law spouse: intervention $n=40(53.3 \%)$, control $n=41$ (54.7\%); ex-spouse or ex-common-law spouse: intervention $n=14(18.7 \%)$, control $n=11(14.7 \%)$; girlfriend: intervention $n=5(6.7 \%)$, control $n=7(9.3 \%)$; ex-girlfriend: intervention $n=16(21.3)$, control $n=$ $16(21.2 \%)$

Education: (in years) intervention: 11.35 (SD \pm 2.99$)$, control 12.20 (SD \pm 2.55$)$

Interventions

\section{Intervention}

Face-to-face meeting involving usual services of the District Attorney's office and intervention telephone calls ( 6 telephone calls over 8 weeks).

Intervention call: each call began with the safety-promoting behaviour checklist, noting behaviours adopted since the previous call. During the intervention calls, the investigator discussed specific safe- 
McFarlane 2004 (Continued)

ty-promoting behaviours with the participant, suggesting, for example, that she make extra keys, apply for a driver's license, and obtain copies of documents such as a Social Security card and marriage license. The investigator would also suggest hiding money and documents, in an empty tampon container, for example, or entrusting them to a friend, neighbour, or relative. Discussion also involved creating a code for use with family and friends as a signal for the need for help, removing weapons from the home, and enlisting neighbours to call the police if they overheard an altercation

\section{Control}

Usual services of the family-violence unit of the District Attorney's office, which included counselling on promoting safety and information on social services and legal resources.

Timing of follow-up: $3,6,12$, and 18 months

Notes Funding: NS

\section{Risk of bias}

\begin{tabular}{lll}
\hline Bias & Authors' judgement & Support for judgement \\
\hline $\begin{array}{l}\text { Random sequence genera- } \\
\text { tion (selection bias) }\end{array}$ & Unclear risk & $\begin{array}{l}\text { Not random allocation, but by day of the week. It is not clear if there could be a } \\
\text { systematic bias in this method of allocation }\end{array}$ \\
\hline $\begin{array}{l}\text { Allocation concealment } \\
\text { (selection bias) }\end{array}$ & High risk & $\begin{array}{l}\text { Not known. There may have been some systematic bias related to the day of } \\
\text { the week for allocation - staffing perhaps, payday in the week or the month, } \\
\text { Weekend effect? }\end{array}$ \\
\hline $\begin{array}{l}\text { Blinding (performance } \\
\text { bias and detection bias): } \\
\text { all outcomes }\end{array}$ & High risk & $\begin{array}{l}\text { It would not be possible to blind the researcher or the participant from the in- } \\
\text { tervention }\end{array}$ \\
\hline
\end{tabular}

Incomplete outcome da- Low risk

All but 1 participant was present at the 18-month follow-up

ta (attrition bias): all out-

comes

Selective reporting (re- Low risk Dependent on recall of the participants. Some risk of participant bias
porting bias)

\begin{tabular}{lll}
\hline $\begin{array}{l}\text { Baseline measurement of } \\
\text { outcome measures }\end{array}$ & Low risk & There were few significant differences between the 2 groups \\
\hline $\begin{array}{l}\text { Reliability of outcome } \\
\text { measures }\end{array}$ & High risk & $\begin{array}{l}\text { Safety-planning behaviours very much context dependent, different for each } \\
\text { individual }\end{array}$ \\
\hline $\begin{array}{l}\text { Protection against conta- } \\
\text { mination }\end{array}$ & High risk & $\begin{array}{l}\text { Women were recruited from the same attorney's office. It may be possible for } \\
\text { the women to have known about the intervention. Study authors also report- } \\
\text { ed that there was an element of safety planning in the control group as part of } \\
\text { standard care. }\end{array}$ \\
\hline
\end{tabular}

\begin{tabular}{|c|c|c|}
\hline $\begin{array}{l}\text { How valuable is the re- } \\
\text { search (richness of the da- } \\
\text { ta for the review research } \\
\text { questions)? }\end{array}$ & Unclear risk & $\begin{array}{l}\text { Moderate value in terms of this review. There was a limited amount of advoca- } \\
\text { cy in terms of empowerment or actively connecting the women to or facilitat- } \\
\text { ing access to community resources. }\end{array}$ \\
\hline
\end{tabular}

questions)?

\section{McFarlane 2006}

$\begin{array}{ll}\text { Methods } & \begin{array}{l}\text { Design: cluster-RCT } \\ \text { Randomisation method: allocated by week of recruitment }\end{array}\end{array}$


McFarlane 2006 (Continued)

\section{Analysis by ITT: no \\ Power calculation: yes}

\section{Participants}

Setting: recruited in 2 primary care public health clinics and 2 Women, Infants, and Children clinics (WICs)

Country: USA

\section{Inclusion criteria}

- Women

- Aged 18-44 years

- English or Spanish speaking

- 4Identified by nurse as physically or sexually abused by an intimate partner within last 12 months

Exclusion criteria: none stated

Participant numbers: numbers recruited: 360 (180 intervention group, 180 control group); number of dropouts: 41 (19 intervention group, 22 control group); numbers analysed (and percentage of recruited): 319 (161 (89\%) intervention group, 158 (88\%) control group)

Age: mean 30 years intervention group, mean 31 years control group

Ethnicity: $12 \%$ white, $28 \%$ black, $60 \%$ Latina, $1 \%$ other; immigration status: born in USA $56.3 \%$ of control and $43.5 \%$ of intervention

Socioeconomic status indicators (annual household income): 32\%, < USD 5000; 21\%, USD 5000-10,000; 31\%, USD 10,000-20,000; $17 \%$, > USD 20,000

Education: $49 \%<$ high school diploma; $29 \%$ high school graduate; $22 \%>$ high school

Relationship status: current spouse $59.2 \%$ controls and $71.5 \%$ intervention

$48.1 \%$ of controls and $49.1 \%$ of intervention group lived with abuser

\section{Intervention}

Nurse case management empowering abused women by increasing independence/control: focus on protection/safety, enhanced choice-making/problem solving; nurse facilitates this by giving anticipatory guidance and guided referrals tailored to woman's individual needs; sessions lasted for 20 min, on average, and were provided at baseline and at 6-month intervals for 2 years

\section{Control}

Provision of a referral card listing a safety plan and sources for IPV services; no counselling, education, referrals or other services were offered

\section{Notes}

Grant from the Agency for Healthcare Research and Quality RO1HS11079

Participant recruitment began in February 2001 and ended in June 2002

\section{Risk of bias}

\begin{tabular}{lll}
\hline Bias & Authors' judgement & Support for judgement \\
\hline $\begin{array}{l}\text { Random sequence genera- } \\
\text { tion (selection bias) }\end{array}$ & Low risk & $\begin{array}{l}\text { Quote: “... [E]ach week of the study was randomised by a computer-generat- } \\
\text { ed process so that each woman consenting would be assigned to an interven- } \\
\text { tion group based on the week in which she was assessed for abuse” }\end{array}$ \\
\hline $\begin{array}{l}\text { Allocation concealment } \\
\text { (selection bias) }\end{array}$ & High risk & $\begin{array}{l}\text { The randomisation was completed by the project manager and presented to } \\
\text { the research nurses responsible for recruitment at the beginning of each week }\end{array}$ \\
\hline $\begin{array}{l}\text { Blinding (performance } \\
\text { bias and detection bias): } \\
\text { all outcomes }\end{array}$ & High risk & $\begin{array}{l}\text { Blinding of participants and key personnel (people providing the interven- } \\
\text { tion) was not possible. The outcome assessors also provided the intervention, } \\
\text { hence assessment was not blinded }\end{array}$ \\
\hline
\end{tabular}


McFarlane 2006 (Continued)

Incomplete outcome da- Low risk $\quad 19 / 180$ (11\%) missing from intervention group, 22/180 (12\%) missing from conta (attrition bias): all outcomes trol group. Reason given for attrition was that the participants could not be contacted. There was no difference in trial arm

\section{Selective reporting (re- Low risk All measures discussed in the 'Methods' section of the paper were also dis- porting bias) cussed in the 'Results' section}

Baseline measurement of Low risk outcome measures

Demographics of the treatment groups were similar except that more women in the case management group who were Hispanic were born outside of the USA. Acculturation scores for Hispanics, however, were the same between groups. The equivalence in acculturation scores across groups suggested birth outside the USA would not interact with the assigned treatment, and thus, country of origin was not used as a covariate in the analysis

$\begin{array}{ll}\begin{array}{l}\text { Reliability of outcome Low risk } \\ \text { measures }\end{array} & \begin{array}{l}4 \text { of the outcomes (including the primary outcomes of abuse) were reliable. No } \\ \text { data were provided on the reliability of the scale used to measure the use of } \\ \text { community resources }\end{array}\end{array}$

\section{Protection against conta- High risk} mination

The same healthcare professionals managed both women in the intervention and the control arms of the trial

How valuable is the re- Unclear risk Moderate value

search (richness of the da-

ta for the review research

questions)?

\section{Merchant 2015}

\begin{tabular}{|c|c|}
\hline Methods & Qualitative interviews \\
\hline \multirow[t]{8}{*}{ Participants } & Country: USA \\
\hline & Setting: shelters \\
\hline & Participant numbers: 19 current and former shelter workers from nine shelters in the south-western \\
\hline & ${ }^{2}$ \\
\hline & Ethnicity: 7 white, 7 Hispanic, and 5 African-American \\
\hline & Age: from $23-61$, though most were in their late 30 s or early $40 \mathrm{~s}$ \\
\hline & $\begin{array}{l}\text { Experience: } 15 \text { of those who responded were currently working in shelters, while } 3 \text { were previous em- } \\
\text { ployees who had left shelter employment } 5-6 \text { years prior to this study after } 3,6 \text {, and } 10 \text { years of employ- } \\
\text { ment, respectively. } 3 \text { current advocates had previously worked in another shelter. Positions of partic- } \\
\text { ipants included } 2 \text { childcare workers, } 1 \text { legal advocate, } 8 \text { case mangers/advocates, } 4 \text { advocate supervi- } \\
\text { sors, } 1 \text { hotline advocate, } 1 \text { therapist, and } 1 \text { mixed-position advocate. Length of time working in a shel- } \\
\text { ter ranged from 1-12 years, with an average of } 5.4 \text { years of experience. }\end{array}$ \\
\hline & $\begin{array}{l}\text { Education: } 4 \text { had Master's degrees, } 2 \text { had taken some graduate courses, } 7 \text { had bachelor's degrees, } 2 \\
\text { had associate's degrees, } 2 \text { had attended some college, } 1 \text { had a high school diploma, and } 1 \text { had }<\text { a high } \\
\text { school diploma }\end{array}$ \\
\hline Interventions & Shelter advocacy non-specified \\
\hline
\end{tabular}

\section{Risk of bias}

A realist review of which advocacy interventions work for which abused women under what circumstances (Review) 
Merchant 2015 (Continued)

Bias Authors' judgement Support for judgement

How valuable is the re-

High risk

search (richness of the da-

ta for the review research

questions)?

Was there a clear state-

Low risk

ment of the aims of the re-

search?

Was the research design Low risk
appropriate to address the
aims of the research?

\title{
Was the recruitment strat- Low risk
}

egy appropriate to the

aims of the research?

\section{Has the relationship be- Low risk}

tween researcher and par-

ticipants been adequately

considered?

\begin{abstract}
Is a qualitative methodol- Low risk
ogy appropriate?
\end{abstract}

Have ethical issues been Unclear risk No ethical issues discussed
taken into consideration?

Were the data collected in Low risk a way that addressed the research issue?

\section{Was the data analysis suf- Low risk}

ficiently rigorous?

Is there a clear statement Low risk

of findings?

\section{Muelleman 1999}

Methods

Participants
Case-control study: quasi-experimental study with a consecutive sample, before-after trial design

\section{Country: USA \\ Setting: ED in a country hospital}

Inclusion criteria: intervention (named BRIDGE): after a woman was identified as having acute injuries as a result of DV, she was asked if she wanted to meet with an advocate to discuss options for dealing with her situation. Control group: retrospectively identified by the presence of ICD diagnostic code 995.81 (adult maltreatment syndrome) in 1 of 3 diagnostic fields on the computerised ED log. The medical records were then reviewed to ensure the assailant was a former or current husband or boyfriend.

Participant numbers: during the first 6 months of the BRIDGE programme, 183 women were identified as injured by DV and offered advocate meeting. The after group consisted of the 105 (57\%) who agreed to meet with an advocate. 63 women who met with the advocate consented to police report follow-up.

There were also 27 controls 
Muelleman 1999 (Continued)

Age: $\geq 18$ years

Ethnicity: there were more black women in the BRIDGE group ( $75 \%$ versus $61 \%, P=0.05$ )

Education: no difference in mean income and education as reflected by respective ZIP codes

\section{Intervention}

BRIDGE ED-based advocacy, with the goal of 'bridging' battered women from the ED to community resources. It was thought that if an advocate from the DV community met with the woman while she was in the ED, she would be more likely to use these resources after her visit. After a woman was identified as having acute injuries as a result of DV, she was asked if she wanted to meet with an advocate. If so, the advocate was paged and arrived within 30 min to discuss the incident, address safety issues, educate the woman about the cycle of violence, and inform her of resources available in the community. The resources available included calls to the police, orders of protection, shelters, and counselling. The advocate also requested informed consent for permission to obtain police record follow-up information. The meeting usually lasted $1.5 \mathrm{~h}$.

Control

Women who had an ICD diagnostic code of 995.81 but were not offered a chance to meet with the advocate. They were offered an information sheet with resource phone numbers on it.

Timing of follow-up: pre-post

Notes Funding: none stated

\section{Risk of bias}

\section{Bias}

Authors' judgement Support for judgement

Random sequence genera- High risk No random allocation

tion (selection bias)

\begin{tabular}{ll}
\hline $\begin{array}{l}\text { Allocation concealment } \\
\text { (selection bias) }\end{array}$ & Low risk
\end{tabular}

Blinding (performance High risk It was not possible to blind the participants or researchers to the intervention.
bias and detection bias):
all outcomes

\begin{tabular}{lll}
\hline $\begin{array}{l}\text { Incomplete outcome da- } \\
\text { ta (attrition bias): all out- } \\
\text { comes }\end{array}$ & Unclear risk & $\begin{array}{l}\text { Its not clear how many dropouts there were and if there were differences be- } \\
\text { tween groups }\end{array}$ \\
\hline $\begin{array}{l}\text { Selective reporting (re- } \\
\text { porting bias) }\end{array}$ & High risk & $\begin{array}{l}\text { While some measures were from case records, the others depended on victim } \\
\text { recall. }\end{array}$ \\
\hline
\end{tabular}

Baseline measurement of Low risk These were mainly gathered from administrative data
outcome measures

\begin{tabular}{|c|c|c|}
\hline $\begin{array}{l}\text { Reliability of outcome } \\
\text { measures }\end{array}$ & High risk & $\begin{array}{l}\text { It is not clear whether use of shelters, ED visits or police contracts or orders of } \\
\text { protection are positive or negative, beneficial or harmful }\end{array}$ \\
\hline
\end{tabular}

\begin{tabular}{lll}
$\begin{array}{l}\text { Protection against conta- } \\
\text { mination }\end{array}$ & Low risk & As the control group was identified form records \\
\hline How valuable is the re- & Low risk & The study is of low value
\end{tabular}

How valuable is the re- $\quad$ Low risk $\quad$ The study is of low value
search (richness of the da-


Muelleman 1999 (Continued)

ta for the review research

questions)?

Murray 2015

\begin{tabular}{|c|c|}
\hline Methods & Focus groups \\
\hline \multirow[t]{6}{*}{ Participants } & Country: USA \\
\hline & Setting: DV shelters \\
\hline & Participant numbers: 62 participants (from a total of 9 focus groups) with DV shelter providers \\
\hline & $\begin{array}{l}\text { Ethnicity: white: 37; African American/African/black: 13; Hispanic/Latina/Latino: 5; biracial/multiracial } \\
\text { 4; Asian/Asian American: 1; not reported: } 2\end{array}$ \\
\hline & $\begin{array}{l}\text { Experience: clinical/counselling: 5; community education: 2; advocate/case manager: 17; support staff } \\
\text { (e.g. administrative assistant): 2; administration (e.g. Executive Director): 6; programme co-ordinator: } \\
\text { 18; intern: 6; law enforcement: 5; not reported: } 1\end{array}$ \\
\hline & $\begin{array}{l}\text { Education: high school diploma: 4; some college: } 3 \text {; associate's degree: } 6 \text {; bachelor's degree: } 33 \text {; Mas- } \\
\text { ter's degree: 14; Doctoral degree: } 1 \text {; not reported: } 1\end{array}$ \\
\hline
\end{tabular}

Interventions Shelter advocacy non-specified

\section{Notes}

\section{Risk of bias}

Bias Authors' judgement Support for judgement

How valuable is the re- Low risk Participants described as service providers more broadly than advocacy
search (richness of the da-
ta for the review research
questions)?

Was there a clear state- $\quad$ Low risk
ment of the aims of the re-
search?

Was the research design Low risk
appropriate to address the
aims of the research?

aims of the research?

\section{Was the recruitment strat- Low risk}

egy appropriate to the aims of the research?
Has the relationship be- tween researcher and par- ticipants been adequately considered?
High risk

Is a qualitative methodol- Low risk
ogy appropriate?


Murray 2015 (Continued)

Were the data collected in Low risk a way that addressed the research issue?

Was the data analysis suf- Low risk

ficiently rigorous?

Is there a clear statement Low risk

of findings?

\section{O'Brien 2016}

Methods Self-completion questionnaire. Single group, before and after

\begin{tabular}{ll}
\hline Participants & Country: Australia \\
Setting: a community welfare organisation called DAWN \\
Inclusion criteria: clients referred from other programmes in the same organisation (called Doncare), \\
women's refuges, welfare services and police \\
Participant numbers: 36 (50\% of recruited)
\end{tabular}

Children: most clients had dependent children

\begin{tabular}{ll}
\hline Interventions & Advocacy: Doncare Angel for Women Network (DAWN). Volunteers ('Angels') are matched with a client \\
to provide in-home social support, assistance with practical issues, and companionship.
\end{tabular}

Notes This was not a rigorous research study, more of a service audit. Funding: NS

\section{Risk of bias}

\begin{tabular}{lll}
\hline Bias & Authors' judgement & Support for judgement \\
\hline $\begin{array}{l}\text { Random sequence genera- } \\
\text { tion (selection bias) }\end{array}$ & High risk & Before and after cohort study \\
\hline $\begin{array}{l}\text { Allocation concealment } \\
\text { (selection bias) }\end{array}$ & High risk & Before and after cohort study \\
\hline $\begin{array}{l}\text { Blinding (performance } \\
\text { bias and detection bias): } \\
\text { all outcomes }\end{array}$ & High risk & Before and after cohort study \\
\hline $\begin{array}{l}\text { Incomplete outcome da- } \\
\text { ta (attrition bias): all out- } \\
\text { comes }\end{array}$ & High risk & Data on 50\% of the original sample \\
\hline $\begin{array}{l}\text { Selective reporting (re- } \\
\text { porting bias) }\end{array}$ & Unclear risk & Not well defined study a priori \\
\hline $\begin{array}{l}\text { Baseline measurement of } \\
\text { outcome measures }\end{array}$ & Low risk & \\
\hline $\begin{array}{l}\text { Reliability of outcome } \\
\text { measures }\end{array}$ & Low risk & Mostly validated \\
\hline
\end{tabular}


O'Brien 2016 (Continued)

Protection against conta- High risk $\quad$ Before and after cohort study
mination

How valuable is the re- Low risk Low value

search (richness of the da-

ta for the review research

questions)?

Owen 2015

\begin{tabular}{|c|c|}
\hline Methods & Qualitative interviews \\
\hline \multirow[t]{4}{*}{ Participants } & Country: Australia \\
\hline & $\begin{array}{l}\text { Setting: rural service providers from criminal justice agencies (police and courts), health and welfare } \\
\text { services across } 12 \text { Local Government Areas ranging in population size from } 3000 \text { to 55,000 people in } \\
\text { New South Wales } \\
\text { Participant numbers: } 49 \text { service providers }\end{array}$ \\
\hline & Gender: $16 \%$ male and $84 \%$ female \\
\hline & Ethnicity: NS \\
\hline Interventions & Not specified \\
\hline Notes & Funding: NS \\
\hline
\end{tabular}

\section{Risk of bias}

Bias Authors' judgement Support for judgement

How valuable is the re- High risk

search (richness of the da-

ta for the review research

questions)?

Was there a clear state- Low risk
ment of the aims of the re-
search?

\begin{tabular}{|c|c|c|}
\hline $\begin{array}{l}\text { Was the research design } \\
\text { appropriate to address the } \\
\text { aims of the research? }\end{array}$ & Low risk & $\begin{array}{l}\text { Yes. Study was interested in the role of the rural service providers being a } \\
\text { member of the community as well as a professional in that community. }\end{array}$ \\
\hline $\begin{array}{l}\text { Was the recruitment strat- } \\
\text { egy appropriate to the } \\
\text { aims of the research? }\end{array}$ & Low risk & $\begin{array}{l}\text { Given the geographical spread of the service providers in rural areas, they used } \\
\text { an appropriate recruitment strategy. }\end{array}$ \\
\hline $\begin{array}{l}\text { Has the relationship be- } \\
\text { tween researcher and par- } \\
\text { ticipants been adequately } \\
\text { considered? }\end{array}$ & Low risk & $\begin{array}{l}\text { Anonymity was assured given that they would likely be identifiable in their rur- } \\
\text { al home towns. }\end{array}$ \\
\hline
\end{tabular}

Is a qualitative methodol- Low risk

ogy appropriate? 
Owen 2015 (Continued)

Have ethical issues been Low risk taken into consideration?

Were the data collected in Unclear risk Somewhat. Participants are invited to speculate on the reasoning of women a way that addressed the who use (and do not use) their services.

research issue?

Was the data analysis suf- High risk

ficiently rigorous?

Limited by only 1 person interpreting the themes from the interviews.

Is there a clear statement Low risk

of findings?

Perez 2012

Methods Survey

Participants Country: USA

Setting: battered women's shelters, urban medium-sized Midwestern city

Inclusion criteria: residents of the battered women's shelter and had a documented incident of abuse

from an intimate partner as defined by their responses to the CTS2

Participant numbers: 227 over 4 years

Age: $18-64$

Relationship to abuser: married or cohabiting $84.2 \%$

Ethnicity: $45.8 \%$ African American, $37.0 \%$ white, $8.4 \%$ Hispanic and $8.8 \%$ other race.

Socioeconomic status indicators: unemployed $74 \%$, receiving public assistance $59.5 \%$

Education: high school diploma or equivalent $75.3 \%$

$\begin{array}{ll}\text { Interventions } & \text { Services offered by the shelter system include emergency housing, access to basic resources, case } \\ \text { management, support group, and advocacy programmes. } 1 \text { shelter in the system provided emergency } \\ \text { crisis stabilisation, whereas the other provided apartment-style transitional living space. }\end{array}$

Notes Not just advocacy. Funding: NIMH grant K23 MH067648 and pilot funds from the Summa-Kent State centre for the Treatment and Study of Traumatic Stress

\section{Risk of bias}

\begin{tabular}{lll}
\hline Bias & Authors' judgement & Support for judgement \\
\hline $\begin{array}{l}\text { Representativeness of the } \\
\text { sample }\end{array}$ & Unclear risk & No comparison made with total shelter residents \\
\hline Adequacy of response rate & Unclear risk & NS \\
\hline $\begin{array}{l}\text { Missing data within com- } \\
\text { pleted questionnaires }\end{array}$ & Low risk & $\begin{array}{l}<1 \% \text { of the total data was missing and no participant had significant missing } \\
\text { data on any single measure }\end{array}$ \\
\hline $\begin{array}{l}\text { Conduct of pilot testing } \\
\text { Established validity of the }\end{array}$ & Low risk & Validated measures \\
\hline \hline
\end{tabular}

A realist review of which advocacy interventions work for which abused women under what circumstances (Review) 
Prosman 2014

Methods A pre-post study and semi-structured interviews

Participants

Country: Netherlands

Setting: 32 family practices in the urban area of Rotterdam, a multi-ethnic city

Inclusion criteria: identified abused women with children

Participants: $42 / 98$ family doctors invited agreed to participate, 66 abused women referred to the study but 3 women could not be reached. Lost contact with 3 more women after 1 visit. Another 10 women were referred to mental health care or a social worker after 2 visits. 4 women had only baseline assessment, left the programme and were referred to mental health care due to severe PTSD. 3 women with baseline assessment were lost to follow-up after 6 visits. Thus 43 women (65\%) finished the entire support programme

For the interviews, 21 women consented to be interviewed by telephone, 4 could not be reached and 3 decided not to participate; so final number was 14 women

Ethnicity: the 43 participants of the intervention programme itself (MeMoSA) came from Turkey (10), Morocco (3), Surinam (12), the Netherlands (9), The Antilles (3), Iraq (1), Former Yugoslavia (2), Africa (said to be Algiers, Cape Verde, Goa, though Goa is actually in India) (3). The women interviewed about their experience of MeMoSA ( $n=14)$ came from the same spread of locations (Turkey (3), Morocco (2), Surinam (2), the Netherlands (2), The Antilles (1), Iraq (1), Former Yugoslavia (2), Africa (1).

Age: participants of the intervention programme MeMoSA 18-25 years $(n=8), 26-35$ years $(n=14)$, $36-45$ years $(n=14),>45$ years $(n=6)$. Interviewed women $18-25$ years $(n=2), 26-35$ years $(n=6), 36-45$ years $(n=4),>45$ years $(n=2)$

Children: participants of the intervention programme MeMoSA: 16 had 1 child, 23 had 2-3 children, 4 had $\geq 4$. Interviewed women: 4 had 1 child, 8 had $2-3$ and 2 had $\geq 4$.

Education: mentor mothers were educated for social or health care, such as doctor assistants and social workers. Most women were migrants with lower education.

Interventions Mentor support comprising 16 weekly home visits by a trained mentor mother with the following topics and activities.

- Dealing with IPV (safety planning, recognising, dealing with aggressive behaviour)

- Recognising unacceptable and dangerous behaviour

- Developing a safety plan

- Educating psychological (cognitive and emotional) consequences of IPV

- Evaluating own cognitions of guilt and shame

- Coping with depressive symptoms (based on CBT)

- Inventory of sad mood related to activities

- Evaluating possibilities to influence own mood

- Supporting to implement

- Strengthening social network (finding (volunteering) job, going back to school)

- Inventory opportunities to strengthen personal support system

- Developing, implementing and evaluating a plan for education or finding a job

- (4) Accepting professional mental health assistance and parenting support

- Inventory barriers to visit mental health care (in consultation with their family doctor)

- Visiting mental health care together

- Increasing awareness of the impact of exposure to IPV on children's health

- When needed, involve teachers and preventive health care or (school) social work-parenting support 
Prosman 2014 (Continued)

Funding by the Public Health Authority of the City of Rotterdam, Innovation Foundation Kinderpostzegel fonds (Children's Stamp Fund) and Stichting Volkskracht (Foundation for People's Strength)

\section{Risk of bias}

\begin{tabular}{lll}
\hline Bias & Authors' judgement & Support for judgement \\
\hline $\begin{array}{l}\text { Random sequence genera- } \\
\text { tion (selection bias) }\end{array}$ & High risk & Pre-post study \\
\hline $\begin{array}{l}\text { Allocation concealment } \\
\text { (selection bias) }\end{array}$ & High risk & Pre-post study \\
\hline $\begin{array}{l}\text { Blinding (performance } \\
\text { bias and detection bias): } \\
\text { all outcomes }\end{array}$ & High risk & Pre-post study \\
\hline $\begin{array}{l}\text { Incomplete outcome da- } \\
\text { ta (attrition bias): all out- } \\
\text { comes }\end{array}$ & Low risk & $\begin{array}{l}\text { Characteristics regarding age, country of origin, education and number of chil- } \\
\text { dren showed no significant differences between completers ( } \mathrm{n}=43 \text { ) and non- } \\
\text { completers ( } \mathrm{n}=23)\end{array}$ \\
\hline
\end{tabular}

Selective reporting (re- Low risk porting bias)

\section{Baseline measurement of Low risk outcome measures}

Reliability of outcome Low risk
measures

\begin{tabular}{l}
$\begin{array}{l}\text { Protection against conta- Unclear risk Possible contamination though spread across } 42 \text { doctors } \\
\text { mination }\end{array}$ \\
\hline
\end{tabular}

How valuable is the re- High risk

search (richness of the da-

ta for the review research questions)?

Reina 2015

Methods Qualitative interviews

Participants Country: USA

Setting: a nonprofit DV and sexual assault agency in a metropolitan area of central lowa

Inclusion criteria: Latina immigrants in a metropolitan area of lowa, who had experienced DV, had previously contacted an anti-violence organisation, and had used its services

Participant numbers: total 10 (4 of these in focus group)

Ethnicity: immigrants from Mexico and Central/South America. Lived in the USA from 5-14 years. No participants had legal residency status when they experienced partner abuse, though at the time of the study, nine were in the process of getting visas and 1 was initiating the process. All Latina participants were native Spanish speakers and 2 reported having limited English.

Age: $25-42$ years 
Reina 2015 (Continued)

Socioeconomic status indicators: some participants had either low-skill jobs or were unemployed when they were interviewed, and all were unemployed when they faced partner abuse.

Relationship status: at the time of the study, most women were separated or had divorced from their partners or husbands.

\begin{tabular}{ll}
\hline Interventions & Non-specified \\
\hline Notes &
\end{tabular}

\section{Risk of bias}

\begin{tabular}{|c|c|c|}
\hline Bias & Authors' judgement & Support for judgement \\
\hline $\begin{array}{l}\text { Was there a clear state- } \\
\text { ment of the aims of the re- } \\
\text { search? }\end{array}$ & Low risk & \\
\hline
\end{tabular}

Was the research design
appropriate to address the
aims of the research?

Low risk aims of the research?

$\begin{array}{ll}\begin{array}{l}\text { Was the recruitment strat- } \\ \text { egy appropriate to the }\end{array} & \begin{array}{l}\text { Recruitment strategy via a service provider gatekeeper. There may be an ele- } \\ \text { aims of the research? }\end{array}\end{array}$

Has the relationship be- Low risk

tween researcher and par-

ticipants been adequately considered?

Is a qualitative methodol- Low risk
ogy appropriate?

Have ethical issues been Low risk
taken into consideration?

Were the data collected in Low risk
a way that addressed the research issue?

\section{Was the data analysis suf- Low risk} ficiently rigorous?

Is there a clear statement Low risk
of findings?

\section{Rodgers 2017}

\begin{tabular}{ll}
\hline Methods & Small feasibility RCT \\
\hline Participants & $\begin{array}{l}\text { Country: USA } \\
\text { Setting: community health worker outreach in four urban community health clinics } \\
\text { Participant numbers: a total of } 29 \text { women were identified as eligible for the feasibility study. Of the } 19 \\
(66 \%) \text { women who enrolled in the study, } 10 \text { were randomised to the intervention group and } 9 \text { were ran- }\end{array}$
\end{tabular}


domised to enhanced social work care in the clinic. 15 (79\%) of the 19 women in the study completed the 3-month follow-up interview; 9 of the intervention participants and 6 of the usual care group

The demographic data reported below come from a larger demonstration project $(n=58)$ that was not relevant to our review.

Ethnicity: 90\% African American ( $n=52)$

Age: mean age of African Americans $=36.2$ years $(S D=11.8)$; mean age of participants in feasibility study was 34.5 years $(S D=9.94)$

Socioeconomic status indicators: unemployed 68\%; and 53\% reported incomes of $\leq$ USD 10,000

Children: all intervention participants had children, although many of the children were staying with family members or in foster homes.

Education: high school education or less $74 \%$

Abuse: high-risk category/on the DAS tool, $84 \%$ scored $\geq 18$, which is consistent with the highest level of danger

Relationship status: mostly single (74\%). At the time of enrolment, 4 women were living with their abusive partner; 3 were transient and lived with their children, friends, or family members; and 3 were inpatients in rehabilitation centres

\section{Intervention}

The usual care plus follow-up by a community-based outreach worker, CHW. The CHW served as a peer educator and advocate by

- counselling abused participants on safety planning and access to DV community services

- assisting participants in obtaining access to other needed social and economic resources

- providing intensive emotional and social support on a weekly basis.

Depending on the individual client's needs and desires, the CHW conducted weekly sessions via phone or in person. Assistance included going to court with the participant to help obtain a personal protection order or facilitating contact with resources for safe housing or substance abuse treatment. The $\mathrm{CHW}$ carried a cell phone at all times and was available for crisis management if needed. The $\mathrm{CHW}$ worked intensively with each participant for 3 months.

\section{Control}

Clinic-based Family Health Advocate (FHA) intervention alone for routine IPV screening and a brief motivational intervention
Most of the intervention participants had experienced drug or alcohol problems during their lifetimes; 4 indicated they had used illegal drugs in the past year. 3 of these women were inpatients in drug and alcohol rehabilitation programmes at the time of enrolment. Most had depression and PTSD. This project was funded by the National Institute on Minority Health and Health Disparities (NIMHD; RC1MD-004415).

\section{Risk of bias}

\begin{tabular}{lll}
\hline Bias & Authors' judgement & Support for judgement \\
\hline $\begin{array}{l}\text { Random sequence genera- } \\
\text { tion (selection bias) }\end{array}$ & High risk & Feasibility study that did not clarify procedures \\
\hline
\end{tabular}

Allocation concealment High risk

(selection bias) 
Rodgers 2017 (Continued)

Blinding (performance bias and detection bias): all outcomes
High risk

\begin{tabular}{|c|c|c|}
\hline $\begin{array}{l}\text { Incomplete outcome da- } \\
\text { ta (attrition bias): all out- } \\
\text { comes }\end{array}$ & Low risk & Longitudinal follow-up with 9/10 (90\%) \\
\hline $\begin{array}{l}\text { Selective reporting (re- } \\
\text { porting bias) }\end{array}$ & Unclear risk & $\begin{array}{l}\text { Outcomes not formally specified; narrative of cases even though a feasibility } \\
\text { trial }\end{array}$ \\
\hline $\begin{array}{l}\text { Baseline measurement of } \\
\text { outcome measures }\end{array}$ & Low risk & \\
\hline $\begin{array}{l}\text { Reliability of outcome } \\
\text { measures }\end{array}$ & High risk & Qualitative consideration with no formal tools \\
\hline $\begin{array}{l}\text { How valuable is the re- } \\
\text { search (richness of the da- } \\
\text { ta for the review research } \\
\text { questions)? }\end{array}$ & Low risk & Low value \\
\hline
\end{tabular}

Scheer 2018

\begin{tabular}{ll}
\hline Methods & Survey-based correlational analysis \\
\hline Participants & Country: USA \\
Setting: community & $\begin{array}{l}\text { Inclusion criteria: self-identified sexual and gender minority adults who reported currently experienc- } \\
\text { ing or having experienced some form of IPV within the past year, and who were currently seeking or } \\
\text { who had sought services related to IPV experiences and its aftermath within the past year. }\end{array}$
\end{tabular}

\section{Particant numbers: 227}

Age: $18-78$ years $(M=27.83, S D=9.74)$

Ethnicity: white (60.4\%), biracial or multiracial (18.9\%), Middle Eastern (4.4\%), Hispanic or Latino/a (4.0\%), 'other' (2.5\%), Asian or Asian American (2.6\%), Native American or Alaska Native (2.6\%), African American or Black (1.3\%), and Hawaiian/Pacific Islander (0.4\%)

Education: 9th-11th grade (0.9\%), high school graduate or GED equivalent (5.7\%), vocational school $(3.5 \%)$, some college $(30.4 \%)$, college graduate $(37.4 \%)$, advanced degree $(9.7 \%)$, and graduate degree $(12.3 \%)$

Socioeconomic status indicators: a small percentage of participants reported that they do not worry about paying for things they want and need (7.5\%), 24.2\% reported that they can easily pay their bills but need to be careful, $43.2 \%$ can pay their regular bills but a bill that was bigger than usual would cause a hardship, $18.9 \%$ reported that they have trouble paying their regular bills, and $6.2 \%$ reported that they simply cannot pay their bills

Relationship status: participants were single or not dating (28.6\%), and in an intimate relationship $(71.4 \%)$

\section{Notes}


Scheer 2018 (Continued)

Risk of bias

\begin{tabular}{|c|c|c|}
\hline Bias & Authors' judgement & Support for judgement \\
\hline $\begin{array}{l}\text { How valuable is the re- } \\
\text { search (richness of the da- } \\
\text { ta for the review research } \\
\text { questions)? }\end{array}$ & Low risk & $\begin{array}{l}\text { Study focus was on gender tissues but included mechanisms analysis that } \\
\text { helped to develop the theory }\end{array}$ \\
\hline $\begin{array}{l}\text { Representativeness of the } \\
\text { sample }\end{array}$ & Unclear risk & Participants were recruited from national and local online forums and listservs \\
\hline $\begin{array}{l}\text { Missing data within com- } \\
\text { pleted questionnaires }\end{array}$ & Low risk & $\begin{array}{l}\text { Imputation used; there was minimal to moderate missing data across the in- } \\
\text { cluded measures (from } 4.0 \% \text { for sexual orientation to } 29.5 \% \text { for race) }\end{array}$ \\
\hline Conduct of pilot testing & Low risk & \\
\hline $\begin{array}{l}\text { Established validity of the } \\
\text { survey instrument }\end{array}$ & Low risk & \\
\hline
\end{tabular}

Shorey 2014

\begin{tabular}{ll}
\hline Methods & Systematic review \\
\hline Participants & NS \\
\hline Interventions & Interventions for IPV \\
\hline
\end{tabular}

Notes

\section{Risk of bias}

\begin{tabular}{lll}
\hline Bias & Authors' judgement & Support for judgement \\
\hline $\begin{array}{l}\text { Random sequence genera- } \\
\text { tion (selection bias) }\end{array}$ & Unclear risk & NS \\
\hline
\end{tabular}

Silva-Martinez 2016

\begin{tabular}{ll}
\hline Methods & Qualitative face-to-face interviews \\
\hline Participants & $\begin{array}{l}\text { Country: USA } \\
\text { Setting: community } \\
\text { Inclusion criteria: advocates delivering financial literacy programme } \\
\text { Participant numbers: } 19 \text { advocates from } 15 \text { programmes }\end{array}$ \\
\hline Interventions & $\begin{array}{l}\text { Financial literacy advocacy programme "Moving Ahead Through Financial Management". The curricu- } \\
\text { lum was created to help survivors identify the signs of economic abuse, increase their financial knowl- } \\
\text { edge, enhance their ability to manage their finances, and obtain the confidence they need to rebuild } \\
\text { their financial lives (www.clicktoempower.org). }\end{array}$
\end{tabular}


Silva-Martinez 2016 (Continued)

\title{
Risk of bias
}

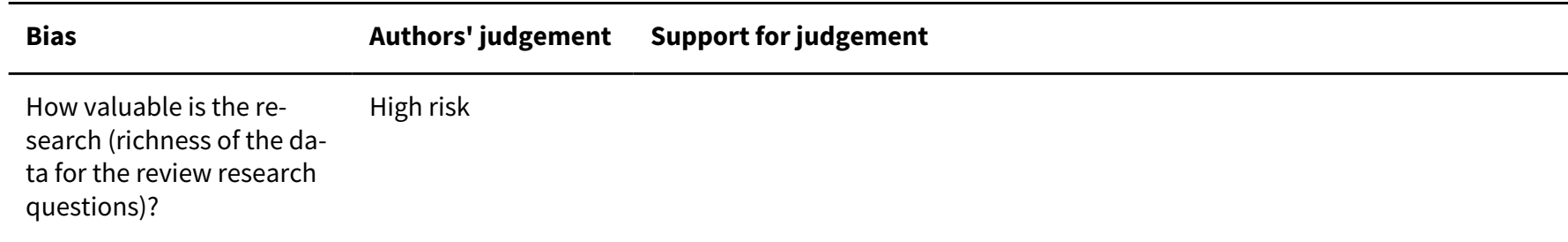

\section{Was there a clear state- Low risk} ment of the aims of the research?

\section{Was the research design appropriate to address the aims of the research?}

Low risk

Was the recruitment strat- Low risk egy appropriate to the aims of the research?
Yes. All 15 programmes were represented

Yes. Information about the programme, and also the advocate's own personal experiences were explored.

tween researcher and par-

Low risk

ticipants been adequately

considered?

\begin{abstract}
Is a qualitative methodol- Low risk
ogy appropriate?
\end{abstract}

\begin{tabular}{ll}
\hline $\begin{array}{l}\text { Have ethical issues been } \\
\text { taken into consideration? }\end{array}$ & Though ethics approval granted \\
\hline $\begin{array}{l}\text { Were the data collected in Low risk } \\
\text { a way that addressed the } \\
\text { research issue? }\end{array}$ & \\
\hline
\end{tabular}

\section{Was the data analysis suf- Low risk}

ficiently rigorous?

Is there a clear statement Low risk
of findings?

of findings?

Slattery 2009

Methods Cross-sectional survey

Participants

\section{Country: USA}

Setting: workplaces of DV advocates

Participant numbers: 79 out of 148 eligible

Age: $19-65(M=36, S D=12)$

Ethnicity: white (114; 77.6\%); Hispanic/Latina (11; 7.5\%); black/African (9; 6.1\%); Asian American (4;2.7\%); Native American (8; 5.4\%) 
Socioeconomic status indicators: income < USD 10,000, 10.6\%; 10,000-19,999, 9.8\%; 20,000-29,999, $39.5 \%$; 30,000-39,999, 33.8\%; 40,000-49,000, 5.6\%; 50,000-59,000, 0.7\%

Education: high school diploma to doctorate. $78 \%$ graduated from college and of these $30.4 \%$ held a Masters degree.

Experience: $1-2$ years $29.9 \%, 3-528.4 \%, 6-1031.2 \%, 10-2510.5 \%$

Workplace setting: shelter $29.1 \%$, DV service $18.2 \%$, court/legal service $16.9 \%$, crisis centre $12.2 \%$, community health centre $10.1 \%$, social service agency $8.1 \%$, hospital $5.4 \%$

\begin{tabular}{ll}
\hline Interventions & Advocacy non-specified \\
\hline Notes & Staff but with experience of abuse frequent: survivor status: survivor of abuse $55.4 \%$, IPV $25.5 \%$, child \\
& sexual abuse $19.9 \%$, child witness to violence $18.8 \%$, rape/sexual assault $17.7 \%$, child physical/emo- \\
& tional abuse $5 \%$, no abuse history $44.6 \%$. \\
& Funding: Boston College Lynch School of Education for a Summer Dissertation Development Grant
\end{tabular}

\title{
Risk of bias
}

\begin{tabular}{|c|c|c|}
\hline Bias & Authors' judgement & Support for judgement \\
\hline $\begin{array}{l}\text { How valuable is the re- } \\
\text { search (richness of the da- } \\
\text { ta for the review research } \\
\text { questions)? }\end{array}$ & High risk & \\
\hline $\begin{array}{l}\text { Representativeness of the } \\
\text { sample }\end{array}$ & Unclear risk & DV advocates working in diverse settings \\
\hline Adequacy of response rate & Unclear risk & Potential sample is not clear to work out the response rate. \\
\hline $\begin{array}{l}\text { Missing data within com- } \\
\text { pleted questionnaires }\end{array}$ & Unclear risk & Missing data not reported \\
\hline Conduct of pilot testing & High risk & No mention of pilot testing \\
\hline $\begin{array}{l}\text { Established validity of the } \\
\text { survey instrument }\end{array}$ & Unclear risk & Valid survey instruments used \\
\hline
\end{tabular}

Song 2010

Methods 1 group pre-post test with interviews and case records analysis

Participants

\author{
Country: Taiwan \\ Setting: community \\ Participants: 72 eligible, 65 began study, 26 the 1st follow-up and 6 the 2 nd follow-up \\ Socioeconomic status indicators: just over a third (34.9\%) lived in their own house, $20.6 \%$ lived in a \\ rented place, $30.2 \%$ stayed in a shelter and $14.3 \%$ in other facilities. Employment status: $20 \%$ worked \\ full-time, $29.2 \%$ did not work, $10.8 \%$ held a part-time job, $9.2 \%$ worked irregularly and $6.2 \%$ were \\ housewives. Just over a third (36.7\%) had no income, $25 \%$ had a monthly income of < USD 468 and \\ 28.3\% had monthly income between USD 469 and USD 937
}

Age: mean age 38.91 (median $=38.50 ; \mathrm{SD}=8.53)$, with a range of $22-59$ years 
Song 2010 (Continued)

Children: most had either 2 (37.1\%) or 4 children (22.6\%)

Education: most to either senior high (42.2\%) or junior high school (31.3\%) level. 8 (12.5\%) held a college or masters degree.

Relationship status: $78.5 \%$ were abused by their current spouse, $15.4 \%$ by their former spouse, $3.1 \%$ by their cohabiting partner and $3.1 \%$ by others

Type of advocacy:
Interventions
- informal support network development
- strengths-based
Survivor-defined practice
Content of the intervention:
- In-person meetings and conversation by phone; the case manager met clients and key members in
their network (mean =1.20 times/month), but more services were provided by telephone (mean =1.47
times) and by meeting in the agency (mean = 1.53 times)
- developing self-awareness; covering daily living, financial insurance, occupation, recreation etc. Case
managers demonstrated unconditional acceptance and positive regard for the clients
- talked to family and friends on their behalf; the sources of the clients' network included relatives,
friends, children's school teachers, local police, leaders of community organisations and religious
groups. "A few case managers worked with the key members in the clients' informal support system
by deliberately engaging them as the change agents. However, since some clients either did not want
to reveal their status as a victim of violence or had exhausted almost every possible supporter in
their network, they might have little interest in further exploring or using their natural support sys-
tem" (quote, p 25)

Notes

\section{Risk of bias}

\begin{tabular}{lll}
\hline Bias & Authors' judgement & Support for judgement \\
\hline $\begin{array}{l}\text { Random sequence genera- } \\
\text { tion (selection bias) }\end{array}$ & High risk & 1 group pre-post test \\
\hline $\begin{array}{l}\text { Allocation concealment } \\
\text { (selection bias) }\end{array}$ & High risk & 1 group pre-post test \\
\hline $\begin{array}{l}\text { Blinding (performance } \\
\text { bias and detection bias): } \\
\text { all outcomes }\end{array}$ & High risk & 1 group pre-post test \\
\hline $\begin{array}{l}\text { Incomplete outcome da- } \\
\text { ta (attrition bias): all out- } \\
\text { comes }\end{array}$ & Low risk & $\begin{array}{l}\text { The 26 participants who completed the second assessment were not signifi- } \\
\text { cantly different from those who did not (n=39) }\end{array}$ \\
\hline $\begin{array}{l}\text { Selective reporting (re- } \\
\text { porting bias) }\end{array}$ & Low risk & \\
\hline $\begin{array}{l}\text { Baseline measurement of } \\
\text { outcome measures }\end{array}$ & Low risk \\
\hline $\begin{array}{l}\text { Reliability of outcome } \\
\text { measures }\end{array}$ & Low risk & \\
\hline
\end{tabular}


Song 2010 (Continued)

Protection against conta- High risk mination

How valuable is the re- High risk search (richness of the da-

ta for the review research questions)?

\section{Song 2012}

\begin{tabular}{ll}
\hline Methods & Survey \\
\hline Participants & Country: Taiwan \\
& $\begin{array}{l}\text { Setting: centres of Prevention and Intervention for DV and private sectors in Taiwan clients contacted } \\
\text { by social workers }\end{array}$
\end{tabular}

Participant numbers: 392 questionnaires were given to the clients through their social worker; 191 participants responded

Age: mean 39.30 years (SD 9.65)

Relationship status: 124 (65.3\%) married, living with abuser 73 (38.4\%)

Education: < high school 73 (38.8); high school 83 (44.1); college 32 (17.0)

Socioeconomic status indicators: 50 (26.2\%) unemployed, mostly low-income

Abuse: physical abuse 160 (84.2\%); verbal abuse 161 (84.7\%); emotional abuse 127 (66.8); sexual abuse 47 (24.7).

Abused at least once a week $58(31.0 \%)$

Interventions DV services non-specified

\section{Notes}

\section{Risk of bias}

Bias Authors' judgement Support for judgement

How valuable is the re- Low risk

search (richness of the da-

ta for the review research

questions)?

Was there a clear state- Low risk

ment of the aims of the re-

search?

$\begin{aligned} & \text { Was the research design } \\ & \text { appropriate to address the } \\ & \text { aims of the research? }\end{aligned}$
$\begin{aligned} & \text { Was the recruitment strat- } \\ & \text { egy appropriate to the } \\ & \text { aims of the research? }\end{aligned}$


Song 2012 (Continued)

Has the relationship be- Unclear risk Little information tween researcher and participants been adequately considered?

\section{Is a qualitative methodol- Low risk} ogy appropriate?

Have ethical issues been High risk
taken into consideration?

taken into consideration?

Were the data collected in High risk Case managers, rather than independent researchers collected data.
a way that addressed the research issue?

Was the data analysis suf- Unclear risk ficiently rigorous?

Quotes were all positive, not clear how many women shared these views.

\section{Is there a clear statement Low risk}

of findings?

\section{Stover 2010}

Methods Quasi-experimental controlled comparison

\section{Participants}

\section{Country: USA}

Setting: 5 policing districts in the city of New Haven, Connecticut

Inclusion criteria: based on screening of police reports generated November 2004-October 2005

DVHVI

- An altercation between a male and female intimate partner (IPV) resulting in criminal charges against the male perpetrator

- Arrest of the male perpetrator made or an arrest warrant pending

- Female victim aged $\geq 18$ years

\section{Exclusion criteria}

- Non-IPV cases (e.g. brother-sister, mother-child domestics, same sex)

- DV cases that resulted in a dual arrest or arrest of the female

- partner

- Non-female victims or victims aged $<18$

- Victims who did not speak English or Spanish fluently

Participant numbers: the final sample of 107 women was recruited from a pool of 430 women $(n=198$ DVHVI users and $n=232$ non-DVHIVI area) who were eligible to participate; 52 women given the DVHVI and 55 controls

Ethnicity: 27.8\% Hispanic, 53.7\% African American, and 13\% white

Age: mean 30

Socioeconomic status indicators: primarily low-income with limited education, and most were unemployed 
Relationship status: $18 \%$ were married to the perpetrator, $16 \%$ were living together but not married, $28 \%$ were in a relationship but not living with the perpetrator, and $39 \%$ were ex-partners

Children: a median of 2 with a mean age of 7.5

The aim is to monitor victim safety, improve victims' understanding and enforcement of court orders, increase access to information and concrete services, and provide psychological screening, acute psychological support, and access to treatment for victims and their children.

Women who lived in the 5 policing districts that implemented the DVHVI received the intervention, whereas control women in the remaining 5 districts received standard police services following a 911 (emergency) call to the police for DV.

Notes

\section{Risk of bias}

\begin{tabular}{lll}
\hline Bias & Authors' judgement & Support for judgement \\
\hline $\begin{array}{l}\text { Random sequence genera- } \\
\text { tion (selection bias) }\end{array}$ & High risk & Seems simply consecutive cases till quota \\
\hline $\begin{array}{l}\text { Allocation concealment } \\
\text { (selection bias) }\end{array}$ & High risk & \\
\hline $\begin{array}{l}\text { Blinding (performance } \\
\text { bias and detection bias): } \\
\text { all outcomes }\end{array}$ & High risk & \\
\hline $\begin{array}{l}\text { Incomplete outcome da- } \\
\text { ta (attrition bias): all out- } \\
\text { comes }\end{array}$ & Unclear risk & $\begin{array}{l}\text { Cases were recruited from the pool of potential research participants until the } \\
\text { goal of } 50 / \text { group was achieved. However, of the 430 women eligible to par- } \\
\text { ticipate in the research study, 25\% completed, 24\% declined, } 42 \% \text { were not } \\
\text { reached either due to inaccurate contact information on the police report or } \\
\text { lack of response to phone messages/mailings, and 9\% agreed to participate, } \\
\text { then did not show up. Research participants were less likely to be married or } \\
\text { living with the perpetrator at the time of the domestic incident, and they had } \\
\text { cases with less severe police charges. This resulted in a sample of research } \\
\text { participants who were either dating or ex-partners with perpetrators who had } \\
\text { lower-level severity violence as a group. }\end{array}$
\end{tabular}

Selective reporting (re- Low risk
porting bias)

\begin{tabular}{ll}
\hline $\begin{array}{l}\text { Baseline measurement of } \\
\text { outcome measures }\end{array}$ & Tow risk \\
& $\begin{array}{l}\text { Tween the groups at baseline were ethnicity }(c 2=17.85, P<.001) \text { and perpetra- } \\
\text { tor criminal histories }(T=1.88, P<.10) . \text { Hence used as covariate }\end{array}$
\end{tabular}

Reliability of outcome Low risk
measures

\begin{tabular}{lll}
\hline $\begin{array}{l}\text { Protection against conta- } \\
\text { mination }\end{array}$ & Low risk & Single visit \\
\hline $\begin{array}{l}\text { How valuable is the re- } \\
\text { search (richness of the da- } \\
\text { ta for the review research } \\
\text { questions)? }\end{array}$ & Unclear risk & Moderate value, fills a gap in knowledge \\
\hline
\end{tabular}


Stylianou 2018

\begin{tabular}{ll} 
Methods & $\begin{array}{l}\text { Ethnography: the first session between family court advocates and IPV survivors were recorded across } \\
5 \text { family court programmes }\end{array}$ \\
\hline Participants & $\begin{array}{l}\text { Country: USA } \\
\text { Setting: } 5 \text { family court programmes operated by } 1 \text { victim service agency located in a northeastern city } \\
\text { in the USA }\end{array}$
\end{tabular}
in the USA

Participant numbers: purposive sampling was utilised to recruit survivors across all 5 family court programmes; 22 agreed to participate, 13 did so ranging from 1-4 sessions/advocate

No other data specified

Interventions The first session between family court advocates and IPV survivors

\section{Notes}

\section{Risk of bias}

\begin{tabular}{lll}
\hline Bias & Authors' judgement & Support for judgement \\
\hline $\begin{array}{ll}\text { How valuable is the re- } \\
\text { search (richness of the da- } \\
\text { ta for the review research } \\
\text { questions)? }\end{array}$ & High risk & $\begin{array}{l}\text { Of specific value to family court advocates. Was limited to } 1 \text { geographical area } \\
\text { and service providers, may be limited in terms of transferability }\end{array}$ \\
\hline
\end{tabular}

\section{Was there a clear state- Low risk}

ment of the aims of the re-

search?

\begin{abstract}
Was the research design appropriate to address the aims of the research?
\end{abstract}

\section{Low risk}

Was the recruitment strat- High risk egy appropriate to the aims of the research?

Recruiting people in court lobbies, and waiting areas may have been a little insensitive to their immediate context.
Has the relationship be- tween researcher and par-
High risk ticipants been adequately considered?
Is a qualitative methodol- Low risk ogy appropriate?

Were the data collected in Low risk a way that addressed the research issue?

Was the data analysis suf- Low risk
ficiently rigorous?

Is there a clear statement Low risk

of findings? 
Sudderth 2017

\begin{tabular}{ll}
\hline Methods & Interviews \\
\hline Participants & Country: New Zealand \\
& Setting: shelters \\
& Participant numbers: 24 shelters in the sample, 19 (79\%) were National Collective of Independent \\
Women's Refuges (NCIWR) members, and 5 (21\%) were unaffiliated. 6 (25\%) were specifically for Māori \\
women, although some refuges had specific services and providers for Māori women within their own \\
agency, that is, "parallel services." \\
No other data specified \\
\hline Interventions & Non-specified \\
\hline Notes &
\end{tabular}

\section{Risk of bias}

Bias Authors' judgement Support for judgement

How valuable is the re-

High risk

search (richness of the da-

ta for the review research

questions)?

Was there a clear state- Low risk
ment of the aims of the re-
search?

Was the research design Low risk
appropriate to address the

aims of the research?

\begin{tabular}{|c|c|c|}
\hline $\begin{array}{l}\text { Was the recruitment strat- } \\
\text { egy appropriate to the } \\
\text { aims of the research? }\end{array}$ & High risk & $\begin{array}{l}\text { Partly. Only views of service providers in this study. Sometime the inclusion } \\
\text { of community members sounded like formal support on the cheap. It wasn't } \\
\text { clear who these community members were, which would likely affect the } \\
\text { views of women (from personal friends to police) }\end{array}$ \\
\hline
\end{tabular}

Has the relationship between researcher and parHigh risk ticipants been adequately considered?

\begin{tabular}{l}
$\begin{array}{l}\text { Is a qualitative methodol- Low risk } \\
\text { ogy appropriate? }\end{array}$ \\
$\begin{array}{l}\text { Have ethical issues been } \\
\text { taken into consideration? }\end{array}$ \\
$\begin{array}{l}\text { Were the data collected in risk } \\
\text { a way that addressed the } \\
\text { research issue? }\end{array}$ \\
$\begin{array}{l}\text { Was the data analysis suf- } \\
\text { ficiently rigorous? }\end{array}$ \\
\hline
\end{tabular}


Sudderth 2017 (Continued)

Is there a clear statement of findings?

Sullivan 1991

$\begin{array}{ll}\text { Methods } & \text { Randomisation method: random allocation with ratio of 2:1 (intervention: control), no stratification } \\ & \text { Analysis by ITT: no } \\ & \text { Power calculation: no }\end{array}$

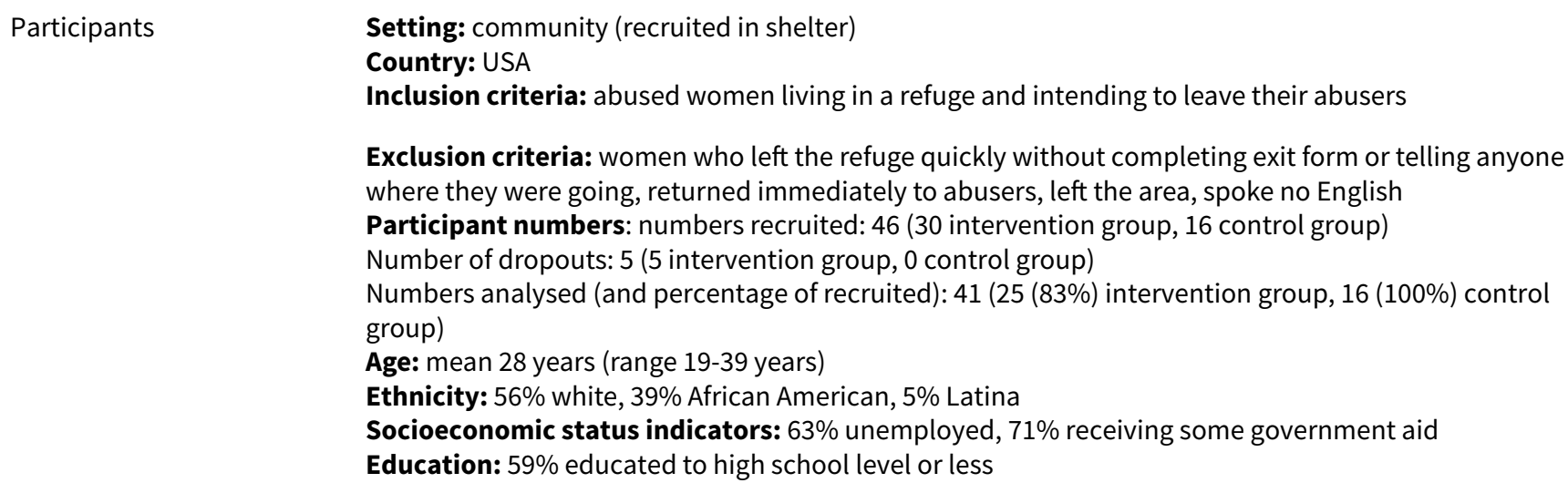

Exclusion criteria: women who left the refuge quickly without completing exit form or telling anyone where they were going, returned immediately to abusers, left the area, spoke no English

Participant numbers: numbers recruited: 46 (30 intervention group, 16 control group) Number of dropouts: 5 (5 intervention group, 0 control group) Numbers analysed (and percentage of recruited): 41 (25 (83\%) intervention group, $16(100 \%)$ control group)

Age: mean 28 years (range 19-39 years)

Ethnicity: 56\% white, 39\% African American, 5\% Latina

Socioeconomic status indicators: $63 \%$ unemployed, $71 \%$ receiving some government aid Education: $59 \%$ educated to high school level or less

\section{Interventions Intervention}

Intensive 1-1 services with a trained paraprofessional advocate who assisted women in accessing needed community resources within 1 week of leaving the shelter; 10 weeks of advocacy, usually meeting twice a week: 4-6 $\mathrm{h}$ in person and another $2 \mathrm{~h}$ telephone contact

Control

Women received standard shelter care and no additional post-shelter service

$\begin{array}{ll}\text { Notes } & \text { Partially funded by the George Gund Foundation and the Michigan Department of Mental Health } \\ \text { Data were collected between } 1986 \text { and } 1988\end{array}$
Data were collected between 1986 and 1988

\section{Risk of bias}

\begin{tabular}{|c|c|c|}
\hline Bias & Authors' judgement & Support for judgement \\
\hline \multirow[t]{2}{*}{$\begin{array}{l}\text { Random sequence genera- } \\
\text { tion (selection bias) }\end{array}$} & Unclear risk & $\begin{array}{l}\text { Quote: "... [W]omen were randomly assigned to either of the two conditions, } \\
\text { stratifying for order. Two-thirds were randomly assigned to the [intervention], } \\
\text { and 1-third to the control condition." }\end{array}$ \\
\hline & & No further information was provided \\
\hline $\begin{array}{l}\text { Allocation concealment } \\
\text { (selection bias) }\end{array}$ & Unclear risk & $\begin{array}{l}\text { No information was provided about who performed the allocation or the pro- } \\
\text { cedure used }\end{array}$ \\
\hline $\begin{array}{l}\text { Blinding (performance } \\
\text { bias and detection bias): } \\
\text { all outcomes }\end{array}$ & High risk & $\begin{array}{l}\text { Blinding of participants and key personnel (people providing the intervention) } \\
\text { was not possible. Also, the outcome assessors were not blinded as at data } \\
\text { collection they had to ask intervention group participants what they thought } \\
\text { about the advocacy process }\end{array}$ \\
\hline $\begin{array}{l}\text { Incomplete outcome da- } \\
\text { ta (attrition bias): all out- } \\
\text { comes }\end{array}$ & High risk & $\begin{array}{l}\text { Attrition differs markedly by group: } 5 / 30(17 \%) \text { missing from intervention } \\
\text { group, } 0 / 16(0 \%) \text { missing from control group. Reason given for attrition was } \\
\text { that } 5 \text { participants were no longer 'eligible', as they had to receive at least } 3 \\
\text { weeks of services and had dropped out ( } 3 \text { of these because they had returned } \\
\text { to their abusers). This may affect outcome effect size. However we note that }\end{array}$ \\
\hline
\end{tabular}


Sullivan 1991 (Continued)

the poor attrition is a function of the study design, where women had to attend at least 3 advocacy sessions to be eligible, which could not apply to the controls

\begin{tabular}{lll}
\hline $\begin{array}{l}\text { Selective reporting (re- } \\
\text { porting bias) }\end{array}$ & Low risk & $\begin{array}{l}\text { All measures discussed in the 'Methods' section of the paper were also dis- } \\
\text { cussed in the 'Results' section }\end{array}$ \\
\hline $\begin{array}{l}\text { Baseline measurement of } \\
\text { outcome measures }\end{array}$ & Low risk & $\begin{array}{l}\text { The investigators stated that the groups did not differ on severity of abuse; ef- } \\
\text { fectiveness in accessing resources was not measured at baseline }\end{array}$ \\
\hline $\begin{array}{l}\text { Reliability of outcome } \\
\text { measures }\end{array}$ & Low risk & Outcome measures were reliable \\
\hline $\begin{array}{l}\text { Protection against conta- } \\
\text { mination }\end{array}$ & Unclear risk & $\begin{array}{l}\text { There might have been communication between women in different arms of } \\
\text { the trial, as all were recruited from the same shelter }\end{array}$ \\
\hline
\end{tabular}

Sullivan 2002

\begin{tabular}{ll} 
Methods & Randomisation method: NS \\
& Analysis by ITT: no \\
& Power calculation: NS \\
\hline
\end{tabular}

Participants

Country: USA

Setting: community - working in the family's home (recruited primarily in shelter)

Inclusion criteria:

- Physical violence from an intimate partner or ex-partner in previous 4 months

- Intended to stay in area for 8 months

- At least 1 child aged 7-11 years living with them

- At least 1 of these required to participate

Exclusion criteria: none stated

Participant numbers: numbers recruited: 80 (45 intervention group, 35 control group)

Number of dropouts: 4 (trial arm not stated)

Numbers analysed (and percentage of recruited): 78 (98\%) as some data imputed (45 (56\%) intervention group, 33 (41\%) control group)

Age: mean 31 years

Ethnicity: 49\% white, 39\% African American, 5\% Latina, 5\% multiracial, 1\% Asian, 1\% Native American Socioeconomic status indicators: mean income USD 1200/month, $88 \%$ receiving government aid, $44 \%$ employed

Education: none stated

Multi-component advocacy intervention to improve the well-being of mothers and self-confidence of their children, and to protect against continued violence; 3 components:

- Advocacy for abused mothers, to help mothers generate, mobilise, and access community resources

- Advocacy for children (similar to above)

- Support and education group for the children: children attended a 10-week support and education group; intervention based on individual needs of mother and child, full programme lasted 16 weeks, families saw advocates for a median $8 \mathrm{~h} /$ week, averaging $5 \mathrm{~h}$ with children and an additional $3 \mathrm{~h}$ with the women 
Sullivan 2002 (Continued)

\section{Control}

Women had usual access to services available to community residents

Notes Breakdown of numbers recruited: (Rivas 2015)

Funded by the U.S. National Institute of Mental Health. Data were collected 1990-1997

\section{Risk of bias}

Bias Authors' judgement Support for judgement

Random sequence genera- Unclear risk tion (selection bias)

Quote: “... [W]omen were randomly assigned to either the experimental or the control condition."

No other information was provided

Allocation concealment Unclear risk No information was provided.

(selection bias)

\begin{tabular}{|c|c|c|}
\hline $\begin{array}{l}\text { Blinding (performance } \\
\text { bias and detection bias): } \\
\text { all outcomes }\end{array}$ & Unclear risk & $\begin{array}{l}\text { Blinding of participants and key personnel (people providing the intervention) } \\
\text { was not possible. No information was provided in relation to the outcome as- } \\
\text { sessors }\end{array}$ \\
\hline
\end{tabular}

\begin{tabular}{ll}
\hline $\begin{array}{l}\text { Incomplete outcome da- } \\
\text { ta (attrition bias): all out- }\end{array}$ & Low risk \\
$\begin{array}{ll}\text { comes } & \text { marticipants dropped out, not stated by trial arm. However, where possible, } \\
& \text { missing data were imputed resulting in 0/45 (0\%) 'missing' from intervention } \\
& \text { group, } 2 / 35(6 \%) \text { 'missing' from control group. Reasons given for attrition were } \\
& \text { that } 2 \text { women declined further involvement and } 2 \text { could not be located (not re- } \\
\text { ported by trial arm) }\end{array}$
\end{tabular}

\begin{tabular}{|c|c|c|}
\hline $\begin{array}{l}\text { Selective reporting (re- } \\
\text { porting bias) }\end{array}$ & Low risk & $\begin{array}{l}\text { All measures discussed in the 'Methods' section of the paper were also dis- } \\
\text { cussed in the 'Results' section }\end{array}$ \\
\hline
\end{tabular}

\begin{tabular}{lll}
\hline $\begin{array}{l}\text { Baseline measurement of } \\
\text { outcome measures }\end{array}$ & Low risk & $\begin{array}{l}\text { The investigators stated that the groups did not differ on any of the outcome } \\
\text { variables }\end{array}$ \\
\hline $\begin{array}{l}\text { Reliability of outcome } \\
\text { measures }\end{array}$ & Low risk & Outcome measures were reliable \\
\hline $\begin{array}{l}\text { Protection against conta- } \\
\text { mination }\end{array}$ & Unclear risk & $\begin{array}{l}\text { It is possible that there might have been communication between women in } \\
\text { different arms of the trial }\end{array}$ \\
\hline
\end{tabular}

Sullivan 2018

\begin{tabular}{ll}
\hline Methods & Pre-post \\
\hline Participants & Country: USA \\
& Setting: shelters \\
& Inclusion criteria: DV shelter residents from 1/4 programmes in Ohio who had been in shelter for at \\
& least 14 days \\
& Participant numbers: 106 provided baseline data, 60 provided both baseline and follow-up, 3 had \\
& missing data so analysed sample = 57. 54 participants provided demographic information \\
& Ethnicity: $70.4 \%$ white women, $18.6 \%$ African/African American/black, 3.7\% Hispanic/Latina, 3.7\% Na- \\
tive American/Alaskan Native, and 3.7\% multiracial women. All reported English as their primary lan- \\
guage, and all but 1 were born in the USA
\end{tabular}


Sullivan 2018 (Continued)

Age: $19-59$, with an average of 39.8 years (SD 9.81)

Relationship status: single $=55.4 \%$

Education: ranged from having < an 8th grade education to having an advanced degree, with most participants having a high school diploma/GED (37\%) or some college (25.9\%)

\begin{tabular}{|c|c|c|}
\hline Interventions & \multicolumn{2}{|c|}{ Trauma-informed care including advocacy } \\
\hline Notes & \multicolumn{2}{|c|}{ While this study includes all shelter services the focus was on advocate-survivor interactions } \\
\hline \multicolumn{3}{|l|}{ Risk of bias } \\
\hline Bias & Authors' judgement & Support for judgement \\
\hline $\begin{array}{l}\text { Random sequence genera- } \\
\text { tion (selection bias) }\end{array}$ & High risk & Pre-post study \\
\hline $\begin{array}{l}\text { Allocation concealment } \\
\text { (selection bias) }\end{array}$ & High risk & Pre-post study \\
\hline $\begin{array}{l}\text { Blinding (performance } \\
\text { bias and detection bias): } \\
\text { all outcomes }\end{array}$ & High risk & Pre-post study \\
\hline $\begin{array}{l}\text { Incomplete outcome da- } \\
\text { ta (attrition bias): all out- } \\
\text { comes }\end{array}$ & High risk & 106 started, 57 analysed; 56 with complete info \\
\hline $\begin{array}{l}\text { Selective reporting (re- } \\
\text { porting bias) }\end{array}$ & Low risk & \\
\hline $\begin{array}{l}\text { Baseline measurement of } \\
\text { outcome measures }\end{array}$ & Low risk & \\
\hline $\begin{array}{l}\text { Reliability of outcome } \\
\text { measures }\end{array}$ & Low risk & \\
\hline $\begin{array}{l}\text { Protection against conta- } \\
\text { mination }\end{array}$ & Low risk & \\
\hline $\begin{array}{l}\text { How valuable is the re- } \\
\text { search (richness of the da- } \\
\text { ta for the review research } \\
\text { questions)? }\end{array}$ & Unclear risk & Moderate value, specific focus \\
\hline
\end{tabular}

\section{Sullivan 2019}

Methods Qualitative interviews

Participants Country: USA

Setting: diverse community agencies: areas with very limited affordable housing or with more options available to them; agencies in large urban cities, a university town, mid-sized cities, and small, rural communities

Inclusion criteria: professional contacts of the first author 
Sullivan 2019 (Continued)

- known to assist survivors with housing

- worked with diverse survivors from different areas of the country

Participant numbers: 11 advocates from 3 different regions of the USA (Northwest, Midwest, East) were invited and took part

Ethnicity: 4 were white, 3 were Latina, 2 were African American, 1 was Native American, and 1 was Asian

Age: ranged from mid-20s-mid-50s

Experience: worked as advocates from 2.5-16 years (average 8 years). 3 worked in culturally specific agencies (focused on immigrants and refugees, LGBTQ communities, and immigrant Latinas, respectively)

\begin{tabular}{ll}
\hline Interventions & Advocacy including housing assistance \\
\hline Notes & All but 2 of the interviews were conducted in person, with the remaining 2 by phone
\end{tabular}

\section{Risk of bias}

\begin{tabular}{lll}
\hline Bias & Authors' judgement & Support for judgement \\
\hline $\begin{array}{l}\text { How valuable is the re- } \\
\text { search (richness of the da- } \\
\text { ta for the review research } \\
\text { questions)? }\end{array}$ & Low risk & $\begin{array}{l}\text { Study looked at a small number of advocates' views of barriers to housing in } \\
\text { the USA. There may be limited transferability }\end{array}$ \\
\hline
\end{tabular}

\section{Was there a clear state- Low risk} ment of the aims of the research?

\begin{tabular}{|c|c|c|}
\hline $\begin{array}{l}\text { Was the research design } \\
\text { appropriate to address the } \\
\text { aims of the research? }\end{array}$ & High risk & $\begin{array}{l}\text { Advocates were asked to recall a representative case. But advocates pointed } \\
\text { out that every case is different. The topic guide may have limited the range of } \\
\text { answers. }\end{array}$ \\
\hline
\end{tabular}

Was the recruitment strat- Unclear risk NS
egy appropriate to the
aims of the research?

Has the relationship be- $\quad$ High risk No
tween researcher and par-
ticipants been adequately
considered?

Is a qualitative methodol- Low risk
ogy appropriate?

Have ethical issues been
taken into consideration?

Was the data analysis suf- Low risk

ficiently rigorous? 
Sullivan 2019 (Continued)

Is there a clear statement Low risk of findings?

\section{Taft 2011}

Methods

Randomisation method: cluster-randomised - not stated explicitly how clusters were generated, but allocations were concealed in opaque envelopes and randomly selected by a person outside the study; GP clinics were randomised by number of GPs participating (1 or 2), Maternal and Child Health (MCH) clinics were randomised by team (stratified by numbers of births/local government area)

Analysis by ITT: no (study authors state it was ITT analyses based on original allocations and not on care received, but only data for the women completing the study were analysed)

Power calculation: yes, but the required number of 350 women in each trial arm not attained

Participants Country: Australia

Setting: recruited in primary care clinics - general practices offering shared care (care for pregnant women shared between local GP and maternity hospital) and $\mathrm{MCH}$ clinics

Country: Australia

Inclusion criteria: women aged $\geq 16$ years attending clinics, pregnant or with at least 1 child $\leq 5$ years, disclosed IPV or were psychosocially distressed (and symptoms suggested IPV); study authors were contacted and provided data for moderately to severely abused women only. (Clinic inclusion criteria: GP clinics offering shared care and MCH clinics)

Exclusion criteria: women with serious mental illnesses and not taking medication, inadequate English or Vietmanese for informed consent

Numbers recruited: 141 abused subset (93 intervention group, 48 control group). Numbers dropouts: 38 abused subset (22 intervention group, 16 control group). Numbers analysed (and percentage of recruited): 103 abused subset (71 (76\%) intervention group; 32 (67\%) control group)

Indicators below are for total sample of $n=141$

Age: mean 32 years

Ethnicity: born overseas: 36\% intervention group, 32\% control group

Socioeconomic status indicators: income from welfare/pension: $62 \%$ intervention group, $53 \%$ control group; healthcare (subsidy) card: $74 \%$ intervention group, $70 \%$ control group

Education: (year 12 or less): $47 \%$ intervention group, $51 \%$ control group

Children: all pregnant

Interventions Intervention

MOSAIC model: combined social support, advocacy, and antenatal mentoring: non-judgemental listening/support/friendship; maintaining contact/support (weekly on average) through phone calls, home visiting/outings; assistance in developing safety strategies appropriate to women's circumstances; developing a trusting relationship/modelling a sense of hope; providing parenting information/support; providing information/assisting referral to community services/resources (especially family violence services) for women and children; intervention delivered by trained and supported nonprofessional mentor mothers for up to 12 months

\section{Control}

Women in the control group received an information sheet for mothers; this included family violence service contact details

Notes

Multi-component intervention: advocacy and parenting support for abused mothers. Child-related measures not included in this review

Development and piloting of the MOSAIC intervention model and the design of the trial occurred over a period of 3 years (2001-2003)

Research funding: National Health and Medical Research Council, Victorian Health Promotion Foundation (VicHealth)

Implementation funding: Victorian Government Community Support Grants Fund, beyondblue - the depression initiative. Angela Taft supported by a Victorian Health Promotion Foundation (VicHealth) 


\section{Risk of bias}

\begin{tabular}{lll}
\hline Bias & Authors' judgement & Support for judgement \\
\hline $\begin{array}{l}\text { Random sequence genera- } \\
\text { tion (selection bias) }\end{array}$ & High risk & $\begin{array}{l}\text { Cluster-randomised: randomisation occurred at 2 public meetings to which } \\
\text { GPS/ MCH team leaders were invited "to ensure the fairness of the process and } \\
\text { to check the contents of the envelopes prior to being sealed" - but no details } \\
\text { about the randomisation procedure used. A large percentage of clinics in both } \\
\text { arms did not refer any potential participants }\end{array}$ \\
&
\end{tabular}

Allocation concealment High risk
(selection bias)

Clinic allocations (as cluster-randomised) "were concealed in opaque en(selection bias) velopes and randomly selected by someone outside the study". However, staff recruiting individual participants were aware of allocation status, and study authors report selection bias because of poor participation by control clinics

$\begin{array}{ll}\begin{array}{l}\text { Blinding (performance } \\ \text { bias and detection bias): }\end{array} & \text { High risk } \\ \begin{array}{ll}\text { all outcomes } & \text { Blinding of participants and key personnel (people providing the intervention) } \\ & \text { was not possible. Research staff were not blinded as they "fully briefed partic- } \\ & \text { ipants in the intervention arm about the mentoring pro- gramme, negotiated } \\ \text { informed consent and collected follow-up data about mentoring from partici- } \\ \text { pants in the intervention arm" }\end{array}\end{array}$

\begin{tabular}{|c|c|c|}
\hline $\begin{array}{l}\text { Incomplete outcome da- } \\
\text { ta (attrition bias): all out- } \\
\text { comes }\end{array}$ & High risk & $\begin{array}{l}\text { Large attrition: } 43 / 106 \text { clinics did not refer any potential participants ( } 35 \% \\
\text { intervention versus } 42 \% \text { control). Of those women recruited, } 22 / 93(24 \%) \\
\text { missing from abused subset of intervention group, } 16 / 48(33 \%) \text { missing from } \\
\text { abused subset of control group. Reasons for attrition not stated. No test for } \\
\text { differences across groups }\end{array}$ \\
\hline
\end{tabular}

\begin{tabular}{|c|c|c|}
\hline $\begin{array}{l}\text { Selective reporting (re- } \\
\text { porting bias) }\end{array}$ & Low risk & $\begin{array}{l}\text { All measures discussed in the 'Methods' section of the paper also discussed in } \\
\text { the 'Results' section }\end{array}$ \\
\hline
\end{tabular}

\begin{tabular}{lll}
\hline $\begin{array}{l}\text { Baseline measurement of } \\
\text { outcome measures }\end{array}$ & Low risk & $\begin{array}{l}\text { No statistics given, but additional information provided by study authors for } \\
\text { the abused subset indicates the groups were similar in terms of severity of } \\
\text { abuse, depression and well-being, although the intervention group did report } \\
\text { slightly less social support }\end{array}$ \\
\hline $\begin{array}{l}\text { Reliability of outcome } \\
\text { measures }\end{array}$ & Low risk & $\begin{array}{l}\text { Outcome measures were reliable } \\
\begin{array}{l}\text { Protection against conta- } \\
\text { mination }\end{array}\end{array}$ \\
\hline
\end{tabular}

\section{Taha 2015}

\begin{tabular}{ll}
\hline Methods & RCT \\
\hline Participants & Country: USA \\
& Setting: \\
& Inclusion criteria: women aged $18-64$, who self-identified as African American and reported IPV expo- \\
sure and at least 1 suicide attempt within the past 12 months & \\
& Participant numbers: 217 women recruited; 89 (41\%; 45 randomised to the intervention Grady Nia \\
& Project (Nia) and 44 randomised to control (treatment as usual (TAU)) participated in the post-interven-
\end{tabular}


Taha 2015 (Continued)

tion assessment. Only data from these 89 completers were analysed in this study. Rates of attrition for women in intervention and control were $62.5 \%$ and $49.4 \%$ respectively.

Socioeconomic status indicators: the income of approximately $98 \%$ of the sample (based on a 12 month aggregate of individual monthly income) was below the poverty threshold of USD 11,670/year for a single-person household

\section{Intervention}

Women in intervention participated in a culturally-sensitive group intervention led by 2 co-therapists, 1 African American and 1 non-African American woman. 10 weekly 2-h group meetings. The primary intervention targets included such factors as cognitive processes and resource mobilisation.

\section{Controls}

Encouraged to attend free weekly suicide and IPV support groups that were led by 2 study team members. The support groups were hour-long, nonmanualised sessions that were non-specific in treatment orientation. Group leaders acted predominantly as discussion facilitators. All women in the control condition had access to the hospital's psychiatric emergency services that were open $24 \mathrm{~h}, 7$ days/week as well as standard psychiatric and medical care offered by the hospital. Standard care included a medical and/or psychiatric evaluation, outpatient psychotherapy, psychiatric medication management, inpatient treatment, follow-up medical care, and referrals to community agencies (battered women's shelters and suicide/IPV crisis hotlines).

Notes

Although a group intervention, elements of advocacy were individualised and the study provided useful information

\section{Risk of bias}

\section{Bias}

\section{Authors' judgement Support for judgement}

Random sequence genera- Unclear risk tion (selection bias)

Randomised to intervention or control, using a 2 (intervention) to 1 (control) assignment but details not stated

Incomplete outcome data (attrition bias): all out-

Unclear risk

Rates of attrition for women in intervention and control were $62.5 \%$ and $49.4 \%$ comes respectively. There were no pre-intervention differences between the 2 groups regarding major demographic factors or additional treatments. Independent-samples t-tests were run to determine if there were differences in demographic characteristics between women who fully participated in intervention and control and those that prematurely terminated. Only 1 significant finding emerged; women that prematurely terminated from intervention had higher levels of homelessness $(\mathrm{M}=0.61, \mathrm{SD}=0.50)$ than women who fully participated $(\mathrm{M}=0.40, \mathrm{SD}=0.50), \mathrm{M}=0.21(95 \% \mathrm{Cl} 0.03$ to 0.40$), \mathrm{T}(118)=2.29, \mathrm{P}=0.023$. Because of high attrition rates, controlled for group differences by adding pre-intervention scores as covariates for all subsequent analyses.

Selective reporting (re-

Low risk porting bias)

Baseline measurement of Unclear risk outcome measures

\begin{abstract}
There were no pre-intervention differences between the 2 groups regarding major demographic factors or additional treatments. Independent-samples $\mathrm{t}$-tests were run to determine if there were differences in demographic characteristics between women who fully participated in intervention and control and those that prematurely terminated. Only 1 significant finding emerged; women that prematurely terminated from intervention had higher levels of homelessness $(\mathrm{M}=0.61, \mathrm{SD}=0.50)$ than women who fully participated $(\mathrm{M}=$ $0.40, \mathrm{SD}=0.50), \mathrm{M}=0.21(95 \% \mathrm{Cl} 0.03$ to 0.40$), \mathrm{T}(118)=2.29, \mathrm{P}=0.023$. Because of high attrition rates, controlled for group differences by adding pre-intervention scores as covariates for all subsequent analyses
\end{abstract}


Taha 2015 (Continued)

$\begin{aligned} & \text { Reliability of outcome Low risk Good Cronbach's alphas } \\ & \text { measures }\end{aligned}$
S

Protection against conta- Low risk Control involved some form of more generalised intervention
mination

How valuable is the re- $\quad$ High risk High value
search (richness of the da-
ta for the review research
questions)?

\section{Thiara 2009}

\begin{tabular}{ll}
\hline Methods & $\begin{array}{l}\text { Service evaluation: mixed methods including secondary data analysis and qualitative feedback by } \\
\text { abused women }\end{array}$
\end{tabular}

\section{Participants}

\section{Country: UK}

Setting: Refuge (shelter) Southwark Advocacy Project (RSAP)

Participants: feedback from 38 women (55\% completed face to face and $42 \%$ by telephone), case analysis of 295 women supported by the RSAP, $65 \%$ of whom were referred to the service and $35 \%$ directly contacted by the advocates in court; interviews and discussions with all strategic and operational project staff and other multi-agency players $(n=15)$.

Ethnicity: 91/295 (31\%) were white British; 28 (9.5\%) black - Caribbean, British, other; 23 (7.8\%) Black African and with a diverse range of other ethnicities. The majority of women were British/EU nationals $(n=152)$

Age: mode aged $<44$ years

Abuse: reported DV offences for April 2006-March 2007 numbered 4377, accounting for $20 \%$ of violent crime, with the majority of victims being female and aged $<45$ years

Women had experienced a range of abuse, with physical and emotional abuse the most common.

Children: In over half $(n=167)$ of the 295 cases, women had no children. In feedback sample, 30/38 women had children

Relationship status: at the time of contact with RSAP, the majority of women were living at home without the abuser (92\%), and a minority were either living in a refuge (5\%) or in a homeless hostel (2.5\%).

\section{Intervention}

The RSAP, funded by Southwark Council in London, was focused on the provision of court-based support to women going through the criminal justice system, an area not covered by existing agencies in the Borough.

The following were the key services offered to women: being kept informed about case; general telephone support; being accompanied to court; risk assessment and safety plan; requesting special measures; pre-trial visit to court; victim impact statements; criminal restraining orders; referral to other agencies. The advocates were extremely proactive in their attempts to reach all women going through the courts and offer them support.

\section{Notes}

Pilot service funded for a year; the RSAP was implemented from November 2006 to the end of March 2008 and the evaluation was conducted from May 2007-March 2008.

\section{Risk of bias}

A realist review of which advocacy interventions work for which abused women under what circumstances (Review) 
Thiara 2009 (Continued)

\begin{tabular}{lll} 
Bias & Authors' judgement & Support for judgement \\
\hline $\begin{array}{l}\text { Was the research design } \\
\begin{array}{l}\text { appropriate to address the } \\
\text { aims of the research? }\end{array}\end{array}$ & Low risk & \\
\hline $\begin{array}{l}\text { Was the recruitment strat- } \\
\text { egy appropriate to the }\end{array}$ & Unclear risk & No details given \\
aims of the research? & & \\
\hline
\end{tabular}

Has the relationship be-

High risk

tween researcher and par-

ticipants been adequately

considered?

\begin{tabular}{|c|c|c|}
\hline $\begin{array}{l}\text { Is a qualitative methodol- } \\
\text { ogy appropriate? }\end{array}$ & Unclear risk & Somewhat appropriate. Women's views described as feedback \\
\hline $\begin{array}{l}\text { Have ethical issues been } \\
\text { taken into consideration? }\end{array}$ & Low risk & \\
\hline $\begin{array}{l}\text { Were the data collected in } \\
\text { a way that addressed the } \\
\text { research issue? }\end{array}$ & Low risk & \\
\hline $\begin{array}{l}\text { Was the data analysis suf- } \\
\text { ficiently rigorous? }\end{array}$ & Low risk & \\
\hline $\begin{array}{l}\text { Is there a clear statement } \\
\text { of findings? }\end{array}$ & Unclear risk & $\begin{array}{l}\text { There are some direct quotes, but it's not clear which respondent is speaking, } \\
\text { or if this represents a range of respondents or only } 1 \text { or a few. }\end{array}$ \\
\hline
\end{tabular}

\section{Tiwari 2005}

$\begin{array}{ll}\text { Methods } & \text { Randomisation method: generated by computer and concealed in consecutively numbered sealed en- } \\ \text { velopes by a researcher not involved in the study } \\ \text { Analysis by ITT: yes } \\ \text { Power calculation: yes }\end{array}$

\section{Participants}

\section{Country: China (Hong Kong)}

Setting: public hospital antenatal clinic

\section{Inclusion criteria:}

- Pregnant women

- Aged $\geq 18$ years

- <30 weeks' gestation

- Attending first antenatal appointment

- Identified as abused by an intimate partner within last 12 months

Exclusion criteria: abuser was not a male partner

Participant numbers: numbers recruited: 110 (55 intervention group, 55 control group)

Number of dropouts: 4 (4 intervention group, 0 control group)

Numbers analysed (and percentage of recruited): 106 (51 (93\%) intervention group, 55 (100\%) control

group)

Age: mean 30 years intervention group, mean 31 years control group

Ethnicity: all Chinese 
Tiwari 2005 (Continued)

Socioeconomic status indicators: intervention group: 13\% < HKD 10000; 28\% HKD 10,000-20,000; 55.5\% > HKD 20,000; control group: 18.6\% < HKD 10,000; 39\% HKD 10,000-20,000; 36.6\% > HKD 20,000 (as reported in the paper)

Education background: none stated

$\begin{array}{ll}\text { Interventions } & \text { Intervention } \\ \text { Intervention based on empowerment to enhance abused women's independence and control; con- } \\ \text { sisted of advice in the areas of safety, decision making and problem solving; helped women to posi- } \\ \text { tively value themselves; intervention delivered by a senior researcher (a midwife with a degree in coun- } \\ \text { selling) and lasted about } 30 \text { min, an information brochure also provided } \\ \text { Control } \\ \text { Women received standard care: a wallet-sized card with information on community resources for } \\ \text { abused women, which included shelter hotlines, law enforcement, social services and non-government } \\ \text { organisations } \\ \text { This study was supported by a Low Budget High Impact Research Grant awarded by the University of } \\ \text { Hong Kong } \\ \text { The study was undertaken May 2002-July } 2003\end{array}$

\section{Risk of bias}

\begin{tabular}{lll}
\hline Bias & Authors' judgement & Support for judgement \\
\hline $\begin{array}{l}\text { Random sequence genera- } \\
\text { tion (selection bias) }\end{array}$ & Low risk & The allocation schedule was computer-generated \\
\hline $\begin{array}{l}\text { Allocation concealment } \\
\text { (selection bias) }\end{array}$ & Low risk & $\begin{array}{l}\text { Allocation was concealed by the use of consecutively numbered, sealed en- } \\
\text { velopes, a researcher not involved in the study carried out this task }\end{array}$ \\
\hline $\begin{array}{l}\text { Blinding (performance } \\
\text { bias and detection bias): } \\
\text { all outcomes }\end{array}$ & Low risk & $\begin{array}{l}\text { Blinding of participants and key personnel (people providing the intervention) } \\
\text { was not possible. Outcome assessors were blinded to study design/hypothe- } \\
\text { ses and group allocation, and this appeared to be successful (questions on sat- } \\
\text { isfaction with care not asked until all outcome data collected, no participant } \\
\text { revealed their randomisation status early). Data analysed by people not in- } \\
\text { volved in the design of the trial }\end{array}$
\end{tabular}

Incomplete outcome da- Low risk ta (attrition bias): all outcomes
4/55 (7\%) missing from intervention group, 0/55 missing from control group $(0 \%)$. Reason given for attrition was that the participants could not be traced

\begin{tabular}{lll}
\hline $\begin{array}{l}\text { Selective reporting (re- } \\
\text { porting bias) }\end{array}$ & Low risk & $\begin{array}{l}\text { All measures discussed in the 'Methods' section of the paper were also dis- } \\
\text { cussed in the 'Results' section }\end{array}$ \\
\hline $\begin{array}{l}\text { Baseline measurement of } \\
\text { outcome measures }\end{array}$ & Low risk & $\begin{array}{l}\text { No specific information on between-group differences was provided, although } \\
\text { the means and standard deviations seemed comparable. }\end{array}$ \\
\hline $\begin{array}{l}\text { Reliability of outcome } \\
\text { measures }\end{array}$ & Low risk & Outcome measures were reliable \\
\hline $\begin{array}{l}\text { Protection against conta- } \\
\text { mination }\end{array}$ & Low risk & $\begin{array}{l}\text { Only } 1 \text { clinic, but study investigators took active steps to minimise bias: per- } \\
\text { son administering the intervention was separated from all control group par- } \\
\text { ticipants, clinic waiting times minimised to reduce the chance of the } 2 \text { groups } \\
\text { meeting, allocation status not recorded in the medical record }\end{array}$ \\
\hline
\end{tabular}


Tiwari 2010

Methods

Randomisation method: generated by computer and concealed in serially numbered, opaque envelopes by a researcher not involved in participant recruitment

Analysis by ITT: yes

Power calculation: yes
Country: China (Hong Kong)

Setting: community centre providing a range of social, health, child care, educational, and recreational services for users of all age groups

\section{Inclusion criteria:}

- Community-dwelling Chinese women

- Aged $\geq 18$ years

- Residing or working in 1 of the districts covered by the community centre

- Screening positive for IPV within last 12 months

Exclusion criteria: women not communicating in Cantonese or Putonghua (the 2 main dialects in Hong Kong)

Participant numbers: numbers recruited: 200 (100 intervention group, 100 control group)

Number of dropouts: none

Numbers analysed (and percentage of recruited): 200 (100 (100\%) intervention group, 100 (100\%) control group)

Age: mean 38 years intervention group, 38 years control group

Ethnicity: all Chinese (born in Hong Kong: 33\% intervention group, $43 \%$ control group; born in mainland China: $65 \%$ intervention group, $56 \%$ control group)

Socioeconomic status indicators: employed: $30 \%$ intervention group, 32\% control group; experiencing financial hardship: $72 \%$ intervention group, $73 \%$ control group; in need of financial support: $65 \%$ intervention group, 58\% control group; receiving comprehensive social security assistance: $33 \%$ intervention group, $9 \%$ control group

Education background: $\leq 6$ th grade: $25 \%$ intervention group, $30 \%$ control group; 7 th-13th grade: $71 \%$ intervention group, $65 \%$ control group; postsecondary education: $4 \%$ intervention group, $5 \%$ control group

\section{Interventions Intervention (2 components)}

- Empowerment: included information about the cycle of violence, protection and safety, options and resources available, choice making and problem solving; this was a one-off, face-to-face session delivered by a social worker and lasted about $30 \mathrm{~min}$ (range 20-45 min).

- Telephone social support: included non-judgemental listening, discussion of needs, offering information or referral to other professionals/services as required; consisted of 12 scheduled weekly telephone calls lasting about 10-20 min each call, and 24-h access to a hotline for additional social support. In addition, women could receive the usual care services offered by the centre or its outreach sites.

\section{Control}

Women in the control group received the usual community services provided by the centre or its outreach sites; women could decide on the uptake of services according to their own needs (child care, healthcare and promotion, recreational facilities); at the time of the study, there was no provision of standard care for abused women in the community except crisis intervention for those severely abused

Notes

This study was supported by the Health and Health Services Research Fund awarded by the Food and Health Bureau of the Hong Kong SAR Government (Project \# 04060741) Study Start Date: February 2007, Study Completion Date: June 2009, Primary Completion Date: December 2008 (Final data collection date for primary outcome measure)

\section{Risk of bias}


Tiwari 2010 (Continued)

\begin{tabular}{lll} 
Bias & Authors' judgement & Support for judgement \\
\hline $\begin{array}{l}\text { Random sequence genera- } \\
\text { tion (selection bias) }\end{array}$ & Low risk & $\begin{array}{l}\text { Participants randomised (1:1) to groups "according to a list of random permu- } \\
\text { tations prepared by computer-generated blocked randomisation performed } \\
\text { by a research staff member who had not been involved in participant recruit- } \\
\text { ment" }\end{array}$ \\
\hline $\begin{array}{l}\text { Allocation concealment } \\
\text { (selection bias) }\end{array}$ & Low risk & $\begin{array}{l}\text { Quote: "The block size was kept secure by the randomiser, and the order of al- } \\
\text { location was centrally controlled to avoid any bias in selection. The allocation } \\
\text { sequence was concealed in opaque envelopes. At the time of randomisation, } \\
\text { the research assistant who had successfully recruited a participant called the } \\
\text { site investigator, who then opened the envelope containing the group assign- } \\
\text { ment. To ensure random assignment ... no detail was provided to the site in- } \\
\text { vestigator about the identity of the participant" }\end{array}$
\end{tabular}

Blinding (performance Low risk

bias and detection bias):

all outcomes
Blinding of participants and key personnel (people providing the intervention) was not possible. Assessors were blinded; they "were not involved in the design of the study, did not know the study hypotheses, and were blinded to group assignment" ... this appeared to be sustained "because none of the assessors knew the group assignment of the participants until they came to the last question, which solicited the participants' evaluation of the intervention or usual community services"

\begin{tabular}{|c|c|c|}
\hline $\begin{array}{l}\text { Incomplete outcome da- } \\
\text { ta (attrition bias): all out- } \\
\text { comes }\end{array}$ & Low risk & There were no missing data \\
\hline $\begin{array}{l}\text { Selective reporting (re- } \\
\text { porting bias) }\end{array}$ & Low risk & $\begin{array}{l}\text { All measures discussed in the 'Methods' sections of the papers also discussed } \\
\text { in the 'Results' sections }\end{array}$ \\
\hline $\begin{array}{l}\text { Baseline measurement of } \\
\text { outcome measures }\end{array}$ & Low risk & There were no significant differences on any of the outcome measures \\
\hline $\begin{array}{l}\text { Reliability of outcome } \\
\text { measures }\end{array}$ & Low risk & Outcome measures were reliable \\
\hline $\begin{array}{l}\text { Protection against conta- } \\
\text { mination }\end{array}$ & Unclear risk & $\begin{array}{l}\text { Participation was solicited through several means but primarily through cen- } \\
\text { tre events/publicity, so there were some opportunities for women in the } 2 \\
\text { arms to meet - even though the centre served a large population (approxi- } \\
\text { mately } 800,000 \text { ) }\end{array}$ \\
\hline
\end{tabular}

Trevillion 2013

\begin{tabular}{|c|c|}
\hline Methods & $\begin{array}{l}\text { Quasi-experimental controlled design within } 5 \text { community mental health teams ( } 3 \text { intervention and } 2 \\
\text { control teams). Qualitative Interviews, process evaluations, economic evaluation }\end{array}$ \\
\hline Participants & $\begin{array}{l}\text { Country: UK } \\
\text { Setting: Community Mental Health Teams (CMHTs) } \\
\text { Inclusion criteria: male and female CMHT service users (aged } \geq 18 \text { years) experiencing DV in the previ- } \\
\text { ous year, and scoring above the cut-off point (i.e... } \geq 3 \text { ) on the CAS } \\
\text { Exclusion criteria: service users deemed by clinicians to be too unwell to enter the study and those } \\
\text { living outside the catchment area } \\
\text { Abuse: total violence score (CAS) } 32.0(23.0-44.0) \text { at baseline } \\
\text { Participant numbers: } 5 \text { CMHTs recruited to the study ( } 3=\text { intervention, } 2=\text { control (usual care)). The } \\
5 \text { CMHTs had a caseload of around } 1220 \text { service users: approximately } 600 \text { cases in the control arm and } \\
620 \text { in the intervention arm. Of } 139 \text { service users assessed for eligibility, } 83 \text { did not meet the inclusion } \\
\text { criteria leaving } 52 \text { eligible; } 13 \text { declined to participate ( } 10 \text { intervention and } 3 \text { control arm participants). }\end{array}$ \\
\hline
\end{tabular}


Trevillion 2013 (Continued)

Unable to establish contact with 8 service users. Recruitment of service users via care-co-ordinators was high in the intervention group (21/27) but low in the control arm (1/7); 4 self-referrals were recruited in both arms. During researcher screening sessions a total of 87 service users ( $n=37$ in the intervention arm and $n=50$ in the control arm) were interviewed: 4 of the 5 eligible service users in the intervention arm and 2 of the 4 in the control arm agreed to participate.

$27 / 29$ intervention and $7 / 7$ control participants completed interviews at baseline and 3 months' follow-up.

Age: service users' mean age was 38 years

Ethnicity: $13(38 \%)$ were white and $21(62 \%)$ were of BAME origin

Children: $11 / 34$ women

Relationship status: most service users were single/separated or divorced and 7 (21\%) were married; $17(50 \%)$ lived alone and 12 (35\%) with a partner and/or children

Provision of integrated DV advocacy for service users modified for this study and delivered by DV advisors (seconded from a local voluntary sector organisation).

Advocacy comprised emotional and practical support, including DV education, facilitation of support groups, safety planning and legal/housing support.

In this study, each CMHT had a named advisor who was available to discuss/take referrals and feed outcomes back to the team and to regularly attend clinical meetings to discuss cases and provide DV education.

Content of the intervention:

- $4 \mathrm{~h}$ of DV training for clinicians

- DV manual for clinicians

- $6 \mathrm{~h}$ mental health training

- Referral pathway

- Advocacy (see above)

- Information campaign

\section{Controls}

Service users in the 2 control CMHTs received usual care, which could include referral to DV services.

Notes $\quad$ Focus of study included clinician training

Funded by the National Institute for Health Research (NIHR) under its Research for Patient Benefit (RfPB) Programme (PB-PG-0906-11026).

Primary diagnoses included depressive disorder $(n=13(39 \%))$, bipolar disorder $(n=5(15 \%))$ and schizophrenia and related disorders $(n=6(18 \%))$

Includes economic analysis

\section{Risk of bias}

\begin{tabular}{lll}
\hline Bias & Authors' judgement & Support for judgement \\
\hline $\begin{array}{l}\text { Random sequence genera- } \\
\text { tion (selection bias) }\end{array}$ & High risk & $\begin{array}{l}\text { Quasi-experimental controlled design: as this was a pilot study did not ran- } \\
\text { domly allocate teams and instead determined allocation by grouping teams } \\
\text { that shared the same building (to avoid problems of contamination) }\end{array}$ \\
\hline
\end{tabular}

$\begin{array}{lll}\text { Allocation concealment } & \text { High risk } & \text { As this was a pilot study did not randomly allocate teams } \\ \text { (selection bias) }\end{array}$


Trevillion 2013 (Continued)
Blinding (performance
High risk
As this was a pilot study did not randomly allocate teams

bias and detection bias):

all outcomes

\begin{tabular}{|c|c|c|}
\hline $\begin{array}{l}\text { Incomplete outcome da- } \\
\text { ta (attrition bias): all out- } \\
\text { comes }\end{array}$ & Unclear risk & $\begin{array}{l}\text { Results are presented on the } 34 \text { service users ( } 27 \text { intervention and } 7 \text { control } \\
\text { participants) who completed interviews at baseline and } 3 \text { months' follow-up } \\
\text { out of the } 52 \text { eligible and } 36 \text { recruited }\end{array}$ \\
\hline
\end{tabular}

Selective reporting (re- Low risk
porting bias)
participants) who completed interviews at baseline and 3 months' follow-up of the 52 eligible and 36 recruited

\begin{tabular}{|c|c|c|}
\hline $\begin{array}{l}\text { Baseline measurement of } \\
\text { outcome measures }\end{array}$ & Low risk & \\
\hline $\begin{array}{l}\text { Reliability of outcome } \\
\text { measures }\end{array}$ & Low risk & \\
\hline $\begin{array}{l}\text { Protection against conta- } \\
\text { mination }\end{array}$ & Low risk & $\begin{array}{l}\text { As this was a pilot study did not randomly allocate teams and instead deter- } \\
\text { mined allocation by grouping teams that shared the same building (to avoid } \\
\text { problems of contamination). }\end{array}$ \\
\hline
\end{tabular}

How valuable is the re- Low risk Lalue, focus of study included clinician training
search (richness of the da-
ta for the review research
questions)?

\title{
Tutty 1996
}

Methods Pre-post study with in-depth interviews

\section{Participants}

\author{
Country: Canada \\ Setting: follow-up shelter services \\ Inclusion criteria: NS \\ Exclusion criteria: NS \\ Participant numbers: workers provided the demographic characteristics and answered rating scales \\ regarding safety, self-esteem, and use of social supports for 60 women \\ Standardised measures of social support and perceived stress were completed by a subset of 28 \\ women when they entered the programmes and 3 months later. \\ In-depth interviews with 31 of the 60 women \\ Ethnicity: $n=8$ Aboriginal, the remainder had recently immigrated from countries such as the Philip- \\ pines, India, and Germany. \\ Age: average age 32.5 years (range 18-60) \\ Abuse: the women reported having separated previously an average of 4 times (SD $=5)$ with a range of \\ 0-20 separations; before they entered the emergency shelter, $87 \%(n=50)$ reported having been both \\ physically and emotionally abused. Of these, $35 \%$ or 21 women also reported having been sexually \\ abused by their partner. The police had intervened in $55 \%$ of the total cases $(n=33)$ and weapons or \\ objects had been used in the abuse of $41 \%$ of the women $(n=24)$.
}

Socioeconomic status indicators: income. Almost $2 / 3$ of the women (65\%) were receiving social assistance or worked part-time with a social assistance supplement.

Education: average level completed was Grade $11(\mathrm{SD}=2.3)$ with a range of 1 year of school to 14

Children: $75 \%$ of the women had children ranging in age from 3 months to adults of 30 years 
Tutty 1996 (Continued)

Relationship status: $57 \%$ of the women $(n=32)$ described themselves as separated or divorced, $28.3 \%$, single $(n=17), 11.7 \%$ married, and $6.7 \%$ as common-law

Interventions

The major goal of follow-up programmes is to respond to the diverse needs of each woman and to coordinate the support services required for her to remain independent and safe. The issues that follow-up workers face range from procuring basic needs, such as income, housing, and furnishings, to help with the legal system, educational opportunities, job training, or employment. Because a considerable proportion of abused women flee their homes to escape their partners, they often move to a new community where they know no-one. A major focus is helping the woman to plan how to respond to her ex-partner, particularly if he has child visitation rights or has been threatening her.

Follow-up programmes as reported here differ from the advocacy programmes for former shelter resi-
dents that are relatively common across North America in several ways. Advocacy programmes are pri-
marily directed toward helping assaulted women connect with community resources.
Although advocacy is certainly an important role of workers in follow-up programmes, it is only 1 facet
of the service.
A second distinction is that many advocacy programmes use volunteers to deliver the services rather
than professionals

\section{Risk of bias}

\begin{tabular}{|c|c|c|}
\hline Bias & Authors' judgement & Support for judgement \\
\hline $\begin{array}{l}\text { Random sequence genera- } \\
\text { tion (selection bias) }\end{array}$ & High risk & Pre-post study \\
\hline $\begin{array}{l}\text { Allocation concealment } \\
\text { (selection bias) }\end{array}$ & High risk & Pre-post study \\
\hline $\begin{array}{l}\text { Blinding (performance } \\
\text { bias and detection bias): } \\
\text { all outcomes }\end{array}$ & High risk & Pre-post study \\
\hline $\begin{array}{l}\text { Incomplete outcome da- } \\
\text { ta (attrition bias): all out- } \\
\text { comes }\end{array}$ & Low risk & \\
\hline $\begin{array}{l}\text { Selective reporting (re- } \\
\text { porting bias) }\end{array}$ & High risk & $\begin{array}{l}\text { Only } 1 \text { of the follow-up programme co-ordinators was interested in evaluating } \\
\text { self-esteem as an outcome variable. }\end{array}$ \\
\hline $\begin{array}{l}\text { Baseline measurement of } \\
\text { outcome measures }\end{array}$ & Low risk & Pre-post study \\
\hline $\begin{array}{l}\text { Reliability of outcome } \\
\text { measures }\end{array}$ & Low risk & \\
\hline $\begin{array}{l}\text { Protection against conta- } \\
\text { mination }\end{array}$ & Low risk & Pre-post study of existing programme \\
\hline $\begin{array}{l}\text { How valuable is the re- } \\
\text { search (richness of the da- } \\
\text { ta for the review research } \\
\text { questions)? }\end{array}$ & Low risk & Low value \\
\hline
\end{tabular}


Umeda 2017

\begin{tabular}{ll}
\hline Methods & Semi-structured interview \\
\hline Participants & Country: Japan \\
& Setting: mixed community settings \\
& Participant numbers: 8 expert professionals with extensive experience in the care of IPV victims \\
& $\begin{array}{l}\text { Experience: participation in a national network of IPV advocacy (from obstetrics and gynaecology, } \\
\text { psychological counselling, women's shelter, and public referral services for IPV survivors). Average du- } \\
\text { ration of working with IPV survivors: } 13.5 \text { years }\end{array}$ \\
\hline Interventions & Advocacy non-specified \\
\hline Notes & Interviews were conducted from August-December in 2010
\end{tabular}

\section{Risk of bias}

Bias Authors' judgement Support for judgement

How valuable is the re-

High risk

search (richness of the da-

ta for the review research questions)?

\section{Was there a clear state- Low risk ment of the aims of the re-} search?

\section{Was the research design appropriate to address the aims of the research?}

\section{Low risk}

Was the recruitment strat- Low risk egy appropriate to the aims of the research?
Yes. Snowballing recruitment till diversity of perspectives reached.

\footnotetext{
Has the relationship be- Low risk

tween researcher and par-

ticipants been adequately

considered?
}

\begin{tabular}{ll}
\hline $\begin{array}{l}\text { Is a qualitative methodol- } \\
\text { ogy appropriate? }\end{array}$ & Low risk \\
\hline $\begin{array}{l}\text { Have ethical issues been } \\
\text { taken into consideration? }\end{array}$ & Low risk \\
\hline $\begin{array}{l}\text { Were the data collected in } \\
\text { a way that addressed the } \\
\text { research issue? }\end{array}$ & Low risk \\
\hline $\begin{array}{l}\text { Was the data analysis suf- } \\
\text { ficiently rigorous? }\end{array}$ & Low risk \\
\hline $\begin{array}{l}\text { Is there a clear statement } \\
\text { of findings? }\end{array}$ & Low risk \\
\end{tabular}


Weisz 1999

\begin{tabular}{ll}
\hline Methods & Qualitative focus groups and interviews \\
\hline Participants & Country: USA \\
Setting: court-ordered treatment with Family Shelter Service (FSS) \\
Inclusion criteria: DV survivors \\
Exclusion criteria: NS \\
Participant numbers: $\mathrm{n}=11$ women survivors; 3 focus groups with FSS staff: $1 \times 3$ advocates, $1 \times 7$ shel- \\
ter workers and the programme director, 1 x 6 FSS staff members including the agency director, the \\
programme director, counsellors, and advocates. \\
Age: interviewees $27-45$ years \\
Ethnicity: 2 interviewees African American, 1 Puerto Rican, and the others were non-Hispanic or white. \\
Relationship status: 8 interviewees were married to their abusers during most of the abusive incidents \\
they described, and 3 were in an intimate non-married relationship. At the time of the interviews, 2 of \\
the women were married and planned to remain married to the men who abused them. 2 women were \\
in the process of getting divorced from their abusers; 4 were already divorced.
\end{tabular}

Children: all of the women interviewees had 1-4 children

Socioeconomic status indicators: 4 women interviewees worked full-time outside the home, and 2 worked part-time and had professional training. The others worked in blue-collar or office jobs. 5 women were not working outside the home.

Interventions

The DuPage County DV Protocol, instituted in 1989, provides for mandatory arrest of batterers with probable cause, no-drop prosecution, and frequent use of court-ordered treatment for batterers. Intervention: active outreach and support of victims was topic of study.

Advocates contacted victims following a police intervention at their house, whether or not there was an arrest. The advocates offered support, gave women information about the legal system, and informed them about counselling and advocacy services. Advocates assisted women in obtaining protective orders and gave support during prosecution of batterers.

Advocates provided survivors with information regarding definitions of illegal behaviour and appropriate times to call the police.

They gave women information on protective orders and prosecution and provided emotional support during stressful court appearances.

Notes Funding NS

\section{Risk of bias}

\section{Bias \\ Authors' judgement Support for judgement}

How valuable is the re- Low risk search (richness of the data for the review research questions)?
This is a very old study. The context of understanding DV as a legal issue and court responses to DV is relatively new.

\section{Was there a clear state- Low risk}

ment of the aims of the re-

search?

Was the research design

Low risk

appropriate to address the

aims of the research? 
Weisz 1999 (Continued)

Was the recruitment strat- High risk Via a gatekeeper. Study authors recognise this could lead to a selection bias egy appropriate to the towards women still involved or connected to the service, and likely hold more aims of the research? positive views.

\begin{tabular}{|c|c|c|}
\hline $\begin{array}{l}\text { Has the relationship be- } \\
\text { tween researcher and par- }\end{array}$ & High risk & $\begin{array}{l}\text { No. Study author gave the women their personal telephone number, rather } \\
\text { than numbers of any professionals. }\end{array}$ \\
\hline
\end{tabular}
ticipants been adequately considered?

\section{Is a qualitative methodol- Low risk ogy appropriate?}

\begin{tabular}{ll}
\hline $\begin{array}{l}\text { Have ethical issues been } \\
\text { taken into consideration? }\end{array}$ & High risk
\end{tabular}

Were the data collected in Low risk
a way that addressed the

a way that addressed the

research issue?

Was the data analysis suf- High risk No formal qualitative methods used.
ficiently rigorous?

ficiently rigorous?
Is there a clear statement Low risk
of findings?

of findings?

White 2019

\begin{tabular}{|c|c|}
\hline Methods & Qualitative 'conversations' and theoretical discussions \\
\hline \multirow[t]{2}{*}{ Participants } & Country: USA \\
\hline & $\begin{array}{l}\text { Setting: community } \\
\text { Inclusion criteria: leaders in the fields of DV and sexual assault from various areas } \\
\text { Exclusion criteria: NS } \\
\text { Participant numbers: } 72\end{array}$ \\
\hline Interventions & NS \\
\hline Notes & Study took place $2014-2016$ \\
\hline \multicolumn{2}{|l|}{ Risk of bias } \\
\hline Bias & Authors' judgement Support for judgement \\
\hline $\begin{array}{l}\text { How valuable is the re- } \\
\text { search (richness of the da- } \\
\text { ta for the review research } \\
\text { questions)? }\end{array}$ & Low risk \\
\hline $\begin{array}{l}\text { Was there a clear state- } \\
\text { ment of the aims of the re- } \\
\text { search? }\end{array}$ & Low risk \\
\hline
\end{tabular}


White 2019 (Continued)

\section{Was the research design appropriate to address the aims of the research?}

Low risk

\author{
Was the recruitment strat- Low risk \\ egy appropriate to the \\ aims of the research?

Is a qualitative methodol- Low risk
ogy appropriate?

\begin{tabular}{|c|c|c|}
\hline $\begin{array}{l}\text { Were the data collected in } \\
\text { a way that addressed the } \\
\text { research issue? }\end{array}$ & Unclear risk & NS \\
\hline $\begin{array}{l}\text { Was the data analysis suf- } \\
\text { ficiently rigorous? }\end{array}$ & Uncle & $\begin{array}{l}\text { Grounded theory is described as the guiding principle, but no further detail on } \\
\text { methods of analysis are given. }\end{array}$ \\
\hline
\end{tabular}

Grounded theory is described as the guiding principle, but no further detail on methods of analysis are given.

\section{Is there a clear statement Low risk} of findings?

\section{Wies 2008}

\begin{tabular}{ll}
\hline Wies 2008 & Participant observation and interviews \\
\hline Pethods & $\begin{array}{l}\text { Country: USA } \\
\text { Setting: the Battered Women's programme (BWP) and the DV centre in a medium-sized metropolitan } \\
\text { area in the state of Kentucky, USA }\end{array}$ \\
& $\begin{array}{l}\text { Participant numbers: semi-structured, in-depth interviews: 20 BWP advocates, } 15 \text { DV centre advo- } \\
\text { cates, and 14 oral history participants (ie people active in past feminist battered women's movement), } \\
\text { totaling } 49 \text { interviews }\end{array}$ \\
\hline Interventions & Shelter advocacy non-specified \\
\hline Notes & Data collection May 2004-December 2005
\end{tabular}

\section{Risk of bias}

\begin{tabular}{lll}
\hline Bias & Authors' judgement & Support for judgement \\
\hline $\begin{array}{l}\text { How valuable is the re- } \\
\text { search (richness of the da- } \\
\text { ta for the review research } \\
\text { questions)? }\end{array}$ & Low risk & The study is a history of professionalisation of advocacy. \\
\hline $\begin{array}{l}\text { Was there a clear state- } \\
\text { ment of the aims of the re- }\end{array}$ & Low risk & \\
search? & & \\
\hline $\begin{array}{l}\text { Was the research design } \\
\text { appropriate to address the } \\
\text { aims of the research? }\end{array}$ & Unclear risk & $\begin{array}{l}\text { Not certain. Method is described as participant observation. Later, issue of } \\
\text { maintaining boundaries is discussed }\end{array}$ \\
\hline
\end{tabular}


Wies 2008 (Continued)

Was the recruitment strat- Unclear risk Not clear. Working as an advocate themselves, the study author can gain egy appropriate to the greater access to advocates.

aims of the research?

$\begin{array}{ll}\begin{array}{l}\text { Has the relationship be- } \\ \text { tween researcher and par- }\end{array} & \text { Low risk }\end{array}$
ticipants been adequately considered?
Is a qualitative methodol- Low risk
ogy appropriate?

\section{Have ethical issues been High risk \\ taken into consideration?}

\begin{tabular}{|c|c|c|}
\hline $\begin{array}{l}\text { Were the data collected in } \\
\text { a way that addressed the }\end{array}$ & Unclear risk & $\begin{array}{l}\text { The focus is } 1 \text { geographical area and service provider. The professionalisation } \\
\text { under examination may be relevant to this site only. }\end{array}$ \\
\hline
\end{tabular}

research issue?

\section{Was the data analysis suf- High risk \\ ficiently rigorous?}

Is there a clear statement Low risk
of findings?

of findings?

Wong 2013

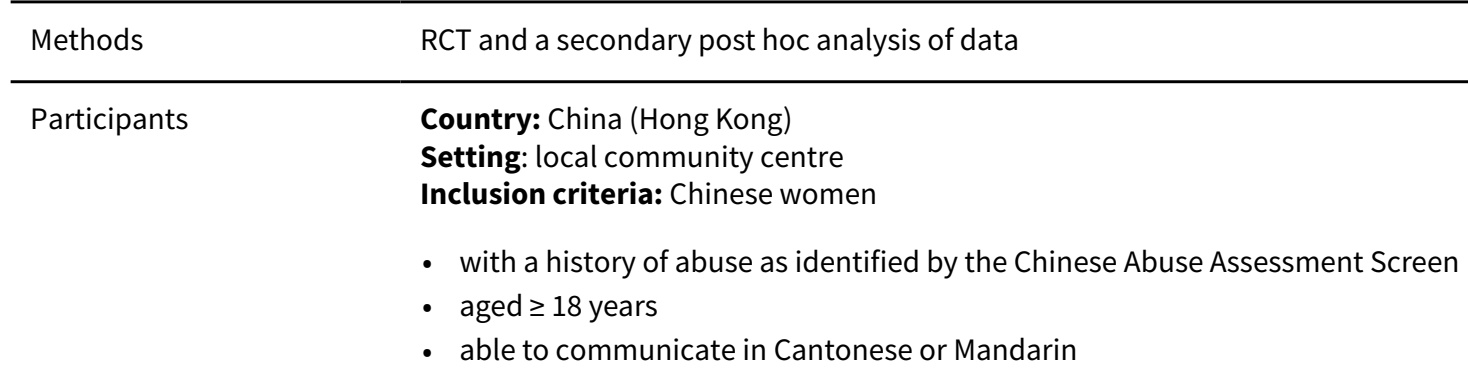

Participant numbers: $\mathrm{n}=200,100$ in each study arm. Among the 1753 potential participants, 202 $(11.5 \%)$ screened positive for IPV. 2 declined to participate. The remaining 200 participants were randomised to either the intervention or control group. There was no loss to follow-up and no report of harm to the participants.

Ethnicity: $n=142$ (62\%) Mainland China; $n=60$ (30.5\%) immigrants

Socioeconomic status indicators: immigrant women had lower employment status $(n=11,18.3 \%, P=$ 0.012)

Age: mean $38.1 \pm 7.21$ years. Immigrant women were much younger ( mean age $=35.6, S D=4.8, P=$ 0.001)

Children: nearly half had $\leq 1$ child; immigrant women had more children $(P=0.003)$

Relationship status: $90 \%$ were married. Immigrant women were more likely to stay married or continue to cohabit with their partners $(n=58,97.7 \%, P=0.001)$ during the study

Interventions Intervention

12-week advocacy intervention. 
The intervention consisted of 1 × 30-min face-to-face empowerment training session followed by 12 weeks of telephone social support. The focus was to increase women's safety through the recognition of increased danger, paired with a safety plan developed for individual use. Women were given information about the cycle of violence, facts, and options regarding partner violence, legal protection orders, filing for criminal charges, and community resources for abused women, for them to make their own decisions. Each woman was given an empowerment pamphlet to reinforce the information as used in Tiwari 2005.

The design of the empowerment training was originally developed by McFarlane 1997 and is based on Dutton's empowerment model that includes protection and enhanced choice-making and problem-solving.

The telephone social support involved 12 scheduled weekly phone calls. The 12 telephone sessions were decided as a balance between the maximum number that would not overwhelm the participants, and the minimum number needed for sufficient social support based on the experience of the investigator team. The telephone social support was based on Cohen's Social Support Theory to enhance the cultural congruence with respect to addressing the cultural difference between abused Chinese women and abused women in Western countries. It aimed to provide tangible and perceived social support through social relationships to promote health and well-being.

\section{Controls}

Usual community services provided by the community centre including health, social, educational, and recreational services.

\section{Risk of bias}

\begin{tabular}{|c|c|c|}
\hline Bias & Authors' judgement & Support for judgement \\
\hline $\begin{array}{l}\text { Random sequence genera- } \\
\text { tion (selection bias) }\end{array}$ & Unclear risk & NS how done \\
\hline $\begin{array}{l}\text { Allocation concealment } \\
\text { (selection bias) }\end{array}$ & Unclear risk & NS how done \\
\hline $\begin{array}{l}\text { Blinding (performance } \\
\text { bias and detection bias): } \\
\text { all outcomes }\end{array}$ & Unclear risk & NS how done \\
\hline $\begin{array}{l}\text { Incomplete outcome da- } \\
\text { ta (attrition bias): all out- } \\
\text { comes }\end{array}$ & Low risk & No participants were lost to follow-up \\
\hline $\begin{array}{l}\text { Selective reporting (re- } \\
\text { porting bias) }\end{array}$ & Low risk & \\
\hline $\begin{array}{l}\text { Baseline measurement of } \\
\text { outcome measures }\end{array}$ & Unclear risk & $\begin{array}{l}\text { At baseline, the intervention and control groups were comparable in all re- } \\
\text { spects, except that significantly more women in the intervention than control } \\
\text { group received comprehensive social security assistance (CSSA) (33\% vs 9\%, P } \\
<0.001) \text { ) }\end{array}$ \\
\hline
\end{tabular}

Reliability of outcome Low risk
measures

measures

Protection against conta- Low risk Controls had some form of intervention
mination

mination 
Wong 2013 (Continued)

How valuable is the re

Methods Qualitative interviews

\section{Participants}

\section{Country: USA}

Setting: IPV(IPV)-focused agencies: rural: 7 urban: 15

Inclusion criteria: people who had been working in direct DV services $\geq 1$ year were eligible to participate. Participants needed to be currently working in a direct service role with survivors. The study was open to people working in a direct service role that was not specifically psychotherapy. Advocates were asked about survivor status as part of the interview protocol, but it was not an eligibility criterion.

Participants: 22 advocates completed 21 interviews in 2 states ( 2 advocates were interviewed as a pair)

Ethnicity: 14 white, 5 Latina, and 3 as a person of colour in additional categories.

Age: $22-62$

Experience: An advocate's time in a current position ranged from three months to 20 years, and overall time working with survivors of intimate partner violence ranged from 1.5 to 32 years.

\section{Interventions}

Notes

18 participants identified some history of abuse. This included child witnessing of violence, experience of sexual assault, and some form of intimate partner abuse as a teenager or adult. 7 worked in rural locations.

\section{Risk of bias}

\section{Bias}

Authors' judgement Support for judgement

How valuable is the research (richness of the da-

High risk

ta for the review research questions)?

\section{Was there a clear state- Low risk} ment of the aims of the research?

Was the research design Low risk
appropriate to address the
aims of the research?

$\begin{array}{ll}\text { Was the recruitment strat- Low risk } & \text { Rural and urban } \\ \text { egy appropriate to the } & \end{array}$
aims of the research?

\footnotetext{
Is a qualitative methodol- Low risk ogy appropriate?
} 
Wood 2014 (Continued)

Were the data collected in

Low risk a way that addressed the research issue?

Was the data analysis suf- Low risk

ficiently rigorous?

Is there a clear statement Low risk

of findings?

\section{Wuest 2015}

Methods

Mixed methods over the 3 papers: single-group, pre-post intervention study with chart review data and interviews for process evaluation

\section{Participants}

\section{Country: Canada}

Setting: Women's Equality Branch enabled partnering with DV outreach programmes in 2 urban and 2 rural communities

Inclusion criteria: all women who had separated from abusive partners

Exclusion criteria: women were not excluded on the basis of their substance use or mental health issues, common exclusionary criteria for DV programmes.

Participant numbers: pre-post study: 52 abused women of whom 42 completed data collection; interviews with $18(43 \%)$ of the 42, as purposefully sampled participants, and all 9 interventionists Age: mean 41.7 (pre-post study)

Ethnicity: NS

Abuse: mean duration in years 9.7 (pre-post study)

Intervention was developed across the 4 sites, using interviews with 20 women with current or past exposure to partner abuse, and 27 nurse home visitors employed within the same 4 sites the women had used ( 22 of the 27 nurse home visitors participated in both of the development focus groups conducted at their site) (see Jack paper, secondary reference for Wuest 2015).

The study iHEAL: a theory-based, primary healthcare intervention provided in partnership by a Registered Nurse (RN) generalist and DV support worker at each site. Outreach workers committed to 3 sessions/participant as in-kind services. RNs were expected to meet about 11 times with each participant. For each participant, decisions regarding who would offer each component of the iHEAL were based on interventionist expertise and availability, and the woman's priorities. Aims of advocacy were to improve women's quality of life and health after leaving an abusive partner by enhancing women's capacity and reducing intrusion.

Notes

\section{Risk of bias}

\begin{tabular}{lll}
\hline Bias & Authors' judgement & Support for judgement \\
\hline $\begin{array}{l}\text { Random sequence genera- } \\
\text { tion (selection bias) }\end{array}$ & High risk & Pre-post study \\
\hline $\begin{array}{l}\text { Allocation concealment } \\
\text { (selection bias) }\end{array}$ & High risk & Pre-post study \\
\hline $\begin{array}{l}\text { Blinding (performance } \\
\text { bias and detection bias): } \\
\text { all outcomes }\end{array}$ & High risk & Pre-post study \\
\hline
\end{tabular}


Wuest 2015 (Continued)

Incomplete outcome da- Unclear risk 52 women were recruited; 10 (19\%) did not complete the study: 2 declined afta (attrition bias): all out- ter baseline data collection, 6 withdrew during the intervention, and 2 comcomes pleted the iHEAL but could not be located for the 12-month data collection. Withdrawal did not differ by site.

Selective reporting (re- Low risk
porting bias)

Baseline measurement of Low risk

outcome measures

\begin{tabular}{ll}
\hline $\begin{array}{l}\text { Reliability of outcome } \\
\text { measures }\end{array}$ & Low risk \\
& months, except for the Mastery Scale, for which Cronbach alpha was $0.78, .69$, \\
& and 0.77 respectively.
\end{tabular}

\section{Protection against conta- High risk} mination

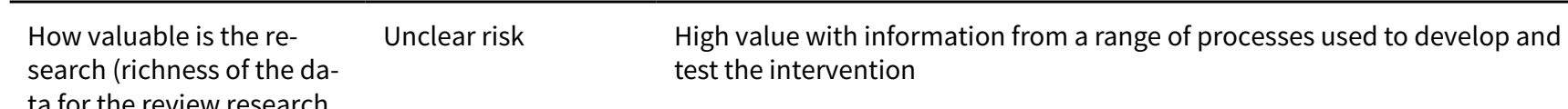

ta for the review research

questions)?

\section{Zweig 2007}

\begin{tabular}{ll}
\hline Methods Survey \\
\hline
\end{tabular}

Participants Country: USA

Setting: co-ordinated community response - community agencies

Participant numbers: 26 communities across 8 states ( 2 in Colorado, 4 in Illinois, 3 in Massachusetts, 3 in Pennsylvania, 3 in Texas, 4 in Vermont, 3 in Washington, and 4 in West Virginia); telephone interviews with 1509 women from these

Ethnicity: white non-Hispanic (80\%); about $8 \%$ were Hispanic, $5 \%$ were black non-Hispanic, 5\% were biracial non-Hispanic, $1 \%$ was Asian/Pacific Islander, and $1 \%$ was Native American

Socioeconomic status indicators: $29 \%$ of the women had personal incomes of < USD 5000, but only $3 \%$ of women lived in households with incomes that low. $20 \%$ of the sample lived in households with incomes between USD 35,000 and USD 50,000, and another 24\% in households with incomes between USD 50,000 and USD 80,000

Relationship status: most women were married $(27 \%)$ or separated (39\%)

Interventions

The STOP Violence Against Women Formula Grants programme to develop and strengthen victim services and to support communities in implementing systems change through the co-ordinated efforts of multiple agencies, so that victims encounter a positive and effective response from the criminal and civil legal systems, and from other agencies offering services and supports.

Notes Interviews June 2001-February 2002

\section{Risk of bias}

\begin{tabular}{lll}
\hline Bias & Authors' judgement & Support for judgement \\
\hline
\end{tabular}

Reliability of outcome

High risk

measures 
Zweig 2007 (Continued)

$\begin{aligned} & \text { Representativeness of the Low risk } \\ & \text { sample }\end{aligned} \quad \begin{aligned} & \text { A wide diversity in the geographic settings of the study communities and in the } \\ & \text { level of interaction occurring between community agencies }\end{aligned}$
sample level of interaction occurring between community agencies

\begin{tabular}{lll}
\hline Conduct of pilot testing $\quad$ High risk $\quad$ None undertaken \\
\hline
\end{tabular}

Established validity of the High risk

survey instrument

BAME: black and minority ethnic; BRFSS: Behavioral Risk Factor Surveillance System; BSI: Brief Symptom Inventory; CAS: Composite Abuse Scale; CBT: cognitive behavioural therapy; CDC: Centres for Disease Control and Prevention; CES-D: Centre for Epidemiological Studies-Depression scale; CHW: community health worker; CTI: critical time intervention; CTS: Conflict Tactics Scale; CTS2: Conflict Tactics Scale revised; DA(S): Danger Assessment (score); DOVE: Domestic Violence Enhanced Home Visitation Programme; DSM-IV: Diagnostic and Statistical Manual of Mental Disorders fourth edition; DTS: Davidson Trauma Scale; DV: domestic violence; DVHVI: Domestic Violence Home Visit Intervention; ED: emergency department; EDVA: emergency department victim advocacy; EPDS: Edinburgh Postnatal Depression Scale; GED: General Educational Development; ICD: International Statistical Classification of Diseases and Related Health Problems; IDVA: independent domestic violence advocacy; IPA: Index of Psychological Abuse; IPV: intimate partner violence; ISA: Index of Spouse Abuse; ISEL: Interpersonal Support Evaluation List; ITT: intention-to-treat; LDVP: Latina Domestic Violence Programme; LGBTQ: lesbian, gay, bisexual, transsexual, queer; M: mean; MCH: Maternal Child Health (clinics); MOSAIC: Mothers' Advocates in the Community; MOVERS: Measure of Victims' Empowerment Related to Safety; NFP: Nurse-Family Partnership; NGO: non-governmental organisation; NS: not stated; PMWI: Psychological Maltreatment of Women Inventory; PTSD: post-traumatic stress disorder; RCT: randomised controlled trial; ROAD: Reaching Out About Depression; SCID: Structured Clinical Interview for Diagnostic and Statistical Manual of Mental Disorders; SD: standard deviation; SEI: Self-Esteem Inventory; SES: socio-economic status; SF-36: Short-form-36; SVAWS: Severity of Violence Against Women Scale; WEB: Women's Experience with Battering

\section{Characteristics of excluded studies [ordered by study ID]}

\begin{tabular}{|c|c|}
\hline Study & Reason for exclusion \\
\hline Anderson 2012 & Type of data: not associated with an intervention; abstract concepts \\
\hline Andrews 2011 & Focus on substance abuse. No separate data for IPV \\
\hline Bair-Merritt 2010 & Focus on families not abused women \\
\hline Basu 2009 & $\begin{array}{l}\text { Group intervention not fitting advocacy as we have defined it and with no relevance to our research } \\
\text { questions }\end{array}$ \\
\hline Becker 2008 & Focus on parenting and children. No separate data for IPV \\
\hline Bennett 2007 & $\begin{array}{l}\text { Focus on drug abuse and co-ordinated community response and with no relevance to our research } \\
\text { questions }\end{array}$ \\
\hline Camacho 2008 & Type of data: criminal justice outcomes rather than specific features of advocacy \\
\hline Carlson 2012 & Focus on HIV. No separate data for IPV \\
\hline Champion 2007 & Focus on HIV. No separate data for IPV \\
\hline Clarke 2013 & Co-ordinated community response, not advocacy as we have defined it and includes perpetrator \\
\hline Cupples 2011 & Not abused women \\
\hline Davidson 2012 & Not advocacy as we have defined it. Not within an advocacy programme \\
\hline Denne 2012 & Advocacy is for perpetrators \\
\hline
\end{tabular}




\begin{tabular}{|c|c|}
\hline Study & Reason for exclusion \\
\hline Donovan 2010 & $\begin{array}{l}\text { Service evaluation (what works evalutaion; not research) with insufficient data to answer our re- } \\
\text { search questions }\end{array}$ \\
\hline El-Mohandes 2011 & Focus on birth outcomes. No separate data for IPV \\
\hline Feder 2011 & Not advocacy as we have defined it \\
\hline Fox 2018 & Not advocacy as we have defined it \\
\hline Giocolea 2015 & Not advocacy as we have defined it \\
\hline Goldblatt 2009 & Focus on family lives of advocates \\
\hline Graham-Bermann 2013 & Focus on parenting. Not advocacy as we have defined it \\
\hline Hart 2013 & Background information on IPV for practitioners \\
\hline Hovmand 2009 & Methodology paper \\
\hline Howard 2017 & About HIV. No abused women \\
\hline Jack 2015 & Not abused women. Not advocacy as we have defined it \\
\hline Juillard 2016 & Not just IPV. No separate data on IPV \\
\hline Kramer 2012 & Focus on parenting. Not advocacy as we have defined it \\
\hline Kyegombe 2014 & Focus on HIV. No separate data for IPV \\
\hline Lorenzo 2018 & Not advocacy as we have defined it \\
\hline Mantler 2017 & Not advocacy as we have defined it. About shelter change \\
\hline McFeely 2017 & Health visitors. Not advocacy as we have defined it \\
\hline McKean 2004 & Not advocacy as we have defined it. Capacity building of services \\
\hline McLelland 2008 & Not advocacy as we have defined it \\
\hline McNamara 1997 & Not clear what case management entailed (i.e. whether it included advocacy as we have defined it) \\
\hline Miller 2011 & Not advocacy as we have defined it \\
\hline Miller 2014 & Not advocacy as we have defined it \\
\hline Mitchell 2006 & $\begin{array}{l}\text { Not advocacy as we have defined it or with useful information even though it considers the concept } \\
\text { of coping }\end{array}$ \\
\hline Nichols 2013 & Theoretical and with no relevance to our research questions \\
\hline Rhodes 2014 & $\begin{array}{l}\text { Focus on problem drinking and with no relevance to our research questions. No separate data for } \\
\text { IPV }\end{array}$ \\
\hline Saggurti 2014 & Focus on HIV. No separate data for IPV \\
\hline
\end{tabular}




\begin{tabular}{ll}
\hline Study & Reason for exclusion \\
\hline Samuels-Dennis 2013 & Both IPV and child abuse. No separate data for IPV \\
\hline Simmons 2011 & Not advocacy as we have defined it but access to services \\
\hline Valpied 2014 & Not advocacy as we have defined it \\
\hline Wagman 2015 & Focus on HIV. No separate data for IPV \\
\hline Zlotnick 2011 & Psychotherapy only. Not advocacy as we have defined it \\
\hline
\end{tabular}

IPV: intimate partner violence

Characteristics of studies awaiting assessment [ordered by study ID]

Harris 2002

Notes The abstract says this paper reports on a collaboration between a US domestic violence shelter and city hospitals to provide crisis counsellors to abused patients during their ED visits. Services include on-site counselling, assistance with filling out protection orders and with making police reports, and referral of many of the women to shelter and/or support groups. As we cannot retrieve the full text we have not been able to evaluate the relevance of this paper (Harris 2002). MF. Advocacy for battered women: implications for a coordinated community response. In: Shepard MF, Pence EL, editor(s). Coordinating Community Responses to Domestic Violence: Lessons from Duluth and Beyond. Thousand Oaks, CA: Sage, 1999:115-25

ED: emergency department

Characteristics of ongoing studies [ordered by study ID]

\section{NCT03016481}

Trial name or title Realizing opportunities for self-supported improvement (ROSSI) (ROSE-SAFE)

Starting date $\quad 15$ April 2017

Name: Iwona Juskiewicz, MD, MPH

Address:

Telephone: 585-275-4961

Email: iwona_juskiewicz@urmc.rochester.edu 
NCT03016481 (Continued)

Exclusion criteria: if they have active psychosis or alcohol or substance dependence based on 3month threshold on the Mini-International Neuropsychiatric Interview (MINI), or who are unable to understand the consent form or study procedures, are imminently suicidal, or are currently working with a CHW

Participant numbers: 300

Age: $\geq 18$ years

Ethnicity: NS

Intervention: active comparator: the CHW - Personalized Support for Progress

Participants will meet with their CHW to prioritise their needs detected by the Promote psychosocial screening and identify their preferences for intervention using a secure, online card sorting tool called Optimal Sort, allowing them both to begin to identify goals. The prioritisation survey results provide subjects a personalised package of services that map onto the 4 main intervention options: legal help, problem solving therapy, medical consultation, or social services.

Control: care as usual- Social Worker

Based on their assessment and the participants' needs, brief onsite interventions are provided and consist of empowerment-focused advocacy, IPV education, community referrals, and safety planning. Participants determine follow-up. This will be a short-term interaction as routinely practiced across hospital settings.

Outcomes: patient safety ( 0 and 6 months); depression ( 0 and 9 months); quality of life ( 0 and 12 months)

Trial registration number: NCT03016481 against women

Starting date 24 August 2017

Contact information

Name: Nancy Glass, PhD, MPH

Address: RNJohns Hopkins University

Telephone: $410-614-2849$

Email: nglass1@jhu.edu

Setting: primary and reproductive health clinics in 4 states (Arizona, Massachusetts, Pennsylvania and West Virginia)

\section{Inclusion criteria:}

- Seeking healthcare at $1 / 14$ partner clinics

- Ability to complete a survey on a device (computer/tablet/smartphone in English or Spanish)

- Access to safe device (as defined above) if doing surveys online

- Has a safe email address or safe phone number

- Is not acutely ill

\section{Exclusion criteria:}

- Male 
- Not seeking healthcare at $1 / 9$ partner clinics

- Aged $<18$ years

- Aged $>59$ years

- Cannot read/speak English or Spanish

- No access to safe device if doing surveys online

- Does not have a safe email address or safe phone number

- Acutely ill

Participant numbers: 6000

Age: $18-59$ years

\section{Ethnicity: NS}

Intervention: clinic provider/staff training on implementing universal education into clinical practice to:

- provide all women information about IPV/sexual assault, harm reduction, and available safety resources

- provide trauma-informed counselling to women who disclose IPV/sexual assault; includes access to the myPlan safety decision aid to develop a tailored safety action plan

- provide warm referrals in partnership with local DV and sexual assault advocacy agencies

- integrate documentation and quality improvement templates and measures into clinical settings.

Control: standard clinical practice

Outcomes: change in use of safety behaviours, change in self-efficacy to use harm reduction strategies both at baseline and 6 months, provider behaviour at baseline (discussing healthy and unhealthy relationships during the visit, and referring to resources)

Trial registration number: NCT03259646

Tol 2017

$\begin{array}{ll}\text { Trial name or title } & \begin{array}{l}\text { Nguvu: evaluating an integrated approach to reduce intimate partner violence and psychological } \\ \text { distress in refugees in Tanzania }\end{array}\end{array}$
distress in refugees in Tanzania

\begin{tabular}{ll}
\hline Starting date & October 2014 \\
\hline Contact information & Name: Dr Wietse Tol \\
& Address: Johns Hopkins Bloomberg School of Public Health, 624 North Broadway, Rm. 863, Balti- \\
more, 21205, USA & Email: wtol@jhu.edu \\
\hline Country: Tanzania & Setting: Nyarugusu refugee camp \\
& Inclusion criteria: \\
& - Women \\
- & $\geq 18$ years \\
- & Married, living with a partner, or in a relationship in the last 12 months \\
- Current member of a registered local women's group \\
- Screened positive for a past-year history of IPV (physical or sexual)
\end{tabular}


Tol 2017 (Continued)

- Screened positive for a moderate or severe level of psychological distress

\section{Exclusion criteria:}

- Current imminent risk of suicide

- Observable signs of severe (neuro)psychiatric disorder that impedes participation in a group intervention (e.g. acute psychosis; severe substance misuse).

Women at immediate physical risk of injury from IPV and other forms of protection risks will be included in the study and will be linked to protection services as part of standard operating procedures in Nyarugusu.

Participant numbers: 400

Education and training: refugee incentive workers in Nyarugusu have been trained to deliver the intervention by two expatriate trainers and a Tanzanian clinical psychologist

Age: $>18$ years

Ethnicity: refugees from the Democratic Republic of the Congo

Intervention: the Nguvu intervention - an 8-session intervention consisting of 1 individual initial session, followed by 7 group sessions, delivered once/ week over 8 weeks. Week 1 : individual session with a participant and a facilitator to set expectations for the intervention, begin a discussion surrounding IPV and psychological distress, conduct a thorough danger assessment, and develop a safety plan. Weeks 2-7 are based on Cognitive Processing Therapy, to develop the woman's skills and ability to identify and understand the relationship between a potentially traumatic event, thoughts and feelings. The final session is a group advocacy session that revisits the safety plans developed in session one and provides a forum for discussion on how to improve one's safety plan and share safety and coping strategies that have been effective for other group members.

Control: the treatment-as-usual control condition is access to standard mental health and protection services.

Outcomes: measures made before the start of the intervention, 1 week after the intervention period (i.e. approximately a 9-week interval) and 12 weeks after the intervention. The primary outcomes are psychological distress and IPV. Secondary outcomes: functional impairment, measured using items developed through qualitative research. Mediators include social support, coping and service utilisation. Moderators include previous exposure to potentially traumatic events, ongoing potentially traumatic events and marital status.

Trial registration number: ISRCTN65771265

CHW: community health worker; CPS: child protective services; DV: domestic violence; IPV: intimate partner violence; NS: not stated; URMC: University of Rochester Medical Center

\section{ADDITIONAL TABLES}

\section{Table 1. Changes from protocol}

\begin{tabular}{ll}
\hline In protocol & Reason not undertaken \\
\hline $\begin{array}{l}\text { Stakeholder panel to adjudicate on any de- } \\
\text { cisions where the two reviewers could not } \\
\text { agree }\end{array}$ & Not needed \\
\hline EMMIE to be used for quality assessment & Adopted frameworks already used within Cochrane Reviews instead \\
\hline $\begin{array}{l}\text { All included studies need to consider inter- } \\
\text { ventions for women who have experienced }\end{array}$ & $\begin{array}{l}\text { In Phase 2 searching we made the decision to also include some other similar inter- } \\
\text { ventions (e.g. where advocacy was only one of several types of intervention given in }\end{array}$
\end{tabular}

A realist review of which advocacy interventions work for which abused women under what circumstances (Review) 
Table 1. Changes from protocol (Continued) partner abuse according to our specific definition of advocacy a shelter, where advocacy was given in a group intervention, where psychotherapy formed a large part of the intervention, and co-ordinated multi-agency work), if the study provided information relevant to our aims, and we have made it clear which studies these are.

Table 2. Essential principle 1: advocacy that takes into account the influence of the abuser on the woman will be more effective in reducing or ending the woman's tolerance of his abuse and coercive control

Essential principle 1: advocacy that takes into account the influence of the abuser on the woman will be more effective in reducing or ending the woman's tolerance of his abuse and coercive control

Hypothesis 1.1: many women will be economically, physically, legally or emotionally dependent on the abuser and this needs to be addressed within advocacy to make it less challenging for the woman to change the abusive relationship

Hypothesis 1.2: the trade-offs (balance of the immediate and long-term losses and gains) of the woman leaving the abusive relationship or staying need to be part of the woman's decision-making process and addressed by advocacy

Hypothesis 1.3: the impact of the woman's risk or severity (or both) of abuse on advocacy outcomes depends on the outcome being considered and hence the goals of the advocacy, but a higher severity of abuse may require extended advocacy

\begin{tabular}{|c|c|c|c|c|c|}
\hline Contexts $a$ & $\begin{array}{l}\text { Enabling mechanisms } \\
\text { (EM) and disabling } \\
\text { mechanisms (DM) with } \\
\text { respect to use of advo- } \\
\text { cacy } a\end{array}$ & $\begin{array}{l}\text { Effect of advocacy itself (en- } \\
\text { abling (EMfA1) and disabling } \\
\text { (DMfA) mechanisms) }^{a}\end{array}$ & $\begin{array}{l}\text { Relevant } \\
\text { advocacy } \\
\text { strategies }\end{array}$ & $\begin{array}{l}\text { Potential an- } \\
\text { ticipated out- } \\
\text { comes of ad- } \\
\text { vocacy }{ }^{a}\end{array}$ & $\begin{array}{l}\text { Potential } \\
\text { unanticipat- } \\
\text { ed outcomes } \\
\text { of advocacy } a\end{array}$ \\
\hline $\begin{array}{l}\text { Individual } \\
\text { Coercive con- } \\
\text { trol (emo- } \\
\text { tional depen- } \\
\text { dence) H1.1 } \\
\text { and H1.2 } \\
\text { Woman phys- } \\
\text { ically/legal- } \\
\text { ly/econom- } \\
\text { ically de- } \\
\text { pendent on } \\
\text { abuser H1.1 } \\
\text { and H1.2 } \\
\text { due to: } \\
\text { - low income } \\
\text { - low SES } \\
\text { (education) } \\
\text { - and/or } \\
\text { mental or } \\
\text { physical } \\
\text { health } \\
\text { problems } \\
\text { or disabili- } \\
\text { ty } \\
\text { - and/or un- } \\
\text { document- } \\
\text { ed immi- }\end{array}$ & $\begin{array}{l}\text { Enabling mechanisms } \\
\text { EM1: if an abused } \\
\text { woman is physical- } \\
\text { ly/legally/economical- } \\
\text { ly dependent on her } \\
\text { abuser but is of a high- } \\
\text { er SES/has a job/is well } \\
\text { educated and able to } \\
\text { research her options } \\
\text { and navigate the sys- } \\
\text { tems and elude the } \\
\text { abuser (e.g. by seeing } \\
\text { services when he thinks } \\
\text { she has gone to work) } \\
\text { then she has a wider } \\
\text { set of choices available } \\
\text { to her to end or reduce } \\
\text { the abuse and seek help } \\
\text { from services according } \\
\text { to her need. H1.1 } \\
\text { or } \\
\text { When the woman does } \\
\text { not live with her abuser, } \\
\text { she is more easily able } \\
\text { to evade him to seek } \\
\text { help and to hide her } \\
\text { help-seeking from him, } \\
\text { which gives her a wider } \\
\text { set of choices available }\end{array}$ & $\begin{array}{l}\text { Enabling mechanisms from ad- } \\
\text { vocacy } \\
\text { EMfA1: if the advocacy increases } \\
\text { the woman's capabilities to use } \\
\text { resources (from a clearer under- } \\
\text { standing of what support is avail- } \\
\text { able to her that is suitable for her } \\
\text { needs/dependencies, and help in } \\
\text { accessing this), then her ability to } \\
\text { choose to end or reduce the abuse } \\
\text { and seek help from services ac- } \\
\text { cording to her need will increase. } \\
\text { H1.1 and H1.2 } \\
\text { or } \\
\text { EMfA2: if the advocacy increas- } \\
\text { es the woman's self-esteem and } \\
\text { self-efficacy and develops in her } \\
\text { the confidence in her ability to be- } \\
\text { gin and to safely sustain changes } \\
\text { to the relationship and/or seek } \\
\text { help from services and/or im- } \\
\text { prove her circumstances. H1.1 and } \\
\text { H1.2. If the advocacy increases the } \\
\text { woman's self-esteem and self-effi- } \\
\text { cacy and develops her confidence } \\
\text { in herself, then there will be an in- } \\
\text { crease in her self-perceived and } \\
\text { actual ability to begin and to safe- } \\
\text { ly sustain changes to the relation- }\end{array}$ & $\begin{array}{l}\text { Education and } \\
\text { information } \\
\text { on abuse and } \\
\text { relationships } \\
\text { Education and } \\
\text { information } \\
\text { on women's } \\
\text { rights and } \\
\text { sources of } \\
\text { help } \\
\text { Active referral } \\
\text { to, and help } \\
\text { in accessing, } \\
\text { other services } \\
\text { Self-efficacy } \\
\text { and self-es- } \\
\text { teem develop- } \\
\text { ment } \\
\text { Recommen- } \\
\text { dations } \\
\text { Goal setting } \\
\text { and planning }\end{array}$ & $\begin{array}{l}\text { Proximal/in- } \\
\text { termediate } \\
\text { Access to/use } \\
\text { of resources. } \\
\text { H1.1 and H1.2 } \\
\text { Taking steps } \\
\text { to end the } \\
\text { abuse, such as } \\
\text { prosecuting } \\
\text { her abuser. } \\
\text { H1.1 and H1.2 } \\
\text { Enrolment } \\
\text { on education } \\
\text { courses/gain- } \\
\text { ing employ- } \\
\text { ment. H1.1 } \\
\text { Reduced } \\
\text { abuse/ } \\
\text { greater safety } \\
\text { (woman may } \\
\text { remain in rela- } \\
\text { tionship). H1.1 } \\
\text { and H1.2 } \\
\text { Distal } \\
\text { Reduced } \\
\text { abuse/greater }\end{array}$ & $\begin{array}{l}\text { Proximal/in- } \\
\text { termediate } \\
\text { Lack of confi- } \\
\text { dence in ser- } \\
\text { vices. H1.1 } \\
\text { and H1.2 } \\
\text { Overconfi- } \\
\text { dence in ser- } \\
\text { vices leading } \\
\text { to lack of sat- } \\
\text { isfaction with } \\
\text { them. H1.1 } \\
\text { and H1.2 } \\
\text { Psychologi- } \\
\text { cal distress or } \\
\text { other psycho- } \\
\text { logical or psy- } \\
\text { chosocial out- } \\
\text { comes. H1.1 } \\
\text { and H1.2 and } \\
\text { H1.3 } \\
\text { Limited ac- } \\
\text { cess to/use } \\
\text { of resources } \\
\text { H1.1 and H1.2 } \\
\text { Continued } \\
\text { or increased } \\
\text { abuse/stalk- }\end{array}$ \\
\hline
\end{tabular}


Table 2. Essential principle 1: advocacy that takes into account the influence of the abuser on the woman will be more effective in reducing or ending the woman's tolerance of his abuse and coercive control (Continued) gration sta- to her to end or reduce tus the abuse and seek help

- perceptions of de- to her need. H1.3 pendence

Women lacking English language skills $\mathrm{H} 1.2$

Women experiencing severe sexual or physical abuse and/ or living with abuser $\mathrm{H} 1.3$

\section{Consequences}

EM1: this leads to the woman's seeking appropriate help from services. $\mathrm{H} 1.1$

\section{Disenabling mecha- nisms}

DM1: if an abused woman is physically/legally/economically dependent on her ship and/or seek help from services and/or improve her circumstances. $\mathrm{H} 1.1$ and $\mathrm{H} 1.2$

\section{Consequences} abuser and has limited resources with which to provide for herself or support herself without her abuser, then her ability to choose to end or reduce the abuse and seek help from services according to her need is limited, and she will fear the potential loss of her home and the close ties with family and friends, even though she may have high expectations of the helpfulness of services for her without these constraints. $\mathrm{H} 1.1$ and $\mathrm{H} 1.2$

or

DM2: if an abused woman is emotionally dependent on her abuser because the coercive control has lowered her perceptions of her own value and capabilities, she will perceive her ability to choose to begin and to sustain changes to the relationship (e.g. to end or reduce the abuse) and/or seek help from services as limited and will fear the potential loss of her home and the close ties with family and friends. $\mathrm{H} 1.1$ and $\mathrm{H} 1.2$
EMfA1 and EMfA2: in both cases, this leads to the woman's decreased fear of the consequences of ending the relationship/limiting the abuse and reduction in her learned helplessness (including deeply held beliefs and values that may be culturally shaped) and leads her to take steps to make the changes, which may include seeking help from services.

EMfA3: if the advocacy shifts an abused woman's understandings and perceptions of her emotional dependencies and commitment / investment in the abusive relationship, through education on abuse and healthy relationships, and awareness and assessment of her risks from the abuse, underlying reasons for and triggers of abuse and the way the man controls her through her dependences, then her perceptions of her need to take safety precautions and make use of services will be changed. $\mathrm{H} 1.1$ and $\mathrm{H} 1.2$

\section{Consequences}

EMfA3: this leads to the woman's increased use of safety behaviours and the development of a safety plan, which may include seeking help from services. $\mathrm{H} 1.1$ and $\mathrm{H} 1.2$

EMfA4: if the advocacy shifts an abused woman's goals and priorities about her life and the abusive relationship, through education on abuse and healthy relationships, awareness and assessment of her risks from the abuse, an improved/restored self-identity and the capacity to balance risks and benefits of different choices, she will

fear the consequences of continued abuse more than any reduction in circumstances. $\mathrm{H} 1.2$

Consequences

EMfA4: This leads to the woman's decreased fear of the consequences of ending the relation-

Assessment of safety. H1.1

ing/death. risk of repeat and $\mathrm{H} 1.2 \quad \mathrm{H} 1.1, \mathrm{H} 1.2$ and abuse $\mathrm{H} 1.3$

Safety plan-

Distal

ning

Women-centred housing and refuges

Continued abuse/stalking/death $\mathrm{H} 1.1, \mathrm{H} 1.2$ and $\mathrm{H} 1.3$

A realist review of which advocacy interventions work for which abused women under what circumstances (Review) 
Table 2. Essential principle 1: advocacy that takes into account the influence of the abuser on the woman will be more effective in reducing or ending the woman's tolerance of his abuse and coercive control (Continued)

or

DM3: if an abused woman is experiencing moderate to severe physical or sexual abuse from her abuser, she will have a limited set of options to end or reduce the abuse and/ or seek help from services and will fear retribution from her abuser if she does so. $\mathrm{H} 1.3$

\section{Consequences}

DM1, DM2 and DM3: in all 3 cases, this leads her to delay/avoid seeking help, and to put up with her situation and adopt a wait-and-see position with respect to its evolution and to develop unfavourable psychosocial and psychological responses to the abuse (e.g. depression). $\mathrm{H} 1.1, \mathrm{H} 1.2$ and $\mathrm{H} 1.3$ ship/limiting the abuse and she

takes steps to do so, which may in-

clude seeking help from services and developing personal action, implementation and evaluation goals/plans. $\mathrm{H} 1.2$

EMfA5: If an abused woman is experiencing moderate to severe physical or sexual abuse from her abuser, and she is supported in understanding the risks to herself and the choice set of options available to her to change the relationship then her perceptions of her need to take safety precautions and make use of services and her fear of the abuser will be heightened. $\mathrm{H} 1.3$

\section{Consequences}

EMfA5:This leads to her active and engaged help-seeking to reduce the risks to herself from the abuse whilst also minimising the effects of retribution from the man. $\mathrm{H} 1.3$

EMfA6:If the advocate educates the community about IPV and about available services for survivors, this increases the capabilities of the community and the woman's informal network to be supportive to the woman. $\mathrm{H} 1.2$

or

EMfA6:If the advocate provides emotional support for members of the woman's social networks who are themselves emotionally affected by the woman's abuse, this increases their capabilities regarding abuse. $\mathrm{H} 1.2$

Consequences

EMfA6:This leads to an increase in local informal network support for the woman and the woman's social capital, which decreases her anxiety and depression, PTSD symptoms and suicide attempts (as responses to the abuse) and benefits her well-being. $\mathrm{H} 1.2$

EMfA7:When a woman is provided with social support by advocacy and her well-being improves as a result, she will feel more hopeful 
Table 2. Essential principle 1: advocacy that takes into account the influence of the abuser on the woman will be more effective in reducing or ending the woman's tolerance of his abuse and coercive control (Continued)

and confident in developing her capabilities to make changes to her situation. $\mathrm{H} 1.2$

Consequences

EMfA7:This leads to her making active improvements in her situation in a spiral of positive gains. $\mathrm{H} 1.2$

EMfA8:When an advocate develops knowledge or awareness of cultural humility through exposure to relevant groups or training she will realise that leaving the relationship is not necessarily the best option for a woman.

\section{Consequences}

EMfA8:This leads to her exploring with the woman in a womanfocused way what options the woman prefers and she no longer pressures the woman to leave the relationship. $\mathrm{H} 1.2$

EMfA9:If an advocate helps the woman to develop the capabilities to look for work or education, then the woman will view her future outside of the abusive relationship more positively. $\mathrm{H} 1.1$

\section{Consequences}

EMfA9:This will give her the confidence and the motivation to enrol on a course or gain employment. H1.1

\section{Disenabling mechanisms from} advocacy

DMfA1: if the woman reduces her dependencies on the abuser, the man will feel that his male status (e.g. as a provider) is threatened. $\mathrm{H} 1.1$ and $\mathrm{H} 1.2$

Consequences

DMfA1: this will lead him to escalate the abuse to regain control over the woman. $\mathrm{H} 1.1$ and $\mathrm{H} 1.2$

\begin{tabular}{|c|c|c|c|c|c|}
\hline Local & $\begin{array}{l}\text { Disenabling mecha- } \\
\text { nisms }\end{array}$ & $\begin{array}{l}\text { Enabling mechanisms from ad- } \\
\text { vocacy }\end{array}$ & $\begin{array}{l}\text { Structural ad- } \\
\text { vocacy }\end{array}$ & $\begin{array}{l}\text { Proximal/in- } \\
\text { termediate }\end{array}$ & $\begin{array}{l}\text { Proximal/in- } \\
\text { termediate }\end{array}$ \\
\hline $\begin{array}{l}\text { availability } \\
\text { of alternative } \\
\text { options. } \mathrm{H} 1.1\end{array}$ & $\begin{array}{l}\text { DM4: if an abused } \\
\text { woman is physical- } \\
\text { ly/legally/economical- } \\
\text { ly/emotionally depen- }\end{array}$ & $\begin{array}{l}\text { EMfA10: if the advocates under- } \\
\text { stand the ways of working of the } \\
\text { systems and bureaucratic process- } \\
\text { es they and the woman need to }\end{array}$ & $\begin{array}{l}\text { Woman-cen- } \\
\text { tred focus } \\
\text { Goal setting }\end{array}$ & $\begin{array}{l}\text { Access to/use } \\
\text { of resources. } \\
\mathrm{H} 1.1 \text { and } \mathrm{H} 1.2\end{array}$ & $\begin{array}{l}\text { Lack of confi- } \\
\text { dence in ser- } \\
\text { vices. } \mathrm{H} 1.1 \\
\text { and } \mathrm{H} 1.2\end{array}$ \\
\hline
\end{tabular}

A realist review of which advocacy interventions work for which abused women under what circumstances (Review) 
Table 2. Essential principle 1: advocacy that takes into account the influence of the abuser on the woman will be more effective in reducing or ending the woman's tolerance of his abuse and coercive control (Continued)

Previous experiences of services. $\mathrm{H} 1.1$

Level of social support. H1.2

Co-ordinated community response

Geography (small, closed, rural community); cultural/patriarchal norms/ Latin Marianism that act to keep domestic violence hidden (if woman wishes to maintain her status in the community). $\mathrm{H} 1.2$ dent on her abuser and has limited resources with which to provide for herself or support herself without her abuser, and previous negative experience of help-seeking to deal with this, then she will perceive her ability to choose to end or reduce the abuse and seek help from services according to her need as particularly diminished. H1.1 and $\mathrm{H} 1.2$

\section{Consequences}

DM4: This will lead her to develop a learned helplessness about her situation and a lack of confidence in any services as being able to help her and so she will not seek help. $\mathrm{H} 1.1$ and $\mathrm{H} 1.2$

DM5: if an abused woman is physically/legally/economically dependent on her abuser and has limited resources with which to provide for herself or support herself without her abuser, then her ability to choose to end or reduce the abuse and seek help from services according to her need is limited, and she will fear the potential loss of her home and the close ties with family and friends, even though she may have high expectations of the helpfulness of services for her without these constraints. H1.1 and $\mathrm{H} 1.2$

or

DM6: If an abused woman, through geography, is marginalised in the employment market, locked navigate and are able to work within them to effect change or overcome them, and are able to share these capabilities with the abused woman, then she will develop the capabilities to do so herself. $\mathrm{H} 1.1$ and $\mathrm{H} 1.2$

\section{Consequences}

EMfA10: this leads to the woman's increased confidence in involving these services and she takes steps to do so. $\mathrm{H} 1.1$ and $\mathrm{H} 1.2$

EMfA11: if an advocate educates an abused woman about healthy social relationships (not just intimate relationships) the woman develops new 'templates' for thinking about informal network relationships that may help her. $\mathrm{H} 1.2$

Consequences

EMfA11: this leads to the woman taking steps to change or develop informal networks that will be supportive. $\mathrm{H} 1.1$ and $\mathrm{H} 1.2$

EmfA12: If the advocate supports the woman in developing goals and plans to improve her situation such as plans to get work or education, then the woman will feel more confident about her abilities to gain these.

Consequences

EmfA12: This will lead her to gain work or enrol on a course.

\section{Disenabling mechanisms from advocacy}

DMfA2: If the advocacy only provides criminal justice options this limits the woman's increased choice of options to end the abuse to measures that directly constrain the man and that will show him she is taking steps against him. $\mathrm{H} 1.3$

\section{Consequences}

DMfA2: This may lead the woman to become afraid that her abuser will punish her for her actions, or she may feel confidence in the criminal justice system to end the abuse, which may include using this as leverage, for example to

Court-focused support

Women centred housing and refuges

Culturally appropriate services

Education on relationships and the in-

volvement of social networks

(a)

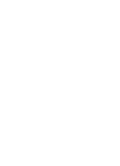


Table 2. Essential principle 1: advocacy that takes into account the influence of the abuser on the woman will be more effective in reducing or ending the woman's tolerance of his abuse and coercive control (Continued)

out of the inheritance structure and limited in their education or careers because of family or community responsibilities, and has limited resources with which to provide for herself or support herself without her abuser, then her ability to choose to end or reduce the abuse and seek help from services or her community according to her need is limited, and she will fear the potential loss of her home and community. $\mathrm{H} 1.2$

\section{Consequences}

DM 5 and 6: this leads her to delay/avoid seeking help, and to put up with her situation and adopt a wait-and-see position with respect to its evolution and to develop unfavourable psychosocial and psychological responses to the abuse (e.g. depression). $\mathrm{H} 1.2$ make the man fearful of impris-

onment or a criminal record if the

abuse continues. $\mathrm{H} 1.3$

DMfA3: if the advocate does not manage the woman's (high or low) expectations of what advocacy can achieve, and the woman's capabilities do not increase as quickly as she anticipates (or at all), or structural or local barriers are not removed to the access to or use of services, her choices will continue to be constrained.

\section{Consequences}

DMfA3: this will lead her to abandon the advocacy or to have reinforcement of her learned helplessness (if developed from previous service encounters and from the coercive control).

DMfA4: When an advocate has to deal with poor infrastructure, limited services and local social patriarchal attitudes her choices of what she can do for the woman are constrained. H1.1

\section{Consequences}

DMfA4: This leads her to draw from a limited set of choices in supporting the women and therefore the woman is not effectively supported. $\mathrm{H} 1.1$

\section{Structural \\ Disenabling mecha- nisms}

Eligibility and rights to state support (e.g. immigration status). $\mathrm{H} 1.1$ and $\mathrm{H} 1.2$

Cultural/patriarchal norms/ Latin Marianism

\section{DM7: if an abused} woman is an undocumented migrant and fears immigration officials, then her ability to choose to end or reduce the abuse and seek help from services according to her need is limited, even though she may have high expectations of the helpfulness of services for her without these constraints. $\mathrm{H} 1.1$

Consequences

\section{Enabling mechanisms from ad- vocacy}

EMfA13: if the advocates understand the ways of working of the systems and bureaucratic processes they and the woman need to navigate and are able to work within them to effect change or overcome them, and are able to share these capabilities with the abused woman, then she will develop the capabilities to do so herself.

Consequences

EMfA13: this leads to the woman's increased confidence in involving these services and she takes steps to do so, which may include using this as leverage, for example us-

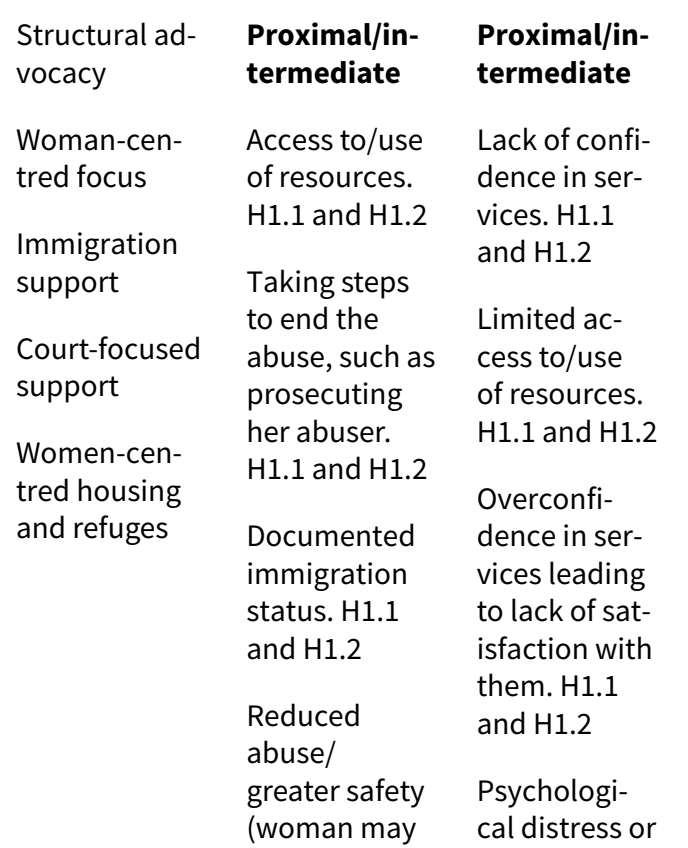


Table 2. Essential principle 1: advocacy that takes into account the influence of the abuser on the woman will be more effective in reducing or ending the woman's tolerance of his abuse and coercive control (Continued)

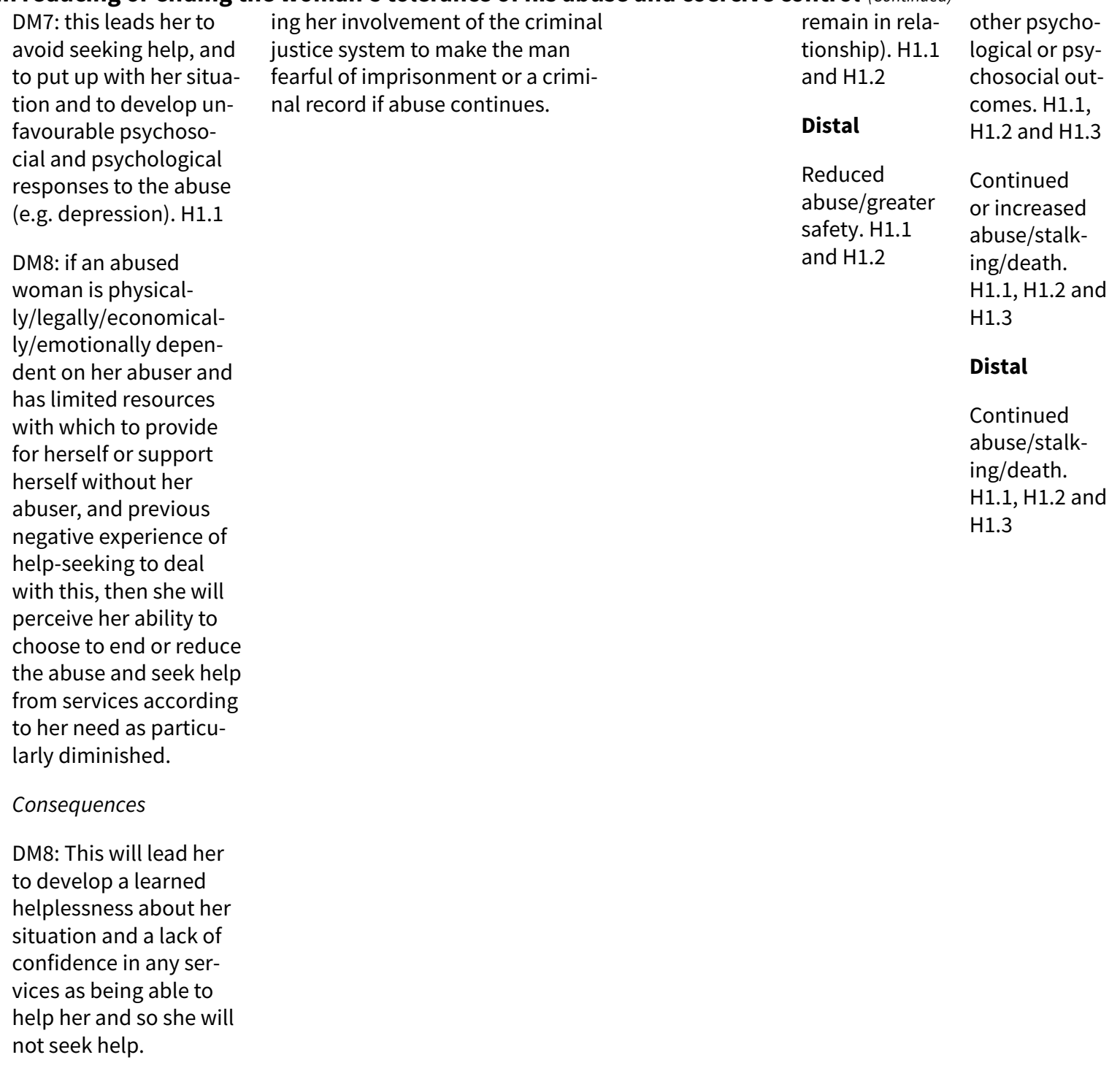

DM: disabling mechanism; DMfA: disabling mechanisms from advocacy; EM: enabling mechanism; EMfA: enabling mechanisms from advocacy; H: hypothesis; IPV: intimate partner violence/abuse; PTSD: post-traumatic stress disorder; SES: socioeconomic status

aNote, each comment (CMO configuration) is linked to a hypothesis at the top of the table. Statements are labelled as enabling and disenabling mechanisms, with numbers to facilitate.

Table 3. Findings related to essential principle 1: the influence of the abuser on the woman must be taken into account in advocacy work as it may lead the woman to tolerate his abuse and coercive control

\begin{tabular}{llll}
\hline Review finding & $\begin{array}{l}\text { CERQual: assess- } \\
\text { ment of confi- } \\
\text { dence in the evi- } \\
\text { dence }\end{array}$ & Explanation of CERQual assessment & $\begin{array}{l}\text { Studies contributing to the re- } \\
\text { view finding }\end{array}$ \\
\hline $\begin{array}{l}\text { Many women will } \\
\text { be economically, } \\
\text { physically, legal- } \\
\text { ly or emotional- }\end{array}$ & $\begin{array}{l}\text { Moderate confi- } \\
\text { dence }\end{array}$ & $\begin{array}{l}\text { We graded this finding moderate confidence } \\
\text { because: there were only 10 studies and two of } \\
\text { these had a high risk of bias (Kendall 2009; Sulli- } \\
\text { van 2018); there was only moderate coherence }\end{array}$ & $\begin{array}{l}\text { Bacchus 2016a; Curry 2006; } \\
\text { Dendall 2009; Reina 2015; Sul- }\end{array}$ \\
\hline
\end{tabular}

A realist review of which advocacy interventions work for which abused women under what circumstances (Review) 
Table 3. Findings related to essential principle 1: the influence of the abuser on the woman must be taken into account in advocacy work as it may lead the woman to tolerate his abuse and coercive control (Continued)

ly dependent on

the abuser and this

needs to be ad-

dressed within advocacy to make it less challenging for the woman to change the abusive relationship

The trade-offs (bat-

The trade-offs (balance of the immediate and long-term losses and gains) of the woman leaving the abusive relationship or staying need to be part of the woman's decision-making process and addressed by advocacy (some studies had not found the association with economic dependence but studies of advocates, women and modelling cohered);

studies were all relevant and with adequate data but there was over-reliance on 1 study (DePrince 2012). livan 2018; Tiwari 2005; Wong 2013; Wood 2014
High confidence

$$
\begin{aligned}
& \text { We graded this finding as high confidence be- } \\
& \text { cause there were } 31 \text { studies of which } 22 \text { were } \\
& \text { studies with low risk of bias (with possible risk of } \\
& \text { bias from Bahadir-Yilmaz 2018; Constantino 2005; } \\
& \text { Gillum 2009; Howarth 2016; McFarlane 2000; Mc- } \\
& \text { Farlane 2006; Prosman 2014; Song 2012; Stover } \\
& \text { 2010; Sullivan 2018), studies took place across } \\
& \text { countries and settings; there was strong coher- } \\
& \text { ence across studies, modelling work, experimen- } \\
& \text { tal studies, and studies of women and advocates } \\
& \text { all concurred, and all studies were relevant, with } \\
& \text { adequate and mostly rich data. However, there } \\
& \text { is some concern regarding measure of percep- } \\
& \text { tion of access to resources vs actual access, and } \\
& \text { what access suggests in different settings (e.g. are } \\
& \text { reduced calls to the police a positive or negative } \\
& \text { outcome?). }
\end{aligned}
$$

Moderate confidence

\begin{abstract}
We graded this finding as moderate confidence because there were 53 studies across countries and settings, with mostly low risk of bias, with relevant studies some of which provided rich data, though there was a possible risk of bias from $\mathrm{Ba}$ hadir-Yilmaz 2018; Bell 2001; Casey 2007; Davis 2006; Davis 2007; Gillum 2009; Kendall 2009; Krasnoff 2002; McFarlane 1997; McFarlane 2000; McFarlane 2004; McFarlane 2006; Muelleman 1999; Prosman 2014; Song 2010; Song 2012; Stover 2010; Sullivan 2019; Taft 2011; Tutty 1996, and abuse severity was often conjecture by reviewers (danger of circular reasoning), coherence was low (a dose-response was suggested but may depend on setting; models and views studies provided stronger evidence; and some brief interventions were effective despite the overall finding).
\end{abstract}

Bacchus 2016a; Bacchus 2016b; Bahadir-Yilmaz 2018; Bybee 2005; Briones-Vozmediano 2014; Coker 2012; Constantino 2005; Curry 2006; Cripe 2010; Coy 2011; DePrince 2012; Dunn 2007; Gillum 2009; Goodman 2016a; Goodman 2016b; Howarth 2016; Johnson 2015; McFarlane 2000; McFarlane 2006; Merchant 2015; Murray 2015; Owen 2015; Perez 2012; Prosman 2014; Reina 2015; Silva-Martinez 2016; Song 2012; Stover 2010; Sudderth 2017; Sullivan 2018; Tiwari 2010

Bacchus 2016a; Bacchus 2016b; Bader 2014; Bahadir-Yilmaz 2018; Bell 2001; Bybee 2005; Casey 2007; Coker 2012; Constantino 2005; Coy 2011; Cripe 2010; Curry 2006; Davis 2006; Davis 2007; DePrince 2012 ; Ekstrom 2015; Ganz 2015; Gillum 2009; Goodman 2016a; Goodman 2016b; Hathaway 2008; Howarth 2016; Hughes 2017; Hyman 2001; Kendall 2009; Kenyon 2016; Kiely 2010; Krasnoff 2002; Lako 2018; Lea 2016; McFarlane 1997; McFarlane 2000; McFarlane 2004; McFarlane 2006; Merchant 2015; Muelleman 1999; Perez 2012; Prosman 2014; Song 2010; Song 2012; Stover 2010; Sudderth 2017; Sullivan 2019; Sullivan 2002; Stylianou 2018; Taft 2011; Taha 2015; Thiara 2009; Tiwari 2005; Tiwari 2010; Tutty 1996; Wies 2008; Wong 2013 
Table 4. Essential principle 2: there is a need for holistic, tailored advocacy intervention plans that recognise the individual risks and needs of vulnerable and marginalised women such as those from minority groups or from rural areas

Essential principle 2: there is a need for holistic, tailored advocacy intervention plans that recognise the individual risks and needs of vulnerable and marginalised women such as those from minority groups or from rural areas

Hypothesis 2.1: advocates find it challenging to remove structural barriers to resource access despite the value of this

Hypothesis 2.2: once marginalised women access advocacy they are best supported by advocates with cultural humility, knowledge of how to tackle specific issues for these women, and awareness of intersectionalities

Hypothesis 2.3: helping the woman should also prove mutually beneficial for the advocate

\begin{tabular}{llllll}
\hline Contexts & $\begin{array}{l}\text { Enabling (EM) and dis- } \\
\text { abling mechanisms } \\
\text { (DM) with respect to } \\
\text { use of advocacya }\end{array}$ & $\begin{array}{l}\text { Effect of advocacy itself (en- } \\
\text { abling (EMfA) and disabling } \\
\text { (DMfA) mechanisms) }\end{array}$ & $\begin{array}{l}\text { Relevant ad- } \\
\text { vocacy strate- } \\
\text { gies/compo- } \\
\text { nents }\end{array}$ & $\begin{array}{l}\text { Potential an- } \\
\text { ticipated out- } \\
\text { comes of ad- } \\
\text { vocacya } a\end{array}$ & $\begin{array}{l}\text { Potential } \\
\text { enticipat- } \\
\text { of advocacy } a\end{array}$ \\
\hline
\end{tabular}

Mechanisms for abused women

\begin{tabular}{|c|c|c|c|c|c|}
\hline $\begin{array}{l}\text { Legal/eco- } \\
\text { nomic de- } \\
\text { pendence on } \\
\text { abuser (e.g. } \\
\text { undocument- } \\
\text { ed immigra- } \\
\text { tion status or } \\
\text { a rural woman } \\
\text { who through } \\
\text { geography, is } \\
\text { marginalised } \\
\text { in the employ- } \\
\text { ment market, } \\
\text { locked out } \\
\text { of the inher- } \\
\text { itance struc- } \\
\text { ture and lim- } \\
\text { ited in her ed- } \\
\text { ucation or ca- } \\
\text { reer because } \\
\text { of family or } \\
\text { community } \\
\text { responsibili- } \\
\text { ties) } \\
\text { Lack of Eng- } \\
\text { lish lan- } \\
\text { guage/avail- } \\
\text { ability/type of } \\
\text { interpreters } \\
\text { Immigration } \\
\text { status }\end{array}$ & $\begin{array}{l}\text { Disenabling mecha- } \\
\text { nisms } \\
\text { DM1: if an abused } \\
\text { woman is legally/eco- } \\
\text { nomically dependent } \\
\text { on her abuser and has } \\
\text { limited resources with } \\
\text { which to provide for } \\
\text { herself or support her- } \\
\text { self without her abuser } \\
\text { and/or is not fluent in } \\
\text { English, then her abili- } \\
\text { ty to choose to end or } \\
\text { reduce the abuse and } \\
\text { seek help from services } \\
\text { according to her need } \\
\text { is limited, and she will } \\
\text { fear the potential loss } \\
\text { of her home and the } \\
\text { close ties with family } \\
\text { and friends and com- } \\
\text { munity, even though } \\
\text { she may have high ex- } \\
\text { pectations of the help- } \\
\text { fulness of services for } \\
\text { her without these con- } \\
\text { straints. H2.1 } \\
\text { Consequences } \\
\text { DM1: this leads her to } \\
\text { delay/avoid seeking } \\
\text { help, to resort to hid- } \\
\text { ing the abuse or oth- } \\
\text { erwise continuing as } \\
\text { she has been (not using } \\
\text { services) and to put up } \\
\text { with her situation and } \\
\text { adopt a wait-and-see } \\
\text { position with respect to }\end{array}$ & $\begin{array}{l}\text { EMfA1: if the advocacy increases } \\
\text { the woman's capabilities to use } \\
\text { resources (from a clearer un- } \\
\text { derstanding of what support is } \\
\text { available to her that is suitable } \\
\text { for her needs/dependences, } \\
\text { and help in accessing this), then } \\
\text { her ability to choose to end or } \\
\text { reduce the abuse and seek help } \\
\text { from services according to her } \\
\text { need will increase. H2.1 } \\
\text { or } \\
\text { EMfA2: if the advocacy increas- } \\
\text { es the woman's self-esteem and } \\
\text { self-efficacy and develops her } \\
\text { confidence, then there will be } \\
\text { an increase in her self-perceived } \\
\text { and actual ability to begin and } \\
\text { to safely sustain changes to the } \\
\text { relationship and/or seek help } \\
\text { from services and/or improve } \\
\text { her circumstances. H2.1 } \\
\text { Consequences } \\
\text { EMfA1 and EMfA2: in both cas- } \\
\text { es, this leads to the woman's } \\
\text { decreased fear of the conse- } \\
\text { quences of ending the relation- } \\
\text { ship/limiting the abuse and re- } \\
\text { duction in her learned helpless- } \\
\text { ness (including deeply held be- } \\
\text { liefs and values that may be cul- } \\
\text { turally shaped) and leads her to } \\
\text { take steps to make the changes, } \\
\text { which may include seeking help } \\
\text { from services. }\end{array}$ & $\begin{array}{l}\text { Culturally sensi- } \\
\text { tive counselling, } \\
\text { spiritual and net- } \\
\text { work support } \\
\text { BAME specialist } \\
\text { Advocacy train- } \\
\text { ing, supervision } \\
\text { and support } \\
\text { Childcare and } \\
\text { money for trans- } \\
\text { portation } \\
\text { Language ser- } \\
\text { vices } \\
\text { Immigration sup- } \\
\text { port } \\
\text { Woman-centred } \\
\text { focus } \\
\text { Education and } \\
\text { information on }\end{array}$ & $\begin{array}{l}\text { Reduced } \\
\text { abuse/ } \\
\text { greater safety } \\
\text { (woman may } \\
\text { remain in rela- } \\
\text { tionship). H2.1 } \\
\text { and H2.2 } \\
\text { Distal } \\
\text { Reduced } \\
\text { abuse/greater } \\
\text { safety. H2.1 } \\
\text { and H2.2 } \\
\text { Fewer symp- } \\
\text { toms of de- } \\
\text { pression and } \\
\text { PTSD. H2.1 } \\
\text { and H2.2 }\end{array}$ & $\begin{array}{l}\text { Lack of confi- } \\
\text { dence in ser- } \\
\text { vices. H2.1 } \\
\text { and H2.2 } \\
\text { Overconfi- } \\
\text { dence in ser- } \\
\text { vices leading } \\
\text { to lack of sat- } \\
\text { isfaction with } \\
\text { them (expec- } \\
\text { tations not } \\
\text { met). H2.1 and } \\
\mathrm{H} 2.2 \\
\text { Limited ac- } \\
\text { cess to/use } \\
\text { of resources. } \\
\mathrm{H} 2.1 \text { and H2.2 } \\
\text { Short-term } \\
\text { losses (e.g. } \\
\text { family home, } \\
\text { friends, } \\
\text { family, fi- } \\
\text { nances/tem- } \\
\text { porary reduc- } \\
\text { tion in circum- } \\
\text { stances as the } \\
\text { woman ad- } \\
\text { justs to the } \\
\text { changes, she } \\
\text { has made to } \\
\text { her life). H2.1, } \\
\mathrm{H} 2.2 \text { and H2.3 }\end{array}$ \\
\hline
\end{tabular}

A realist review of which advocacy interventions work for which abused women under what circumstances (Review) 
Table 4. Essential principle 2: there is a need for holistic, tailored advocacy intervention plans that recognise the individual risks and needs of vulnerable and marginalised women such as those from minority groups or from rural

areas (Continued)

its evolution and to develop unfavourable psychosocial and psychological responses to the abuse (e.g. depression). $\mathrm{H} 2.1$

DM2: if an abused woman is legally/economically dependent limited resources with which to provide for herself or support herself without her abuser and/or is not fluent in English, and has previous negative experience of help-seeking to deal with this, then she will perceive her ability to choose to end or reduce the abuse and seek help from services according to her need as particularly diminished. $\mathrm{H} 2.1$ and $\mathrm{H} 2.2$

Consequences

DM2: This will lead her to develop a learned helplessness about her situation and a lack of confidence in any services as being able to help her and so she will not seek help. $\mathrm{H} 2.1$ and $\mathrm{H} 2.2$ on her abuser and has

EMfA3: if the advocacy shifts an abused woman's understandings and perceptions of her emotional dependencies and commitment/investment in the abusive relationship and community pressures, through education on abuse and healthy relationships, and awareness and assessment of her risks from the abuse, then her perceptions of her need to make changes in her life will shift. H2.1

\section{Consequences}

EMfA3: this leads the woman to shift her priorities from preserving her position in the community to preserving herself as she develops a better understanding of abuse and relationships. $\mathrm{H} 2.1$

\section{Disenabling mecha- nisms}

Areas without specialist services or with services that are very under-resourced (e.g. with limited possibility of appropriate onward referrals)

\section{Geography} (small, closed rural community)
DM3: if an abused woman is physically/legally/economically dependent on her abuser and has limited resources with which to provide for herself or support herself without her abuser, then her ability to choose to end or reduce the abuse and seek help from services according to her need is limited, and she will fear the potential loss of her home and

\section{Enabling mechanisms from advocacy}

EMfA4: When women migrate from a country with limited services for IPV or when they perceive their new country as a land of opportunity, they will have confidence in the choices made available to them through advocacy and the impact of these services. $\mathrm{H} 2.1$

\section{Consequences}

EMfA4: This will lead them to use and engage with these services. $\mathrm{H} 2.1$ abuse and relationships

Education and information on women's rights and sources of help

Active referral to, and help in accessing, other services

Self-efficacy and self-esteem development

Recommendations

Goal setting and planning

Assessment of risk of repeat abuse

Safety planning other psychological or psychosocial outcomes. H2.1, $\mathrm{H} 2.2$ and $\mathrm{H} 2.3$

Continued or increased abuse/stalking/death $\mathrm{H} 2.1, \mathrm{H} 2.2$ and $\mathrm{H} 2.3$

Attrition. H2.1, $\mathrm{H} 2.2$ and $\mathrm{H} 2.3$

Non-engagement. H2.1, $\mathrm{H} 2.2$ and $\mathrm{H} 2.3$

Distal

Continued abuse/stalking/death. $\mathrm{H} 2.1, \mathrm{H} 2.2$ and $\mathrm{H} 2.3$
Culturally sensitive interventions

Targeted outreach services that recognise the diversity of immigrant cultural, religious and other identity-based groups

Culturally appropriate housing support

Culturally sensitive counselling,

\section{Proximal/in- Proximal/in termediate termediate}

Access to/use Lack of confiof resources. dence in ser$\mathrm{H} 2.1$ and $\mathrm{H} 2.2$ vices. $\mathrm{H} 2.1$ and $\mathrm{H} 2.2$

Documented immigration status. $\mathrm{H} 2.1$ and $\mathrm{H} 2.2$

Taking steps to end the abuse, such as prosecuting her abuser. $\mathrm{H} 2.1$ and $\mathrm{H} 2.2$
Overconfidence in services leading to lack of satisfaction with them (expectations not met). $\mathrm{H} 2.1$ and $\mathrm{H} 2.2$

Short-term losses (e.g. 
Table 4. Essential principle 2: there is a need for holistic, tailored advocacy intervention plans that recognise the individual risks and needs of vulnerable and marginalised women such as those from minority groups or from rural

areas (Continued)

Cultural/patriarchal norms/ Latin Marianism that act to keep domestic violence hidden (if woman wishes to maintain her status in the community)

Undocumented immigration status

Distance to intervention the close ties with family and friends, even though she may have high expectations of the helpfulness of services for her without these constraints. $\mathrm{H} 2.1$

\section{Consequences}

DM3: this leads her to delay/avoid seeking help, and to put up with her situation and adopt a wait-and-see position with respect to its evolution and to develop unfavourable psychosocial and psychological responses to the abuse (e.g. depression). The woman may be unwilling to accept she is at greater risk if she does not make active changes to the relationship as a form of active denial so as not to damage her status quo.

\section{$\mathrm{H} 2.1$}

DM4: if an abused woman is physically/legally/economically/emotionally dependent on her abuser and has limited resources with which to provide for herself or support herself without her abuser, and previous negative experience of help-seeking to deal with this, then she will perceive her ability to choose to end or reduce the abuse and seek help from services according to her need as particularly diminished. $\mathrm{H} 2.1$

\section{Consequences}

DM4: this will lead her to develop a learned helplessness about her situation and a lack of confidence in any services as being able to
EMfA5: When the advocate attends social events with the woman, this boosts the woman's emotional capital and coping mechanisms. $\mathrm{H} 2.1$

\section{Consequences}

EMfA5: This improves the woman's mental health, her well-being, which gives her the feeling of empowerment to improve other aspects of her life.

\section{$\mathrm{H} 2.1$}

EMfA6: When services are nearby, or if means of transportation/childcare are available, and funded, then a woman's ability to choose to seek help according to her need is strengthened. $\mathrm{H} 2.1$

\section{Consequences}

EMfA6: This improves her access to services. $\mathrm{H} 2.1$

EMfA7: If an abused woman has a social network within the community that allows her to gain access to financial and/or material resources or other support, or if the advocacy provides this, then her ability to choose to seek services according to her need is strengthened. $\mathrm{H} 2.1$

\section{Consequences}

EMfA7: This improves her access to services. $\mathrm{H} 2.1$

\section{Disenabling mechanisms}

DMfA1: If a woman from a patriarchal or closed community seeks advocacy and services are so resource-constrained that they cannot guarantee her privacy, there may be inadvertent disclosure of her abuse/helpseeking. H2.1

\section{Consequences}

DMfA1: This will lead to the woman being shamed, embarrassed or humiliated or otherwise condemned by her local communitv and mav also lead

\author{
spiritual and net- \\ work support
}

BAME specialist

Advocacy training, supervision and support

Childcare and money for transportation

\section{Language ser- vices}

Immigration support

Formal partnership protocols for advocacy and co-ordinated services

Woman-centred focus

\section{Education and} information on abuse and relationships

Education and information on women's rights and sources of help

Active referral to, and help in accessing, other services

Self-efficacy and self-esteem development

Recommendations

Goal setting and planning

Assessment of risk of repeat abuse

Safety planning
Reduced

abuse/ greater safety (woman may remain in relationship). $\mathrm{H} 2.1$ and $\mathrm{H} 2.2$

Distal

Reduced abuse/greater safety. $\mathrm{H} 2.1$ and $\mathrm{H} 2.2$

Fewer symptoms of depression and PTSD. H2.1 and $\mathrm{H} 2.2$

(a)

family home, friends, family, finances). $\mathrm{H} 2.3$

Psychological distress and shame or other psychological or psychosocial outcomes. H2.1, $\mathrm{H} 2.2$ and $\mathrm{H} 2.3$

Disengagement with services. $\mathrm{H} 2.1$

Limited access to/use of resources. $\mathrm{H} 2.1$ and $\mathrm{H} 2.2$

Continued or increased abuse/stalking/death. $\mathrm{H} 2.1, \mathrm{H} 2.2$ and $\mathrm{H} 2.3$

Distal

Continued abuse/stalking/death. $\mathrm{H} 2.1, \mathrm{H} 2.2$ and $\mathrm{H} 2.3$ 
Table 4. Essential principle 2: there is a need for holistic, tailored advocacy intervention plans that recognise the individual risks and needs of vulnerable and marginalised women such as those from minority groups or from rural

areas (Continued)

help her and so she will not seek help. $\mathrm{H} 2.1$

DM5: If an abused women is in a situation of geographic vulnerability, regarding proximity/availability of services and of means of transportation, then women's ability to choose to seek help according to their need is constrained. $\mathrm{H} 2.1$

\section{Consequences}

DM5: this leads her to delay/avoid seeking help, to resort to hiding the abuse or otherwise continuing as she has been (not using services) and to put up with her situation and adopt a wait-and-see position with respect to its evolution and to develop unfavourable psychosocial and psychological responses to the abuse (e.g. depression). $\mathrm{H} 2.1$

DM6: If an abused woman, through geography, is marginalised in the employment market, locked out of the inheritance structure and limited in her education or career because of family or community responsibilities, and has limited resources with which to provide for herself or support herself without her abuser, then her ability to choose to end or reduce the abuse and seek help from services or her community according to her need is limited, and she will fear the potential loss of her home and community. $\mathrm{H} 2.1$ to an escalation of the abuse. $\mathrm{H} 2.1$

DMfA2: When the woman has children not yet school age and there is no childcare at IPV services, and the woman does not want to talk about the issues in front of her children, or the woman is distracted by them, then the woman's engagement with advocacy and her development of her capabilities will be reduced. $\mathrm{H} 2.1$

\section{Consequences}

DMfA2:This will lead her to use advocacy less, or less effectively and it may also lead her to become disappointed in services. $\mathrm{H} 2.1$

DMfA3:If the woman has to take her children to advocacy visits because of a lack of childcare and is afraid the children will reveal her help-seeking to the abuser, she may become fearful that he will harm her and the children. $\mathrm{H} 2.1$

\section{Consequences}

DMfA3:This will lead her to disengage from and avoid advocacy and help-seeking. $\mathrm{H} 2.1$ 
Table 4. Essential principle 2: there is a need for holistic, tailored advocacy intervention plans that recognise the individual risks and needs of vulnerable and marginalised women such as those from minority groups or from rural areas (Continued)

\author{
Consequences
}

DM6: This leads her to delay/avoid seeking help, and to put up with her situation and adopt a wait-and-see position with respect to its evolution and to develop unfavourable psychosocial and psychological responses to the abuse (e.g. depression). The woman may be unwilling to accept she is at greater risk if she does not make active changes to the relationship as a form of active denial so as not to damage her status quo.

\title{
$\mathrm{H} 2.1$
}

DM7: Where there is a discrepancy between sociocultural norms and beliefs, on one hand, and advocacy approaches on the other, then a woman's ability to choose to seek advocacy and services according to her need is limited. H2.1

\section{Consequences}

DM7: This leads to feelings of shame and stigma if she continues and therefore encourages domestic or traditional care practices. $\mathrm{H} 2.1$

DM8: If the woman's informal networks feel overwhelmed / unskilled/unsupported in helping her with her abuse or if they feel constrained by the same cultural or geographical factors as the abused women, they will lack the capabilities to help her.

Consequences 
Table 4. Essential principle 2: there is a need for holistic, tailored advocacy intervention plans that recognise the individual risks and needs of vulnerable and marginalised women such as those from minority groups or from rural areas (Continued)

DM8:This leads to the woman becoming even more marginalised and to feelings of learned helplessness and hopelessness and she will feel she has no choice but to maintain the status quo.

\begin{tabular}{|c|c|}
\hline Structural & $\begin{array}{l}\text { Disenabling mecha- } \\
\text { nisms }\end{array}$ \\
\hline $\begin{array}{l}\text { Cultural/patri- } \\
\text { archal norms }\end{array}$ & DM9: if an abused \\
\hline $\begin{array}{l}\text { Structural } \\
\text { barriers to } \\
\text { access of re- } \\
\text { sources for } \\
\text { some women } \\
\text { (inequalities) }\end{array}$ & $\begin{array}{l}\text { mented migrant and } \\
\text { fears immigration offi- } \\
\text { cials, then her ability to } \\
\text { choose to end or reduce } \\
\text { the abuse and seek help } \\
\text { from services accord- }\end{array}$ \\
\hline $\begin{array}{l}\text { Eligibility and } \\
\text { rights to state } \\
\text { support (e.g. } \\
\text { immigration } \\
\text { status) }\end{array}$ & $\begin{array}{l}\text { ing to her need is lim- } \\
\text { ited, even though she } \\
\text { may have high expecta- } \\
\text { tions of the helpfulness } \\
\text { of services for her with- } \\
\text { out these constraints. } \\
\text { H2.1 }\end{array}$ \\
\hline
\end{tabular}

\section{Consequences}

DM9: this leads her to avoid seeking help, and to put up with her situation and to develop unfavourable psychosocial and psychological responses to the abuse (e.g. depression). H2.1

DM10: if an abused woman is physically/legally/economically/emotionally dependent on her abuser and has limited resources with which to provide for herself or support herself without her abuser, and previous negative experience of help-seeking to deal with this, then she will perceive her ability to choose to end or reduce the abuse and seek help from services according to her need as particularly diminished. H2.1

\section{Enabling mechanisms from advocacy}

EMfA8: if the advocates understand the ways of working of the systems and bureaucratic processes they and the woman need to navigate and are able to work within them to effect change or overcome them, and are able to share these capabilities with the abused woman, then she will develop the capabilities to do so herself. $\mathrm{H} 2.1$ and $\mathrm{H} 2.2$

\section{Consequences}

EMfA8: this leads to the woman's increased confidence in involving these services and she takes steps to do so. $\mathrm{H} 2.1$

and $\mathrm{H} 2.2$ tive counselling spiritual and network support

BAME specialist

Advocacy training, supervision and support

Childcare and money for transportation

Language services

Immigration support

Formal partnership protocols for advocacy and coordinated services

\section{Proximal/in- Proximal/in- termediate termediate}

Access to/use Lack of confiof resources. dence in ser$\mathrm{H} 2.1$ and $\mathrm{H} 2.2$ vices. $\mathrm{H} 2.1$ and $\mathrm{H} 2.2$

Taking steps to end the abuse, such as prosecuting her abuser. $\mathrm{H} 2.1$ and $\mathrm{H} 2.2$

Documented immigration status. $\mathrm{H} 2.1$ and $\mathrm{H} 2.2$

Reduced abuse/ greater safety (woman may remain in relationship). $\mathrm{H} 2.1$ and $\mathrm{H} 2.2$

\section{Distal}

Reduced abuse/greater safety. H2.1 and $\mathrm{H} 2.2$

\section{Limited ac-} cess to/use of resources. $\mathrm{H} 2.1$ and $\mathrm{H} 2.2$

Overconfidence in services leading to lack of satisfaction with them (expectations not met). $\mathrm{H} 2.1$ and $\mathrm{H} 2.2$

\section{Psychologi-} cal distress or other psychological or psychosocial outcomes. H2.1, $\mathrm{H} 2.2$ and $\mathrm{H} 2.3$

Continued or increased abuse/stalking/death. $\mathrm{H} 2.1, \mathrm{H} 2.2$ and $\mathrm{H} 2.3$

Disengagement with services. $\mathrm{H} 2.1$

Distal

Continued abuse/stalking/death. $\mathrm{H} 2.1, \mathrm{H} 2.2$ and $\mathrm{H} 2.3$ 
Table 4. Essential principle 2: there is a need for holistic, tailored advocacy intervention plans that recognise the individual risks and needs of vulnerable and marginalised women such as those from minority groups or from rural

areas (Continued)

\section{Consequences}

DM10: this will lead her to develop a learned helplessness about her situation and a lack of confidence in any services as being able to help her and so she will not seek help. $\mathrm{H} 2.1$
Woman-centred

focus

Education and

information on abuse and relationships

Education and information on women's rights and sources of help

Active referral to, and help in accessing, other services

Self-efficacy and self-esteem development

Recommendations

Goal setting and planning

Assessment of risk of repeat abuse

Safety planning

\section{Mechanisms for advocates}

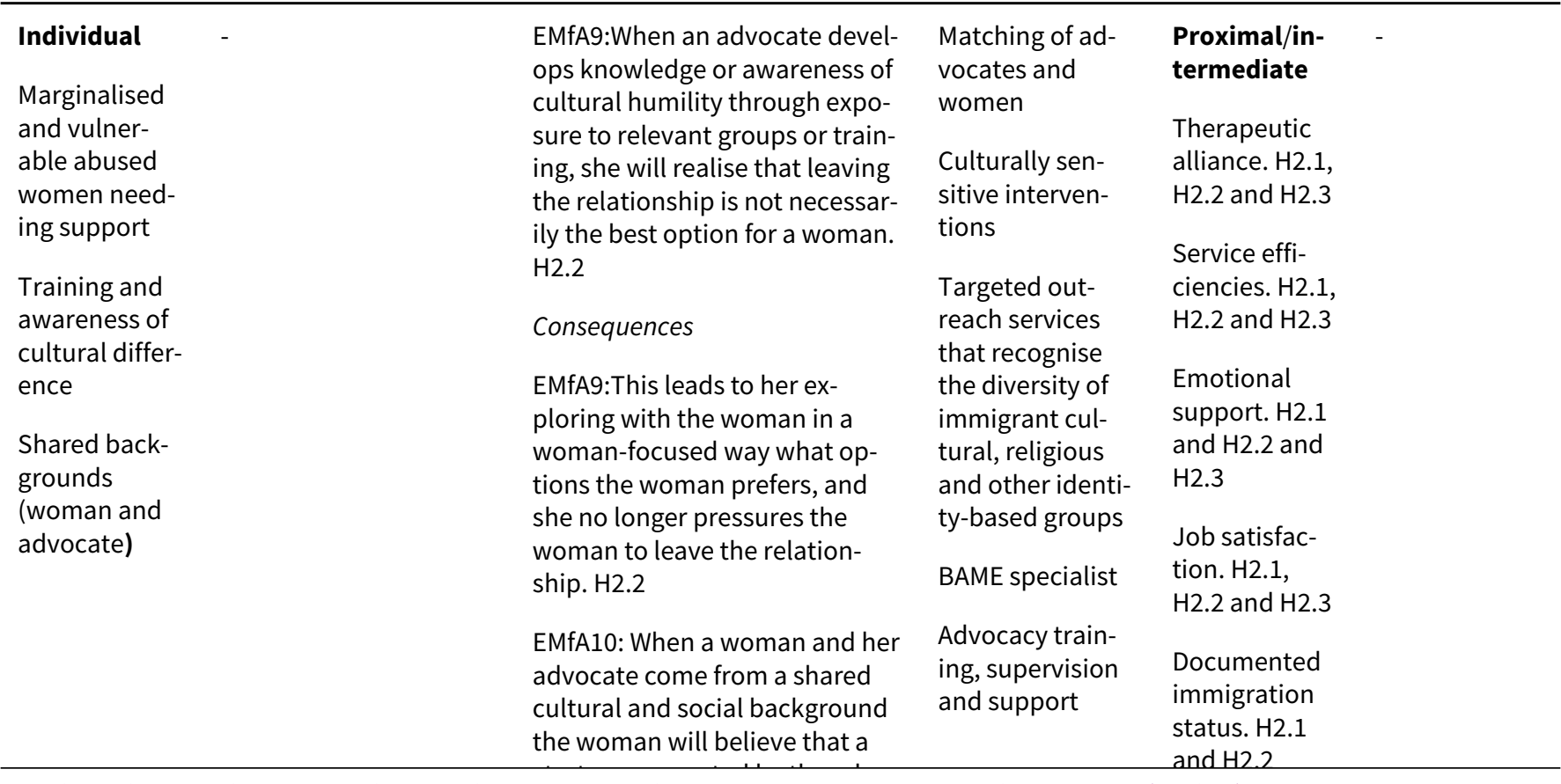


Table 4. Essential principle 2: there is a need for holistic, tailored advocacy intervention plans that recognise the individual risks and needs of vulnerable and marginalised women such as those from minority groups or from rural

areas (Continued)

cate will be effective for her in achieving the outcomes she desires. $\mathrm{H} 2.3$

Consequences

EMfA10:

This leads to greater engagement with the advocacy. $\mathrm{H} 2.3$
Immigration sup-

port

Woman-centred

focus

Education and information on abuse and relationships

Education and information on women's rights and sources of help

Active referral to, and help in woman's accessing, other services

Self-efficacy and self-esteem development for woman

Recommendations

Goal setting and planning

Assessment of risk of repeat abuse

Safety planning

Local
Local ways
of working of
services and
availability of
resources
Culturally ap-
propriate re-
sources
Local train-
ing, protocols,
case supervi-
sion and de-
briefs

EMfA11: if the advocates understand the ways of working of the systems and bureaucratic processes they and the woman need to navigate and are able to work within them to effect change or overcome them, and are able to share these capabilities with the abused woman, then she will develop the capabilities to do so herself. $\mathrm{H} 2.1$, $\mathrm{H} 2.2$ and $\mathrm{H} 2.3$

\section{Consequences}

EMfA11: this leads to the woman's increased confidence in involving these services and she takes steps to do so. The woman overcomes fear of immigration systems and depor-
Culturally sensitive interventions

Targeted outreach services that recognise the diversity of immigrant cultural, religious and other identity-based groups

Culturally appropriate housing support

Culturally sensitive counselling, spiritual and network support
Reduced

abuse/

greater safety

(woman may

remain in rela-

tionship). $\mathrm{H} 2.1$

and $\mathrm{H} 2.2$

\section{Proximal/in- termediate}

Therapeutic alliance. $\mathrm{H} 2.1$, $\mathrm{H} 2.2$ and $\mathrm{H} 2.3$

Service efficiencies. $\mathrm{H} 2.1$, $\mathrm{H} 2.2$ and $\mathrm{H} 2.3$

Woman's engagement with advocacy. $\mathrm{H} 2.1, \mathrm{H} 2.2$ and $\mathrm{H} 2.3$

Women's access to/use of resources. $\mathrm{H} 2.1$ and $\mathrm{H} 2.2$
Proximal/intermediate

Dissatisfaction with their work/staff turnover/ burn-out. $\mathrm{H} 2.1, \mathrm{H} 2.2$ and $\mathrm{H} 2.3$

Reduced or inefficient services/lack of fidelity to intervention. $\mathrm{H} 2.1, \mathrm{H} 2.2$ and $\mathrm{H} 2.3$ 
Table 4. Essential principle 2: there is a need for holistic, tailored advocacy intervention plans that recognise the individual risks and needs of vulnerable and marginalised women such as those from minority groups or from rural

areas (Continued)

tation by developing trust in advocate, being educated about processes and supported with the system. $\mathrm{H} 2.1, \mathrm{H} 2.2$ and $\mathrm{H} 2.3$

EMfA12: When advocates are uncertain about how to help women, they may develop their own capabilities through reference to written protocols (especially when they have built-in flexibility to be adapted not only to suit individual women but also to suit an advocate's individual ways of working).

\section{Consequences}

EMfA12:This leads to advocates having more confidence in supporting the women appropriately and less fear of doing the wrong thing and the consequences of this, and also provide them with more time to focus on other aspects of their work.

EMfA13: When advocates lack confidence in their capabilities, this can be increased by case supervision and debriefs, through shared emotional support, shared strategies for practical support and confidence building, and pressure to develop competencies.

\section{Consequences}

EMfA13:This leads to the advocates' developing practice and consistency in services as well as advocates feeling more supported

\section{Disenabling}

DMfA5: When the abused woman comes from an ethnic minority group and there is an absence of systematic and ongoing culturally sensitive institutional interventions that the advocate can refer her to, the woman may become disappointed in the advocacy. $\mathrm{H} 2.1$
BAME specialist

Advocacy train-

ing, supervision and support

Childcare and money for transportation

Language services

Immigration support

Formal partnership protocols for advocacy and co-ordinated services

Woman-centred focus

Education and information on women's rights and sources of help

Active referral to, and help in accessing, other services

Advocate self-efficacy and selfesteem development
Emotional

support. H2.1, $\mathrm{H} 2.2$ and $\mathrm{H} 2.3$

Job satisfaction. H2.1, $\mathrm{H} 2.2$ and $\mathrm{H} 2.3$

Multi-agency co-operation. $\mathrm{H} 2.1, \mathrm{H} 2.2$ and $\mathrm{H} 2.3$

Consequences 
Table 4. Essential principle 2: there is a need for holistic, tailored advocacy intervention plans that recognise the individual risks and needs of vulnerable and marginalised women such as those from minority groups or from rural

areas (Continued)

DMfA5: this will lead her to develop a learned helplessness about her situation and a lack of confidence in any services as being able to help her and so she will not seek further help. H2.1

DMfA6: When an advocate lacks cultural understandings and humility and capabilities she may worry about sanctions from funders, policymakers and the justice system or retribution by or alienation of immigrant groups if she makes cultural mistakes. $\mathrm{H} 2.1, \mathrm{H} 2.2$ and $\mathrm{H} 2.3$

\section{Consequences}

DMfA6:This leads her to draw from a limited set of choices in supporting the women and therefore the woman is not effectively supported. $\mathrm{H} 2.1, \mathrm{H} 2.2$ and $\mathrm{H} 2.3$

\section{Structural \\ Cultural/patri- \\ archal norms \\ Structural \\ barriers to \\ access of re- \\ sources for \\ some women \\ (inequalities) \\ Eligibility and rights to state support}

National training, protocols, guidance

\section{EMfA14: if the advocate is able to successfully challenge and overcome structural barriers to advocate and women's resource use (e.g. through women's lan- guage lessons, culturally appro- priate services, increased ser- vice access), then her capabili- ties to be effective in her work referring abused women to oth- er agencies will be increased. $\mathrm{H} 2.1$ and $\mathrm{H} 2.3$ \\ Consequences}

EMfA14: this will lead to enhanced advocacy and enhanced service use by abused women and their greater engagement with and benefit from services as a community of practice. This will also lead to enhanced advocate job satisfaction and less burn-out and advocate staff turnover. $\mathrm{H} 2.1$ and $\mathrm{H} 2.3$
Change-based structural advocacy

Culturally sensitive interventions

\section{Targeted out-} reach services that recognise the diversity of immigrant cultural, religious and other identity-based groups

Culturally appropriate housing support

Culturally sensitive counselling, spiritual and network support

BAME specialist

Advocacy training, supervision and support

\section{Proximal/in- Proximal/in- termediate termediate \\ Women's ac- cess to/use \\ Staff turnover/ $\mathrm{H} 2.1$ and $\mathrm{H} 2.2 \quad \mathrm{H} 2.3$}

Woman's engagement with advocacy. $\mathrm{H} 2.1, \mathrm{H} 2.2$ and $\mathrm{H} 2.3$

Service efficiencies. $\mathrm{H} 2.1$, $\mathrm{H} 2.2$ and $\mathrm{H} 2.3$

Job satisfaction. $\mathrm{H} 2.1$, $\mathrm{H} 2.2$ and $\mathrm{H} 2.3$

Multi-agency co-operation. $\mathrm{H} 2.1, \mathrm{H} 2.2$ and $\mathrm{H} 2.3$ 
Table 4. Essential principle 2: there is a need for holistic, tailored advocacy intervention plans that recognise the individual risks and needs of vulnerable and marginalised women such as those from minority groups or from rural areas (Continued)

$$
\begin{aligned}
& \text { Childcare and } \\
& \text { money for trans- } \\
& \text { portation } \\
& \text { Language ser- } \\
& \text { vices } \\
& \text { Immigration sup- } \\
& \text { port } \\
& \text { Formal partner- } \\
& \text { ship protocols } \\
& \text { for advocacy and } \\
& \text { co-ordinated ser- } \\
& \text { vices } \\
& \text { Active referral } \\
& \text { to, and help in } \\
& \text { accessing, other } \\
& \text { services } \\
& \text { Advocate self-ef- } \\
& \text { ficacy and self- } \\
& \text { esteem develop- } \\
& \text { ment }
\end{aligned}
$$

\begin{tabular}{|c|c|c|c|}
\hline Review finding & $\begin{array}{l}\text { CERQual assess- } \\
\text { ment of confi- } \\
\text { dence in the evi- } \\
\text { dence }\end{array}$ & Explanation of CERQual assessment & $\begin{array}{l}\text { Studies contributing to the re- } \\
\text { view finding }\end{array}$ \\
\hline $\begin{array}{l}\text { Advocates find it } \\
\text { challenging to re- } \\
\text { move structural } \\
\text { barriers to resource } \\
\text { access despite the } \\
\text { value of this }\end{array}$ & $\begin{array}{l}\text { Moderate confi- } \\
\text { dence }\end{array}$ & $\begin{array}{l}\text { We graded this finding moderate confidence be- } \\
\text { cause there were } 19 \text { good-quality studies and } 1 \\
\text { poor-quality study (Coy 2011), there was good co- } \\
\text { herence (including 'deviant' cases), studies were } \\
\text { relevant studies and there was moderate-quality } \\
\text { data, though bias towards qualitative research (al- } \\
\text { beit rich) and grey literature }\end{array}$ & $\begin{array}{l}\text { Bacchus 2016a; Briones-Vozme- } \\
\text { diano 2014; Burnett 2012; Coy } \\
\text { 2011; DePrince 2012; Donnelly } \\
\text { 2005; Garcia-Leeds 2017; Good- } \\
\text { man 2016b; Grant 2012; John- } \\
\text { son 2014; Kapur 2017; Logan } \\
\text { 2018; Lynch 2013; Macy 2018; } \\
\text { McFarlane 2000; Owen 2015; } \\
\text { Reina 2015; Rodgers 2017; Sil- } \\
\text { va-Martinez 2016; Thiara } 2009\end{array}$ \\
\hline $\begin{array}{l}\text { Once marginalised } \\
\text { women access ad- } \\
\text { vocacy they are } \\
\text { best supported }\end{array}$ & $\begin{array}{l}\text { Moderate confi- } \\
\text { dence }\end{array}$ & $\begin{array}{l}\text { We graded this finding moderate confidence be- } \\
\text { cause there were } 16 \text { mostly good-quality studies } \\
\text { across countries but they did not represent crim- } \\
\text { inal justice settings well and there was low risk of }\end{array}$ & $\begin{array}{l}\text { Briones-Vozmediano 2014; De- } \\
\text { Prince 2012; Donnelly 2005, Gar- } \\
\text { cia-Leeds 2017; Gillum 2008; } \\
\text { Goodman 2016a; Logan 2018; }\end{array}$ \\
\hline
\end{tabular}

BAME: Black, Asian and minority ethnic groups; DM: disabling mechanism; DMfA: disabling mechanisms from advocacy; EM: enabling mechanism; EMfA: enabling mechanisms from advocacy; H: hypothesis; IPV: intimate partner violence/abuse; PTSD: posttraumatic stress disorder

aNote, each comment (CMO configuration) is linked to a hypothesis at the top of the table. Statements are labelled as enabling and disenabling mechanisms, with numbers to facilitate.

Table 5. Findings related to essential principle 2: there is a need for holistic, tailored advocacy intervention plans that recognise the individual risks and needs of vulnerable and marginalised women such as those from minority groups or from rural areas 
Table 5. Findings related to essential principle 2: there is a need for holistic, tailored advocacy intervention plans that recognise the individual risks and needs of vulnerable and marginalised women such as those from minority

\section{groups or from rural areas (Continued)}

by advocates with

cultural humility, knowledge of how to tackle specif-

ic issues for these women, and awareness of intersectionalities bias from Prosman 2014, Sullivan 2019 and Trevillion 2013. Further, there was strong coherence across studies of all types, and 4 focused on ethnicity in their study design from the viewpoint of needing cultural humility. All were relevant studies, of advocacy for abused women from marginalised groups. There were some rich studies.
Lyon 2011; Matthew 2016; Prosman 2014; Sudderth 2017; Sullivan 2019; Thiara 2009; Trevillion 2013; White 2019; Wong 2013

$\begin{array}{llll}\begin{array}{l}\text { Helping the woman } \\ \text { should also }\end{array} & \begin{array}{l}\text { Moderate confi- } \\ \text { dence }\end{array} & \begin{array}{l}\text { We graded this finding moderate confidence be- } \\ \text { cause there were 16 mostly good-quality studies } \\ \text { across countries, coherence was good and they }\end{array} & \begin{array}{l}\text { Bacchus 2007; Coy 2011; Gar- } \\ \text { cia-Leeds 2017; Goodman } \\ \text { 2016a; Hidalgo 2016; Kapur }\end{array} \\ \begin{array}{ll}\text { prove mutually } \\ \text { beneficial for }\end{array} & \begin{array}{l}\text { were limited settings and a small risk of bias from } \\ \text { Coy 2011, O'Brien 2016, Prosman 2014, and Song }\end{array} & \begin{array}{l}\text { Lynch 2017; Lako 2018; Logan 2018; } \\ \text { man 2014; Reina 2015; Sil- }\end{array} \\ \text { the advocate } & \begin{array}{l}\text { 2010, and the data were moderately strong (with } \\ \text { va-Martinez 2016; Song 2010; }\end{array} & \text { Thiara 2009; Wong 2013 }\end{array}$

CERQual: confidence in the evidence from reviews of qualitative research

Table 6. Essential principle 3: whether an abused woman was pregnant or had children could affect her engagement with advocacy and possibly also lead to some adverse outcomes

Essential principle 3: whether an abused woman was pregnant or had children could affect her engagement with advocacy and possibly also lead to some adverse outcomes

Hypothesis 3.1: women with children are more likely to engage with advocacy in order to support them

Hypothesis 3.2: women may be reluctant to use advocacy services because of perceived risks to themselves and their children from taking action against the abuser

Hypothesis 3.3: women are more likely to engage with advocacy when childcare facilities are provided

Hypothesis 3.4: women who are pregnant are easier for advocates to access

\begin{tabular}{|c|c|c|c|c|c|}
\hline Contexts & $\begin{array}{l}\text { Enabling (EM) } \\
\text { and disabling } \\
\text { mechanisms } \\
\text { (DM) with re- } \\
\text { spect to use } \\
\text { of advocacya }\end{array}$ & $\begin{array}{l}\text { Effect of advocacy itself (enabling (EMfA) } \\
\text { and disabling (DMfA) mechanisms }{ }^{a}\end{array}$ & $\begin{array}{l}\text { Relevant } \\
\text { advoca- } \\
\text { cy strate- } \\
\text { gies/compo- } \\
\text { nents }\end{array}$ & $\begin{array}{l}\text { Potential an- } \\
\text { ticipated out- } \\
\text { comes of ad- } \\
\text { vocacya }\end{array}$ & $\begin{array}{l}\text { Potential } \\
\text { unanticipat- } \\
\text { ed outcomes } \\
\text { of advocacy }\end{array}$ \\
\hline $\begin{array}{l}\text { Individual } \\
\text { Having chil- } \\
\text { dren } \\
\text { Being preg- } \\
\text { nant } \\
\text { Risk of abuse } \\
\text { Type of abuse }\end{array}$ & $\begin{array}{l}\text { Enabling } \\
\text { mechanisms } \\
\text { EM1: if a } \\
\text { woman is } \\
\text { pregnant for } \\
\text { the first time, } \\
\text { she will have } \\
\text { fewer con- } \\
\text { straints on her } \\
\text { movements } \\
\text { than a preg- } \\
\text { nant woman }\end{array}$ & $\begin{array}{l}\text { Enabling mechanisms from advocacy } \\
\text { EMfA1: the more children a woman has, or } \\
\text { the greater the risk she perceives them to be } \\
\text { in from the abuse, and the more concerned } \\
\text { she is about this, the more she will feel that } \\
\text { the sharing of her experiences with advo- } \\
\text { cates will lead to greater safety and better } \\
\text { outcomes for her children. H3.1 } \\
\text { or } \\
\text { EMfA2: if a woman is concerned that her } \\
\text { abuser will find out she is seeking help, but }\end{array}$ & $\begin{array}{l}\text { Outreach } \\
\text { Home visits } \\
\text { Mentor moth- } \\
\text { ers } \\
\text { Video advice } \\
\text { Education and } \\
\text { information } \\
\text { on abuse and } \\
\text { relationships }\end{array}$ & $\begin{array}{l}\begin{array}{l}\text { Proximal/in- } \\
\text { termediate }\end{array} \\
\text { Time spent } \\
\text { with the ad- } \\
\text { vocate. H3.1, } \\
\text { H3.3 and H3.4 } \\
\text { Service use. } \\
\text { H3.1, H3.3 and } \\
\text { H3.4 }\end{array}$ & $\begin{array}{l}\begin{array}{l}\text { Proximal/in- } \\
\text { termediate }\end{array} \\
\text { Lack of confi- } \\
\text { dence in ser- } \\
\text { vices. H3.2 } \\
\text { and H3.3 } \\
\text { Limited ac- } \\
\text { cess to/use } \\
\text { of resources. } \\
\text { H3.3 }\end{array}$ \\
\hline
\end{tabular}


Table 6. Essential principle 3: whether an abused woman was pregnant or had children could affect her engagement with advocacy and possibly also lead to some adverse outcomes (Continued)

with other children. H3.4

Consequences

EM1: This will make it easier for her to attend all advocacy sessions. H3.4 weighs up the risks to her children versus herself in this, she may choose to put the safety of her children first. H3.1 or

EMfA3: if a woman learns to recognise IPV from advocacy while she is pregnant, her maternal instincts to protect her unborn child will increase her sensitivity to abuse. H3.1

\section{Consequences}

EMfA1, EMfA2 and EMfA3: this makes her more likely to use advocacy and safety plans and behaviours and to stay engaged with these. H.3.1

EMfA4: if a woman who is pregnant or has children is experiencing mild abuse and is not aware she is, a brief intervention may make her aware of this for the first time. H3.2

\section{Consequences}

EMfA4: this leads her to want to protect her children from harm and so will prompt her to adopt safety behaviours and a safety plan. H3.2

EMfA5: when an advocate is also a woman's midwife or is a mentor mother who has already been through what the woman has and managed to deal with it, the woman is likely to trust them as having her interests at heart and as being experts. H3.4

\section{Consequences}

EMfA5: this will encourage the woman to follow their advice. H.3.4

\section{Disenabling mechanisms from advocacy}

DMfA1: if a woman who has a baby takes steps to change her relationship with the abuser, the man may be particularly sensitive to this because of factors associated with having a child such as poor sleep, and jealousy. H3.2

\section{Consequences}

DMfA1: this will lead him to reassert his control over the woman by escalating the abuse. H3.2

DMfA2: if the woman with children is made aware of the abuse, she may develop feelings of guilt or low mood about previous exposure of any children. $\mathrm{H} 3.2$
Assessment of risk of repeat abuse

Safety planning

Recommendations

Empathetic, non-judgmental listening

Help with telephone calls

to end the abuse, such as prosecuting her abuser. $\mathrm{H} 3.1, \mathrm{H} 3.3$ and H3.4

Reduced abuse/greater safety.

$\mathrm{H} 3.1, \mathrm{H} 3.3$ and H3.4

Distal

Reduced abuse/greater safety. H3.1, H3.3 and H3.4
Overconfidence in services leading to lack of satisfaction with them. H3.1

Removal of children from family/harm to children. H3.1-H3.4

Continued or increased abuse/stalking/ death. H3.1-H3.4

Distal

Removal of children from family/Harm to children. H3.1-H3.4

Continued abuse/stalking/ death. H3.1-H3.4 
Table 6. Essential principle 3: whether an abused woman was pregnant or had children could affect her engagement with advocacy and possibly also lead to some adverse outcomes (Continued)

Consequences

DMfA2: this may undermine her use of services and lead to her dropping out or a need for more complex support. H3.2

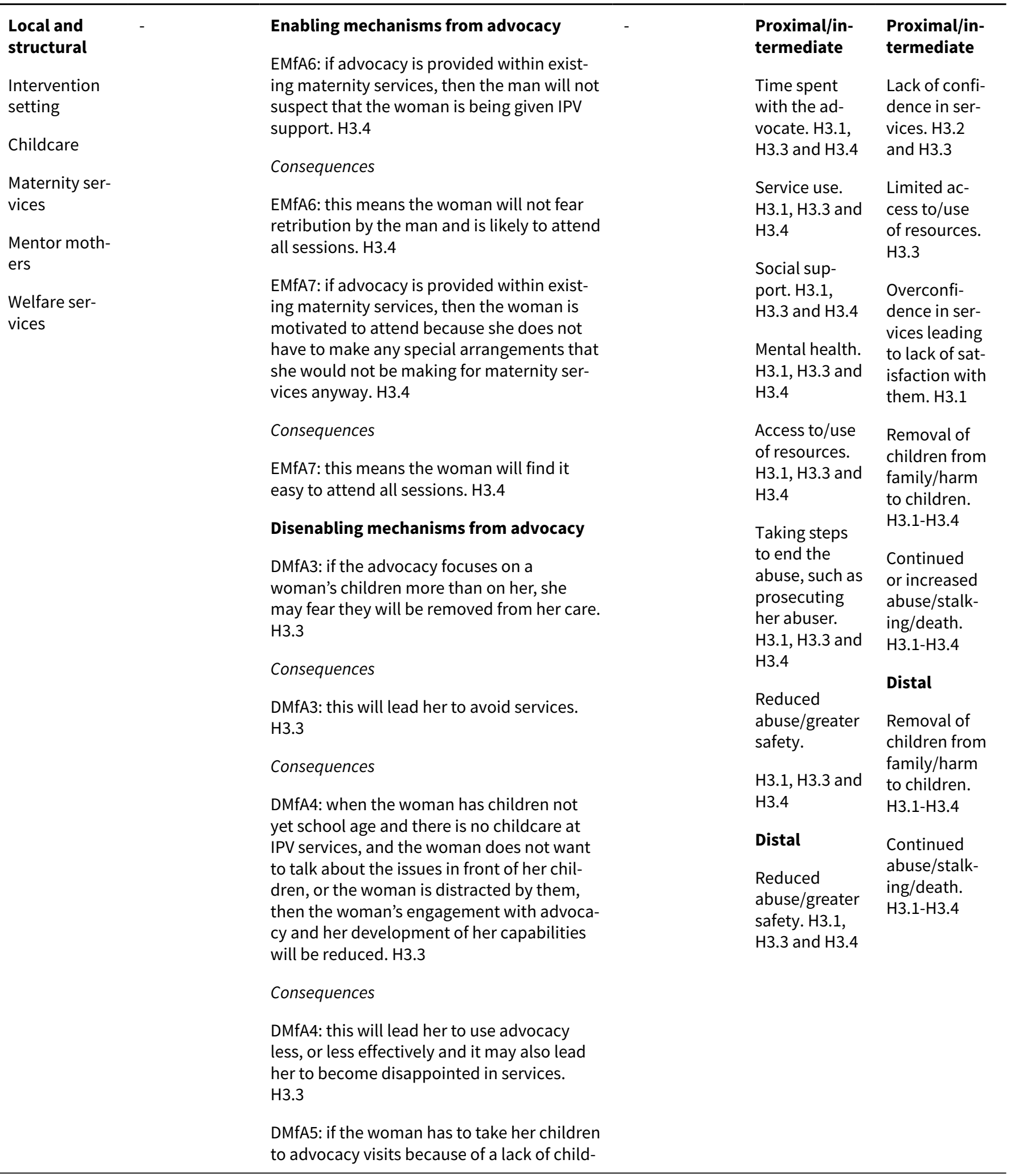


Table 6. Essential principle 3: whether an abused woman was pregnant or had children could affect her engagement with advocacy and possibly also lead to some adverse outcomes (Continued)

\author{
care and is afraid the children will reveal her \\ help-seeking to the abuser, she may become \\ fearful that he will harm her and the chil- \\ dren. $\mathrm{H} 3.3$ \\ Consequences \\ DMfA5: this will lead her to disengage from \\ and avoid advocacy and help-seeking. H3.3
}

DM: disabling mechanism; DMfA: disabling mechanisms from advocacy; EM: enabling mechanism; EMfA: enabling mechanisms from advocacy; H: hypothesis; IPV: intimate partner violence/abuse

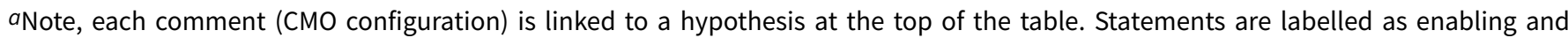
disenabling mechanisms, with numbers to facilitate.

Table 7. Findings related to essential principle 3: whether an abused woman was pregnant or had children could affect her engagement with advocacy and possibly also lead to some adverse outcomes

\begin{tabular}{|c|c|c|c|}
\hline Review finding & $\begin{array}{l}\text { CERQual assess- } \\
\text { ment of confi- } \\
\text { dence in the evi- } \\
\text { dence }\end{array}$ & Explanation of CERQual assessment & $\begin{array}{l}\text { Studies contributing } \\
\text { to the review finding }\end{array}$ \\
\hline $\begin{array}{l}\text { Women with children } \\
\text { are more likely to engage } \\
\text { with advocacy in order to } \\
\text { support them }\end{array}$ & Low confidence & $\begin{array}{l}2 \text { relevant studies with weak data and risk of bias } \\
\text { from } 1 \text { of the } 2\end{array}$ & $\begin{array}{l}\text { Howarth 2016; Stover } \\
2010\end{array}$ \\
\hline $\begin{array}{l}\text { Women may be reluctant } \\
\text { to use advocacy services } \\
\text { because of perceived risks } \\
\text { to themselves and their } \\
\text { children from taking action } \\
\text { against the abuser }\end{array}$ & Low confidence & $\begin{array}{l}\text { Only } 5 \text { relevant studies that did not focus on the } \\
\text { risks, so not of direct relevance, and } 1 \text { had (low) } \\
\text { risk of bias. }\end{array}$ & $\begin{array}{l}\text { Hidalgo 2016; } \\
\text { Humphreys 2002; } \\
\text { Shorey 2014; Sullivan } \\
\text { 2006; Thiara } 2016\end{array}$ \\
\hline $\begin{array}{l}\text { Women are more likely to } \\
\text { engage with advocacy when } \\
\text { childcare facilities are pro- } \\
\text { vided }\end{array}$ & Low confidence & $\begin{array}{l}\text { Only } 2 \text { relevant studies, though both focused partly } \\
\text { on this issue with coherence }\end{array}$ & Macy 2018; Owen 2015 \\
\hline $\begin{array}{l}\text { Women who are pregnant } \\
\text { are easier for advocates to } \\
\text { access }\end{array}$ & Low confidence & $\begin{array}{l}4 \text { studies were relevant though } 13 \text { mostly good- } \\
\text { quality studies considered pregnant women. Some } \\
\text { risk of bias from Howarth 2016, McFarlane } 1997 \text { Mc- } \\
\text { Farlane } 2000 \text { and Taft 2011, including one (Howarth } \\
\text { 2016), that considered the issue explicitly. There } \\
\text { was poor coherence - } 1 \text { study shows potential neg- } \\
\text { ative outcomes, the other interventions suggest } \\
\text { positive outcomes. Data were moderately rich. }\end{array}$ & $\begin{array}{l}\text { Bacchus 2016a; Bac- } \\
\text { chus 2016b; Cripe 2010; } \\
\text { Curry 2006; Feder 2018; } \\
\text { Howarth 2016; Kiely } \\
\text { 2010; Macy 2018; McFar- } \\
\text { lane 1997; McFarlane } \\
\text { 2000; Owen 2015; Taft } \\
\text { 2011; Tiwari 2005 }\end{array}$ \\
\hline
\end{tabular}

CERQual: confidence in the evidence from reviews of qualitative research 
Table 8. Essential principle 4: a good advocate-survivor relationship is important for effective advocacy, as predicted by underpinning theories

Essential principle 4: a good advocate-survivor relationship is important for effective advocacy, as predicted by underpinning theories

Hypothesis 4.1: qualities of a good advocate-survivor relationship correspond with what women need for empowerment

Hypothesis 4.2: advocacy can be offered across diverse disciplines provided that advocate competency is assured through training

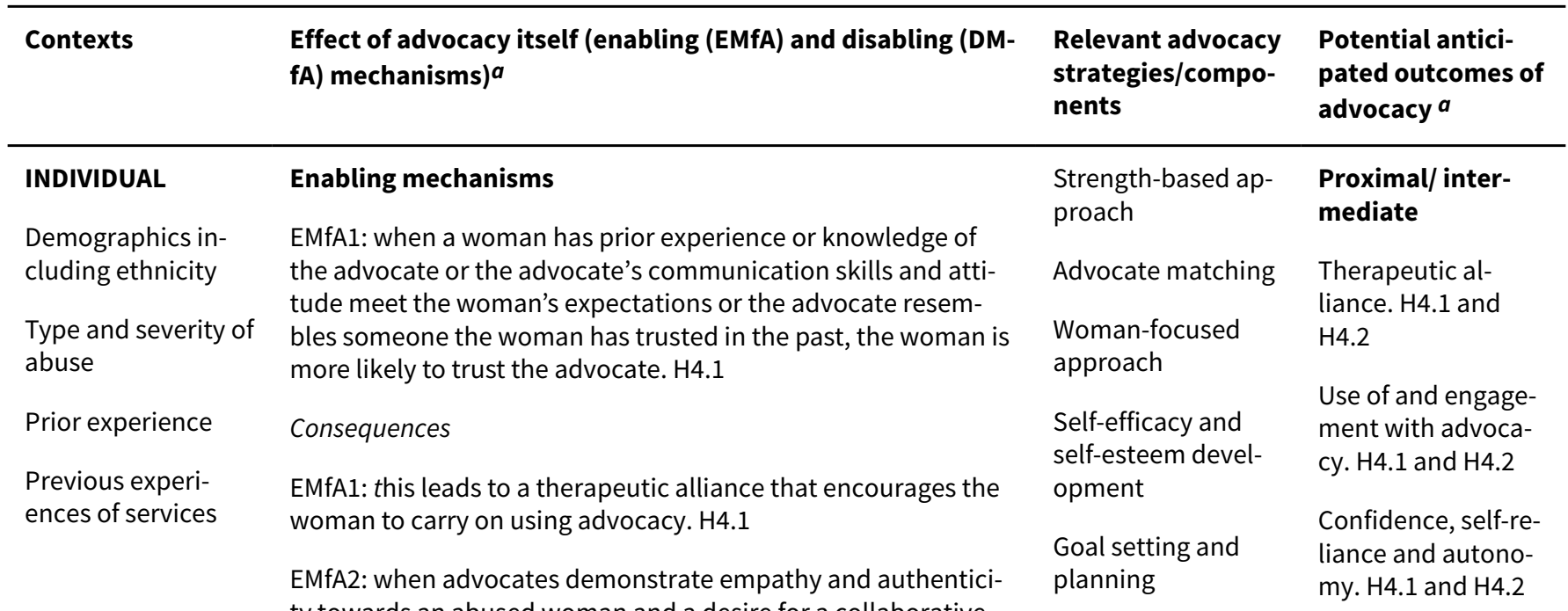
ty towards an abused woman and a desire for a collaborative, if not equal, relationship with her, the woman will be more likely to trust the advocate and the advocate will be more likely to avoid power imbalances despite their specialised knowledge. H4.1

Consequences

EMfA2: this therapeutic alliance leads to alignment of goals between the advocate and woman, empowering her and helping her to gain or regain confidence, self-reliance and autonomy and encourages the woman to carry on using advocacy. H4.1

EMfA3: when advocates have training or experience, they develop an understanding that recovery or life choices for IPV survivors could be markedly different from what the advocates themselves might prioritise. $\mathrm{H} 4.1$

\section{Consequences}

EMfA3: this leads to more woman-focused care in which goals are determined by the woman herself and a strong therapeutic alliance. H4.1

EMfA4: when advocates have training or experience, they develop an understanding of the difficult, time-consuming, nonlinear process of decision making for the women. H4.1

\section{Consequences}

EMfA4: this leads them to avoid pressuring women into choices the woman might regret. $\mathrm{H} 4.1$

EMfA5: when women are involved in shaping the advocacy process, they feel a personal investment that is aligned with their personal beliefs and values. H4.1 
Table 8. Essential principle 4: a good advocate-survivor relationship is important for effective advocacy, as predicted by underpinning theories (Continued)

Consequences

EMfA5: this increases their perception that service provision is helpful and survivor-defined. H4.1

EMfA6: when advocates draw on women's strengths this increases their belief in their own capabilities and values. H4.1

Consequences

EMfA6: this leads them to believe they can do what is needed to move ahead. H4.1

EMfA7: when advocates engage with 'the woman's agenda' and help her with chores on the day as a way of sharing and demonstrating non-judgmental empathy, they are more likely to be trusted. H4.1

\section{Consequences}

EMfA7: this leads to a therapeutic alliance that encourages the woman to carry on using advocacy.

H4.1

\section{Local}

Values of community to which woman belongs
EMfA8: when a woman and her advocate come from a shared cultural and social background the woman will believe that a strategy suggested by the advocate will be effective for her in achieving the outcomes she desires, especially when the advocate is also a survivor of abuse (also then acting as role models). H4.1

Consequences

EMfA8: this leads to greater engagement with the advocacy. H4.1

\section{Proximal/interme- diate}

Therapeutic alliance. $\mathrm{H} 4.1$ and $\mathrm{H} 4.2$

Use of and engagement with advocacy. H4.1 and $\mathrm{H} 4.2$

Confidence, self-reliance and autonomy. $\mathrm{H} 4.1$ and $\mathrm{H} 4.2$

\section{Structural}

Competency standards

\begin{abstract}
EMfA9: standardisation of minimum training requirements or competencies ensures that advocates across disciplines can work to the same standards. $\mathrm{H} 4.2$
\end{abstract}

\section{Consequences}

EMfA9: this increases the consistency of advocacy and the satisfaction women are likely to have with it. H4.2
Cross-disciplinary training and competency standards development for advocates

\section{Proximal/interme- diate \\ Therapeutic al- liance. $\mathrm{H} 4.1$ and $\mathrm{H} 4.2$ \\ Use of and engage- ment with advoca- cy. $\mathrm{H} 4.1$ and $\mathrm{H} 4.2$ \\ Trained advocates. H4. 2}

DMfA: disabling mechanisms from advocacy; EMfA: enabling mechanisms from advocacy; H: hypothesis; IPV: intimate partner violence/abuse

a Note, each comment (CMO configuration) is linked to a hypothesis at the top of table. Statements are labelled as enabling and disenabling mechanisms, with numbers to facilitate understanding. 
Table 9. Findings related to essential principle 4: a good advocate-survivor relationship is important for effective advocacy, as predicted by underpinning theories

$\begin{array}{llll}\text { Review finding } & \begin{array}{l}\text { CERQual assess- } \\ \text { ment of confi- } \\ \text { dence in the evi- }\end{array} & \text { Explanation of CERQual assessment } & \begin{array}{l}\text { Studies contributing to the review } \\ \text { finding }\end{array}\end{array}$

dence

\begin{tabular}{|c|c|c|}
\hline $\begin{array}{l}\text { Qualities of a good } \\
\text { advocate-survivor } \\
\text { relationship corre- } \\
\text { spond with what } \\
\text { women need for }\end{array}$ & High confidence & $\begin{array}{l}21 \text { relevant studies of often good-quality } \\
\text { across settings (though some small risk of } \\
\text { bias from Bell 2001; Kelly 1999; Lyon 2011; } \\
\text { O'Brien 2016; Prosman 2014; Song 2010; Song } \\
\text { 2012; Stover 2010; Zweig 2007. }\end{array}$ \\
\hline
\end{tabular}

empowerment
Strong coherence of qualities across a range of studies of advocates and abused women, and strong data from a variety of types of studies

\author{
Bacchus 2016a; Bacchus 2016b; Bell \\ 2001; Curry 2006; Goodman 2009; \\ Goodman 2016a; Hughes 2017; John- \\ son 2014; Kelly 1999; Kulkarni 2015; \\ Lako 2018; Lyon 2011; O'Brien 2016; \\ Prosman 2014; Song 2010; Song 2012; \\ Stover 2010; Thiara 2009; Umeda \\ 2017; Wood 2014; Zweig 2007
}

\begin{tabular}{|c|c|c|}
\hline $\begin{array}{l}\text { Advocacy can be } \\
\text { offered across di- } \\
\text { verse disciplines } \\
\text { provided that advo- } \\
\text { cate competency } \\
\text { is assured through } \\
\text { training }\end{array}$ & High confidence & $\begin{array}{l}41 \text { generally good-quality studies (some risk } \\
\text { of bias from Bahadir-Yilmaz 2018; Bell 2001; } \\
\text { Casey 2007; Constantino 2005; Gillum 2009; } \\
\text { Kelly 1999; Kendall 2009; Krasnoff 2002; Mc- } \\
\text { Farlane 2000; McFarlane 2006; Muelleman } \\
\text { 1999; Rodgers 2017; Song 2010; Stover 2010; } \\
\text { Trevillion 2013; Tutty 1996; Wuest 2015). }\end{array}$ \\
\hline
\end{tabular}

Strong coherence across effectiveness studies but also qualitative studies and other designs

Relevant studies, of advocates or of effectiveness studies involving advocacy for abused women with descriptions of the advocates.

Moderate-quality data (no studies were designed to answer the question as to whether advocates from different disciplines may have differences in impact)
Bacchus 2016a; Bacchus 2016b; Bahadir-Yilmaz 2018; Bell 2001; Bybee 2005; Casey 2007; Constantino 2005; Cripe 2010; Curry 2006; DePrince 2012; Garcia-Leeds 2017; Gillum 2009; Gupta 2017; Hathaway 2008; Hidalgo 2016; Hyman 2001; Infanti 2015; Kelly 1999; Kendall 2009; Kiely 2010; Krasnoff 2002; Lako 2018; Lea 2016.; Logan 2018; Macy 2018; McFarlane 2000; McFarlane 2006; Muelleman 1999; Reina 2015; Rodgers 2017; Song 2010; Stover 2010; Sullivan 2002; Thiara 2009; Tiwari 2005; Tiwari 2010; Trevillion 2013; Tutty 1996; Wong 2013; Wood 2014; Wuest 2015

CERQual: confidence in the evidence from reviews of qualitative research

Table 10. Essential principle 5: advocates have needs and requirements too in striving for effectiveness

\section{Essential principle 5: advocates have needs and requirements too in striving for effectiveness}

Hypothesis 5.1: advocacy takes time to be effective

Hypothesis 5.2: economic resources are needed to preserve the consistency and availability of advocacy services

Hypothesis 5.3: the personal nature of advocacy work makes it challenging for advocates to know how to manage personal-professional boundaries safely, which has implications for the well-being of the advocates and the abused women

Hypothesis 5.4: there is a need for advocates to balance competing roles and duties

Hypothesis 5.5: advocates take on emotional labour, which needs to be acknowledged

Hypothesis 5.6: in it together. The importance of support, connection and shared understandings within and across organisations (common language, common tools, common goals...) 
Table 10. Essential principle 5: advocates have needs and requirements too in striving for effectiveness (Continued)

\section{Contexts}

\section{Relevant ad- vocacy strate- gies/compo-} nents

\section{Enabling mechanisms from advocacy}

\section{Individual}

Competencies

Training

Time

Stressful or uncertain situations port. H5.1

\section{Consequences}

EMfA1: when advocates see an abused woman over a long period, they are able to develop a better therapeutic relationship and build up more skills and knowledge in the abused woman and undertake protracted processes such as housing sup-

EMfA1: this leads to disclosures and confidences from the woman, the advocate's better holistic understanding of the woman's case and the woman's perceptions of greater support, optimism about her situation, and increased capabilities. H5.1

EMfA2: when advocates feel emotionally drained because of their work they may focus on positive aspects of the job. H5.5

\section{Consequences}

EMfA2: this helps advocates to manage their emotions and prevent burnout. H5.5

\section{Disenabling mechanisms from advocacy}

DMfA1: when a woman leaves advocacy early for structural or personal reasons, the advocate feels frustrated or ineffective. H5.1

Consequences

DMfA1: this leads the advocate to be dissatisfied with their work and leave their job/suffer burnout. H5.1

DMfA2: when advocates feel traumatised by their work, they may take time away from their work or leave their job to avoid burnout. H5.5

\section{Consequences}

DMfA2: this leads to lack of continuity of care for abused women. H5.5

DMfA3: when advocates deal with difficult situations in a counselling or advocacy setting, they may doubt their skills and competencies. H5.5

\section{Consequences}

DMfA3: this leads them to develop feelings of reduced personal accomplishment and self-confidence and thence burnout. H5.5

Brief interven-
tion
Community out-
reach

Safety planning and referral

Follow-up and longer-term advocacy

Crisis intervention

Providing information and options

Survivor-focused perspective

Ways to manage emotions

\section{Potential antici- pated outcomes of advocacy $a$ \\ ential unan- ticipated out- comes of advo- cacy ${ }^{a}$}

\section{Proximal/inter- Proximal/ inter- mediate mediate}

Therapeutic alliance. H5.1-H5.6

Emotional support. H5.3-H5.6

Dissatisfaction with their work/ staff turnover/ burnout. H5.1H5.6

Job satisfaction. H5.1-H5.6

Reduced services/lack of fidelity to intervention. H5.1H5. 6 
Table 10. Essential principle 5: advocates have needs and requirements too in striving for effectiveness (Continued)

Local
Intervention set-
ting
Patriarchal
norms
Resource,
organisation-
al, programme
and peer support
constraints

constraints

\section{Enabling mechanisms from advocacy}

EMFA3: when boundaries are defined in protocols this gives the advocate a structured approach to follow. H5.3

\section{Consequences}

EMfA3: this leads to improved emotional and physical safety of woman and staff. H5.3

EMfA4: when advocates obtain support from within their organisation, they feel less stressed. H5.3 and 5.5

\section{Consequences}

EMfA4: this leads them to get more satisfaction from their work. H5.3 and 5.5

\section{Disenabling mechanisms}

DMfA4: when advocates are over-stretched or otherwise lacking in resources (e.g. because of organisational or funding constraints) they are limited in the ways they can support abused women. H5.2 and $\mathrm{H} 5.4$

\section{Consequences}

DMfA4: this may lead them to choose to cut corners in their work because of wanting to get something accomplished, or to struggle with roles and duties and respond to women's needs reactively, in order to provide the woman with the most critical help. $\mathrm{H} 5.2$ and $\mathrm{H} 5.4$

DMfA5: when an advocate accompanies women into the community, she is worried about finding the right balance between empowering and disempowering the women. $\mathrm{H} 5.3$

\section{Consequences}

DMfA5: this leads the advocate to become stressed or suffer burnout. $\mathrm{H} 5.3$ and $\mathrm{H} 5.5$

DMfA6: when advocates talk about their role outside of work, they may experience occupational stigma. $\mathrm{H} 5.5$

\section{Consequences}

DMfA6: this leads to a reduction in the advocates' sense of self, sense of work, and willingness to share with others outside of work. H5.5

DMfA7: when advocates disclose the work they do to others, the advocates are perceived as extraordinarily capable of managing traumatic material, with exceptional resilience. $\mathrm{H} 5.5$
Brief interven-

tion

Community outreach

Safety planning and referral

Follow-up and longer-term advocacy

Crisis intervention

Providing information and options

Survivor-focused perspective

Staff support and training

\section{Ways to manage} emotions

Inter- and intra-organisational working and knowledge exchange

\section{Proximal/inter- Proximal/inter- mediate mediate}

Therapeutic al- Dissatisfaction liance. H5.1-H5.6 with their work/ staff turnover/ burnout. H5.1-

Emotional and physical safety of woman and staff. H5. 3 and H5.5

Emotional support. H5.3-H5.6

Job satisfaction. H5.6

Reduced services/lack of fidelity to intervention. H5.1H5.6 
Table 10. Essential principle 5: advocates have needs and requirements too in striving for effectiveness (Continued) DMfA7: this precludes them from finding emotional support. H5.5

DMfA8: when advocates encounter structural and intra-organisational issues this constrains the work they can do with outside agencies. H5.6

Consequences

DMfA8: this makes it stressful for advocates to negotiate collaborations with outside agencies and creates a need for more knowledge exchange across and more efficient resource use across organisations. H5.6

Structural
Resource con-
straints and aus-
terity measures
Policy
Organisational
collaboration

\section{Enabling mechanisms from advocacy}

EMfA5: when the impact of advocacy recedes, two years or more after the woman completes it, booster shots of advocacy may be needed for reinforcement and reminding. H5.1

\section{Consequences}

EMfA5: these could lead to women continuing safety behaviours and focusing on the changes in their circumstances to maintain impact in the longer term. H5.1

\section{Disenabling mechanisms from advocacy}

DMfA9: When advocates are over-stretched or otherwise lacking in resources (e.g. because of organisational or funding constraints) they are limited in the ways they can support abused women. H5.2 and $\mathrm{H} 5.4$

Consequences

DMfA9: this may lead them to choose to cut corners in their work because of wanting to get something accomplished, or to struggle with roles and duties and respond to women's needs reactively, in order to provide the woman with the most critical help. H5.2 and H5.4

DMfA10: when advocates encounter structural and intra-organisational issues this constrains the work they can do with outside agencies. H5.6

\section{Consequences}

DMfA10: this makes it stressful for advocates to negotiate collaborations with outside agencies and creates a need for more knowledge exchange across and more efficient resource use across organisations. H5.6
Brief interven-

tion

Community out-

reach

Safety planning and referral

Follow-up and longer-term advocacy

Crisis intervention

Providing information and options

Survivor-focused perspective

Staff support and training

\section{Ways to manage} emotions

Inter- and intra-organisational working and knowledge exchange

\section{Proximal/inter- Proximal/inter- mediate mediate}

Therapeutic al- Dissatisfaction liance. H5.1-H5.6 with their work/ staff turnover/ Emotional sup- burnout. H5.1port. H5.3-H5.6 H5.6

Job satisfaction. Reduced serH5.1-H5.6

Multi-agency cooperation. H5.6 vices/lack of fidelity to intervention. H5.1H5.6

DMfA: disabling mechanisms from advocacy; EMfA: enabling mechanisms from advocacy; H: hypothesis

a Note, each comment (CMO configuration) is linked to a hypothesis at the top of table. Statements are labelled as enabling and disenabling mechanisms, with numbers to facilitate understanding. 
Table 11. Findings related to essential principle 5: advocates have needs and requirements too in striving for effectiveness

\begin{tabular}{ll}
\hline Review finding & $\begin{array}{l}\text { CERQual assess- } \\
\text { ment of confi- } \\
\text { dence in the evi- } \\
\text { dence }\end{array}$
\end{tabular}

\author{
Studies contributing to the review find- \\ ing
}

$\begin{array}{ll}\text { Advocacy takes } & \text { Moderate confi- } \\ \text { time to be effective } & \text { dence }\end{array}$

Advocacy takes

\begin{abstract}
44 generally good-quality studies (some risk of bias from Bahadir-Yilmaz 2018; Bell 2001; Casey 2007; Constantino 2005; Gillum 2009; Howarth 2016; Kelly 1999; Kendall 2009; Krasnoff 2002; McFarlane 2000; McFarlane 2004; McFarlane 2006; Muelleman 1999; Prosman 2014; Rodgers 2017; Song 2010; Song 2012; Stover 2010; Sullivan 2019; Taft 2011; Trevillion 2013; Tutty 1996; Wuest 2015)
\end{abstract}

Moderate coherence (some brief interventions are effective, but advocates and women cohere)

Relevant studies

Strong data

\section{Economic re-} sources are needed to preserve the consistency and availability of advocacy services

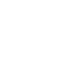

\section{Moderate confi- \\ Moder
dence}

\section{9 good-quality studies across countries (some risk of bias from Kelly 1999; Pros- man 2014; Weisz 1999) \\ Strong coherence across studies \\ Relevant studies}

Weak data - some rich studies but many are qualitative studies of advocates who might be biased
Bacchus 2016a; Bacchus 2016b; Bahadir-Yilmaz 2018; Bell 2001; Bybee 2005; Casey 2007; Constantino 2005; Cripe 2010; Coker 2012; Curry 2006; DePrince 2012; Feder 2018; Goodman 2016a; Gillum 2009; Gupta 2017; Howarth 2016; Hyman 2001; Kelly 1999; Kendall 2009; Kiely 2010; Kolb 2008; Krasnoff 2002; Lako 2018; McFarlane 2000; McFarlane 2004; McFarlane 2006; Merchant 2015; Muelleman 1999; Perez 2012; Prosman 2014; Rodgers 2017; Song 2010; Song 2012; Stover 2010; Sullivan 1991; Sullivan 2002; Sullivan 2019; Taft 2011; Tiwari 2005; Tiwari 2010; Trevillion 2013; Tutty 1996; Wong 2013; Wuest 2015
Bacchus 2007; Briones-Vozmediano 2014; Burnett 2012; Goodman 2016a; Goodman 2016b; Grant 2012; Kapur 2017; Kelly 1999; Lako 2018; Logan 2018; Lynch 2013; Macy 2018; Magruder 2017; Merchant 2015; Murray 2015; Prosman 2014; Silva-Martinez 2016; Weisz 1999; Wood 2014

Garcia-Leeds 2017; Goodman 2009; Goodman 2016a; Johnson 2014; Kolb 2008; Lako 2018; Merchant 2015; O'Brien 2016; Umeda 2017; Wies 2008; Wood 2014
11 good-quality studies across countries (risk of bias from 1 poor-quality study O'Brien 2016, but this did not contribute substantially)
Coherence good
Relevant studies
Rich data despite low number of studies
ture of advoca-
cy work makes it
difficult for advo-
cates to know how
to manage per-
sonal-profession-
al boundaries safe- ly, which has im- plications for the
High confidence well-being of the advocates and the abused women

Advocates need to balance competing roles and duties
High confidence

\author{
8 good-quality studies (risk of bias from \\ Kelly 1999) \\ Coherence good
}

Relevant studies
Burnett 2012; Coy 2011; Dunn 2007; Goodman 2016b; Kelly 1999; Lako 2018; Merchant 2015; Silva-Martinez 2016 
Table 11. Findings related to essential principle 5: advocates have needs and requirements too in striving for effectiveness (Continued)

Rich data despite low number of studies

\begin{tabular}{|c|c|c|c|}
\hline $\begin{array}{l}\text { Advocates take on } \\
\text { emotional labour, } \\
\text { which needs to be } \\
\text { acknowledged }\end{array}$ & High confidence & $\begin{array}{l}17 \text { mostly good-quality studies (risk } \\
\text { of bias from Bemiller 2010; Kelly 1999; } \\
\text { O'Brien 2016; Prosman 2014; Slattery } \\
\text { 2009) } \\
\text { Strong coherence across studies }\end{array}$ & $\begin{array}{l}\text { Bacchus 2016b; Babin 2012; Bemiller 2010; } \\
\text { Burnett 2012; Ganz 2015; Grant 2012; Kelly } \\
\text { 1999; Kolb 2008; Kulkarni 2015; Merchant } \\
\text { 2015; Garcia-Leeds 2017; Kapur 2017; } \\
\text { O'Brien 2016; Prosman 2014; Slattery 2009; } \\
\text { Wies 2008; Wood } 2014\end{array}$ \\
\hline
\end{tabular}

Relevant studies

Strong data - some rich qualitative stud-

ies and also models

In it together. The
importance of sup-
port, connection
and shared under-
standings within
and across organ-
isations (common
language, com-
mon tools, com-
mon goals...)

High confidence

22 mostly good-quality studies (some
risk of bias from Bell 2001; Howarth
2016; Kelly 1999; O'Brien 2016, Prosman
2014; Song 2010)
Strong coherence across studies
Relevant studies
Strong data across many settings

Bell 2001; Burnett 2012; Coy 2011; DePrince 2012; Goodman 2016a; Howarth 2016; Infanti 2015; Kapur 2017; Kelly 1999; Kulkarni 2015; Lako 2018; Lynch 2013; Magruder 2017; Merchant 2015; Murray 2015; O'Brien 2016; Prosman 2014; Silva-Martinez 2016; Song 2010; Thiara 2009; White 2019; Wood 2014

CERQual: confidence in the evidence from reviews of qualitative research

Table 12. Essential principle 6: the type of setting affects the theoretical underpinnings and the type and focus of the advocacy being offered and the women's responses

Essential principle 6: the type of setting affects the theoretical underpinnings and the type and focus of the advocacy being offered and the women's responses

Hypothesis 6.1: advocacy delivered across different settings offers its own specific characteristics, advantages and issues

Hypothesis 6.2: urban and rural areas are very differently resourced and have very different community cultures, which will shape the form of advocacy services

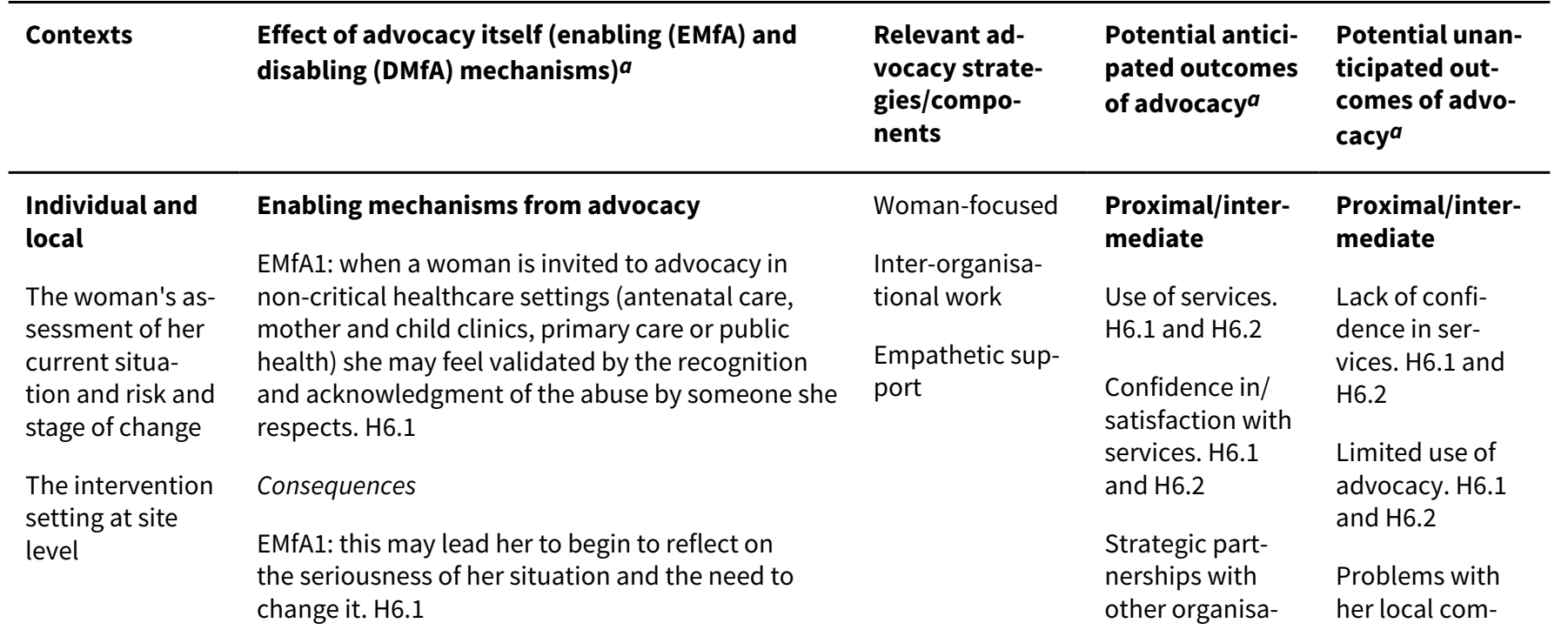

A realist review of which advocacy interventions work for which abused women under what circumstances (Review) 
Table 12. Essential principle 6: the type of setting affects the theoretical underpinnings and the type and focus of the advocacy being offered and the women's responses (Continued)

Poor infrastructure, limited services and local social attitudes

EMfA2: when a woman goes to a shelter, she is likely to have experienced abuse severe enough to induce her to leave home. H6.1

\section{Consequences}

EMfA2: this may make her receptive to interventions of long duration, such as finding housing. H6.1

EMfA3: when police officers accompanied by an advocate make unannounced home visits this may give the woman confidence in the police. H6.1

Consequences

EMfA3: this may lead to the woman contacting the police more often about abusive episodes. H6.1

EMfA4: when the perpetrator of abuse is cautioned or arrested by the police, this may give the woman trust in the 'strong arm of the law' and the use of force from the police to protect the woman from the perpetrator of her abuse. $\mathrm{H} 6.1$

Consequences

EMfA4: this may lead to the woman contacting the police more often about abusive episodes. H6.1

EMfA5: if advocates make clear to women that they are independent of the police the women are more likely to trust them. H6.1

Consequences

EMfA5: this leads to more use of advocacy for judicial support. H6.1

EMfA6: when an advocate is located in a formal setting, credibility by association is conferred on the advocate. H6.1

\section{Consequences}

EMfA6: this leads the woman to trust the competency of the advocate and make use of their services. H6.1

EMfA7: when services are rural, they have to deliver services well beyond their mandate and stretch their resources. $\mathrm{H} 6.2$

Consequences

EMfA7: this makes strategic partnerships with other rural organisations a matter of survival. H6.2

\section{Disenabling mechanisms from advocacy}

DMfA1: when a woman is invited to advocacy in non-critical healthcare settings (antenatal care, mother and child clinics, primary care or public health) from which she has not sought help for

\begin{tabular}{|c|c|}
\hline tions. $\mathrm{H} 6.1$ & munity or with \\
\hline $\mathrm{H} 6.2$ & $\begin{array}{l}\text { social services } \\
\text { or perceptions }\end{array}$ \\
\hline $\begin{array}{l}\text { Reduced abuse/ } \\
\text { greater safe- } \\
\text { ty }\end{array}$ & $\begin{array}{l}\text { of this. } \mathrm{H} 6.1 \text { and } \\
\mathrm{H} 6.2\end{array}$ \\
\hline
\end{tabular}

Distal

Woman's safety compromised -

Reduced abuse/ continued or ingreater safety. $\mathrm{H} 6.1$ and $\mathrm{H} 6.2$ creased abuse/ stalking/death. $\mathrm{H} 6.1$ and $\mathrm{H} 6.2$

Advocate dissatisfaction with their work/ staff turnover/ burnout. H6.2

Reduced services/lack of fidelity to intervention. H6.2

\section{Distal}

Problems with her local community or with social services or perceptions of this. H6.1 and H6.2

Continued abuse/stalking/death H6.1 and $\mathrm{H} 6.2$ 
Table 12. Essential principle 6: the type of setting affects the theoretical underpinnings and the type and focus of the advocacy being offered and the women's responses (Continued)

abuse, there may be a mismatch between what she

is offered and her stage of change. H6.1

Consequences

DMfA1: this leads her to reject the advocacy. H6.1

DMfA2: when police officers accompanied by an advocate make unannounced home visits the perpetrator of abuse may not be aware of police involvement or may resent it. $\mathrm{H} 6.1$

\section{Consequences}

DMfA2: this may antagonise the perpetrator of the abuse and lead to the woman's safety being compromised and her diminished use of the police. H6.1

DMfA3: unsolicited help may represent an often unwanted intrusion into the lives of the women that intersects negatively with broader societal structures. H6.1

Consequences

DMfA3: this may lead the woman to have problems with her local community or with social services or to perceive that she might have. $\mathrm{H} 6.1$

DMfA4: when an advocate has to deal with poor infrastructure, limited services and local social patriarchal attitudes her choices of what she can do for the woman are constrained. H6.1

Consequences

DMfA4: this leads her to draw from a limited set of choices in supporting the women and therefore the woman is not effectively supported. H6.1

DMfA5: when an advocate has to deal with poor infrastructure, limited services and local social patriarchal attitudes she will experience exclusion and over-onerous workloads $\mathrm{H} 6.2$

Consequences

DMfA5: this leads her to a reduction in her own well-being and job dissatisfaction and burnout. H6.2

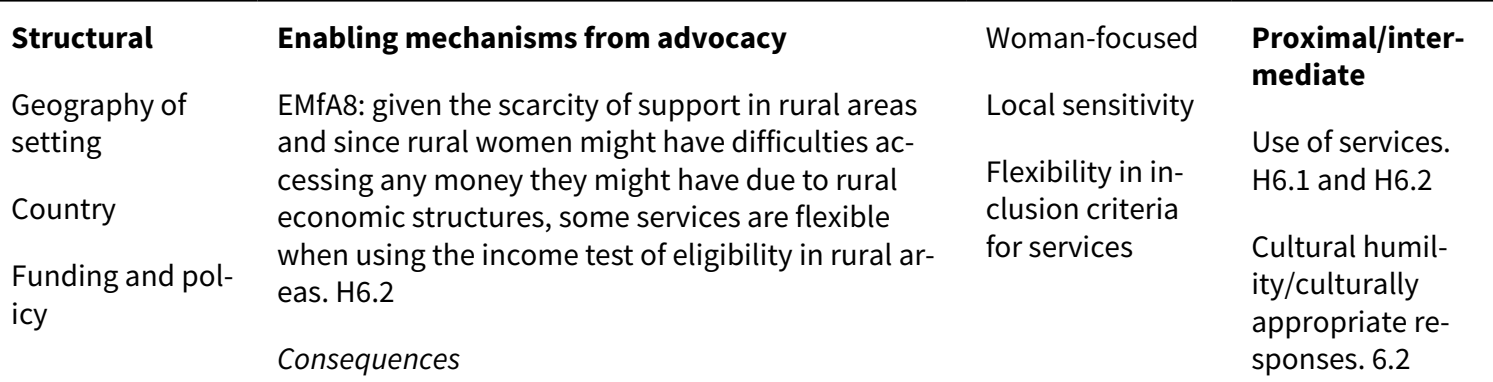


Table 12. Essential principle 6: the type of setting affects the theoretical underpinnings and the type and focus of the advocacy being offered and the women's responses (Continued)

EMfA8: this enables women who need support to

access services. $\mathrm{H} 6.2$

EMfA9: when advocates are sensitive to the setting in which they work they will appreciate that the intersection of country and internal geographical setting (e.g. rural/urban or type of healthcare system) is associated with a variety of differences in the demographics of service users, the people or organisations they hear about services from and the type of advocacy support preferred. $\mathrm{H} 6.2$

Consequences

EMfA9: this leads them to woman-focused care. H6.2

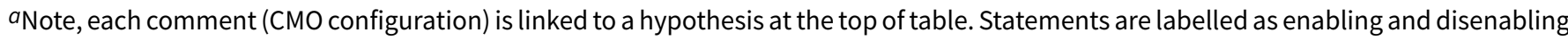
mechanisms, with numbers to facilitate understanding.

Table 13. Findings related to essential principle 6: the type of setting affects the theoretical underpinnings and the type and focus of the advocacy being offered and the women's responses

$\begin{array}{lll}\text { Review finding } & \begin{array}{l}\text { CERQual assess- } \\ \text { ment of confi- } \\ \text { dence in the evi- } \\ \text { dence }\end{array} & \begin{array}{l}\text { Explanation of CERQual assess- } \\ \text { ment }\end{array}\end{array}$

Advocacy delivered across different settings offers its own specific characteristics, advantages and issues
High confidence

67 often good-quality studies (some risk of bias from Bahadir-Yilmaz 2018; Bell 2001; Casey 2007; Constantino 2005; Howarth 2016; Kendall 2009; Kelly 1999; Krasnoff 2002; McFarlane 1997; McFarlane 2000; McFarlane 2004; McFarlane 2006; Muelleman 1999; O'Brien 2016; Prosman 2014; Rodgers 2017; Song 2010; Stover 2010; Taft 2011; Trevillion 2013; Tutty 1996; Weisz 1999; Wuest 2015)

Strong coherence

Relevant studies

Strong data
Bacchus 2016a; Bacchus 2016b; Bahadir-Yilmaz 2018; Bell 2001; Briones-Vozmediano 2014; Burnett 2012; Bybee 2005; Casey 2007; Coker 2012; Constantino 2005; Coy 2011; Cripe 2010; Curry 2006; DePrince 2012; Dunn 2007; Ekstrom 2015; Feder 2018; Ganz 2015; Garcia-Leeds 2017; Gillum 2008; Goodman 2016a; Gupta 2017; Howarth 2016; Hyman 2001; Infanti 2015; Johnson 2014; Kapur 2017; Kelly 1999; Kendall 2009; Kiely 2010; Krasnoff 2002; Kulkarni 2015; Lako 2018; Lea 2016; Logan 2018; Lynch 2013; Macy 2018; McDermott 2004; McFarlane 1997; McFarlane 2000; McFarlane 2004; McFarlane 2006; Merchant 2015; Muelleman 1999; Murray 2015; O'Brien 2016; Owen 2015; Prosman 2014; Reina 2015; Rodgers 2017; Silva-Martinez 2016; Song 2010; Stover 2010; Sudderth 2017; Sullivan 2002; Taft 2011; Taha 2015; Thiara 2009; Tiwari 2005; Tiwari 2010; Trevillion 2013; Tutty 1996; Umeda 2017; Weisz 1999; Wong 2013; Wood 2014; Wuest 2015

Bacchus 2016a; Burnett 2012; Coy 2011; Johnson 2014; Lynch 2013; Lyon 2011; Macy 2018; Owen 2015; Sullivan 2019; Thiara 2009; Umeda 2017; Wuest 2015
Urban and rural areas are very differently resourced and have very different community cultures, which will shape the form of advocacy services
High confidence

12 often good-quality studies across countries and settings (some risk of bias from Lyon 2011; Sullivan 2019; Wuest 2015)

Strong coherence across studies

Very relevant studies 
Table 13. Findings related to essential principle 6: the type of setting affects the theoretical underpinnings and the type and focus of the advocacy being offered and the women's responses (Continued)

Strong data, rich in-depth qual-

itative studies and also 2 RCTs

and 2 community-based studies

from the grey literature

CERQual: confidence in the evidence from reviews of qualitative research

\section{AP PEN DICES}

Appendix 1. Glossary

\begin{tabular}{ll}
\hline Term & Definition \\
\hline Active ingredients & $\begin{array}{l}\text { Those elements of an intervention that have an effect on the target population (intended or unin- } \\
\text { tended, helpful or not) }\end{array}$ \\
\hline
\end{tabular}

Backward citation tracking The process of identifying and screening for inclusion additional studies of interest from the reference lists of included studies.

\section{Complex intervention An intervention in which the causal pathway is dependent on interacting components between the} intervention and the intermediate and final outcomes.

$\begin{array}{ll}\text { Cluster searching } & \text { The systematic use of several search techniques to identify papers or other research outputs for } \\ \text { a single study. These may be directly related ('sibling' outputs) or indirectly related ('kinship' out- } \\ \text { puts). }\end{array}$

Controlling behaviours

Behaviours used by an abuser that are intended to create inferiority dependency by isolating a person from sources of support, exploiting them for personal gain, depriving them of their independence and possibility of resistance and escape, and regulating everything they do.

\section{Coercive control}

The use of abusive acts in ways that create anticipatory fear in the woman and a feeling that she is under constant surveillance. It leads the woman to internalise the abuser's rules, acquiescing to them and following them, even when out of his sight, for fear of further abuse. The abuser's threats of physical abuse can become as effective as actual physical acts, reducing the outward evidence that could help the woman to access help.

\section{Forward citation tracking}

The process of identifying and screening for inclusion additional studies of interest that cite an included study since publication.
An output that is related indirectly to another. Kinship outputs may be linked theoretically or contextually (including by provenance) or may be linked to a common antecedent study.

\section{Mechanisms of effect}

The processes by which the active ingredients or components of an intervention are 'activated'

\section{Moderators}

These may be a property of the intervention itself or a result of the context of its delivery; for example, the people who deliver it, the setting of delivery, other features of the context in which it is delivered, and other responses to the resources offered by the intervention in a specific context to a specific group of people.

\section{Mediators}

The steps in the chain of events (or intermediate outcomes) that occur between an intervention and the final outcomes 


\section{Appendix 2. Search strategies}

These intentionally excluded articles that considered both domestic violence and child abuse per se as major study focus.

\section{Cochrane Central Register of Controlled Trials (CENTRAL) in the Cochrane Library}

$\# 1$ (battered womn)

\#2MeSH descriptor: [Battered Women] explode all trees

\#3MeSH descriptor: [Domestic Violence] this term only

\#4MeSH descriptor: [Spouse Abuse] this term only

\#5abuse near/3 (woman or women)

\#6abuse* near/3 partner*

\#7abuse* near/3 spouse*

\#8wife near/3 batter* or wives near/3 batter*

\#9wife* near $/ 3$ abuse* or wives near $/ 3$ abuse*

\#10violen ${ }^{\star}$ near/3 partner* or violen* near/3 spous*

$\# 11$ violen ${ }^{\star}$ near $/ 3$ date or violen* near $/ 3$ dating

$\# 12 \# 1$ or \#2 or \#3 or \#4 or \#5 or \#6 or \#7 or \#8 or \#9 or \#10 or \#11

$\# 13$ child abuse

\#14MeSH descriptor: [Child Abuse] this term only

\#15MeSH descriptor: [Child Abuse, Sexual] this term only

$\# 16 \# 13$ or \#14 or \#15

$\# 17 \# 12$ not \#16

\#18wom*n or female*

\#19MeSH descriptor: [Women] this term only

\#20adolescen ${ }^{*}$ or teen ${ }^{*}$

$\# 21 \mathrm{MeSH}$ descriptor: [Adolescent] this term only

\#22\#18 or \#19 or \#20 or \#21

\#23advocacy

\#24MeSH descriptor:

\#25MeSH descriptor:

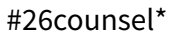

\#27MeSH descriptor:

\#28social work

\#29MeSH descriptor:

\#30mentor*

\#31MeSH descriptor:

\#32crisis intervention

\#33MeSH descriptor:

\#34risk assessment

\#35MeSH descriptor:

\#36MeSH descriptor:

\#37social welfare

\#38social support

\#39MeSH descriptor:

\#40help seeking

\#41information giving

\#42give near/3 information

\#43advice giving

\#44give near/3 advice or giving near/3 advice

\#45patient education

\#46MeSH descriptor: [Patient Education as Topic] this term only

\#47MeSH descriptor: [Health Education] explode all trees

\#48safety

\#49MeSH descriptor: [Safety] explode all trees

\#50women's health

\#51MeSH descriptor: [Women's Health] explode all trees 
$\# 52 \# 23$ or \#24 or \#25 or \#26 or \#27 or \#28 or \#29 or \#30 or \#31 or \#32 or \#33 or \#34 or \#35 or \#36 or \#37 or \#38 or \#39 or \#40 or \#41 or \#42 or \#43 or \#44 or \#45 or \#46 or \#47 or \#48 or \#49 or \#50 or \#51

\#53\#17 and \#22 and \#52

\section{MEDLINE Ovid}

1 Battered Women/

2 Domestic Violence/

3 Spouse abuse/

4 battered women.tw.

5 (abus\$ adj3 partner\$).tw.

6 (abus\$ adj3 wom\#n\$).tw.

7 (abus $\$$ adj3 spous\$).tw.

8 ((wife or wives) adj3 batter\$).tw.

9 ((wife or wives) adj3 abuse\$).tw.

10 (violen\$ adj3 partner\$).tw.

11 (violen\$ adj3 spous\$).tw.

12 (violen\$ adj3 (date or dating)).tw.

13 or/1-12

14 exp child abuse/

15 child $\$$ abus $\$$.tw.

16 (child adj3 abus\$ adj3 sex\$).tw.

17 or/14-16

1813 not 17

19 Women/

20 Females/

21 (woman or women or female\$).tw.

22 (adolescen\$ or teen\$).tw.

23 Adolescent/

24 or/19-23

25 Advocacy.tw.

26 exp Patient Advocacy/

27 exp Consumer Advocacy/

28 mentor\$.tw.

29 exp Mentors/

30 exp Crisis Intervention/

31 Crisis Intervention.tw.

32 exp Patient Advocacy/

33 exp Consumer Advocacy/

34 exp Counseling/

35 counsel\$.tw.

36 Social Work/

37 social work\$.tw.

38 exp Risk Assessment/

39 risk assessment.tw.

40 exp Social Welfare/

41 social welfare.tw.

42 Social Support/

43 social support.tw.

44 help seeking.tw.

45 information giving.tw.

46 giving information.tw.

47 (giv\$ adj3 information).tw.

48 advice giving.tw.

49 ((give or giving) adj3 advice).tw.

50 Patient Education as Topic/

51 exp Health Education/

52 patient educat\$.tw.

53 health educat\$.tw.

54 exp Safety/

55 safety.tw.

56 womens health.tw. 
57 Women's Health/

58 or $/ 25-57$

5918 and 24 and 58

\section{Embase Ovid}

1 battered women.tw.

2 Battered Women/

3 domestic violence/ or partner violence/ or family violence/ or battering/ 4 (abuse adj3 (woman or women)).tw.

5 (abuse $\$$ adj3 partner\$).tw.

6 (abuse $\$$ adj3 spouse\$).tw.

7 ((wife or wives) adj3 batter\$).tw.

8 ((wife or wives) adj3 abuse\$).tw.

9 (violen\$ adj3 partner\$).tw.

10 (violen\$ adj3 spous\$).tw.

11 (violen\$ adj3 (date or dating)).tw.

12 or/1-11

13 child abuse.tw.

14 child abuse/ or child abuse, sexual/

1512 not (13 or 14)

16 (woman or women or female\$).tw.

17 Women/

18 Female/

19 (adolescen\$ or teen\$).tw.

20 Adolescent/

21 or/16-20

22 advocacy.tw.

23 exp Patient Advocacy/

24 exp Consumer Advocacy/

25 counsel\$.tw.

26 exp Patient Counseling/

27 social work.tw.

28 exp Social Work/

29 mentor\$.tw.

31 crisis intervention.tw.

32 exp Crisis Intervention/

33 risk assessment.tw.

34 exp Risk Assessment/

35 exp Social Welfare/

36 social welfare.tw.

37 social support.tw.

38 exp social support/

39 help seeking.tw.

40 information giving.tw.

41 (give adj3 information).tw.

42 advice giving.tw. (127)

43 ((give or giving) adj3 advice).tw.

44 patient education.tw.

45 exp Patient Education/

46 exp Health Education/

47 safety.tw.

48 exp Safety/

49 patient safety/

50 Womens health.tw.

51 or/22-50

5215 and 21 and 51

\section{PsycINFO Ovid}

1 Battered Females/

2 Domestic Violence/

3 Partner Abuse/

4 Intimate Partner Violence/

A realist review of which advocacy interventions work for which abused women under what circumstances (Review) 
5 battered women*.tw.

6 (abus\$ adj5 partner\$).tw.

7 (abus\$ adj5 wom\#n\$).tw.

8 (abus\$ adj5 spous\$).tw.

9 ((wife or wives) adj5 batter\$).tw.

10 ((wife or wives) adj5 abus\$).tw.

11 (violen\$ adj5 partner\$).tw.

12 (violen\$ adj5 spous\$).tw.

13 (violen\$ adj5 (date or dating)).tw.

14 or/1-13

15 exp child abuse/

16 child\$ abus\$.tw.

17 (child adj3 abus\$ adj3 sex\$).tw.

18 or/15-17

1914 not 18

20 exp Human Females/

21 human females/ or mothers/ or wives/

22 (WOM\#N or GIRL\$ or FEMALE\$).tw.

23 (adolescen\$ or teen\$).tw.

24 adolescence $1317 \mathrm{yrs}$.ag.

2521 or 22 or 23 or 24

26 Advocacy/

27 Advocacy.tw.

28 mentor/

29 mentor\$.tw.

30 exp Crisis Intervention/

31 Crisis Intervention.tw.

32 exp Risk Assessment/

33 risk assessment.tw.

34 social casework/

35 social welfare\$.tw.

36 social support/

37 social support.tw.

38 help seeking behavior/

39 help seeking.tw.

40 (giv\$ adj3 (advice\$ or information\$)).tw. 41 patient education.tw.

42 client education/

43 health education/

44 exp safety/

45 safety\$.tw.

46 womens health.tw.

47 or/26-46

4819 and 25 and 47

\section{PsycArticles Ovid}

(((Battered adj Female) OR (Domestic adj Violence) OR (Partner adj Abuse) OR (Partner adj Violence) OR (battered adj women) OR (battered adj woman) OR ((abuse OR abused) adj partner) OR ((abuse OR abused) adj (women OR woman)) OR ((abuse OR abused) adj (spouse OR spousal)) OR ((wife or wives) adj (batter OR Battered)) OR ((wife or wives) adj (abuse OR abused)) OR (violence or violent) adj partner) OR ((violence or violent) adj (spouse OR spousal)) OR (violence or violent) adj (date or dating)) NOT (child adj abuse)) AND (Advocacy OR mentor OR mentors OR (Crisis adj Intervention) OR (risk adj assessment) OR (social adj welfare) OR (social adj support) OR (help adj seeking) OR advice OR information OR education OR safety OR (women's adj health))

\section{ASSIA ProQuest}

$\left(\left(\left(\mathrm{KW}=\left(\left(\right.\right.\right.\right.\right.$ abuse ${ }^{\star}$ within 3 (wom?n or partner ${ }^{\star}$ or spous ${ }^{\star}$ or wife or wives $\left.)\right)$ or (batter ${ }^{\star}$ within 3 (wom?n or partner ${ }^{\star}$ or spous ${ }^{\star}$ or wife or wives)) or (violen* within 3 (wom?n or partner* or spous* or wife or wives))) orkW=((family violence) or (domestic violence) or (dat ${ }^{\star}$ violence))) and not $\left(\mathrm{KW}=\left(\left(\right.\right.\right.$ child $^{*}$ within 3 abuse) or (child* within 3 sex $^{*}$ within 3 abuse $)$ or (child* within 3 maltreatment $\left.\left.\left.)\right)\right)\right)$ and $\left(\left(\mathrm{KW}=\left(\left(\right.\right.\right.\right.$ wom? $\mathrm{n}$ or female ${ }^{\star}$ or mother $\left.{ }^{\star}\right)$ or (adolescen ${ }^{\star}$ or teen $\left.\left.\left.{ }^{\star}\right)\right)\right)$ and $\left(\left(\mathrm{KW}=\left(\left(\right.\right.\right.\right.$ advocacy or counsel $^{\star}$ or mentor $\left.{ }^{\star}\right)$ or (crisis within 3 (intervention or management)) or (risk within 3 assessment)) or KW=((social within 3 (support or welfare)) or (help within 3 seek $\left.^{\star}\right)$ or (giv* within 3 (information or advice))) orkW=((safety) or (education within 3 (patient or health) )))) 


\section{CINAHL PLUS EBSCOhost (Cumulative Index to Nursing and Allied Health Literature)}

S49 S8 and S18 and S48

$\mathrm{S} 48 \mathrm{~S} 19$ or S20 or S21 or S22 or S23 or S24 or S25 or S26 or S27 or S28 or S29 or S30 or S31 or S32 or S33 or S34 or S35 or S36 or S37 or $\mathrm{S} 38$ or $\mathrm{S} 39$ or $\mathrm{S} 40$ or $\mathrm{S} 41$ or $\mathrm{S} 42$ or $\mathrm{S} 43$ or $\mathrm{S} 44$ or $\mathrm{S} 45$ or $\mathrm{S} 46$ or $\mathrm{S} 47$

S47 women* health service*

S46 (MH "Women's Health Services")

S45 (MH "Women's Health")

S44 social work*

S43 (MH "Social Work+")

S42 (MH "Patient Safety+") OR (MH "Safety+")

S41 safety

S40 patient $^{\star}$ education

S39 health education

S38 (MH "Health Education+")

S37 (MH "Patient Education+")

S36 (giv information $^{\star}$ ) or (give* advice*)

S35 help seek ${ }^{\star}$ or help seek ${ }^{*}$

S34 (MH "Help Seeking Behavior")

S33 (MH "Social Networks")

S32 (MH "Support, Psychosocial") OR (MH "Social Capital") S31 social welfare*

S30 (MH "Social Welfare")

S29 risk assessment*

S28 crisis intervention*

S27 (MH "Risk Assessment")

S26 (MH "Crisis Intervention+")

S25 mentor*

S24 (MH "Mentorship")

S23 S21 or S22

S22 counsel $^{*}$

S21 (MH "Counseling")

S20 advocacy

S19 (MH "Consumer Advocacy") OR (MH "Patient Advocacy")

$\mathrm{S} 18 \mathrm{~S} 13$ or $\mathrm{S} 14$ or $\mathrm{S} 15$ or $\mathrm{S} 16$ or $\mathrm{S} 17$

S17 teen* or adolescen*

S16 AG adolescent

S15 (MH "Mothers")

S14 (MH "Women")

S13 woman* or women* or female*

S12 S8 NOT S11

S11 S9 or S10

S9 (MH "Child Abuse") OR (MH "Child Abuse, Sexual")

S8 S1 or S2 or S3 or S4 or S5 or S6 or S7

S7 (violen* N5 partner ${ }^{\star}$ ) or (violen ${ }^{\star}$ N5 spous ${ }^{\star}$ ) or (violen* N5 date)

S6 (wife ${ }^{\star}$ 5 batter $^{\star}$ ) or (wife ${ }^{\star}$ N5 abus ${ }^{\star}$ ) or (wives ${ }^{\star}$ N5 batter ${ }^{\star}$ )

S5 (ABUS* N5 WOMEN*) or (ABUS* N5 Woman*) OR (Abus* N5 PARTNER*) OR (ABUS* N5 SPOUS*)

S4 battered women ${ }^{\star}$

S3 (MH "Domestic Violence")

S2 (MH "Intimate Partner Violence")

S1 (MH "Battered Women")

\section{Social Sciences Citation Index Web of Science (SSCI)}

Lemmatization $=$ Off

\# 6 \#5 AND \#4 AND \#3

\# $5 \mathrm{TS}=\left(\right.$ advoca $\left.^{\star}\right)$ or TS=(counsel $\left.{ }^{\star}\right)$ or TS=(mentor $\left.{ }^{\star}\right)$ or TS=(Risk SAME Assessment) or TS=(social welfare) or TS==(Social SAME- Support) or $\mathrm{TS}=\left(\right.$ help $^{\star}$ SAME seek ${ }^{*}$ or TS=(giv* SAME advice) or TS=(Patient SAME Education) or TS=(safety)

\# 4 TS $=\left(\right.$ adolescen $^{\star}$ or teen $\left.{ }^{\star}\right)$ or TS $=\left(\right.$ mother $\left.^{\star}\right)$ or TS $=\left(\right.$ female $\left.{ }^{\star}\right)$ or TS $=\left(\right.$ wom $\left.^{\star} n\right)$

\# 3 \#1 not \#2

\# 2 TS=(child SAME abuse) or TS=(child ${ }^{\star}$ SAME abuse* SAME sexual $\left.{ }^{\star}\right)$

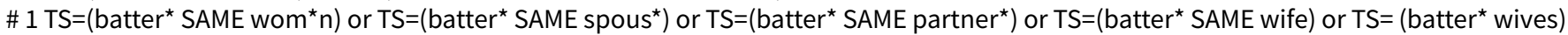
or TS=(abuse* SAME wom ${ }^{\star} n$ ) or TS=(abuse* SAME spous* $)$ or TS=(abuse* SAME partner ${ }^{\star}$ ) or TS=(abuse* SAME wife) or TS=(abuse* wives) 
or TS=(violen* SAME womn) or TS=(violen* SAME spous*) or TS=(violen* SAME partner $\left.{ }^{\star}\right)$ or TS= (violen* SAME wife) or TS=(violen* wives) or TS=(domestic violence) or TS=(dat ${ }^{\star}$ SAME violen $\left.{ }^{\star}\right)$

\section{International Bibliography of Social Sciences ProQuest (IBSS)}

((TI(crisis near/3 (intervention or management)) or $\mathrm{TI}($ risk near/3 assessment) or $\mathrm{TI}($ social near/3 (support or welfare)) or $\mathrm{TI}($ help near/3 seek $^{\star}$ ) or $\mathrm{TI}\left(\right.$ giv $^{\star}$ near/3 (information or advice))) OR (AB(crisis near/3 (intervention or management)) or $\mathrm{AB}$ (risk near/3 assessment) or $A B$ (social near/3 (support or welfare)) or $A B$ (help near/3 seek*) or AB (giv* near/3 (information or advice)))) AND $\left(\left(\left(T I\left(\left(\right.\right.\right.\right.\right.$ wom $^{\star} n$ or female* or mother $\left.{ }^{\star}\right)$ or (adolescen* or teen*))) OR $\left(\mathrm{AB}\left(\left(\right.\right.\right.$ wom $^{\star} \mathrm{n}$ or female* or mother $\left.{ }^{\star}\right)$ or (adolescen* or teen $\left.\left.\left.\left.{ }^{\star}\right)\right)\right)\right)$ AND $((\operatorname{TI}(($ family violence) or (domestic violence) or (dat* violence))) OR (AB((family violence) or (domestic violence) or (dat* violence))) OR ((TI((violen* near/3 (wom ${ }^{\star} n$

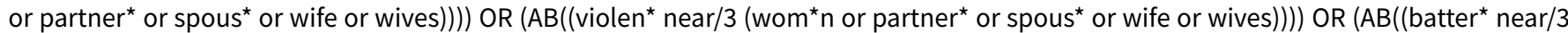

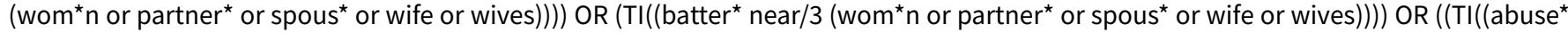
near/3 (wom ${ }^{\star} n$ or partner* or spous* or wife or wives $\left.\left.\left.)\right)\right)\right)$ OR $(\mathrm{AB}(($ abuse* near/3 (womn or partner* or spous* or wife or wives)) ))) OR $\left(\mathrm{TI}\left(\left(\right.\right.\right.$ abuse ${ }^{\star}$ near/3 (wom ${ }^{\star}$ n or partner or spous $^{\star}$ or wife or wives $\left.\left.)\right)\right)$ ) OR $\left(\mathrm{AB}\left(\left(\right.\right.\right.$ abuse ${ }^{\star}$ near/3 (wom ${ }^{\star}$ n or partner ${ }^{\star}$ or spous ${ }^{\star}$ or wife or wives $)))))$ AND $\left(\mathrm{TI}\left(\left(\right.\right.\right.$ wom $^{\star} n$ or female ${ }^{\star}$ or mother $\left.{ }^{\star}\right)$ or $\left(\right.$ adolescen $^{\star}$ or teen $\left.\left.\left.{ }^{\star}\right)\right)\right)$ AND $\left(\mathrm{AB}\left(\left(\right.\right.\right.$ wom $^{\star}$ n or female* or mother $\left.{ }^{\star}\right)$ or $\left(\right.$ adolescen $^{\star}$ or teen*))))

\section{Health Management Information Consortium Ovid (HMIC)}

1 domestic violence/ or partner abuse/

2 ((violen\$ adj3 dat\$) or (violen\$ adj3 domestic) or (violen\$ adj3 family) or ((violen\$ adj3 spous\$) or (violen\$ adj3 partner\$) or (violen\$ adj3

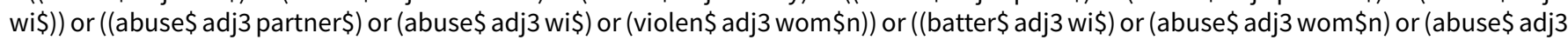
spous\$)) or ((batter\$ adj3 wom\#n) or (batter\$ adj3 spous\$) or (batter\$adj3 partner\$))).mp. [mp=title, other title, abstract, heading words] 31 or 2

4 Crisis intervention/

5 exp risk assessment/

6 counselling/

7 social welfare/

8 Social work/

9 Patient education/

10 Health education/

11 Mentoring/

12 Social support/

13 Safety/

14 exp Womens health/

15 advocacy/or patient advocacy/

16 Advocacy.tw.

17 mentor\$.tw.

18 Crisis Intervention.tw.

19 counsel\$.tw.

20 social work\$.tw.

21 risk assessment.tw.

22 social welfare.tw.

23 social support.tw.

24 help seeking.tw.

25 information giving.tw.

26 giv\$ information.tw.

27 advice giving.tw.

28 giv $\$$ advice.tw.

29 patient educat\$.tw.

30 health educat\$.tw.

31 safety.tw.

32 womens health.tw.

33 or $/ 4-32$

343 and 33

\section{Maternity and Infant Care Ovid}

1 battered women.tw.

2 Domestic violence.de.

3 (abus\$ adj3 (women or woman or spous\$)).tw.

4 (ABUS\$ adj3 (PARTNER\$ or WIFE or WIVES)).tw.

5 (BATTER $\$$ adj3 (PARTNER\$ or WIFE or WIVES)).tw.

6 (batter\$ adj3 (women or woman or spous\$)).tw.

A realist review of which advocacy interventions work for which abused women under what circumstances (Review) 
7 (VIOLEN\$ adj3 (PARTNER\$ or WIFE or WIVES)).tw. 8 (violen\$ adj3 (woman or women or spous\$)).tw.

9 (violen\$ adj3 dat\$).tw.

10 or/1-9

11 Child abuse.de.

12 (child adj3 abus\$).tw.

1311 or 12

1410 not 13

Database of Abstracts of Reviews of Effects (DARE), part of the Cochrane Library

\#1(battered wom ${ }^{\star}$ ):ti,ab

\#2MeSH descriptor: [Battered Women] explode all trees \#3MeSH descriptor: [Domestic Violence] this term only \#4MeSH descriptor: [Spouse Abuse] this term only

\#5(abuse near/3 (woman or women)):ti,ab

\#6(abuse near/3 partner $^{\star}$ ):ti,ab

\#7(abuse ${ }^{\star}$ near/3 spouse*):ti,ab

\#8(wife near/3 batter ${ }^{\star}$ or wives near/3 batter ${ }^{\star}$ ):ti,ab

\#9(wife ${ }^{\star}$ near $/ 3$ abuse* or wives near $/ 3$ abuse ${ }^{\star}$ ):ti,ab

\#10(violen* near/3 partner* or violen* near/3 spous*):ti,ab

$\# 11$ (violen ${ }^{\star}$ near/3 date or violen* near/3 dating):ti,ab

$\# 12 \# 1$ or \#2 or \#3 or \#4 or \#5 or \#6 or \#7 or \#8 or \#9 or \#10 or \#11

\#13(child next abuse):ti,ab

\#14MeSH descriptor: [Child Abuse] this term only

\#15MeSH descriptor: [Child Abuse, Sexual] this term only

$\# 16 \# 13$ or \#14 or \#15

$\# 17 \# 12$ not \#16

\#18(wom ${ }^{\star}$ or female $\left.{ }^{\star}\right):$ ti,ab

\#19MeSH descriptor: [Women] this term only

\#20(adolescen* or teen ${ }^{\star}$ ):ti,ab

$\# 21 \mathrm{MeSH}$ descriptor: [Adolescent] this term only

\#22\#18 or \#19 or \#20 or \#21

\#23advocacy:ti,ab

\#24MeSH descriptor: [Patient Advocacy] this term only

\#25MeSH descriptor: [Consumer Advocacy] explode all trees

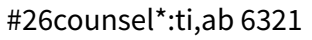

\#27MeSH descriptor: [Counseling] explode all trees

$\# 28$ social work:ti,ab

\#29MeSH descriptor: [Social Work] explode all trees

\#30mentor*:ti,ab

\#31MeSH descriptor: [Mentors] this term only

\#32crisis intervention:ti,ab

\#33MeSH descriptor: [Crisis Intervention] this term only

\#34risk assessment:ti,ab

\#35MeSH descriptor: [Risk Assessment] this term only

\#36MeSH descriptor: [Social Welfare] explode all trees

\#37social welfare:ti,ab

\#38social support:ti,ab

\#39MeSH descriptor: [Social Support] this term only

\#40help seeking:ti,ab

\#41information giving:ti,ab

\#42give near/3 information:ti,ab

\#43advice giving:ti,ab

\#44(give near/3 advice or giving near/3 advice):ti,ab

\#45(patient education):ti,ab

\#46MeSH descriptor: [Patient Education as Topic] this term only

\#47MeSH descriptor: [Health Education] explode all trees

\#48safety:ti,ab 55764

\#49MeSH descriptor: [Safety] explode all trees

\#50women's health:ti,ab

\#51MeSH descriptor: [Women's Health] explode all trees 
$\# 52 \# 23$ or \#24 or \#25 or \#26 or \#27 or \#28 or \#29 or \#30 or \#31 or \#32 or \#33 or \#34 or \#35 or \#36 or \#37 or \#38 or \#39 or \#40 or \#41 or \#42 or \#43 or \#44 or \#45 or \#46 or \#47 or \#48 or \#49 or \#50 or \#51

$\# 53 \# 17$ and \#22 and \#52

\#54\#53

\section{Cochrane Database of Systematic Reviews (CDSR); part of the Cochrane Library}

$\# 1$ (battered wom $\left.{ }^{\star} n\right): t i, a b$

\#2MeSH descriptor: [Battered Women] explode all trees

\#3MeSH descriptor: [Domestic Violence] this term only

\#4MeSH descriptor: [Spouse Abuse] this term only

\#5(abuse near/3 (woman or women)):ti,ab

\#6(abuse* near/3 partner*):ti,ab

$\# 7$ (abuse* near/3 spouse $\left.{ }^{\star}\right):$ ti,ab

\#8(wife near/3 batter ${ }^{\star}$ or wives near/3 batter $\left.{ }^{\star}\right): t i, a b$

\#9(wife ${ }^{\star}$ near/3 abuse ${ }^{\star}$ or wives near/3 abuse $\left.{ }^{\star}\right): \mathrm{ti}, \mathrm{ab}$

\#10(violen* near/3 partner ${ }^{\star}$ or violen* near/3 spous ${ }^{\star}$ ):ti,ab \#11(violen* near/3 date or violen ${ }^{\star}$ near/3 dating):ti,ab

$\# 12 \# 1$ or \#2 or \#3 or \#4 or \#5 or \#6 or \#7 or \#8 or \#9 or \#10 or \#11 \#13(child next abuse):ti,ab

\#14MeSH descriptor: [Child Abuse] this term only

\#15MeSH descriptor: [Child Abuse, Sexual] this term only

$\# 16 \# 13$ or \#14 or \#15

$\# 17 \# 12$ not \#16

\#18(wom ${ }^{\star}$ or female $\left.{ }^{\star}\right):$ ti,ab

\#19MeSH descriptor: [Women] this term only

\#20(adolescen ${ }^{\star}$ or teen ${ }^{\star}$ ):ti,ab

$\# 21 \mathrm{MeSH}$ descriptor: [Adolescent] this term only

\#22\#18 or \#19 or \#20 or \#21

\#23advocacy:ti,ab

\#24MeSH descriptor: [Patient Advocacy] this term only \#25MeSH descriptor: [Consumer Advocacy] explode all trees \#26counsel*:ti,ab

\#27MeSH descriptor: [Counseling] explode all trees \#28social work:ti,ab

\#29MeSH descriptor: [Social Work] explode all trees \#30mentor*:ti,ab

\#31MeSH descriptor: [Mentors] this term only \#32crisis intervention:ti,ab

\#33MeSH descriptor: [Crisis Intervention] this term only \#34risk assessment:ti,ab

\#35MeSH descriptor: [Risk Assessment] this term only \#36MeSH descriptor: [Social Welfare] explode all trees \#37social welfare:ti,ab

\#38social support:ti,ab

\#39MeSH descriptor: [Social Support] this term only

\#40help seeking:ti,ab

\#41information giving:ti,ab

\#42give near/3 information:ti,ab

\#43advice giving:ti,ab

\#44(give near/3 advice or giving near/3 advice):ti,ab

\#45(patient education):ti,ab

\#46MeSH descriptor: [Patient Education as Topic] this term only

\#47MeSH descriptor: [Health Education] explode all trees

\#48safety:ti,ab 55764

\#49MeSH descriptor: [Safety] explode all trees

\#50women's health:ti,ab

\#51MeSH descriptor: [Women's Health] explode all trees

$\# 52 \# 23$ or \#24 or \#25 or \#26 or \#27 or \#28 or \#29 or \#30 or \#31 or \#32 or \#33 or \#34 or \#35 or \#36 or \#37 or \#38 or \#39 or \#40 or \#41 or \#42 or \#43 or \#44 or \#45 or \#46 or \#47 or \#48 or \#49 or \#50 or \#51

$\# 53 \# 17$ and \#22 and \#52

\#54\#53

\section{OpenGrey}

violence OR abuse AND (Advocacy OR advocate OR mentor OR mentors OR (Crisis Intervention) OR (risk assessment) OR (social welfare) OR (social support) OR (help seeking) OR advice OR information OR education OR safety OR (women's health))ities.

\section{Dissertations \& Theses ProQuest}

ti(((domestic violence) or (wife abuse) OR (battered women) OR (spouse abuse) OR (partner abuse) OR (domestic abuse)) AND (Advocacy OR advocate OR mentor OR mentors OR (Crisis Intervention) OR (risk assessment) OR (social welfare) OR (social support) OR (help seeking) OR advice OR information OR education OR safety OR (women's health))) 


\section{WHO International Clinical Trials Registry Platform (ICTRP; apps.who.int/trialsearch/)}

Condition: domestic violen* OR domestic abus ${ }^{\star}$ OR partner violen* OR partner abus ${ }^{\star}$ OR spouse violen ${ }^{\star}$ or spouse abus ${ }^{\star}$ OR battered women

UK Clinical Research Network Study Portfolio (UKCRN)/UK Clinical Trials Gateway (now known as Be Part of Research; bepartofresearch.nihr.ac.uk)

Domestic violence

\section{Appendix 3. Figures of essential principles}

Figure 5.

Figure 5. Essential principle 1. Key Dark green hexagons = negative outcomes Light green circles $=$ positive outcomes

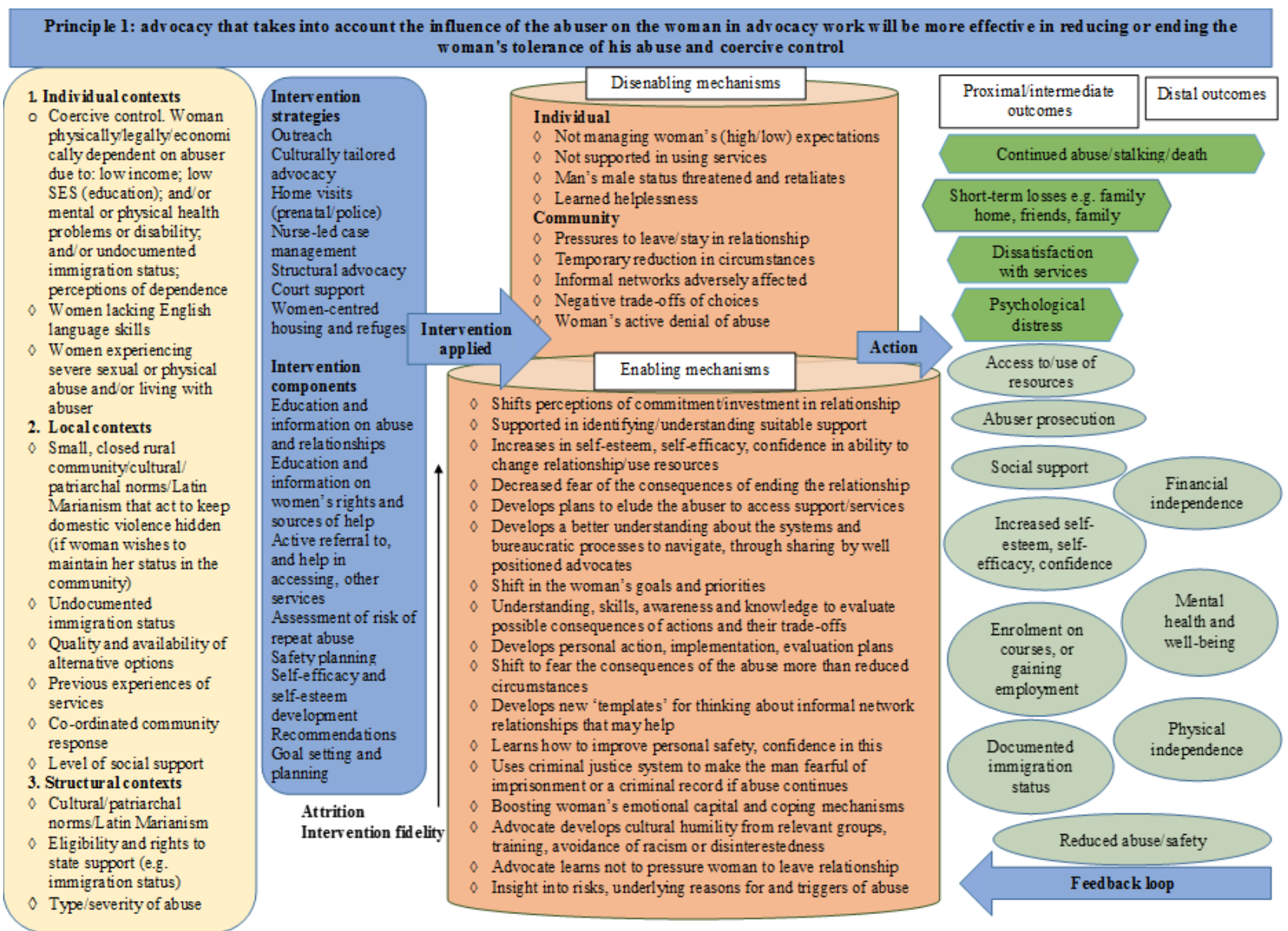

Figure 6. 
Figure 6. Essential principle 2; mechanisms for women. Key Dark green hexagons = negative outcomes Light green circles = positive outcomes Abbreviations: BAME: black, Asian and minority ethnic; CJS: criminal justice system

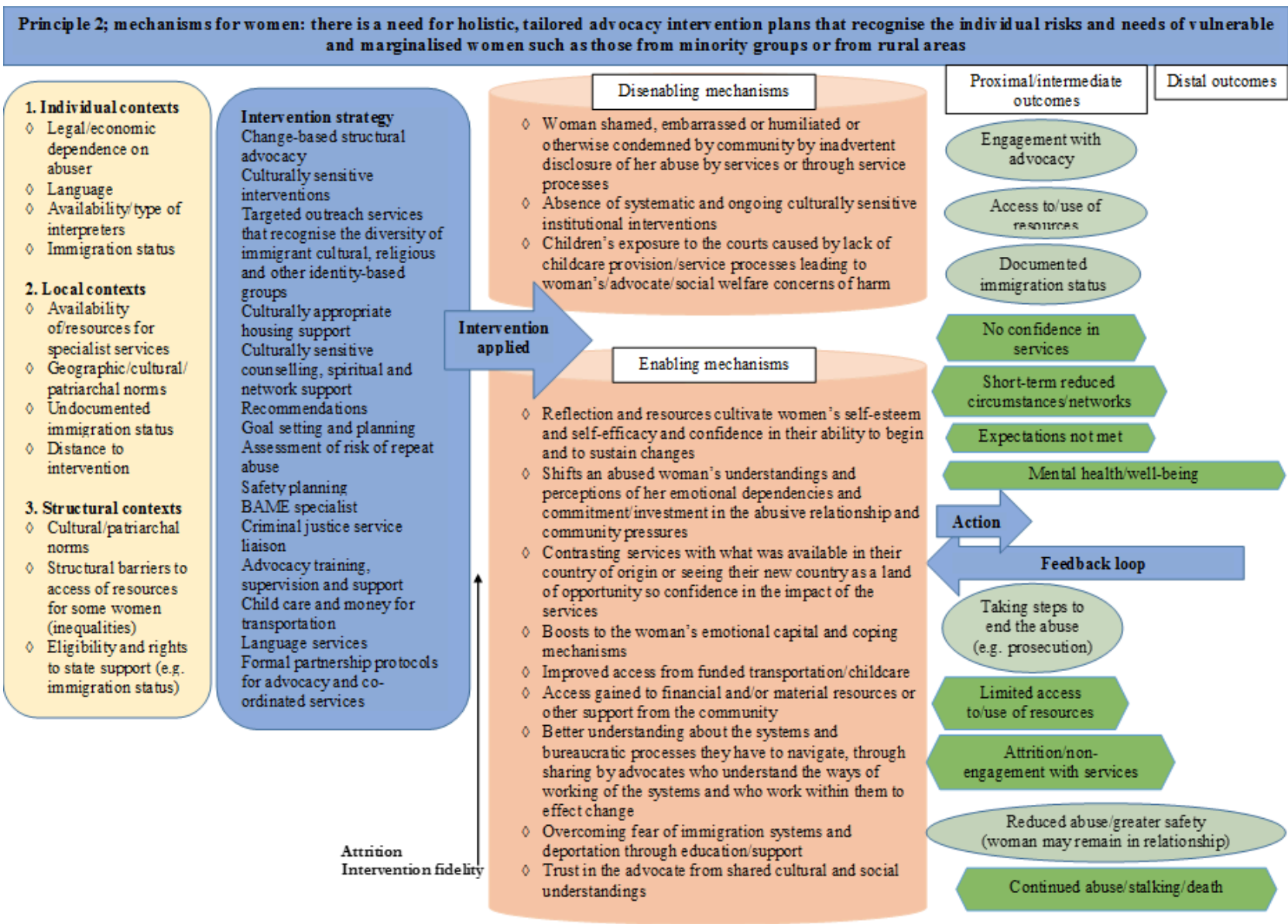

Figure 7. 
Figure 7. Essential principle 2; mechanisms for advocates. Key Dark green hexagons = negative outcomes Light green circles = positive outcomes Abbreviations: BAME: black, Asian and minority ethnic; CJS: criminal justice system

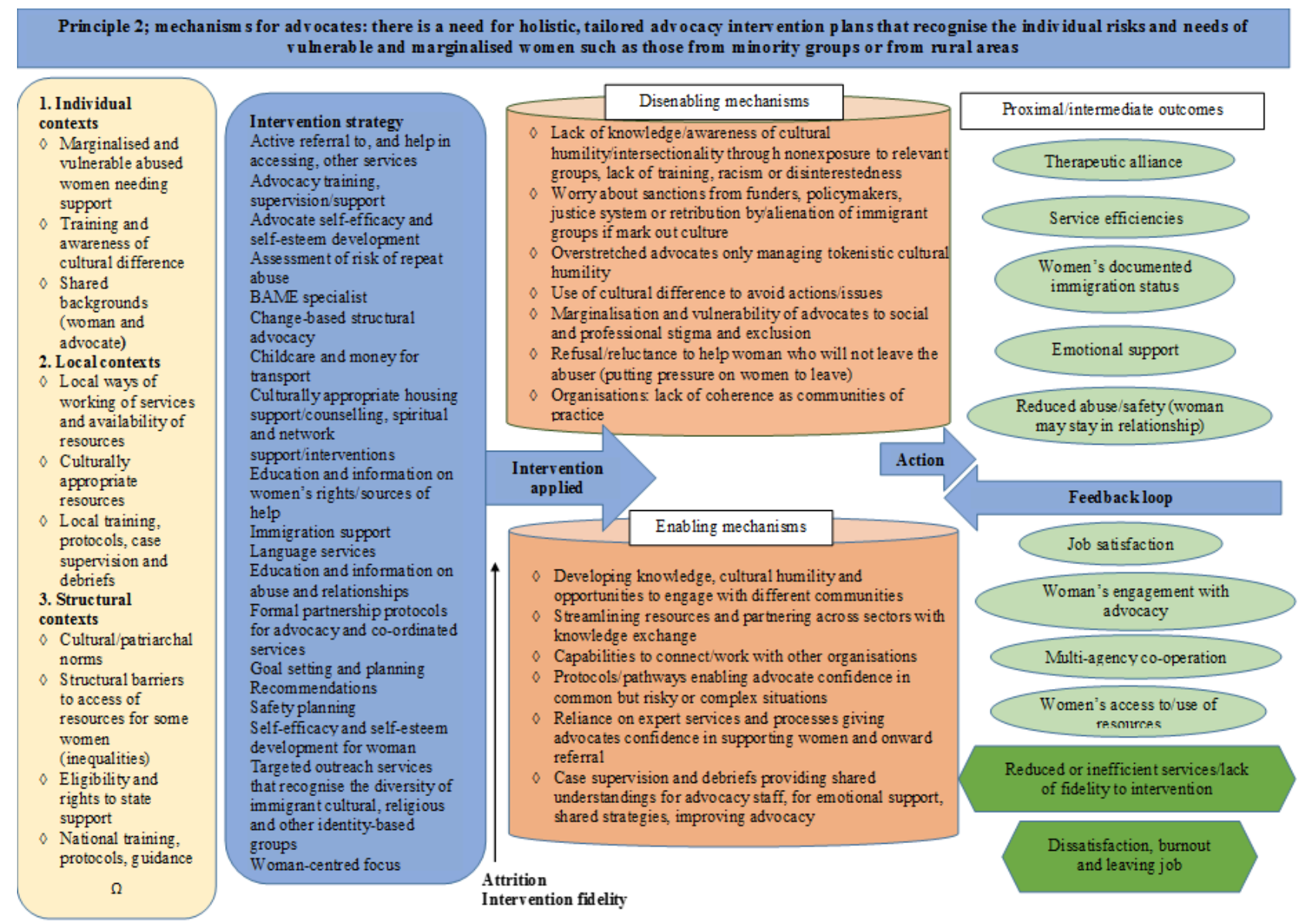

Figure 8. 
Figure 8. Essential principle 3. Key Dark green hexagons = negative outcomes Light green circles $=$ positive outcomes

Principle 3: whether an abused woman was pregnant or had child ren could affect her engagement with advocacy and possibly also lead to some adverse outcomes

\begin{tabular}{|l|} 
1. Individ ual \\
contexts \\
0 Having children \\
0 Being pregnant \\
0 Risk of abuse \\
0 Type of abuse \\
2. Local and \\
structural contexts \\
0 Intervention setting \\
0 Childcare \\
0 Welfare services \\
0 Prenatal/m aternity \\
services \\
0 Mentor $m$ others \\
\hline
\end{tabular}

Attrition

Interv ention fidelity

Assessment of

risk of repeat

abuse

Safety planning

Recomm endati

ons

Empathetic,

non-judgmental

listening

Help with

telephone calls

\section{Disenabling mechanism}

- Lack of childcare at agencies providing challenges for the w om an and risks to the children and her self

Unwilling to acknowledge risks of abuse due to coercive control fear of man/leamed helplessness/fear of social welf are removing children from her care/perceived risk of harm to them

- Feelings of guilt or low mood about previous ex posure of any children, undermining intervention attem pts (e.g dropout)

0 Services wanting to spend time on the safety and wellbeing of the children $m$ ay appear to ex clude the wom an or make her fearful of social welf are services separating her from her children

- Factors associated with having a child (poor sleep, jealousy, for ex ample) that led him to reassert his control over the wom an

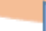

Interv entio

applied

Enabling mecharisms

Women with heightened maternal instincts/em otions developing awareness they are abused, the risks to the children and advocacy packaged in with prenatal care so the abuser will not be aware of it and the woman needs no special arrangem ents

- Weighing the risk of 'doing nothing' against the potential harm, abduction, or removal of their children if they take action

- Professionalism of / shared understandings with midwives/mentor mothers engendering trust and confidence

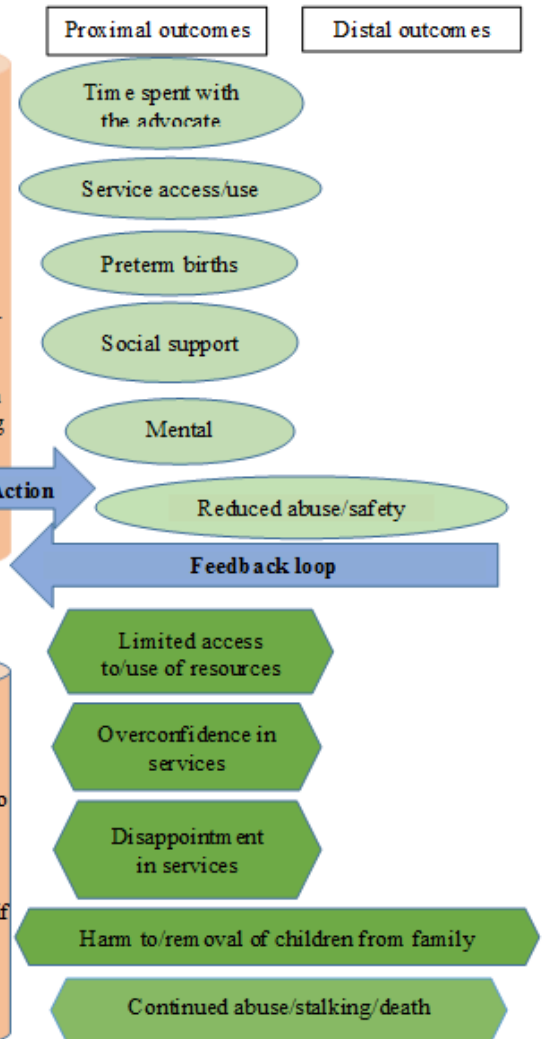

Figure 9. 
Figure 9. Essential principle 4. Key Dark green hexagons = negative outcomes Light green circles = positive outcomes Abbreviations: BAME: black, Asian and minority ethnic

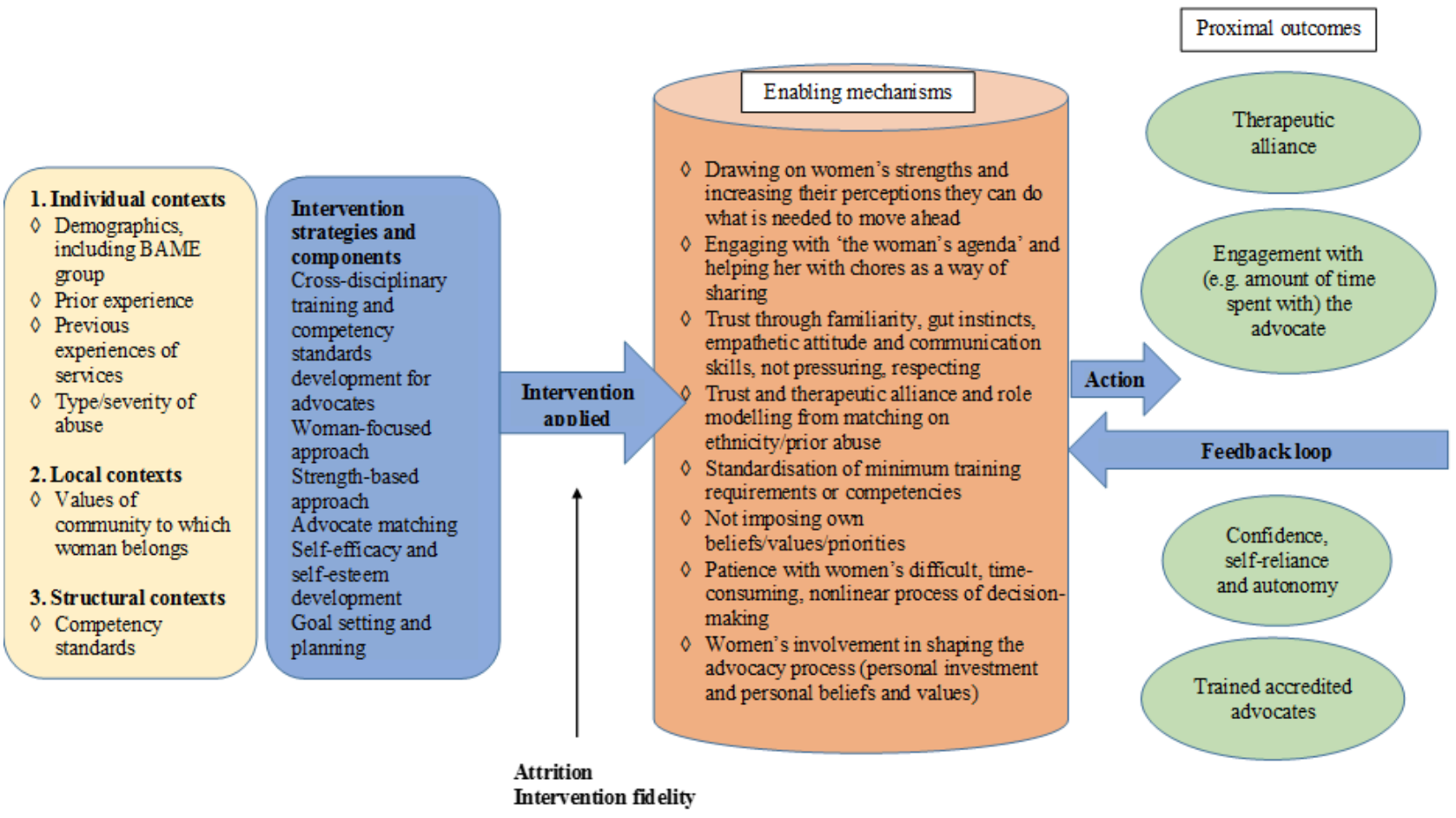

Figure 10. 
Figure 10. Essential principle 5. Key Dark green hexagons = negative outcomes Light green circles $=$ positive outcomes

1. Individual contexts

$\checkmark$ Competencies

$\checkmark$ Training

Time

- Stresful or

uncertain situations

2. Local contexts

0 Intervention setting

- Patriarchal norm s

$\checkmark$ Resource,

organisational,

programme and peer

support constraints

3. Structural contexts

- Resource constraints and austerity

measures

- Policy

Organisational

collaboration
Interv ention

strategy

Brief intervention

Comm unity

outreach

Saf ety planning

and referral

Follow-up and

longer-term

advocacy

Crisis

intervention

Providing

inform ation and

options

Survivor-focused

perspective

Staff support and

training

Ways to $m$ anage

emotions

Inter- and intra-

organisational

working and

knowledge

ex change

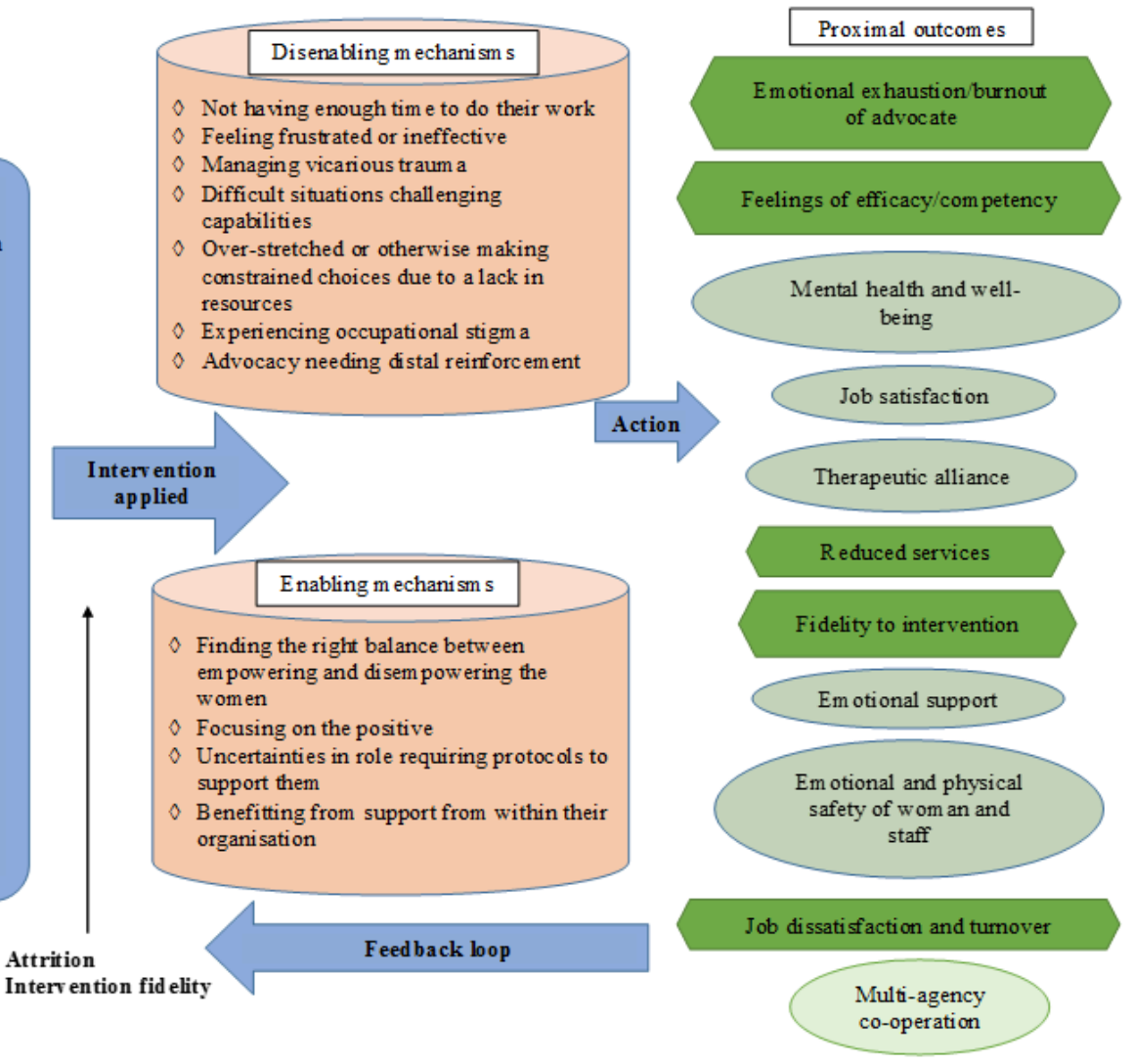

Figure 11. 
Figure 11. Essential principle 6. Key Dark green hexagons = negative outcomes Light green circles $=$ positive outcomes

Principle 6: the typ e of setting affects the theoretical und erpinnings and the type and focus of the adv ocacy being offered and the women's responses

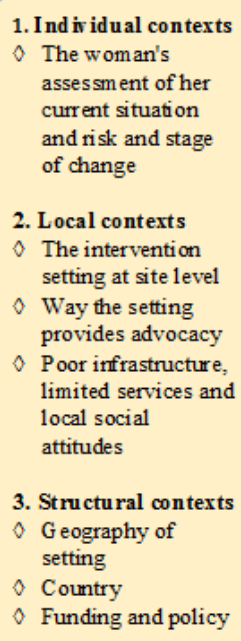

1. Ind in idual contexts

The woman's

assessment of her

current situation

and risk and stage

of change

2. Local contexts

- The intervention

setting at site level

$\checkmark$ Way the setting

provides advocacy

$\checkmark$ Poor infrastructure,

limited services and

local social

attitudes

3. Structural contexts

$\checkmark$ G eography of

setting

$\checkmark$ Country

$\checkmark$ Funding and policy

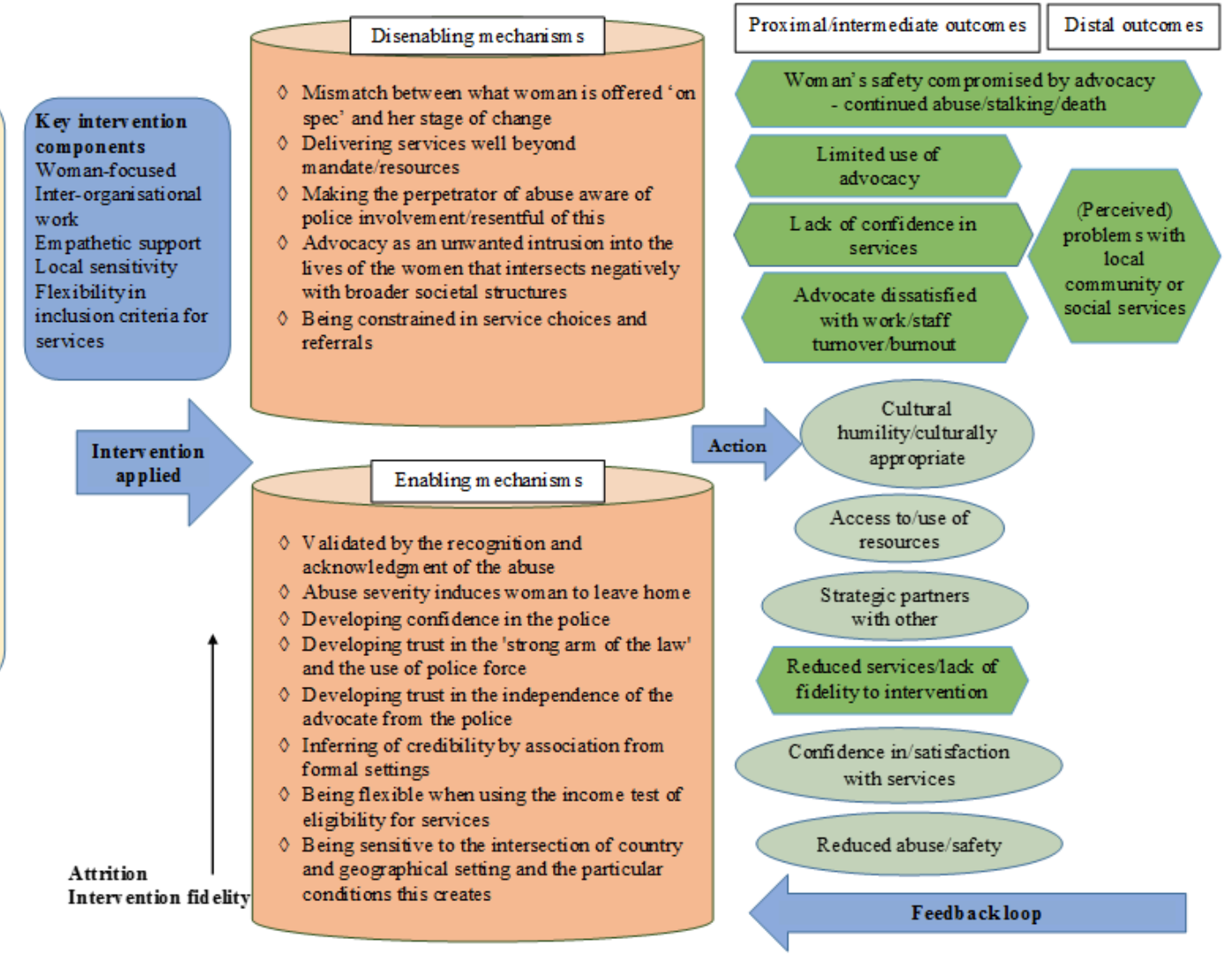

\section{Appendix 4. Logic model of advocacy}

See Figure 12 for tentative logic model of advocacy interventions for abused women. 
Figure 12. Tentative logic model for advocacy interventions Abbreviations: EP: essential principle; IPV: intimate partner violence

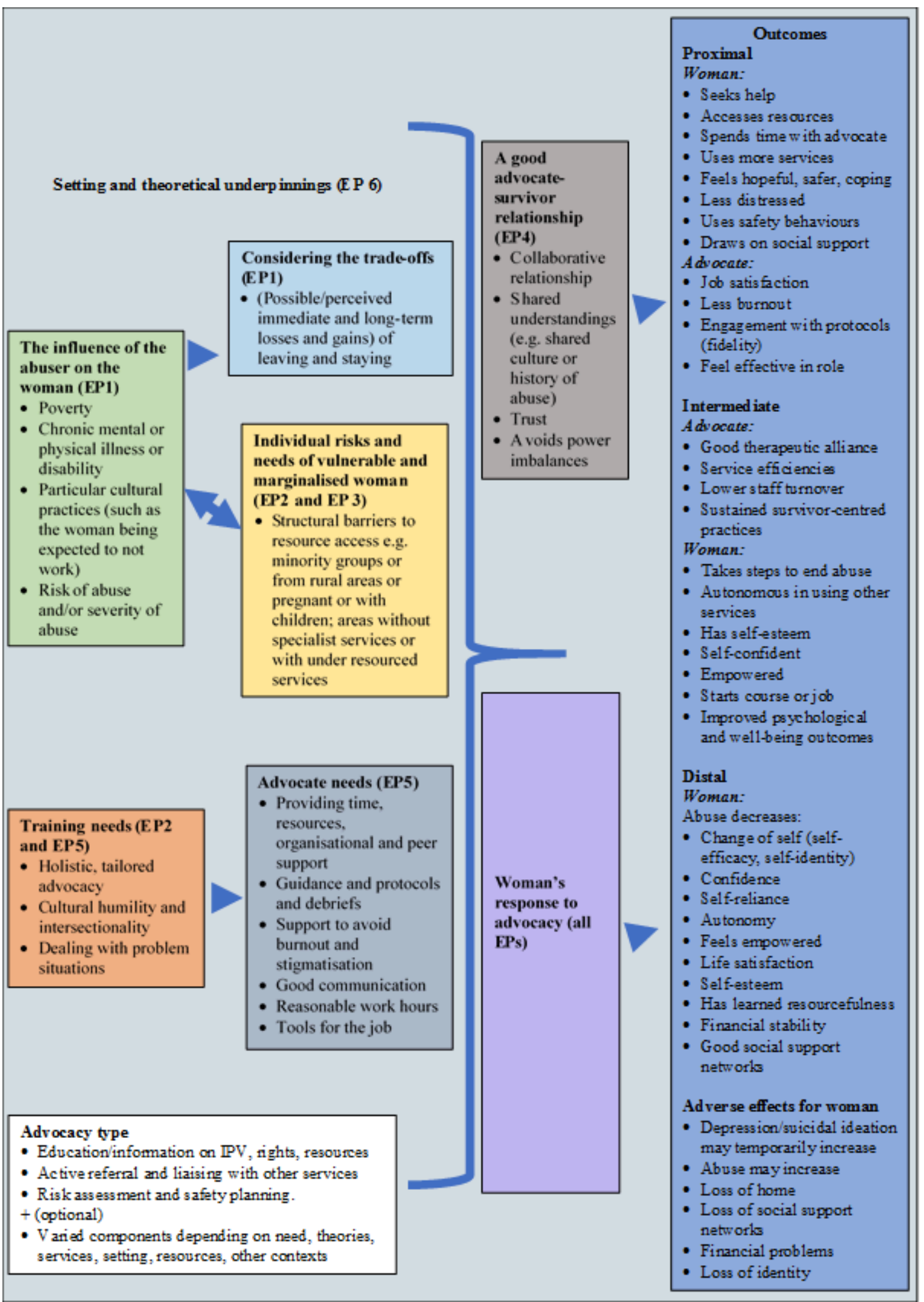




\section{Appendix 5. Overall summary model of advocacy}

Figure 13.

Figure 13. Overall summary model. Abbreviations: IPV: intimate partner violence

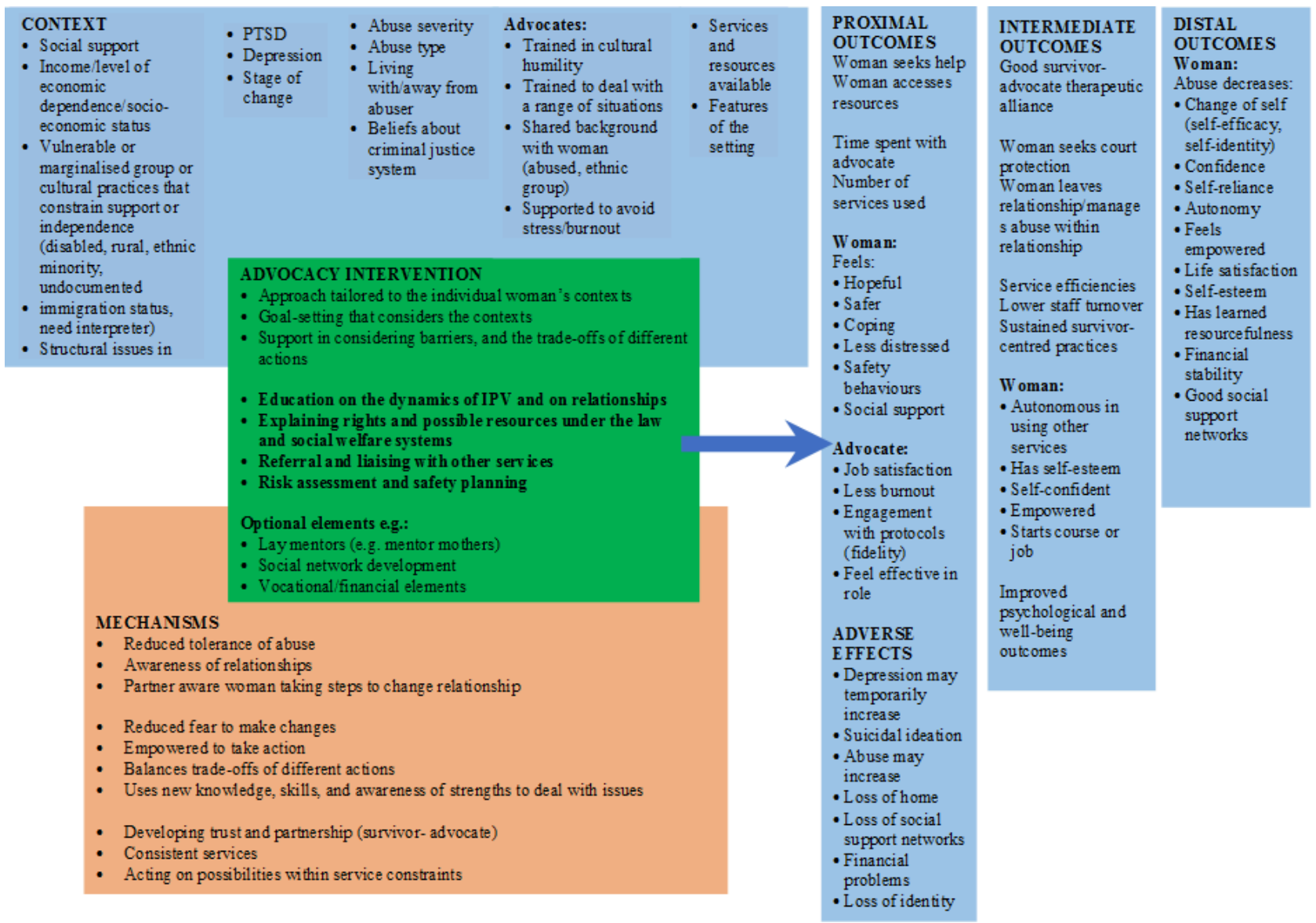

\section{CONTRIBUTIONS OFAUTHORS}

Carol Rivas (CR) had the idea for the study and wrote the first draft of the protocol and the first draft of the review. CR was the lead reviewer and is the guarantor of the review.

Carol Vigurs (CV) contributed substantially to further drafts of the protocol, including tables and assessments of risk of bias, and was the second review author.

Lucia Yeo (LY) undertook data extractions and some of the analyses.

Jacqui Cameron (JC) acted as a third reviewer, undertaking extractions, and constructing tables and conducting 'Risk of bias' analyses, and developing interpretations and contributing to the text of the final review.

\section{DECLARATIONS OF INTEREST}

This review was partly funded by the NIHR Cochrane Incentive Scheme 2017 (project reference 17/62/37). The views and opinions are those of the author and do not necessarily reflect those of the NIHR, NHS, or the Department of Health (England).

Carol Rivas (CR) worked at Queen Mary University of London on the Ramsay 2009 review of advocacy interventions; this institution received payment from Socialforsksnings Institut (SFI) Nordic Campbell Centre Denmark, for submitting an edited version of the 2009 review to the Campbell Collaboration. CR is a co-applicant on a small study funded by a UCL Knowledge Exchange grant intended to improve the use of evidence on domestic violence, with a focus on the College of Policing Crime Reduction Toolkit (whatworks.college.police.uk/toolkit/ Pages/Toolkit.aspx). CR is a member of a team currently looking at core outcome sets and with two workstreams, in the Children and Families Policy Research Unit (CPRU) (www.ucl.ac.uk/children-policy-research/), and Violence, Abuse and Mental Health (VAMH) Network (www.vamhn.co.uk/), which is funded by UK Research and Innovation (UKRI; formerly Research Councils UK (RCUK)), CR is the main supervisor of an ESRC-funded PhD student exploring court-based decision making concerning recidivism and imprisoned perpetrators of abuse.

A realist review of which advocacy interventions work for which abused women under what circumstances (Review) 
Carol Vigurs has been funded by the Educational and Scientific Research Council (ESRC) for reviews of domestic violence perpetrator programme and the UK College of Policing for a first responses study.

Lucia Yeo - none known.

Jacqui Cameron is funded by The Centre of Research Excellence to promote Safer Families (Safer Families Centre) (www.saferfamilies.org.au/). It is a five-year programme (2017 to 2022), funded by the National Health and Medical Research Council (NHMRC) in Australia. Jacqui Cameron is undertaking a PhD on the knowledge translation of domestic violence research, supported by an Australian Government Research Training Program Scholarship.

\section{SOURCES OF SUPPORT}

\section{Internal sources}

- None, Other.

- Carol Rivas, UK. University College London, salary

- Carol Vigurs, UK. University College London, salary

\section{External sources}

- None, Other.

- Natioanl Institute for Health Research (NIHR), UK.

This work was funded, in part, by the NIHR Cochrane Incentive Scheme 2017 (project reference 17/62/37). The views expressed are those of the authors and not necessarily those of the NIHR or the Department of Health and Social Care.

\section{DIFFERENCES BETWEEN PROTOCOLANDREVIEW}

We have not included EMMIE tables in our quality assessment, though we used EMMIE to structure our data extractions.

\section{IN DEX TERMS}

\section{Medical Subject Headings (MeSH)}

${ }^{*}$ Crime Victims [economics] [education] [psychology]; *Patient Advocacy [education]; Health Services Needs and Demand; Qualitative Research; Randomized Controlled Trials as Topic; Safety; Spouse Abuse [economics] [*prevention \& control] [psychology]; Surveys and Questionnaires

\section{MeSH check words}

Adolescent; Adult; Female; Humans 